\title{
Assessment and Exploitation of the Inherent Value of Waste Electrical and Electronic Equipment (WEEE) for Circular Economy
}

\author{
Charles, Rhys G.
}

How to cite:

Charles, Rhys G. (2018) Assessment and Exploitation of the Inherent Value of Waste Electrical and Electronic Equipment (WEEE) for Circular Economy. Doctoral thesis, Swansea University.

http://cronfa.swan.ac.uk/Record/cronfa39601

Use policy:

This item is brought to you by Swansea University. Any person downloading material is agreeing to abide by the terms of the repository licence: copies of full text items may be used or reproduced in any format or medium, without prior permission for personal research or study, educational or non-commercial purposes only. The copyright for any work remains with the original author unless otherwise specified. The full-text must not be sold in any format or medium without the formal permission of the copyright holder. Permission for multiple reproductions should be obtained from the original author.

Authors are personally responsible for adhering to copyright and publisher restrictions when uploading content to the repository.

Please link to the metadata record in the Swansea University repository, Cronfa (link given in the citation reference above.)

http://www.swansea.ac.uk/library/researchsupport/ris-support/ 


\title{
Assessment and Exploitation of the Inherent Value of Waste Electrical and Electronic Equipment (WEEE) for Circular Economy
}

\author{
Rhys G. Charles
}

Submitted to Swansea University in fulfilment of the requirements for the Degree of Doctor of Engineering

Swansea University 


\begin{abstract}
Waste electrical and electronic equipment (WEEE) represents a global environmental and resource-efficiency crisis. However, WEEE is a valuable urban mine of economically, strategically and environmentally important materials e.g. precious metals (PMs) and critical raw materials (CRMs). Economic value derived from WEEE can drive solutions to the 'WEEE problem' which are conducive to circular economy, enhance global resource-efficiency, and generate environmental and social benefits. This thesis examines the value of WEEE, and methods for its exploitation to the benefit of global sustainability. The 'WEEE problem' is examined in the context of global sustainability, considering environmental \& resourceefficiency implications and linear resources use by the electrical \& electronic equipment (EEE) industry. Solutions are considered which exploit WEEE as an 'urban mine' and embrace circular economy.

Within this context, recycling potential of future WEEE is evaluated through projections of PM \& Cu content of PCBs, based on temporal trends in historic RAM modules. CRMs are then identified in WEEE and methods of enhancing their recovery through intervention in preprocessing stages of recycling are evaluated. An industrial symbiosis process which recovers Pt from waste thermocouples for use in solar cells is presented as an example of the greater value generation potential offered by circular economy and the potential of such processes to overcome barriers to CRM recovery. Challenges and opportunities in lifecycle optimisation of printable photovoltaics for circular economy is considered as a means of enhancing the industrial ecology of this industry to avoid WEEE generation, reduce primary materials demand and enhance the value derived from these technologies at all stages of their lifecycles. Appropriate battery selection for solar off-grid systems in South Africa is then considered, demonstrating that greater value can be derived from EEE for local economies if compatibility of technologies with local skills and infrastructure for in-use and EoL management.
\end{abstract}




\section{Declaration}

This work has not previously been accepted in substance for any degree and is not being concurrently submitted in candidature for any degree.

Signed ..................................................................... (candidate)

Date

This thesis is the result of my own investigations, except where otherwise stated. Where correction services have been used, the extent and nature of the correction is clearly marked in a footnote(s).

Other sources are acknowledged by footnotes giving explicit references. A bibliography is appended.

Signed

(candidate)

Date

I hereby give consent for my thesis, if accepted, to be available for photocopying and for inter-library loan, and for the title and summary to be made available to outside organisations.

Signed (candidate)

Date 
This thesis is dedicated in loving memory to Mrs Pauline Elizabeth Holley (1930-2017) 


\section{Contents}

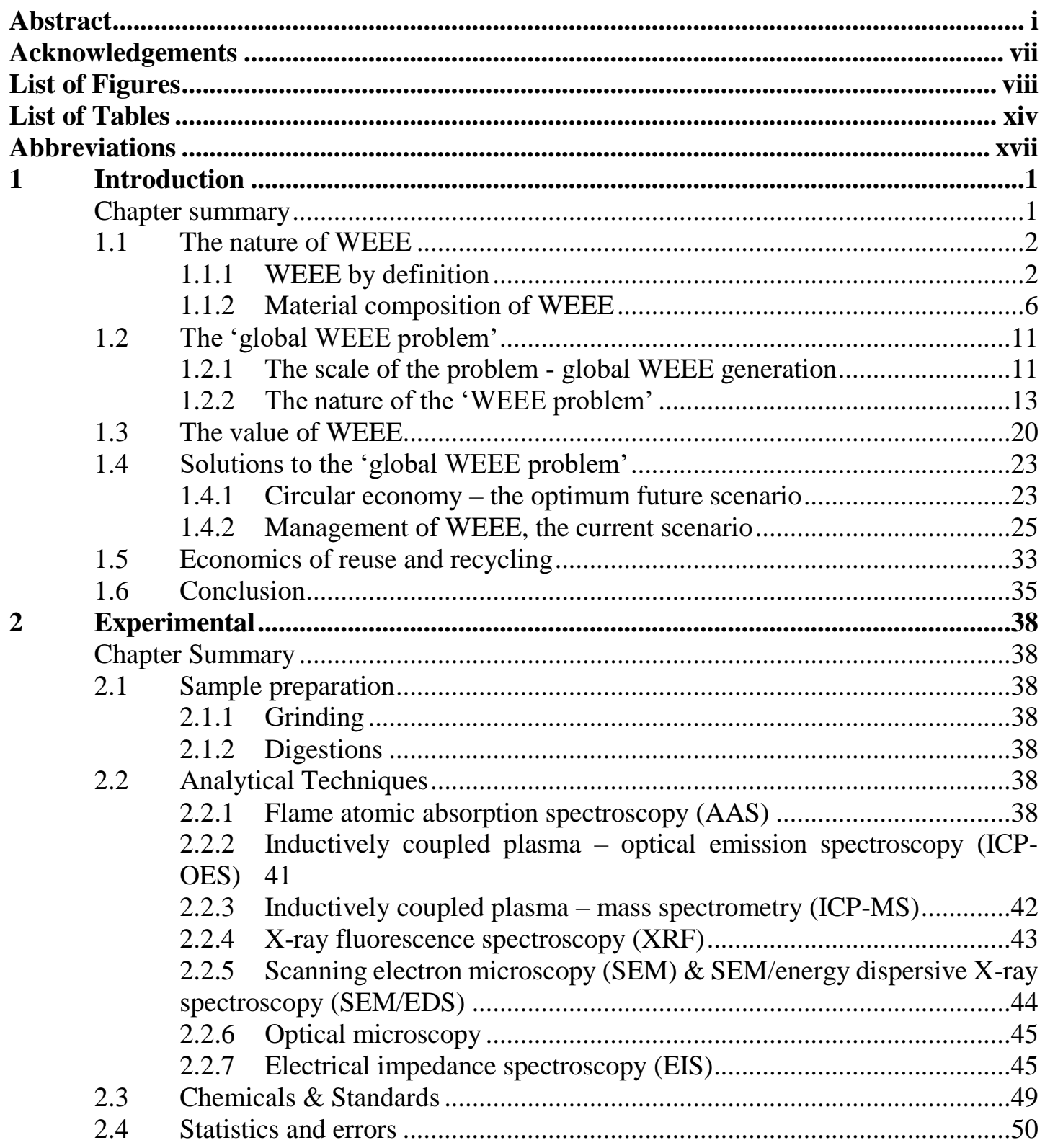

3 Temporal Trends in Precious Metal Content of WEEE - Implications for Future Recycling Potential ${ }^{144}$.....................................................................................................51

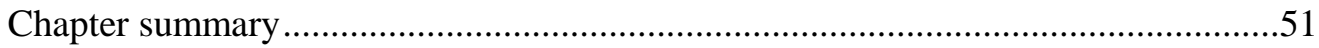

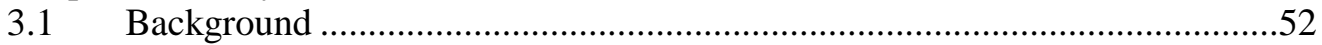

3.1.1 Criticality of precious metals and copper .......................................52

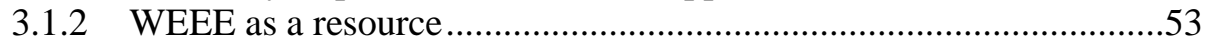

3.1.3 The recycling process.....................................................................54

3.1.4 Market values of metals .................................................................55

3.1.5 Waste management strategies ........................................................56

3.1.6 Outlook for waste management and viability of WEEE recycling ....56

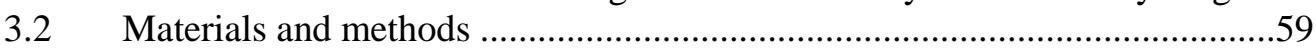

3.2.1 Evaluation of historic trends in composition and value of DRAM

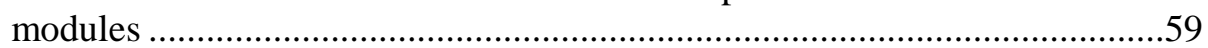

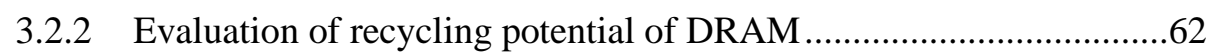

3.2.3 Projections of future recycling potential..........................................62

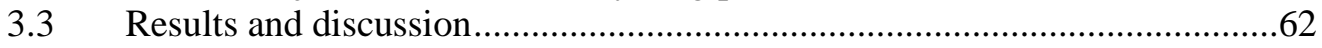


3.3.1 Historic trends in composition and value of DRAM modules ...........62

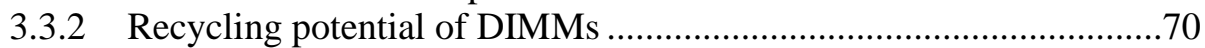

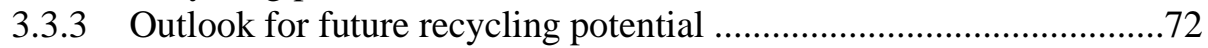

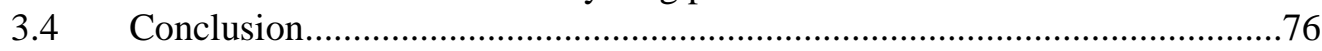

4 Recovery of Critical Raw Materials from WEEE - Wales........................................79

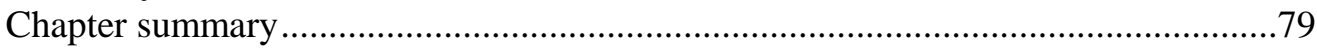

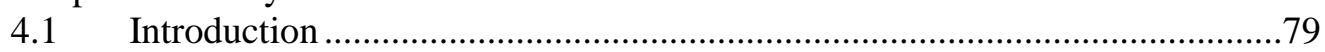

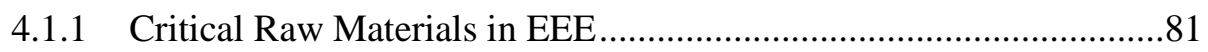

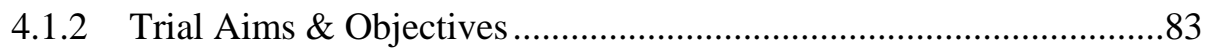

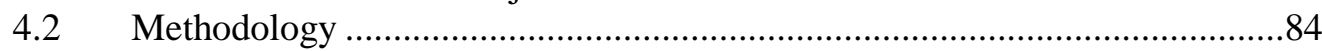

4.2.1 Selection of Samples for analysis .......................................................84

4.2.2 Evaluation of procedures for qualitative \& quantitative determination

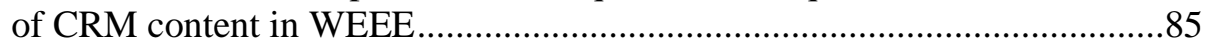

4.2.3 Identification of Critical Materials in Samples ..................................87

4.2.4 Evaluation of Processes for Implementation......................................87

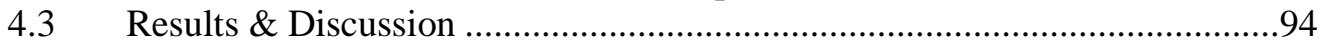

4.3.1 Selection of Samples for analysis ...................................................94

4.3.2 Identification of Critical Materials in Samples .................................95

4.3.3 Evaluation of processes for isolation of CRMs from WEEE............123

4.3.4 Cost Benefit Analysis ....................................................................149

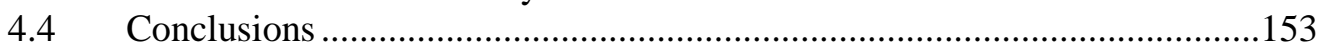

5 Platinized counter-electrodes for dye-sensitised solar cells from waste thermocouples: a case study for resource-efficiency, industrial symbiosis and circular

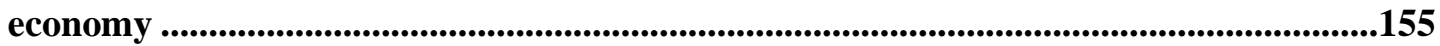

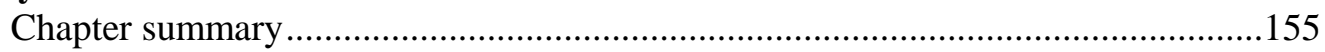

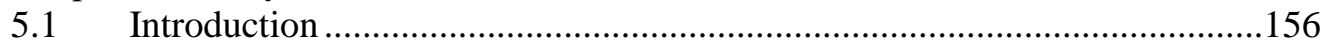

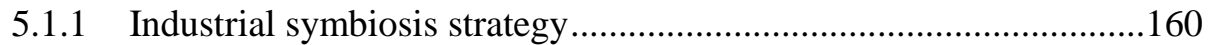

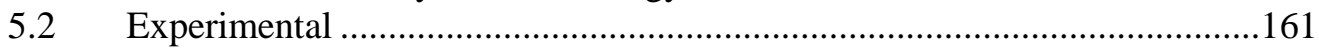

5.2.1 Chloroplatinic acid synthesis .....................................................162

5.2.2 Fabrication and characterization of platinized counter-electrodes...163

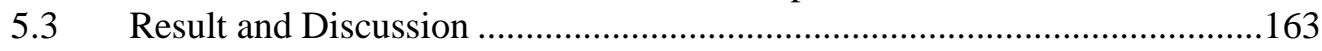

5.3.1 Isolation of filaments from waste thermocouples ...........................163

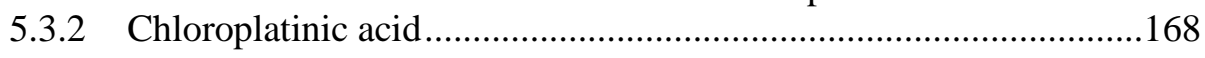

5.3.3 Assessment of suitability for DSSC electrode manufacture ..............168

5.3.4 Supply and environmental impact..................................................169

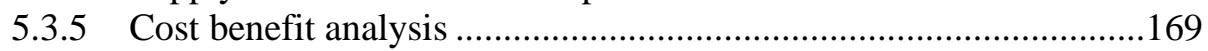

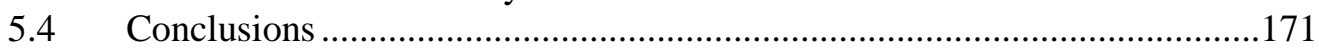

6 Third Generation Photovoltaics - Early Intervention for Circular Economy and

a Sustainable Future .......................................................................................................................173

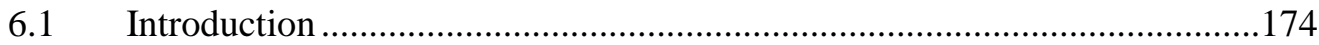

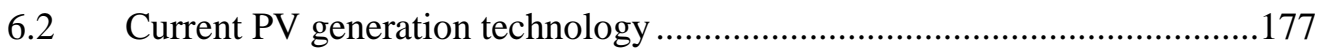

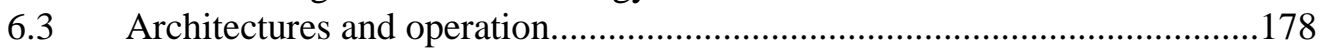

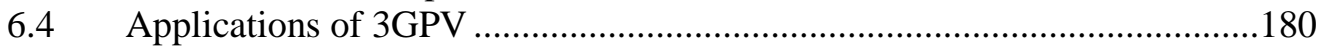

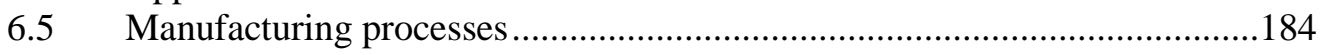

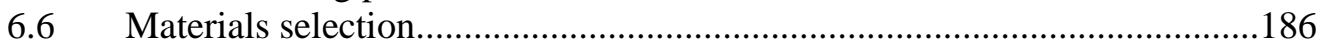

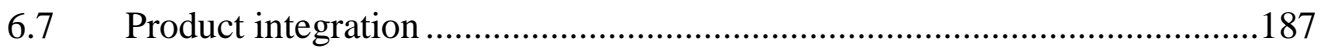

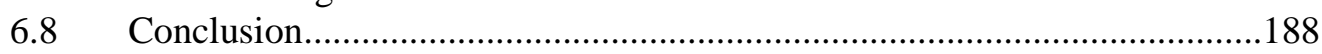

$7 \quad$ Sustainable Solar Energy Storage for Rural Africa .................................................190

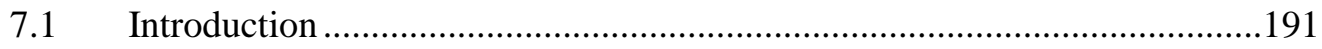

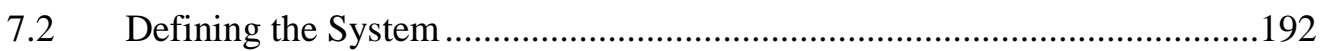

7.3 Battery Choices \& Cost........................................................................195

7.4 Carbon Footprint and Lifecycle Impact Consideration ................................196

7.5 Resource-efficiency and Circular Economy................................................198

7.5.1 Critical Materials ........................................................................198

7.5.2 End-of-life prospects \& compatibility with circular economy..........198 


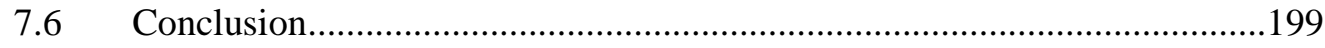

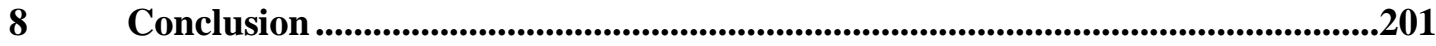

Appendix A - Evaluation of Fischerscope XDAL.......................................................................210

Glossary ........................................................................................................................................217

References.....................................................................................................................................218 


\section{Acknowledgements}

First and foremost, I wish to express my gratitude to my great friend and mentor, Dr Peter Douglas for his supervision of this project. Working with Peter has been a pleasure and an honour. His advice and guidance in matters of science and life in general have been invaluable. Thank you, Peter. Thanks also to Helen Balshaw, working with you has been a pleasure, as have our travels. Special thanks to Dr Matthew Davies, Dr David Penney, Gareth Liversage, Ian Matthews, Mark Dowling, Prof David Worsley, Dr Ian Mabbett, Dr Trystan Watson and Prof Margaret Bates for exceptional support throughout this project.

I wish to acknowledge and thank the following for their contribution to work presented in specific chapters:

Chapter 3: Dr Ingrid Hallin, Plymouth University for confidence intervals of linear regression analyses of metal content in future RAM modules.

Chapter 4: Mark Dowling and Giraffe Innovations for collaboration on 'Recovery of Critical Raw Materials - Wales'; WRAP for funding this project. I also wish to acknowledge: G\&P, Clarity Environmental, Budget Pack for expert advice on battery recycling; Nick Trillwood of Delta Products for expert advice on industrial recycling of $\mathrm{Ta}$ and $\mathrm{Nb}$ surface mount components; BASF precious metals for precious metal assays, and advice on recycling potential of assayed fractions; CEM for the assistance offered for the microwave ashing of PCBs; Fischer Instrumentation for loan of the XDAL instrument; and ChemTest Ltd for ICPMS analyses of Yttrium and Neodymium in samples.

Chapter 5: Dr Dyfyr Davies and Mr Richard Vaughan of Weartech International Ltd for assistance in gathering waste thermocouple samples and provision of information to support the work; Dr Jamie Douglas for SEM analysis of platinized counter-electrodes; Dr Simon Curling, Bangor University Biocomposites Centre for conducting ICP-OES analyses of chloroplatinic acid samples; Dr Jenny Baker for support on interpretation of EIS results; and Swansea University and the EPSRC Impact Acceleration Account for funding.

Chapter 6: All participants of the '3rd Generation Photovoltaics: Early Intervention for Circular Economy and a Sustainable Future' sandpit, hosted at the SPECIFIC-IKC, Baglan, South Wales on 7th-8th of April 2016; Dr Matthew Davies for co-chairing the event; and Swansea University and the EPSRC Impact Acceleration Account for funding.

Chapter 7: Thanks to participants for their contributions to the 'Eco-Design of Printable Photovoltaics - the Building of an African Infrastructure' workshop, Durban, South Africa, 10-1 $1^{\text {th }}$ January 2017; Special thanks to Prof Bice Martincigh, Prof Vincent Nyamori and Edwin 'Tonde' Mombeshora for support in organising the workshop and your hospitality; Swansea University and EPSRC for internal GCRF pump priming funding for the workshop; Sêr Solar and Prof David Worsley for funding out attendance

Finally, I would like to thank my beautiful wife Rhiannon, and my wonderful children Alex, James, Ingrid and Magnus for all your love and support, and for putting up with me during writing up. I love you all so much, and can't wait to spend more time with you. 


\section{List of Figures}

Figure 1.1 Flow of EEE into WEEE (adapted from StEP, 2014) ${ }^{10}$

Figure 1.2 Breakdown by category of WEEE collected in the EU in 2014 (data from Statistical Office of the EC, 2016) ${ }^{11}$

Figure 1.3 Masses of WEEE collected in EU member states in 2014 with compositional breakdown by category (data from Statistical Office of the EC, 2016) ${ }^{11}$

Figure 1.4 General material compositions of WEEE by mass given by EMPA, Switzerland (top); and the European Topic Centre on Resource and waste management (bottom) ${ }^{3}$.

Figure 1.5 Material composition by mass of four e-waste categories: a) large household appliances; b) small household appliances; c) ICT and consumer electronics; d) lamps ${ }^{17} \ldots . . .7$

Figure 1.6 Mass proportions of polymers found in WEEE plastics (ABS: acrylonitrilebutadiene-styrene; HIPS: high impact polystyrene; PA: polyamide; PBT: poly(butyleneterephthalate); PC: polycarbonate; PP: polypropylene; PPE: polyphenylene ether; PS: polystyrene; PVC: poly(vinyl chloride) ${ }^{15}$.

Figure 1.7 Worldwide Google searches for the term 'iPhone slow' correlated with release dates of new iPhone models (100=peak search volume) (Figure used with permission from statista.com $)^{41}$

Figure 1.8 Material flows in the linear 'take-make-use-dispose' economy 15

Figure 1.9 Human Development Index vs Ecological Footprint of the World's Nations in 2007. Taken from UNEP, 2012. ${ }^{50}$

Figure 1.10 Correlation between resource use and GDP/captia of countries(image courtesy of Accenture, permission pending ${ }^{49}$.

Figure 1.11 Schematic criticality plot for evaluation of materials criticality (adapted with permission from Frenzel et al., 2017) $)^{54}$

Figure 1.12 Elemental resources used in smartphones and times until their depletion based upon current rates of extraction from known reserves (adapted from Clark et al., with permission $)^{55}$ 18

Figure 1.13 The metal wheel-a schematic illustration of the geological relationships between different industrial metals and their co- and by-products; criticality is assigned according to the EU20. (C IOP Publishing. Reproduced with permission. All rights reserved) ${ }^{54}$

Figure 1.14. Estimated value of major metals contained in WEEE in Australia in 2014: (A) total WEEE; (B) waste PCBs (reused with permission from Elsevier) ${ }^{66}$

Figure 1.15 Revenue breakdown by material from WEEE adapted from (Sodhi and Reimer, 2001.30

Figure 1.16 Material flows within the circular economy (adapted from Ellen MacArthur Foundation $)^{47}$

Figure 1.17 The waste hierarchy with refurbishment, repair, upgrade, remanufacturing and repurposing operations incorporated.

Figure 1.18 The recycling process chain with special focus on recovery of precious metals and copper (adapted from Chancerel et al.) ${ }^{106}$

Figure 1.19 Electrical and electronic equipment (EEE) put on the market and waste EEE collected and treated in the EU from 2008-to 2014) ${ }^{110}$.... 
Figure 1.20 Total collection rate for WEEE in 2014 as a percentage of the average weight of EEE put on the market in the three preceding years (2011-13), (2014 collection data estimated for Cyprus) $)^{110}$ 30

Figure 1.21 The 'Grade-Recovery Function' which results in losses of target precious metals and other trace elements as larger bulk fractions are removed from shredded WEEE in automated pre-processing, (02006, IEEE (re-printed, with permission, from Hagelüken, $2006)^{18}$.......

Figure 1.22 Flowsheet of Umicore's integrated metals smelter/refinery. (C2006, IEEE (reprinted, with permission, from Hagelüken,2006) ${ }^{18}$

Figure 1.23 Qualitative cost function in WEEE recycling interface optimisation, IEEE (Reprinted, with permission, from Hagelüken, 2006) ${ }^{18}$.

Figure 2.1 Electronic transition from ground state $\left(E_{0}\right)$ to an excited state $\left(E_{1}\right)$ resulting from absorption of a photon of energy $E$ and wavelength $(\lambda)$

Figure 2.2 Electronic decay processes resulting in emission lines

Figure 2.3 The processes involved in X-ray fluorescence(adapted from Fifield). ${ }^{135}$

Figure 2.4 Sinusoidal wave forms of applied AC potential (E) and current response (I) in phase with one another (left); and out of phase by the phase shift angle, $\theta$ (right).

Figure 2.5 Example Nyquist plot, test frequency, $\omega$ is varied from high to low frequency, each frequency leads to an extra point on the curve, where the curve crosses the $\mathrm{x}$-axis the impedance is resistance only, vector indicates the magnitude of impedance, $|Z|$. The simple circuit is composed of an ideal resistor in parallel with an ideal capacitor. 47

Figure 2.6 Equivalent circuit fit for 'dummy cell' EIS data using a Randles circuit with short Warburg element (Ws1), showing Rct and Cdl 48

Figure 3.1 Annual gold production from scrap \& average gold price. Data from O'Connell et al. ${ }^{150,161}$

Figure 3.2 Anatomy of PC DRAM module and locations of precious metals and copper. ${ }^{144}$

Figure 3.3. Timeline of DRAM technologies in PCs POM. M is the Module Memory Capacity (MB); the vertical blocks ( $\square$ ) show the year of introduction of RAM type (data from Mueller, 2011; Ögren et al.). ${ }^{177,} 178$ The horizontal lines show the period when PCs POM were available with memory capacity M (data for from Polsson, 2013). ${ }^{176}$ 63

Figure 3.4 Gold content of PC DRAM modules (dashed line represents best estimate of time period in which module was on the market (Figure 3.3); modules $\mathbf{2}$ and $\mathbf{3}$, have tin rather than gold edge contacts

Figure 3.5 Image used for comparison of contact surface areas of DDR (bottom) and DDR2 (top) DRAM DIMMs.

Figure 3.6 Variation in palladium content of PC DRAM modules over time. The dashed line represents best estimate of period in which module was on the market (Figure 3.3); the solid line shows the temporal trend in Pd content.

Figure 3.7 Variation in silver content of PC DRAM samples over time (dashed line represents best estimate of time period in which module was on the market (Figure 3.3).

Figure 3.8 Variation in Copper content of PC DIMM samples over time (dashed lines, represents best estimate of period in which module was on the market (Figure 3.4); solid line shows the temporal trend in copper content in DIMMs). 68

Figure 3.9 Inherent value of DRAM samples, a) per module and b) per tonne, showing value contributions of PMs and copper. 
Figure 3.10 Confidence intervals for projections of future quantities of gold in future waste

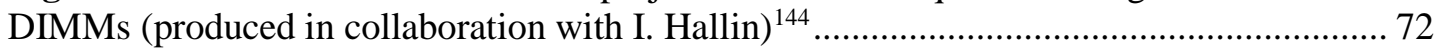

Figure 3.11 Confidence intervals for projected quantities of silver in future waste DIMMs (produced in collaboration with I. Hallin) ${ }^{144}$ 73

Figure 3.12 Confidence intervals for projected quantities of copper in future waste DIMMs (produced in collaboration with I. Hallin) ${ }^{144}$

Figure 3.13 Confidence intervals for projected quantities of palladium in future waste DIMMs (produced in collaboration with I. Hallin) ${ }^{144}$.

Figure 4.1 Supply risk versus economic importance of 41 materials evaluated for the EU showing the EU14 CRMs ${ }^{100}$

Figure 4.2 Analysed x-ray emission spectrum of a powder sample and measured relative mass percentages of elements. 86

Figure 4.3 Removing edge contacts from server boards with a Rubi ND-200 wet saw / electric tile ccutter.

Figure 4.4 Sony Walkman Following Liquid Nitrogen Treatment (left), cracked open to reveal internal CRM bearing components (right). .............................................................. 90

Figure 4.5 PCB placed on to the surface of liquid solder in a small solder bath 91

Figure 4.6 Magnetrons (top left) and isolated magnets (top right) and ferrite cores of the filter (bottom right).... 96

Figure 4.7 XRF spectra of magnetron magnets indicating the presence of Co. 96

Figure 4.8 XRF spectra of ferrite cores from the magnetron filters indicating the presence of Co. 96

Figure 4.9 Typical hard disk drive (HDD) 97

Figure 4.10 X-ray emission spectrum of NIB magnetic alloy showing the presence of Nd 98

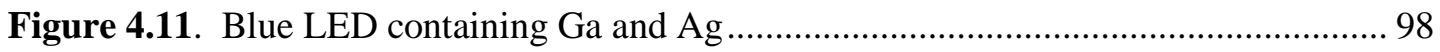

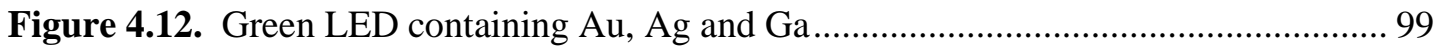

Figure 4.13 Laptop Li-ion battery pack and isolated LIB cylinder cells and PCB. ............. 99

Figure 4.14 Disassembly of LCD Screen and isolation of housing, PCBs and LCD sandwich.

.

Figure 4.15 X-ray emission spectrum of CCFL phosphor containing yttrium. ................. 100

Figure 4.16 AZ91D Mg alloy laptop LCD screen housing .............................................. 101

Figure 4.17 X-ray emission Spectra of AZ91D Mg alloy laptop LCD screen housing showing

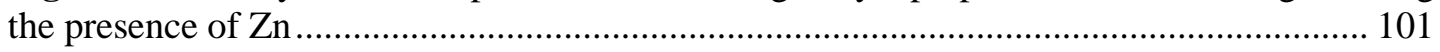

Figure 4.18 Disassembly of Nokia 6230i mobile phone ................................................. 102

Figure 4.19. Connectors on screens of mobile phone and MP3 player............................ 102

Figure 4.20 X-ray emission spectrum of the magnetic alloy in mobile phone speaker magnets.

Figure 4.21 X-ray emission spectrum of gold contacts of mobile phone sample .............. 103

Figure 4.22 X-ray emission spectrum of SD card contacts.............................................. 104

Figure 4.23 XRF spectrum of SD card plastic containing Sb ......................................... 104

Figure 4.24 Disassembly of Sony NWZ-s639F digital media player into its component parts 
Figure 4.25 RSA anti-hacking security dongle (top) with top vies (bottom left) and bottom view (bottom right) of its encapsulated internal components. ........................................... 106

Figure 4.26. Sanyo Cybershot digital camera and its isolated PCB and Li-ion battery ..... 107

Figure 4.27 Example LIBs. From left to right, cylinder cells from laptop battery packs, and packet cells from cameras, MP3 players and mobile phones 107

Figure 4.28 Li-ion cylinder cells from laptop battery packs cut open to reveal internal electrodes (top) and x-ray emission spectra of electrode material (bottom) 108

Figure 4.29 X-ray emission spectrum of electrodes in Nokia 6230i mobile phone Li-ion battery 108

Figure 4.30 Edge contacts on a PC graphics card 109

Figure 4.31 HDMI connectors on a TV PCB (left) and the gold coated contacts within (right) 110

Figure 4.32 HDMI ports on a TV PCB and their X-ray emission spectrum indicating the presence of gold $(9.71 \mathrm{keV})$... 110

Figure 4.33 Plated $\mathrm{Au}$ on the surface of mobile phone $\mathrm{PCB}$ 111

Figure 4.34 X-ray emission spectrum of $\mathrm{Pb}$ free solder containing $\mathrm{Ag}$ on the $\mathrm{PCB}$ of a digital media player (MP3 player)

Figure 4.35 Examples of surface mount chip resistors

Figure 4.36 X-ray emission spectrum of a chip resistor SMD containing $\mathrm{Ru}, \mathrm{Pd}, \mathrm{Ag} \& \mathrm{Sb}$

Figure 4.37 ICs on HDD PCB

Figure 4.38 X-ray emission spectrum of an IC showing the presence of $\mathrm{Au} \& \mathrm{Ag}$. 113

Figure 4.39 Typical capacitors and XRF analysis showing presence of yttrium (Y)

Figure 4.40 X-ray emission spectra of typical blue MLCC indicating the presence of Pd.114

Figure 4.41 MLCC containing $\mathrm{Nb} \& \mathrm{Ag}$ 115

Figure 4.42 Red MLCC containing $\mathrm{Nb} \& \mathrm{Pd}$ 115

Figure 4.43 Typical $\mathrm{x}$-ray emission spectrum of large (top) and small (bottom) MLCCs containing iridium $\mathrm{Nb} \& \mathrm{Ir}$.....

Figure 4.44 X-ray emission spectrum of small outline transistor containing $\mathrm{Au}, \mathrm{Ag}$ and $\mathrm{Sb}$ 116

Figure 4.45 Typical SMD transistor containing $\mathrm{Au}, \mathrm{Ag}$ and $\mathrm{Sb}$ 116

Figure 4.46 X-ray emission spectrum of coloured 3-pin small outline transistors containing $\mathrm{Au}, \mathrm{Ag}$, and $\mathrm{Ga}$, present on server board sample. 117

Figure 4.47 Typical tantalum capacitor SMDs from samples.....

Figure 4.48 X-ray mission spectrum of exposed Ta metal of typical Ta-capacitor SMD revealing the presence of and measured $\mathrm{X}$-ray emission spectrum

Figure 4.49 X-ray emission spectrum of a typical chip array SMD containing Ag and $\mathrm{Ru}$ 118

Figure 4.50 X-ray emission spectrum of brown inductors on mobile phone PCBs containing $\mathrm{Nb}, \mathrm{Pd} \& \mathrm{Ag}$

Figure 4.51 X-ray emission spectrum of grey inductors on mobile phone PCBs containing Ag.. 
Figure 4.52 X-ray emission spectrum of purple inductors on mobile phone PCBs containing $\mathrm{Ag} \& \mathrm{Co}$.

Figure 4.53 X-ray emission spectrum of typical polarized moulded body diode containing $\mathrm{Sb}$

Figure 4.54 Colour coded CRM distribution in TV main PCB ……............................. 122

Figure 4.55 Sanyo domestic microwave magnetron .................................................... 124

Figure 4.56 LCD screen with the CCFL containing portion cut away.............................. 130

Figure 4.57 Liquid nitrogen cooling and smashing of MP3 player................................... 132

Figure 4.58 Cryo-cracking of a Sony Cybershot digital camera to isolate its PCB ............ 134

Figure 4.59 Sony Xacti Full HD 10 MP digital video camera sample post cryo-treatment135

Figure 4.60. Cryo-cracking of Nokia 6310i mobile phones sample to isolate SM bearing

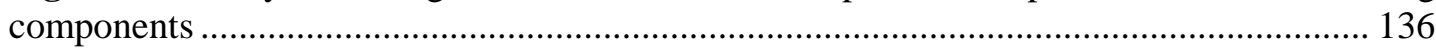

Figure 4.61 Isolation of contacts from Nokia 6230i mobile phone PCB and housing....... 140

Figure 4.62 Gold edge connectors removed from graphics card ...................................... 140

Figure 4.63 Ashed gold edge contacts isolated from graphics card PCB …..................... 146

Figure 5.1 Industrial symbiosis process for fabrication of dye-sensitised solar cell (DSSC) counter-electrode catalysts from waste thermocouples 156

Figure 5.2 Recycling rates of metals highlighted as critical in recent assessments (adapted from Graedel et al., 2011; Reck \& Graedel, 2012) $21,53,60,100,118$. 157

Figure 5.3 Waste Heraeus Electro-Nite Positherm expendable immersion thermocouple (schematic diagram adapted from Van der Perre) ${ }^{241}$.... 159

Figure 5.4 Material flows in a circular economy drawing on secondary raw materials from the wider circular economy through industrial symbiosis (adapted from Ellen McArthur Foundation, 2013) $)^{47}$ 159

Figure 5.5 Structure of a Dye-Sensitised Solar Cell (DSSC) 161

Figure 5.6 Procedure for synthesising DSSC counter electrodes from waste thermocouples (WTCs). The process includes 3 stages: 1) isolation of Pt filaments from WTCs; 2) synthesis of chloroplatinic acid from filaments; 3) creation of DSSC counter electrodes ................... 162

Figure 5.7 Electrochemical symmetrical 'dummy cells' used for EIS measurements....... 163

Figure 5.8 Waste thermocouple in poor condition with exposed filaments and alloy compositions as determined by SEM/EDS. 164

Figure 5.9 Diameter measurements of thin (left) and thick (right) waste thermocouple filaments from SEM images at 1,200 and 1,800 time magnification respectively 165

Figure 5.10 Elemental map of $\mathrm{Pt}$ in thin waste thermocouple filament (top) with corresponding X-ray emission spectra and alloy composition analysis (bottom) produced using SEM/EDS. 166

Figure 5.11 Elemental map of $\mathrm{Pt}$ and $\mathrm{Rh}$ in thin waste thermocouple filament (top) with corresponding X-ray emission spectra and alloy composition analysis (bottom) produced using SEM/EDS/ 167

Figure 5.12 Industrial symbiosis operations and associated costs of each operation indicated by letters in square brackets: [a] Labour and overheads; [b] Landfill tax + gate fee; [c] transport; [d] price of filaments $([\mathrm{a}+\mathrm{b}]) ;[\mathrm{e}]$ chemicals + energy; [f] total cost of chloroplatinic acid $([\mathrm{c}+\mathrm{d}+\mathrm{e}])$ 170 
Figure 6.1 The commercialisation challenge for photovoltaics 176

Figure 6.2 a) Dye-sensitised solar cell (DSSC - Grätzel Cell); b) solid-state dye-sensitised solar cell (ssDSSC) (adapted from Snaith, 2013) 265 . 178

Figure 6.3 Example architectures of Peroskite solar cells: a) perovskite based mesosuperstructured solar cell (MSSC); b) porous perovskite heterojunction; c) perovskite p-i-n heterojunction (adapted from Snaith, 2013; ${ }^{265}$ HTM: hole transport material 180

Figure 6.4 Organic photovoltaic (OPV) solar cell device architecture 180

Figure 6.5 The DSSC 'GCell' produced by G24Power (top left) and its product intergrated applications in: solar backpacks (top right), ipad keyboard folios (bottom left), and ibeacons (bottom right); images coutesy of www.gcell.com ${ }^{274}$. 181

Figure 6.6 Sony Hana-Akari (flower lamps) for indoor use powered by glass based DSSCs in the external flower decorations. 182

Figure 6.7 Coloured DSSC façade of the Swisstech Convention Centre.

Figure 6.8 Structure of a tandem perovskite-Si solar cell (adapted from Oxford PV) ${ }^{275} \ldots 183$

Figure 6.9 Heliatek's OPV Heliafilms ${ }^{\circledR}$ (top left) suitable for: retrofitting buildings (top right; , product integration in mobile phone chargers (bottom left), and solar tape for retrofitting products (bottom right). ${ }^{276}$ 183

Figure 6.10 Principle of roll-to-roll production of planar p-i-n perovskite cell as shown in Figure $6.3 \mathrm{c}$. 184

Figure 6.11 Recycling procedure for perovskite solar cells. (I) Removal of Au electrode with adhesive tape. (II) Removal of the HTM by immersing in chlorobenzene. (III) Transformation of the perovskite into methylammonium iodide (MAI) and $\mathrm{PbI}_{2}$ and extraction of MAI in water. (IV, V) Removal of $\mathrm{PbI}_{2}$ and $\mathrm{TiO}_{2}$ using dimethylformamide (DMF). (VI) Preparation of a new $\mathrm{TiO}_{2}$ film. (VII) Formation of the perovskite film on recycled FTO from recycled $\mathrm{PbI}_{2}$. (VIII) Preparation of the HTM layer. (IX) Evaporation of the Au top electrode. (Reprinted with permission from Binek et al., ${ }^{283}$ Copyright (2016) American Chemical Society). 185

Figure 6.12 Modified DSSC for replacement of dye and electrolyte. 188

Figure 7.1 Integrated technologies on the SPECIFIC 'Active Classroom' . 192

Figure 7.2 Off-grid solar energy structure with $1.4 \mathrm{kWp}$ of integrated PV in an orphanage in Mutende, Lulamba, Zambia 192

Figure 7.3 An estimated cost of an off-grid DC, PV system in Durban, South Africa, consisting of $600 \mathrm{Wp}$ silicon module, a $12 \mathrm{~V}, 250 \mathrm{Ah}$ lead-acid battery and a charge controller and cables (top); and breakdown of cost of system ( $€$ ) over 20 years (bottom) 194

Figure 7.4 Carbon footprint of batteries for the system including replacements (based on data from Baumann et al. ${ }^{310}$. VRLA - valve regulated lead-acid; LTO - lithium-iron-phosphate with lithium titanate anode; LFP - lithium-iron-phosphate with carbon anode; LMO - lithiummanganese-oxide; NCM - lithium-nickel-cobalt-manganese; NCA - lithium nickel-cobaltaluminium-oxide). 196

Figure A.1 X-ray emission spectrum of 100\%-TaCap powder... 211

Figure A.2 Comparison of spectra obtained from the same area of $100 \%$ TaCap with $2 \mathrm{~s}$ (blue) and 300s (yellow) scan times, showing improved S/N with increased scan time; blue $2 \mathrm{~s}$ scan time spectra is jagged and noisy compared to the yellow 300s scan

Figure A.3 Comparison of X-ray emission spectra of 100\%-TaCap powder measured with 16 s (yellow) and 300s (blue) scan times 212

Figure A.4 Ground Ta capacitor powder containing 39.5 wt $\%$ metallic $\mathrm{Zn}$... 215 
Figure A.5 X-ray emission spectrum of 35.9 wt $\%$-Zn sample obtained with 32 s scan time

\section{List of Tables}

Table 1.1 Definitions of WEEE

Table 1.2 Categories of (W)EEE used in the EU as set out in Annex I of the WEEE directive ${ }^{4}$

Table 1.3 'High-tech' metals' and their driving emerging technologies ${ }^{22}$......................... 8

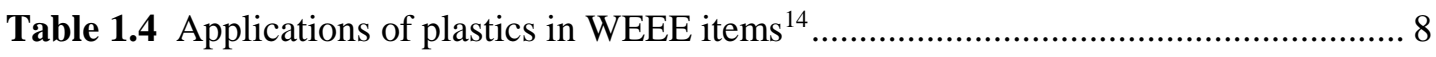

Table 1.5 Non-exhaustive list of hazardous substances found in and arising from WEEE ${ }^{25-29}$

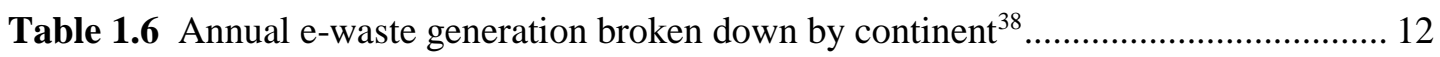

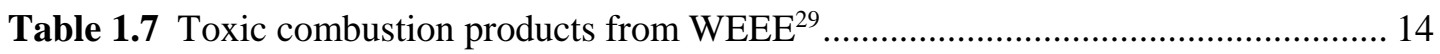

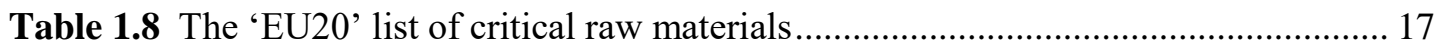

Table 1.9 Growth in element use projected to 2030 in emerging technologies. The 2006 and 2030 indicators show the proportion of demand compared to 2006 production e.g. Ga: indicator $2006=28 / 152=0.18$ and indicator $2030=603 / 152=3.97^{57}$

Table 1.10 Primary carbon footprint of selected elements in WEEE goods ${ }^{16}$............ 22

Table 1.11 Example breakdown of revenue received for PCBs by Metech Recycling UK ${ }^{22}$

Table 2.1 Samples analysed by AAS, relevant sections of the thesis, elements analysed and sample preparation procedures.

Table 2.2 Parameters for Analysis of individual elements by AAS

Table 2.3 Samples analysed by ICP-MS, relevant sections of the thesis, elements analysed and sample preparation procedures

Table 2.4 Positions of emission peaks in XRF analysis...

Table 2.5 Equivalent circuit elements, their current-voltage relationships, impedances and phase shift properties

Table 2.6 Metal foils used as standards for XRF evaluation 50

Table 3.1 DRAM modules analysed during author's MRes project. ${ }^{22}$

Table 3.2 Precious metal and copper content of PC DRAM samples 1-1522 (errors given as ranges for triplicate analyses), ${ }^{a}$ and calculated average composition for DIMMs and SIMMs

Table 3.3 Literature values for precious metal and copper content of PC PCBs compared to calculated average composition of analysed DRAM samples. (Errors quoted are standarderrors of the mean)

Table 3.4 Estimated quantities of precious metals and copper in globally generated waste DIMMs in PCs POM 2013, their values and proportions of global demand, demand from the EEE sector and global secondary production each represents (calculated from average metal contents of samples 4-15, data provided in O'Connell et al., Statista, 2015) 150, 151, 153, 154, 18371 
Table 3.5 Projections of future recycling potential of various WEEE items (based on data

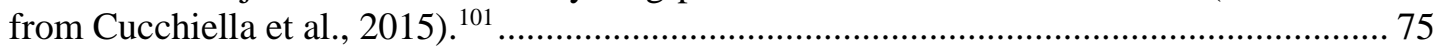

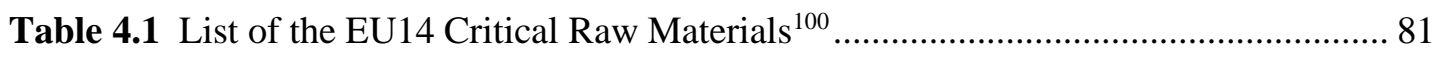

Table 4.2 Major Applications of Critical Raw Materials in the EEE sector ${ }^{51,144,189}$............ 82

Table 4.3 Overheads for Metech by percentage split ....................................................... 93

Table 4.4 Samples for analysis supplied by Metech Recycling (UK) Ltd .......................... 95

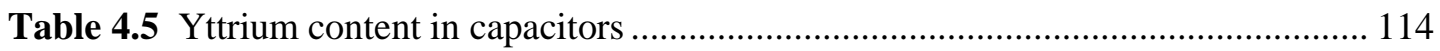

Table 4.6 Average CRM content of $100 \mu \mathrm{F}$ 10V 107A Ta-capacitors................................ 118

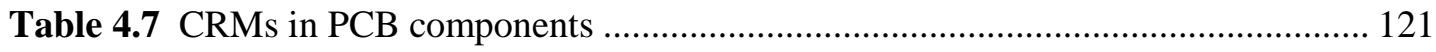

Table 4.8 Summary of identified CRMs in trial samples ................................................. 123

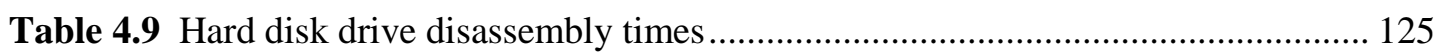

Table 4.10 Times taken for manual disassembly of CRM bearing components of Li-ion

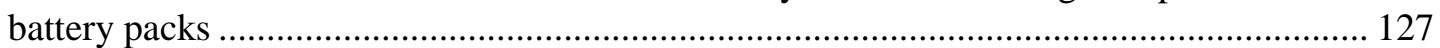

Table 4.11 Cobalt content of Laptop Li-ion batteries ..................................................... 128

Table 4.12 Time to manually isolate CRM bearing components of LCD displays............ 129

Table 4.13 Comparison of best times for LCD screen disassembly methods.................... 130

Table 4.14 MP3 player disassembly times .................................................................... 132

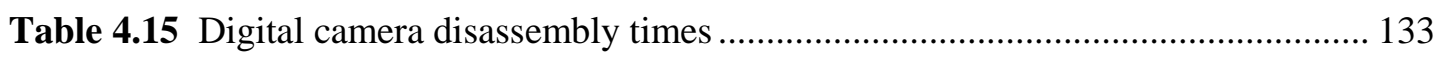

Table 4.16 Time to isolate CRM bearing components of mobile phones using manual

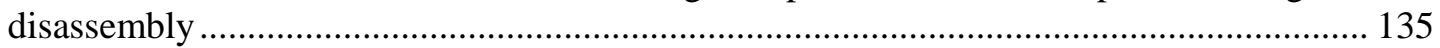

Table 4.17 Results of product disassembly following liquid nitrogen treatment and cracking 136

Table 4.18 Breakdown of costs for cryo-cracking ......................................................... 137

Table 4.19 Cobalt content of Li-ion batteries................................................................. 137

Table 4.20 Gold and copper quantity in samples and the value of these metals (based on market values, not revenue received from refiners) ............................................................ 140

Table 4.21 Time to isolate gold edge contacts from PCB devices using a guillotine and cost of operation.

Table 4.22 Time to isolate gold edge contacts from PCB devices using a wet tile cutter and cost of the operation (based on energy demand and time of process). 141

Table 4.23 Time taken for manual removal of components from PCBs and process cost. 142

Table 4.24 Time taken for isolation of components from PCBs and process cost

Table 4.25 Time taken for isolation of components from PCBs, energy demand and process cost using a paint stripping gun.

Table 4.26 Time for isolation of SMDs from the surfaces of PCBs using a solder bath ... 144

Table 4.27 Value of isolated components from PCB samples when sent for refining.... 145

Table 4.28 Mass reductions and savings on treatment charges achieved for materials with microwave ashing

Table 4.29 Value gained from microwave ashing of 1 tonne of isolated contacts from samples 
Table 4.30 SMDs and CRMs contained in sieved fractions

Table 4.31 No of each PCB sample investigated required to generate $500 \mathrm{~kg}$ of ash from contacts, calculated costs of isolating contacts from this quantity of PCBs, and revenue received for this material based on BASF refining charges (issued to Metech in 2012)..... 150

Table 4.32 Cost-benefit analysis of isolating and ashing contacts to produce $500 \mathrm{~kg}$ of ash from the quantities of analysed samples indicated in Table 4.31 ...................................... 150

Table 4.33 Costs for isolation of PCBs from items using various disassembly techniques 151

Table 4.34 Per item costs of isolating SMDs from PCBs with various techniques 152

Table 4.35 Cost benefit of best cost options for disassembly and isolation of SMDs from PCBs in whole samples 152

Table 5.1 Global demand for platinum by end use sector in 2015 and estimated end-of-life (EoL) recycling rates. 158

Table 5.2 PGM content of chloroplatinic acid and Pt recovery yield from WTCs and 99.998\% Pt wire 168

Table 5.3 Average charge transfer resistances $\left(\mathrm{R}_{\mathrm{CT}}\right)$, double layer capacitances $\left(\mathrm{C}_{\mathrm{dl}}\right)$ and exchange current densities $\left(\mathrm{j}_{0}\right)$ at the electrolyte | electrode interface for electrodes in 'dummy cells' created with platinum from different source; and corresponding $\mathrm{j}_{0}$ expressed per unit concentration of Pt in IPA used for creation of electrodes $\left(\mathrm{j}_{0} /[\mathrm{Pt}]\right)$. 169

Table 5.4 Cost analysis for production of $1 \mathrm{~g}$ chloroplatinic acid from WTCs 170

Table 5.5 Comparative cost of platinum as chloroplatinic acid synthesised from WTCs and purchased in minimum and maximum available quantities 171

Table 7.1 Example daily energy consumption for small domestic appliances 193

Table 7.2 Globally installed stationary energy storage capacity by battery type (data from Baumann et al. $)^{310}$..... 195

Table 7.3 Comparative costs of commercially available batteries over 20-year lifetime of system 195

Table 7.4 Supply risks of materials in batteries, those highlighted in orange have been identified as CRMs in recent assesments. 198

Table A.1 Detection of metals through steel and aluminium foils 210

Table A.2 Variations in measured relative mass percentages (rel. \%) of detected elements in $100 \%$-TaCap powder with scan time.

Table A.3 Repeat measurements of relative masses of detected elements in a single area of $100 \%$-TaCap powder using $16 \mathrm{~s}$ scans.

Table A.4 Relative percentage masses of detected elements at 12 sites of $100 \%$-TaCap powder measured with $16 \mathrm{~s}$ scan times.

Table A.5 Average relative mass percentage composition of elements calculated from 12 repeat $16 \mathrm{~s}$ scans on different areas of samples

Table A.6 rel.\% of elements detected in 12 sample locations in $39.5 \% \mathrm{Zn}$ sample using $32 \mathrm{~s}$ scan times and calculated wt $\%$ of elements in the sample. 


\section{Abbreviations}

\begin{tabular}{|c|c|c|c|}
\hline 3GPV & $3^{\text {rd }}$ Generation Photovoltaics & EVA & Ethylene Vinyl Acetate \\
\hline \multirow[t]{2}{*}{ AAS: } & Atomic Absorption & FPM & Fast Page Mode (RAM) \\
\hline & Spectroscopy & GHG & Greenhouse Gas \\
\hline \multirow[t]{2}{*}{ AATF } & Approved Authorized & HCL & Hollow Cathode Lamp \\
\hline & & HDD & Hard Disk Drive \\
\hline ABS & $\begin{array}{l}\text { Acrylonitrile Butadiene } \\
\text { Styrene (plastic) }\end{array}$ & HDI & Human Development Index \\
\hline \multirow{3}{*}{ BATRRT } & Best Available Treatment, & HIPS & High Impact Polystyrene \\
\hline & Recovery and Recycling & HTM & Hole Transport Material \\
\hline & Techniques & IC & Integrated Circuit \\
\hline BIPV & $\begin{array}{l}\text { Building Integrated } \\
\text { Photovoltaics }\end{array}$ & ICP-OES & $\begin{array}{l}\text { Inductively Coupled Plasma - } \\
\text { Optical Emission Spectroscopy }\end{array}$ \\
\hline \multirow{2}{*}{$\begin{array}{l}\text { BFRs } \\
\text { CBA }\end{array}$} & Brominated Flame Retardants & ICP-MS & Inductively Coupled Plasma - \\
\hline & Cost-Benefit Analysis & & Mass Spectrometry \\
\hline \multirow[t]{2}{*}{ CCFL } & Cold Cathode Fluorescent & IPA & Isopropyl alcohol \\
\hline & Lamp & IPR & Individual Producer \\
\hline $\mathbf{C E}$ & Circular Economy & & Responsibility \\
\hline \multirow{3}{*}{$\begin{array}{l}\text { CFC } \\
\text { CIGS }\end{array}$} & Chlorofluorocarbon & ITO & Indium Doped Tin Oxide \\
\hline & Copper-Indium-Gallium- & LCA & Lifecycle Analysis \\
\hline & Diselinide (PV) & LCD & Liquid Crystal Display \\
\hline cps & Counts per Second & LCOE & Levelised Cost Of Electricity \\
\hline CRMs & Critical Raw Materials & LED & Light Emitting Diode \\
\hline CNCs & Cellulose Nanocrystals & LFP & Lithium-Iron-Yttrium- \\
\hline COGs & Cut-Off Grades & & Phosphate Battery \\
\hline CRMs & Critical Raw Materials & LIB & Lithium-Ion Battery \\
\hline CRT & Cathode Ray Tube & MAI & Methylammonium Iodide \\
\hline DDR & Double Data Rate (SDRAM) & MFA & Material Flow Analysis \\
\hline DEHP & Di(ethylhexyl) Phthalate & MLCCs & Multi-Layer Ceramic Capacitor \\
\hline \multirow[t]{2}{*}{ DfRR } & $\begin{array}{l}\text { Design for Reuse, } \\
\text { (Refurbishment) And }\end{array}$ & MSSCs & $\begin{array}{l}\text { Mesosuperstructured Solar } \\
\text { Cells }\end{array}$ \\
\hline & Recycling & NIB & Neodymium-Iron-Boron (alloy \\
\hline \multirow{2}{*}{$\begin{array}{l}\text { DIMMs } \\
\text { DMF }\end{array}$} & Dual In-line Memory Modules & & permanent magnets) \\
\hline & dimethylformamide & OPV & Organic Photovoltaics \\
\hline & Dynamic RAM & P3HT & 3-hexylpolythiophene \\
\hline $\begin{array}{l}\text { DRAM } \\
\text { DSSC }\end{array}$ & Dye-sensitised Solar Cell & PA & Polyamide \\
\hline EDO & Extended Data Output (RAM) & PBB & Polybrominated Biphenyls \\
\hline \multirow[t]{2}{*}{ EDS } & Energy Dispersive X-Ray & PBT & Poly(Butyleneterephthalate) \\
\hline & Spectroscopy & PC & Polycarbonate \\
\hline \multirow[t]{2}{*}{ EEE } & Electrical and Electronic & PCB & Printed Circuit Board \\
\hline & Equipment & РCBM & Phenyl-C61-Butyric Methyl \\
\hline EoL & End-of-Life & & Ester \\
\hline EPBT & Energy Payback Time & PCE & Power Conversion Efficiency \\
\hline \multirow[t]{2}{*}{ EPR } & Extended Producer & PDPE & Polybrominated dipheyl ethers \\
\hline & Responsibility & PET & Polyethylene terephthalate \\
\hline \multirow{2}{*}{$\begin{array}{l}\text { ETL } \\
\text { ETRD }\end{array}$} & Electron Transport Layer & PM & Precious Metal \\
\hline & $\begin{array}{l}\text { Emerging Technologies Raw } \\
\text { Material Demand }\end{array}$ & PMR & $\begin{array}{l}\text { Material for Precious Metal } \\
\text { Recovery }\end{array}$ \\
\hline $\mathbf{E U}$ & European Union & POM & Placed on the Market \\
\hline
\end{tabular}




\begin{tabular}{|c|c|}
\hline $\mathbf{P P}$ & Polypropylene \\
\hline PPE & Polyphenylene ether \\
\hline PS & Polystyrene \\
\hline PPV & Printable Photovoltaics \\
\hline PV & Photovoltaics \\
\hline PVC & Polyvinyl Chloride \\
\hline PVD & Physical Vapour Deposition \\
\hline PVDF & Polyvinyldiene Fluoride \\
\hline $\mathbf{R} 2 \mathbf{R}$ & Roll-to-Roll (production) \\
\hline REEE & Reusable EEE \\
\hline RSA & Republic of South Africa \\
\hline REMs & Rare Earth Metals \\
\hline RSD & Relative Standard Deviation \\
\hline SD & Standard Deviation \\
\hline SDA & Small Domestic Appliances \\
\hline SDR & Single Data Rate (Sdram) \\
\hline SDRAM: & Synchronous DRAM \\
\hline sem & Standard Error on the Mean \\
\hline SEM & Scanning Electron Microscopy \\
\hline SIMMs & $\begin{array}{l}\text { Single In-Line Memory } \\
\text { Module }\end{array}$ \\
\hline SMD & Surface Mounted Device \\
\hline ssDSSC & Solid-State DSSC \\
\hline TBBA & Tetrabromo-Bisphenol-A \\
\hline TCO & Transparent Conductive Oxide \\
\hline wDIMMS & Waste DIMMs \\
\hline WEEE & $\begin{array}{l}\text { Waste Electrical \& Electronic } \\
\text { Equipment }\end{array}$ \\
\hline wPCs & Waste PCs \\
\hline WRAP & $\begin{array}{l}\text { Waste \& Resources Action } \\
\text { Programme }\end{array}$ \\
\hline WTC & Waste Thermocouple \\
\hline XRF & $\begin{array}{l}\text { X-Ray Fluorescence } \\
\text { Spectroscopy }\end{array}$ \\
\hline
\end{tabular}




\section{Introduction}

\section{もったいない}

\section{Chapter summary}

WEEE represents a considerable sustainability challenge for humanity. Most of the WEEE generated around the globe unaccounted for and its improper treatment by informal sectors has resulted in significant impacts on the environment and human health. In addition, the linear throughput of materials used by the EEE industry to manufacture new products to replace WEEE, including critical raw materials which are recovered with low efficiency or not at all from WEEE, represents a resource-efficiency crisis which must be urgently addressed. The ever-growing volumes of WEEE around the globe are also a significant secondary resource of important valuable materials. If exploited effectively, WEEE presents opportunities to address global resource-efficiency challenges and mitigate materials criticality. Significant economic, environmental and social benefits can be derived by exploiting the inherent value of WEEE, particularly through reuse and efficient recovery of valuable metals such as precious metals, PGMs and copper.

Circular economy represents a solution to the global WEEE problem and a means to enhance the industrial ecology of the EEE industry through reuse \& recycling of EEE and its components, and through provision of secondary raw materials/components for manufacturing. This provides a means of exploiting greater value from WEEE through novel methods of valorisation which present economic, social and environmental benefits over traditional recycling processes. To drive circular material flows sufficient economic value must be derived from WEEE to cover costs incurred throughout recycling process chains. This requires suitable recovery processes to be available; product design to accommodate circular practices; and appropriate reverse logistics, business models and legislation to be in place. Much progress has been made around the world in establishing suitable legislation to govern WEEE management. This has resulted in establishment of collection systems, and efficient recovery processes for economically and environmentally important metals, particularly 
precious metals and copper concentrated in PCBs. However, much progress is still required to address low collection rates and exports to regions where WEEE is recycled by the informal sector resulting in significant impacts on populations, ecosystems and the environment. Losses of potentially recoverable materials in pre-processing and recovery stages of recycling which limit derivable value from WEEE and the ability to efficiently recover 'high-tech' metals must also be addressed in the interests of global resource-efficiency.

To overcome these issues, greater knowledge of the composition of items within the waste stream and the available value therein is required to plan future EoL management. In addition, recovery processes which derive greater value and are more conducive to circular economy are required for viable recovery of materials which are currently lost in recycling. For the future, optimisation of product design and whole product lifecycles is necessary to ensure problems encountered in managing WEEE today are avoided, and greater value can be derived from material resources contained in WEEE. Greater education and awareness of the material content of items and available end-of-life processes is necessary to enable consumers to make informed decisions when selecting technologies that will result in better outcomes at end-of-life.

\subsection{The nature of WEEE}

Electrical and electronic equipment (EEE) has permeated into all aspects of our lives resulting in prosperity for billions. EEE includes the smartphones in our pockets, toys and computer games with which our children play, tablets and laptop computers. The internet itself upon which society has become increasingly dependent is hosted on a global network of servers. Low-carbon technologies for global sustainability are being rapidly and widely deployed around the world. EEE has enabled the digital revolution and provides the means of addressing the global knowledge gap, energy access gap, and to mitigate global climate change. But for all the benefits of EEE, its use leaves mankind with a considerable problem: waste electrical and electronic equipment (WEEE) - the planet's fastest growing waste stream. ${ }^{1,2}$

\subsubsection{WEEE by definition}

WEEE, electronic waste (e-waste) and e-scrap are terms used interchangeably to describe electrical and electronic equipment (EEE) which has been discarded by its owners and is perceived to be useless. ${ }^{3}$ However, due to inconsistency in the global use of these terms, numerous definitions of WEEE exist (Table 1.1).4-8 In an attempt to reconcile some of these 
inconsistencies and introduce global conformity, the StEP initiative recently published guidance on the use of a new non-legal global definition of EEE...

"Any household or business item with circuitry or electrical components with power or battery supply."

and e-waste...

"E-waste is a terms used to cover items of all types of electrical and electronic equipment (EEE) and its parts that have been discarded by the owner as waste without the intention for re-use." 6

Table 1.1 Definitions of WEEE

\begin{tabular}{|c|c|}
\hline Source & Definition of WEEE \\
\hline $\mathrm{EC}^{4,9}$ & $\begin{array}{l}\text { "Electrical or electronic equipment which is waste within the meaning of Article 3(1) } \\
\text { of Directive 2008/98/EC, including all components, sub-assemblies and } \\
\text { consumables which are part of the product at the time of discarding" and "electrical } \\
\text { and electronic equipment' or 'EEE' means equipment which is dependent on electric } \\
\text { currents or electromagnetic fields in order to work properly and equipment for the } \\
\text { generation, transfer and measurement of such currents and fields and designed for } \\
\text { use with a voltage rating not exceeding } 1000 \text { volts for alternating current and } 1500 \\
\text { volts for direct current" - WEEE directive, 2012/19/EU. }\end{array}$ \\
\hline & $\begin{array}{l}\text { 'waste' means any substance or object which the holder discards or intends or is } \\
\text { required to discard" - Waste framework directive, 2008/98/EC. }\end{array}$ \\
\hline $\begin{array}{l}\text { StEP } \\
\text { Initiative. }^{6}\end{array}$ & $\begin{array}{l}\text { "A term used to cover almost all types of electrical and electronic equipment (EEE) } \\
\text { that has or could enter the waste stream. Although e-waste is a general term, it can } \\
\text { be considered to cover almost any household or business item with circuitry or } \\
\text { electrical components with power or battery supply." }\end{array}$ \\
\hline OECD. ${ }^{7}$ & "Any appliance using an electric power supply that has reached its end of life." \\
\hline $\begin{array}{l}\text { Basel Action } \\
\text { Network. }{ }^{8}\end{array}$ & $\begin{array}{l}\text { "E-waste encompasses a broad and growing range of electronic devices ranging } \\
\text { from large household appliances such as refrigerators and air-cons to hand-held cell } \\
\text { phones and personal stereos." }\end{array}$ \\
\hline
\end{tabular}

All definitions suggest that EEE becomes 'waste' EEE once the user chooses to discard it. Therefore, it is the owners of EEE, their perception of its value and functionality, and the decisions they make regarding what to do with items for which they no longer have a use, which determine the point at which EEE becomes 'WEEE'. The situation can best be described diagrammatically (Figure 1.1). EEE is manufactured and distributed to users. Once the user no longer wishes to keep the device they make a decision about whether or not to discard it. If the device has been leased, it may be returned to the company from which it was leased for reuse with another user. Likewise, items owned by current users which are perceived to have retained value can be sold, traded or donated for use by another user. If the owner chooses not to sell, donate or return items to lease companies then they discard items (i.e. get rid of it as 
waste), at which point EEE enters the end-of-life (EoL) phase of its lifecycle and becomes WEEE. Once received by the waste management sector, a decision is made whether the item is suitable for reuse or not. Reusable EEE (REEE) may be refurbished and sold to a new user. WEEE that has no value for re-use is recycled for recovery of valuable materials and disposal of hazardous and non-recyclable components.

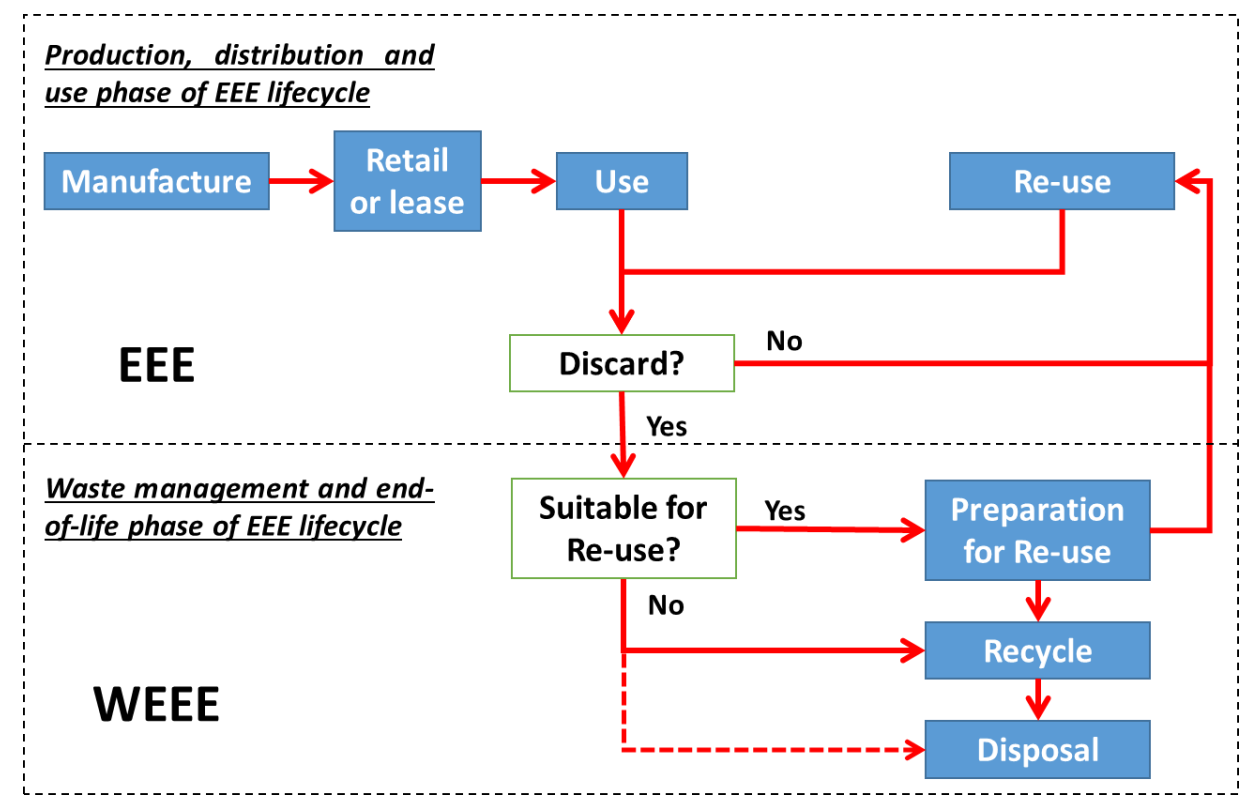

Figure 1.1 Flow of EEE into WEEE (adapted from StEP, 2014) $)^{10}$

An enormous variety of items fall within the definitions of EEE \& WEEE, which are often grouped into categories such as those used in the EU for statutory reporting of quantities of EEE placed on the market and WEEE collected and treated (Table 1.2). A breakdown of WEEE collected in the EU by category is shown in Figure 1.2, and quantities of WEEE collected in each member state are given in Figure 1.3.

Table 1.2 Categories of (W)EEE used in the EU as set out in Annex I of the WEEE directive 4

\begin{tabular}{lll}
\hline Category* & Example items \\
\hline 1 & Large household appliances & White goods (ex. cooling equipment) \\
2 & Small household appliances & Vacuums, toasters, irons \\
3 & IT and telecoms equipment & Computers, printers, phones (screens included) \\
4 & Consumer equipment \& PV panels & Radios, electronic musical instruments, \\
5 & Lighting equipment & Fluorescent lamps, discharge lamps \\
6 & Electrical and electronic tools & Drills, sewing machines, saws \\
7 & Toys, leisure and sports equipment & Games consoles, coin slot machines \\
8 & Medical devices & Dialysis machines, ventilators \\
9 & Monitoring and control instruments & Smoke detectors, thermostats, scales \\
10 & Automatic dispensers & ATMs, vending machines \\
\hline$*$ for a non-exhaustive list of items that fall in each category refer to Annex II of the WEEE directive
\end{tabular}




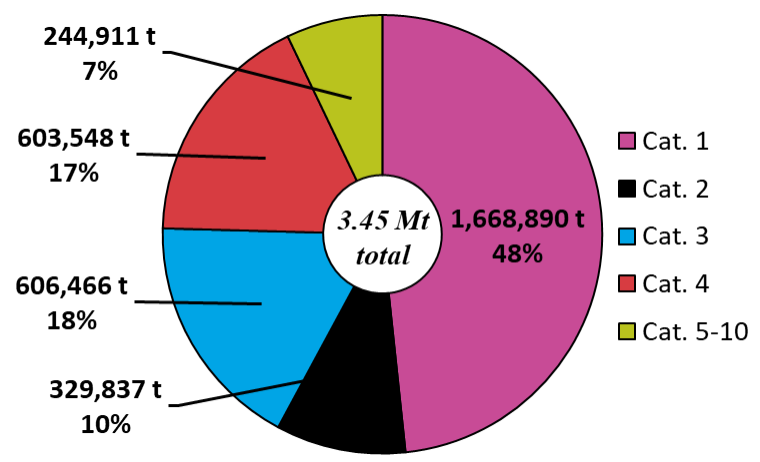

Figure 1.2 Breakdown by category of WEEE collected in the EU in 2014 (data from Statistical Office of the $E C, 2016)^{11}$

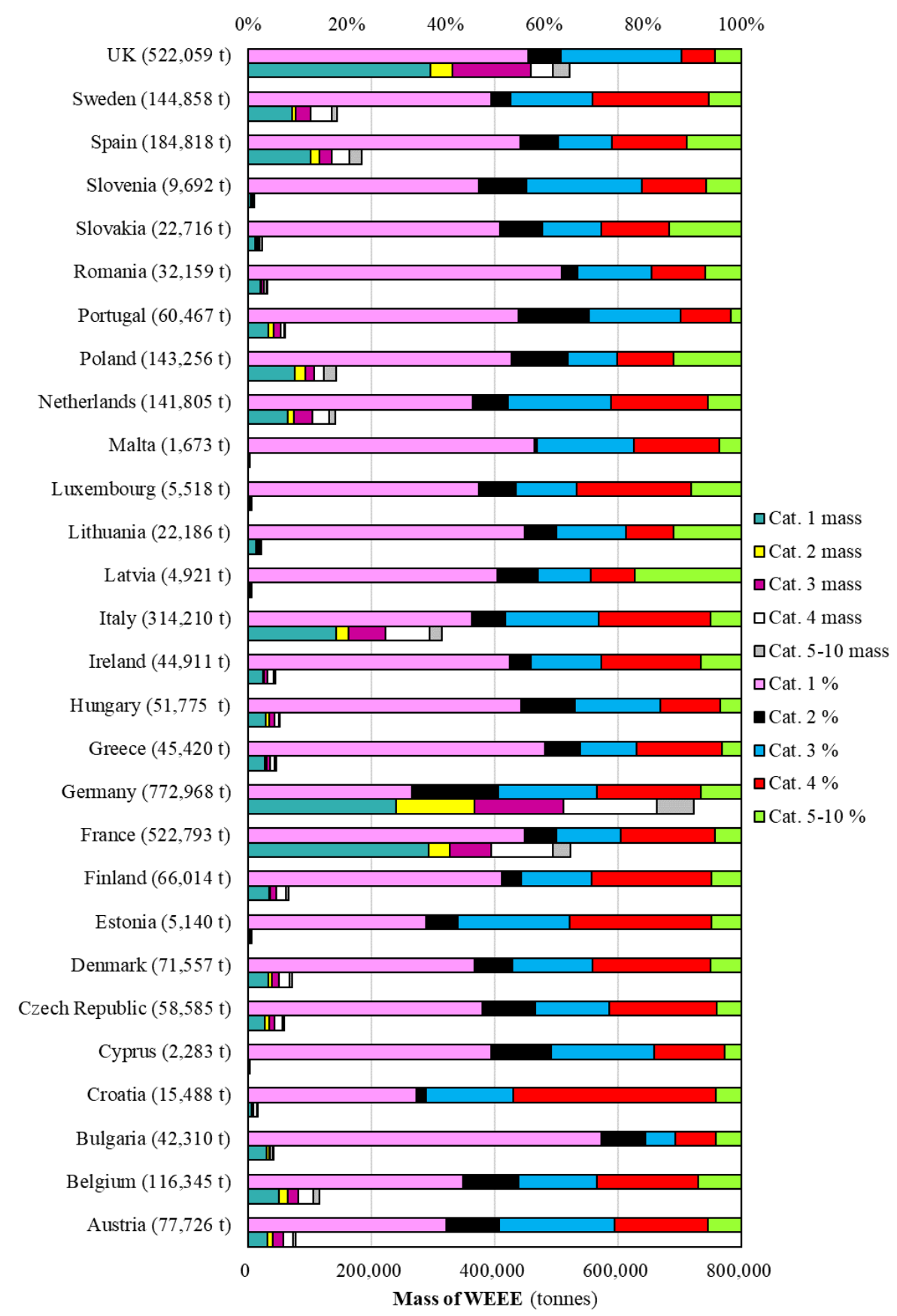

Figure 1.3 Masses of WEEE collected in EU member states in 2014 with compositional breakdown by category (data from Statistical Office of the EC, 2016) $)^{11}$ 
Each category, and different kinds of equipment within each category, often require distinct and specific waste management strategies at EoL, because of differences in design, structure and material compositions.

\subsubsection{Material composition of WEEE}

It is difficult to determine an overall composition for WEEE, due to compositional differences between WEEE types, the rapidly evolving nature of items in the waste stream and lack of available data from many regions of the world. However, some general compositions which have been proposed are shown in Figure 1.4.
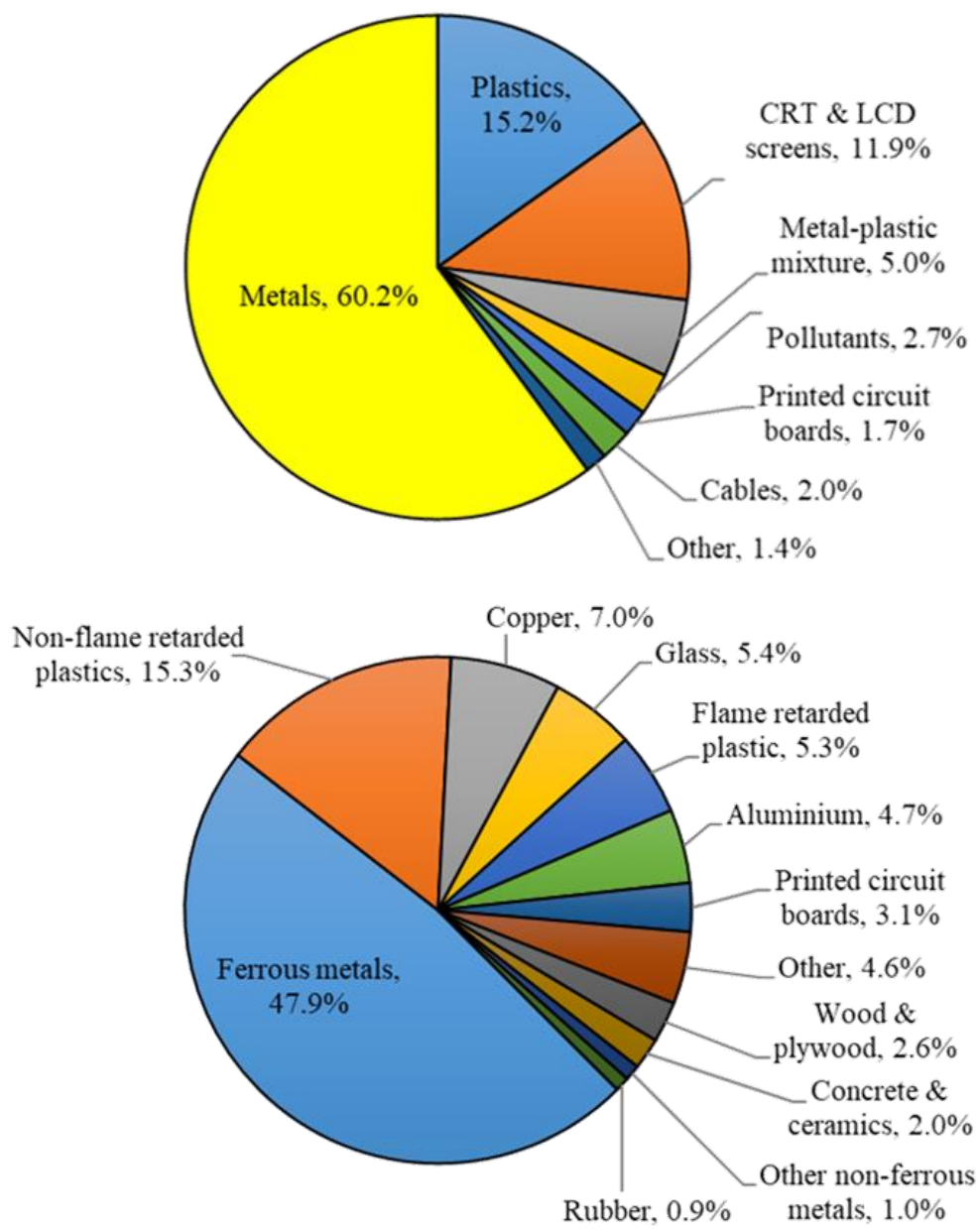

Figure 1.4 General material compositions of WEEE by mass given by EMPA, Switzerland (top); and the European Topic Centre on Resource and waste management (bottom $)^{3}$

It is easier to give general compositions for WEEE categories or particular items (Figure 1.5). The material composition, even of the same kind of WEEE (e.g. different mobile phones or PCs ), varies enormously, and WEEE is a complex heterogeneous combination of materials, ${ }^{12}$ 
the major mass fractions being bulk metals (aluminium, copper and ferrous), plastics ${ }^{13-15}$ and glass. Individual items can contain up to 60 elements. ${ }^{16}$
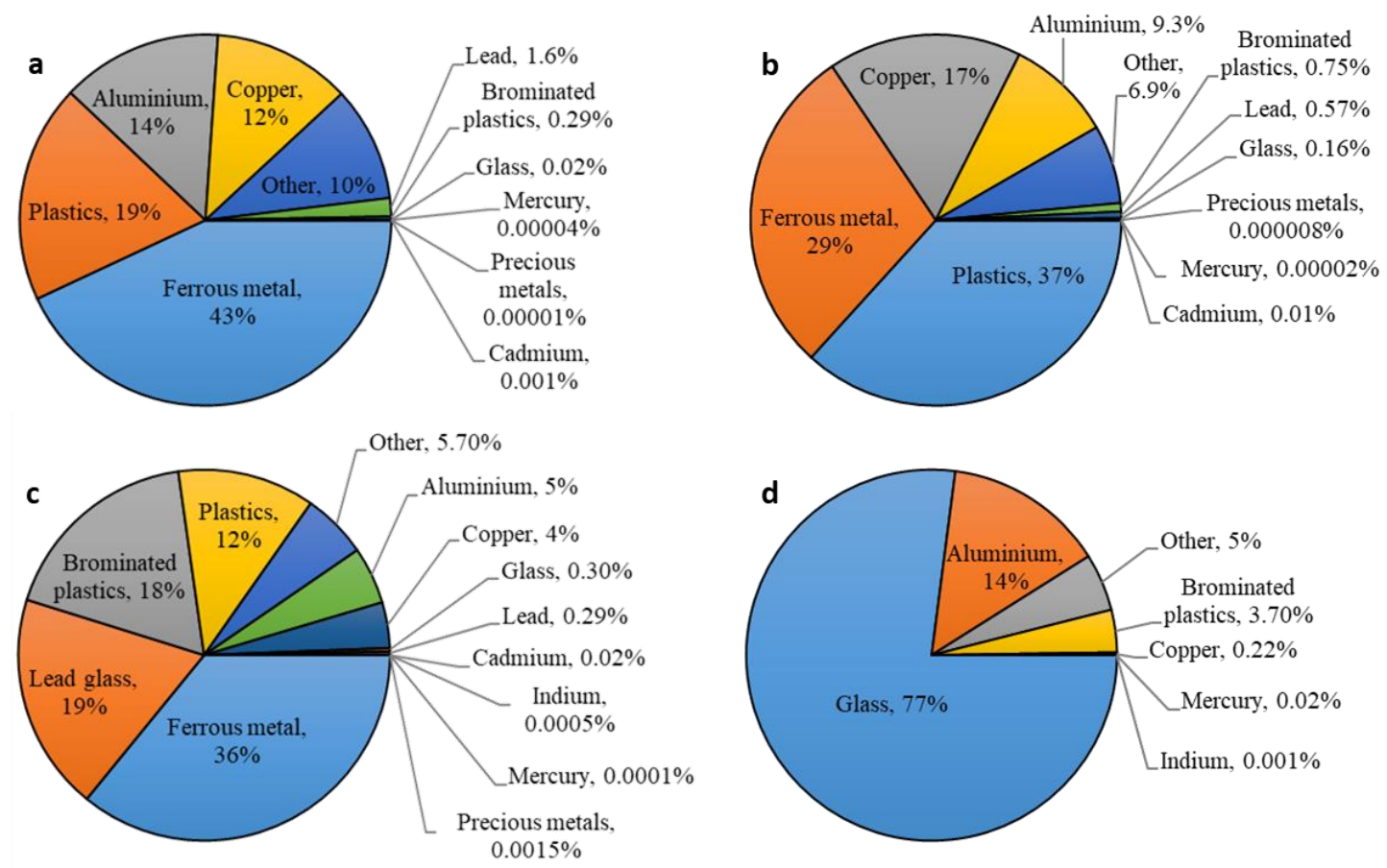

Figure 1.5 Material composition by mass of four e-waste categories: a) large household appliances; b) small household appliances; c) ICT and consumer electronics; d) lamps ${ }^{17}$

Metals - A large number of metals are found in WEEE. In addition to bulk metals and alloys such as, aluminium, brass, copper, and steel, which are used in structural components of EEE, cables, motors, heat sinks etc. and which form the major mass fraction of the waste stream, there are also trace amounts of 'high-tech metals. ${ }^{18}$ These are metals which are vital components of EEE and typically used in high-tech applications. These include precious metals (PMs) and critical raw materials (CRMs). ${ }^{19,20}$

Critical Raw Materials - In recent years, demand has risen for CRMs such as rare earth metals (REMs) used in permanent magnets, indium used in LCD screens; and metals in printed circuit board components such as tantalum, gallium and platinum group metals (PGMs). Although only used in small quantities in EEE, these metals are often crucial to the functionality of devices with few viable options for substitution. Global demand for these materials is rising rapidly due to growth in demand for EEE and use of these metals in many emerging technologies including 'green' technologies (Table 1.3). Economic impacts associated with supply shortages of these elements would be significant. This fact, coupled with rising demand and the possibility of supply bottlenecks has resulted in these elements gaining 'critical' status 
from numerous studies in recent years. ${ }^{21}$ (Materials criticality is discussed further in Section 1.2.2)

Table 1.3 'High-tech' metals' and their driving emerging technologies ${ }^{22}$

\begin{tabular}{cl}
\hline Raw Material & \multicolumn{1}{c}{ Emerging Technologies } \\
\hline $\mathrm{Ga}$ & Thin layer photovoltaics, ICs, LEDs \\
$\mathrm{Li}$ & Li-ion batteries \\
$\mathrm{Nd}$ & Permanent magnets, laser technology \\
$\mathrm{In}$ & Displays, thin layer photovoltaics \\
$\mathrm{Ge}$ & Fibre optic cable, IR optical technologies \\
$\mathrm{Pt}$ & Fuel cells, catalysts \\
$\mathrm{Ta}$ & Micro capacitors, medical technology \\
$\mathrm{Ag}$ & RFID, lead-free soft solder \\
$\mathrm{Co}$ & Lithium-ion batteries, synthetic fuels \\
$\mathrm{Pd}$ & Catalysts, seawater desalination \\
$\mathrm{Ti}$ & Seawater desalination, implants \\
$\mathrm{Cu}$ & Efficient electric motors, RFID \\
$\mathrm{Nb}$ & Micro capacitors, ferroalloys \\
$\mathrm{Sb}$ & Flame retardant, dopant in silicon wafers \\
$\mathrm{Cr}$ & Seawater desalination, marine technologies \\
$\mathrm{REMs}$ & Permanent magnets for wind turbines \& electric vehicles \\
\hline
\end{tabular}

Plastics - Plastics are widely used in EEE for insulation, noise reduction, sealing, housings, interior structural parts, functional parts and interior electronic components (Table 1.4). ${ }^{14} 15$ Acrylonitrile butadiene styrene (ABS), polycarbonate (PC), PC/ABS polymer blends and high impact polystyrene (HIPS) are used predominantly in housings and make up the majority of plastics in WEEE. (Figure 1.6). Polypropylene (PP) is commonly used for internal components of household appliances such as dishwashers, and PVC is used for insulating cables \& wiring. ${ }^{14}$ Of the plastics used in WEEE, about a third are flame-retarded, $~ 60 \%$ with non-halogenated flame retardants and $\sim 40 \%$ with halogenated flame-retardants. ${ }^{15}$

Table 1.4 Applications of plastics in WEEE items ${ }^{14}$

\begin{tabular}{ll}
\hline Polymer & Application \\
\hline ABS & Housings of phones, SDA; microwaves; flat screens \& certain monitors \\
& Enclosures \& internal parts of ICT equipment \\
HIPS & Internal refrigerator components (liners, shelves) \\
& Housings of SDA, data processing and consumer electronics \\
PC & Housings of SDA \& ICT equipment \\
Epoxy resins & PCBs \\
PP & Internal components of dishwashers \& washing machines, castings of SDA \\
PPO (HIPS/PE) & Housings of TVs, monitors and some SDA \\
& TV, computer, printer \& copier components \\
PC/ABS & Housings of ICT equipment and some SDA (e.g. kettles, shavers) \\
PVC & Cable sheathing \\
\hline
\end{tabular}

SDA: small domestic appliances; PCBs: printed circuit boards 


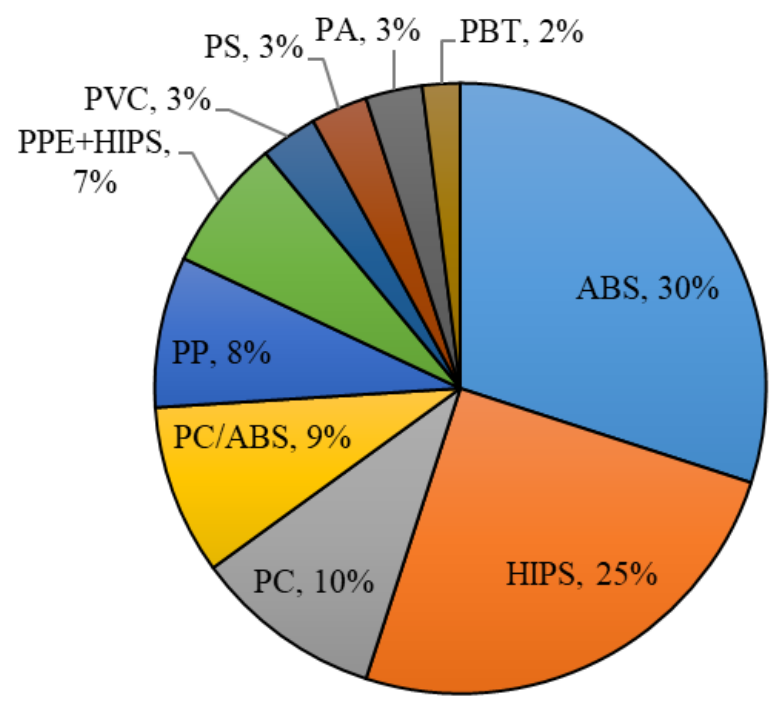

Figure 1.6 Mass proportions of polymers found in WEEE plastics (ABS: acrylonitrile-butadienestyrene; HIPS: high impact polystyrene; PA: polyamide; PBT: poly(butyleneterephthalate); PC: polycarbonate; PP: polypropylene; PPE: polyphenylene ether; PS: polystyrene; PVC: poly(vinyl chloride $)^{15}$

Hazardous materials - WEEE is hazardous because it contains a variety of toxic substances including heavy metals such as $\mathrm{Hg}, \mathrm{Cd}$ and $\mathrm{Pb}$, and halogenated flame retardants such a polybrominated diphenyl ethers (PBDEs) and tertrabromobisphenol A (TBBA). ${ }^{23,24} \mathrm{~A}$ list of hazardous substances in WEEE and their location in items is given in Table 1.5. 
Table 1.5 Non-exhaustive list of hazardous substances found in and arising from WEEE

\begin{tabular}{l} 
Contaminant \\
\hline Halogenated compounds \\
Brominated flame retardants (BFRs): \\
- Polybrominated diphenyl ethers (PDPE) \\
- Polybrominated biphenyls (PBB) \\
- Tetrabromo-bisphenol-A (TBBA) \\
Polychlorinated biphenyls \\
Chlorofluorocarbon (CFC) \\
PVC (polyvinyl chloride)
\end{tabular}

\section{Plasticisers}

Phthalates e.g DEHP

\section{Metals}

Antimony $(\mathrm{Sb})$

Arsenic (As)

Barium (Ba)

Beryllium (Be)

Cadmium (Cd)

Chromium VI (Cr VI)

Gallium (Ga)

Indium (In)

Lead $(\mathrm{Pb})$

Mercury $(\mathrm{Hg})$

Nickel (Ni)

Selenium (Se)

Silver (Ag)

Tin (Sn)

Zinc (Zn)

REMs

Radio-active substances

Americium (Am)

\section{Relationship with WEEE}

Thermoplastic components \& cable insulation. TBBA is widely used in PCBs \& ca

Condensers, transformers

Cooling units (fridges, air-cons), insulation foam

Cable insulation; Encapsulating material for SMDs

Plasticiser in PVC

Flame retardants in plastics \& PCBs $\left(\mathrm{SbO}_{3}\right)$; CRT \& lamp phosphors; Diodes Dopant for $\mathrm{Si}$; doping agent in transistors and PCBs; GaAs semiconductor in LED Getters in CRTs; vacuum tubes of CRT displays

Power supply boxes containing silicon controlled rectifiers; X-ray lenses; motherb NiCd batteries, phosphor in CRTs (CdS); printer inks/toners; plastics; contacts \& s Data tapes; floppy disks; corrosion inhibitor and hardening element for metallic ho Semiconductors e.g. GaAs in LEDs \& ICs

Indium doped tin oxide ITO for LCDs and thin-film photovoltaics (PV); transistors $\mathrm{PbO}$ as radiation shield in $\mathrm{CRT}$ glass; $\mathrm{Pb}$-acid batteries; solder on PCBs; stabilisers LCD backlights; alkali batteries; mercury wetted switches/relays in old mainframe NiCd \&NiMH batteries; electron gun in CRTs; surface plating for PCBs and electr Rectifiers; photo drums in older photocopier; PCBs

Wiring, switches, numerous SMDs; Connectors; $\mathrm{Pb}$-free solder Solder; LCD screens (ITO)

Zinc sulphide mixed with rare earths in fluorescent layer of CRT screens; used in P $\mathrm{Y}, \mathrm{Eu}, \mathrm{La}, \mathrm{Tb}$ used in phosphors of lamps and CRTs; Nd, Dy, Sm in permanent ma

Smoke detectors (active sensing element); medical equipment For details of hazards associated with materials found in WEEE see EMPA, 2009; Greenpeace, 2005; and Robinson, 2009. ${ }^{17,25,29}$ 


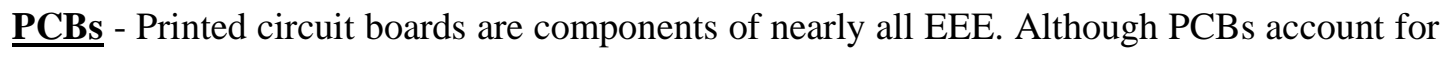
a small proportion of the mass of WEEE overall, they represent the most valuable portion of the waste stream because they contain precious metals which can be present at 40-50 times higher concentration than naturally occurring ores. ${ }^{30}$ According to UNU, 320 tons of gold and 7500 tons of silver are consumed annually in the manufacturing processes of PCs, cell phones, tablets and other items, adding a value of $\$ 21$ billion to the urban mine. ${ }^{31}$ Generally, PCBs are composed of $40 \%$ metals, $30 \%$ plastics and $30 \%$ ceramics $^{32}$ The most common PCBs, used in higher value items such as phones and computers, are FR-4 PCBs made from fibre glass reinforced epoxy resin. FR-2 boards are common in lower value items and power boards, and are composed of cellulose paper reinforced with phenolic resin. ${ }^{33}$ The most common metal in PCBs is copper, used to form the electrical circuits on the PCB, present in $\sim 15-30 \mathrm{wt} \%$ depending on the nature of the item. ${ }^{22,34}$. Nickel and zinc are also present in significant quantities, along with lead and tin in solder alloys used to attach surface mount device (SMD) components to boards.

\subsection{The 'global WEEE problem'}

\subsubsection{The scale of the problem - global WEEE generation}

WEEE is the fastest growing waste stream on the planet ${ }^{1,2}$, it accounts for $5 \%$ of all municipal solid waste ${ }^{3}$ with global generation currently at 48.9 million tonnes/year ${ }^{31}$ and increasing by 3-5\% annually. ${ }^{35}$ Within the EU, annual WEEE generation has exceeded 10 million tonnes/year with a projected increase to 12 million tonnes/year by $2020 .{ }^{36}$ In the UK WEEE generation has reached $\sim 1.5$ million tonnes/year. ${ }^{37} \mathrm{~A}$ breakdown of global e-waste generation by continent and per inhabitant in each continent is given in Table 1.6. This data highlights that WEEE is a global problem and that WEEE generation is concentrated in regions of greatest economic development. Per region, the most WEEE is generated in Asia, however per capita Europe leads WEEE generation, followed by Oceania. There is a direct correlation between GDP per capita and WEEE per capita. ${ }^{38}$ However, although the majority of WEEE is generated in OECD member countries, growth in the rate of WEEE generation in the developing world exceeds that in developed regions where consumer electronics markets are saturated. Rapid market penetration of electronics in developing nations with high populations will significantly increase the rate of WEEE generation in these regions, with WEEE generation in developing countries likely to soon exceed that in the developed world..$^{3,39}$ 
Table 1.6 Annual e-waste generation broken down by continent ${ }^{38}$

\begin{tabular}{lcc}
\hline Continent & Amount (million tonnes) & Amount (kg/inh) \\
\hline Africa & 1.9 & 1.7 \\
Americas (north \& South) & 11.7 & 12.2 \\
Asia & 16.0 & 3.7 \\
Europe & 11.6 & 15.6 \\
Oceania (Australia) & 0.6 & 15.2 \\
\hline
\end{tabular}

\section{Why is WEEE generated?}

The reasons that EEE enters the waste stream as WEEE are numerous. Damage to items and malfunctioning components is a common cause. Obsolescence is another. This is exemplified by trends for TV and display equipment in recent years. CRT displays have been replaced with flat screens (plasma and LCD technologies), which has driven generation of waste CRTs with many devices discarded by users while still functional. This rapid replacement of CRTs has occurred due to a combination of factors. Despite being available since 1988, rapid decreases in the price of flat screens since 2003 made them more affordable to consumers, resulting in rapid growth in sales..$^{40}$ Another reason for rapid replacement of technology is shorter lifetimes of new technologies. The lifespan of flat screens is only one third that of CRTs. Aesthetics changes such as the screen size may also result in replacement, with increasingly large screens becoming ever more popular as costs fall. Upgrade of existing technologies with new functionality and upgrade of peripheral technologies results in rapid replacement of devices by consumers who view current products as outdated. Continuing with the example of TVs, new video formats e.g. 4k replacing 1080p, which previously replaced 720p; storage media e.g. Blu-ray replacing DVD which replaced VHS; and signal transmissions changes e.g. the digital switchover, ${ }^{2}$ and the use of internet cables, WiFi or satellite in place of the traditional roof mounted TV aerial and cable have all resulted in replacement of functioning equipment. Incorporation of new functionality is also a driver for replacement as was seen following incorporation of VHS and then DVD players into CRT TVs. The increasing popularity of 'smart' TVs and 3D TVs as their cost falls will no doubt result in replacement of functional TVs into the future.

It has been suggested that consumer perception of their products in light of new updated products hitting the market may result in replacement of EEE. This has been proposed as the cause for the increase in the frequency with which the term 'iPhone slow' is searched on Google at the time new iPhone models hit the market; the 'slow iPhone phenomenon' (Figure 1.7).$^{41}$ Interestingly, the same trend is not observed for Android based devices such as the Samsung Galaxy. This suggests user perception of iPhone performance is not psychological, but rather devices are really running more slowly. Devices which have been in use for a period 
of time can be slowed down if basic 'housekeeping' is not periodically conducted. Additionally, the release of the iPhone always coincides with an update to Apple's operating system (iOS), which may be optimized for newer hardware and therefore result in a 'slowing' of older devices which receive the OS update. ${ }^{42}$ This is an example of how software development can result in consumer perception that devices are outdated, which could be an important factor in WEEE generation from computing products such as tablets, laptops and PCs.

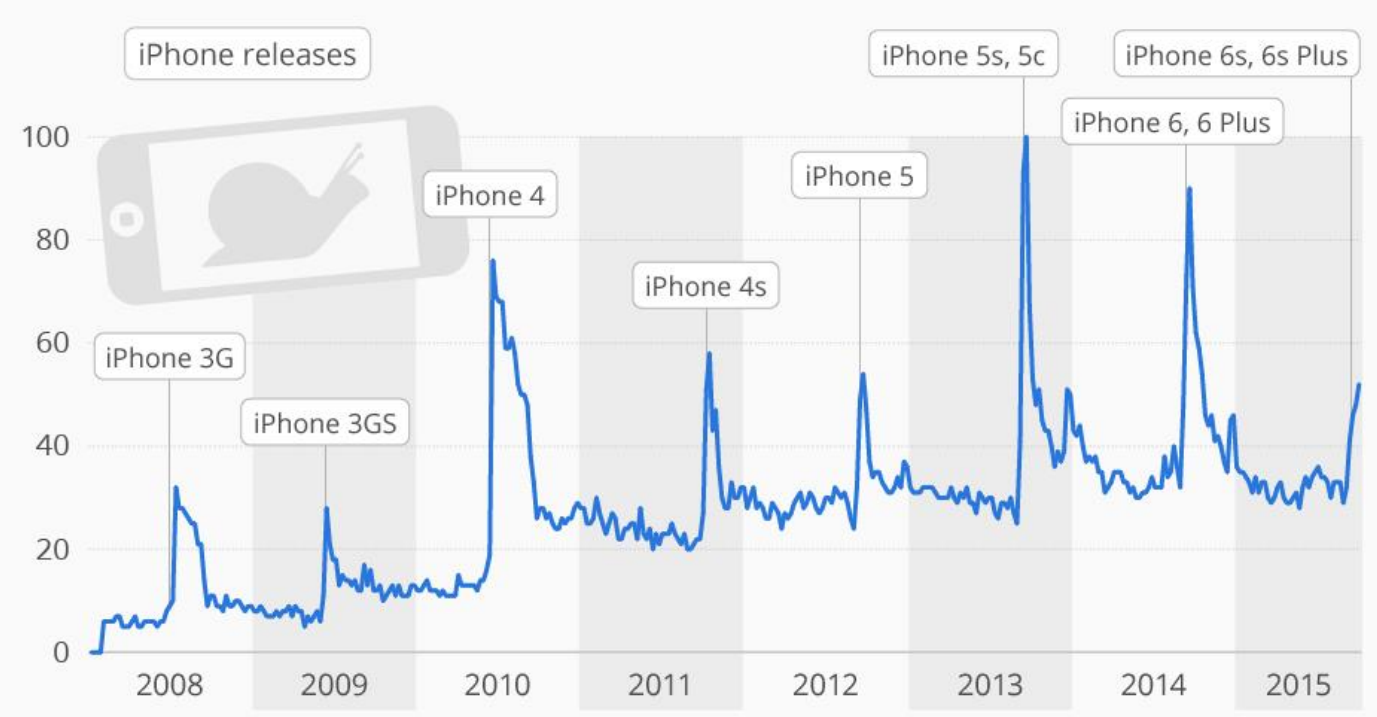

Figure 1.7 Worldwide Google searches for the term 'iPhone slow' correlated with release dates of new iPhone models $(100=\text { peak search volume) (Figure used with permission from statista.com })^{41}$

\subsubsection{The nature of the 'WEEE problem'}

\section{Impacts on human health \& the environment}

The majority of WEEE is either landfilled or exported to parts of the world where it is recycled in a crude and inappropriate manner. ${ }^{43}$ When this occurs, hazardous components of WEEE accumulate in the environment and within food chains. ${ }^{25,}{ }^{44}$ Persistent hazardous chemicals such as dioxins and other hazardous substances are generated as combustion products from 'open burning' of circuit boards and cables, a practice common in the developing world to recover valuable metals (Table 1.7). As a result, the improper treatment and management of WEEE possess considerable risks to human health and the environment. ${ }^{24}$ Much research has been conducted to determine the impacts on human health associated with WEEE, and this has been reviewed by Grant et al. ${ }^{45}$ 
Table 1.7 Toxic combustion products from $\mathrm{WEEE}^{29}$

\begin{tabular}{ll}
\hline Hazardous compound & Generation mechanism \\
\hline Polycyclic aromatic hydrocarbons (PAHs) & $\bullet \quad$ Combustion product \\
Polyhalogenated aromatic hydrocarbons (PHAHs) & $\bullet \quad$ Low temperature combustion product \\
$\begin{array}{l}\text { Polychlorinated dibenzo-p-dioxins (PCDDs) } \\
\text { Polychlorinated dibenzofurans (PCDFs) }\end{array}$ & $\bullet \quad \begin{array}{l}\text { Low temperature combustion products } \\
\text { of PVC \& other plastics }\end{array}$ \\
\hline
\end{tabular}

Nowhere have the detrimental impacts of poor recycling practice impacted the environment and local population to a greater extent than in Guiyu City and its surrounding region in Guangdong province, China. This is the largest WEEE recycling site in the world. Here environmentally detrimental 'backyard' WEEE recycling practices are widespread, including open burning to reduce volumes and recover metals, and open acid digestion of components to recover precious metals. Waste acid containing high levels of heavy metals is dumped directly into the soil and rivers. Solder containing $\mathrm{Pb}$ and $\mathrm{Sn}$ is melted over makeshift coal grills to liberate valuable components form PCBs. These ongoing practices have resulted in contamination of the air, soil and water systems. Elevated levels of dioxins have been found in human milk, placentas and hair at sufficient levels to pose a serious health risk. Transfer of polychlorinated biphenyls via the food chain and water system to humans has been observed. Recycling workers have been shown to have median PDBE levels of $126 \mathrm{ng} / \mathrm{L}$ in their blood serum, with local residents in Guiyu at $35 \mathrm{ng} / \mathrm{L}, \sim 13$ and 3.5 time higher than residents in nearby regions. respectively. Samples of human hair from towns in the region demonstrate high levels of PBBs, PBDEs and polychlorinated biphenyls. Tragically, children in the region have significantly elevated blood levels of $\mathrm{Pb}$ and $\mathrm{Cd}$, and lower cognitive abilities than children from nearby areas. Elevated levels of polychlorinated biphenyls are also found in breast milk. Elevated levels of $\mathrm{Cr}$ in umbilical cord blood due to exposure of mothers' to WEEE recycling correlates with DNA damage, which may result from exposure to Cr or other toxic substances from WEEE. Recycling workers in the region have a twenty times higher chromosomal aberration rate than normal. This all suggests that WEEE has been a source of genetic mutation and cytogenetic damage for the population. ${ }^{25}$

A major factor contributing to such impacts around the world is illegal exports of WEEE from the developed countries to Africa and Asia where it is treated in an inappropriate manner by the informal sector. ${ }^{8,24,25,43,46}$ Such practices persist due to a lack of education on the impacts of such practices, high unemployment rates, and a lack of regulation and enforcement of WEEE management. Proper management of WEEE, particularly when hazardous materials need to be properly processed, is costly and for this reason much material is illegally exported. 


\section{Implications for global resource-efficiency}

Currently the EEE industry is based primarily on linear economic models, with growth in the sector coupled to the linear throughput of material resources (Figure 1.8). Like all manufacturing based on the 'take-make-use-dispose' model, creation of new EEE requires finite natural resources to be mined and refined, which are then used in manufacturing to create products which find their way to consumers who dispose of them as waste at the end of their useful life. This process reduces natural capital, and at each stage of the process energy produced from fossil fuels is consumed and environmental emissions and waste energy and materials are generated. This contributes to global warming and climate change, as well as acidification of the oceans. ${ }^{47}$ Such models are no longer sustainable i.e. they fail to meet the needs of the present while maintaining the ability of future generations to meet their own needs. ${ }^{48}$

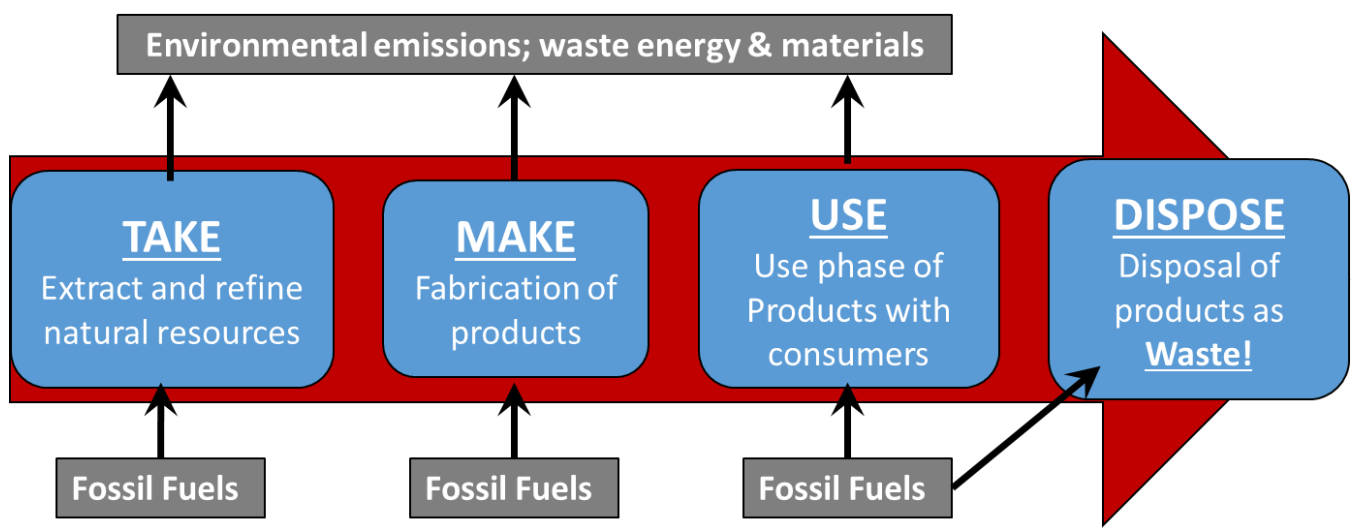

Figure 1.8 Material flows in the linear 'take-make-use-dispose' economy

Figure 1.9 indicates the position of countries according to their human development index (HDI), roughly equivalent to standard of living, and ecological footprint per capita. The dashed horizontal lines represent the planet's available biocapacity per person. As countries have developed economically they have moved across the plot according to the trend indicated by the red arrow. The increasing environmental impact with increasing HDI correlates strongly with resource use as demonstrated by Figure $1.10 .{ }^{49}$ For global sustainability, all nations must be within the green area highlighted in Figure 1.9, in which citizens would be living well, within the limits of the planet. The data suggests firstly, that current levels of resource consumption are unsustainable, and secondly, that a resource-efficient alternative to linear economic models, which decouples economic development from the use of finite resources, is necessary. 


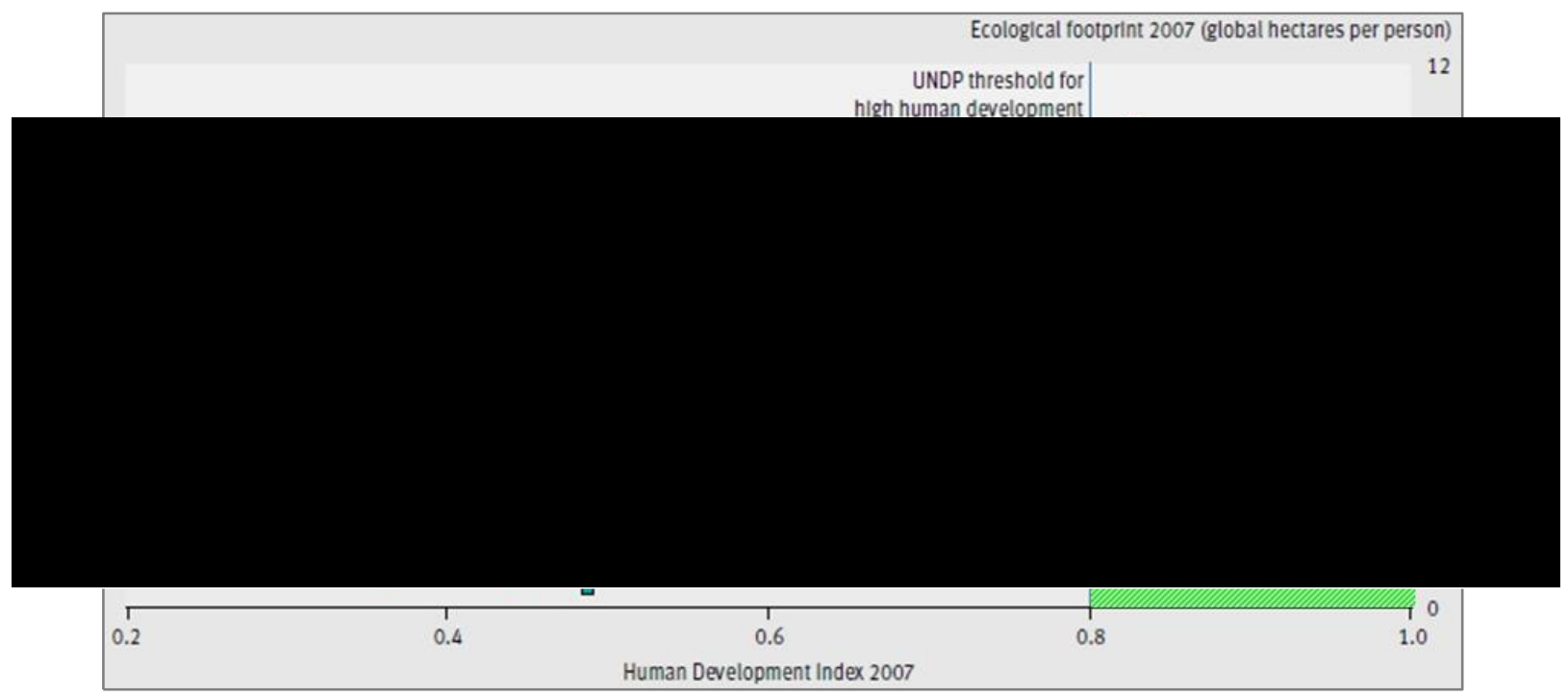

Figure 1.9 Human Development Index vs Ecological Footprint of the World's Nations in 2007. Taken from UNEP, 2012. ${ }^{50}$

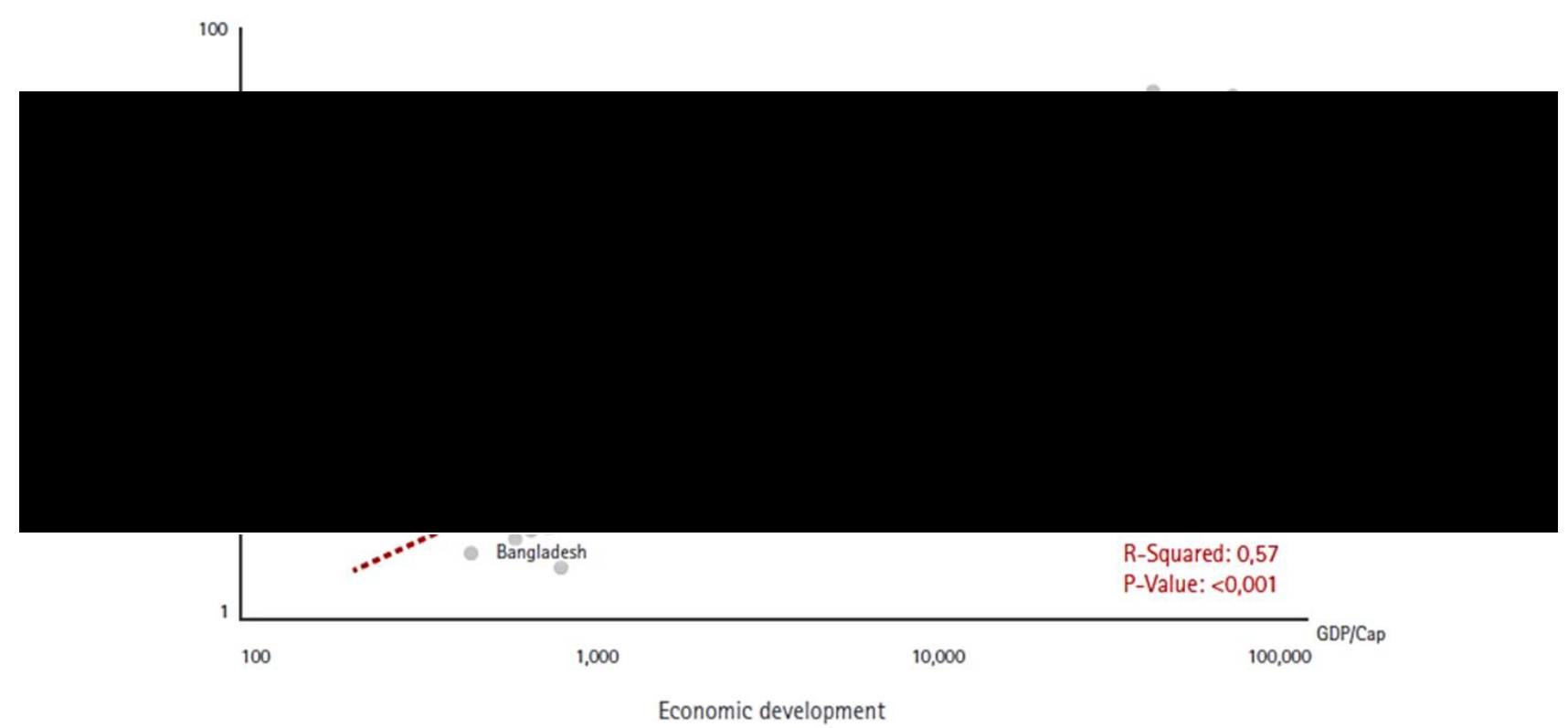

Figure 1.10 Correlation between resource use and GDP/captia of countries(image courtesy of Accenture, permission pending $)^{49}$

The correlation between resource consumption, economic development and WEEE generation is clear. As the planet's fastest growing waste stream, the contribution of WEEE to the unsustainable use of resources by society must be addressed. The rate at which EEE is discarded contributes to global resource depletion; discarded items must be replaced with new ones manufactured from raw materials resulting in accelerated resource consumption and pollution. ${ }^{2}$ For example, producing a single $32 \mathrm{MB}$ RAM module requires $32 \mathrm{~L}$ of water, 1.6 
$\mathrm{kg}$ of fossil fuels, $700 \mathrm{~g}$ of gasses and $72 \mathrm{~g}$ of various different chemicals. ${ }^{36}$, and at least 240 $\mathrm{kg}$ of fossil fuels are consumed in manufacturing a whole PC and monitor. ${ }^{50}$

\section{Materials criticality}

In recent years, the issue of materials 'criticality' has received a great deal of attention from governments and industry around the world in light of growing concerns over global resource security. Numerous assessments have been conducted to evaluate the economic importance of particular materials to economies, sectors and organisations, and the risk of future supply bottlenecks for each material (Figure 1.11). ${ }^{21,51,52}$ Those materials found to have high risk to supply and high economic impacts associated with supply shortage are determined to be 'critical' raw materials (CRMs). A prominent example of a criticality evaluation is the recent 2013 evaluation by the EC which generated the 'EU20' list of critical raw materials (Table $1.8) .^{53}$

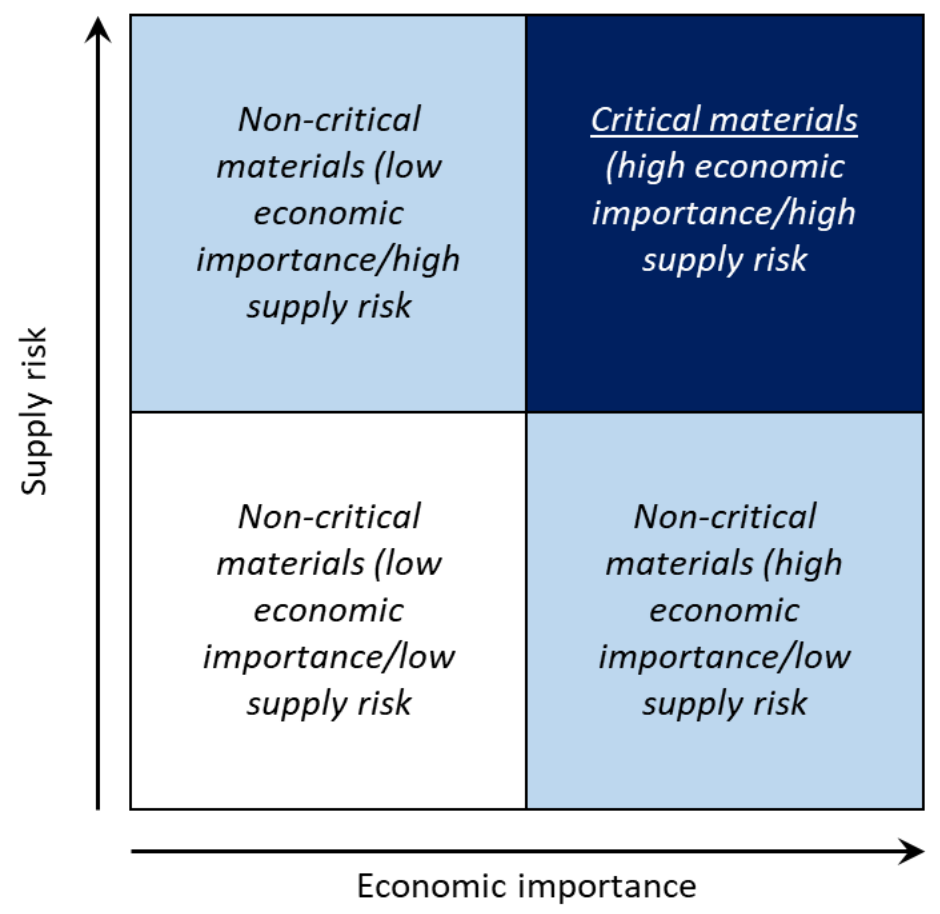

Figure 1.11 Schematic criticality plot for evaluation of materials criticality (adapted with permission from Frenzel et al., 2017) ${ }^{54}$

Table 1.8 The 'EU20' list of critical raw materials

\begin{tabular}{ccccc}
\hline Antimony & Beryllium & Borates & Chromium & Cobalt \\
Coking coal & Fluorspar & Gallium & Germanium & Indium \\
Magnesite & Magnesium & Natural Graphite & Niobium & PGMs \\
Phosphate Rock & REMs (Heavy) & REMs (Light) & Silicon Metal & Tungsten \\
\hline
\end{tabular}

REMs: Rare earth metals; PGMs: platinum group metals 
Technology metals are consistently found to be critical, for a number of reasons. Some are scarce in the earth's crust, such as Ga, In, and Sb which are used in smartphones (Figure 1.12).

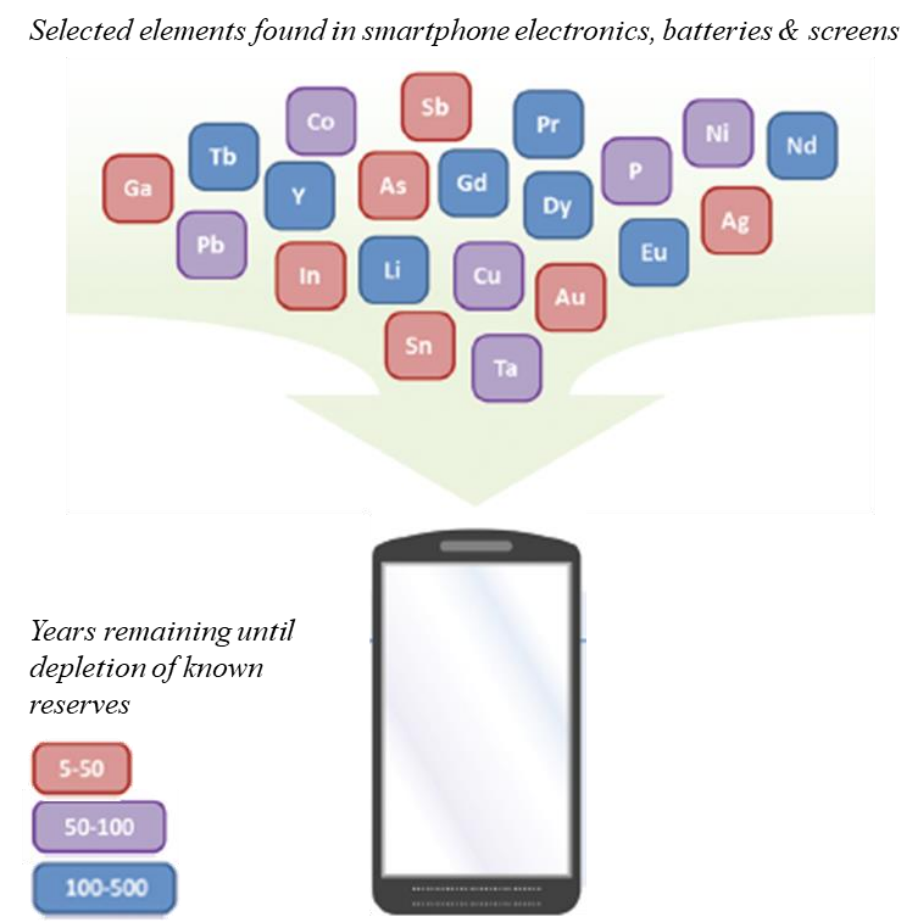

Figure 1.12 Elemental resources used in smartphones and times until their depletion based upon current rates of extraction from known reserves (adapted from Clark et al., with permission) ${ }^{55}$

Global reserves for these metals are likely to be exhausted within the next decade based on current rates of consumption and known reserves. ${ }^{55}$ Production for many CRMs is concentrated in single regions of the globe, for example China is responsible for $>95 \%$ of rare earth metals (REMs) production and has recently imposed export restrictions. Secondly, these materials are often produced as by-products of other mining operations e.g. indium which is a by-product of refining $\mathrm{Zn}$ and $\mathrm{Pb}$ sulphide ores (Figure 1.13). For this reason, global production cannot easily respond to rising demand. Rather, the production of these materials is dependent upon the markets of the major target metals mined. ${ }^{56}$ Often CRMs are produced in regions of conflict or political instability, which adds to supply risk e.g. over half of the world's cobalt is supplied from DRC. 


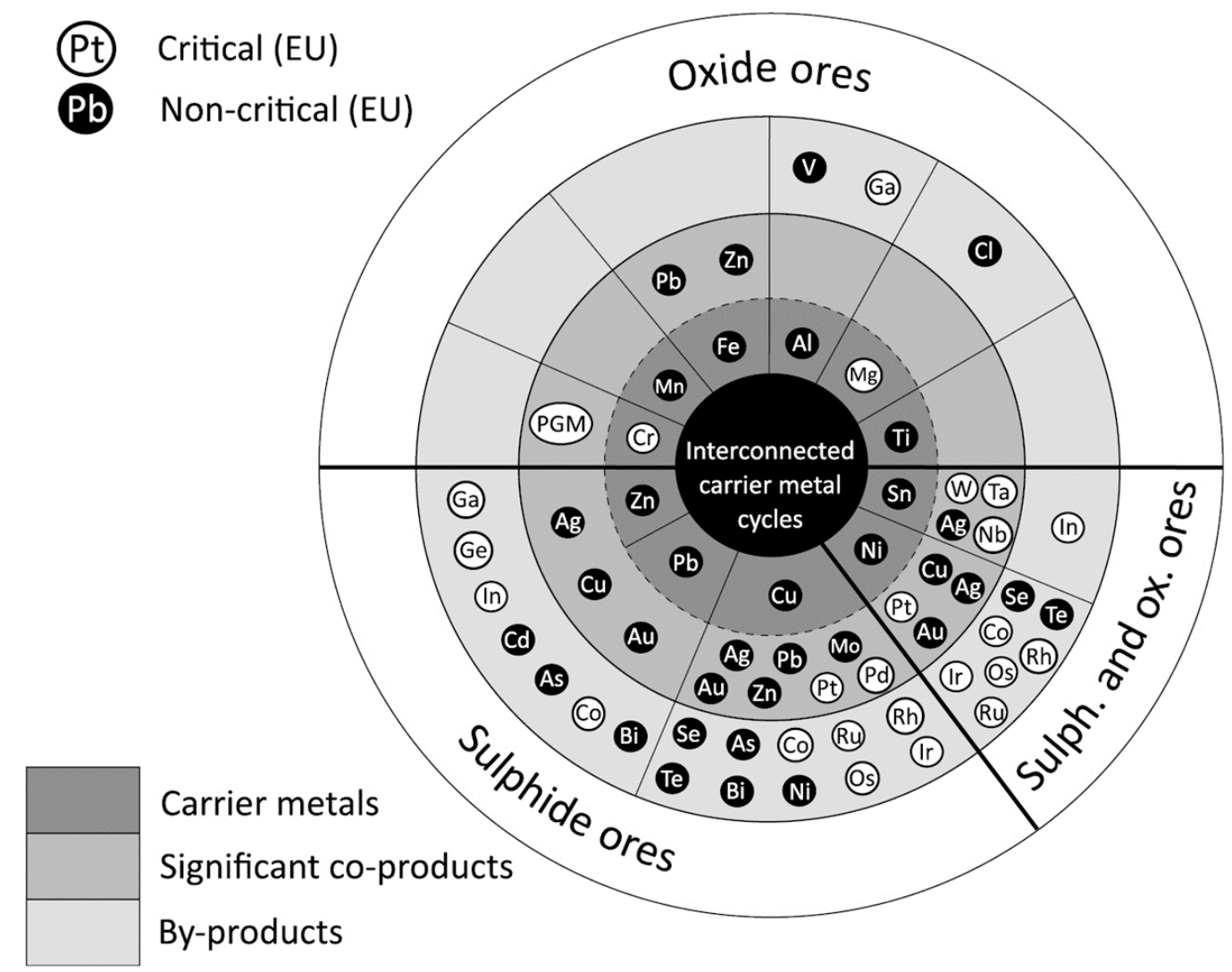

Figure 1.13 The metal wheel - a schematic illustration of the geological relationships between different industrial metals and their co- and by-products; criticality is assigned according to the EU20. (C) IOP Publishing. Reproduced with permission. All rights reserved $)^{54}$

Table 1.9 Growth in element use projected to 2030 in emerging technologies. The 2006 and 2030 indicators show the proportion of demand compared to 2006 production e.g. Ga: indicator $2006=$ $28 / 152=0.18$ and indicator $2030=603 / 152=3.97^{57}$

\begin{tabular}{|c|c|c|c|c|c|}
\hline Raw material & $\begin{array}{c}\text { Production }^{\mathbf{A}} \\
\text { (t) }\end{array}$ & $\begin{array}{c}\text { Demand } 2006 \\
\text { (t) }\end{array}$ & $\begin{array}{c}\text { Demand } 2030 \\
\text { (t) }\end{array}$ & $\begin{array}{c}\text { Indicator } \\
2006\end{array}$ & $\begin{array}{c}\text { Indicator } \\
2030\end{array}$ \\
\hline Gallium & $152^{\mathrm{E}}$ & 28 & 603 & $0.18^{\mathrm{A}}$ & $3.97^{\mathrm{A}}$ \\
\hline Indium & 581 & 234 & 1,911 & $0.40^{\mathrm{A}}$ & $3.29^{\mathrm{A}}$ \\
\hline Germanium & 100 & 28 & 220 & $0.28^{\mathrm{A}}$ & $2.20^{\mathrm{A}}$ \\
\hline Neodymium $^{\mathrm{F}}$ & 16,800 & 400 & 27,900 & $0,23^{\mathrm{A}}$ & $1.66^{\mathrm{A}}$ \\
\hline Platinum $^{\mathrm{G}}$ & 255 & very small & 345 & - & $1.35^{\mathrm{A}}$ \\
\hline Tantalum & 1,384 & 551 & 1,410 & $0.40^{\mathrm{A}}$ & $1.02^{\mathrm{A}}$ \\
\hline Silver & 19,051 & 5,342 & 15,823 & $0.28^{\mathrm{A}}$ & $0.83^{\mathrm{A}}$ \\
\hline Cobalt & 62,279 & 12,820 & 26,860 & $0.21^{\mathrm{A}}$ & $0,43^{\mathrm{A}}$ \\
\hline Palladium $^{\mathrm{G}}$ & 267 & 23 & 77 & $0.09^{\mathrm{A}}$ & $0.29^{\mathrm{A}}$ \\
\hline Titanium & $7,211,000^{\mathrm{C}}$ & 15,397 & 58,148 & 0.08 & 0.29 \\
\hline Copper & $15,093,000$ & $1,410,000$ & $3,696,070$ & 0.09 & 0.24 \\
\hline Ruthenium $^{\mathrm{G}}$ & $29^{\mathrm{E}}$ & 0 & 1 & 0 & 0.03 \\
\hline Niobium & 44,531 & 288 & 1,410 & 0.01 & 0.03 \\
\hline Antimony & 172,223 & 28 & 71 & $<0.01$ & $<0.01$ \\
\hline Chromium & $19,825,713^{\mathrm{B}}$ & 11,250 & 41,900 & $<0.01$ & $<0.01$ \\
\hline
\end{tabular}

${ }^{\mathrm{A}}$ Data updated by the BGR based on new information; ${ }^{\mathrm{B}}$ Chromite; ${ }^{\mathrm{C}}$ Ore concentrate; ${ }^{\mathrm{D}}$ Consumption; ${ }^{\mathrm{E}}$ Estimation of full production in China and Russia; ${ }^{\mathrm{F}}$ rare earth metal; ${ }^{\mathrm{G}}$ platinum group metals 
Table 1.9 sets out estimates of demand for various scarce technology metals and their compounds used in emerging sustainable technologies; its final column shows how 2030 demand for such metals compares to 2006 demand and 2006 production for all uses. At current rates of production, by 2030 demand for $\mathrm{Ga}, \mathrm{In}, \mathrm{Ge}, \mathrm{Nd}, \mathrm{Pt}$ and Ta will have exceeded levels of production as they were in 2006.

Many CRMs are not easily substituted and recycling rates of most CRMs from WEEE are low, ${ }^{58-60}$ which means that use of these metals in EEE results in considerable depletion of finite CRM reserves. This is a problem not only for economies that rely on continued supply of CRMs, but also for humanity's ability to deploy existing and emerging green technologies which are vital to strategies for achieving sustainability with our planet. ${ }^{61,62}$

\subsection{The value of WEEE}

The ever-growing quantities of WEEE present a considerable opportunity if properly managed. WEEE itself is a source of reusable EEE, and a rich secondary material resource. For this reason WEEE is considered an 'urban mine' or 'mine above ground' ${ }^{63-65}$ If effectively exploited, WEEE can help enhance global resource-efficiency, reduce global emissions, mitigate materials criticality, and generate economic and social value. ${ }^{34}$

WEEE has an economic value. This is derived from financial returns following the sale of reusable equipment and components, or recovered materials. The material value of an items is based upon the revenue generated from sale of materials recycled from WEEE which is dependent upon market values of those materials. Figure 1.14 indicates the estimated value of major metals contained within WEEE in Australia, 50\% of which is attributable to precious metals and copper. In PCBs, the proportion of the value represented by these metals is higher still. Recovery of these metals has historically provided $\sim 95 \%$ of the revenue from WEEE recycling (Figure 1.15), and exploitation of this material value has been the essential financial driving force for WEEE recycling to date. 
A)

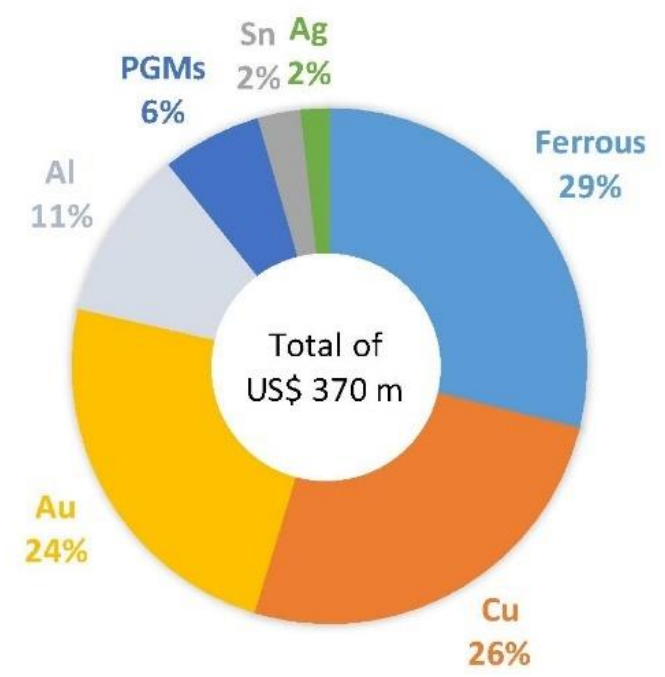

B) Waste PCBs

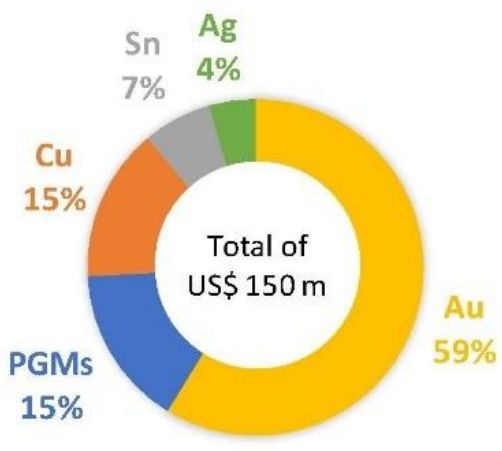

Figure 1.14. Estimated value of major metals contained in WEEE in Australia in 2014: (A) total WEEE; (B) waste PCBs (reused with permission from Elsevier) ${ }^{66}$

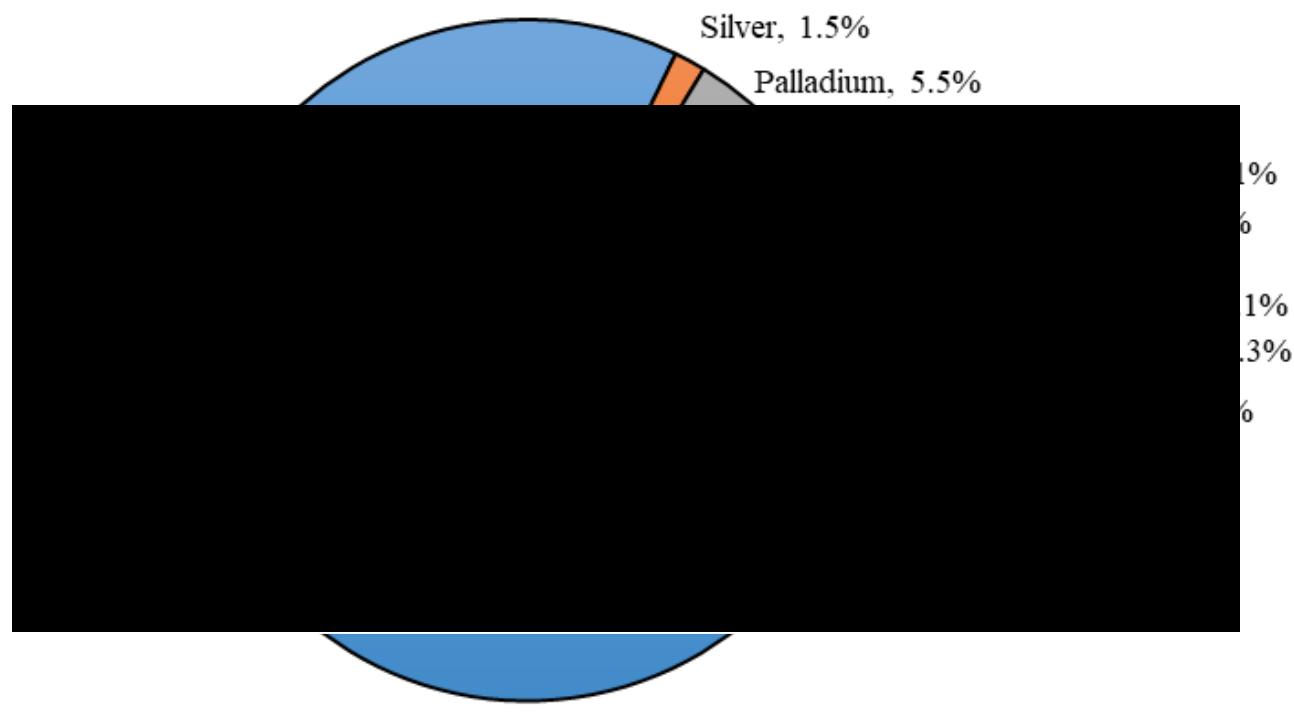

Figure 1.15 Revenue breakdown by material from WEEE adapted from (Sodhi and Reimer, 2001. ${ }^{30}$

Reusable equipment $\&$ components have a value greater than their material value, this is their utility value. The functionality of items grants higher economic value, for example, a functioning reused laptop is worth more than its material value.

There are additional aspects to the value of WEEE, which are not necessarily linked to its economic value. WEEE has strategic value by virtue of its CRM contents. Although the 
material value of CRMs is low, access to such materials mitigates materials criticality and increases economic resilience. Although sale of recovered CRMs will not deliver high financial returns, access to these materials is of importance for reasons previously outlined.

WEEE also has environmental value. Within each item of WEEE, considerable embodied environmental costs exist, associated with lifecycle impacts of its production from primary resources. Reuse of whole items offsets demand for new EEE and avoids the necessity to incur these impacts during the production of new EEE. Recycling of materials represents a lower environmental value as environmental impacts result from the manufacturing process. However, many materials can be recovered at lower environmental cost than producing them from primary resources. This is particularly true for metals with high primary production environmental impacts such as precious and platinum group metals (Table 1.10). ${ }^{67,68}$ Recovery of 70,000 tonnes of metals at Umicore Precious Metals Refining (Hoboken, Belgium) has resulted in a saving of $1 \mathrm{Mt}$ of $\mathrm{CO}_{2}$, i.e. $79 \%$ of the $\mathrm{CO}_{2}$ that would have been generated by primary production. ${ }^{69}$

Table 1.10 Primary carbon footprint of selected elements in WEEE goods ${ }^{16}$

\begin{tabular}{cccc}
\hline Metals used in EEE & $\begin{array}{c}\text { Primary production intensity } \\
\text { (t CO2/t metal) }\end{array}$ & $\begin{array}{c}\text { Demand for EEE } \\
\text { (t/a) }\end{array}$ & $\begin{array}{c}\mathbf{C O}_{2} \text { emissions } \\
\text { (Mt/a) }\end{array}$ \\
\hline $\mathrm{Au}$ & 16,991 & 327 & 5.56 \\
$\mathrm{Pt}$ & 13,954 & 8 & 0.11 \\
$\mathrm{Ru}$ & 13,954 & 16 & 0.22 \\
$\mathrm{Pd}$ & 9,380 & 30 & 0.28 \\
$\mathrm{Ag}$ & 144 & 4,917 & 0.71 \\
$\mathrm{In}$ & 142 & 717 & 0.10 \\
$\mathrm{Sn}$ & 16 & 129,708 & 2.09 \\
$\mathrm{Co}$ & 8 & 16,470 & 0.13 \\
$\mathrm{Cu}$ & 3 & $7,174,000$ & 24.39 \\
\hline
\end{tabular}

WEEE represents a source of social value. The exploitation of the economic value of WEEE will provide employment opportunities within the waste management sector. Finally, WEEE has intangible/emotional value. Despite owners ceasing to need EEE due to replacement with updated technology, they will often be reluctant to part with devices, perhaps because of some emotional attachment or the idea that it 'may come in handy sometime'. In this way WEEE has a unique value to its owner, and many homes have a stockpile of unused EEE. A recent customer behaviour survey conducted in the UK by the Waste \& Resources Action Programme (WRAP) revealed over $60 \%$ of 4,000 participants purchasing new EEE have items at home they no longer use. ${ }^{37}$ 'Stockpiling' of WEEE in this way represents a barrier to recycling. ${ }^{2}$ 
These elements of economic, social and environmental value are synergistic; as value in one area is derived from WEEE, so benefits will be afforded in other areas. Addressing the global WEEE problem and enhancing the sustainability of the EEE sector overall relies upon the ability to exploit economic value from WEEE, and the magnitude of benefits afforded will be related to the economic value derived. ${ }^{48,70}$

\subsection{Solutions to the 'global WEEE problem'}

Any solution to the 'global WEEE problem' must address two issues. Firstly, how can the increasingly large volumes of existing WEEE and EEE in the market place which will soon reach end-of-life (EoL) be managed in a sustainable and responsible manner; and secondly, what can be done to enhance the industrial ecology of the EEE industry and ensure that the complex issues associated with managing current WEEE are avoided when new EEE, yet to emerge on the market, reaches EoL? Any solution must result in maximum possible resourceefficiency whilst minimising environmental impacts.

\subsubsection{Circular economy - the optimum future scenario}

Circular economy (CE) is a concept which describes a sustainable manufacturing economy inspired by natural systems which is restorative by design ${ }^{47} \mathrm{CE}$ is regenerative by design and replaces the concepts of 'end-of-life' (EoL) and 'waste' with 'restoration' and 'resources'. Wastes therefore are viewed as valuable raw material. This represents an alternative to linear economic models with growth decoupled from consumption of finite material resources, and materials retained in circular flows within economies, cycled repeatedly in successive product lifecycles thereby remaining economically productive (Figure 1.16).

In a circular economy products are designed for longevity so that they may be maintained, repaired and upgraded by users to extend their useful life for as long as possible. Products may also be leased to users with ownership maintained by a service provider for collection and take-back for re-use once users cease to require them. If re-use is not possible, products may be sent back to manufacturers for refurbishment or remanufacturing to retain components in new products. Only when re-use of components is no longer possible are they recycled to recover materials for manufacturing new components. Leakage of materials from the economy as waste is minimised through efficient collection of items at EoL. Industrial symbiosis in which by-products of manufacturing in one organisation become the raw materials for manufacturing in another, ${ }^{71}$ is used to minimise demand for primary raw materials. This presents new opportunities for valorisation of WEEE and exploitation of its inherent value. 


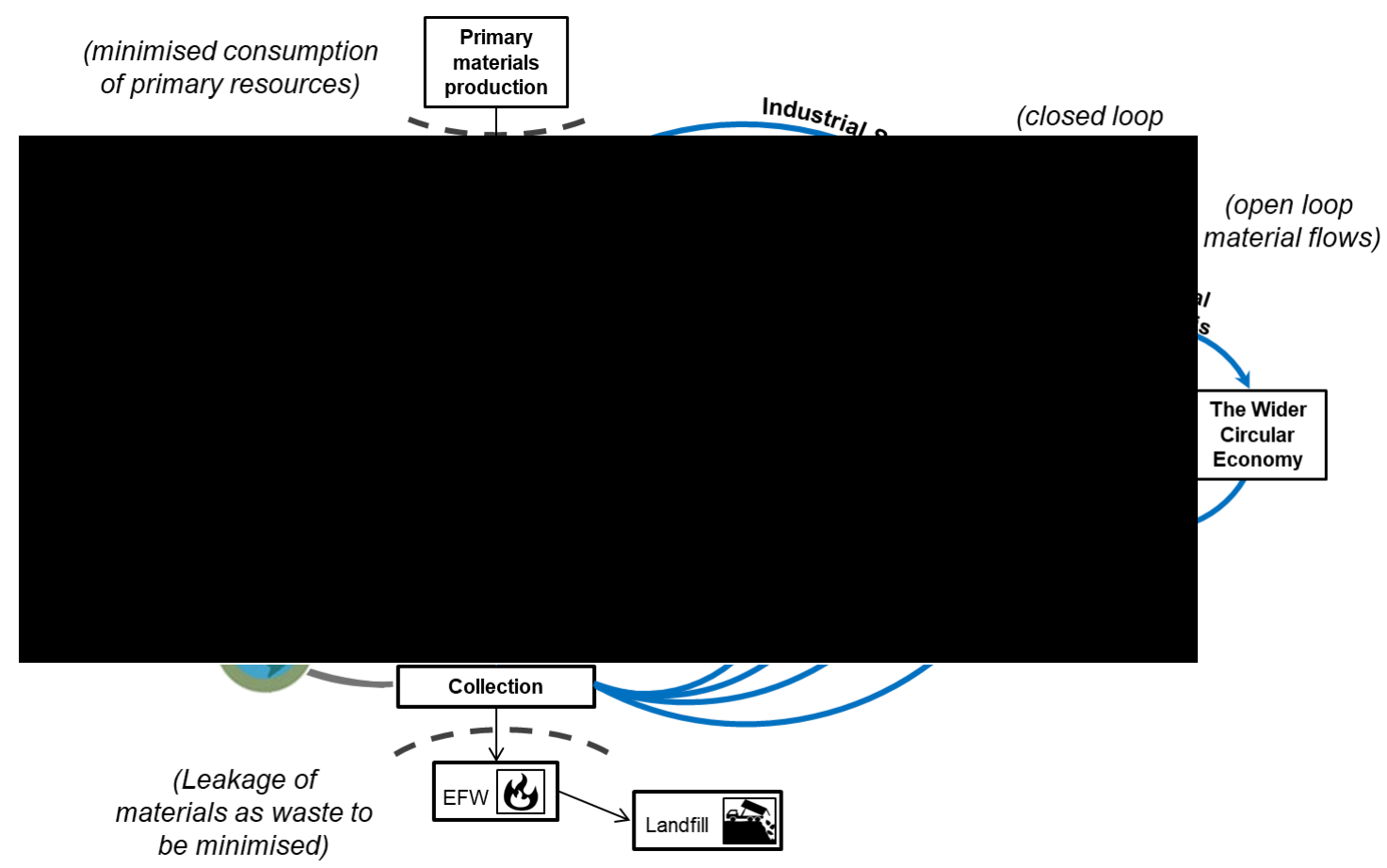

Figure 1.16 Material flows within the circular economy (adapted from Ellen MacArthur Foundation) ${ }^{47}$

Cascading components and materials across multiple product lifecycles presents alternative, often higher value routes for materials compared to traditional recycling, and in this way greater economic value may be derived. Primary raw materials may be replaced with biological materials which are renewable and can go back to the biosphere without negative environmental impacts. Product design to accommodate effective refurbishment, remanufacturing, and efficient disassembly and materials recovery are optimised. Elimination of hazardous materials which are detrimental to human health $\&$ the environment and add high cost to EoL treatment; and the use of renewable energy are key features of circular economy. A conducive legislative environment, appropriate business models which valorise products past their point of initial sale, and appropriate reverse logistics which ensure effective return of products from users are also important for a viable circular economy. ${ }^{49}$

Figure 1.16 shows the circular economy in diagrammatic form. The smaller the closed loop the greater material resources retained, the greater their economic productivity, and the greater retention of embodied economic and environmental value. It has been estimated that a smartphone can be resold for reuse at $\sim 60 \%$ of its original price, far greater value than can be derived through recycling. ${ }^{47}$ In addition, the longer that EEE and its materials are cycled within the economy, the greater the economic, environmental and social benefits. Recovered materials and components are usually obtainable at lower cost than virgin ones, and so materials cost savings for manufacturing will result from circular economy. The economic and 
social potential of circular economy is significant. In Wales alone, a transition to an advanced state of circular economy has been predicted to be worth $£ 2$ bn to the economy and is projected to deliver 30,000 jobs. ${ }^{72,73}$ Recently the EMF conducted a circularity analysis of products with different lifecycles and concluded that medium lived products (including EEE) represent the 'sweet spot' for circularity interventions i.e. it is from these goods that greatest benefits can be derived in a circular economy. ${ }^{47}$

The EC adopted 'the circular economy package' on $2 / 12 / 15$, which included amendments to waste directives and set long term targets to divert material from landfill and increase reuse $\&$ recycling. In the interests of achieving closed loop product lifecycles the package included an action plan to support circular economy at each stage of product lifecycles through manufacturing, use and waste management with emphasis on feeding secondary raw materials back in to the economy. This aims to add momentum to the transition towards circular economy. ${ }^{74}$

\subsubsection{Management of WEEE, the current scenario}

Although product lifecycle optimisation and design to support circular practices may in the future provide the means to overcome the global WEEE problem, the transition to circularity will be gradual. However, the way in which WEEE is currently managed is conducive to circular economy in many respects. Legislation is in place which has resulted in infrastructure for collection and recycling of WEEE. Reuse of many items is common, particularly higher value items such as ICT equipment as a result of political impetus and recognition of the economic and social value. ${ }^{75,76}$ Efficient recovery processes for many materials in WEEE are in place, particularly for copper and precious metals. ${ }^{12,}$ 14, 77-82 Through recovery of these materials from WEEE, much economic value is derived with associated social and environmental benefits. On the other hand, there is much room for improvement in many areas of WEEE management. This is partially due to the fact that recycling of WEEE has been established as a means of dealing with the potential hazards of WEEE, rather than its efficient exploitation as a material resource. The following sections outline the status of legislation, collection and recycling, with a focus on recovery of 'high-tech' metals and identifying areas for improvement in resource-efficiency.

\section{Legislation and policy}

In light of the issues associated with WEEE the EU introduced and recently recast the WEEE directive $e^{4,5}$ and the RoHS directive, ${ }^{83,84}$ which aim to enhance the industrial ecology and resource-efficiency of the EEE industry. The WEEE directive aims to establish infrastructure for appropriate management of WEEE by diversion from landfill to authorised approved 
treatment facilities (AATFs) employing best available treatment, recovery and recycling techniques (BATRRT) ${ }^{85}$ It achieves this by banning landfilling of WEEE, and establishing statutory targets for collection, recycling and preparation for reuse. The WEEE directive places emphasises on the waste hierarchy, prioritising reuse (Figure 1.17). This is all conducive to circular economy, in fact amendments to the directive were a crucial part of the recent EC 'circular economy package' (along with amendments to the waste framework directive, batteries directive and other waste legislation) designed to stimulate progression towards an advanced state of circular economy. ${ }^{86}$ The directive also imposes extended producer responsibility (EPR) on manufacturers and importers of EEE, implementing the 'producer pays' principle by making producers \& importers of EEE responsible for their product EoL costs. This aims to ensure that cost is not a barrier to recycling and also provides a financial incentive to producers to improve product design for reuse, refurbishment and recycling (DfRR) which should reduce EoL costs and the financial burden of compliance. Progress in this regard has been slow however, in part because of the way in which EPR is implemented in member states. This is done in one of two ways: as individual producer responsibility (IPR), or as collective producer responsibility (CPR) as is the case in the UK. IPR makes individual producers directly responsible for the EoL costs of their products, whereas CPR makes producers collectively responsible for EoL costs of all collected WEEE in the categories which their products fall. CPR is less effective in encouraging DfRR than IPR, and therefore represents a barrier to circular economy. ${ }^{72}$

\section{WASTE HIERARCHY}

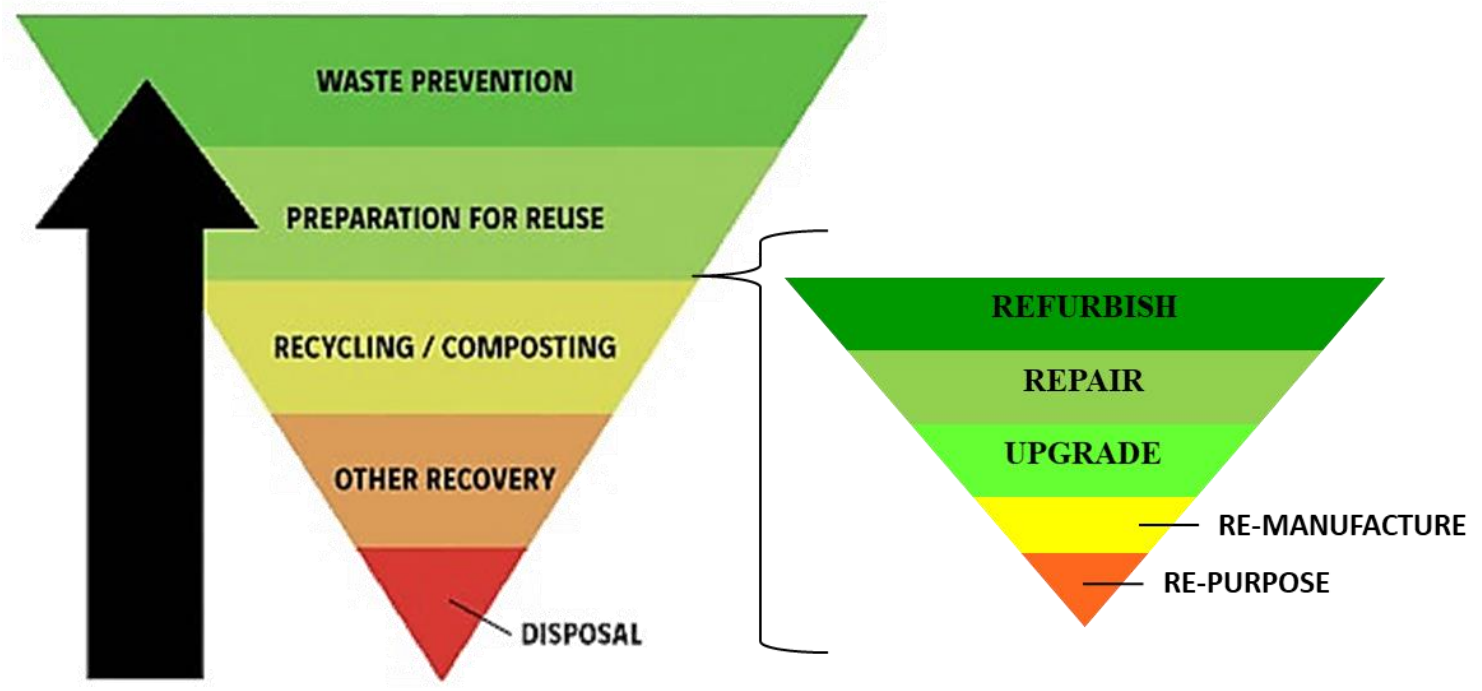

Figure 1.17 The waste hierarchy with refurbishment, repair, upgrade, remanufacturing and repurposing operations incorporated. 
The RoHS directive limits the use of toxic substances in EEE imposing maximum permissible concentrations in new EEE placed on the market. This includes toxic heavy metals such as $\mathrm{Hg}$, $\mathrm{Cd}$ and $\mathrm{Pb}$, and brominated flame retardant compounds. In addition to health and environmental improvements, reduced hazardous material content will also reduce recycling $\operatorname{costs}^{87}$ and make illegal export less likely. This improves the chances of WEEE treatment with best practice in terms of resource-efficiency and environmental outcomes. Globally, many governments have introduced their own WEEE legislations which in the main are based on the WEEE and RoHS directives. ${ }^{31,88-96}$

The 'Resource Efficient Europe Flagship Initiative' is a key pillar of the Europe 2020 strategy for sustainable growth. ${ }^{97}$ More efficient recycling of materials from WEEE is an important component of this initiative. ${ }^{98}$ Much attention has been focussed on WEEE as a secondary resource of CRMs. ${ }^{19,}$ 20, 51, 53, 99-105

\section{WEEE management}

WEEE is managed via the recycling process chain (Figure 1.18).It is collected and delivered to pre-processing facilities (AATFs) where items are sorted, depolluted and disassembled to separate components into distinct material streams (e.g. bulk metals, plastics, batteries, LCDs, cables etc.) rich in target materials of the subsequent recovery processes to which they are sent. ${ }^{106,107}$ Items identified as suitable for reuse are diverted from the recycling stream for direct reuse, or reuse following refurbishment/repair. At this time, little remanufacturing of electronics takes place. Hazardous components such as CRTs, batteries, and fluorescent lamps, are diverted to processes for safe treatment and disposal. Pre-processing is carried out manually, or with automated or semi-automated processes. Precious metal, PGM and copper containing fractions are recovered using pyrometallurgical and/or hydrometallurgical processes. 


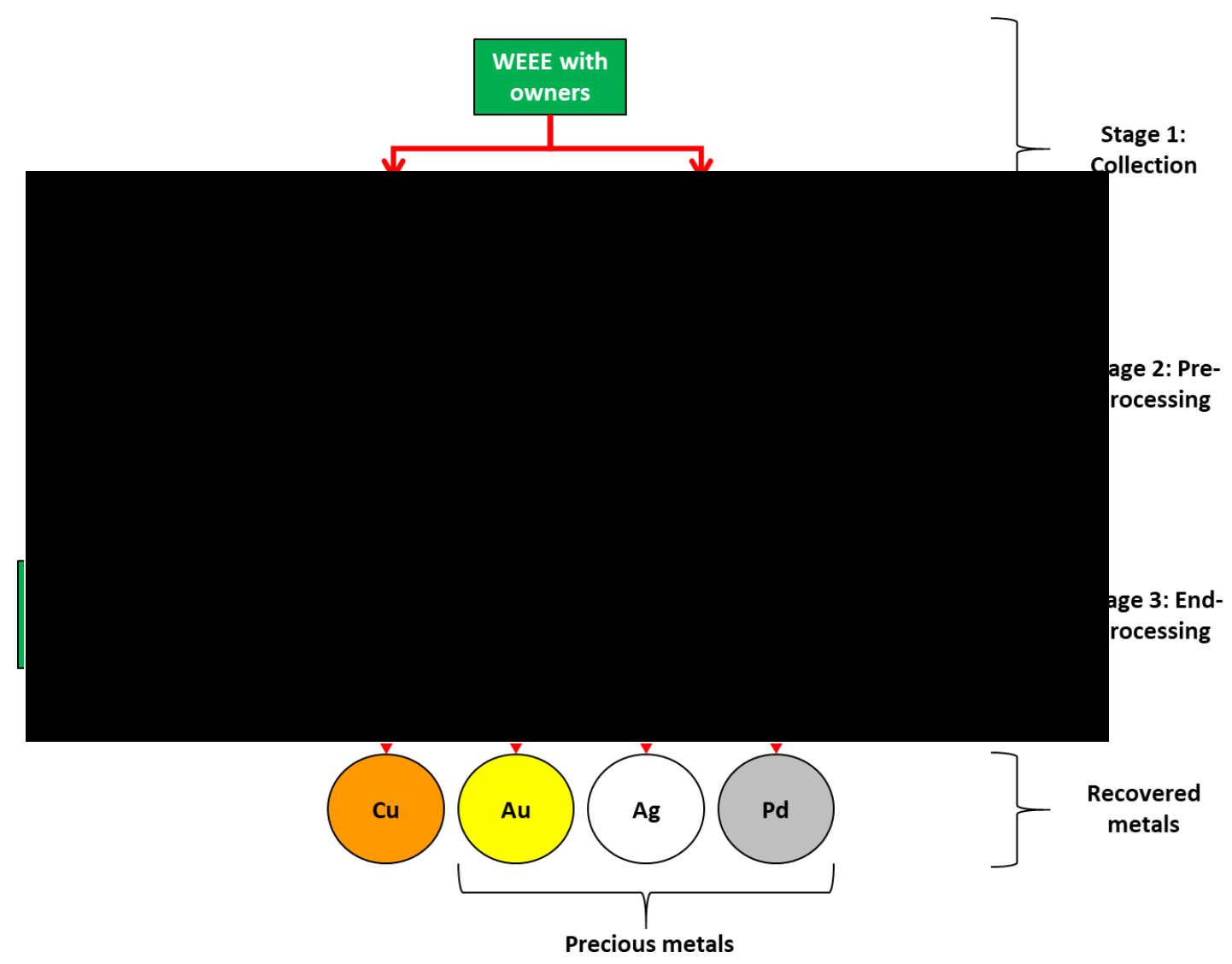

Figure 1.18 The recycling process chain with special focus on recovery of precious metals and copper (adapted from Chancerel et al. $)^{106}$

The efficiency with which WEEE is either reused, or materials are recovered is determined as the product of the efficiencies of individual stages of the process. Inefficiencies in any one stage may have a dramatic impact on bottom-line recovery efficiency of materials. ${ }^{106}$ As shown in Figure 1.19, low collection rates in the EU have been the limiting factor in the rate of reuse and recycling of WEEE. In the developing world, collection rates tend to be high, as the informal sector is generally very efficient in this regard. However, despite high collection rates, bottom-line recovery efficiencies of materials tend to be low due to the poor recovery efficiencies which are achieved with the crude and often hazardous recycling practices used in the informal sector. ${ }^{108}$

A 2012 study conducted by Oakdene Hollins for WRAP examined PM and CRM flows and associated value flows for these materials in WEEE within the UK. This highlighted that uncontrolled disposal of WEEE in residual waste streams results in considerable losses of materials and value from the urban mine. Shredding was shown to result in considerable loss of value, precious metals and CRMs. The report highlights that many CRMs present in materials sent to Europe for recovery are lost in the these recovery processes. ${ }^{109}$ 


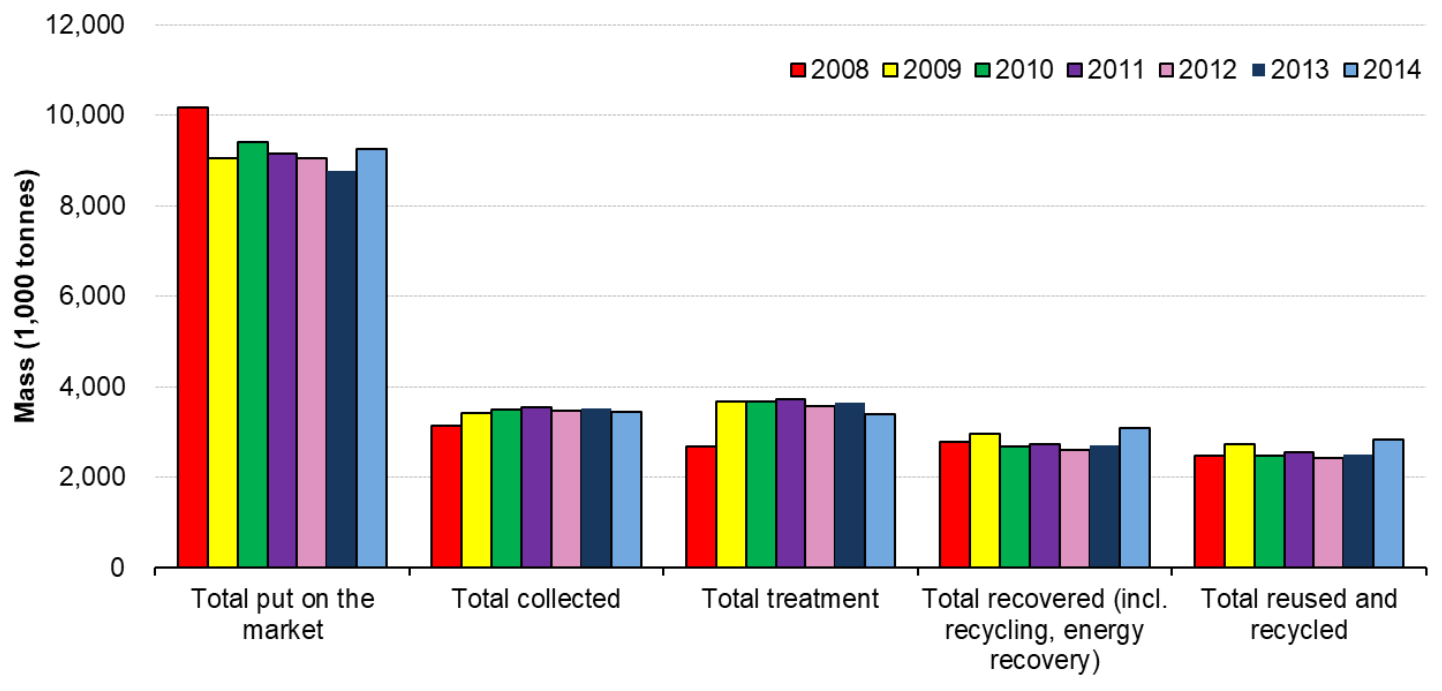

Figure 1.19 Electrical and electronic equipment (EEE) put on the market and waste EEE collected and treated in the EU from 2008-to 2014) ${ }^{110}$

Collection In Europe, EPR and statutory targets for member states imposed by the WEEE directive have resulted in establishment of collection infrastructure. EPR based reverse logistics systems are also operated around the world, with examples in Australia, Japan, Taiwan, South Korea and many of the US states, with numerous other countries, such as India, exploring how EPR may be incorporated into current regulations. ${ }^{111,112}$ Despite this, global collection rates remain low. Much of the world is still to develop sufficient reverse logistics systems and recycling infrastructure, and even those countries which have done so see low collection rates due to illegal exports of WEEE for 'reuse', stockpiling, and loss of items in residual waste streams. ${ }^{113}$ It is estimated that $80 \%$ of global WEEE is exported to Asia, with significant further quantities exported to Africa where BATRRT is not employed. ${ }^{2}$ Much of the UK's household WEEE, particularly small domestic appliances which are easily concealed within household residual waste streams, is still landfilled despite infrastructure for collection. It was estimated that 154,372 tonnes of WEEE was disposed of as residual waste in England during the 2010/11 financial year, ${ }^{114}$ equivalent to about one third of WEEE collected throughout the whole UK during that period. ${ }^{115}$ A 2012 study of residual waste arising in Denmark revealed that WEEE and batteries present constituted $16 \%$ and 39\% of quantities being collected through proper channels at that time, respectivley. ${ }^{113}$

The UK WEEE collection rate in 2016 was $35.6 \%$. Household WEEE is collected through distributor takeback schemes, or household waste recycling centres (civic amenities sites). The majority of WEEE generated is still unaccounted for. Figure 1.20 Indicates that despite infrastructure for collection, few EU member states have exceeded 50\% collection, with many 
considerably lower. Where WEEE is not collected, its inherent material resources and potential economic, social and environmental value are permanently lost.

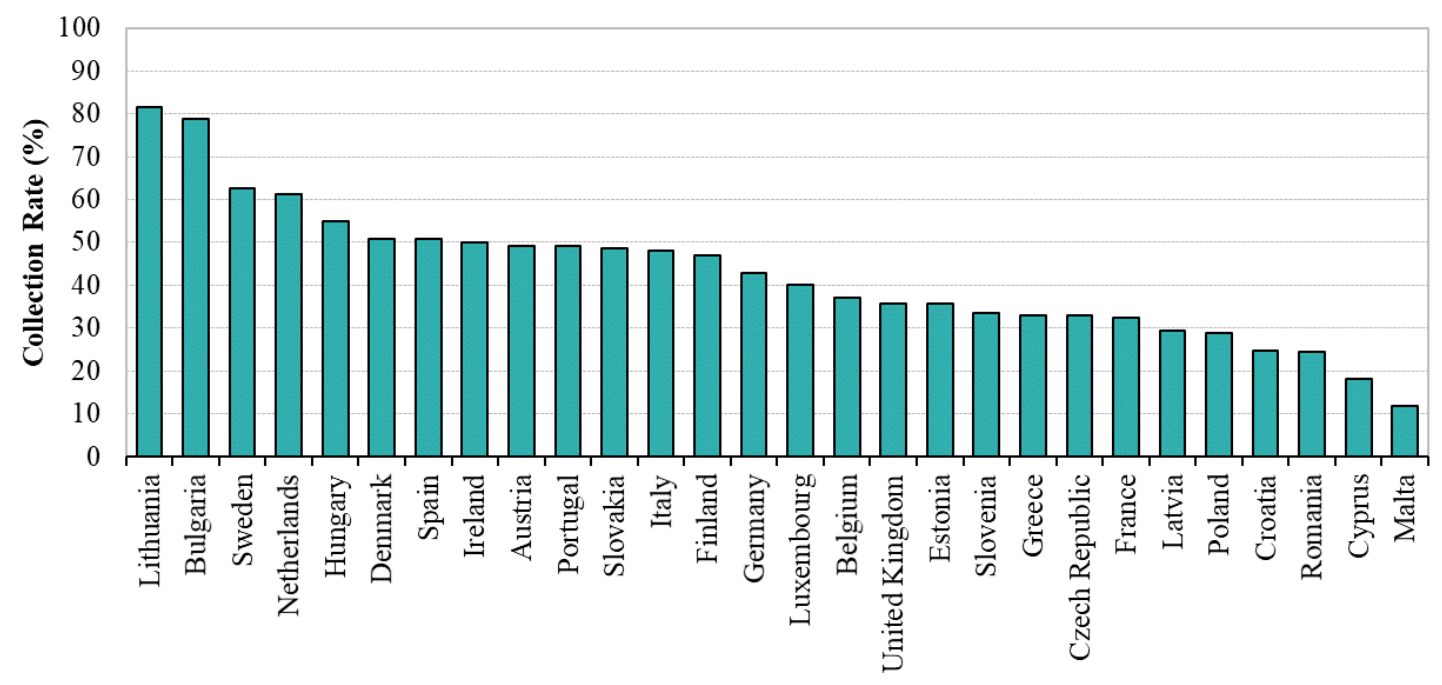

Figure 1.20 Total collection rate for WEEE in 2014 as a percentage of the average weight of EEE put on the market in the three preceding years (2011-13), (2014 collection data estimated for Cyprus) $)^{110}$

\section{The WEEE treatment process}

WEEE treatment consists of two distinct stages: i) pre-processing; and ii) materials recovery. Pre-processing serves a vital function. Items are separated into their constituent material/component fractions, which are then diverted to appropriate processes for materials recovery and hazardous waste disposal. At this stage in recycling, decisions are made about whether items or their components will be reused, and to which processes materials for recovery should be sent. These are critical decisions in determining the value that can be exploited from WEEE.

Materials separation can be conducted with automated, manual or semi-automated processes. Manual pre-processing is relatively costly as it is labour-intensive. Products are manual disassembled into their component parts. This provides the opportunity to harvest components for reuse, and to isolate circuit boards and other components which contain concentrations of precious metals. ${ }^{116}$ Greater value can be recovered from items with high concentrations of precious metals in their PCBs with manual processing than through automated processes, justifying high process costs. Manual processing also gives the opportunity to isolate components in which small amounts of CRMs are present.

Automated recycling involves shredding items (following depollution) with subsequent automated separation of material fractions i.e. magnetic, eddy-current, electrostatic and 
density separation to give ferrous, aluminium, stainless-steel, glass, copper, brass and plastic fractions. ${ }^{12,111}$ Items processed in this way have no chance of reuse. Such processes are relatively cheap, with high throughput and minimal labour requirements. However, they result in significant losses of precious metals as a result of the 'grade recovery function', a principle which applies to ore \& minerals processing and is equally applicable to the processing of WEEE for the recovery of metals (Figure 1.21). As target material content is upgraded in a material flow by separation of non-target materials, the overall recovery yield diminishes. Shredding disperses particles of trace amounts of precious (and critical) materials present throughout the mass of the shred where they adhere to the surfaces of larger particles. At each stage in separation, these metals are lost through unintended co-separation with bulk fractions. Recovery processes for these bulk fractions do not target precious and critical materials and so these are permanently lost. From a resource-efficiency point of view, such processes should be avoided where possible in favour of semi-automated or manual pre-processing. ${ }^{116}$ Grinding also results in dispersal of hazardous materials throughout larger mass fractions, resulting in higher potential toxicity of output fractions than WEEE items prior to shredding. ${ }^{117}$

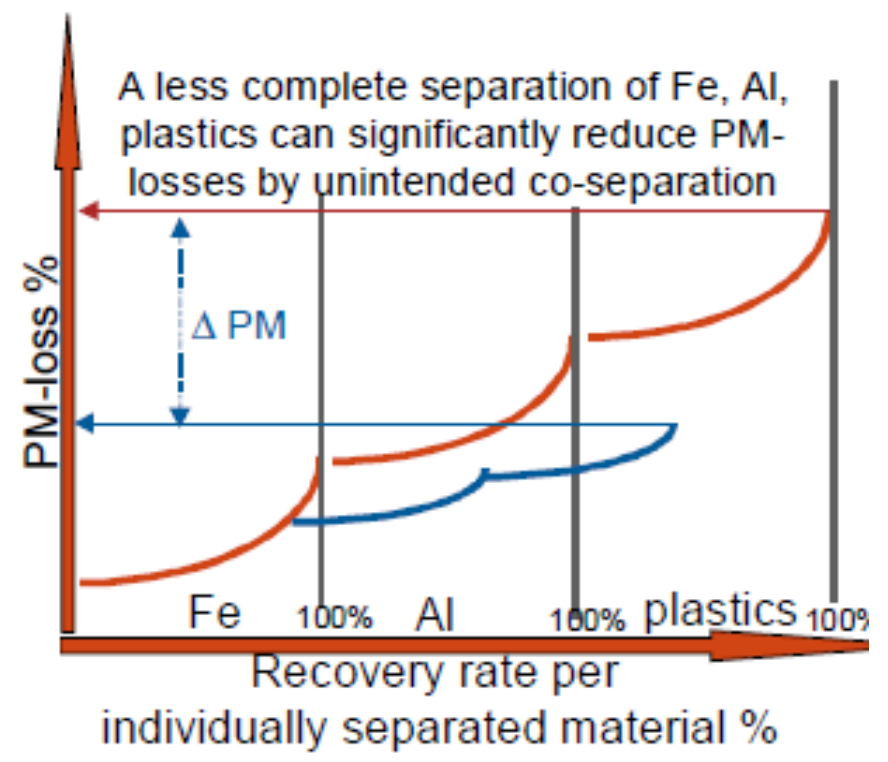

Figure 1.21 The 'Grade-Recovery Function' which results in losses of target precious metals and other trace elements as larger bulk fractions are removed from shredded WEEE in automated pre-processing, (C)2006, IEEE (re-printed, with permission, from Hagelüken, 2006) ${ }^{18}$

The choice of process is largely determined by the recoverable material value of items. Low value WEEE with low precious metal content (e.g. toasters, hair dryers etc.), is recycled with automated processes, and items with high inherent value (e.g. PCs) are processed manually. 
Within the UK, treatment of WEEE is largely dictated by the hazardous materials contained and the specialist processes required to deal with them as required by the WEEE directive. As a result, poor recovery of metal values is a major challenge to the recycling operation. CRMs are often lost due to their dispersion during shredding.

Europe, particularly Germany and Belgium, is using advanced technologies for refining precious metals with very good recovery rates for secondary recovery ( $>98 \%$ for $\mathrm{Au}$ ). Umicore's integrated smelting (pyrometallurical) facility in Hoboken, Belgium is the most advance plant of its kind, smelting PCBs to recover precious metal enriched copper bullion, which is then further refined in hydrometallurgical processes (Figure 1.22). Considerable plant capacity is available which is currently being expanded. Total hydrometallurgical recovery processes are more common in Asia. Many recovery processes require a further mechanical pre-treatment to upgrade materials prior to recovery. This enhances the concentration of target materials, which improves the cost benefit of recovery. However, the 'grade-recovery' function applies, and so losses are also incurred in this upgrading process. WEEE is preprocessed to give the optimum cost-benefit taking account of processing costs, and value of recovered materials (Figure 1.23)

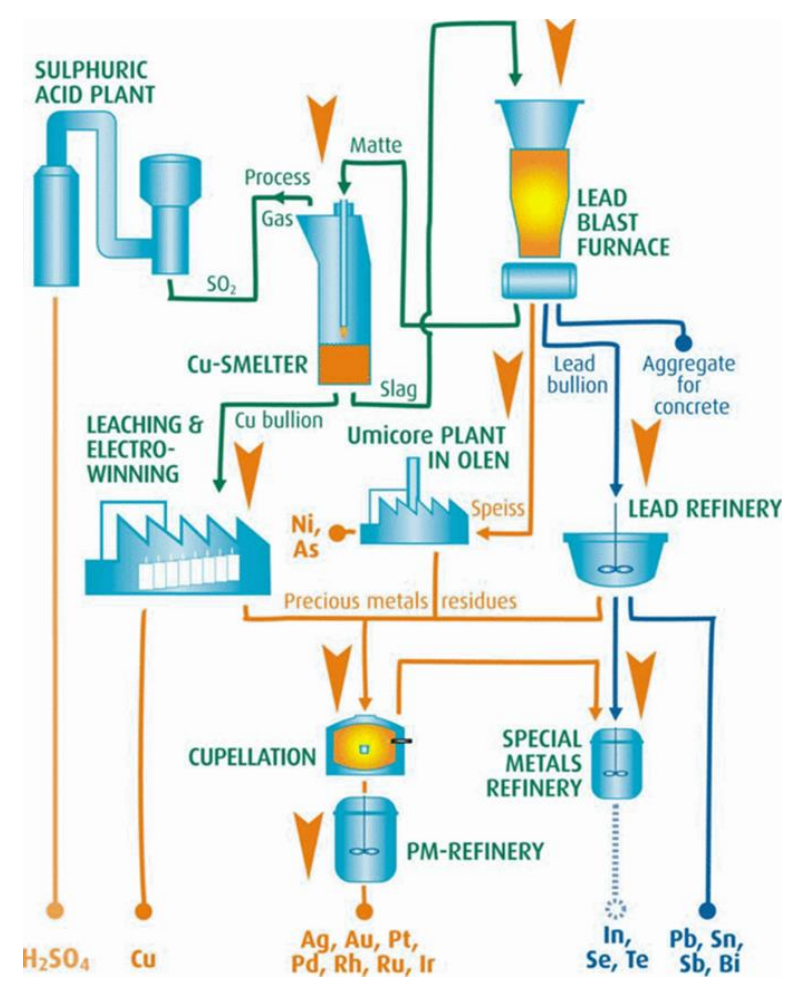

Figure 1.22 Flowsheet of Umicore's integrated metals smelter/refinery. (2006, IEEE (reprinted, with permission, from Hagelüken, 2006) ${ }^{18}$ 


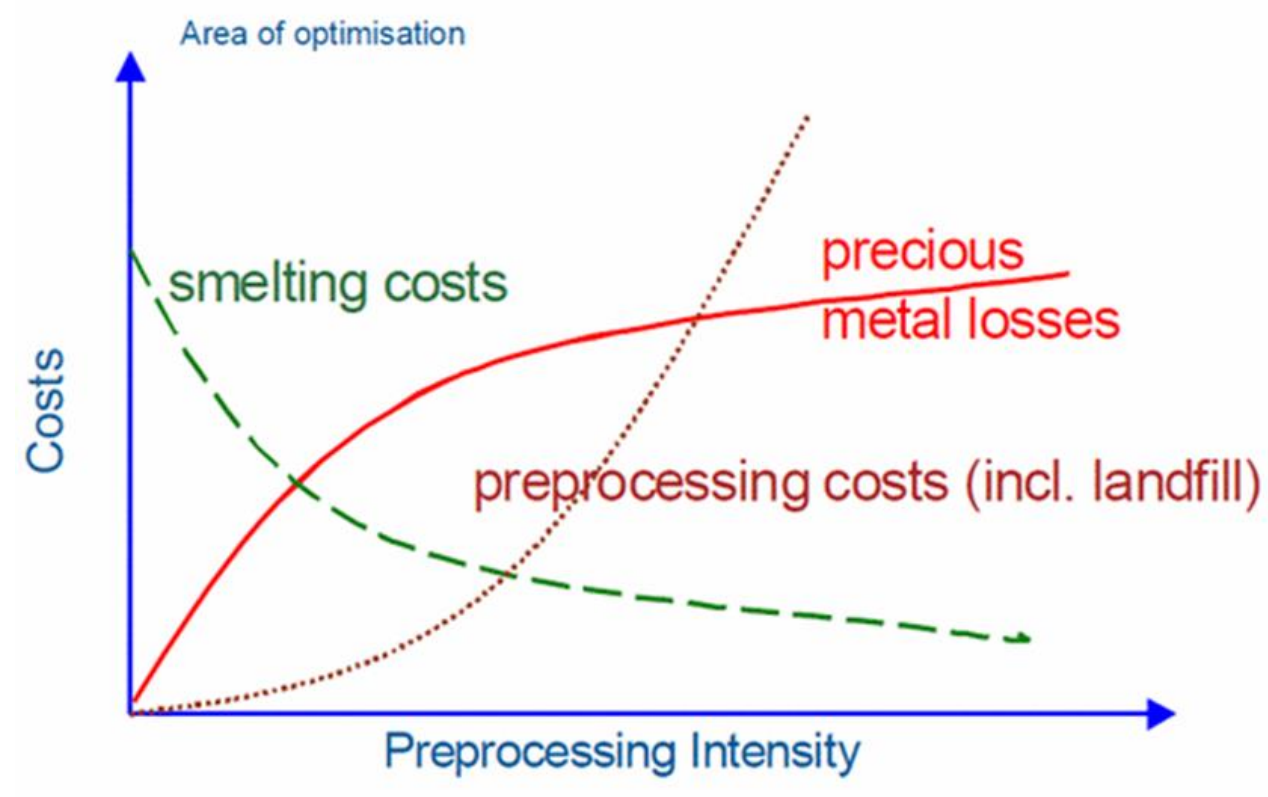

Figure 1.23 Qualitative cost function in WEEE recycling interface optimisation, IEEE (Reprinted, with permission, from Hagelüken, 2006) ${ }^{18}$

Despite high recovery efficiencies for target metals in these processes, many of the other materials present are not recovered either because they are of low value and therefore recovery is uneconomical, or there are thermodynamic limitations to recovery e.g. tungsten which is present in ICs as an interconnect material is lost in slag during smelting. Similar losses will be incurred for other low value critical materials in recovery processes, as indicated by the Oakdene Hollins study. ${ }^{109,118}$

\subsection{Economics of reuse and recycling}

To drive circular material flows in EEE, it is essential that sufficient cost-benefit is achieved through reuse, refurbishment, remanufacturing and recycling. It has been stated by Cossu $\&$ Williams regarding urban mining of WEEE:

"As for natural ores, extraction and processing of anthropogenic stocks is necessary and the generation of an economic benefit is essential." 119

The revenue obtained through sale of reused equipment, or recovered components and materials must be sufficient to cover all costs associated with generating those materials/products. This includes costs of collection and transport between processing sites, process costs and operational overheads, as well as any costs associated with disposal of hazardous materials. In pre-processing, isolating components and materials for downstream processing must be achievable at a cost which can be recouped by sale of these materials for 
recovery. The revenue received for PCBs at precious metal refineries is often the equivalent of the value of recovered materials less treatment and refining charges applied for processing the material (e.g. Table 1.11).

Table 1.11 Example breakdown of revenue received for PCBs by Metech Recycling UK ${ }^{22}$

\begin{tabular}{cc}
\hline Mass & $\mathbf{3 2 8 . 7} \mathbf{~ k g}$ \\
Treatment Charge & $£ 0.525 / \mathrm{kg}=£ 172.57$ \\
Gold recovered & $25.3 \mathrm{~g}$ \\
Gold less minimum deed & $20.4 \mathrm{~g}$ \\
Silver recovered & $262.9 \mathrm{~g}$ \\
Copper recovered & $36 \mathrm{~kg}$ \\
Gold treatment charge & $£ 180 / \mathrm{kg}=£ 3.67$ \\
Silvertreatment charge & $£ 20 / \mathrm{kg}=£ 4.99$ \\
Copper treatment charge & $£ 0.80 / \mathrm{kg}=£ 28.80$ \\
Gold Value & $£ 33.141 / \mathrm{g}=£ 676.07$ \\
Silver Value & $£ 0.63708 / \mathrm{g}=£ 159.07$ \\
Copper Value & $£ 4.899 / \mathrm{kg}=176.36$ \\
Total charges & $£ 210.03$ \\
\hline Total net revenue & $£ 801.47$ \\
\hline
\end{tabular}

Although other metals present e.g. Ru may be recovered by refineries, no value from these materials is returned to the pre-processor. This means that the viability of manual preprocessing is determined by the PM and copper content of PCBs. If this value is insufficient to recoup these costs, automated pre-processing must be conducted, resulting in losses of potentially recoverable value and poorer resource-efficiency outcomes. It is therefore essential that items are pre-processed in the most cost-effective manner possible, with precious metal and copper losses minimised to ensure the maximum value from WEEE is always exploited through efficient recovery of the most valuable constituent materials. In the future, eco-design and DfRR will reduce manual pre-processing costs. ${ }^{120-123}$ As a current solution, the 'best of two worlds' approach has been advocated, whereby WEEE would be manually processed in the developing world where labour costs are low, and material fractions would be recovered in advanced recovery processes in the developed world. ${ }^{124}$ Another option is to automate disassembly with robots, a strategy which has been explored by Apple and by the University of New South Wales seperately. ${ }^{125-127}$ Technological innovations in pre-processing such as this, which reduce processing costs, will be essential to support a viable circular economy for EEE. 


\subsection{Conclusion}

WEEE represents a considerable sustainability challenge which must be urgently addressed in the interests of mitigating the impacts of WEEE on human health \& the environment, and as a means of enhancing global-resource-efficiency. WEEE is a rich secondary source of materials and reusable EEE \& components, and exploitation of economic value from this 'urban mine' will yield synergistic environmental and social benefits. Recovery of materials and components will off-set demand from primary raw materials with which to manufacture new EEE, resulting in preservation of natural capital and avoidance of emissions and waste. WEEE is also rich in critical raw materials which face global supply risks from primary sources, and for which continued supply is essential for sustainable economic growth and meeting demand from emerging technologies.

Circular economy represents a solution to the global WEEE problem and a means to enhance the industrial ecology of the EEE industry through reuse \& recycling of EEE and its components, and through provision of secondary raw materials and components for manufacturing new EEE. This provides a means to exploit greater value from WEEE through novel methods of valorisation which present economic, social and environmental benefits over traditional recycling processes. Driving these circular material flows requires sufficient economic value to be derived from WEEE to cover costs incurred throughout recycling process chains; availability of suitable recovery processes; appropriate product design to accommodate circular practices; and appropriate reverse logistics, business models and legislative environments.

Much progress has been made around the world in establishing suitable legislation to govern the management of WEEE. In addition, collection systems and efficient recovery processes are available for the most economically and environmentally important metallic components of WEEE i.e. precious metals and copper, predominantly concentrated in PCBs. However, much progress is still required. In regions with advanced systems for managing WEEE, much is lost in residual waste streams or illegally exported. Much of the world is still without appropriate infrastructure for the management of WEEE, and great volumes are shipped to such regions where WEEE is recycled by the informal sector using crude 'backyard recycling' operations which have resulted in significant impacts on populations, ecosystems and the environment. Losses of potentially recoverable materials in shredding processes where manual pre-processing is prohibitively expensive, and during recovery processes, limits the economic value that is derived from WEEE and the ability to efficiently recover 'high-tech' metals. To enhance recovery, greater optimization of the interface between pre-processing and recovery is required. 
In order to overcome these issues, greater knowledge of the composition of items within the waste stream and the available value therein is required to plan future EoL management. In addition, recovery processes which derive greater value through circular economy are required for viable recovery of materials that are currently lost due to low economic value. For the future, full lifecycle optimisation of products with their manufacturing and end-of-life processes will be necessary to ensure that problems associated with WEEE management today are avoided in the future, and that greater value can be derived from material resources contained in EEE at end-of-life. Greater awareness of the material content of items, available infrastructure for end-of-life treatment, and education about the 'global WEEE problem' and its solutions is also necessary to help consumers make more informed decisions when selecting technologies for superior resource-efficiency and sustainability outcomes at EoL.

In the following chapters of this thesis the inherent material and economic value of WEEE is investigated, as well as methods of improving resource-efficiency and the derivable value of WEEE at end-of-life. Most cases presented address industrial challenges encountered by Metech Recycling (UK) Ltd. at their AATF in Hirwaun, South Wales.

Chapter 3 discusses the recycling potential of WEEE based on derivable value from precious metals (PMs) and copper in PCBs of items and presents an evaluation of future recycling potential of WEEE through evaluation of temporal trends in PM and copper content in historic PC RAM modules. Identified trends are applied to other WEEE items to project temporal changes in PCB compositions. This data is considered in conjunction with projections of future WEEE arisings in Europe to estimate recoverable value from the waste stream in the future.

Chapter 4 presents the results of an industrial trial conducted on behalf of WRAP which investigated potential techniques for increasing the value derived from treatment of WEEE in Wales through recovery of critical raw materials. This chapter identifies occurrences of CRMs in WEEE, and evaluates processes for isolation of CRM bearing components for downstream recovery. The recycling potential of identified CRMs is discussed and processes for component isolation are evaluated based on cost and revenue generated through sale of material fractions produced. Identified barriers to CRM recovery are discussed with methods to overcome these in the future.

Chapter 5 presents an industrial symbiosis process in which platinum from waste thermocouples filaments is converted to chloroplatinic acid for fabrication of dye-sensitised solar cell (DSSC) counter electrode catalysts. Recovery of platinum from these thermocouples is not viable through traditional recycling routes due to the dissipative nature of the use of the CRM platinum in this application, which exemplifies a major barrier to CRM recovery from 
WEEE. The process developed is used to demonstrate the potential that circular economy holds for enhancing derivable economic value from CRMs in WEEE to drive recovery, the materials costs savings and waste valorisation opportunities afforded to industry, and the environmental and social value resulting.

Chapter 6 details findings of a multi-disciplinary cross-sector sandpit organised and hosted by the author to establish a framework for future research to optimise the lifecycles of printable photovoltaics (PPV) for circular economy. The advantages of lifecycle optimisation for PPV are examined with key aspects of design and materials selection; manufacturing; likely applications of PPV and implication for EoL management; and potential recycling \& refurbishment strategies. Features conducive to circular economy, identified barriers to circularity and priority areas for future research are discussed. This provides a pathway through which the industrial ecology of the PPV industry may be enhanced and growth for the industry decoupled from consumption of material resources resulting in superior resourceefficiency outcomes when these emerging technologies reach end-of-life.

Chapter 7 examines the issue of selection of appropriate battery technologies for off-grid solar energy systems for rural South Africa. During a workshop arranged and co-hosted by the author in Durban, South Africa, which aimed to develop a pan-African multi-disciplinary collaboration of stakeholders for the development of circular economy around off-grid solar systems for rural Africa, batteries selection was considered the most crucial element in solar home system design. A framework for selection of batteries is discussed which takes account of materials content, cost, local available knowledge and infrastructure for management and processing of batteries during use and at EoL in a manner conducive to circular economy which benefits local South African economies. This exemplifies how educated and informed selection of EEE can result in avoided negative impacts at EoL and sustainable economic development with superior resource-efficiency outcomes. 


\section{Experimental}

\section{Chapter Summary}

This chapter outlines materials and methods used in the experimental work presented in this thesis. Details of chemicals and standard materials used, methods of sample preparation and conditions for analyses are outlined. Necessary theory underpinning analytical techniques necessary to interpret results presented is also included. References to the thesis sections to which details apply are included throughout the chapter.

\subsection{Sample preparation}

\subsubsection{Grinding}

All grinding was conducted with an IKA-A10 cutting mill with tungsten carbide cutting blade, suitable for effective grinding of PCBs. ${ }^{128}$ Samples were ground for 15 minutes in a water cooled grinding chamber to prevent overheating. This method is suitable for producing a reasonably homogenised powder ${ }^{129}$ of particle size $<0.2 \mathrm{~mm}$, sufficient for complete leaching of metals from samples. ${ }^{32,34,130,131}$ In general, three $0.1 \mathrm{~g}$ sub-samples of the homogenous powder were taken and subjected to acid digestion.

\subsubsection{Digestions}

To analyse samples by AAS, ICP-OES and ICP-MS, acid digestions were conducted and analysis performed directly on leachate solutions, or dilutions of these solutions to an appropriate concentration range for analysis. The specific conditions for digestion in each case are detailed in subsequent sections.

\subsection{Analytical Techniques}

\subsubsection{Flame atomic absorption spectroscopy (AAS)}

Atomic Absorption Spectroscopy (AAS) was used for determination of metal concentrations in solution. AAS has been used to quantify CRMs in samples discussed in Chapters $4 \& 5$.

Theory - The technique is based upon the phenomenon of atomic absorption i.e. ground state electrons of atoms will absorb visible and/or UV light of energy $E$ causing them to be promoted from ground state $\left(E_{0}\right)$ to an excited state $\left(E_{1}\right)$ (Figure 2.1). The wavelengths $(\lambda)$ and energies of absorbed photons are characteristic of particular elements. 


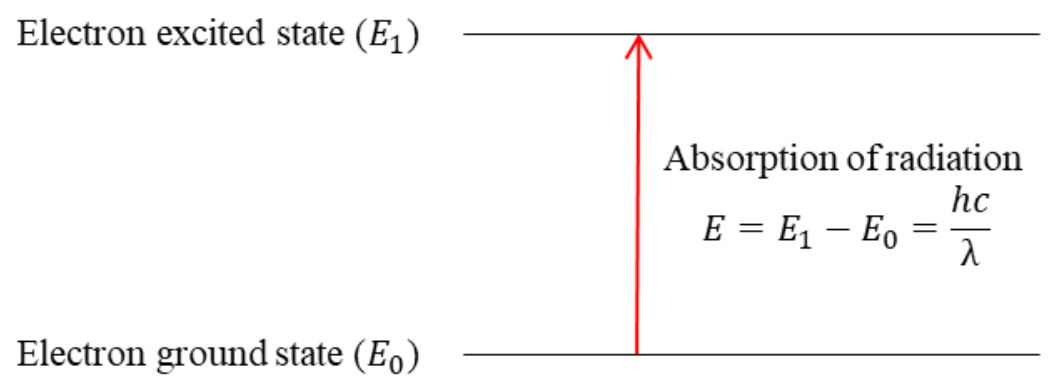

Figure 2.1 Electronic transition from ground state $\left(E_{0}\right)$ to an excited state $\left(E_{1}\right)$ resulting from absorption of a photon of energy $E$ and wavelength $(\lambda)$

Following oxidative digestion of samples, a solution containing metal ions is obtained. The solution containing analyte is aspirated through a nebulizer forming an aerosol which is combined with high pressure acetylene and air in a burner chamber. The mixture is carried through a burner head into a reducing flame where combustion of the mixture occurs. As the sample exits the burner head and enters the flame, desolvation of the nebulized sample occurs, followed by vaporization and reduction of metallic ions to gas phase atoms which are able to absorb incident UV/visible light at characteristic wavelengths $(\lambda)$. A hollow cathode lamp with a cathode made from metal of the analyte of interest is used as a radiation source. This emits all characteristic wavelengths in the absorption spectrum of the analyte. The emitted radiation is focused into a beam which passes through the path length of the flame and then through a monochromator to the detector which measures the intensity of radiation which has passed through the flame. As the beam passes through the flame, some of the radiation is absorbed by atoms of the analyte according to the Beer-Lambert law (Equation 2.1) where $\varepsilon$ is the molar extinction coefficient of the analyte species, $c$ is the concentration of analyte in solution and $l$ is the path length of the flame. This results in reduced intensity of the detected beam $(I)$ in comparison to the intensity in the absence of analyte species $\left(I_{0}\right)$. The absorbance of the sample is then calculated from the intensities of detected radiation in the presence and absence of samples with Equation 2.2

$$
\begin{gathered}
A b s=\varepsilon c l \\
A b s=\log _{10}\left(\frac{I_{0}}{I}\right)
\end{gathered}
$$

Equation 2.1

Equation 2.2

There are a number of factors which can cause experimental deviations from the BeerLambert law and it is usual to calibrate the instrument with a series of solutions of known concentration $(c)$, to obtain the experimental calibration curve. ${ }^{132}$ 
Limitations- The use of an air-acetylene flame limits the range of CRMs which can be quantified by AAS. Some metals form refractory oxides within the temperature range of the flame so determination of their concentrations in solution is not possible. Where this is the case, ICP-OES and ICP-MS, which use a higher temperature argon plasma in place of an airacetylene flame, have been used to quantify CRMs in samples. This was necessary for determination of Rh, Nd and $\mathrm{Y}$.

Analysis - Methods of sample preparation used are indicated in Table 2.1. Digestions were carried out by boiling samples in acid solutions for 3 hours, or until total dissolution of samples was achieved, while maintaining a sample mass/acid volume ratio of $40 \mathrm{~g} / \mathrm{ml} .{ }^{22}, 1333$ subsamples were taken for analysis and 3 repeat readings were obtained for each solution. All measurements were carried out using an air-acetylene flame and Perkin Elmer Lumina Hollow Cathode Lamps (HCLs). Au, Pt and $\mathrm{Zn}$ analyses used single element HCLs, and $\mathrm{Co}$ and $\mathrm{Cu}$ analysis was conducted with a Co-Cr-Cu-Fe-Mn-Ni multi-element HCL. Specific parameters for the analysis of individual elements are given in Table 2.2. All analyses were conducted by calibration with solutions of known concentrations with the exception of Pt analyses which were conducted using the method of multiple standard additions. ${ }^{134}$

Table 2.1 Samples analysed by AAS, relevant sections of the thesis, elements analysed and sample preparation procedures.

\begin{tabular}{|c|c|c|c|}
\hline Sample & Section & Element & Sample preparation \\
\hline Magnetron ferrite core & 4.3 .3 & $\mathrm{Co}$ & Digest whole sample in $1: 1 \mathrm{HCl}$ \\
\hline Magnetron magnet & 4.3 .3 & $\mathrm{Co}$ & Digest $1 \mathrm{~g}$ sample in $1: 1 \mathrm{HCl}$ \\
\hline $\begin{array}{l}\text { Li-ion battery cells (numerous } \\
\text { types) }\end{array}$ & 4.3 .3 & $\mathrm{Co}$ & $\begin{array}{l}\text { Cut open cells and digest electrodes } \\
\text { in } 1: 1 \mathrm{HNO}_{3}\end{array}$ \\
\hline $\begin{array}{l}\text { Contacts from PCBs (numerous } \\
\text { samples) }\end{array}$ & 4.3 .3 & $\mathrm{Au}, \mathrm{Cu}$ & $\begin{array}{l}\text { Digest whole samples in } 1: 1 \text { aqua } \\
\text { regia }\left(3: 1 \mathrm{HCl} / \mathrm{HNO}_{3}\right)\end{array}$ \\
\hline Waste thermocouple filaments & 5.3 .2 & $\mathrm{Pt}$ & $\begin{array}{l}\text { Digest in } 1: 1 \text { aqua regia }(3: 1 \\
\left.\mathrm{HCl} / \mathrm{HNO}_{3} \mathrm{v} / \mathrm{v}\right)\end{array}$ \\
\hline Chloroplatinic acid & 5.3 .2 & $\mathrm{Pt}$ & Dissolve in $5 \% \mathrm{HCl}$ \\
\hline$\sim 5 \mathrm{mM}$ chloroplatinic acid in IPA & 5.3 .3 & $\mathrm{Pt}$ & Dilute $1 / 5$ with IPA \\
\hline $\begin{array}{l}\text { Ta-capacitor powders with } \mathrm{Zn} \text { as } \\
\text { an internal standard }\end{array}$ & Appendix A & $\mathrm{Zn}$ & $\begin{array}{l}\text { Digest } 0.1 \mathrm{~g} \text { sub-samples of powder } \\
\text { in } 1: 1 \mathrm{HCl}(3 \mathrm{sub} \text {-samples analysed } \\
\text { per sample). }\end{array}$ \\
\hline
\end{tabular}


Table 2.2 Parameters for Analysis of individual elements by AAS

\begin{tabular}{|c|c|c|c|c|c|c|c|}
\hline \multirow{2}{*}{ Element } & \multirow{2}{*}{$\begin{array}{c}\lambda \\
(\mathbf{n m})\end{array}$} & \multirow{2}{*}{$\begin{array}{c}\text { Slit } \\
(\mathbf{m m})\end{array}$} & \multicolumn{2}{|c|}{ Flow Rate (L/min) } & \multirow{2}{*}{$\begin{array}{c}\text { Integration } \\
\text { time (s) }\end{array}$} & \multirow{2}{*}{$\begin{array}{c}\text { Read } \\
\text { delay }(s)\end{array}$} & \multirow[t]{2}{*}{ Replicates } \\
\hline & & & Air & Acetylene & & & \\
\hline $\mathrm{Au}$ & 242.80 & $2.7 / 1.35$ & 12.0 & 2.15 & 3 & 5 & 3 \\
\hline Co & 240.73 & $1.8 / 1.35$ & 10.0 & 2.50 & 3 & 5 & 3 \\
\hline $\mathrm{Cu}$ & 324.75 & $2.7 / 0.8$ & 10.0 & 2.50 & 3 & 5 & 3 \\
\hline $\mathrm{Pt}$ & 265.95 & $2.7 / 1.05$ & 10.0 & 2.50 & 3 & 5 & 3 \\
\hline $\mathrm{Zn}$ & 213.86 & $2.7 / 1.8$ & 10.00 & 2.50 & 3 & 5 & 3 \\
\hline
\end{tabular}

\subsubsection{Inductively coupled plasma - optical emission spectroscopy (ICP-OES)}

Analyses of Rh and Pt in synthesised and purchased chloroplatinic acid samples reported in section 5.3.2 were conducted by ICP-OES.

Theory - ICP-OES can determine concentrations of multiple elements concurrently, with detection limits in the sub-ppb range, making it an ideal technique for quantification of metals in WEEE. Leachates from sample digestions (produced in the same way as for AAS) are nebulised and then vaporised in an argon plasma. Atoms/ions within the plasma vapour are excited and emit photons of characteristic wavelengths. (Figure 2.2).

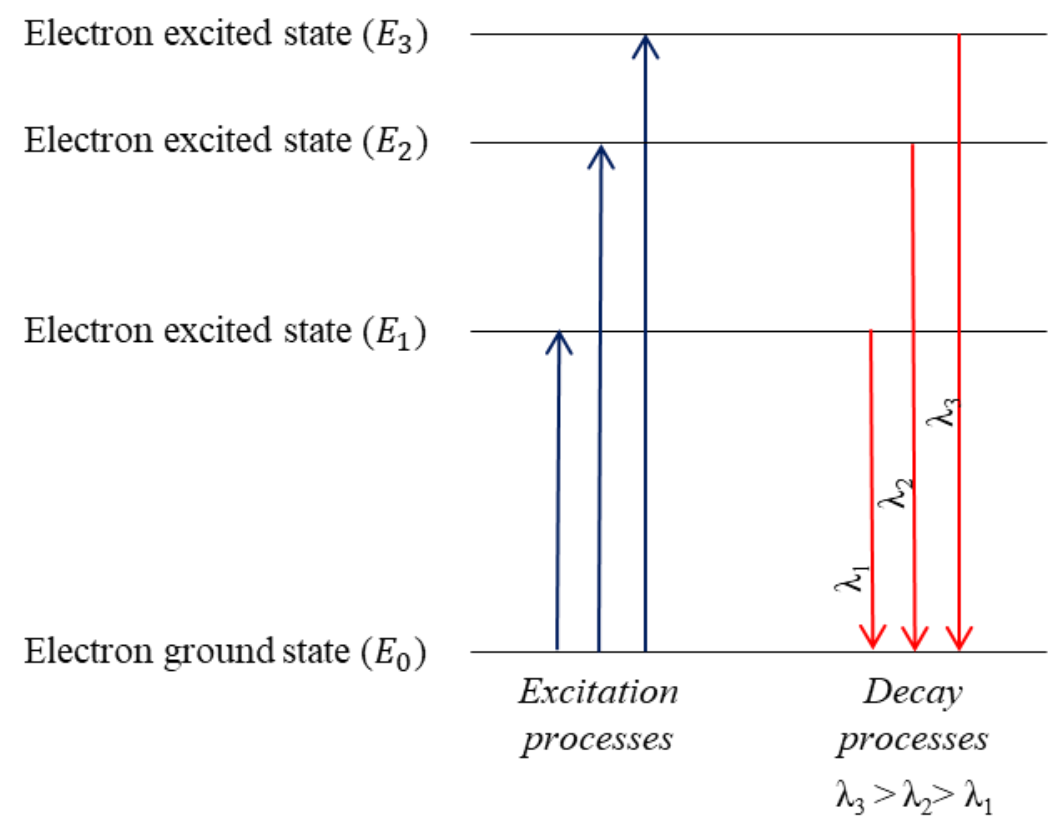

Figure 2.2 Electronic decay processes resulting in emission lines

The spectrometer optics separate the sample emission into its different spectral components enabling element identification by emission wavelength and quantification by emission 
intensity. High plasma temperatures $(6000-8000 \mathrm{~K})$ prevent problems associated with formation of refractory oxides and low excitation efficiencies. The technique is suitable for analysis of elements at lower concentration than is possible with AAS employing an airacetylene flame. ${ }^{132}$

Analysis - Analyses of Rh and Pt in of chloroplatinic acid samples reported in Chapter 4 were performed by Dr Simon Curling, Bangor University Biocomposites Centre commercial analysis service - BC Materials, using an Agilent Varian 710 ES ICP-OES instrument with an axial torch, Varian SPS 3 autosampler, ICP expert II software, and the following operational conditions: plasma flow 15 l/min, nebuliser pressure $240 \mathrm{kPa}$, sample uptake delay $30 \mathrm{~s}$, rinse time $10 \mathrm{~s}$, dry argon as carrier gas. Quantitative analysis was conducted at 3 wavelengths for platinum ( $203.646 \mathrm{~nm}, 214.424 \mathrm{~nm}, 265.945 \mathrm{~nm}-1 \mathrm{st}$ to $3 \mathrm{rd}$ order spectra) with 3 replicates at each wavelength. Results for platinum are taken as the mean result from three wavelengths. Analysis of Rh was conducted at a single wavelength only (343.488 nm - first order spectrum) as poor calibration of $2 \mathrm{nd}$ and $3 \mathrm{rd}$ order spectra were obtained, probably due to interference from other elements present. Analyses were performed in duplicate for each sample. Samples were prepared by dissolution and dilution of chloroplatinic acid with $10 \% \mathrm{HCl}$.

\subsubsection{Inductively coupled plasma - mass spectrometry (ICP-MS)}

Quantitative analysis of Nd and Y in samples reported in Section 4.3.2 was conducted by ICPMS.

Theory - ICP-MS is suitable for simultaneous qualitative and quantitative determination of up to 70 elements, and uses an inductively coupled plasma as an ion source. The technique differs from ICP-OES in the use of a mass spectrometer as detector which increases the sensitivity of the technique to give detection limits in the sub parts per trillion range. Samples are introduced via a nebulizer into the ICP which acts as both atomiser and ioniser. Ions from the plasma are then passed into a mass analyser where they are separated according to their mass to charge ratio $(\mathrm{m} / \mathrm{z})$ by a quadrupole mass spectrometer.

Analysis - Analyses were conducted by ChemTest (Newmarket), an external analytical service. Analyses were conducted using a Perkin Elmer Elan 6100 ICP-MS instrument and Elan instrument control software (version 3.3). A plasma flow rate of $0.92 \mathrm{~L} / \mathrm{min}$; argon carrier gas; sample uptake delay of $40 \mathrm{~s}$; read delay of $20 \mathrm{~s}$; and rinse time of $15 \mathrm{~s}$ were used. Samples for analysis were prepared using the methods given in Table 2.3. Triplicate analyses on each sample were conducted and the result taken as the mean. 
Table 2.3 Samples analysed by ICP-MS, relevant sections of the thesis, elements analysed and sample preparation procedures

\begin{tabular}{lccl}
\hline Sample & Section & Element & Sample preparation \\
\hline LCD display CCFL & 4.3 .2 & $\mathrm{Y}$ & Digest phosphor in $1: 1 \mathrm{HCl}$ \\
MLCCs from PCBs & 4.3 .2 & $\mathrm{Y}$ & Digest whole component in $1: 1 \mathrm{HCl}$ \\
HDD magnets & 4.3 .3 & $\mathrm{Nd}$ & Digest whole sample in $1: 1 \mathrm{HCl}$ \\
Mobile phone speaker magnets & 4.3 .3 & $\mathrm{Nd}$ & Digest whole sample in $1: \mathrm{HCl}$ \\
\hline
\end{tabular}

\subsubsection{X-ray fluorescence spectroscopy $(X R F)$}

$\mathrm{XRF}$ is a non-destructive method for elemental analysis using the characteristic energies of $\mathrm{X}$ rays emitted from an element following bombardment with high energy X-rays. XRF was used to identify the CRMs in WEEE samples discussed in Section 4.3.2. XRF was also used to establish alloy composition of waste thermocouple filaments in Section 5.4.1.

Theory - When atoms at the surface of a sample are bombarded with X-rays, these are absorbed resulting in ejection of an inner shell electron ( $\mathrm{K}$ or $\mathrm{L}$ shell) leaving a 'hole' i.e. a vacant inner orbital, causing the atoms to enter an excited state (Figure 2.3). Atoms then relax when an electron from a higher energy orbital falls in energy to fill the vacant inner orbital, emitted X-rays as it does so. The energy of these emitted X-rays is characteristic of particular elements, so detection of emitted X-rays allows characterisation of elements present in samples. Excitation may result in loss of electrons from K, L or M shells (depending on the species). Electronic transitions into the $\mathrm{K}$ shell result in a $\mathrm{K} \mathrm{X}$-ray emission, transitions into the $\mathrm{L}$ shell result in $\mathrm{L} \mathrm{X}$-ray emissions and so on. ${ }^{135}$
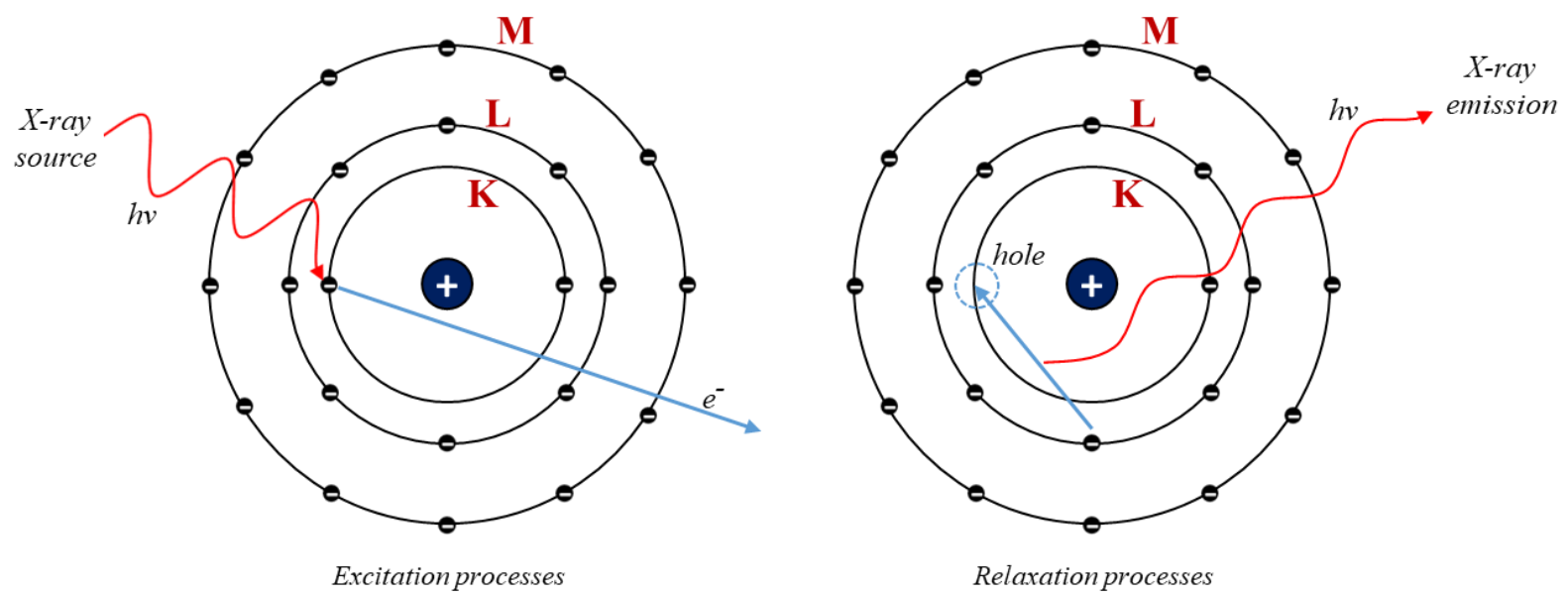

Figure 2.3 The processes involved in X-ray fluorescence(adapted from Fifield). ${ }^{135}$ 
Analysis - All analyses were conducted with a Fischer Instrumentation Fischerscope XDALFD system and Fischer WinFTM software. Manufacturer recommended setting for detection of trace metals were used, i.e. a $50 \mathrm{keV}$ x-ray beam with Al primary filter. A beam with circular cross-section with varying diameter between 0.3 and $1.0 \mathrm{~mm}$ was used. Beam diameter was selected as appropriate for the size of component being analysed, and to focus analysis on particular portions of components. Elements present were identified by emission signals indicated at the characteristic energies given in Table 2.4. Appropriate scan times were established as part of the instrument evaluation (Appendix A). A scan time of $55 \mathrm{~s}$ was used to measure filament alloy compositions reported in section 5.3.1.

Table 2.4 Positions of emission peaks in XRF analysis

\begin{tabular}{cccccc}
\hline Element & $\begin{array}{c}\text { Nature of } \\
\text { electronic } \\
\text { transitions }\end{array}$ & $\begin{array}{c}\text { Emission } \\
\text { energy } \\
(\mathbf{k e V})\end{array}$ & Element & $\begin{array}{c}\text { Nature of } \\
\text { electronic } \\
\text { transitions }\end{array}$ & $\begin{array}{c}\text { Emission } \\
\text { energy } \\
\text { (keV) }\end{array}$ \\
\hline $\mathbf{A g}$ & $\mathrm{K} \alpha_{1}$ & 22.16 & $\mathbf{P d}$ & $\mathrm{K} \alpha_{1}$ & 21.18 \\
& $\mathrm{~K} \beta_{1}$ & 24.94 & & $\mathrm{~K} \beta_{1}$ & 23.82 \\
$\mathbf{A u}$ & $\mathrm{L} \alpha_{1}$ & 9.71 & $\mathbf{P t}$ & $\mathrm{L} \alpha_{1}$ & 9.42 \\
& $\mathrm{~L} \beta_{1}$ & 11.44 & & $\mathrm{~L} \beta_{1}$ & 11.07 \\
$\mathbf{C o}$ & $\mathrm{K} \alpha_{1}$ & 6.93 & $\mathbf{R h}$ & $\mathrm{K} \alpha_{1}$ & 20.22 \\
& $\mathrm{~K} \beta_{1}$ & 7.65 & & $\mathrm{~K} \beta_{1}$ & 22.72 \\
$\mathbf{G a}$ & $\mathrm{K} \alpha_{1}$ & 9.25 & $\mathbf{R u}$ & $\mathrm{K} \alpha_{1}$ & 19.28 \\
& $\mathrm{~K} \beta_{1}$ & 10.27 & & $\mathrm{~K} \beta_{1}$ & 21.67 \\
$\mathbf{G e}$ & $\mathrm{K} \alpha_{1}$ & 9.89 & $\mathbf{N d}$ & $\mathrm{K} \alpha_{1}$ & 16.62 \\
& $\mathrm{~K} \beta_{1}$ & 10.98 & & $\mathrm{~K} \beta_{1}$ & 18.63 \\
$\mathbf{I n}$ & $\mathrm{K} \alpha_{1}$ & 24.21 & $\mathbf{S b}$ & $\mathrm{K} \alpha_{1}$ & 26.36 \\
& $\mathrm{~K} \beta_{1}$ & 22.28 & & $\mathrm{~K} \beta_{1}$ & 29.73 \\
$\mathbf{M g}$ & $\mathrm{K} \alpha_{1}$ & 1.25 & $\mathbf{T a}$ & $\mathrm{L} \alpha_{1}$ & 8.15 \\
& $\mathrm{~K} \beta_{1}$ & 1.30 & & $\mathrm{~L} \beta_{1}$ & 9.34 \\
$\mathbf{N b}$ & $\mathrm{K} \alpha_{1}$ & 16.62 & $\mathbf{W}$ & $\mathrm{L} \alpha_{1}$ & 8.40 \\
& $\mathrm{~K} \beta_{1}$ & 18.63 & & $\mathrm{~L} \beta_{1}$ & 9.67 \\
$\mathbf{N} \mathbf{N d}$ & $\mathrm{L} \alpha_{1}$ & 5.23 & $\mathbf{Y}$ & $\mathrm{K} \alpha_{1}$ & 14.60 \\
& $\mathrm{~L} \beta_{1}$ & 5.72 & & $\mathrm{~K} \beta_{1}$ & 16.74 \\
\hline
\end{tabular}

\subsubsection{Scanning electron microscopy (SEM) \& SEM/energy dispersive X-ray spectroscopy (SEM/EDS)}

Scanning electron microscopy was used to measure diameters of filaments isolated from waste thermocouples and to investigate the platinum particles deposited on the FTO glass as DSSC counter electrode catalysts. SEM/EDS was used for the direct non-destructive analysis of waste thermocouple filaments (Chapter 5). 


\section{Theory}

SEM - SEM is an electron probe method which provides high resolution images of samples by firing a focussed beam of electrons at their surface. Under vacuum, an electron beam is generated by thermionic emission from a tungsten filament in an electron gun and focussed through a series of condenser lenses and apertures into a very small diameter. This is directed at a sample mounted on a stage in a specimen chamber. Rastering of the electron beam across the sample surface using deflection coils results in backscattering and generation of secondary electrons by the sample, which are drawn to a detector by a positive charge. Backscattered electrons result from elastic collisions between incident electrons and electrons or nuclei in atoms of samples, and secondary electrons are created by inelastic collisions with sample electrons. Detection of these electrons reveals surface structure information, topographical contrasts and atomic number contrasts of samples.

SEM/EDS - Bombardment of samples with electrons also results in the generation of X-ray emissions from samples. This occurs in an analogous way to the process of $\mathrm{X}$-ray fluorescence depicted in Figure 2.3 except that it is the incident electrons which excite the atoms rather than an X-ray beam.

Analysis - Analysis of waste thermocouple filaments was conducted with a Hitachi TM3000 table top microscope running BrukerQuantax 70 software for EDS analysis. A $15 \mathrm{kV}$ beam was used. SEM imaging of platinized counter-electrodes was carried out by Dr James Douglas, Department of Materials Science, Oxford University, at $5 \mathrm{kV}$ using a 30 micron aperture, Secondary Electron (SE2) detectors and InLens detectors on a Zeiss Nvision instrument using a Gemini SEM column.

\subsubsection{Optical microscopy}

Visual inspection of thermocouple filaments was conducted with a Hampshire Micro:Wessex WSL1 long arm stereo microscope (Chapter 5).

\subsubsection{Electrical impedance spectroscopy (EIS)}

EIS is used to characterise the electrochemical performance of dye-sensitised solar cell (DSSC) platinum catalysts produced in Chapter 5. The technique reveals kinetic information about electrochemical processes occurring at the surface of the catalyst, and has been use to analyse symmetrical 'dummy cells' composed of pairs of platinized electrodes created from chloroplatinic acid samples to determine charge transfer resistances $\left(R_{C T}\right)$, double-layer capacitances $\left(\mathrm{C}_{\mathrm{dl}}\right)$ and exchange current densities $\left(j_{0}\right)$ at the electrolyte/catalyst interface of electrodes. An overview of the application of EIS in analysis of DSSCs is given by Bisquert \& Fabregat-Santeago. ${ }^{136}$ 
Theory - The impedance of an electrochemical system is a measure of its ability to resist the flow of current. When a sinusoidal AC potential $(E)$ with maximum amplitude $E_{0}$ is applied to an 'ideal resistor', a sinusoidal current response $(I)$ in phase with $E$ results, regardless of the frequency of the applied potential ( $\omega$ ) (Figure 2.6). Real world electrochemical systems have more complex resistive behaviour described by impedance $(\mathrm{Z})$ which is analogous to resistance but varies with $\omega$. Studying the impedance of electrochemical cells with EIS reveals kinetic information about the individual chemical processes. To do this, small AC potentials $(1-10 \mathrm{mV})$ are applied to cells. This result in a pseudo-linear current response i.e. with the same $\omega$ as $E$, but out of phase by an amount described by the phase angle ( $\theta$ ) (Figure 2.4). $E$ can be expressed as a function of time using Equation 2.3 in which $E_{t}$ is the potential at time $t, E_{0}$ is the amplitude and $\omega$ is the radial frequency. The current response $\left(I_{t}\right)$ is described by Equation 2.4. By measuring the sinusoidal wave form of $I$ in response to $E$, $\theta$ can be measured and $Z$ can be calculated using Equation 2.5 which expresses impedance in terms of its magnitude $\left(Z_{0}\right.$.). Using Euler's relationship (Equation 2.6, with $j=V_{-}-1$ ) it is possible to express $Z$ as a complex number using Equation 2.7 in terms of the real $\left(Z^{\prime}\right)$ or restive, and the imaginary $\left(Z^{\prime \prime}\right)$ or capacitive component, of impedance.
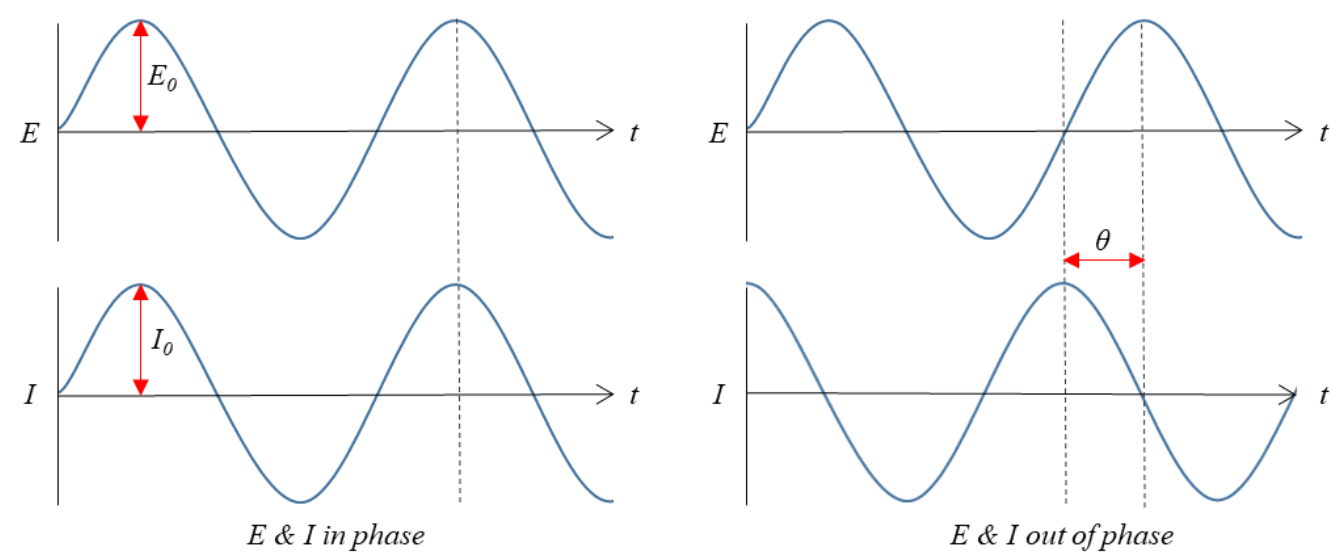

Figure 2.4 Sinusoidal wave forms of applied AC potential (E) and current response (I) in phase with one another (left); and out of phase by the phase shift angle, $\theta$ (right)

$$
\begin{gathered}
E_{t}=E_{0} \sin \omega t \\
I_{t}=I_{0} \sin (\omega t+\theta) \\
Z=\frac{E_{t}}{I_{t}}=Z_{0} \frac{\sin \omega t}{\sin (\omega t+\theta)} \\
e^{(j \theta)}=\cos \theta+j \sin \theta \\
Z(\omega)=\frac{E}{I}=Z_{0} e^{(j \theta)}=Z_{0}(\cos \theta+j \sin \theta)
\end{gathered}
$$

Equation 2.3

Equation 2.4

Equation 2.5

Equation 2.6

Equation 2.7 
Impedance is measured over a range of frequencies, typically 100,000-0.1 Hz. The results of $Z(\omega)$ are related to the real $\left(Z^{\prime}\right)$ and imaginary $\left(Z^{\prime \prime}\right)$ components of impedance by (Equation 2.8), where $|Z|$ is the magnitude of impedance, related to $Z^{\prime}$ and $Z^{\prime \prime}$ by Equation 2.9, and $\theta$ by Equation 2.10.

$$
\begin{aligned}
Z(\omega)=Z^{\prime}+j Z^{\prime \prime} & =(|Z| \cos \theta)+j(|Z| \sin \theta) & & \text { Equation } 2.8 \\
|Z| & =\sqrt{Z^{\prime 2}+Z^{\prime 2}} & & \text { Equation } 2.9 \\
\theta & =\tan ^{-1}\left(\frac{Z^{\prime \prime}}{Z^{\prime}}\right) & & \text { Equation } 2.10
\end{aligned}
$$

The results of impedance at each frequency are generally represented on a Nyquist plot of the real (i.e the resistance) and imaginary (i.e. the capacitance) components of impedance (Figure 2.5). $|Z|$ can be represented as a vector on this plot, which forms an angle, $\arg Z$ with the $Z^{\prime}$ axis. $\mathrm{R}$ is the value of impedance when $\omega=0$, and $\mathrm{C}$ is calculated using $\omega_{\max }=1 / \mathrm{RC}$ when $\arg$ $\mathrm{Z}=45^{\circ} .137$

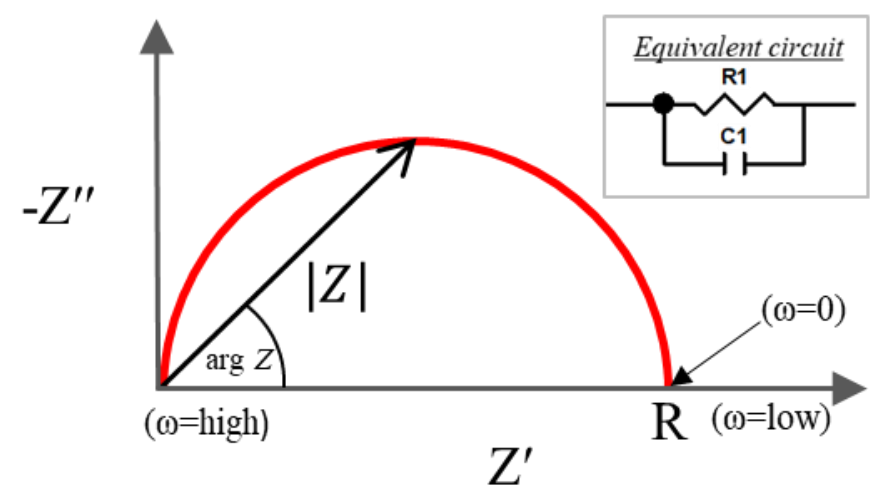

Figure 2.5 Example Nyquist plot, test frequency, $\omega$ is varied from high to low frequency, each frequency leads to an extra point on the curve, where the curve crosses the $x$-axis the impedance is resistance only, vector indicates the magnitude of impedance, $|Z|$. The simple circuit is composed of an ideal resistor in parallel with an ideal capacitor.

EIS data is interpreted by data-fitting an equivalent circuit model. $R$ is the value of impedance when $\omega=0$, and $\mathrm{C}$ is calculated using $\omega_{\max }=1 / \mathrm{RC}$ when $\arg \mathrm{Z}=45^{\circ} .{ }^{137} \mathrm{Common}$ electrical circuit elements such as those shown in Table 2.5 are combined to create a theoretical circuit which is representative of the physical electrochemistry of the system under study. 
Table 2.5 Equivalent circuit elements, their current-voltage relationships, impedances and phase shift properties

\begin{tabular}{|c|c|c|c|c|}
\hline Circuit element & Symbol & I vs E relationship & Impedance & Phase shift effect \\
\hline Resistor & $\sim^{\sim}$ & $E=I R$ & $Z$ & $\theta=0^{\circ}$ \\
\hline Inductor & 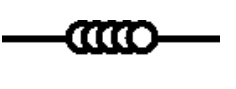 & $E=L \frac{d I}{d t}$ & $Z=j \omega L$ & $\theta=-90^{\circ}$ \\
\hline Capacitor & 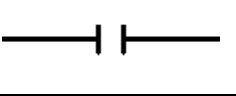 & $I=C \frac{d E}{d t}$ & $Z=\frac{1}{j \omega C}$ & $\theta=90^{\circ}$ \\
\hline
\end{tabular}

For electrochemical systems such as DSSCs, complex combination of multiple components are used to build up equivalent circuits, e.g. diffusion of electrolyte species to sites of reaction at electrode surfaces results in impedance which is represented by a Warburg element. Electrochemical processes at counter-electrodes have been studied with EIS, by creating 'dummy cells' from pairs of counter electrodes. ${ }^{138-142}$ The equivalent circuit model proposed for a cell created from a pair of platinized counter electrodes filled with electrolyte is a Randles circuit with a short Warburg element in which $R s$ is the electrolyte solution resistance of the electrolyte; $C d l$ is the double layer capacitance at the electrode / electrolyte interface; $R c t$ is the charge transfer resistance at electrolyte $\mid$ platinized counter electrode interface; and Ws 1 is a short Warburg diffusion (Figure 2.6). By fitting the data with this equivalent circuit, it is possible to extract value of $R c t$ and $C d l$.

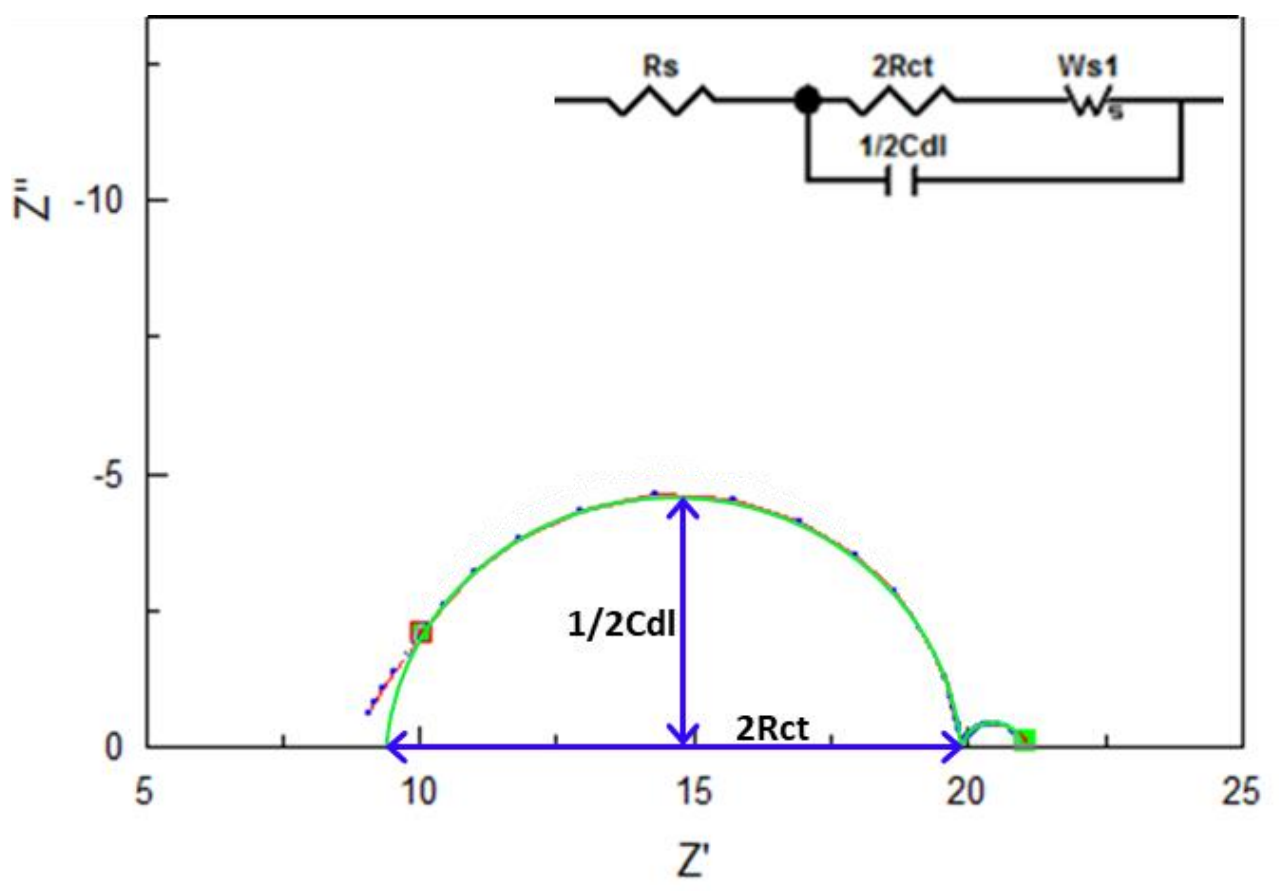

Figure 2.6 Equivalent circuit fit for 'dummy cell' EIS data using a Randles circuit with short Warburg element (Ws1), showing Rct and Cdl 
Analysis - Impedance spectra of dummy cells were measured using a GAMRY Reference 600 Potentiostat at open circuit voltage. An AC voltage of $10 \mathrm{mV}_{\text {rms }}$ was applied over the frequency range 150,000 $-0.1 \mathrm{~Hz}$. Charge transfer resistance $\left(R_{\mathrm{CT}}\right)$ values were measured from Nyquist plots of impedance spectra fitted to a Randles equivalent circuit with a short Warburg element (Figure 2.6) using Z-view software. Each curve was first modelled individually to give an estimate of the values. These values were put into the full model and constrained with only one free variable at a time. Once all the variables had been allowed to move freely the constraints were lifted one at a time until the whole model was free, except for the Ws-P parameter of the Warburg element which was fixed at 0.5 to give a finite Warburg to reflect the finite diffusion within the $25 \mu \mathrm{m}$ distance between electrodes in cells. $R_{\mathrm{ct}}$ values are obtained as half of the real component of impedance for the 'dummy cells'.

\subsection{Chemicals \& Standards}

Acids - $\mathrm{HCl}(31.5-33.0 \%)$ and $\mathrm{HNO}_{3}(69-72 \%)$ were CertiFied AR (analytical reagent) grade, and were purchased from Fisher Chemicals and used as received.

Solvents, substrates and electrolyte materials - (Chapter 5): NSG TEC7 FTO glass (100 $\mathrm{mm} \times 100 \mathrm{~mm} \times 2.3 \mathrm{~mm}, 7 \Omega / \mathrm{sq}$ ); isopropyl alcohol (IPA) ( $\geq 99.7 \%$ ); 1-methyl-3propylimidazolium iodide (98\%); benzimidazole (98\%); iodine (99.8\% trace metal basis); and guanidinium thiocyanate (99\%) were purchased from Sigma-Aldrich. 3-methoxypropionitrile (98\%) was obtained from Acros Organics. All materials were used as received.

Standard solutions - All elemental standard solution used for atomic spectroscopy, ICP-OES and ICP-MS were TraceCERT ${ }^{\circledR}$ single element solutions $(1000 \mathrm{mg} / \mathrm{L})$ purchased from SigmaAldrich and used as received.

Elemental standards - (Chapter 4 \& Appendix A - Evaluation of Fischerscope XDAL The metal foils and their sources are indicated in Table 2.6. Chloroplatinic acid hydrate $(\geq 99.9 \%$ trace metal basis) was purchased from Sigma Aldrich, and 99.998\% platinum wire was purchased from Johnson Matthey. All were used as received. 
Table 2.6 Metal foils used as standards for XRF evaluation

\begin{tabular}{|c|c|}
\hline Metal foil & Source \\
\hline $\begin{array}{l}\text { Co foil, Thickness: } 0.125 \mathrm{~mm} \text {, Purity: } 99.9 \% \text {, Coil width: } 250 \mathrm{~mm} \text {, Temper: } \\
\text { Annealed, } 100 \times 100 \mathrm{~mm}\end{array}$ & Goodfellow \\
\hline $\begin{array}{l}\text { Ge Sheet, Thickness: } 0.5 \mathrm{~mm} \text {, Purity: } 99.999 \% \text {, Condition: Single crystal, } \\
\text { Oriented:(111), Electrical type: N-Type }\end{array}$ & Goodfellow \\
\hline Mg foil, Thickness:0.25 mm, Purity:99.9\%, Temper: As rolled, $25 \times 25 \mathrm{~mm}$ & Goodfellow \\
\hline Ta foil, Thickness:0.04 mm, Purity:99.9\%, Temper: Annealed, coil, $0.1 \mathrm{~m}$ & Goodfellow \\
\hline Pd foil, Thickness:0.1 mm, Purity:99.95\%, Temper: As rolled, $25 \times 25 \mathrm{~mm}$ & Goodfellow \\
\hline Pt foil, Thickness: $0.025 \mathrm{~mm}$, Purity: $99.95 \%$, Temper: As rolled & Goodfellow \\
\hline Y foil, Thickness:0.125 mm, Purity:99.9\%, Temper: As rolled, $25 \times 25 \mathrm{~mm}$ & Goodfellow \\
\hline $\begin{array}{l}\text { Stainless Steel - AISI } 302 \text { Foil, Thickness:0.1 mm, Coil width:300mm, Temper: } \\
\text { Hard, 50x50 mm }\end{array}$ & Goodfellow \\
\hline Ag foil, Thickness:1.0 mm, Purity:99.95+\%, Temper: As rolled, $25 \times 25 \mathrm{~mm}$ & Goodfellow \\
\hline Au foil, Thickness:0.025 mm, Purity:99.95\%, Temper: As rolled, coil, $0.05 \mathrm{~m}$ & Goodfellow \\
\hline In foil, thickness $0.5 \mathrm{~mm}, 99.999 \%$ trace metals basis & Aldrich \\
\hline $\mathrm{Nb}$ foil, thickness $0.025 \mathrm{~mm}, 99.8 \%$ trace metals basis & Aldrich \\
\hline W Foil, foil, thickness $0.05 \mathrm{~mm}, \geq 99.9 \%$ trace metals basis & Aldrich \\
\hline Al foil, $0.1 \mathrm{~mm}$ laboratory reagent, $250 \mathrm{~g}$ & Fisher \\
\hline
\end{tabular}

\subsection{Statistics and errors}

Reported values are the mean of three replicate measurements unless stated otherwise. Error estimates are given as the standard error on the mean (sem). Confidence intervals given in Figures 3.10-3.13 in Chapter 3 were calculated by Dr Ingrid Hallin, Dept of Environmental Science, Plymouth University. ${ }^{143}$ 


\section{Temporal Trends in Precious Metal Content of WEEE - Implications for Future Recycling Potential ${ }^{144}$}

\section{Chapter summary}

Effective and responsible recycling of WEEE is an essential component of any solution to the 'global WEEE problem'. Best-practice recycling is however costly, requiring manual preprocessing and responsible disposal of the plethora of toxic materials found in WEEE items, and incurring significant logistics costs when materials are transported between processing sites, often in different countries. For recycling to remain economically viable, it is essential that recycling potential of WEEE is sustained i.e. "that potential e-waste recycling revenues exceed costs of collection, transportation and processing". ${ }^{145}$ Recoverable quantities of precious metals and copper must therefore remain sufficiently high to permit sufficient value to be derived from recycling of items to finance these operations.

To evaluate future recycling potential of WEEE, quantities of WEEE items generated in the future, their composition, recyclability and future costs of recycling must be considered. It is the purpose of the work presented in this chapter to shed light on the issue at hand and predict future recycling potential of high grade WEEE. Historical trends in PM and copper content of PC DRAM modules, originally placed on the market (POM) over the period 1991-2008 are evaluated. Observed temporal trends in PM and copper content of RAM modules are then applied to other high grade WEEE items to project temporal changes in PCB composition of items, which are considered in conjunction with projections of future waste arisings in Europe to estimate the recoverable value from PCBs in the future waste stream.

Previous results on the chemical analysis of PC RAM modules placed on the market between 1991 and 2008 obtained by the author during his MRes research have been examined in detail and used to provide an assessment of the past temporal trends of PM and copper content of RAM. This assessment has then been used to provide projections of future trends in PM and Cu content in RAM, PCs and WEEE of computing EEE generally.

Total value of precious metals and copper in modules ranged from USD 33,000-54,000/tonne, with an average value across samples of USD 43,700/tonne. Gold and silver content of modules has remained reasonably constant over time, while palladium content has decreased by $20 \%$ due to: miniaturisation of the Multilayer Ceramic Capacitors (MLCCs) in which it is mainly found; reductions in thickness of MLCC internal electrodes; and reductions in Pd 
content of the electrode alloy. Copper content ranges from 1.8-5.1 g/module with a temporal increase observed in DIMMs of 0.23 g/year. Linear extrapolation of this trend suggests an increase in copper content of DRAM modules by $75 \%$ by 2025. The increase in copper is thought to be as a result of increasing number of tracks on DRAM PCBs to support increasing functionality. It is likely that this trend will continue until 2020 despite the potential to reach saturation as additional layers can be added to multi-layered PCBs to support enhanced functionality once individual layers are saturated. Gold represents $85-95 \%$ of potentially recoverable value in modules, so changes in the content of the other metals has had little impact on the recycling value of PC RAM modules or recycling viability.

Limitations to the accuracy of projections of future recycling potential of items suffer from the limited accuracy introduced by use of static compositional data of current devices. This highlights a potential problem for any legislative or waste management measures implemented based upon recycling potential projections which use a static current composition for items. Should any significant changes in the concentration of the key PM metals Ag and Au, in PCBs of items occur as a result of design alterations, materials substitution, or 'thrifting' in manufacturing, recycling potential of items and the future viability of WEEE recycling may be compromised. This demonstrates the value of studying historical trends in the composition of items for more accurate projections of future recycling potential.

\subsection{Background}

\subsubsection{Criticality of precious metals and copper}

The strategic importance of recovering PMs and copper has been well explored; PGMs have been highlighted in the EU14 $4^{100}$ and EU $20^{53}$ critical materials reports and recovery of silver and gold has been shown by the Resource Efficiency Knowledge Transfer Network (KTN) to be of key importance to the UK economy with gold being the most unsecure of 69 metals assessed. ${ }^{146}$ Schneider et al. have shown that PGMs have high economic resource scarcity potential (ESP) and that gold and silver have high abiotic depletion potentials (ADP). ${ }^{147}$ Nasser et al. have shown copper, silver and gold score highly in criticality assessment. ${ }^{148}$ The UK Resource Security Action Plan also identifies gold, silver and copper as unsecure or at risk. ${ }^{149}$

Global demand for gold in 2015 was 4,124 tonnes, approximately 20\% lower than the record demand of 5,087 tonnes reached in 2013. Supply from secondary sources currently accounts for $28 \%$ of global production. EEE accounts for $6 \%$ of global gold demand. ${ }^{150}$ Gold is predominantly used in EEE for contacts and in ICs. ${ }^{69}$ 
Silver demand reached a record high of 36,406 tonnes in 2015, with supply from secondary sources accounting for $12 \%$ of global supply. EEE is the major consuming industry accounting for $21 \%$ of global demand, exceeding supply from secondary sources. ${ }^{151}$ Major applications of silver in EEE include: contacts; multilayer ceramic capacitors (MLCCs); thick film chip resistors; and more recently lead-free solders on circuit boards. ${ }^{69}$ The photovoltaics industry also consumes a significant proportion of Ag for use in bus bars of solar cells. ${ }^{152}$

Global palladium demand in 2015 was 294 tonnes, only 0.5 tonnes below record demand set in 2014. Supply from secondary sources is sufficient to meet $20 \%$ of global demand. EEE accounts for $13 \%$ of global demand ${ }^{153}$ with the major use being manufacture of MLCCs ${ }^{69}$.

In 2015, 21.8 million tonnes of copper were produced globally with $16 \%$ supplied from secondary sources. ${ }^{154}$ The electronics sector is the largest consumer of copper accounting for $\sim 39 \%$ of global demand. ${ }^{155}$ Major applications for copper in EEE include cables, wires, connectors, contacts and conductive tracks such as those on PCBs. ${ }^{69}$

The large proportions of these metals consumed by the EEE industry, and the significance that secondary production has in meeting global demand, emphasises the importance of efficient recovery from WEEE to offsets demand for primary resources. The economic driving force for WEEE recycling has been recovery of material value, $>95 \%$ of which has been attributable to PMs and copper, with $>80 \%$ attributable to gold alone. ${ }^{30}$ Were it not for the presence of sufficiently valuable quantities of PMs in items, recovery of other metals found in PCBs would not be possible. The presence of the PMs grants economic viability to the recovery of certain CRMs which otherwise would be uneconomical. ${ }^{156}$

\subsubsection{WEEE as a resource}

WEEE is a secondary resource and a reserve of speciality metals ${ }^{118}$ i.e.

"a concentration or occurrence of material of intrinsic economic interest in or on the Earth's crust in such form, quality and quantity that there are reasonable prospects for eventual economic extraction". ${ }^{157}$

Useful analogies between primary and secondary resources have been made by others when assessing the potential of scrap materials as metal sources. ${ }^{34,64,158}$ When assessing primary resources, a cut-off grade (COG) is established, below which it is not economically feasible to mine. It is possible to establish similar recycling COGs based upon levels of PMs and copper in WEEE items. These will vary between particular recycling processes and depend upon: total quantities of items available for recycling (governed by number of items placed on the market - POM; and lifespan of items); collection efficiency; recovery efficiencies of target materials; costs of recycling (including costs of labour, plant, energy, transport, and compliance with 
regulation); and value of recovered materials. ${ }^{124}$ Some WEEE items contain gold in concentrations $\sim 200$ times greater than in typical gold ores, e.g. gold can be 0.04 wt\% (400 $\mathrm{g} / \mathrm{t}$ ) of a mobile phone, 200 times greater than typical gold ore $-1-3 \mathrm{~g} / \mathrm{t}$, and 14 times higher than the highest known grade deposit - $28 \mathrm{~g} / \mathrm{t}$ (Tau Tona, RSA). ${ }^{159}$ The cost of recycling an item and the ability to effectively recover precious metals is also significantly influenced by product design.

Concerns have been raised over the future viability of WEEE recycling due to component miniaturisation and substitution of expensive/rare materials in EEE in conjunction with ever rising recycling costs. ${ }^{87,128}$ Data on temporal trends in precious metal (PM) and copper content is important in order to verify these concerns and inform projections of future recycling potential, which is important knowledge for planning future recycling infrastructure and process development. For example, the PM content of mobile phones which have relatively high PM content, has been shown to increase over time as more functions have been added. ${ }^{34}$ This paints a positive picture of likely future recycling potential of such devices. ${ }^{159}$ It has however been suggested that the converse is true for computing technology, which would reduce recycling potential of such items in the future. Little data is available on temporal trends in value of computing technologies, however manufacturing trends may result in a major barrier to recycling as computing equipment currently accounts for a significant proportion of the derivable value in WEEE. ${ }^{159}$

It is the purpose of the work presented in this chapter to contribute to such data through the analysis of PM and copper content of PC DRAM modules placed on the market (POM) over the period 1991-2008. To gain a full understanding of the future viability of recycling, let us first examine the outlook for the individual stages of the recycling process chain and how this may affect viable recycling in the future.

\subsubsection{The recycling process}

Pre-processing - Automated processes involving shredding of items with PCBs inside cause PMs to be dispersed and lost through unintended co-separation with bulk materials fractions. ${ }^{18,}$ 106, 146 Material flow analysis (MFA) studies have shown bottom-line recovery efficiencies of gold and palladium from ICT equipment to be as low as $25 \%$ when such processes are employed. Manual isolation of PCBs achieves the highest bottom-line recovery rates ( 95\% for gold). ${ }^{116}$ Manual pre-processing is costly, with high COGs in comparison to automated pre-processing, but efficient bottom-line recovery of PMs from high grade items justifies the costs, which are greatly affected by product design (DfRR), and the resulting ease of disassembly. ${ }^{156}$ 
When items are difficult to disassemble due to poor design for End-of-Life (EoL), or when low recoverable value prohibits manual disassembly (i.e. item grade $<\mathrm{COG}$ ), semi-automated approaches using coarse crushing to break open housings and hand picking of PCBs has given intermediate pre-processing recovery rates for PMs ( $70 \%$ for gold) at lower cost than manual processes. ${ }^{116}$ As a consequence of the concentration-yield function, ${ }^{18}$ (Section 1.4.2) COGs for WEEE and PM recovery rates are inversely related to the degree of automation used in preprocessing. Therefore, bottom-line recovery efficiencies, COGs, and the viability of WEEE as a PM source, are all largely determined by pre-processing method.

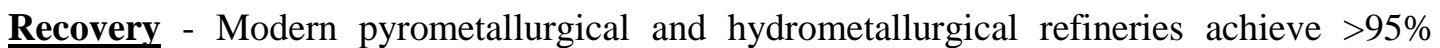
recovery of gold and can recover numerous metals in addition to PMs and copper. Because of high refining efficiencies the recovery process is generally less significant than the method of pre-processing in determining the recycling potential of WEEE. ${ }^{12,78,116}$

\subsubsection{Market values of metals}

Metal recycling is highly dependent upon the vagaries of metal values as shown by the dramatic reduction in quantities of gold supplied from secondary sources in 2013 and 2014 (Figure 3.1), which mirrors annual reductions in the price of gold since 2012. ${ }^{150}$ As metal prices fall, COGs for recycling rise as recoverable value from WEEE and the viability of PM recovery from WEEE is reduced. Since the result of the UK 'Brexit' vote, PM prices have risen dramatically as investors lose faith in falling stocks and hunt for safer investment in gold, silver and palladium. In July 2016, silver and gold values reached a two-year high and the value of palladium rose by $12 \%$, good news for the viability of WEEE recycling. ${ }^{160}$

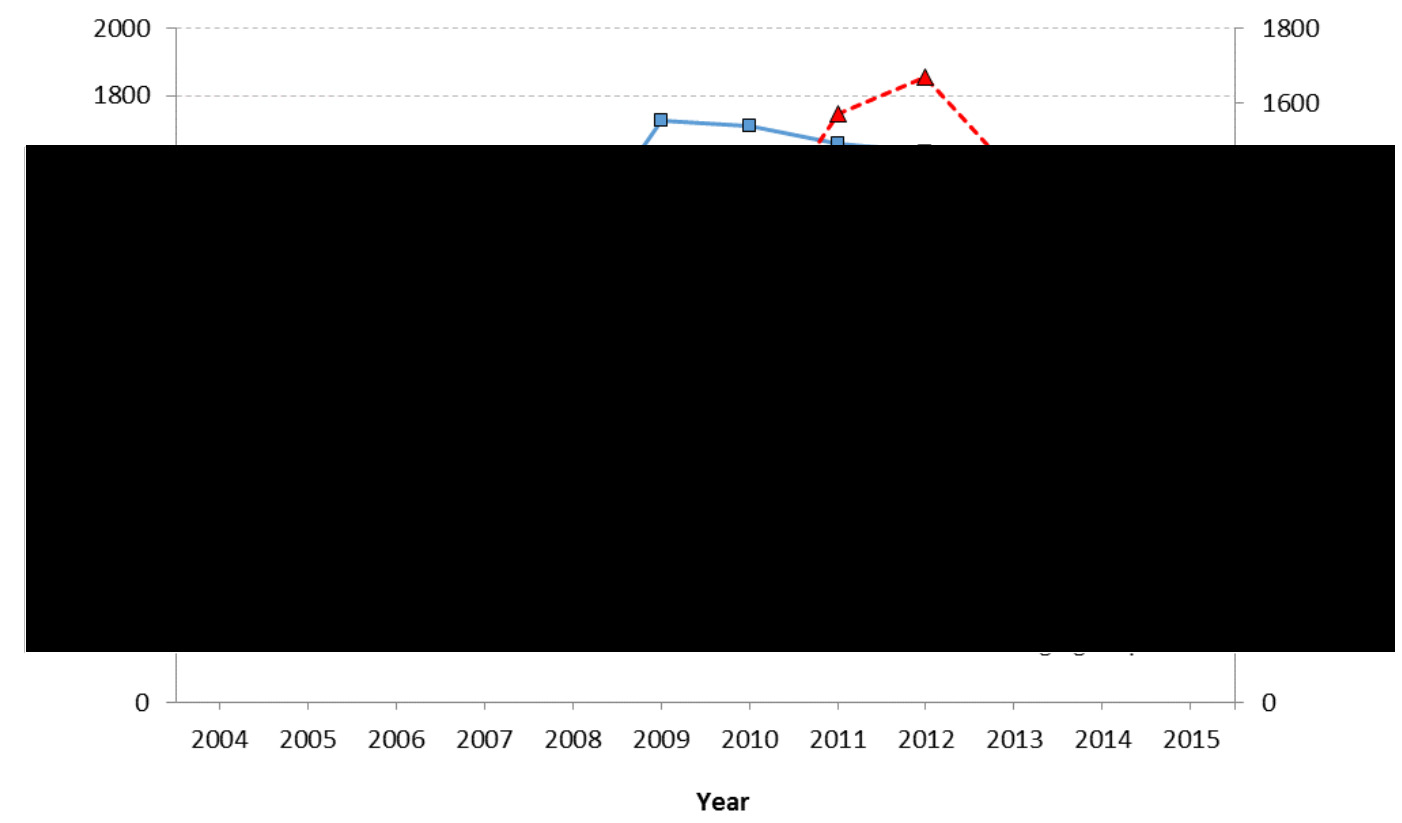

Figure 3.1 Annual gold production from scrap \& average gold price. Data from O'Connell et al. ${ }^{150,161}$ 


\subsubsection{Waste management strategies}

The recycling of circuit boards involves long and complex logistics systems. Take the UK as an example. WEEE is collected via numerous mechanisms including from consumers at household waste recycling centres (HWRCs), through distributor takeback schemes (DTSs), and from businesses by waste management and reverse logistics organizations. WEEE then finds its way to authorized approved treatment facilities (AATFs). Here PCBs are isolated from items and segregated according to grade. These are sold abroad to refiners operating pyrometallurgical and/or hydrometallurgical recovery processes such as Umicore and Boliden in Europe and Mitsubishi Metals in Asia. ${ }^{67,78}$ Often PCBs are sold to brokers acting as intermediaries, who amass sufficient quantities before shipping to refiners. To maintained economic viability of recycling, sufficient value generated from the sale of recovered metals must filter back through the value chain to finance costs incurred at each stage of recycling and during transport between them.

\subsubsection{Outlook for waste management and viability of WEEE recycling}

As global WEEE volumes increase, appropriate waste management will be crucial for mitigating environmental impacts, materials criticality and enhancing global resourceefficiency. The continued viability of WEEE recycling is crucial to global sustainability and management of the growing WEEE problem. This is determined to a large extend by the recycling potential of future WEEE i.e. "that potential e-waste recycling revenues exceed costs of collection, transportation and processing”. ${ }^{145}$ Grades of WEEE items must continue to remain above COGs for recycling also. Doubts over the future viability of WEEE recycling have been raised. Reductions in the value of precious metals present in WEEE and rising overheads for recycling raises COGs for recycling processes. This issue was highlighted as early as $2001{ }^{87}$ Since 2013 costs of recycling have risen by $1.9 \%$ annually, a trend which is expected to continue until 2019. ${ }^{162}$ The rising costs of electronics recycling via BATRRT has led to much WEEE being stockpiled, or exported to regions where recycling and recovery of PMs is conducted in a manner resulting in both low recovery yields and harm to the environment \& human health. This has effectively reduced the size of the 'reserve' of metals present in WEEE, as a smaller proportion of WEEE arising (i.e. the total secondary resource) can be diverted to BATRRT processes. Additionally, the trend in the electronics industry towards miniaturisation and 'thrifting' has reduced quantities of PMs in items, affecting the grade of items and recycling revenues. ${ }^{108,}$ 163, 164

Despite rising recycling costs and diminishing metal content in individual items, volumes of WEEE are increasing rapidly due to greater market penetration of electronics, technological innovation and decreasing product lifetimes. The size of the potential secondary resource is 
therefore increasing and higher collection rates enabled by statutory recycling targets imposed by the growing global number of e-waste legislative instruments (Section 1.4.2) will effectively increase the quantity of WEEE made available for recycling. When larger quantities of WEEE are available, processing of lower grade materials becomes viable. ${ }^{34}$ Resulting economies of scale compensate for reductions in grade of individual items to some extent, although uncertainty exists over how collection costs may scale with collection rate. ${ }^{165}$ Recyclability, i.e. the probability of an item being recycled, taking into account the recycling difficulty of its material components in physical treatment and chemical recovery processes, ${ }^{166}$ will also improve in the future as a result of superior product design for EoL ${ }^{167-169}$ driven by the proliferation of EPR (particularly IPR), restrictions on hazardous substances, and proliferation of product eco-labels. ${ }^{170}$ The expected effect of these factors will be lower recycling costs and greater recovery efficiencies of target metals.

Obtaining information related to future recycling potential of WEEE is essential for the development of legislation and new recycling capacity necessary to deal with ever growing volumes of WEEE. ${ }^{171}$ This valuable information is acquired through 3 types of analyses: projections of mass flow of products over time; product lifespans; and material composition of items. ${ }^{145,172,173}$ This enables quantities of WEEE items arising over time to be projected and the material content and potential recycling revenues to be established (i.e. the size of the potential metals reserve). Over 10 methods and models have been used to estimate WEEE generation in previous studies, ${ }^{174}$ the method of choice depending primarily upon the availability and quality of data. ${ }^{175}$ In addition to these considerations, components within items should also be investigated due to alterations to technologies over time..$^{40}$

Zeng et al. projected the recycling potential of 'new' WEEE arising in China for the period 2010-2030 using the material flow analysis (MFA) and lifespan model of the Weibull function. ${ }^{145}$ Based on previous projections of WEEE arising in Europe, Cucchiella et al. predicted the potential revenues from WEEE recycling in Europe in $2020 .{ }^{172}$ The issue with such projections is that the compositional data of items applied to the mass flow projections are based on current and historical compositional data. Although these studies are robust, insufficient data on temporal changes in metal content of devices is available to allow changes in PM and copper content over time to be taken account of in projections. Both studies indicate a majority of the recoverable value from WEEE into the future will be from copper and PMs, however without data highlighting temporal trends in copper and PM content, it is difficult to take account of the potential effect of miniaturisation and 'thrifting' in manufacturing. It is the purpose of the work presented in this chapter to explore historical temporal changes in PM 
and copper content of DRAM modules, and make projections for future recycling potential of PCBs based on observed trends in RAM.

Precious metals are predominantly found in PCBs of items which are constructed from common surface mount device (SMD) components. Dynamic RAM (DRAM) modules are essential PCB based components of PCs (Figure 3.2). ${ }^{144}$ To investigate historical changes in PM and copper content of PC DRAM modules, samples have been gathered from Metech Recycling (UK) Ltd, dated, and analysed. DRAM is chosen for study as these devices are relatively simple PCBs, containing only a single type of each of those few SMDs which represent the main applications of PMs and copper in PCBs in general i.e. ICs, chip resistors, MLCCs, copper tracks, edge contacts, etc. Following chemical analysis of samples, this simplifies the task of rationalizing observable temporal trends in metal content in terms of changes to common PCB components over time. This task would be difficult for more complex PCBs such as motherboards and mobile phone PCBs with dozens of different kinds of SMD components. Historical temporal trends observed in DRAM, once rationalised against changes in PCB design, can then be used to make predictions on temporal changes in PM and copper content of items into the future.

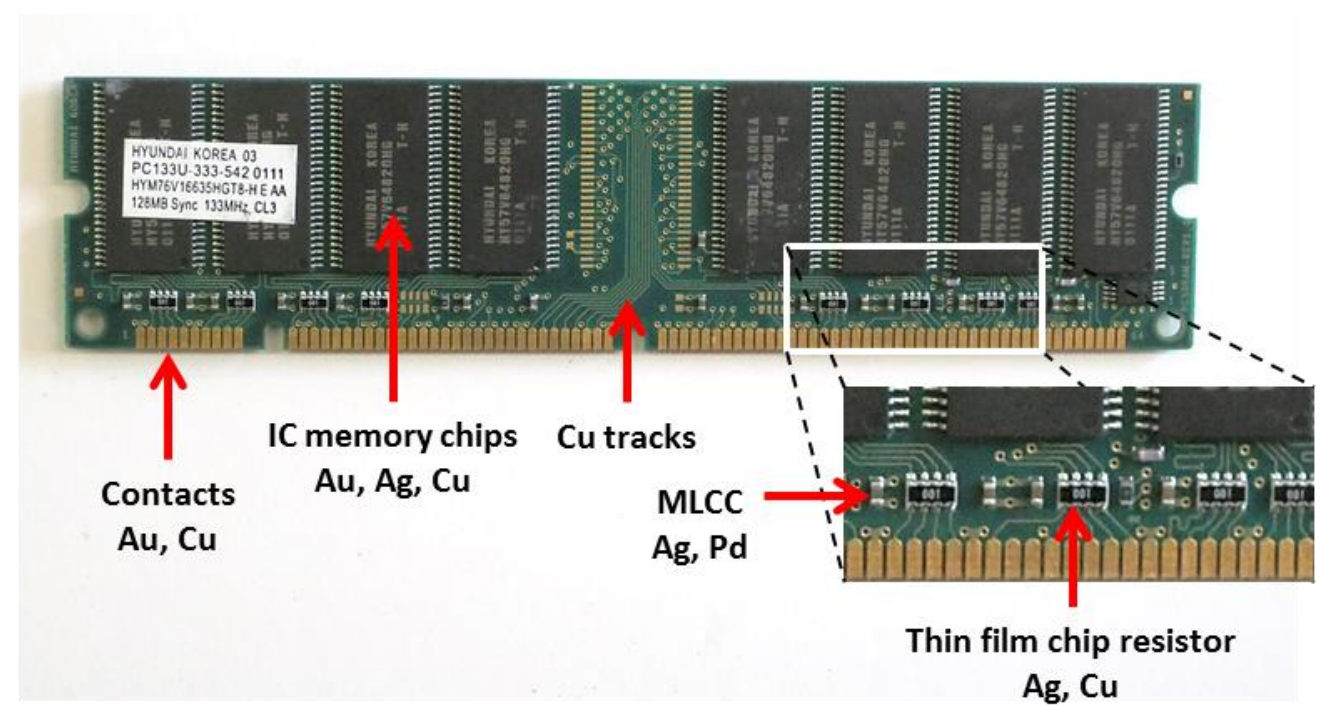

Figure 3.2 Anatomy of PC DRAM module and locations of precious metals and copper. ${ }^{144}$

This work represented the first published compositional data for DRAM modules and temporal data on PM and copper content in PCBs; and the first projections of future changes to PM and copper content of PCBs used with compositional data and product mass flow data to create a picture of future recycling potential which takes account of the potential future impact of 
miniaturisation and 'thrifting' trends in manufacturing. ${ }^{144}$ The value of inclusion of temporal trend data in projections of recycling potential is demonstrated.

\subsection{Materials and methods}

\subsubsection{Evaluation of historic trends in composition and value of DRAM modules}

A range of PC DRAM samples, covering memory sizes and RAM types from 4 MB FPM single in-line memory modules (SIMMs) through to 2 GB DDR3 dual in-line memory modules (DIMMs), were previously gathered from Metech Recycling (UK) Ltd., identified (Table 3.1) and analysed during the author's MRes project (Table 3.2). ${ }^{22}$ Compositional data from this study is used to assess temporal trends in composition of DRAM modules. PCB dimensions of SIMM samples (1-3) are 108.5 x $26.0 \mathrm{~mm}$ while DIMM sample (4-15) PCBs are $133.5 \mathrm{x}$ $31.5 \mathrm{~mm}$. Sample masses range from 13.1-21.9 $\mathrm{g}$ with an average mass of $17.5 \mathrm{~g}$.

Here in a continuation of that work the analytical data is re-examined and reanalysed in detail to extract data on temporal trends in composition and value. In order to create a new timeline of the samples, an investigation of historical data on the size of memory available in PCs POM, and the date of introduction of different RAM technologies was conducted. ${ }^{176-178}$.

Data on PM and copper content of samples were plotted against date of sample introduction based on the DRAM timeline. These plots were then analysed to investigate temporal trends in DRAM sample composition. To rationalise observed trends in Au content of samples, surface areas of edge contacts were compared. This comparison was conducted using images of the samples analysed, in which pairs of RAM modules for comparison were photographed side by side. Using Adobe Photoshop, the areas of the edge contacts in the images can be highlighted and the number of pixels in these areas counted using the pixel counting feature in the software. Comparison of the number of pixels within the areas of contacts in the images of each sample enables a comparison of their surface areas to be made. 
Table 3.1 DRAM modules analysed during author's MRes project. ${ }^{22}$

\begin{tabular}{|c|c|c|c|c|c|}
\hline Sample & Brand & Model & Memory type & Module type & Capacity (MB) \\
\hline 1 & Samsung & KMM5361000G-7 & FPM $^{\mathrm{b}}$ DRAM & 72-pin SIMM ${ }^{\mathrm{g}}$ & 4 \\
\hline 2 & Micron Technology & MEMSIM016AAWW & $\mathrm{EDO}^{\mathrm{c}} \mathrm{DRAM}$ & 72-pin SIMM & 8 \\
\hline 3 & Micron Technology & MT8D432M-6X & EDO DRAM & 72-pin SIMM & 16 \\
\hline 4 & Discovery & DISCOVERY S/N 1212772 & $\begin{array}{l}\text { SDR }^{\mathrm{d}} \\
\text { SDRAM }^{\mathrm{e}}\end{array}$ & PC100 168-pin DIMM ${ }^{\mathrm{h}}$ & 32 \\
\hline 5 & Samsung & KMM366S823CTS-GH & SDR SDRAM & PC100 168-pin DIMM & 64 \\
\hline 6 & Hyundai & $\begin{array}{l}\text { HYM76V16635HGT8-H E } \\
\text { AA }\end{array}$ & SDR SDRAM & PC133 168-pin DIMM & 128 \\
\hline 7 & Infineon & HYS64V16300GU-7.5-C2 & SDR SDRAM & PC133 168-pin DIMM & 128 \\
\hline 8 & Samsung & M368L1716ETM-CB0 & $\begin{array}{l}\text { DDR }^{\mathrm{f}} \\
\text { SDRAM }\end{array}$ & PC2100 184-pin DIMM & 128 \\
\hline 9 & Micron Technology & $\begin{array}{l}\text { MT9VDDT3272LAG- } \\
\text { 265C4 }\end{array}$ & $\begin{array}{l}\text { DDR } \\
\text { SDRAM }\end{array}$ & PC2100 184-pin DIMM & 256 \\
\hline 10 & Kingston Technology & KTH-D530/512 & $\begin{array}{l}\text { DDR } \\
\text { SDRAM }\end{array}$ & PC3200 184-pin DIMM & 512 \\
\hline 11 & Hynix & $\begin{array}{l}\text { HYMD512646PC8J-D3 } \\
\text { AA-C }\end{array}$ & $\begin{array}{l}\text { DDR } \\
\text { SDRAM }\end{array}$ & PC3200 184-pin DIMM & 1024 \\
\hline 12 & Promos Technologies & V916764K24QAFW-E4 & $\begin{array}{l}\text { DDR2 } \\
\text { SDRAM }\end{array}$ & $\begin{array}{l}\text { PC2-4200 240-pin } \\
\text { DIMM }\end{array}$ & 512 \\
\hline 13 & Micron Technology & MT8HTF12864AY-667G1 & $\begin{array}{l}\text { DDR2 } \\
\text { SDRAM }\end{array}$ & $\begin{array}{l}\text { PC2-5300 240-pin } \\
\text { DIMM }\end{array}$ & 1024 \\
\hline 14 & Kingston Technology & $\mathrm{kvr} 800 \mathrm{~d} 2 \mathrm{n} 5 \mathrm{k} 2 / 2 \mathrm{~g}$ & $\begin{array}{l}\text { DDR2 } \\
\text { SDRAM }\end{array}$ & $\begin{array}{l}\text { PC2-6400 240-pin } \\
\text { DIMM }\end{array}$ & 2048 \\
\hline 15 & Samsung & M378B5673FHO-CF8 & $\begin{array}{l}\text { DDR3 } \\
\text { SDRAM }\end{array}$ & $\begin{array}{l}\text { PC3-8500 240-pin } \\
\text { DIMM }\end{array}$ & 2048 \\
\hline
\end{tabular}

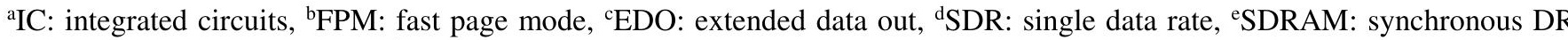
single in-line memory module, hIMM: double inline memory module 
Table 3.2 Precious metal and copper content of PC DRAM samples 1-1522 (errors given as ranges for triplicate analyses ${ }^{a}$, and and SIMMs

\begin{tabular}{|c|c|c|c|c|c|c|c|c|c|}
\hline \multirow{2}{*}{ Sample } & \multirow{2}{*}{ Contacts } & \multicolumn{2}{|c|}{ No. of } & \multicolumn{2}{|c|}{ Gold } & \multicolumn{2}{|c|}{ Palladium } & \multicolumn{2}{|c|}{ Silver } \\
\hline & & ICs & MLCCs & mass/mg & conc/ppm & mass/mg & conc/ppm & mass/mg & conc/ \\
\hline 1 & Gold & 12 & 12 & $20.8( \pm 0.5)$ & $1,144( \pm 26)$ & $3.43( \pm 0.03)$ & $189( \pm 2)$ & $133( \pm 5)$ & 7,321 \\
\hline 2 & Tin & 16 & 16 & $7.2( \pm 0.3)$ & $327( \pm 12)$ & $3.2( \pm 0.6)$ & $150( \pm 30)$ & $62( \pm 3)$ & 2,841 \\
\hline 3 & Tin & 8 & 8 & $\mathbf{3 . 8 ^ { b }}( \pm 0.3)$ & $251^{2}( \pm 17)$ & $1.9^{\mathrm{Ci}}( \pm 0.1)$ & $124^{\mathrm{Ci}}( \pm 8)$ & $54( \pm 1)$ & 3,567 \\
\hline 4 & Gold & 4 & 15 & $10.5( \pm 0.2)$ & $484( \pm 10)$ & $1.49( \pm 0.03)$ & $114( \pm 3)$ & $7.4( \pm 0.3)$ & 562 \\
\hline 5 & Gold & 8 & 39 & $17.8( \pm 0.1)$ & $1,099( \pm 7)$ & $\mathbf{2 . 1} \mathbf{1}^{\mathrm{Cii}}( \pm 0.2)$ & $118^{\mathrm{Cii}}( \pm 11)$ & $24.0( \pm 0.7)$ & 1,329 \\
\hline 6 & Gold & 16 & 34 & $26.8( \pm 0.6)$ & $1,319( \pm 27)$ & $2.1( \pm 0.1)$ & $103( \pm 5)$ & $25( \pm 1)$ & 1,251 \\
\hline 7 & Gold & 8 & 16 & $17.5( \pm 0.4)$ & $1,127( \pm 28)$ & $1.6( \pm 0.1)$ & $102( \pm 8)$ & $13.5( \pm 0.5)$ & 767 \\
\hline 8 & Gold & 8 & 49 & $20.8( \pm 0.3)$ & $1,179( \pm 19)$ & $1.69( \pm 0.08)$ & $96( \pm 5)$ & $14.5( \pm 0.7)$ & 822 \\
\hline 9 & Gold & 8 & 49 & $18.2( \pm 0.5)$ & $1,064( \pm 32)$ & $\mathbf{1 . 0}^{\text {Ciii }}( \pm 0.1)$ & $57^{\text {Ciii }}( \pm 7)$ & $124( \pm 5)$ & 7,232 \\
\hline 10 & Gold & 16 & 42 & $16.8( \pm 0.5)$ & $786( \pm 22)$ & $1.3( \pm 0.07)$ & $59( \pm 3)$ & $27.5( \pm 0.6)$ & 1,290 \\
\hline 11 & Gold & 16 & 39 & $23.4( \pm 0.7)$ & $1,078( \pm 31)$ & 1.0 $^{\mathrm{Civ}}( \pm 0.2)$ & $44^{\text {Civ }}( \pm 9)$ & $33( \pm 1)$ & 1,507 \\
\hline 12 & Gold & 8 & 39 & $15.6( \pm 0.3)$ & $980( \pm 19)$ & $0.59( \pm 0.04)$ & $37( \pm 2)$ & $15.6( \pm 0.7)$ & $979(=$ \\
\hline 13 & Gold & 8 & 27 & $13.2( \pm 0.2)$ & $968( \pm 17)$ & $\mathbf{0 . 6 0}( \pm 0.03)$ & $44( \pm 2)$ & $16.3( \pm 0.4)$ & 1,195 \\
\hline 14 & Gold & 16 & 47 & $18.0( \pm 0.6)$ & $1,098( \pm 36)$ & $\mathbf{0 . 3 9 9}( \pm 0.004)$ & $24.32( \pm 0.05)$ & $20.5( \pm 0.2)$ & 1,248 \\
\hline 15 & Gold & 16 & 114 & $17.8( \pm 0.1)$ & $1,083( \pm 7)$ & $0.58( \pm 0.01)$ & $35.7( \pm 0.4)$ & $19.5( \pm 0.6)$ & 1,208 \\
\hline \multirow{2}{*}{\multicolumn{2}{|c|}{$\begin{array}{c}\text { Average } \\
\text { composition }\end{array}$}} & \multicolumn{2}{|c|}{$\underline{\text { All samples }}(\mathbf{1 - 1 5})$} & 16.5 & 932 & 1.5 & 86 & 39 & 2,208 \\
\hline & & \multicolumn{2}{|c|}{ DIMMs (4-15) } & 18.0 & 1,022 & 1.2 & 70 & 28 & 1,616 \\
\hline
\end{tabular}

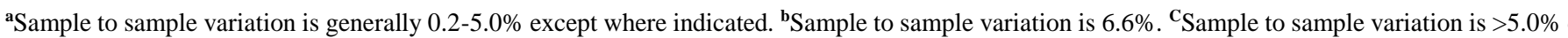
limit of AAS instrument: ${ }^{\mathrm{Ci}_{\text {Sample }}}$ to sample variation of $19.2 \%$; ${ }^{\text {Cii }}$ sample to sample variation $9.1 \%$; ${ }^{\text {Ciii }}$ sample to sample variation of $11.9 \%$; ${ }^{\text {Civ }}$ sample 


\subsubsection{Evaluation of recycling potential of DRAM}

To evaluate recycling potential of metals in RAM, average compositions of RAM modules were calculated from data and compared with compiled compositional data on PC PCBs from available literature. The value of precious metals and copper, as well as the value fractions represented by each of these metals in samples was calculated using the following market values: Au: USD 1221.50/Oz (LBMA 19/2/16 AM); Ag: USD 15.37/Oz (LBMA 19/2/16); Pd: USD 513.00/Oz (LBMA 18/2/16 AM); Cu: USD 4575.00/tonne (LME 18/2/16 Cash buyer). To evaluate the significance of DRAM modules as a global source of PMs and copper, the total quantity of these metals available in waste DRAM generated in 2013 was calculated based on the previously calculated average composition of DRAM, and the quantity of waste DRAM modules arising in 2013. It was assumed that a minimum quantity of waste DRAM modules generated this year would be equivalent to the number of waste PCs arising that year. This was calculated using the WEEE directive assumption that WEEE arising is equivalent to the average of the number of items POM over the previous 3 years. ${ }^{4}$ The value of metals in this quantity of DRAM was calculated. To provide a context to evaluate the significance of quantities of metals in DRAM, total quantities of gold, silver, palladium and copper in waste DRAM generated in 2013 are expressed as percentages of the global demand for these metals, demand from the EEE sector itself, and global supply from secondary sources that year.

\subsubsection{Projections of future recycling potential.}

Projections and confidence intervals for future quantities of metals in waste DRAM were obtained by linear regression analysis carried out in python in collaboration with Dr. Ingrid Hallin, Plymouth University. ${ }^{179-181}$

\subsection{Results and discussion}

\subsubsection{Historic trends in composition and value of DRAM modules}

A timeline of historic DRAM technologies POM based on dates of introduction of RAM technologies and memory sizes is shown in Figure 3.3. This was used to date analysed DRAM samples. Modules were assigned a date equal to the midpoint of the line representing their use over time. For example, sample 4 is a 32 MB PC100 SDRAM module. According to Figure 3.3, such modules were available in PCs for 2 years from the beginning of 1998 until the end of 1999. The sample was therefore assigned a date of 1999 ( \pm 1 year). Once dated, temporal variation in PM and copper content of modules was evaluated for temporal trends. As a means of comparison, Table 3.3 compares average compositions of all RAM samples analysed (115) and average composition of DIMMs only (4-15) with literature values (dated where information was available) for precious metal and copper content, along with average 
composition with per tonne values, and value fractions, for general PC PCBs, rather than just the memory modules described here.

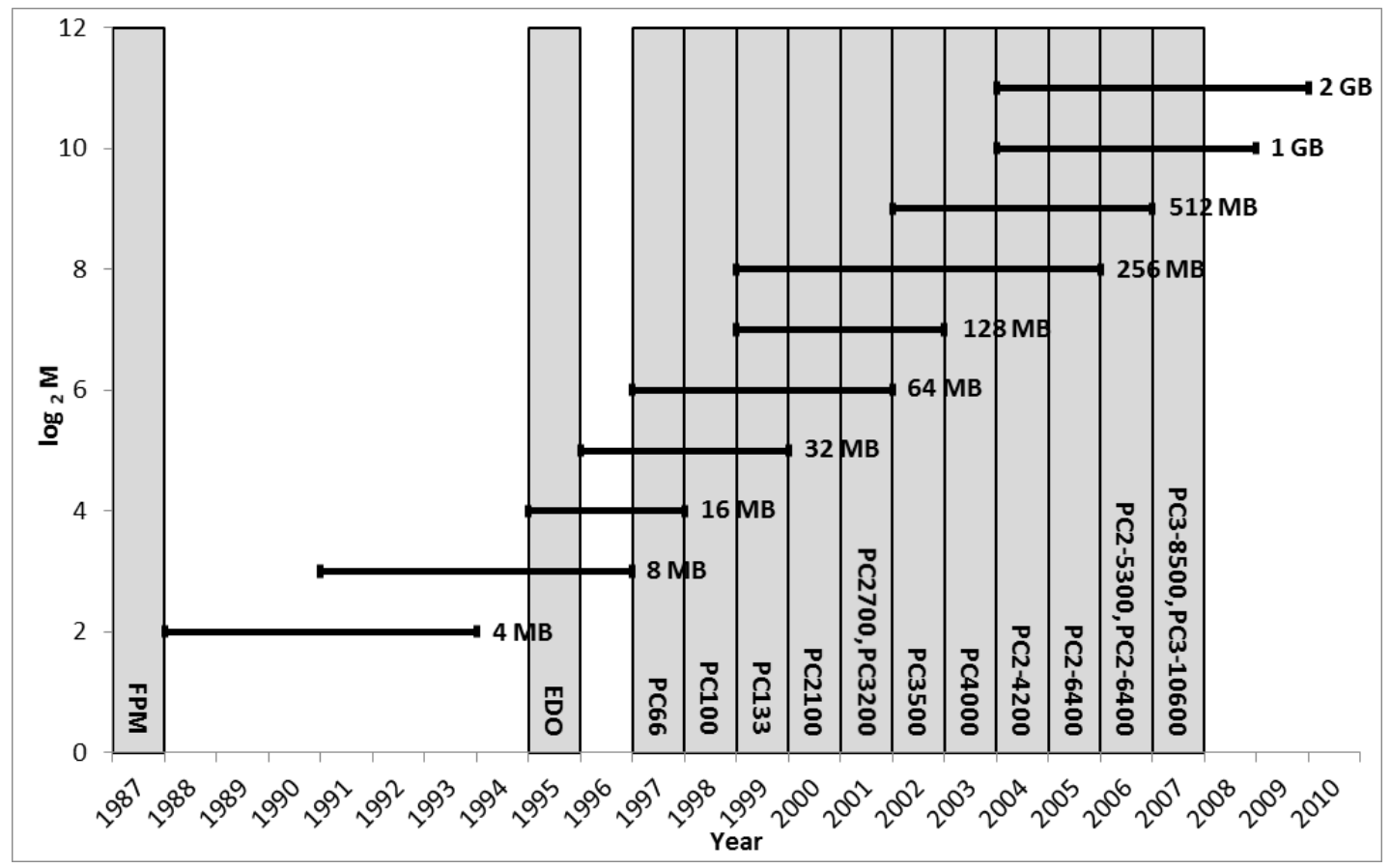

Figure 3.3. Timeline of DRAM technologies in PCs POM. M is the Module Memory Capacity (MB); the vertical blocks ( $\square$ ) show the year of introduction of RAM type (data from Mueller, 2011; Ögren et al.). ${ }^{177,} 178$ The horizontal lines show the period when PCS POM were available with memory capacity M (data for from Polsson, 2013). ${ }^{176}$ 
Table 3.3 Literature values for precious metal and copper content of PC PCBs compared to calculated average composition of analysed DRAM samples. (Errors quoted are standard-errors of the mean)

\begin{tabular}{|c|c|c|c|c|c|}
\hline \multirow{2}{*}{ Source } & \multicolumn{4}{|c|}{ Metal content (ppm) } & \multirow{2}{*}{ Model year } \\
\hline & Au & Ag & Pd & $\mathbf{C u}$ & \\
\hline $\begin{array}{l}\text { Average composition } \\
\text { of DRAM (1-15) }\end{array}$ & $\begin{array}{c}932 \\
( \pm 61)\end{array}$ & $\begin{array}{l}2,208 \\
( \pm 568)\end{array}$ & $\begin{array}{c}86 \\
( \pm 12)\end{array}$ & $\begin{array}{c}196 \times 10^{3} \\
\left( \pm 18 \times 10^{3}\right)\end{array}$ & 1991-2008 \\
\hline $\begin{array}{l}\text { Average composition } \\
\text { of DIMM (4-15) }\end{array}$ & $\begin{array}{l}1,022 \\
( \pm 61)\end{array}$ & $\begin{array}{c}1,789 \\
( \pm 517)\end{array}$ & $\begin{array}{c}70 \\
( \pm 10)\end{array}$ & $\begin{array}{c}200 \times 10^{3} \\
\left( \pm 21 \times 10^{3}\right)\end{array}$ & 1999-2008 \\
\hline $\begin{array}{l}\text { (Oguchi et al., } \\
2011)^{34}\end{array}$ & $\begin{array}{l}220 \\
120 \\
270 \\
270 \\
450 \\
300 \\
130 \\
140\end{array}$ & $\begin{array}{l}570 \\
680 \\
760 \\
510 \\
590 \\
400 \\
230\end{array}$ & $\begin{array}{l}110 \\
160 \\
150\end{array}$ & $\begin{array}{l}210 \times 10^{3} \\
160 \times 10^{3} \\
260 \times 10^{3} \\
340 \times 10^{3} \\
300 \times 10^{3} \\
170 \times 10^{3} \\
200 \times 10^{3} \\
140 \times 10^{3}\end{array}$ & $\begin{array}{c}\text { Unavailable } \\
\text { Unavailable* } \\
1985 \\
1989 \\
1994 \\
1985 \\
1989 \\
1994\end{array}$ \\
\hline $\begin{array}{l}\text { (Chancerel et al., } \\
\text { 2009) }\end{array}$ & $\begin{array}{c}81 \\
250 \\
230 \\
156 \\
300 \\
600\end{array}$ & $\begin{array}{l}905 \\
1000 \\
1000 \\
775 \\
600 \\
700\end{array}$ & $\begin{array}{c}110 \\
90 \\
99 \\
100\end{array}$ & & $\begin{array}{l}\text { Unavailable } \\
\text { Unavailable } \\
\text { Unavailable } \\
\text { Unavailable } \\
\text { Unavailable } \\
\text { Unavailable }\end{array}$ \\
\hline $\begin{array}{l}\text { (Tuncuk et al., } \\
2012)^{12}\end{array}$ & $\begin{array}{c}250 \\
86\end{array}$ & $\begin{array}{c}1000 \\
694\end{array}$ & $\begin{array}{l}110 \\
309\end{array}$ & $\begin{array}{l}200 \times 10^{3} \\
185 \times 10^{3}\end{array}$ & $\begin{array}{l}\text { Unavailable } \\
\text { Unavailable }\end{array}$ \\
\hline $\begin{array}{l}\text { (Yamane et al., } \\
2012)^{131}\end{array}$ & 1300 & 1600 & & $202 \times 10^{3}$ & $\begin{array}{l}\text { Analysis of } 101 \mathrm{~g} \text { sub samples } \\
\text { sample from large batch of } \\
\text { various models of PC PCB. }\end{array}$ \\
\hline $\begin{array}{l}\text { (Cui and Zhang, } \\
2008)^{78}\end{array}$ & $\begin{array}{l}566 \\
250\end{array}$ & $\begin{array}{c}639 \\
1000\end{array}$ & $\begin{array}{l}124 \\
110\end{array}$ & $\begin{array}{l}143 \times 10^{3} \\
200 \times 10^{3}\end{array}$ & $\begin{array}{l}\text { Unavailable } \\
\text { Unavailable }\end{array}$ \\
\hline
\end{tabular}

* average of 8 kinds of $\mathrm{PCB}$

Gold - The gold content of the EDO SIMMs ( $\mathbf{2}$ and $\mathbf{3}$ ) is low (Figure 3.4) due to the use of tin rather than gold in edge contacts. The gold content of the other modules varies from 480-1320 ppm, a factor of 2.6. The average gold content of these modules is $18.2 \mathrm{mg} /$ module (1030 $\mathrm{ppm}$ ). This is a higher gold content than that for PC PCBs generally reported in the literature except by Yamane et al., (Table 3.3), suggesting that RAM modules contain gold in higher concentrations than other PC PCBs such as mother boards and PCI cards.

Comparison of results for modules $\mathbf{2}$ and $\mathbf{3}$ with the rest of the samples shows that the bulk of gold in DRAM is found in the edge contacts. The results do not correlate with the number of ICs or edge contacts on modules. To the naked eye, it appears that the surface areas of edge contacts on SDR, DDR, DDR2 and DDR3 modules are very similar; and measurement of 
contact surface areas of the DDR and DDR2 sample made from photographs (Figure 3.5) using Adobe Photoshop pixel counting function shows them to be within $2 \%$ of one another.

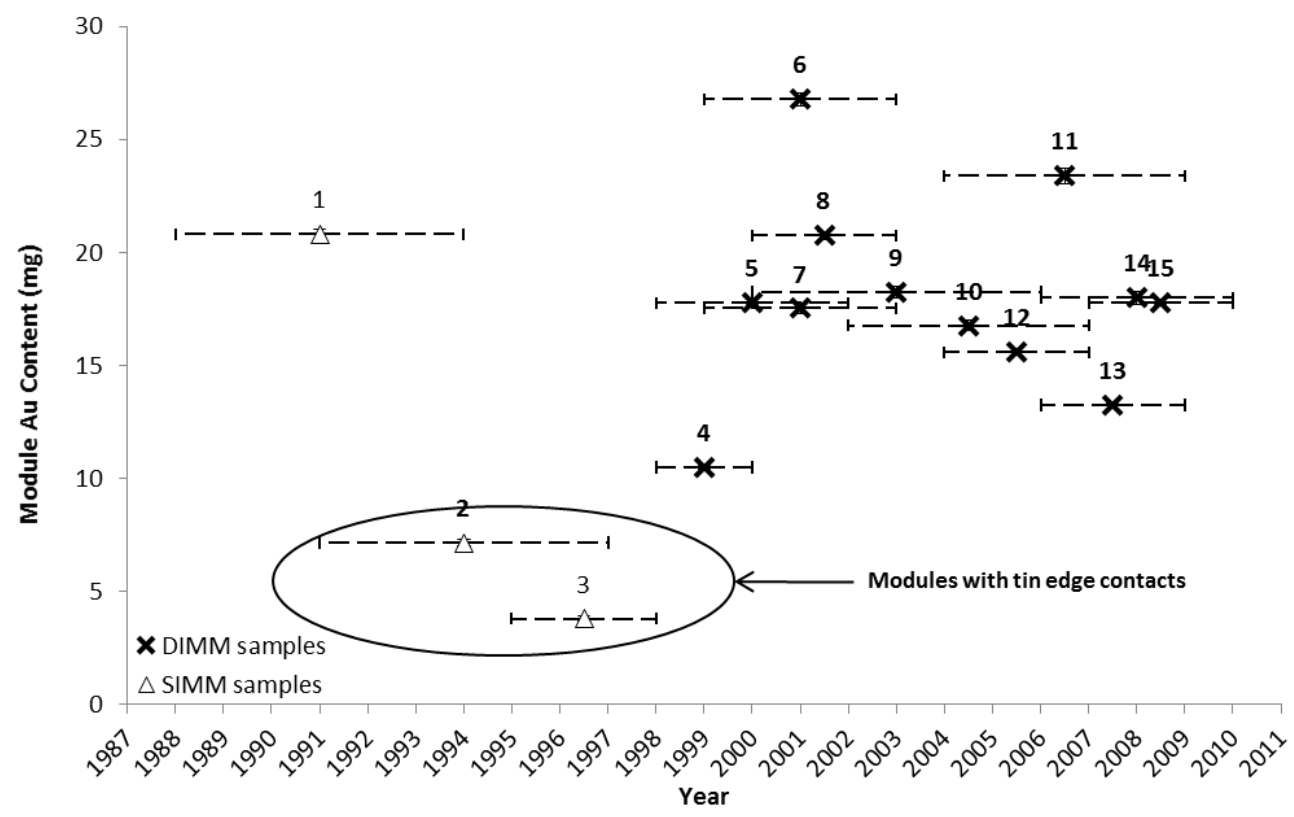

Figure 3.4 Gold content of PC DRAM modules (dashed line represents best estimate of time period in which module was on the market (Figure 3.3); modules 2 and $\mathbf{3}$, have tin rather than gold edge contacts

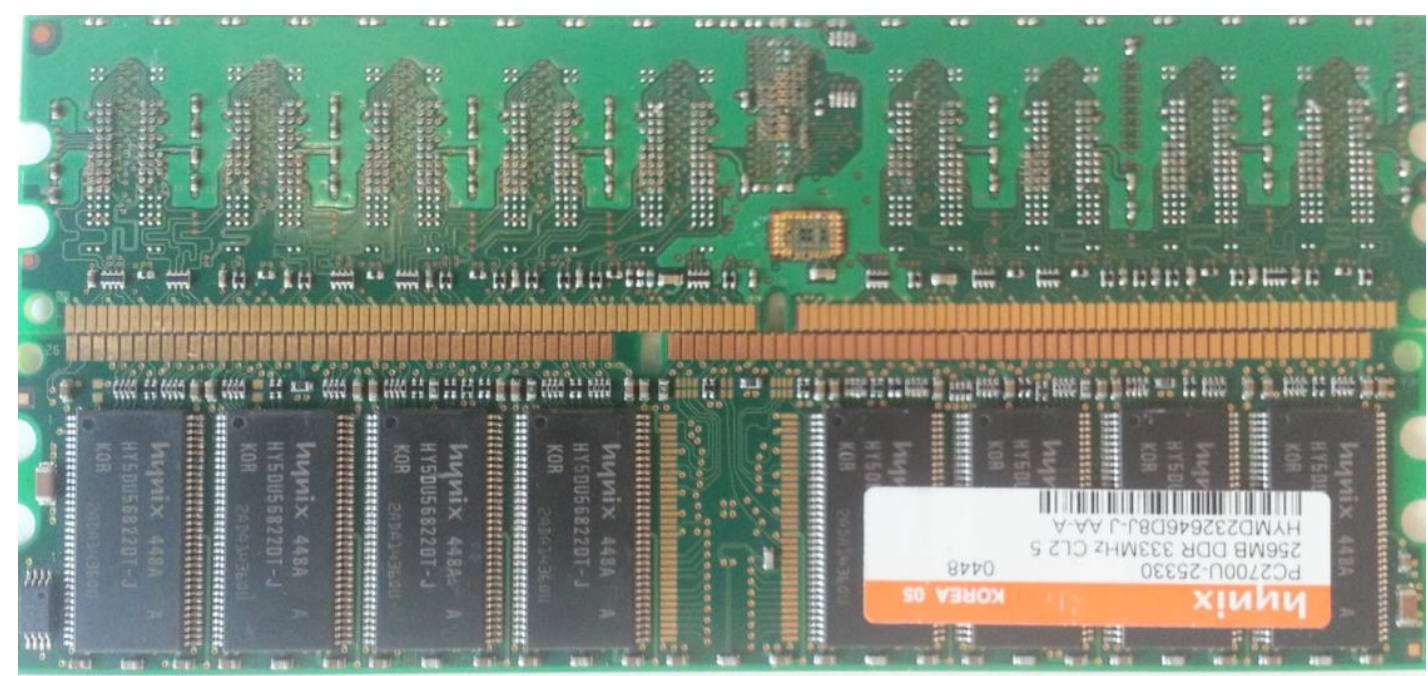

Figure 3.5 Image used for comparison of contact surface areas of DDR (bottom) and DDR2 (top) DRAM DIMMs. 
While gold content varies significantly from module to module, for those with gold edge contacts, no general trend over time is initially obvious. Manufacturers have opted to increase memory using the same module size, and with it roughly the same gold contact areas, rather than maintain memory capacity and reduce the module size. Variation in gold content in DIMMs may be explained by differences in thickness of the Au alloy layer of the contacts plated onto the module during manufacturing. This alloy is electroplated onto the module, and minor differences in the thickness of this layer resulting from variation in technical manufacturing skills, applied voltages, currents and duration of the plating process used in different manufacturing processes around the globe. For such a thin layer of alloy, minor changes to manufacturing parameters could result in the differences in gold content observed across samples.

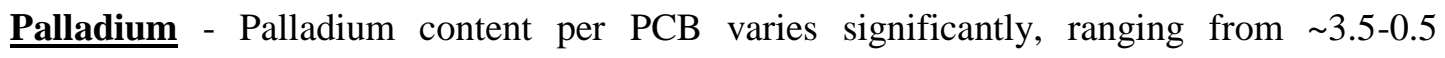
$\mathrm{mg} /$ module, and shows a marked decrease over time (Figure 3.6). The average palladium content $(1.5 \mathrm{mg} /$ module; $86 \mathrm{ppm})$ is somewhat lower than that generally reported in the literature for other PC PCB samples (90-309 ppm, Table 3.3). On these PCBs, Pd is principally found in the multi-layer ceramic capacitors (MLCCs), and while there is no correlation with the number of MLCCs per board (which has generally increased over time), the size of individual MLCCs has been reduced significantly over time. Capacitance improves with smaller units which results in a technical advantage from miniaturisation as well as cost savings for producers. The same is true for thinner layers of Pd in devices. This has enabled manufacturers to achieve additional cost savings by reducing the thickness of the internal electrode plates reducing Pd content further. In the early 1990s 100\% Pd was favoured as the inner electrode material of MLCCs. Since then, AgPd alloys of decreasing Pd content have been developed. By 2004, MLCCs using 2\%Pd alloy were in development. A minimum Pd content of the electrode alloy has now been reached as $100 \%$ silver alloys do not perform well due to technical and structural limitations. ${ }^{182}$ Because of this, a similar decrease in palladium content over time will almost certainly be seen in other PC components and PCB based devices in which MLCCs have been used. 


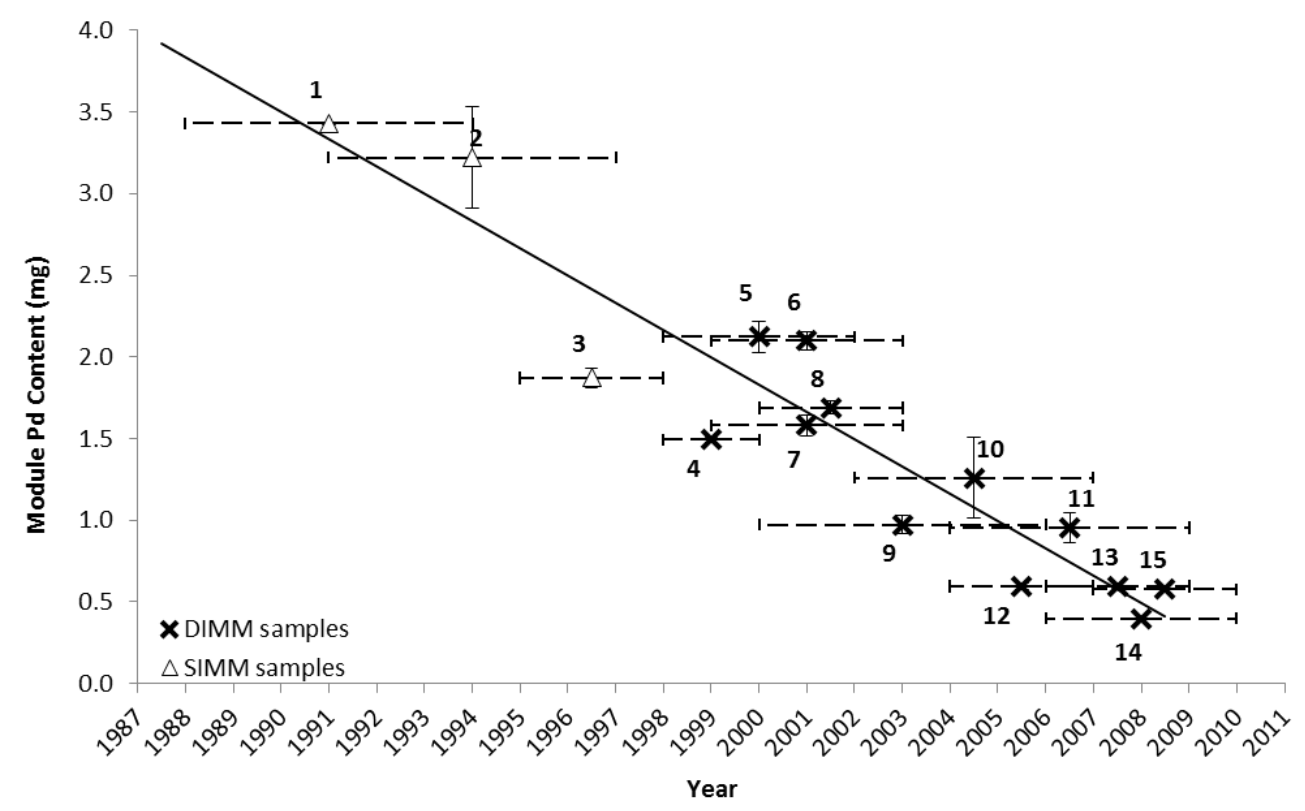

Figure 3.6 Variation in palladium content of PC DRAM modules over time. The dashed line represents best estimate of period in which module was on the market (Figure 3.3); the solid line shows the temporal trend in Pd content.

Platinum - No platinum was detected in any of the samples, which, using our detection limit of $60 \mu \mathrm{g} / \mathrm{L}$ for platinum in solution, puts the quantity of platinum in samples at $<6 \mu \mathrm{g}$ per module $(<0.5 \mathrm{ppm})$. This is unsurprising since platinum is used in PC HDD platters rather than PCBs.

$\underline{\text { Silver }}$ - Silver content of modules varies quite widely from $\sim 10-130 \mathrm{mg} /$ module. In general SIMMs (samples 1-3) contained significantly higher levels of silver $(\sim 50-130 \mathrm{mg})$ than DIMMs (typically $<40 \mathrm{mg}$ ) (Figure 3.7) with the exception of the 256MB PC2100 DIMM (sample 9), which has a very high silver content, a result confirmed by repeat analyses. SIMMs contain significantly higher concentrations of silver than any of the PC PCB samples reported in the literature (Table 3.3). Such modules however have not been utilised in PCs since the introduction of DIMMs (c. 1997) and are now infrequently found in the waste stream. With the exception of sample 9, DIMMs contain relatively high levels of silver to PC PCBs reported by others (Table 3.3). Excluding sample 9, the silver content of DIMMs is low, $\sim 10-40$ $\mathrm{mg} /$ module, with no observable trend over time. 


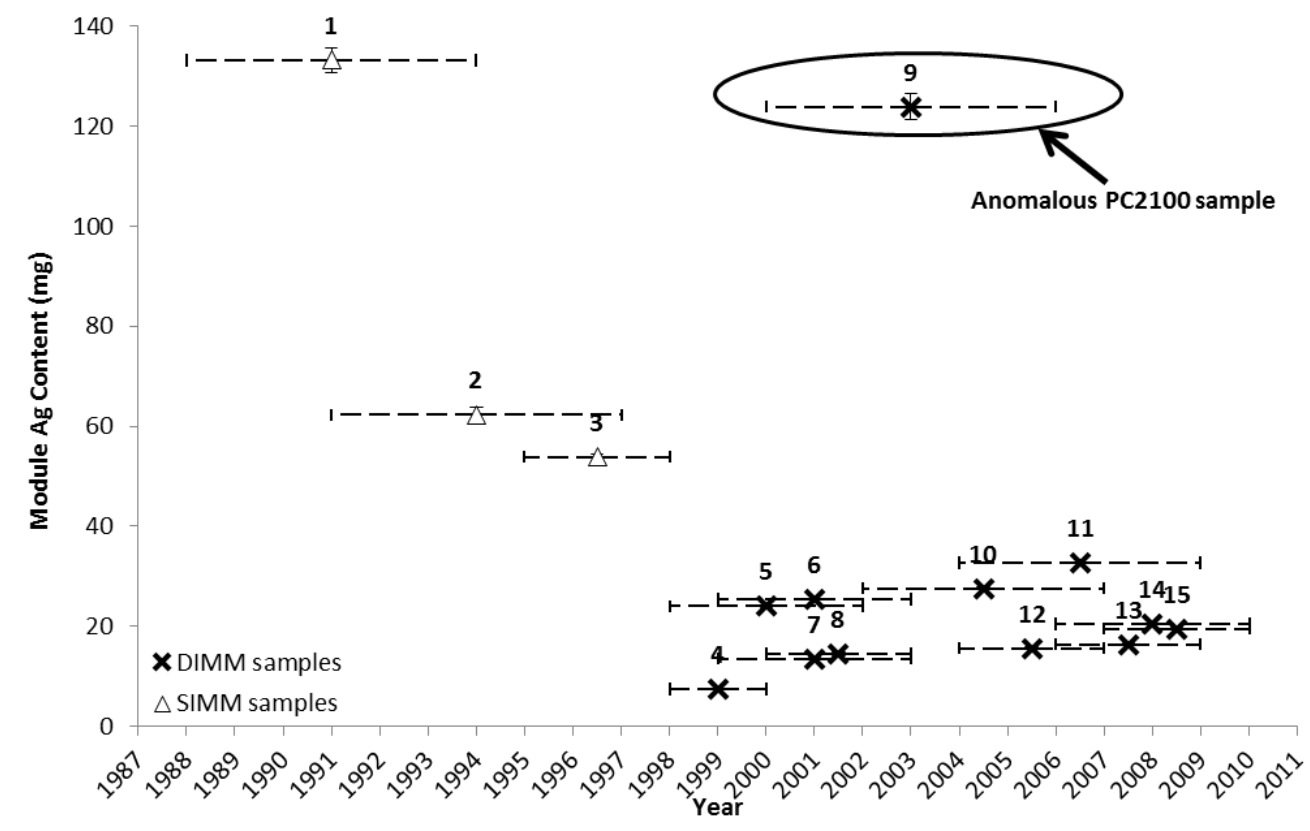

Figure 3.7 Variation in silver content of PC DRAM samples over time (dashed line represents best estimate of time period in which module was on the market (Figure 3.3).

Copper - Copper content ranges from 1.8-5.1 g/module (Figure 3.8). With an average copper content of $\sim 200 \times 10^{3} \mathrm{ppm}$, samples exhibit comparable copper content to PC PCBs reported in the literature (Table 3.4). Taken as a whole, no general trend in copper content of RAM samples is seen. However, if we consider DIMMs alone (Figure 3.8) copper content has generally increased over the years.

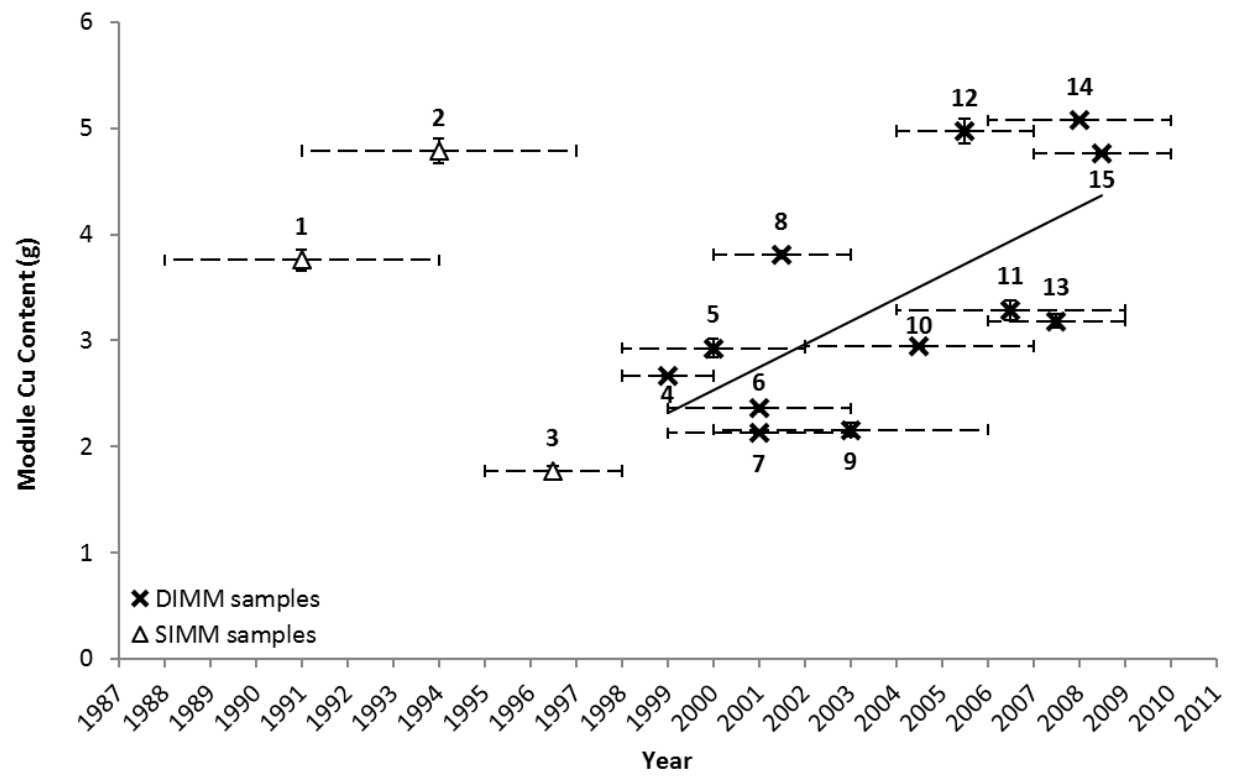

Figure 3.8 Variation in Copper content of PC DIMM samples over time (dashed lines, represents best estimate of period in which module was on the market (Figure 3.4); solid line shows the temporal trend in copper content in DIMMs). 


\section{Temporal trends in value of DRAM modules}

Before discussing the trends in value of RAM modules it is necessary to identify the following two caveats: 1) our analyses do not cover every module used over the time period studied, and 2) we do not have data on the numbers of each individual module produced, or found in the waste stream, which would be necessary to provide a weighted average analysis.

While recognising these two factors following general comments on the trends in value of RAM modules in the waste stream can be made. The value of individual samples per module and per tonne, showing value contributions of PMs and copper, are given in Figure 3.9. Values range from USD $0.23 / \mathrm{g}$ to USD $1.11 / \mathrm{g}$. The two lowest value modules are EDO samples with tin contacts. The more modern DIMM samples (4-15) vary in value by a factor of 2.4 from USD $0.46 / \mathrm{g}$ to USD $1.11 / \mathrm{g}$, with an average value exhibited across all DIMM samples of USD 0.76/g. Gold represents the majority of inherent value in DRAM modules; $85-95 \%$ for the more modern DIMMs (4-15). Over the period represented by the samples there is a small increase in the value fraction of copper and a decrease in the value fraction of palladium. Over time, values and grades of modules have remained relatively stable since the introduction of DIMMs and the elimination of tin edge contacts from module designs. As SIMMs have not been included in PCs since 1997, they no longer represent a significant portion of DRAM in the waste stream. Therefore, the composition of waste DRAM modules generated today is more accurately represented by the average composition of DIMM samples (4-15).

The per tonne values of DRAM samples (Figure 3.9b) follow those for individual modules, and in general are significantly higher than those calculated from average compositional data for PC PCBs available in the literature (Table 3.3). Perhaps what is most important, in terms of the long-term recycling viability of DRAM modules, is that the per tonne values of the most recent module types analysed, DDR2 and DD3 DIMMs (12-15), appear stable. 

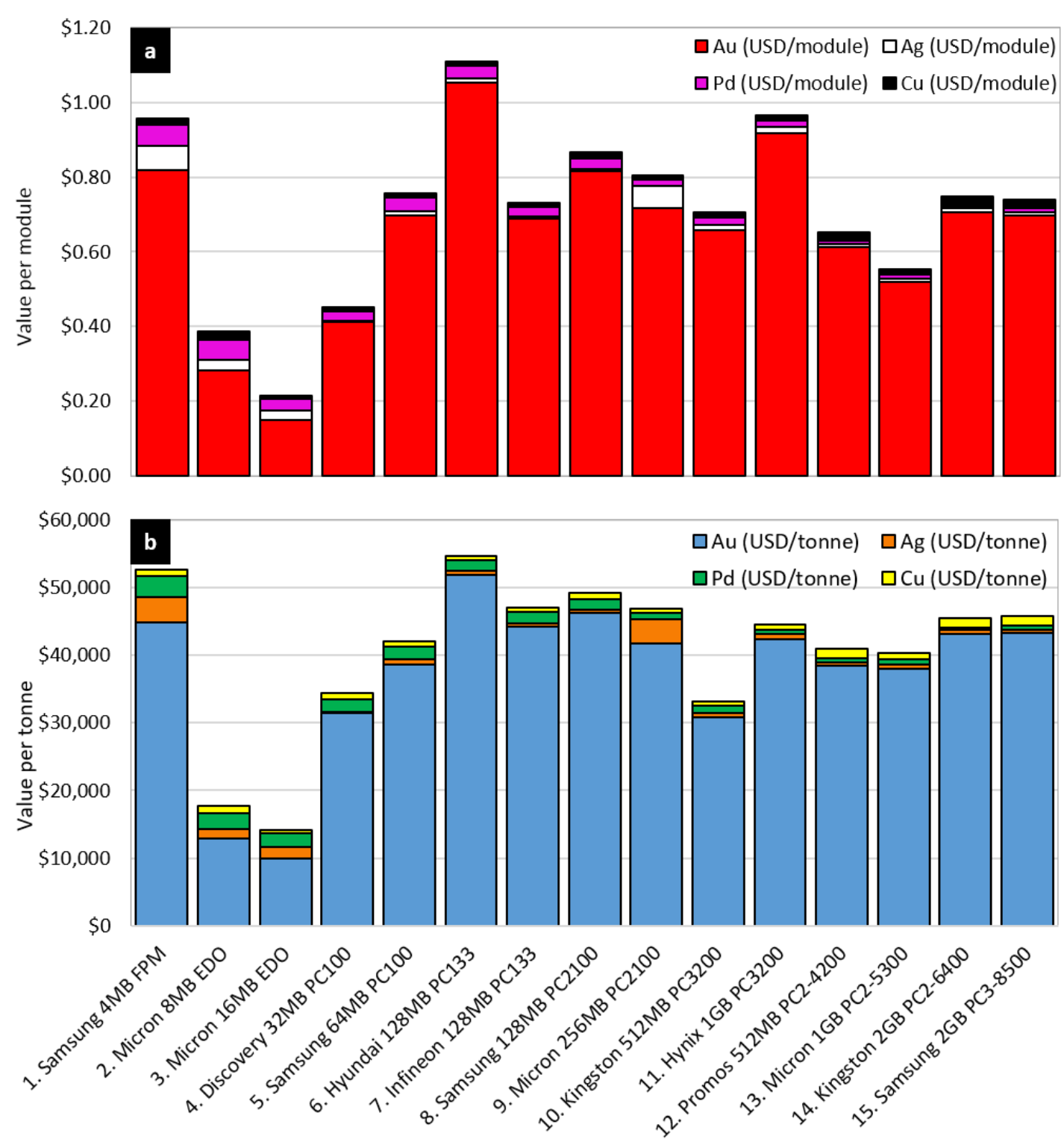

Figure 3.9 Inherent value of DRAM samples, a) per module and b) per tonne, showing value contributions of PMs and copper.

\subsubsection{Recycling potential of DIMMs}

As an indication of the potential contribution of DIMMs to global metal supply and demand (Table 3.4), minimum estimates of total potentially recoverable metals in DRAM in PCs POM in 2013 have been made assuming metal contents are given by the averages of those for samples 4-15 and that each PC POM contains a single RAM module. Assumptions made in the WEEE Directive ${ }^{4}$ and annual PC sales data ${ }^{183}$ suggest that 153.4 million waste PCs were generated globally in 2013. 
Table 3.4 Estimated quantities of precious metals and copper in globally generated waste DIMMs in PCs POM 2013, their values and proportions of global demand, demand from the EEE sector and global secondary production each represents (calculated from average metal contents of samples 4-15, data provided in O'Connell et al., Statista, 2015) 150,151,153, 154, 183

\begin{tabular}{lccccc}
\hline & Au & Ag & Pd & Cu & $\begin{array}{r}\text { Total } \\
\text { Value }\end{array}$ \\
\hline DIMM average composition & $18.0 \mathrm{mg}$ & $28.4 \mathrm{mg}$ & $1.2 \mathrm{mg}$ & $3.4 \mathrm{~g}$ & \\
$\begin{array}{l}\text { Total metal available in DRAM of PCs } \\
\text { sold in 2013 (tonnes) }\end{array}$ & 2.46 & 3.90 & 0.16 & 459 & \\
Market value of metals (million USD) & 96.6 & 1.9 & 2.7 & 2.1 & $\mathbf{1 0 3 . 4}$ \\
Value fraction & $93.5 \%$ & $1.8 \%$ & $2.6 \%$ & $2.0 \%$ & \\
& & & & & \\
As\% of global demand & $0.05 \%$ & $0.34 \%$ & $0.002 \%$ & $0.002 \%$ \\
As\% of demand from EEE sector & $0.83 \%$ & $1.5 \%$ & $0.012 \%$ & $0.006 \%$ \\
As\% of global secondary production & $0.19 \%$ & $2.0 \%$ & $0.009 \%$ & $0.012 \%$ & \\
\hline
\end{tabular}

The actual number of DRAM modules POM is likely to be higher than the number in PCs POM, as modules which malfunction are replaced, and PCs are upgraded. Some PCs contain two modules when placed on the market, so data in Table 3.4 are probably minimum values. It should also be noted, that the quantities presented represent the size of the theoretical 'reserve' of metals in waste arising, and not the quantities that will be available for recovery due to inefficiencies in the collection and pre-processing stages of recycling.

The estimated total value of metals in waste DRAM modules POM in PCs in 2013 was USD 103 million. 2.46 tonnes of gold was present, worth USD 97 million, $93.5 \%$ of total inherent value of the DRAMs. This is equivalent to $0.05 \%$ of global demand for gold, $0.8 \%$ of demand from the EEE sector and $0.2 \%$ of gold supplied from secondary sources that year. 3.9 tonnes of silver was present with a market value of USD 1.9 million and $1.8 \%$ of inherent value. This is equivalent to $0.3 \%$ of global demand, $1.5 \%$ of demand from the EEE sector and $0.19 \%$ of silver supplied from secondary sources. 0.16 tonnes of palladium were present with a market value of USD 2.7 million equal to $2.6 \%$ of inherent value. Palladium in DRAM is equivalent to $0.002 \%$ of global demand, $0.012 \%$ of demand from the EEE sector and $0.009 \%$ of palladium supplied from secondary sources that year. 459 tonnes, USD 2.1 million worth of copper were present in waste DRAM in PCs POM in 2013. This equates to $2 \%$ of inherent value, $0.002 \%$ of global demand, $0.006 \%$ of demand from the EEE sector and just $0.012 \%$ of copper supplied from secondary sources. 


\subsubsection{Outlook for future recycling potential}

DRAM - Figures 3.10-3.13 show projected gold, silver, copper and palladium content of DRAM modules to 2020 from a statistical analysis of DIMM data presented here, with $95 \%$, 90\% and 75\% confidence limits shown as dashed lines. Gold content is projected to remain reasonably stable, while silver content will most likely remain roughly constant or even increase somewhat. Copper content is projected to increase by $\sim 75 \%$. Linear projection of $\mathrm{Pd}$ content in modules shows that Pd should have reached zero in $\sim 2011$ and predicts a negative Pd content by 2020. This is obviously not sensible, as Pd is still used in MLCCs of RAM modules and it is more likely that a minimum value is being approached. It has already been stated that Pd content in the alloy of the MLCC electrodes has already reached a minimum viable content of $2 \%$. It is reasonable to assume that Pd content in individual MLCCs may continue to reduce until a minimum viable size of electrodes is reached, at which point $\mathrm{Pd}$ content of MLCCs will stabilise. If the number of MLCCs per module continues to rise as has been the case to date, then Pd content may begin to increase after this point in proportion to the number of MLCCs mounted on modules, at least until technological innovation results in replacement of Pd in MLCCs, or outright replacement of MLCCs in modules (and other PCBs).

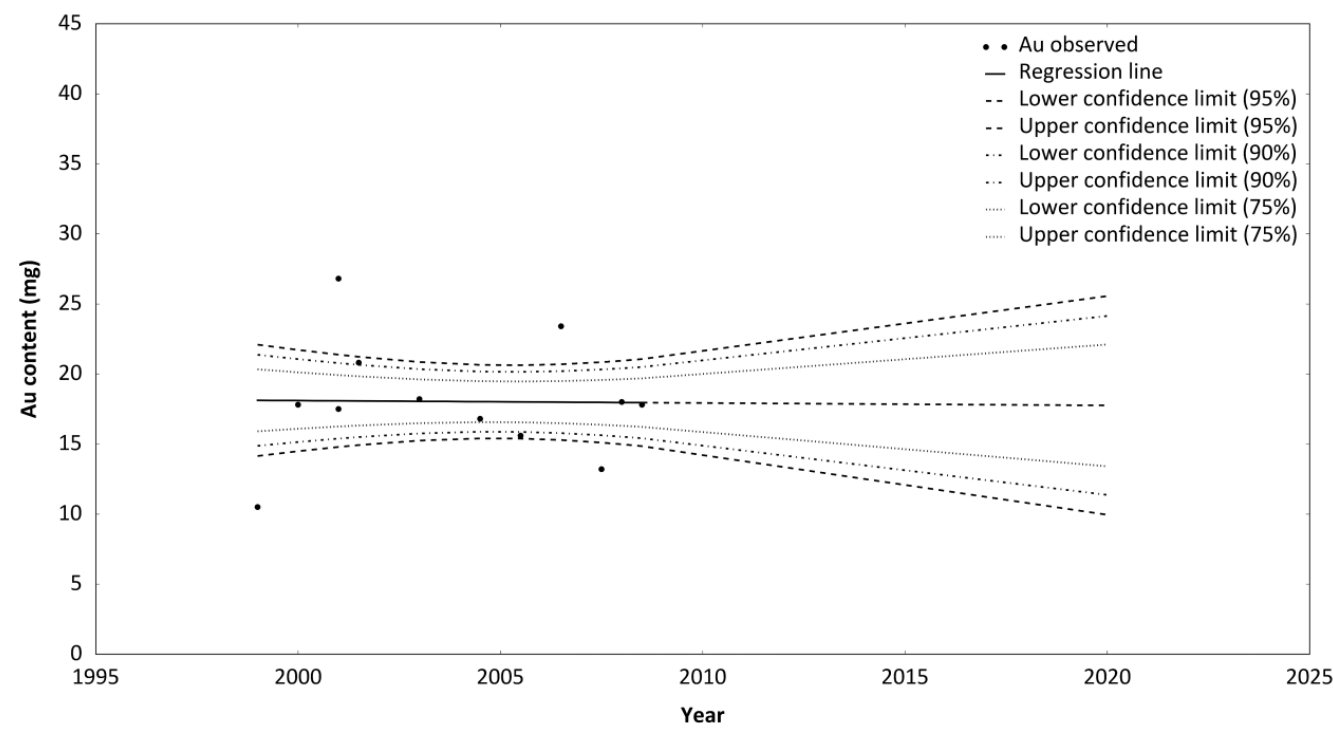

Figure 3.10 Confidence intervals for projections of future quantities of gold in future waste DIMMs

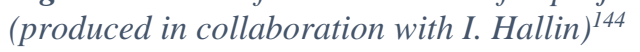




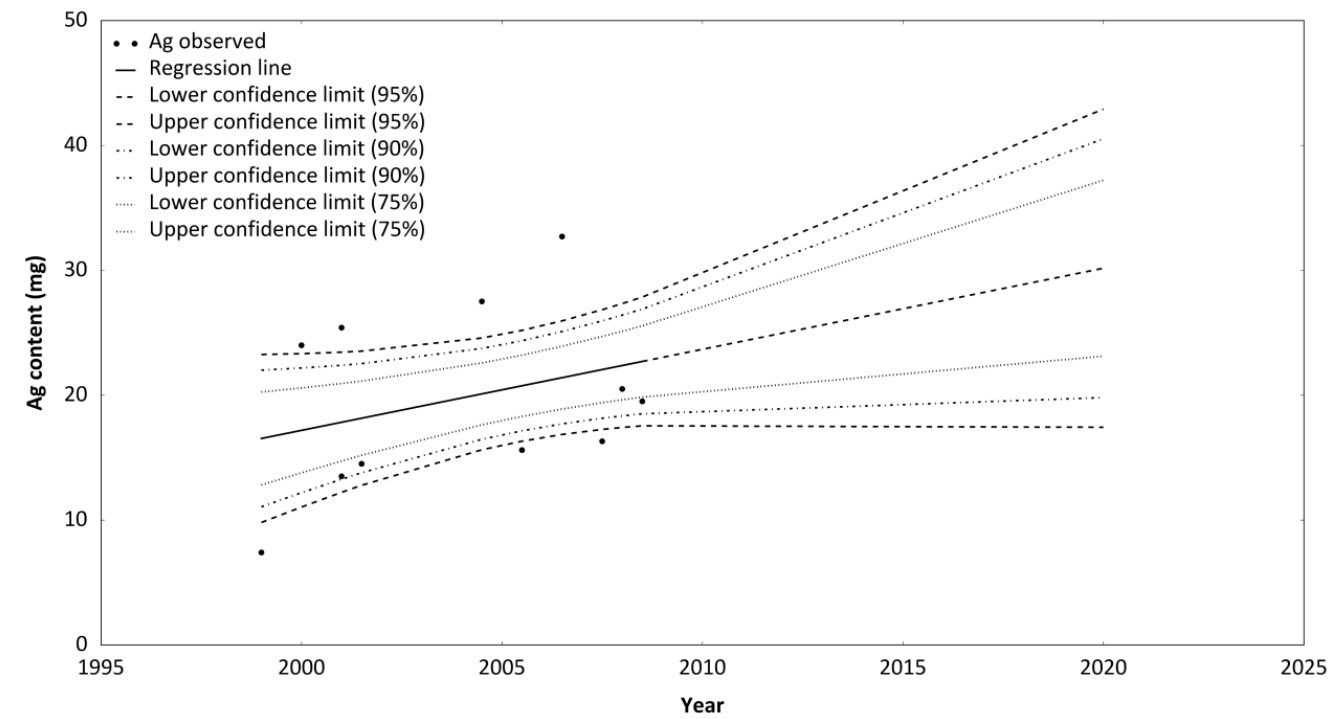

Figure 3.11 Confidence intervals for projected quantities of silver in future waste DIMMs (produced in collaboration with I. Hallin) ${ }^{144}$

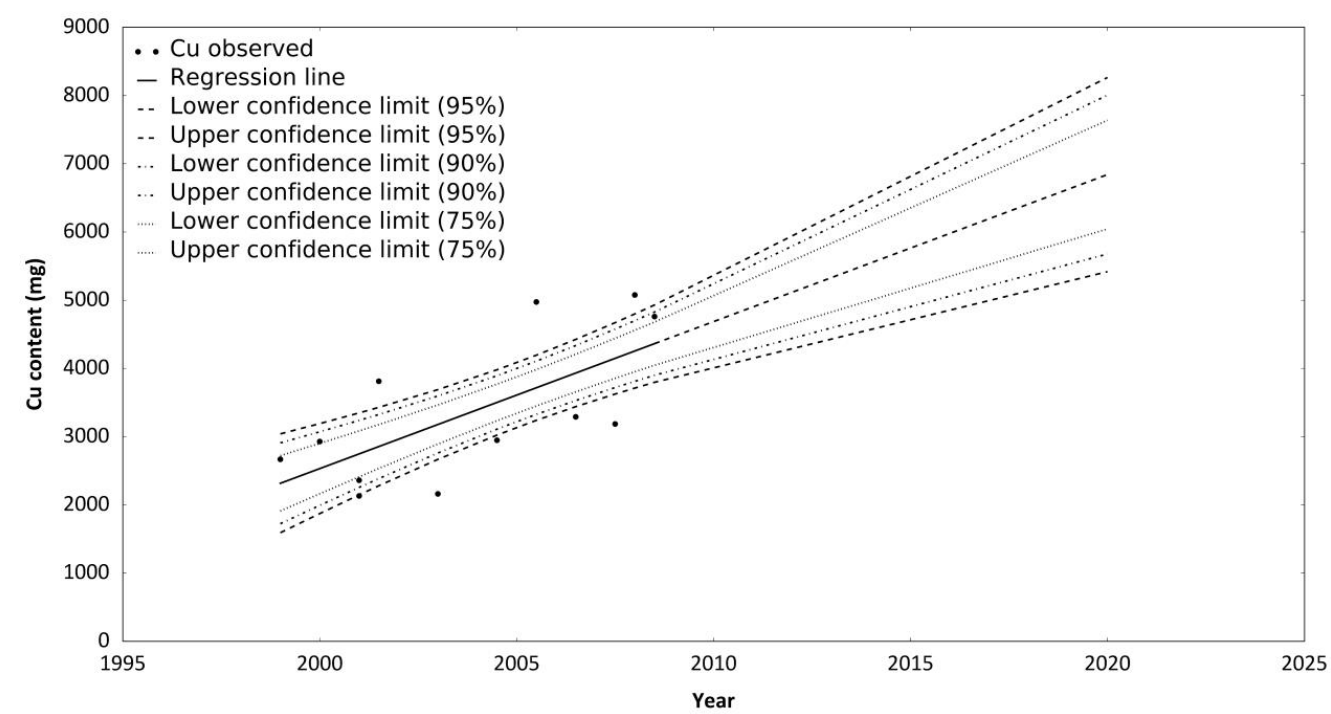

Figure 3.12 Confidence intervals for projected quantities of copper in future waste DIMMs (produced in collaboration with I. Hallin) $)^{144}$

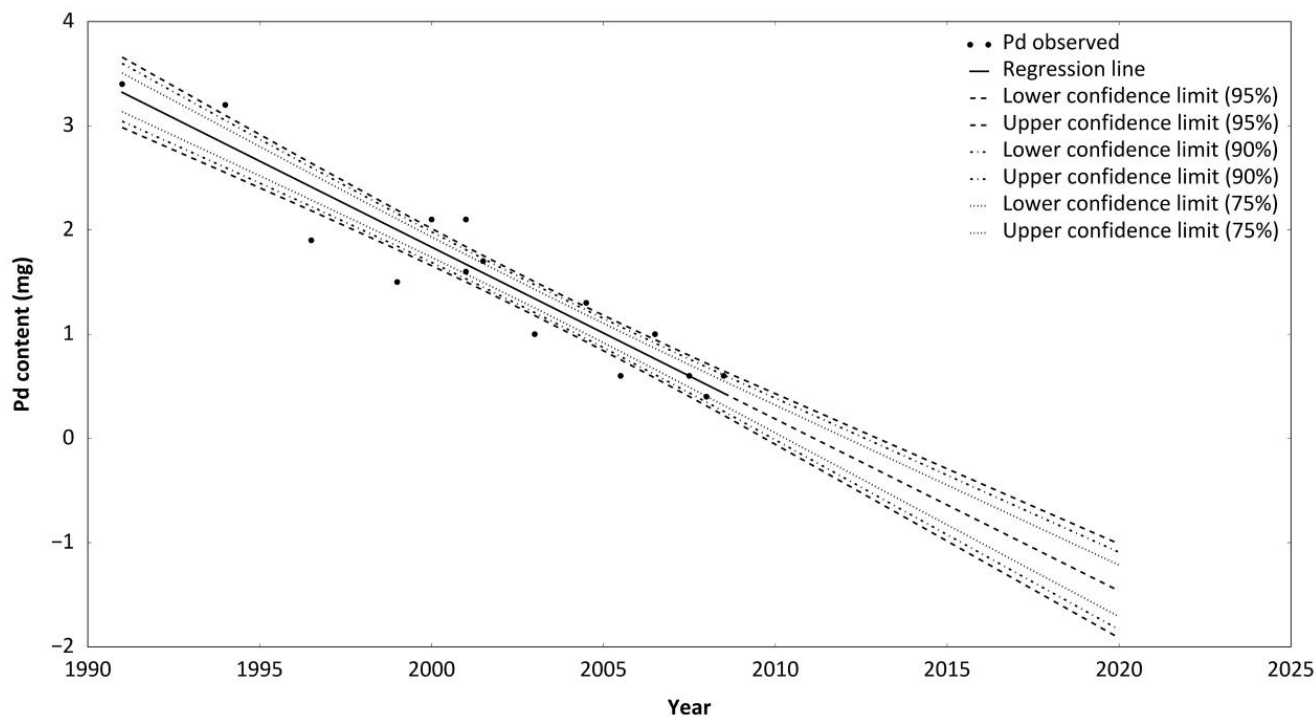

Figure 3.13 Confidence intervals for projected quantities of palladium in future waste DIMMs (produced in collaboration with I. Hallin) ${ }^{144}$ 
General WEEE - Global desktop PC sales are declining due to uptake of laptops, notebooks and tablets. Projections suggest a 28\% reduction in global desktop PC sales over the next 5 years, from 133.9 million units in 2014 to 109.3 million units in $2019 .{ }^{183}$ That said, PC sales in general are rising in the form of laptops and tablets. ${ }^{173}$ This highlights the changing nature of the waste stream in general driven by technological innovation and changes in consumer demands. Projections of recycling potential and recovery potential (i.e. the total amount of potentially recoverable metals in items) of new items in the future as their volumes grow in the waste stream, such as the study by Cucchiella et al. ${ }^{101}$ are important for issues previously discussed. The purpose of the Cucchiella study was to create a system through which the richest items in terms of material value for recovery could be targeted and therefore items in the study are ranked according to the inherent value of PMs and copper in waste items generated in 2020. This study applies available compositional data for numerous WEEE items available in the literature, to quantities of waste items generated in 2014 and a projection of generated waste in 2020 to calculate the potentially recoverable value from waste in 2020 and the growth in recoverable valuable materials in the future. To account for potential changes to composition a sensitivity analysis was performed in which the content of particular materials in items resulting in the majority of inherent value, was varied by $5 \%$.

To highlight the impact that applying observable temporal trends in metal content to projections we have repeated the analysis for 2 different scenarios. Scenario 1 is a base scenario and uses all data available in the previous study to quantify PMs and copper available in selected equipment types including a static composition of items; Scenario 2 repeats the process but takes into account the temporal change in copper content observed in this study of RAM modules, applying a 75\% increase in copper content in 2020 (Table 3.5). For consistency with the original study, average weekly market values for metals from Mar-Aug 2014 are used. Items selected for analysis have their copper and PM content concentrated in PCBs, as temporal data observed for RAM modules is indicative of temporal variations in PCB composition, not whole items which may contain additional copper as cables etc. In contrast to the original study, we have only included the values of PMs and copper in items, neglecting valuable bulk materials such as plastic and aluminium to highlight the impact on the value of copper and PMs in PCBs, which determine recycling potential of PCBs and represent a considerable fraction of recoverable value in items overall.

It can be seen by comparison of the scenario results that applying the increasing trend to copper results in a maximum of a $6 \%$ increase in projected potentially recoverable value for tablets. This is primarily due to the low proportion of recoverable value represented by copper in 
comparison to gold in notebook computers and tablets, and silver in smart phones and cell phones. It is evident then, that any substitution of either of these metals over time through design change of items would result in a considerable mismatch between projections of recycling potential made based on static material content of PCBs in items, and the future reality.

Table 3.5 Projections of future recycling potential of various WEEE items (based on data from Cucchiella et al., 2015). ${ }^{101}$

\begin{tabular}{|c|c|c|c|c|c|c|}
\hline Item & $\begin{array}{c}\text { LCD } \\
\text { notebooks }\end{array}$ & $\begin{array}{c}\text { LED } \\
\text { notebooks }\end{array}$ & $\begin{array}{l}\text { Smart } \\
\text { phones }\end{array}$ & $\begin{array}{c}\text { Cell } \\
\text { phones }\end{array}$ & Tablets & $\begin{array}{l}\text { Avg value Mar- } \\
\text { Aug } 2014(€ / \mathrm{kg})\end{array}$ \\
\hline \multicolumn{7}{|l|}{ Metal } \\
\hline$\overline{\mathrm{Au}(\mathrm{p} p m)}$ & 0.22 & 0.22 & 0.038 & 0.024 & .044 & 34,070 \\
\hline $\mathrm{Ag}(\mathrm{ppm})$ & 0.25 & 0.25 & 0.244 & 1 & .005 & 23,214 \\
\hline $\mathrm{Pd}(\mathrm{ppm})$ & 0.04 & 0.04 & 0.015 & 0.009 & .008 & 514 \\
\hline $\mathrm{Cu}(\mathrm{ppm})$ & 135 & 135 & 14 & 26 & 27 & 5.2 \\
\hline Item weight (kg) & 3.5 & 3.5 & 0.12 & 0.08 & 0.5 & \\
\hline Volume in 2014 (kt) & 80 & 22 & 19 & 11.5 & 5.2 & \\
\hline Volume in $2020(\mathrm{kt})$ & 97 & 45 & 39 & 52 & 10 & \\
\hline \multicolumn{7}{|l|}{2020 Scenario 1} \\
\hline \multicolumn{7}{|l|}{$\overline{\text { Au }}$} \\
\hline Content $(\mathrm{t})$ & 6.1 & 2.8 & 12.4 & 15.6 & 0.9 & \\
\hline Value $(\mathrm{M} €)$ & 208 & 96 & 421 & 531 & 30 & \\
\hline Value fraction & $56 \%$ & $56 \%$ & $19 \%$ & $3 \%$ & $93 \%$ & \\
\hline \multicolumn{7}{|l|}{ Ag } \\
\hline Content $(\mathrm{t})$ & 6.9 & 3.2 & 79 & 650 & 0.1 & \\
\hline Value $(\mathrm{M} €)$ & 161 & 75 & 1841 & 15,089 & 2 & \\
\hline Value fraction & $44 \%$ & $44 \%$ & $81 \%$ & $97 \%$ & $7 \%$ & \\
\hline \multicolumn{7}{|l|}{ Pd } \\
\hline Content (t) & 1.1 & 0.5 & 4.9 & 5.85 & 0.16 & \\
\hline Value $(\mathrm{M} €)$ & 0.6 & 0.3 & 3 & 3 & 0.08 & \\
\hline Value fraction & $0.2 \%$ & $0.2 \%$ & $0.1 \%$ & $0.02 \%$ & $0.3 \%$ & \\
\hline \multicolumn{7}{|l|}{$\mathrm{Cu}$} \\
\hline Content (t) & 3,741 & 1,736 & 4,550 & 16,900 & 540 & \\
\hline Value $(\mathrm{M} €)$ & 19 & 9 & 24 & 88 & 2.81 & \\
\hline Value fraction & $5 \%$ & $5 \%$ & $1 \%$ & $0.6 \%$ & $8 \%$ & \\
\hline Total Value (M€) & 389 & 180 & 2,288 & 15,711 & 35 & \\
\hline \multicolumn{7}{|l|}{2020 Scenario 2} \\
\hline \multicolumn{7}{|l|}{ Au } \\
\hline Content $(\mathrm{t})$ & 6.1 & 2.8 & 12.4 & 15.6 & 0.9 & \\
\hline Value $(\mathrm{M} €)$ & 208 & 96 & 421 & 531 & 30 & \\
\hline Value fraction & $52 \%$ & $52 \%$ & $18 \%$ & $3 \%$ & $80 \%$ & \\
\hline \multicolumn{7}{|l|}{ Ag } \\
\hline Content (t) & 6.9 & 3.2 & 79 & 650 & 0.1 & \\
\hline Value (M€) & 161 & 75 & 1,841 & 15,089 & 2 & \\
\hline Value fraction & $40 \%$ & $40 \%$ & $80 \%$ & $96 \%$ & $6 \%$ & \\
\hline \multicolumn{7}{|l|}{ Pd } \\
\hline Content (t) & 1.1 & 0.5 & 4.9 & 5.85 & 0.16 & \\
\hline Value $(\mathrm{M} €)$ & 0.6 & 0.3 & 3 & 3 & 0.08 & \\
\hline Value fraction & $0.1 \%$ & $0.1 \%$ & $0.1 \%$ & $0.02 \%$ & $0.2 \%$ & \\
\hline \multicolumn{7}{|l|}{$\mathrm{Cu}$} \\
\hline Content $(\mathrm{t})$ & 6,548 & 3,037 & 7,963 & 29,575 & 945 & \\
\hline Value $(\mathrm{M} €)$ & 34 & 16 & 41 & 154 & 5 & \\
\hline Value fraction & $8 \%$ & $8 \%$ & $2 \%$ & $1 \%$ & $13 \%$ & \\
\hline Total Value (M€) & 403 & 187 & 2,306 & 15,777 & 37 & \\
\hline$\%$ increase & $4 \%$ & $4 \%$ & $1 \%$ & $0.4 \%$ & $6 \%$ & \\
\hline
\end{tabular}




\subsection{Conclusion}

Elemental analysis of PC DRAM modules placed on the market over a period of approximately 17 years has shed light on temporal trends in PM and copper content of ICT PCBs with which to assess current and future recycling potential of PC DRAM modules, and PC PCBs in general.

DRAM is of a particularly high grade compared to other PC PCBs with an average composition for DIMMs of $\sim 1,000 \mathrm{pm}$ gold, $\sim 70 \mathrm{ppm}$ palladium, $\sim 1,600 \mathrm{ppm}$ silver and $\sim 200 \times 10^{3} \mathrm{ppm}$ (20 wt \%) copper. The number of RAM modules in PCs POM in 2013 is $\sim 153$ million units which, based on the average composition determined for DIMMs, contained 2.5 tonnes of gold, 3.9 tonnes of silver, $160 \mathrm{~kg}$ of palladium and 459 tonnes of copper with a total market value of USD 103 million. Quantities of gold and silver in DRAM modules have been stable over the time period 2000-2010. DIMMs show an increase in copper content over time, with a doubling of copper content of samples between 1999 and 2010. A decrease in the palladium content of modules at a rate of $5 \%$ per year is observed, which is attributable to miniaturisation of MLCCs over time. The palladium content of modules on the market in 2007 was only $\sim 20 \%$ of that for modules on the market in 1993. It is likely that a minimum Pd content in MLCCs will be reached as Pd content of electrode alloys in these SMDs has reached a minimum, and there is likely to be a limit to how small these electrodes can be made in order to achieve sufficient capacitance for their function. It is probable that in the future the $\mathrm{Pd}$ content of modules (and other PCBs utilising MLCCs) will eventually begin to slowly rise again when additional MLCCs are incorporated to support advanced functionality.

In modern DIMMs, a general increase in copper content of $0.23 \mathrm{~g} /$ module/year is observed over the period 1999-2008, which would result in an increase in copper content of $\sim 75 \%$ if this trend were to continue until 2020. There is some question as to whether such a linear trend can continue, although as PCBs become more complex additional layers are incorporated into multilayer boards and this trend may enable an increasing trend in copper content to continue.

The value of the target metals in DRAM modules analysed ranges from USD 14,00055,000/tonne. Modern DIMMs, which represent the majority of RAM modules available in the waste stream today, vary in value by a factor of 1.65 over the range of USD 33,000$55,000 /$ tonne with an average value across samples of USD 44,000/tonne. The grade of DIMMs is higher than other PC PCBs reported, with the exception of results from Yamane et $a l$. The major value fraction is consistently gold (>90\% average for DIMMs). 
Global sales of desktop PCs and therefore DRAM modules are diminishing as laptops and tablets gain further market share, and thus the total size of the 'reserve' of metals in PC DRAM modules is decreasing and therefore so is their recycling potential. Based on projections of global PC sales, it is likely that the annual number of waste DRAM modules produced will decrease in proportion with PC sales. Total quantities of gold and silver available in globally produced waste DRAM will decrease proportionally, it is likely that palladium content will be reduced significantly, but the amount of copper per DRAM module may well increase enough to compensate for the reduction in PCs sold so that the recoverable quantity of copper remains stable while the concentration of copper increases, which would enhance the viability of its recycling.

We have shown, by replication of projections of PMs and copper in notebooks, smart phones, cell phones and tablets made by Cucchiella et al., ${ }^{101}$ that incorporating temporal trends observed in DRAM modules into projections of recycling potential gives a variation of the total recoverable PM and copper value of $\sim 0.4-6 \%$ for the items considered. Although this is a minor variation the high proportion of recoverable value in laptops and tablets as gold and silver, and silver in smart phones and cell phones, suggest that were temporal variations in these metals to occur in the future major discrepancies between recycling potential projections and the resulting future reality could be considerable, to the detriment of waste management operations and legislation based upon such projections.

We have found no temporal trends in PM content of DRAM which suggest that diminishing PM content in any one particular item will affect its recycling potential in the near future. However, shifts in consumption patterns of consumers away from current technologies to emerging technologies with lower PM content could have a detrimental effect on recycling potential of WEEE overall.

Finally, we note that the shift to cloud based software and data storage means that many of the current components of computers may no longer be necessary. Miniaturisation of computing devices, as has been seen in the shift from the use of conventional desktop PCs to the use of tablets for example, may result in modular components such as DIMMs, being eliminated. Such a shift in the design of devices would result in elimination of large gold contacts and significantly less recoverable value in devices, which would have a significant impact on recycling potential. It should be considered though that this shift to cloud based storage is accompanied by increased volumes of servers in data centres, which are still composed of modular components designed to be swapped out and replaced as necessary, and which generally represent higher grade material than PCs. Previous studies have shown that between 
2008 and 2015 the amount of gold in German data centres would have increased by $61 \%$, reflecting the increase in number of installed servers. ${ }^{184}$ This may mean that the result of the shift to cloud computing simply results in a shift of where to recycle gold from. 


\section{Recovery of Critical Raw Materials from WEEE - Wales}

\section{Chapter summary}

In this chapter, results of a trial conducted August 2012 - March 2013 on behalf of the Waste \& Resources Action Programme (WRAP) entitled 'Critical Materials Recovery - Wales' (project code IMT002-012). The project aimed to investigate potential techniques for increasing the value generated gained from WEEE recycling in Wales through recovery of critical raw materials (CRMs), and to assess the suitability of adopting these techniques on an industrial scale at Metech Recycling (UK) Ltd. The trial was conducted at Metech and Swansea University, in partnership with Giraffe Innovations Ltd. These results formed the basis of the report 'Recovery of Critical Raw Materials (CRMs) - Wales. ${ }^{185}$

The first section of this chapter documents the work undertaken to collect WEEE samples form Metech Recycling for analysis, and to then evaluate the ability of available equipment to identify and quantify CRMs within samples. The second section reports CRMs identified within samples and their components. The final section outlines proposed methods to isolate CRM bearing components, and evaluates these methods in terms of cost-benefit and suitability for implementation at Metech to enhance value derived from CRMs in WEEE. Cost-benefit is only performed for processes which result in a material stream for which a downstream recovery process exists, so that an economic return on isolated materials can be generated. Barriers to recovery of CRMs in WEEE are discussed, along with recommendations for future actions to address these. These include a lack of available recovery infrastructure for CRMS from WEEE, and economic barriers resulting from the dissipative nature of applications of CRMs in EEE.

This work also forms a knowledge base for use by others seeking to develop advanced methods for CRM recovery from WEEE in the future, such as partners involved in ongoing trials under the current 'CRM Recovery' project. ${ }^{186}$

\subsection{Introduction}

Securing access to raw materials is a growing concern globally, and has become a priority for the European Union (EU) which imports nearly all of the non-energy raw materials it consumes. ${ }^{100}$ Non-energy raw materials are linked to all industries and stages of supply chains, and in Europe it is estimated that 30 million jobs depend on these imports..$^{53}$ Green energy \& 
low-carbon technologies are crucial for mitigating climate change and improving/sustaining the environment. Scale of deployment of such technologies as photovoltaics (PV), wind turbines, energy efficient lighting and electric vehicles, may, however, be limited by access to CRMs. Technologies such as these, are vital for mitigating global climate change and currently account for $20 \%$ of global critical raw materials (CRMs) consumption. As clean energy technologies are deployed more widely in the future, their share of global consumption will grow. ${ }^{20,61,187}$ The availability of raw materials is increasingly under pressure due to growing global demand and supply bottlenecks.

To address this, the EU Raw Materials Initiative identified a list of CRMs at EU level. A report was released in 2010 - 'Critical raw materials for the EU: Report of the ad-hoc working group on defining critical raw materials" which puts forward a concept of "criticality", where raw materials are labelled critical when the risks of supply shortage and the associated economic impacts of shortage are high. ${ }^{100}$ Production of CRMs is concentrated within a small number of countries, many of which have resource hungry economies of their own. In $2010,>90 \%$ of rare earth metals (REMs) \& Sb, and $>75 \%$ of Ge and $\mathrm{W}$ was produced in China, $90 \%$ of $\mathrm{Nb}$ was produced in Brazil and $77 \%$ of Pt originated in South Africa. Today, this picture has altered little. In addition, many of the materials deemed critical, particularly the 'high-tech' metals such as In, are by-products of major metals production, and as such the market is not able to immediately respond to growing global demand by increasing supply. Rather the supply of these metals is highly dependent upon the markets and resulting supply of the major metals whose ores these minor metals are associated with (see Figure 1.13). It is also a lengthy process to bring new mines on-line, so rapid solutions to booms in demand for CRMs from primary sources do not exists. ${ }^{188}$ Additionally, conservative policy affecting exports of CRMs such as export restrictions on REMs from China, and political instability in producer nations such as the ongoing conflict in DRC exacerbate CRM supply issues and heighten global resource-security concerns, pushing up market values. ${ }^{100,149}$

In the EU report, 41 materials were assessed. ${ }^{100}$ Figure 4.1 shows these materials in terms of supply risk verses economic importance: The EU report concluded that the 14 raw materials falling within the top right cluster (known as the EU14) were considered "critical" due to their high relative economic importance and high relative supply risk. These CRMs are listed in Table 4.1. Since the initial report the EU14 list was revised in 2013 to give the EU20 list of CRMs, a further revision is due this year (2017) reflecting the fluid and time dependent nature of materials 'criticality'. 


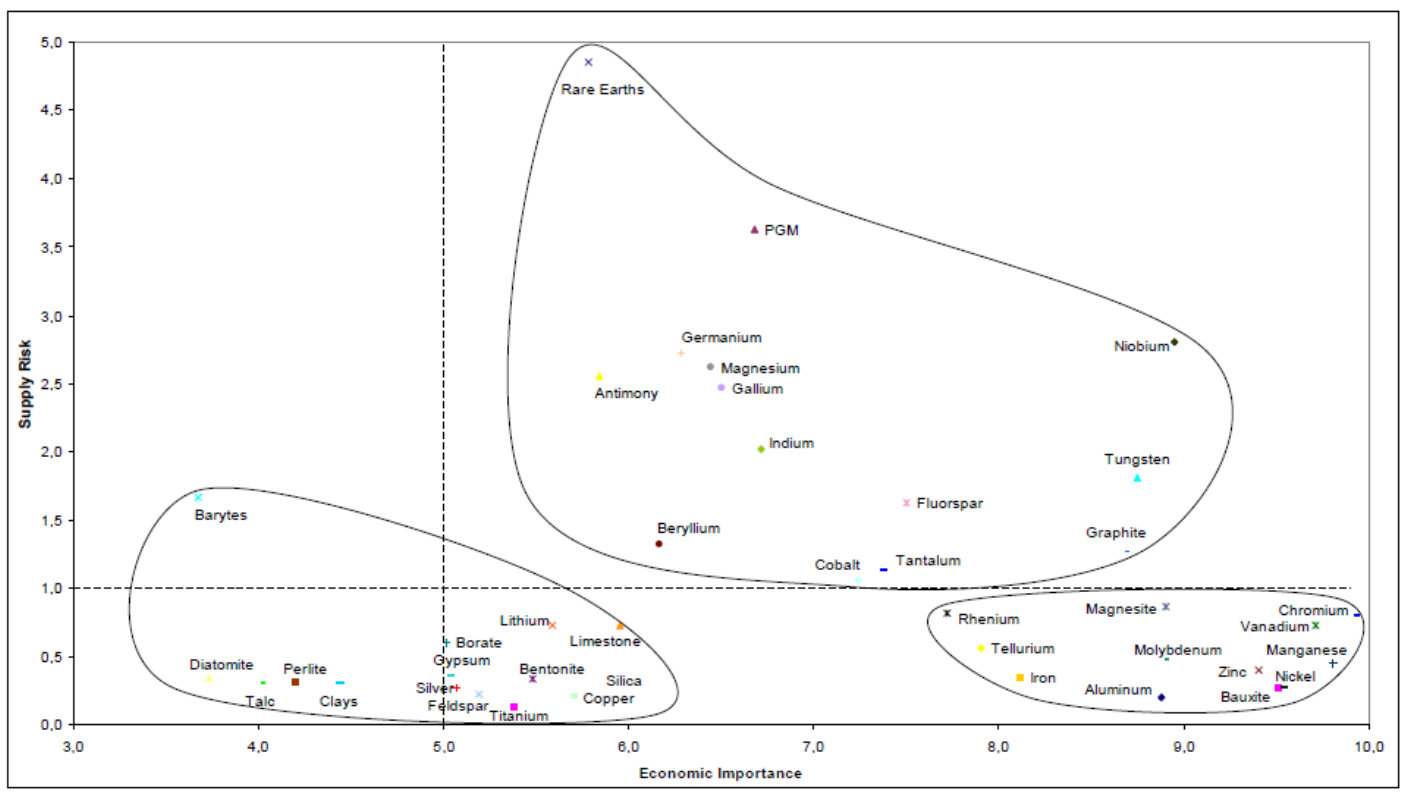

Figure 4.1 Supply risk versus economic importance of 41 materials evaluated for the EU showing the EU14 CRMs ${ }^{100}$

Table 4.1 List of the EU14 Critical Raw Materials ${ }^{100}$

\begin{tabular}{|c|c|c|c|c|c|c|}
\hline Antimony & Beryllium & Cobalt & Fluorspar & Gallium & Germanium & Graphite \\
\hline Indium & Magnesium & Niobium & $\begin{array}{c}\text { Platinum } \\
\text { Group Metals } \\
\text { (PGMs) }\end{array}$ & $\begin{array}{c}\text { Rare Earth } \\
\text { Metals (REMs) }\end{array}$ & Tantalum & Tungsten \\
\hline
\end{tabular}

PGMs: Pt, Pd, Ru, Ir, Rh, Os; REMs: the lanthanide elements and yttrium (Y).

In addition to the EU14, future supply of all materials of economic importance, particularly those which are rare or 'high value', must be considered by the industries/sectors which rely upon them. Priorities applied in criticality assessment differ across sectors and so will the picture of materials criticality. Au \& Ag are excluded from the EU14 despite the criticality of these metals to other sectors (see Section 3.1.1). This study includes Au and Ag as target CRMs with the EU14. It should be noted, the terms precious metals (PMs) and PGMs are generally used interchangeably. However, the EU14 definition of PGMs excludes Au and Ag. To distinguish these sets of metals, further discussion uses the EU14 PGMs definition, and PMs refers only to $\mathrm{Au}$ and $\mathrm{Ag}$.

\subsubsection{Critical Raw Materials in EEE}

All strategically important materials mentioned so far are used extensively by the EEE sector (Table 4.2). For this reason, WEEE represents an important secondary reserve of CRMs. 
Table 4.2 Major Applications of Critical Raw Materials in the EEE sector ${ }^{51,144,189}$

\begin{tabular}{|c|c|}
\hline $\begin{array}{c}\text { Critical Raw } \\
\text { Materials (CRMs) }\end{array}$ & Major End Uses \\
\hline Antimony (Sb) & $\begin{array}{l}\mathrm{SbO}_{3} \text { flame retardants, hardener in } \mathrm{Pb} \text {-acid battery electrodes; used as a } \\
\text { decolourising \& fining agent for glass e.g CRTs, cameras, photocopiers etc. }\end{array}$ \\
\hline Beryllium (Be) & Used in EEE as Be-Cu alloys, widely used in telecoms \& automotive sector \\
\hline Cobalt (Co) & $\begin{array}{l}\text { Mainly used in cathodes of Li-ion batteries, superalloys for jet engines \& } \\
\text { turbines; and SmCo permanent magnets }\end{array}$ \\
\hline Fluorspar & $\begin{array}{l}\text { Acid-grade fluorspar is a source of HF: a precursor in synthesis of almost all } \\
\text { fluoro-chemicals, e.g fluorocarbons refrigerants replacing CFCs; used in IC } \\
\& \text { production }\end{array}$ \\
\hline Gallium (Ga) & Major use in ICs, laser diodes, LEDs, photo-detectors, and PV solar cells \\
\hline Germanium $(\mathrm{Ge})$ & $\begin{array}{l}\text { Used in fibre optics; IR optics; and semiconductors for EEE e.g. phones \& } \\
\text { PV }\end{array}$ \\
\hline Graphite & Natural graphite is used in electrical arc lamp electrodes and batteries \\
\hline Indium (In) & $\begin{array}{l}\text { Indium-doped tin oxide (ITO) is a TCO used in electrodes of flat screens, IR } \\
\text { detectors, high-speed transistor; and PV cells }\end{array}$ \\
\hline Magnesium (Mg) & Used in laptop housing alloys \\
\hline Niobium $(\mathrm{Nb})$ & $\begin{array}{l}\text { Used in the nuclear industry as } \mathrm{Nb}-\mathrm{Zr} \text { for fuel cells;. Nb-Ti-Sn alloy is used } \\
\text { in magnetic coils of MRI equipment. Pure } \mathrm{Nb} \text { metal used in cathodic } \\
\text { protection systems for corrosion resistance }\end{array}$ \\
\hline Tantalum (Ta) & Used in SMD Ta-capacitors, hearing aids \& pacemakers \\
\hline Tungsten (W) & $\begin{array}{l}\text { Used for hard metals for cutting tools (tungsten-carbide); and heat resistant } \\
\text { interconnect alloys in ICs }\end{array}$ \\
\hline \multicolumn{2}{|l|}{$\underline{\text { REMS }}$} \\
\hline Yttrium (Y) & Used in phosphors of CCFLs, CRTs and LEDs \\
\hline Lanthanum (La) & Used in phosphors and electrodes of Li-ion battery electrodes ( $\mathrm{Li}-$. \\
\hline Cerium $(\mathrm{Ce})$ & Used in phosphors \& batteries \\
\hline Praseodymium (Pr) & Magnetic alloys and batteries \\
\hline Neodymium (Nd) & $\begin{array}{l}\text { NdFeB (NIB) magnets for HDDs, wind turbines, headphones and speakers; } \\
\text { electrodes in NiMH batteries }\end{array}$ \\
\hline Samarium (Sm) & Neodymium iron boron ( $\mathrm{NdFeB}$ ) magnets, cobalt magnets ( $\mathrm{SmCo})$, batteries \\
\hline Europium $(\mathrm{Eu})$ & Used in red phosphors \\
\hline Gadolinium (Gd) & Used in phosphors \& permanent magnets \\
\hline Terbium (Tb) & Used in phosphors \& permanent magnets \\
\hline Dysprosium (Dy) & Used in alloy of NIB magnets \\
\hline Erbium (Er) & Used in laser applications \\
\hline \multicolumn{2}{|l|}{ PGMs } \\
\hline Platinum $(\mathrm{Pt})$ & HDD platters and thermocouple wire \\
\hline Osmium (Os) & $\begin{array}{l}\text { Used in Pt alloy for electrical contacts, light bulb filaments and medical } \\
\text { implants }\end{array}$ \\
\hline Iridium (Ir) & $\begin{array}{l}\text { Spark plug tips, iridium crucibles to produce high-quality crystals for } \\
\text { electronics }\end{array}$ \\
\hline Ruthenium $(\mathrm{Ru})$ & Hard disks, chip resistors, flat screen displays, plasma display panels \\
\hline Rhodium (Rh) & LCD glass manufacturing, alloyed with Pt in thermocouple wire \\
\hline Palladium $(\mathrm{Pd})$ & SMDs, mainly MLCCs and hybrid ICs \\
\hline \multicolumn{2}{|l|}{$\underline{\text { Precious Metals }}$} \\
\hline Gold (Au) & ICs and contacts \\
\hline Silver (Ag) & Used in numerous SMDs (ICs, chip resistors, MLCCs etc.) and $\mathrm{Pb}$-free solder \\
\hline Copper $(\mathrm{Cu})$ & $\begin{array}{l}\text { Present in almost all EEE components: connectors, contacts, PCB tracks, } \\
\text { cables, transformers, turbines, electric motors etc. }\end{array}$ \\
\hline
\end{tabular}

In light of concerns over the future supply of CRMs, UK governments have highlighted increased recovery from wastes as a crucial strategy to enhance domestic CRM supply and mitigate issues of resource criticality. In 2012, in response to private sector concerns about the 
availability of raw materials, Defra launched the 'Resource Security Action Plan' to help businesses profit from recovery of PMs, PGMs and other CRMs present in household WEEE, through better segregation and recovery. ${ }^{149}$ The plan recognises the likely increase in demand for CRMs and calls for improvements to product design to support reuse and more efficient recycling of CRMs (DfRR), and substituting primary CRMs with secondary materials available within the economy.

According to the House of Commons report on 'Strategically Important Metals', the UK is recovering substantial quantities of $\mathrm{Pt}, \mathrm{Rh}, \mathrm{Pd}$, and $\mathrm{Au} .{ }^{190}$ Research commissioned by WRAP has shown that the majority of UK treatment is primary treatment with little refining of metals from WEEE to their final pure states. ${ }^{191}$ Rather, metal concentrates are produced and shipped for final refining in Europe, or the Far East. Low collection rates for WEEE prevent potentially recoverable CRMs from entering the recycling process chain. Mass based recovery rates of materials from WEEE which is collected and makes it way to recyclers are high, with $>90 \%$ of materials recovered/re-used. However, this mass based rate of recovery accounts mainly for bulk metals, plastics and glass recovered from shredded materials produced by automated preprocessing. Such mass based reporting of recycling fails to highlight low recovery rates for CRMs which represent only a small mass fraction of the waste stream but a significant proportion of the embodied economic, environmental and strategic value of WEEE. Automated pre-processing in which products are shredded results in dispersion of CRMs throughout bulk material fractions, resulting in losses through unintended co-seperation. ${ }^{62}$ The consequential detrimental effect of this dispersion on recovery rates of PMs in WEEE has been discussed (Section 1.4.2), and is applicable to recovery of CRMs from WEEE in general.

WRAP works with governments and business to deliver practical solutions which improve resource-efficiency. Management of WEEE is a key action area for WRAP who have launched numerous initiatives to improve the industrial ecology of the EEE sector (e.g. eSAP). WRAP is particularly focused on addressing the challenges of CRM recovery from WEEE, and for this reason, in 2012 commissioned the research reported in this chapter (IMT002-012: Recovery of Critical Raw Materials - Wales). ${ }^{185}$ WRAP also leads the ongoing EULife panEuropean 'CRM Recovery' project which aims to investigate the link between WEEE collection methods and CRM recovery rates, whilst investigating novel recovery processes which enhance CRM recovery. ${ }^{186}$

\subsubsection{Trial Aims \& Objectives}

WRAP sought to work with the UK WEEE recycling industry to investigate opportunities in the treatment process to recover CRMs prior to the occurrence of dissipative losses, whilst 
maximising the value generated for recycling businesses. The aim of the trial was to isolate components containing CRMs into fractions for downstream recovery during manual preprocessing, avoiding losses through shredding and in downstream recovery processes which do not target CRMs for recovery. This would increase their recovery rates from WEEE, thereby enhancing the value exploited from WEEE by recyclers.

WRAP commissioned Giraffe Innovations Ltd in partnership with Metech Recycling (UK) Ltd. who operated a manual pre-processing operation at their Approved Authorized Treatment Facility (AATF) in Hirwaun, South Wales. The trials were conducted by the author on behalf of Metech Recycling UK Ltd. who sponsored this EngD. As common with most AATFs, Metech already recovers PCBs which are sent for precious metals recovery (i.e. gold, palladium, silver, and copper are recovered). The aim of the trial was to develop and evaluate processes which could be implemented by Metech to improve CRM recovery rates and increase profitability of recycling operations. The findings of the trial were used as the basis of recommendations to enhance CRM recovery across the sector. ${ }^{185}$

\subsection{Methodology}

The stages undertaken during the trial were as follows. Stage 1 - samples were selected and gathered from WEEE received by Metech for analysis \& use in subsequent disassembly trials. Stage 2 - available equipment for identification and quantification of CRMs within samples, and methods for doing so were evaluated. Stage 3 - CRMs were then identified within samples. Stage 4 - processes for disassembly of WEEE and generation of CRM bearing components as suitable material streams for downstream recovery were evaluated in terms of their suitability for implementation at Metech to enhance value derived from WEEE and CRM recovery rates.

\subsubsection{Selection of Samples for analysis}

WEEE items known to contain CRMs were selected based on: the proportion of the waste stream flowing through Metech each type of equipment represents; prior knowledge of likely composition based on the experience of Metech staff; and a review of available literature. These included items that had previously been problematic to recycle and extract value due to poor product DfRR, or high volume items from which the potential exists to recover regular quantities of CRMs. Replicate samples of WEEE items were collected (up to 12 samples of each item) to provide sufficient numbers for repeat analyses and to investigate a number of recycling methods on each. 


\subsubsection{Evaluation of procedures for qualitative \& quantitative determination of CRM content in WEEE}

For the purposes of the trial it was necessary to: identify CRMs within components; develop a library of these components to inform further processing; perform quantitative analyses to establish target materials content of downstream recovery processes. This information is necessary to establish likely financial returns for material fractions generated, for use in costbenefit analysis (CBA) of proposed recycling processes to establish the economic viability of their implementation at Metech.

\section{$\underline{\text { X-ray Fluorescence Spectroscopy (XRF) }}$}

In order to 'prospect' CRMs in samples, methodical analysis of sample components was necessary. An XRF spectrometer was used to detect CRMs in samples* This is a nondestructive technique which avoids the use of potentially hazardous chemical digestion procedures prior to sample analysis as required for alternative atomic spectroscopy techniques such as AAS or ICP-MS. The method was therefore thought to be suitable for use on-site at Metech by staff who have no formal training in practical chemistry. The instrument selected was the XDAL-FD system, leased from Fischer Instrumentation (GB) Ltd.

The instrument consists of: a movable stage on which whole PCBs are placed; a small X-ray beam (max diameter $1 \mathrm{~mm}$ ) with variable cross-sectional area and shape; and a camera for imaging samples and targeting the $\mathrm{x}$-ray beam. The instrument can determine which elements are present from characteristic energies of emitted x-rays from samples, automatically assigning peaks in the emission spectrum to elements. From the number of emitted $\mathrm{x}$-ray photons detected for each element (i.e the area under the peak corresponding to a particular element, or counts per second -cps) the instrument can quantify the relative abundances of elements present in the scanned area of samples and report the relative mass percentage of each element present (rel.\%), i.e. the sum of the relative masses of detected elements is $100 \%$ (Figure 4.2). Note that this is not the true weight percentage of elements in the sample since the mass of undetected material, such as light elements in plastics etc., is unknown.

\footnotetext{
* for details of the instrument, its operational principles and experimental parameter used please see Section 2.2.4.
} 


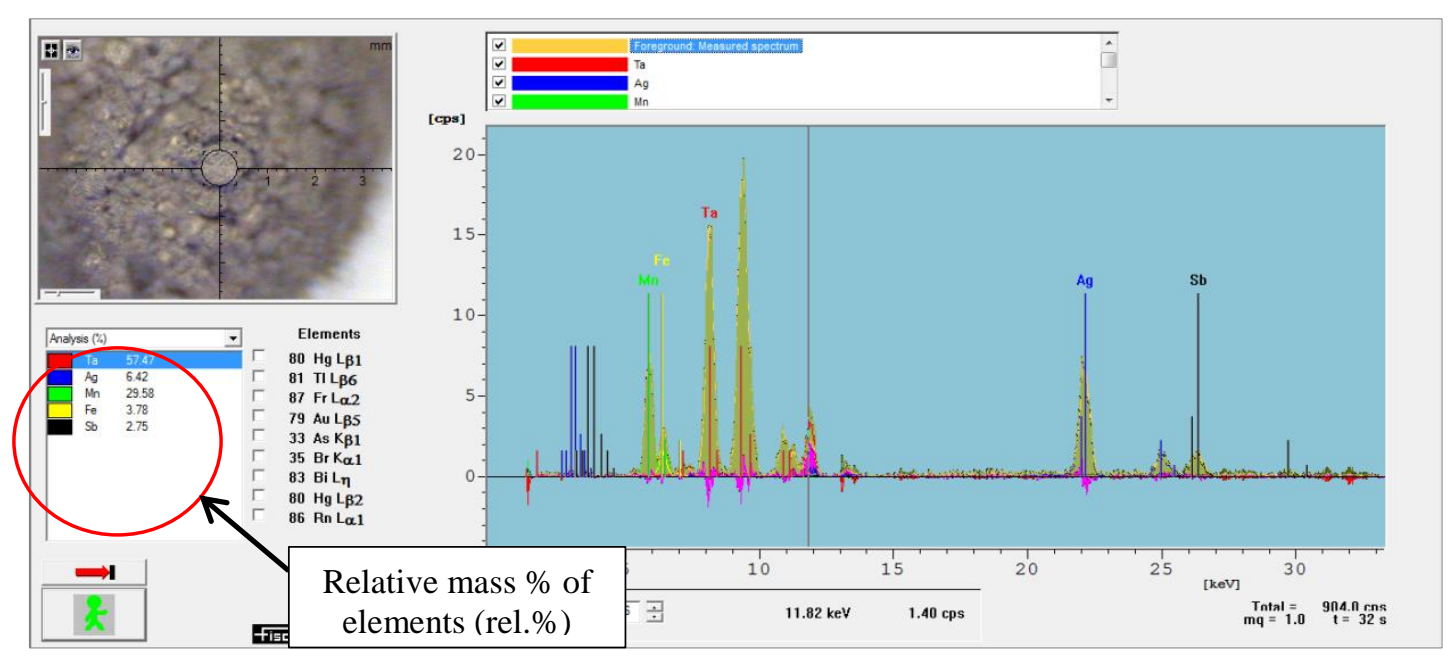

Figure 4.2 Analysed x-ray emission spectrum of a powder sample and measured relative mass percentages of elements

By moving the stage and customizing the beam, individual analyses of SMDs in place on PCB assemblies are possible. Spectra and images of components are also easily obtained with this technique. Although relatively simple in principle, using XRF for qualitative or quantitative analysis is complicated by 'matrix effects' i.e. the X-ray absorption profile of the sample and competitive absorption of both the excitation X-rays and emitted X-rays. ${ }^{135}$ This is particularly problematic for non-homogeneous samples such as WEEE - especially layered structures where the top layer(s) can prevent penetration of X-rays deeper into the sample.

In light of this, it was necessary to evaluate the ability and limitations of the XDAL instrument in detection and quantification of CRMs in WEEE samples, and its suitability for use by Metech. The details of this assessment are given in Appendix A. For the purpose of the work described here the following points on instrument characteristics and performance are significant.

When attempting to detect CRMs in encapsulated components, matrix effects may necessitate grinding or cutting open of components to detect contained CRMs. Where such procedures were necessary to identify CRMs is indicated in the results section. Unless otherwise stated, CRMs were detected with surface scanning of components only. 16s scan times provide best outcome in terms of $\mathrm{S} / \mathrm{N}$ ratio in spectra and time efficiency. Where elements detected represent only a few $\%$ of the overall composition of ground materials (as is the case for most CRMs present), instrument precision for determining these elements is $\sim 10 \%$, too high to base significant alterations to recycling operations upon. The XDAL instrument is an excellent rapid qualitative and/or semi-quantitative method for rapid identification of CRMs in samples. Much calibration and preliminary standardisation work would however be required before the 
XDAL could be used as a reliable quantitative technique with comparable precision to other methods such as AAS, ICP-OES or ICP-MS. This is due to low precision resulting from poor homogeneity in ground samples and large particle sizes relative to beam diameter. Therefore, the XDAL is used to identify CRMs in samples only and where necessary quantitative analysis is conducted with AAS or ICP techniques.

\subsubsection{Identification of Critical Materials in Samples}

\section{Sample disassembly and XRF identification of CRMs in samples}

Disassembly was performed with standard tools, e.g. powered / manual screw drivers, snips, pliers, scalpels, and powered grinding \& cutting tools. XRF was then used to identify CRMs in samples.

\section{Generation of an image library of CRM bearing components}

Following identification of CRMs in PCB components with the XDAL, a library of component images and spectra was compiled to support decision making on further processing. Once a component had been identified as containing target materials, its image was captured directly using the camera of the XDAL and saved with its corresponding $\mathrm{x}$-ray emission spectrum. It was then possible to identify CRM bearing components in PCBs visually by reference to this library. Example images and spectra of components are presented throughout section 4.3.2.

From images of PCBs it was possible to assess: the presence of gold in contacts on PCB surfaces; the number of contacts; and the type and number of components present on the PCB. To verify accuracy of evaluation using the image library, an image of a PCB was taken and a colour coded overlay applied to the image indicating concentrations of target elements. The accuracy of the assessment, PCB components of the boards were methodically analysed and the results compared to the results from visual inspections.

\subsubsection{Evaluation of Processes for Implementation}

\section{Assessment of disassembly and CRM concentration processes}

Processes enabling more rapid disassembly may reduce process costs, which is essential for the recovery of small, low value quantities of CRMs from WEEE. Methods of sample disassembly, isolation and concentration of CRM bearing components as distinct material streams for downstream recovery were developed and evaluated. These included processes for disassembly of whole items and their sub-assemblies e.g. disassembly of mobile phones into PCBs, batteries, screens etc.; and isolation of components from sub-assemblies such as 
removal of SMD components form PCBs with subsequent sieving to separate components by grade \& CRM content.

Following disassembly and PCB isolation, components identified as CRM rich require isolation from whole PCBs in a cost-effective manner to generate material streams suitable for downstream recovery i.e. a CRM pre-concentration method. Several methods were evaluated. Supported by data in the compiled library of X-ray emission spectra and images of components, methods were trialled which enabled isolation of CRM bearing components from PCBs and separation of these components into distinct material streams in which particular CRMs are concentrated. This was an attempt to separate CRMs which are lost in current recovery processes (e.g. Ta capacitors) into material flows of their own for diversion to alternative recovery processes.

The methods trialled were also evaluated in terms of their suitability for scale-up and implementation at Metech Recycling considering aspects such as practicality and compatibility with current environmental permits. It should be noted that Metech's environmental licenses do not permit the use of any liquid based processes. For this reasons techniques involving the use of acids for digestion or flotation processes were excluded from the trial.

For each method, necessary information to perform a cost-benefit analysis (CBA) was captured including: time taken, energy required, costs of necessary equipment and consumables, processing capacity. Output fractions suitable to identified CRM recovery processes were analysed quantitatively to establish a 'value' based on likely returns from sale of materials to refineries. CBA was only performed for processes which generate output fractions for which suitable downstream recovery process for valorisation of these fractions could be identified.

\section{Disassembly \& separation processes}

Manual Disassembly -Processes involved use of the following tools \& equipment.

Precision power cordless screw driver - Used to remove small screws from all devices to disassemble samples into their component parts, particularly for disassembly of phones, MP3 players, cameras, LCD screens.

$\underline{\text { Snips }}$ - Used to cut around surface mounted PCB components to isolate them; to directly cut connections between PCBs and components; to remove surface mounted components from 
TV power boards, cut sections of Mg-alloy casing away for analysis; and open SMD components for XRF analysis of internal CRMs.

Pliers - Used to pull relatively large SMD components \& contacts from PCBs; and crush SMD components prior to XRF analysis of internal CRMs.

Scalpels (various thicknesses) - Used to cut SMDs from PCBs boards when components are connected by a series of thin contacts e.g. ICs; thicker scalpels were used to remove components soldered directly on to PCBs.

Flat head screw driver - Used to scrape low profile soldered SMDs from PCBs when they cannot easily be removed with scalpels.

Dremel multi-tool with grinding and cutting attachments - Used to cut open Li-ion batteries for XRF analysis of internal electrode materials; cut open/grind layers away from SMDs for XRF analysis of internal CRMs; cut open housings of WEEE samples for rapid disassembly as an alternative to unscrewing; and cut away precious metal rich regions of PCBs i.e. those with large regions of Au-alloy such as edge contacts.

$\underline{\text { Hammer }}$ - Used to break open the steel housing of magnetrons and break magnets for rapid isolation without full disassembly.

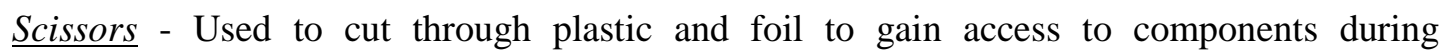
disassembly; and surface stripping SMDs from PCBs.

Standard Precision Screw driver kit - used to remove small screws from devices during disassembly process allowing isolation of specific components of WEEE samples.

Guillotine - Used to cut through PCB fibre-glass laminates of PCBs to isolate gold edge contacts. Method is only suitable for straight cuts and boards with thin, flexible substrates. Guillotine was also used to cut through plastic of LCD housings for rapid disassembly.

Wet Tile Cutter - A Rubi ND-200 Wet Saw / Electric Tile Cutter was used to isolate components from PCBs e.g. edge contacts as shown in Figure 4.3; used as an alternative to the guillotine for rapid disassembly of samples. 


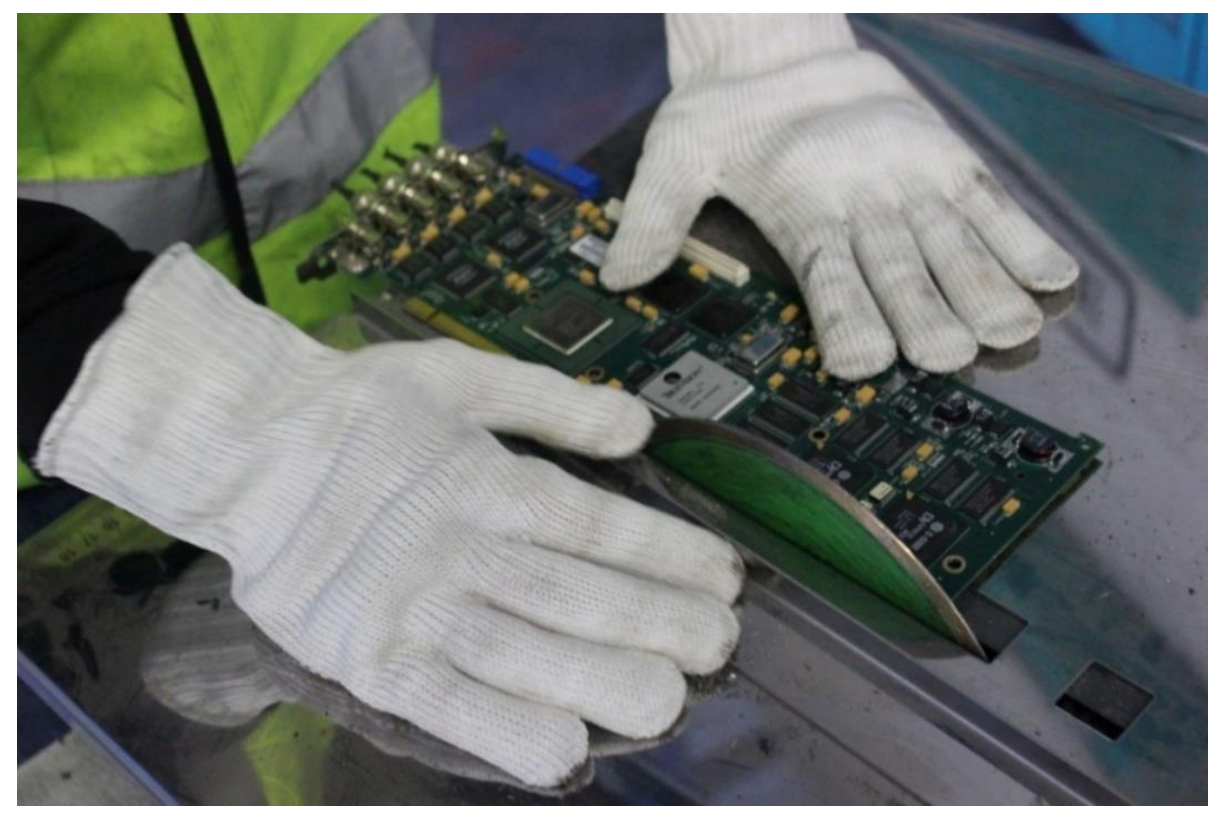

Figure 4.3 Removing edge contacts from server boards with a Rubi ND-200 wet saw / electric tile ccutter

Thermal Methods - Additional processes trialled to enhance the rate and cost-benefit of disassembly and component isolation processes included the following.

Cryo-cracking - Liquid nitrogen was used to embrittle plastic housings and casings of products 7-10 so they could be easily fractured for access to constituent components. Items were submerged in liquid nitrogen for 20 seconds, then removed and struck with a hammer to shatter and remove the casings to allow simple 'cherry picking' of internal CRM bearing components. An example of a Sony Walkman MP3 player following liquid nitrogen treatment and fracturing is shown in Figure 4.4. Cryo-cracking is widely used in plastics recycling. ${ }^{192}$

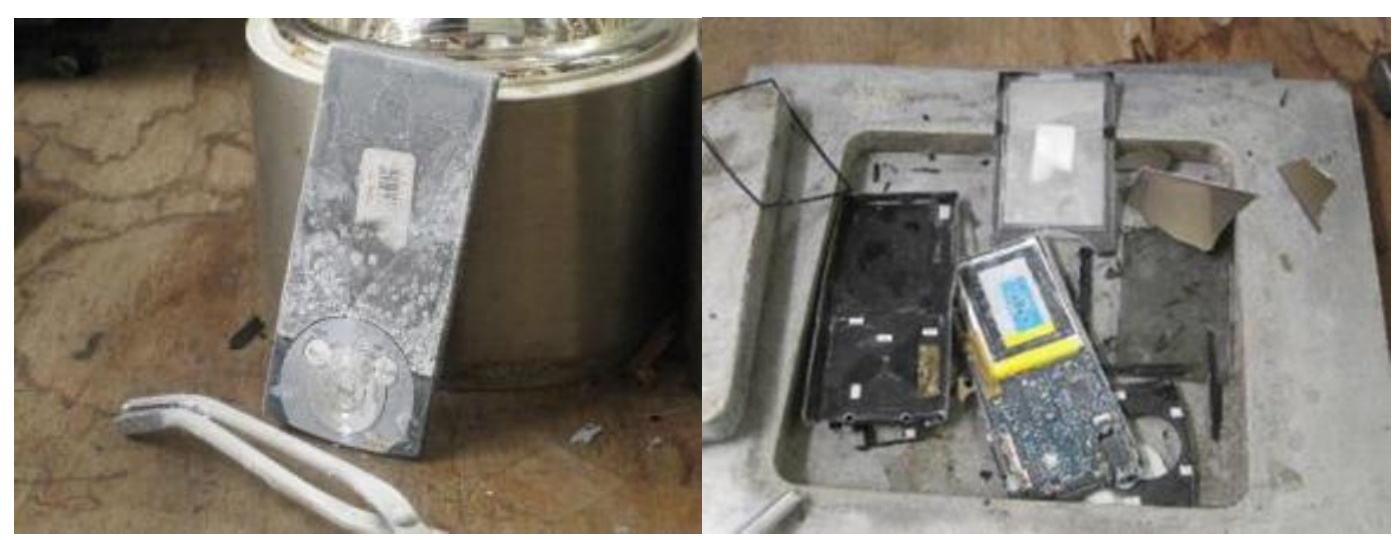

Figure 4.4 Sony Walkman Following Liquid Nitrogen Treatment (left), cracked open to reveal internal CRM bearing components (right). 
De-soldering of PCBs (thermal methods) - Heating methods were investigated to soften solder attaching SMDs to PCBs, enabling their isolation. As thermal treatment of whole PCBs is a complex process, de-soldering methods could only be trialled on a small scale using the following methods.

Blow Torch - PCBs were clamped in place above a beaker and heated using a Dremel 2200 Versaflame blow torch to soften solder and SMDs were scraped from PCB surfaces using a scalpel, collecting in a beaker below.

Soldering Iron - A soldering iron was used to manually de-soldering individual SMDs.

Paint Stripping Gun - A 2kW paint stripping heat gun was used to soften solder in specific areas of PCB assemblies.

Solder Bath - Solder baths are commonly used for soldering PCB assemblies. A variety of boards were placed on the surface of molten solder in a small $500 \mathrm{~W}$ solder bath (Figure 4.5). This softened the solder allowing components to be scraped from PCB surfaces in bulk, or individually removed with tweezers.

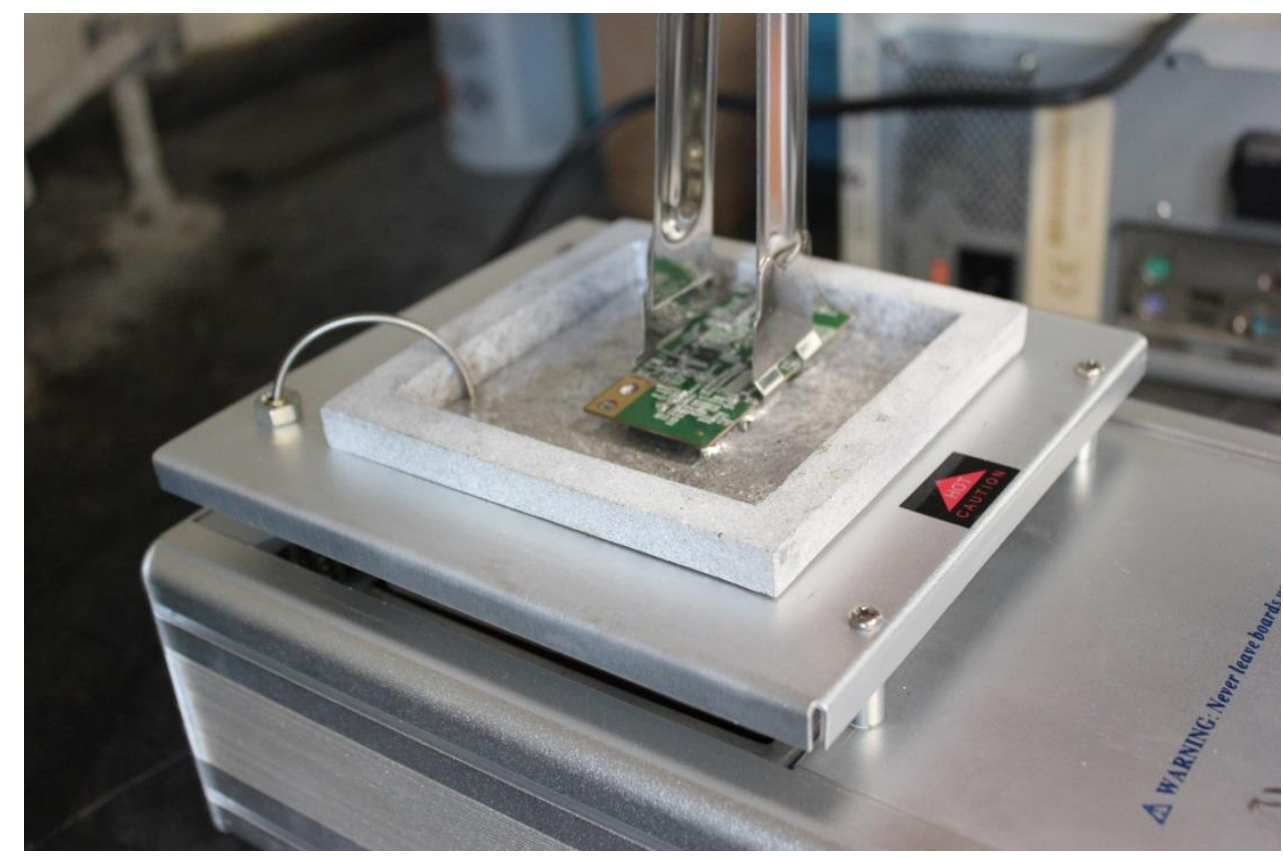

Figure 4.5 PCB placed on to the surface of liquid solder in a small solder bath

NIR oven - An Adphos NIR lab unit with $1 \mathrm{~m}$ heated length was used to heat boards and soften solder. A power setting of $70 \%$, rates of travel through the heated length of between $5 \mathrm{~m} / \mathrm{min}$ 
and $7 \mathrm{~m} / \mathrm{min}$, and heating temperatures of $200-240^{\circ} \mathrm{C}$ used in an attempt to identify optimum conditions.

\section{CRM concentration processes}

Microwave Ashing - Dry ashing is the act of heating a material to a high temperature to volatilize or decompose organic components, in this case epoxy of PCBs, producing an ash containing inorganic components, in this case CRMs and target materials for recovery. Ashing was used to concentrate CRMs in isolated edge contacts. A microwave furnace which was used to ash samples at $800{ }^{\circ} \mathrm{C}$ for 30 minutes within fibre crucibles. Ashing was conducted by CEM using a CEM Phoenix microwave muffle furnace.

Sieving - SMDs were sieved using a range of mesh sizes, to segregate SMDs by size and investigate the suitability of the method to generate material streams in which particular CRMs are concentrated. SMDs isolated from PCBs were combined with virgin SMDs donated by Metech recycling to give an appropriate quantity with which to evaluate the sieving procedure. These were loaded into the top of the stack of sieves of various aperture sizes which was shaken for $60 \mathrm{~s}$. Sieves with the following aperture sizes were used in a stack. $9.52 \mathrm{~mm}, 2.80$ $\mathrm{mm}, 1.68 \mathrm{~mm}, 1.4 \mathrm{~mm}$. SMDs and CRMs contained in each fraction were recorded.

\section{Cost benefit analysis (CBA)}

The second stage of the project was to assess the application of separation processes \& concentration processes on an industrial scale through Cost Benefit Analysis (CBA). The value of material streams generated (Total Benefit - B) was compared to the total costs of its production from WEEE samples (Total costs - A).. Monetary values have been assigned to each cost and benefit identified using data on expenditure and revenues from sale of materials from Metech and other industrial sources, with any assumptions or estimations discussed below.

Total Costs (A)_- Total costs are calculated from: operational overheads absorbed as an hourly labour rate incurred during processing provided by Metech Recycling (UK) Ltd., a full breakdown of which is given in Table 4.3 (£15.838/hour at the time of the assessment (2013, based on operational costs in 2012); chemical purchase costs; costs of electricity consumed ( $£ 0.09546 / \mathrm{kWh}$, taken directly from Metech’s power bill at the time of the trial); refining \& treatment charges for CRM materials fractions produced based on terms provided by BASF precious metals. 
Table 4.3 Overheads for Metech by percentage split

\begin{tabular}{lc}
\hline Overheads & \% Split \\
\hline Transport Costs & 5.45 \\
Disposal Costs & 0.32 \\
Production Facility Rent/Rates & 16.72 \\
Salaries \& Benefits-Production & 28.99 \\
Salaries \& Benefits- Admin \& Sales & 27.95 \\
Rent, Rates \& Insurance & 2.36 \\
Heat \& Lighting & 1.54 \\
Machinery Servicing \& Repair & 0.16 \\
Premises Repair & 0.20 \\
Security System Maintenance \& Rental & 0.25 \\
UK Business Expenses & 1.01 \\
UK Marketing Expenses & 1.14 \\
Postage & 0.06 \\
Telephone & 0.95 \\
Stationery \& Books & 0.32 \\
Professional Fees & 3.74 \\
Equipment Hire & 0.55 \\
Repairs \& Renewals Tools \& Equipment & 0.09 \\
Computer Repairs \& Maintenance & 1.35 \\
Cleaning & 0.73 \\
Bank Charges & 0.55 \\
Subscriptions \& Site Waste Management & 1.48 \\
Clothing Costs & 0.04 \\
Refreshments & 0.10 \\
Miscellaneous Costs & 1.50 \\
Premises Expenses & 0.06 \\
Safety Equipment \& Supplies & 0.07 \\
Production Expenses & 1.54 \\
Other Business Expenses & 0.77 \\
\hline TOTAL & $\mathbf{1 0 0 . 0 0}$ \\
\hline & \\
\hline &
\end{tabular}

Total Benefits (B) - This is based on: the value of recovered component materials (from quotes received from refineries following submission of samples to them for assay and market values for metals at the time of the trial i.e. $\mathrm{Au}-£ 35.9652 / \mathrm{g} ; \mathrm{Cu}-£ 5.46 / \mathrm{kg}$; $\mathrm{Ag}-£ 0.69495 \mathrm{~g}$; Pd $£ 14.3566 / \mathrm{g}$ ), and; avoided refining charges and transport charges because of concentration enhancement of target materials in output fractions.

Cost Benefit (C) -is calculated as the difference in total costs and total benefits $(C=B-A)$. CBA has been carried out on a number of combinations of separation techniques, each specific combination being suitable for a particular sample investigated. E.g. cryo-cracking to isolate PCBs of MP3 players, mobile phones and cameras, with solder bath treatment to isolate SMDs. This enabled identification of the most profitable processing options for samples. 


\section{Notes and Assumptions:}

i) Times taken for processes are obtained from trials on single items and assumed to represent the average ability of Metech employees for a pace of work which can be maintained throughout the day. Cost-benefit analyses are therefore indicative of achievable benefits only, and a time in motion study on site would have to be conducted to verify profitability of proposed processes following scale up and implementation. It is assumed that while an item is being processed e.g. submerged in liquid nitrogen, or being ashed, employees are working on other stages of the processing.

ii) It is assumed no material is lost in any of the separation processes themselves.

iii) It is assumed that recovery rates for metals from ashed and unashed samples are equal and that all refining terms remain the same.

iv) The value of $\mathrm{Ag}, \mathrm{Au}, \mathrm{Pd}$ and $\mathrm{Cu}$ content in isolated fractions were obtained directly from BASF Metals Recycling (refinery) following assay of submitted samples, assuming a recovery rate of $98 \%$ for $\mathrm{Au} \& \mathrm{Cu}, 95 \%$ for $\mathrm{Ag}$ and $85 \%$ for Pd. Refiners only pay the supplier on recovered $\mathrm{Au}, \mathrm{Ag}, \mathrm{Pd}$ and $\mathrm{Cu}$ content, and no rebate is given for other materials present.

v) Treatment charges of $£ 1.80 / \mathrm{kg}$ for material received, and refining charges of $£ 475 / \mathrm{kg}$ for $\mathrm{Pd}, £ 200 / \mathrm{kg} \mathrm{Au}, £ 25 / \mathrm{kg}$ for $\mathrm{Ag}$ are applied by refineries. No refining charge is applied for $\mathrm{Cu}$. (terms supplied by BASF precious metals at the time of this study - Feb 2013).

vi) The financial benefits of separating components are based on production of $500 \mathrm{~kg}$ of components, which is the minimum quantity required for refining.

\subsection{Results \& Discussion}

\subsubsection{Selection of Samples for analysis}

Table 4.4 lists samples used in the trial. Sample 1-6 \& 11-16 are generated by Metech in existing manual disassembly operations. Samples 7-10 are whole products which are not currently disassembled by Metech because it is too expensive to do so. Samples (11- 16) were chosen to demonstrate and evaluate methods for isolation of CRM bearing components from PCBs. 
Table 4.4 Samples for analysis supplied by Metech Recycling (UK) Ltd

\begin{tabular}{llcc}
\hline$\#$ & Sample Item & No. of samples & Type \\
\hline $\mathbf{1}$ & Sanyo domestic microwave magnetron & 12 & Component \\
$\mathbf{2}$ & Hard Disk Drives (assorted 31/2 inch drives) & 12 & Component \\
$\mathbf{3}$ & TV Power boards (2 types) & 6 & Component \\
$\mathbf{4}$ & STB front panel PCBs (2 types) & 12 & Component \\
$\mathbf{5}$ & Sony Li-ion laptop batteries & 11 & Component \\
$\mathbf{6}$ & HP Laptop screens & 12 & Component \\
$\mathbf{7}$ & Mobile Phones (assorted) & 12 & Whole product \\
$\mathbf{8}$ & MP3 players solid state memory (assorted) & 6 & Whole product \\
$\mathbf{9}$ & RSA security anti-hacking dongle & 12 & Whole product \\
$\mathbf{1 0}$ & Digital Cameras (assorted) & 12 & Whole product \\
$\mathbf{1 1}$ & PCB from TV LCD & 5 & Component \\
$\mathbf{1 2}$ & Graphics card & 5 & Component \\
$\mathbf{1 3}$ & Server board & 5 & Component \\
$\mathbf{1 4}$ & Ethernet card & 3 & Component \\
$\mathbf{1 5}$ & Power board from Fridge & 2 & Component \\
$\mathbf{1 6}$ & Main PCB from TV & 1 & Component \\
\hline
\end{tabular}

\subsubsection{Identification of Critical Materials in Samples}

\section{Sanyo domestic microwave magnetron}

Ferrite cores from the filter box and ring magnets as shown in Figures 4.6 were isolated from magnetron samples. Iron oxide and barium or strontium carbonate are used in manufacturing of hard ferrite magnets. ${ }^{193} \mathrm{XRF}$ of the ring magnets shows iron and strontium (Figure 4.7) to be present, with the XRF software indicating the presence of small amounts of critical Co. The $\mathrm{Co} \mathrm{K} \alpha_{1}$ peak (6.93 keV) are masked by the intense $\mathrm{Fe} \mathrm{K} \alpha_{1}$ and $\mathrm{K} \beta_{1}$ peaks (6.40 and $7.06 \mathrm{keV}$ respectively) and the presence of the Co $\mathrm{K} \beta_{1}$ peak $(7.65 \mathrm{keV})$ is not obvious due to its low intensity. XRF shows ferrite cores to be predominantly iron with some $\mathrm{Zn}$ and $\mathrm{Cu}$ and without the strontium carbonate ceramic (Figure 4.8). This probably means that its composed of Ni$\mathrm{Zn}-\mathrm{Cu}$ ferrite. ${ }^{194}$ The presence of cobalt is once again automatically detected by the software, however the same issue with peak masking and low intensity is observed. The presence of Co in each sample was confirmed by AAS, and is discussed in section 4.3.3. 


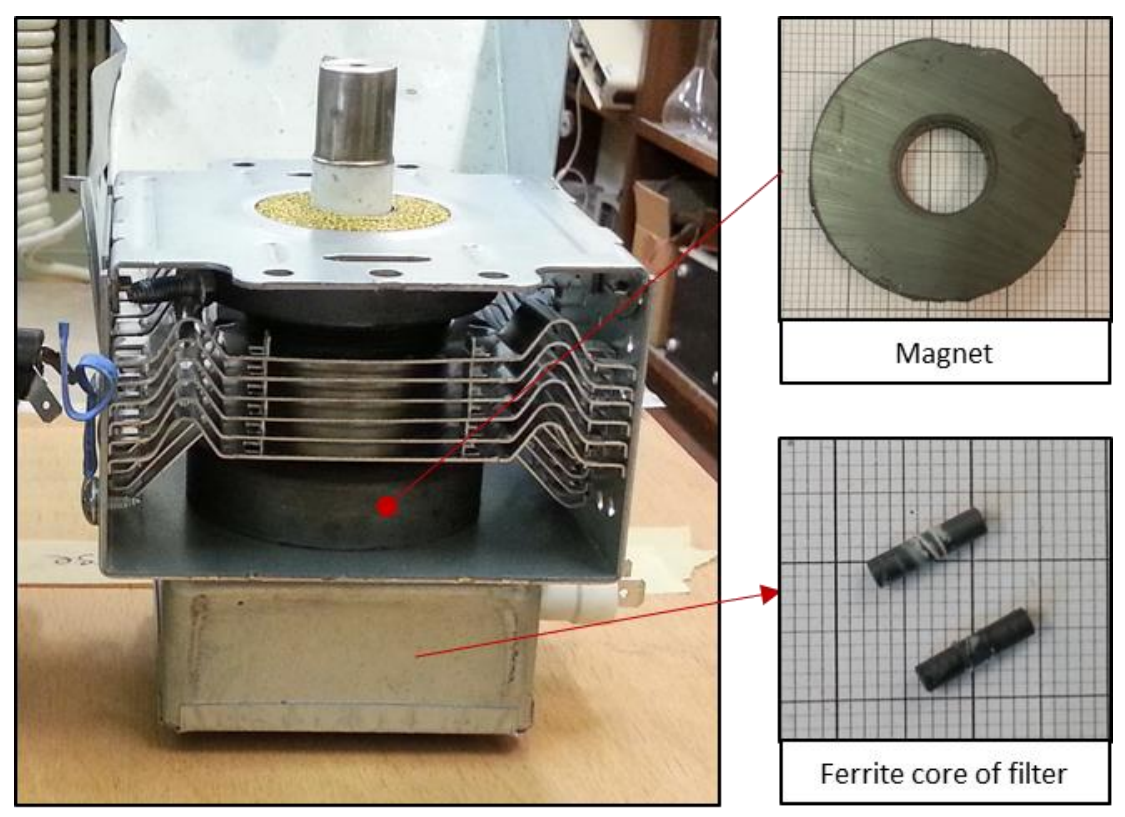

Figure 4.6 Magnetrons (top left) and isolated magnets (top right) and ferrite cores of the filter (bottom right)

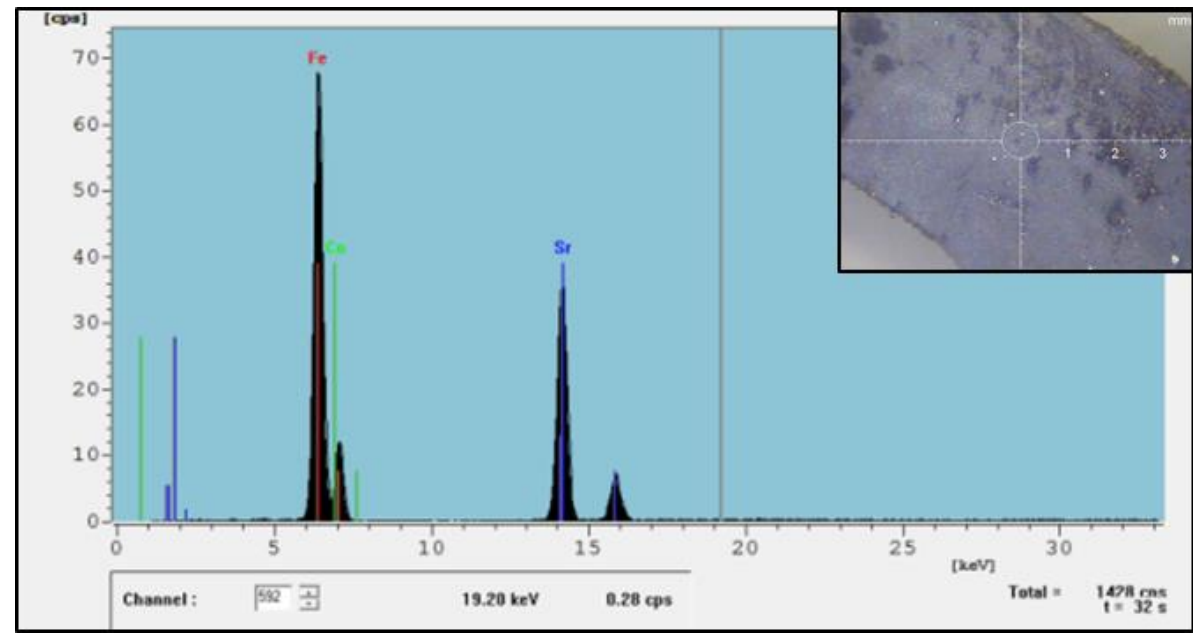

Figure 4.7 XRF spectra of magnetron magnets indicating the presence of Co

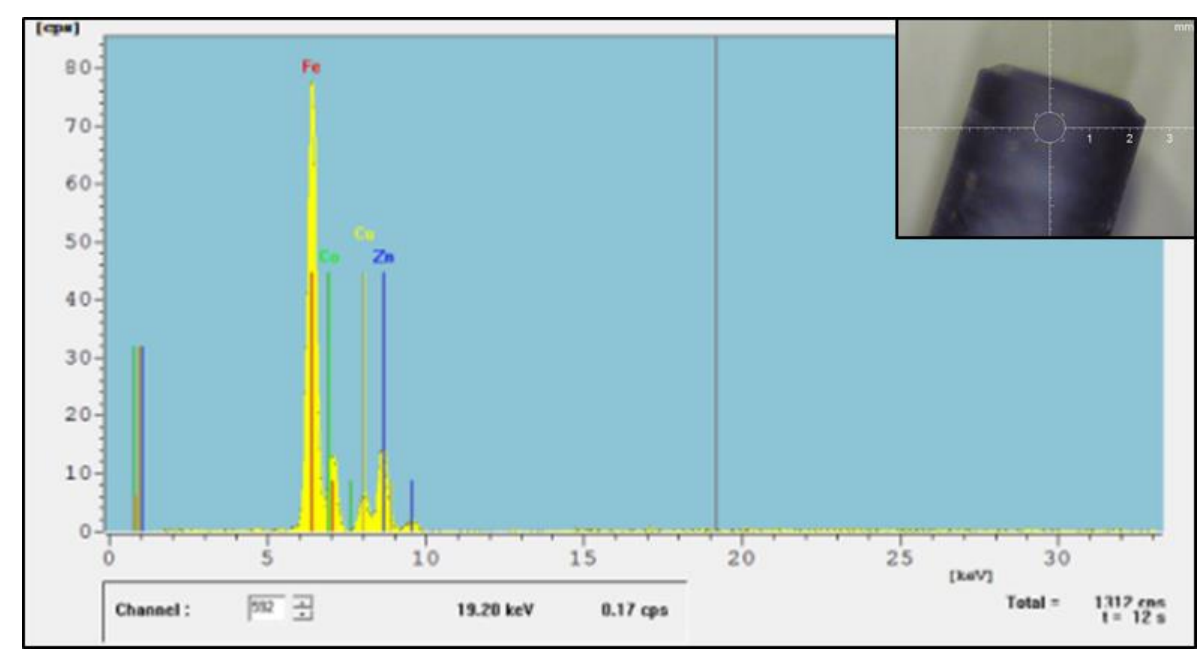

Figure 4.8 XRF spectra of ferrite cores from the magnetron filters indicating the presence of Co 


\section{Hard Disk Drives (HDDs, 31/2 inch)}

CRMs in hard disk drives (HDDs, Figure 4.9) are well known and a comprehensive investigation into the concentrations of CRMs in laptop HDDs ( $2 \frac{1}{2} 2$ inch drives ) based on data available from manufacturers and recyclers was conducted by the Öko-Institut e.V. ${ }^{19}$ The main CRM bearing components are the voice coil accelerator magnets and the PCB of the device. The most common alloy for HDD magnets is $\mathrm{Nd}_{2} \mathrm{Fe}_{14} \mathrm{~B}$ which are known as neodymium iron boron (NIB) magnets; dysprosium may also be present. The platters of the HDD are also reported to contain PGMs. ${ }^{19}$

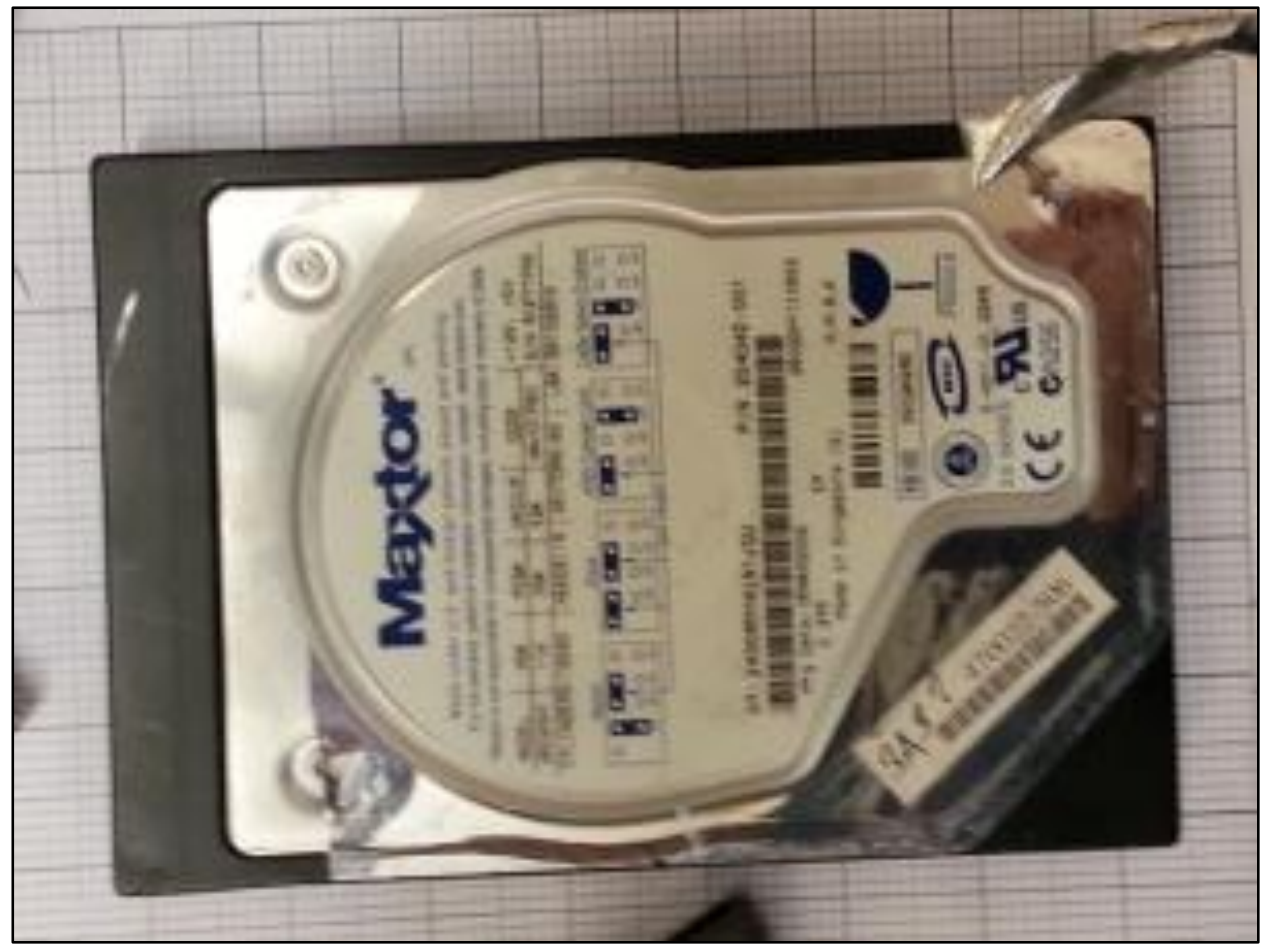

Figure 4.9 Typical hard disk drive (HDD)

Platters - No Pt was detected by XRF in the platters of HDDs.

NIB magnets Two NIB magnets are present in each HDD. XRF analysis of the magnets revealed the presence of $\mathrm{Nd}$ in the alloy, no Dy was detected (Figure 4.10). 


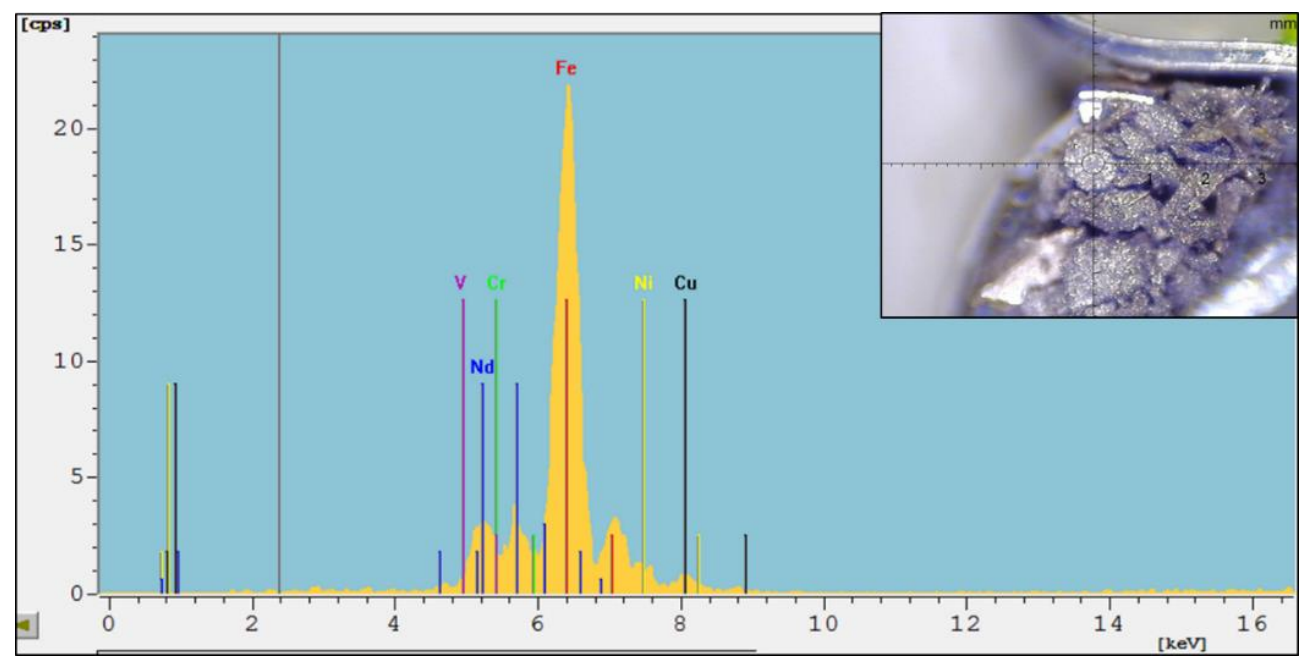

Figure 4.10 X-ray emission spectrum of NIB magnetic alloy showing the presence of Nd

\section{Set top box front panel PCBs}

The STB board is a PCB with a series of mounted blue and green LEDs. Both blue and green LEDs contain $\mathrm{Ga}$ and $\mathrm{Ag}$ (Figures 4.11 \& 4.12), and green LEDs may also contain Au. Ga based semiconductors such as GaAs, GaP and GaAsP are used to create the LED itself. Ag may be used as wiring, or in the solder, and Au is possibly present as connecting wire. The high Br peak may be associated with the polymer cap possibly in a flame retardant.

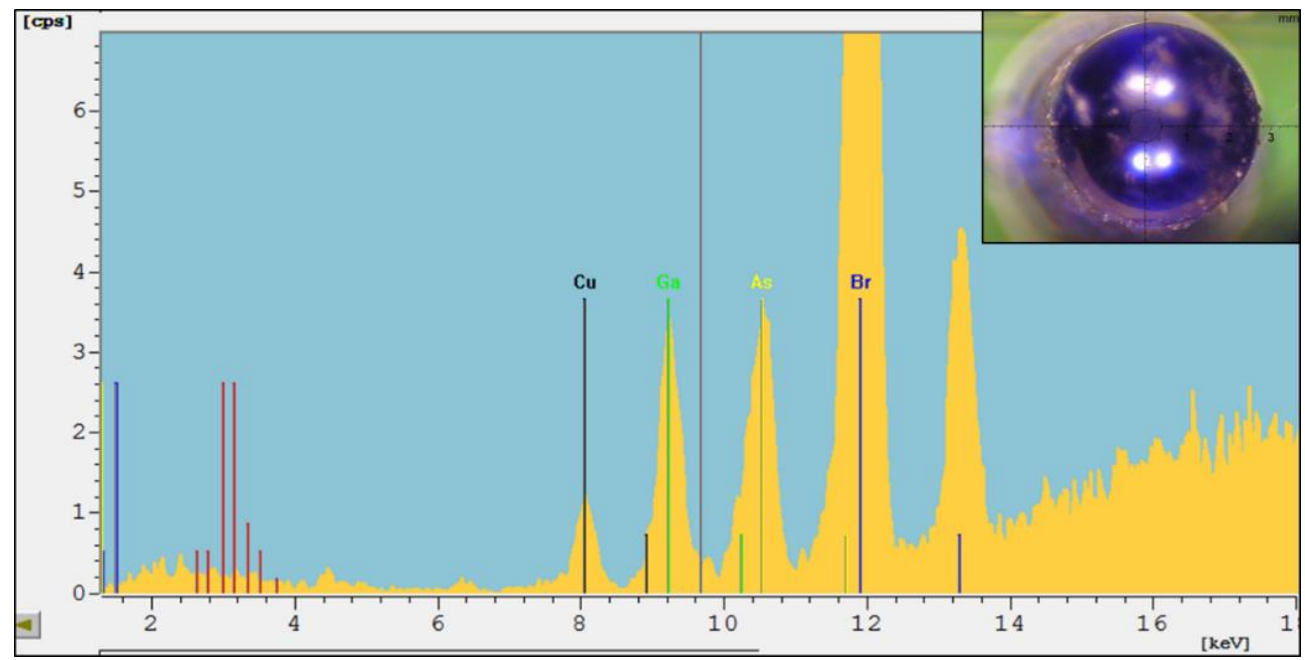

Figure 4.11. Blue LED containing $\mathrm{Ga}$ and $\mathrm{Ag}$ 


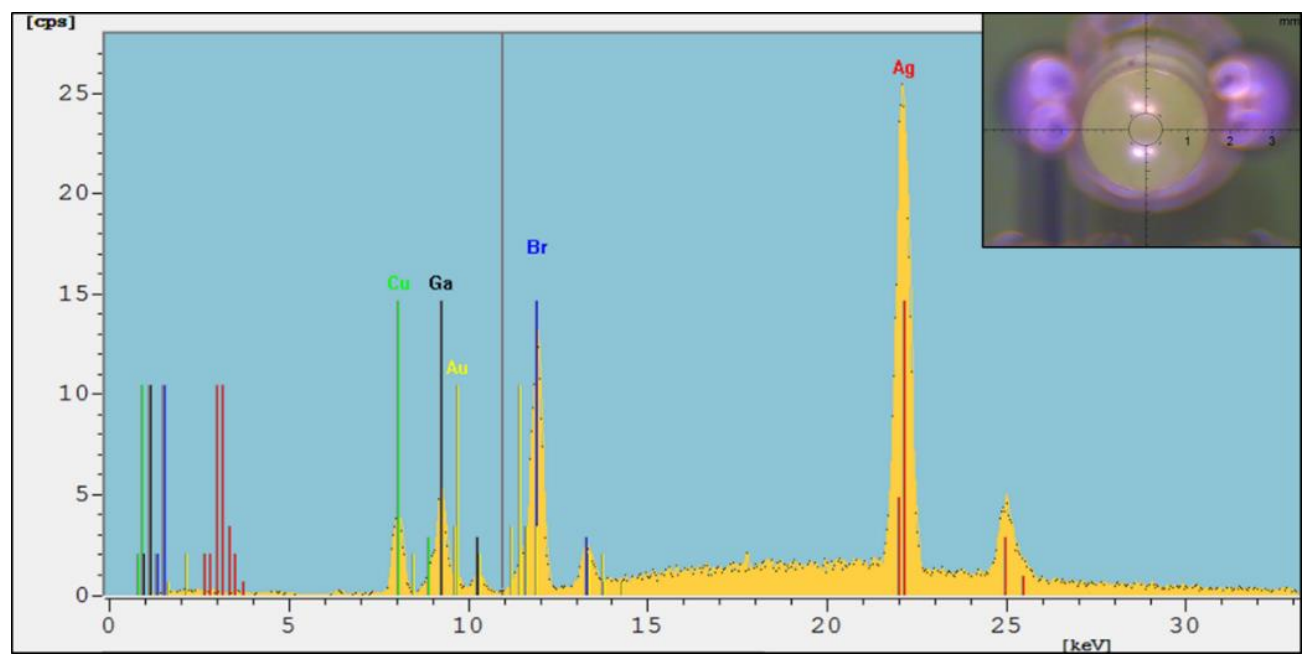

Figure 4.12. Green LED containing $A u, A g$ and $G a$

\section{Sony Li-Ion Laptop Batteries}

Battery packs were disassembled to reveal 6 LIB cylinder cells and a PCB, all of which were found to contain CRMs (Figure 4.13). The PCB was constructed of typical components (LIBs and PCBs are discussed in subsequent sections on common components).

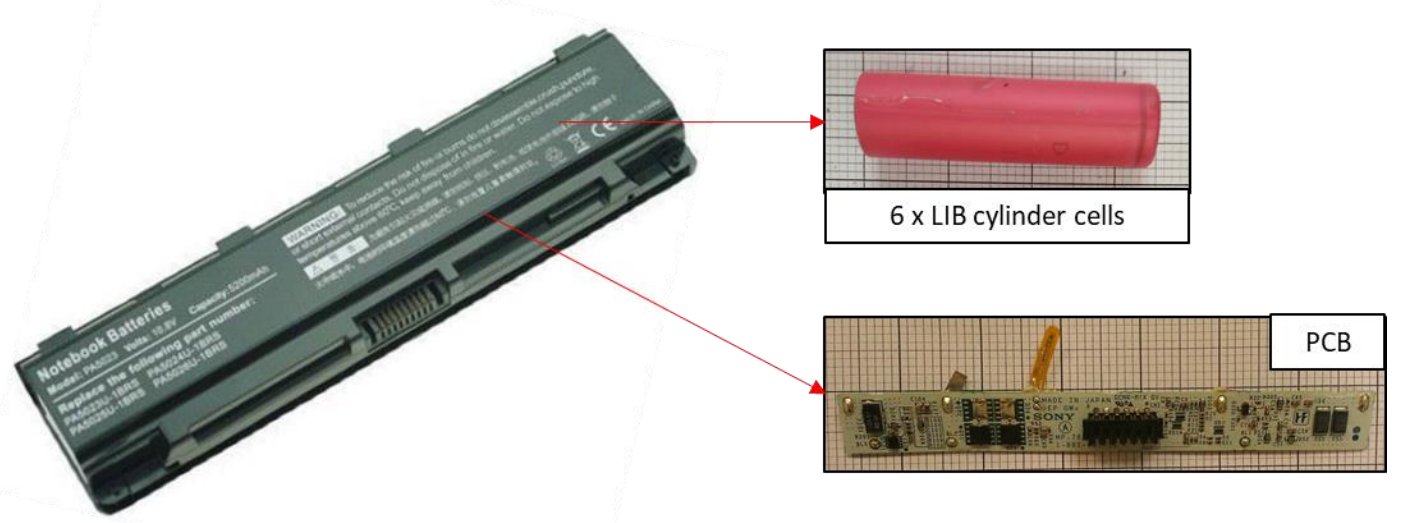

Figure 4.13 Laptop Li-ion battery pack and isolated LIB cylinder cells and PCB.

\section{HP Laptop Screens}

Prior to analysis, HP laptop LCD screens were disassembled into housing materials, major and minor PCBs and the LCD sandwich itself which was further disassembled to remove the cold cathode fluorescent lamp (CCFL) backlight. (Figure 4.14). XRF of components revealed the presence of $\mathrm{Mg}, \mathrm{Y}, \mathrm{Nb}, \mathrm{Sb}, \mathrm{Ru}, \mathrm{Ag}$ and $\mathrm{Au}$ in the screens. 


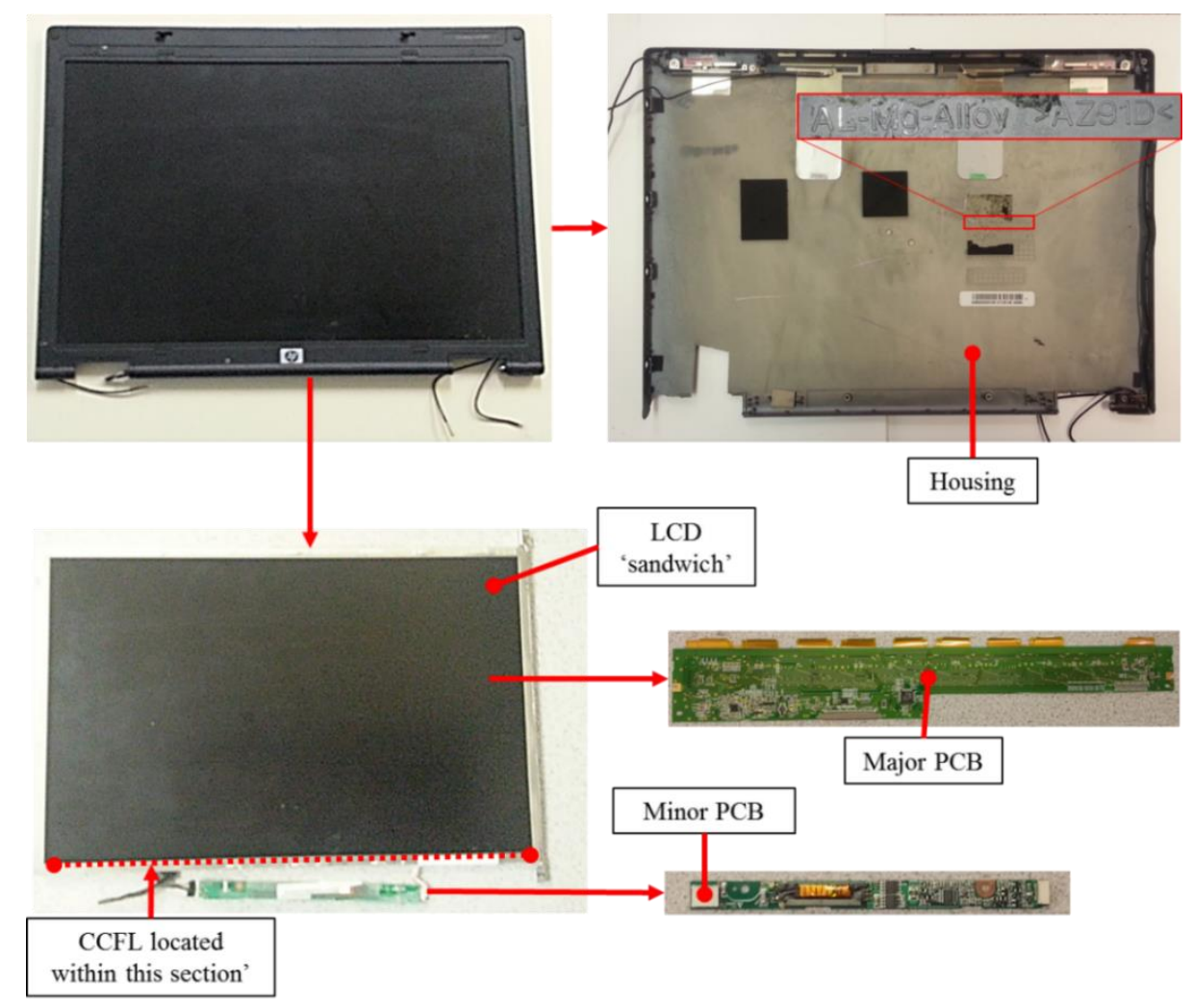

Figure 4.14 Disassembly of LCD Screen and isolation of housing, PCBs and LCD sandwich.

Cold Cathode Fluorescent Lamp (CCFL) - XRF analysis of the CCFL revealed the presence of $\mathrm{Y}$ in the phosphor (at $14.96 \mathrm{keV}$ - Figure 4.15) and $\mathrm{Hg}$ in the terminals of the tube. ICPMS analysis showed each CCFL contains $13 \mathrm{mg}$ of $\mathrm{Y}$ (equivalent to $1.7 \mathrm{wt} \%$ when wires are removed) probably present as the red phosphor $\mathrm{Y}_{2} \mathrm{O}_{3}: \mathrm{Eu}^{3+} \cdot{ }^{99}$ No other CRMs were revealed during XRF analysis suggesting that, although known to be present, quantities of other REEs are below XRF detection limits and present in comparatively low quantities. ${ }^{195}$

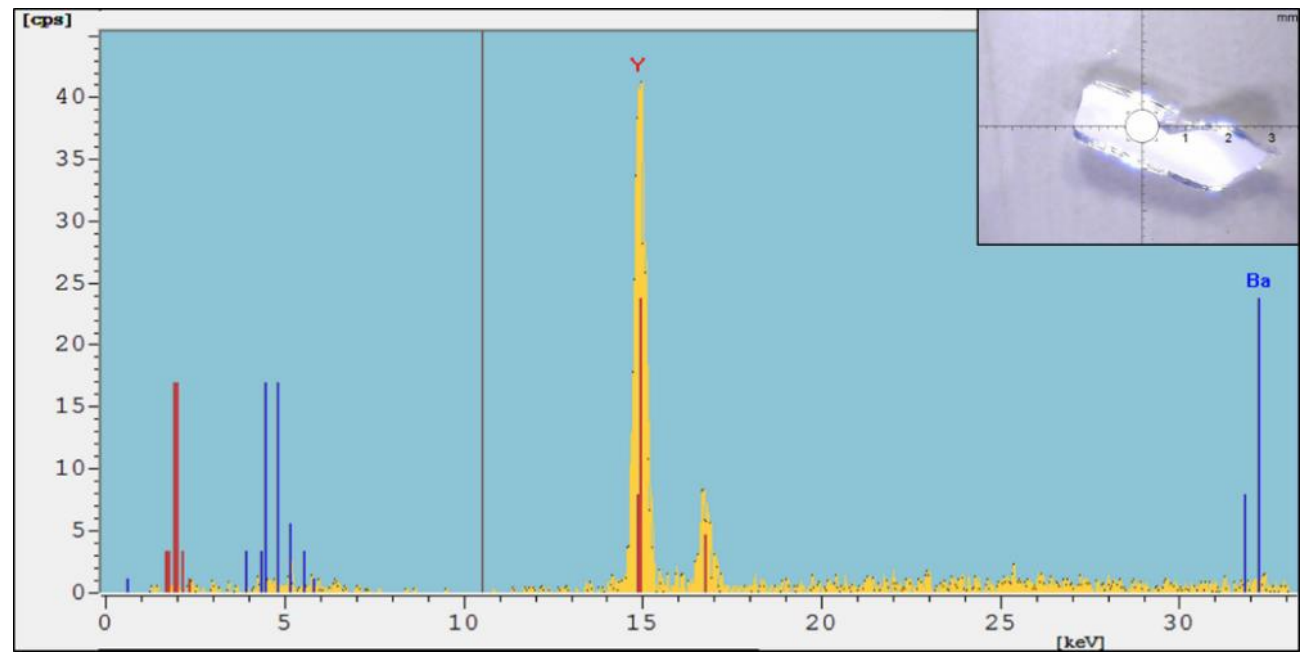

Figure 4.15 X-ray emission spectrum of CCFL phosphor containing yttrium. 
LCD Housings - Housings were labelled as the anodised Mg-alloy AZ91D (Figure 4.16). This means that it should be composed of $90 \% \mathrm{Mg}, 9 \% \mathrm{Al}$, and $1 \% \mathrm{Zn}$. Although the XRF is incapable of detecting the aluminium or magnesium, a single peak at $8.64 \mathrm{keV}$ corresponding to $\mathrm{Zn}$ suggests that AZ91D is the likely alloy (Figure 4.17).

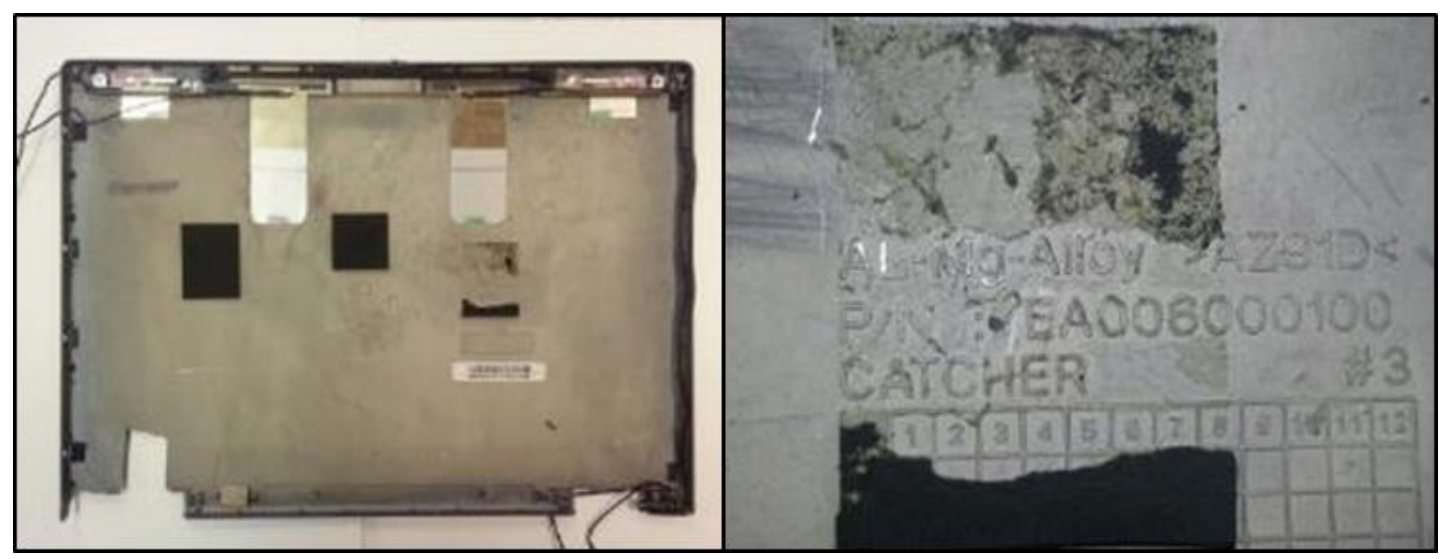

Figure 4.16 AZ91D Mg alloy laptop LCD screen housing

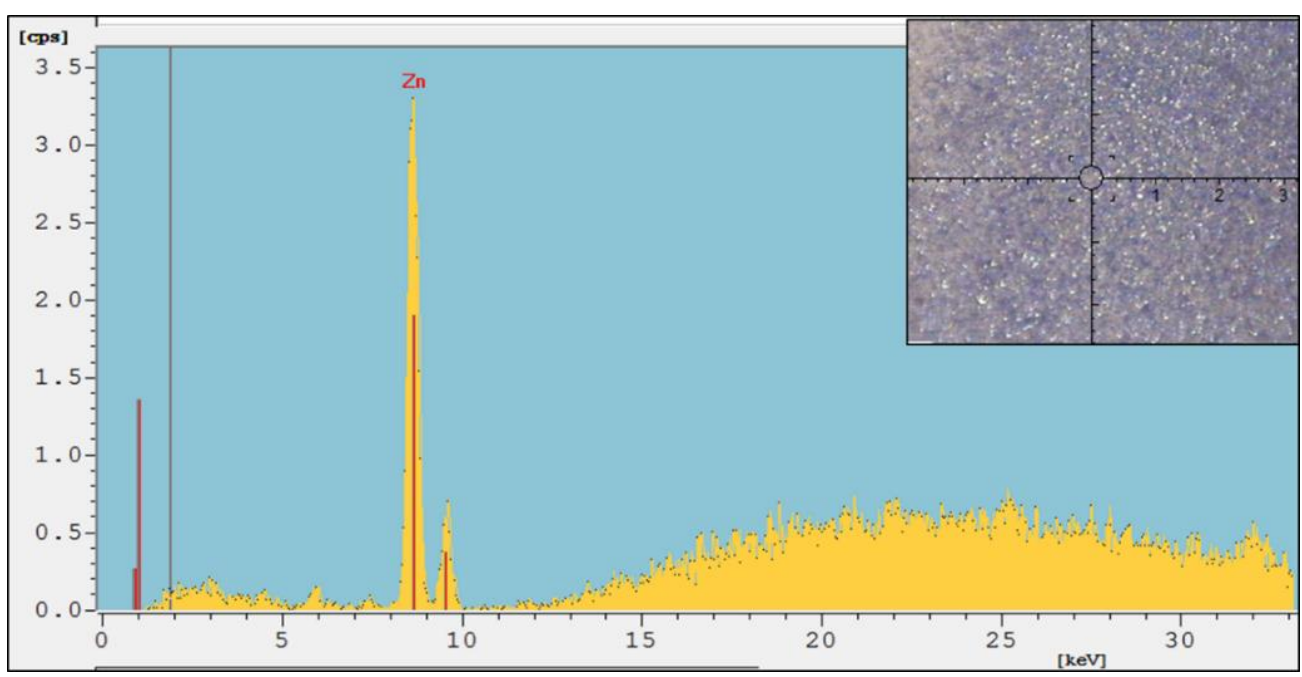

Figure 4.17 X-ray emission Spectra of AZ91D Mg alloy laptop LCD screen housing showing the presence of $\mathrm{Zn}$

Screens - In is known to be present as ITO in screens with a major fraction of global In production consumed by display manufacturing. However, In was undetectable by XRF. The Öko-Institut e.V. have reported a figure of $39 \mathrm{mg}$ of In on a 14-inch display. ${ }^{19} \mathrm{In}$ light of this and the fact that recovery of In from LCDs was not a commercially available process at the time of this study (2012-13) economic recovery of In from this dissipative application was, at the time, unlikely. 


\section{Mobile phones}

Visual inspection of mobile phone samples selected for the trial revealed that they are constructed with common components. Taking a Nokia 6230i as a representative sample, the phone was disassembled to isolate its LIB, charging contacts, SD card, PCB, speaker magnet and screen (Figure 4.18).
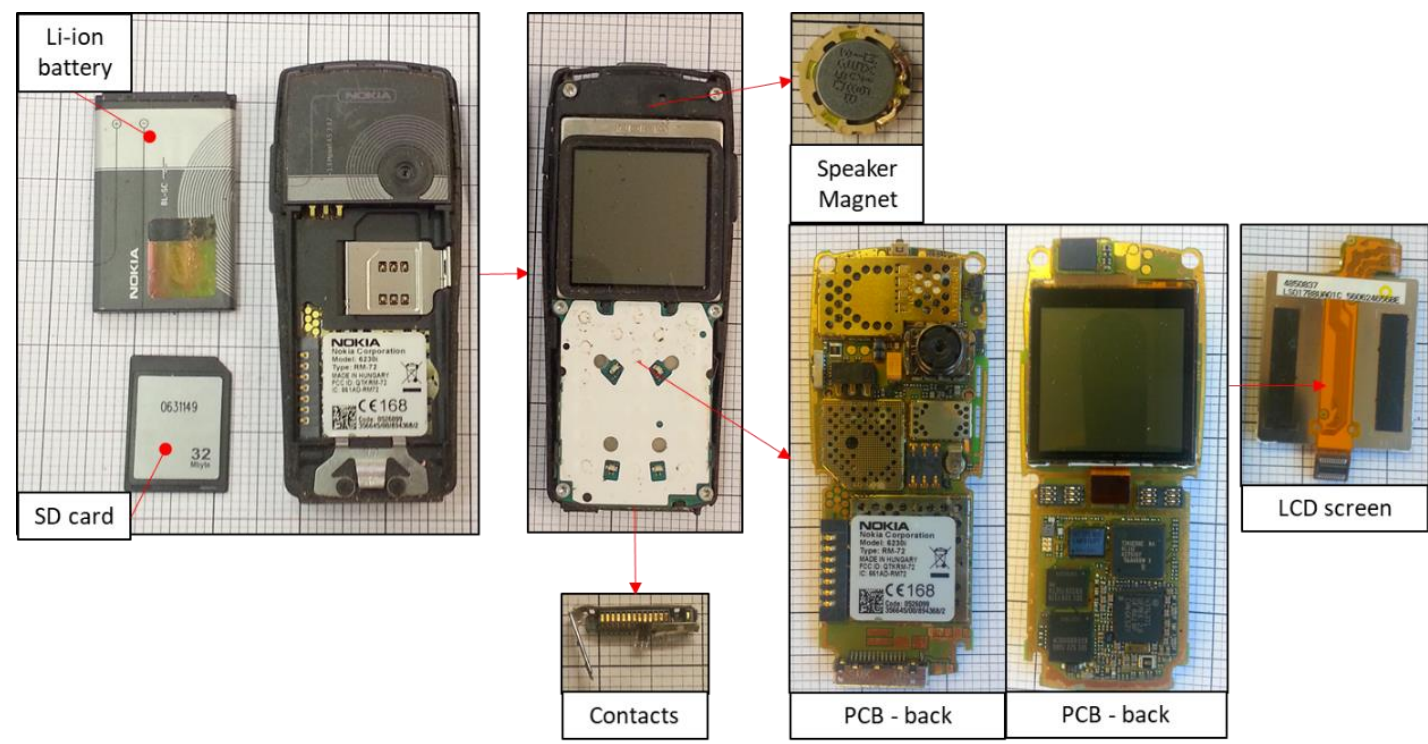

Figure 4.18 Disassembly of Nokia 6230i mobile phone

Screens - screens are attached to the PCBs through a connector which bears SMD components and $\mathrm{Au}$ contacts, common in PCBs (Figure 4.19). The nature of the contacts and components is discussed in the subsequent section about PCBs. Once again it was not possible to detect In on the screen with XRF.

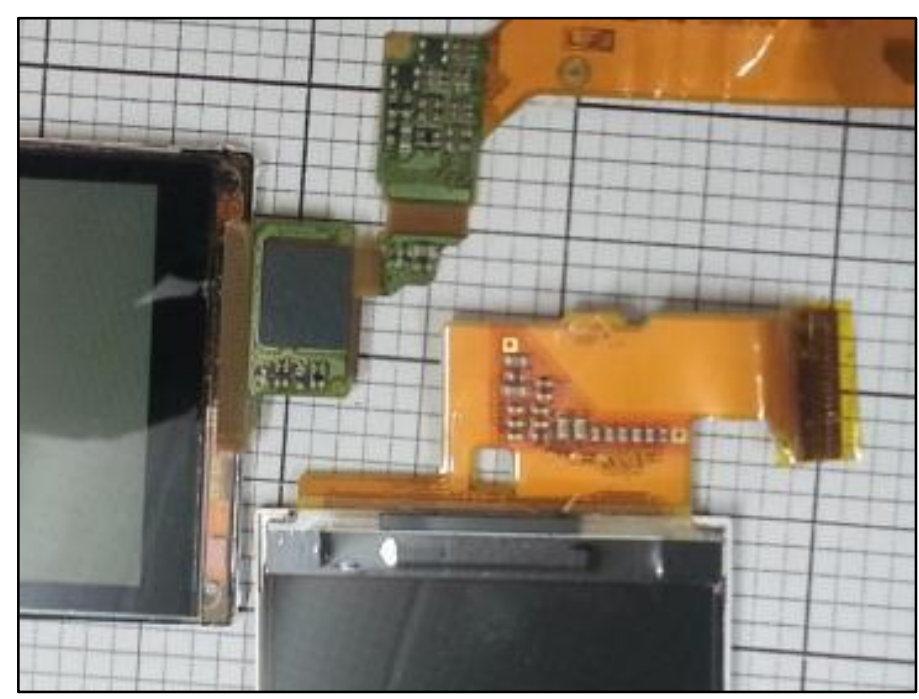

Figure 4.19. Connectors on screens of mobile phone and MP3 player 
Speaker Magnets - speaker magnets were cut open to access the magnetic alloy inside. XRF analysis revealed the presence of $\mathrm{Nd}$ (Figure 4.20).

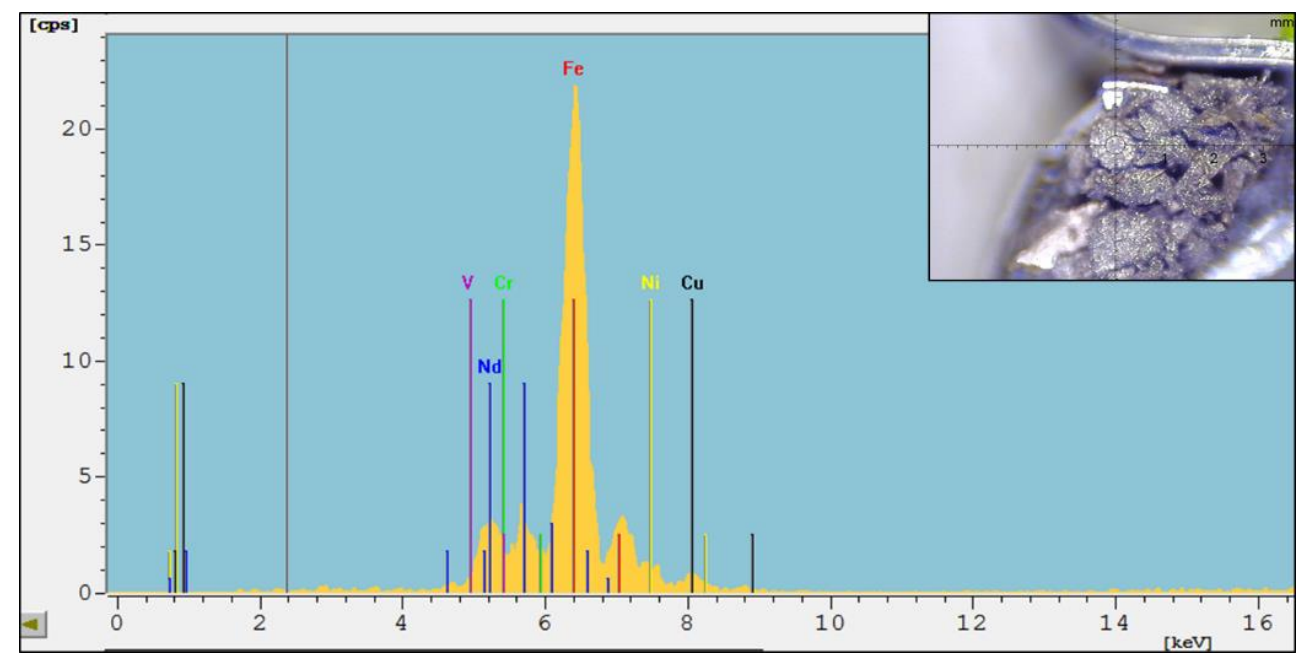

Figure 4.20 X-ray emission spectrum of the magnetic alloy in mobile phone speaker magnets.

Contacts - XRF analysis of gold contacts from the base of the phone reveals $\mathrm{Au}$ and $\mathrm{Pd}$ (Figure 4.21). Au is coated onto the surface of the Ni-Cu alloy of the contacts, and $\mathrm{Pd}$ is probably present in the Au-alloy used as the coating. Numerous sets of contacts were isolated from the phone, including several sets from the PCB and a set which contact the battery. XRF of battery contacts revealed Au but no Pd was detected. Contacts from the PCB are discussed later.

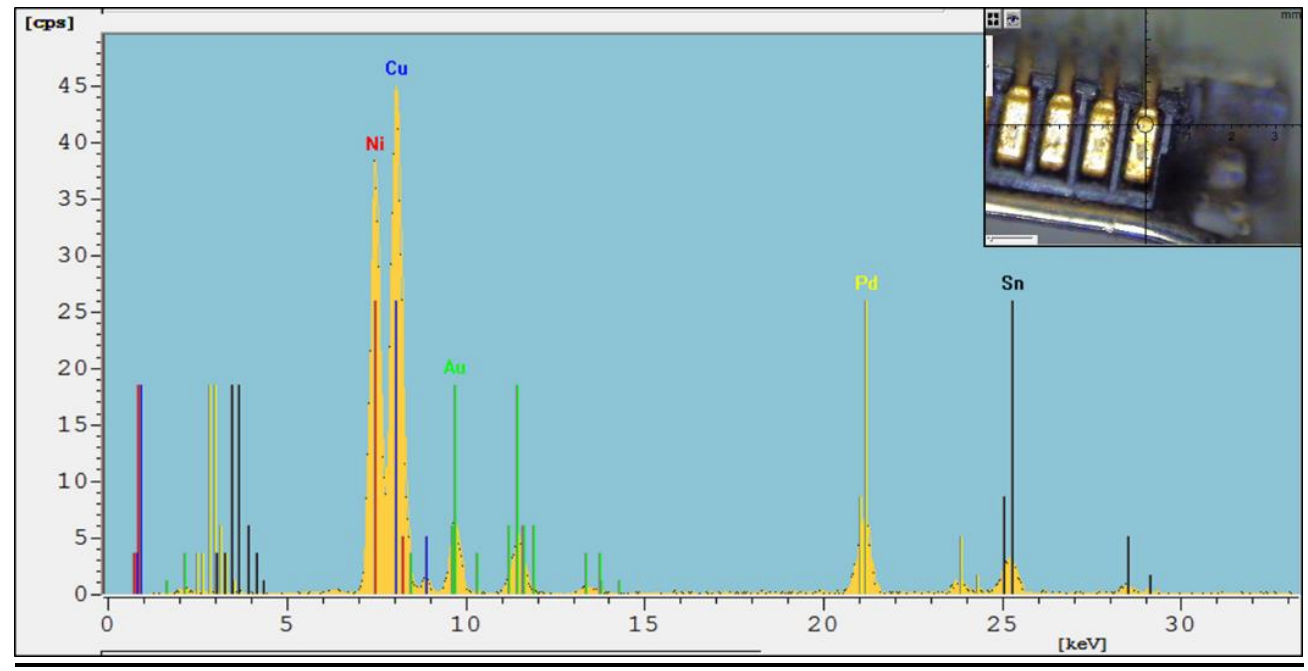

Figure 4.21 X-ray emission spectrum of gold contacts of mobile phone sample 
SD Card - The SD card contacts contained Au (Figure 4.22). SD cards were disassembled in search of additional CRMs but $\mathrm{Sb}$ in the plastic of the SD card housing was the only additional CRM identified (Figure 4.23).

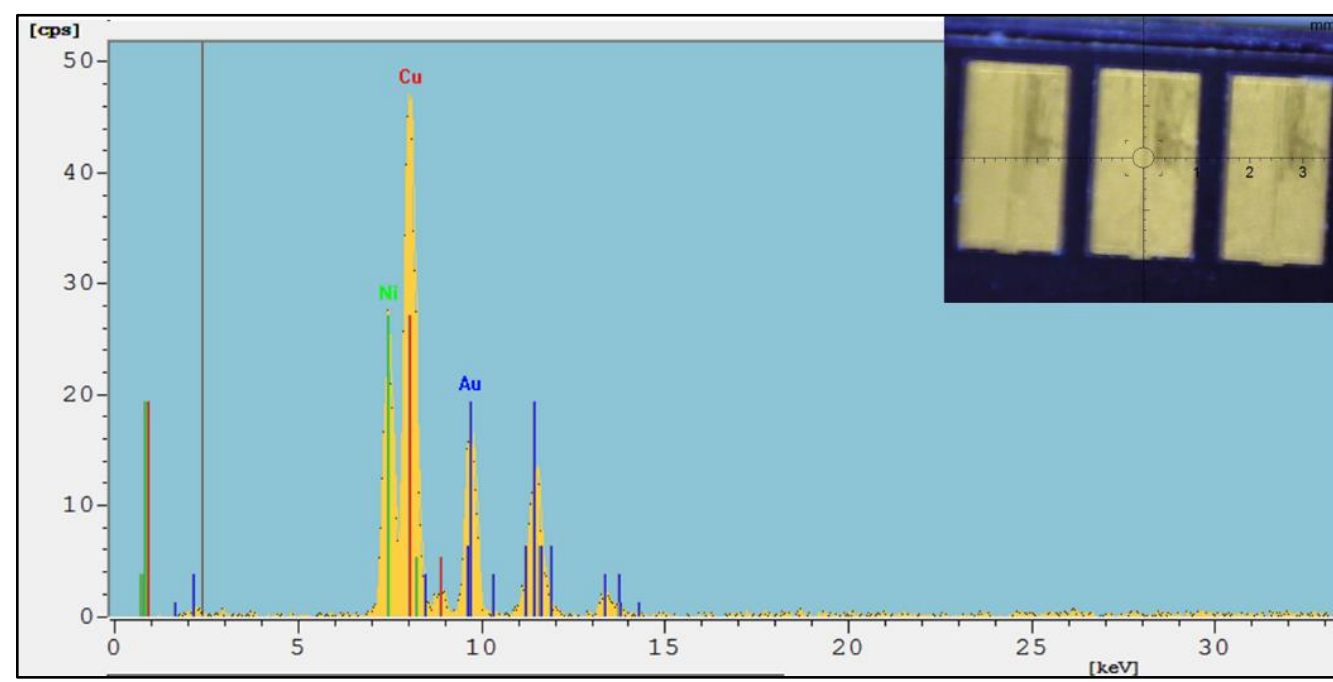

Figure 4.22 X-ray emission spectrum of SD card contacts

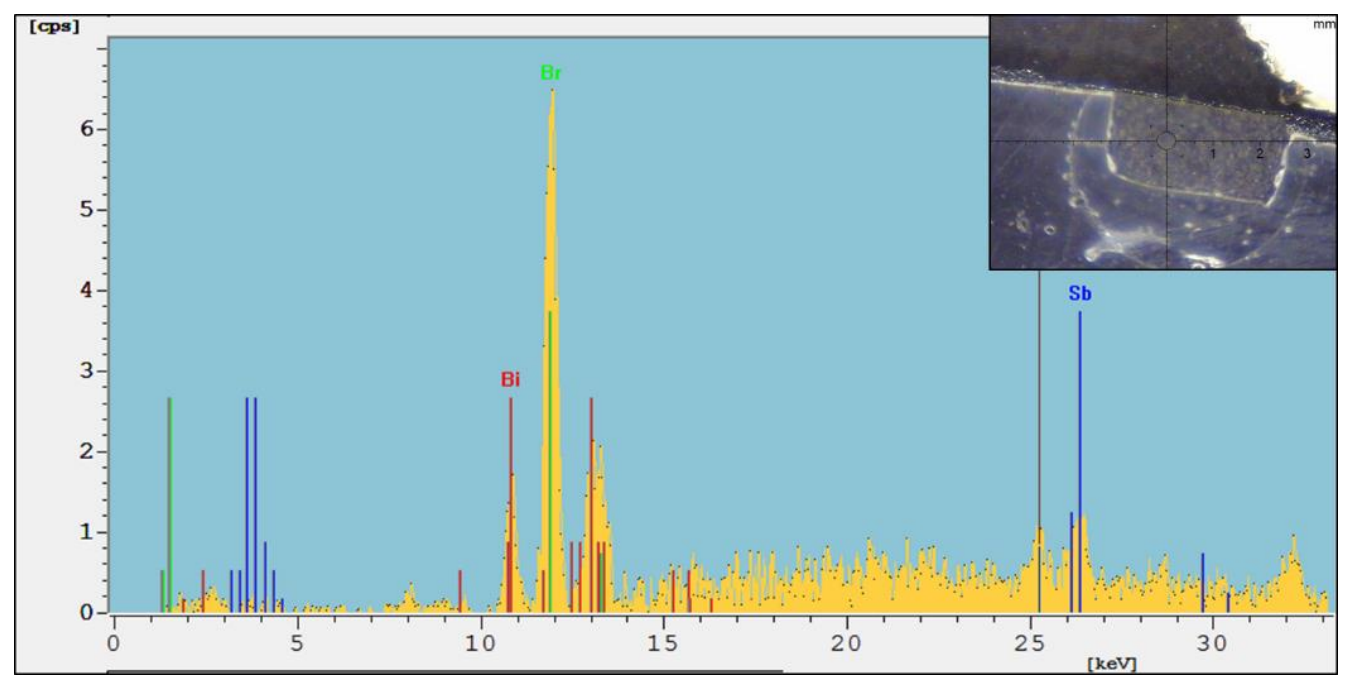

Figure 4.23 XRF spectrum of SD card plastic containing Sb

\section{MP3 players solid state memory}

A Sony NWZ-s639F was disassembled into its housing, two PCBs (a main PCB and minor PCB), its Li-ion battery and its LCD screen (Figure 4.24). Once again, In could not be identified in the screen by XRF, and CRM content of PCBs and batteries is discussed later. 


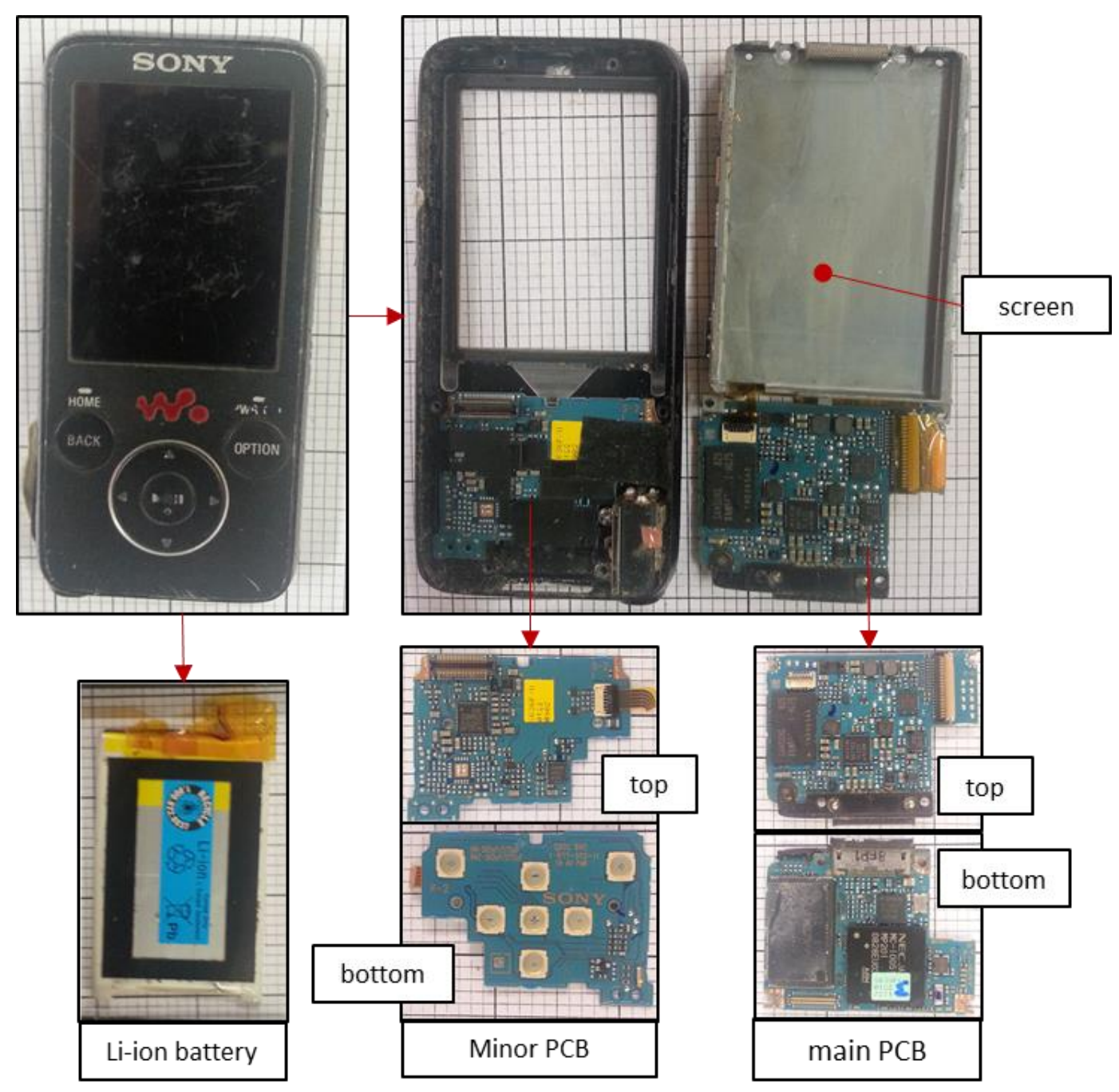

Figure 4.24 Disassembly of Sony NWZ-s639F digital media player into its component parts

\section{$\underline{\text { RSA security anti-hacking dongle }}$}

Disassembly of the dongle was not possible as the housing was a sealed unit. To access the internal electronics a Dremel multi-tool with a cutting disk was used to cut through the hard plastic casing at its edges (Figure 4.25). Internal components including a primary lithium button cell, LCD display and PCB were found encapsulated within the transparent plastic resin. Poor design for end-of-life results in difficult, timely and costly disassembly of these devices. Isolating the encapsulated components is therefore economically unfeasible so samples were not analysed further.

The dongle provides a good example of poor design for end-of-life posing a barrier to recovery of any CRMs present. The Li-battery is a primary cell and so unlikely to contain any EU14 CRMs, however, Li has been highlighted as critical in numerous criticality assessments. ${ }^{21}$ In addition, poor design prohibits depollution of the device prior to recycling by removal of the 
battery. Shredding of the device with its battery in place, despite being environmentally detrimental and forbidden under the WEEE directive is therefore inevitable. This highlights

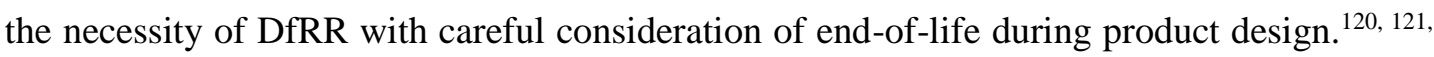
123
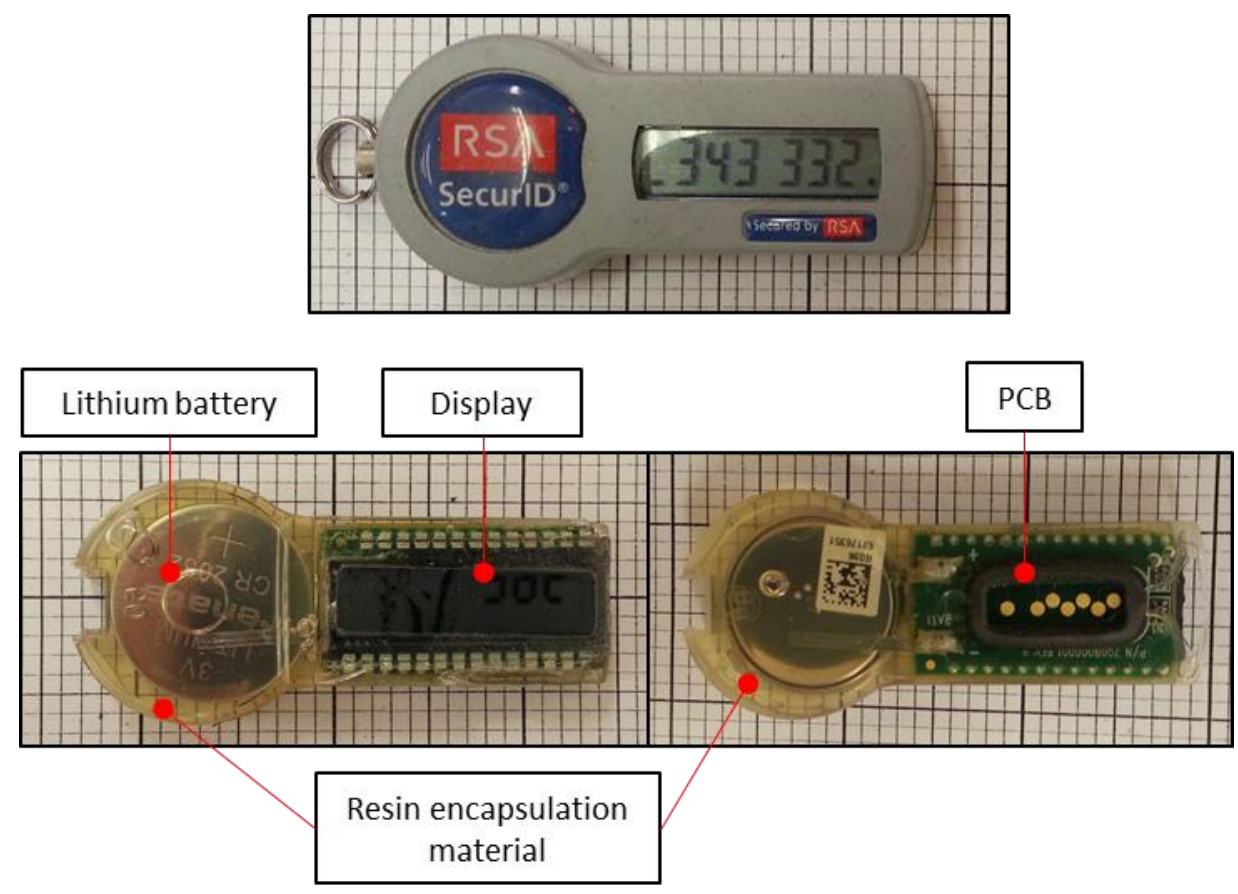

Figure 4.25 RSA anti-hacking security dongle (top) with top vies (bottom left) and bottom view (bottom right) of its encapsulated internal components.

\section{Digital Cameras}

A Sanyo Cybershot digital camera was selected as a representative example of cameras collected for the trial. It was disassembled into its component parts which include a PCB and Li-ion battery (Figure 4.26). No In was detected on the LCD screen. 


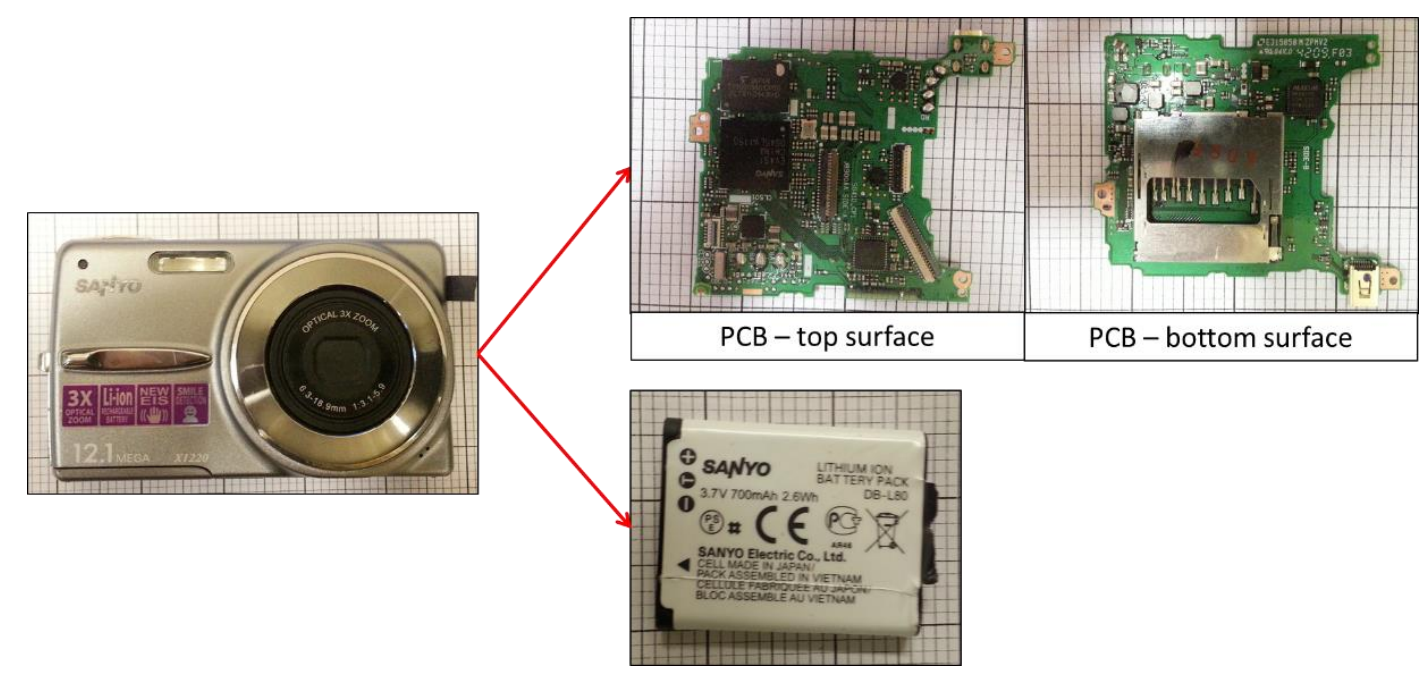

Figure 4.26. Sanyo Cybershot digital camera and its isolated PCB and Li-ion battery

\section{Common components in devices}

\section{Li-ion battery cells}

Lithium ion battery cells were isolated from laptop battery packs, cameras, MP3 players and mobile phones (Figure 4.27). Li-ion batteries cells are manufactured in cylinder or packet (pouch) forms, depending on the way in which electrodes of the cells are folded or rolled. Cell casings prevented $\mathrm{x}$-ray penetrating so the batteries were cut open to expose the internal electrodes for XRF analysis which revealed the presence of Co (Figure 4.28 4.29). This is unsurprising as Co is used in cathodic materials of LIBs. Traditionally this has been lithium cobalt oxide $\left(\mathrm{LiCoO}_{2}\right)$, but newer cathode materials, e.g. $\left(\mathrm{LiCo}_{1-(x+y)} \mathrm{Mn}_{\mathrm{x}} \mathrm{Ni}_{\mathrm{y}} \mathrm{O}_{2}\right)$ contain less Co. ${ }^{196,197}$
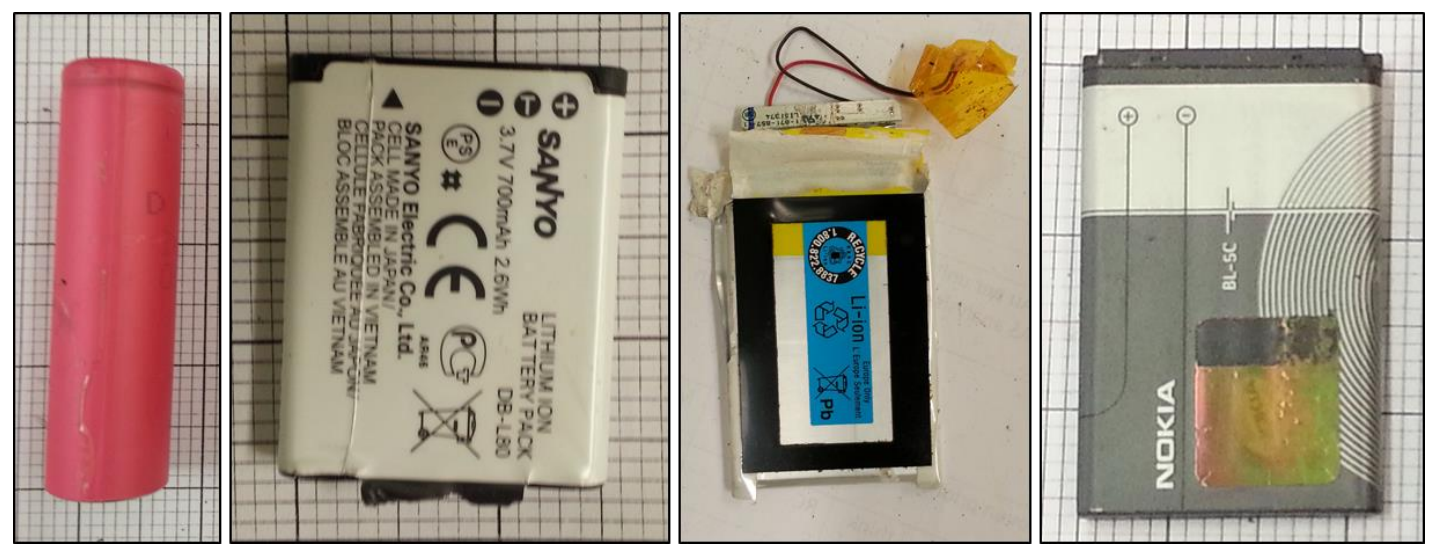

Figure 4.27 Example LIBs. From left to right, cylinder cells from laptop battery packs, and packet cells from cameras, MP3 players and mobile phones 

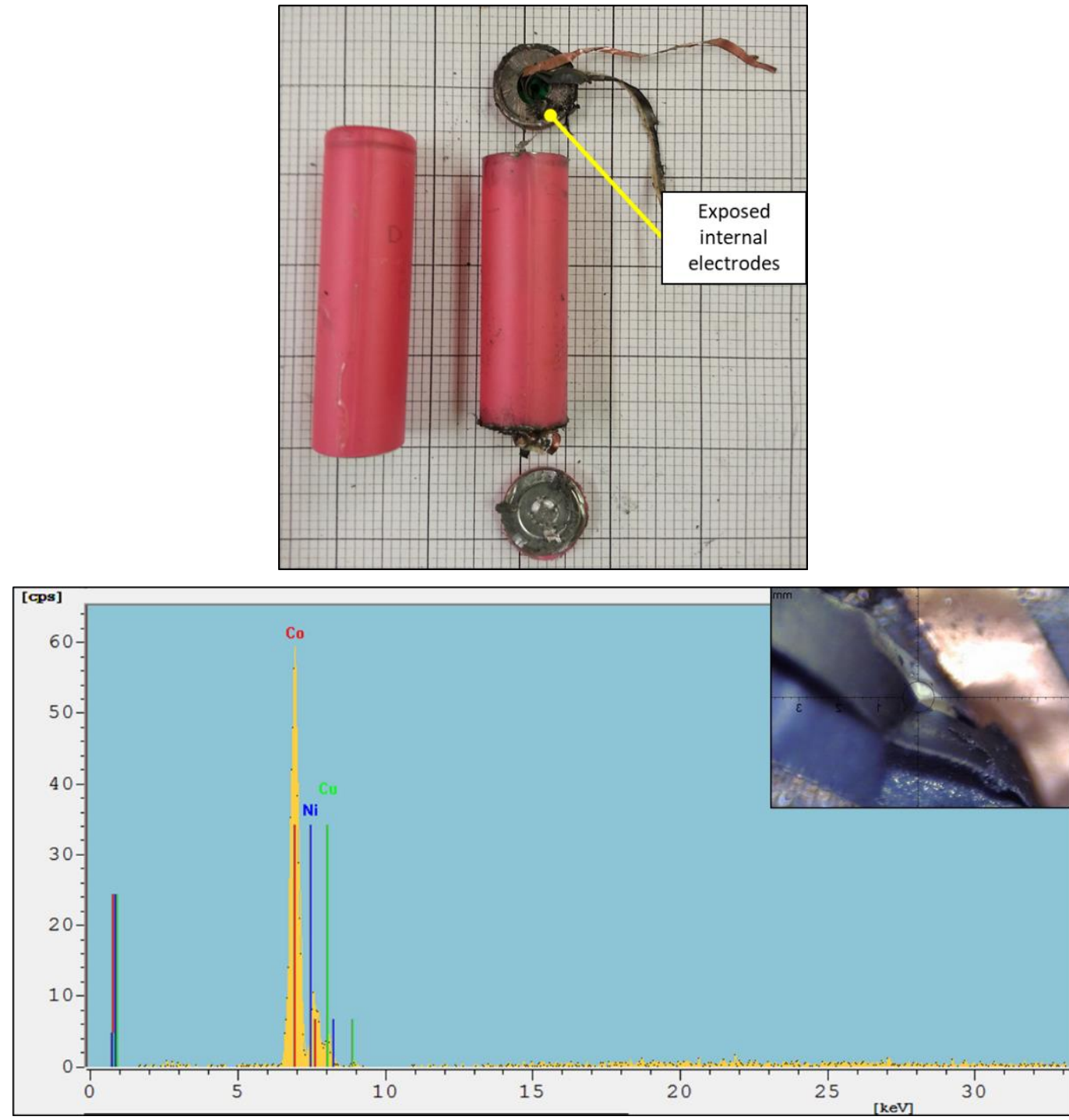

Figure 4.28 Li-ion cylinder cells from laptop battery packs cut open to reveal internal electrodes (top) and $x$-ray emission spectra of electrode material (bottom)

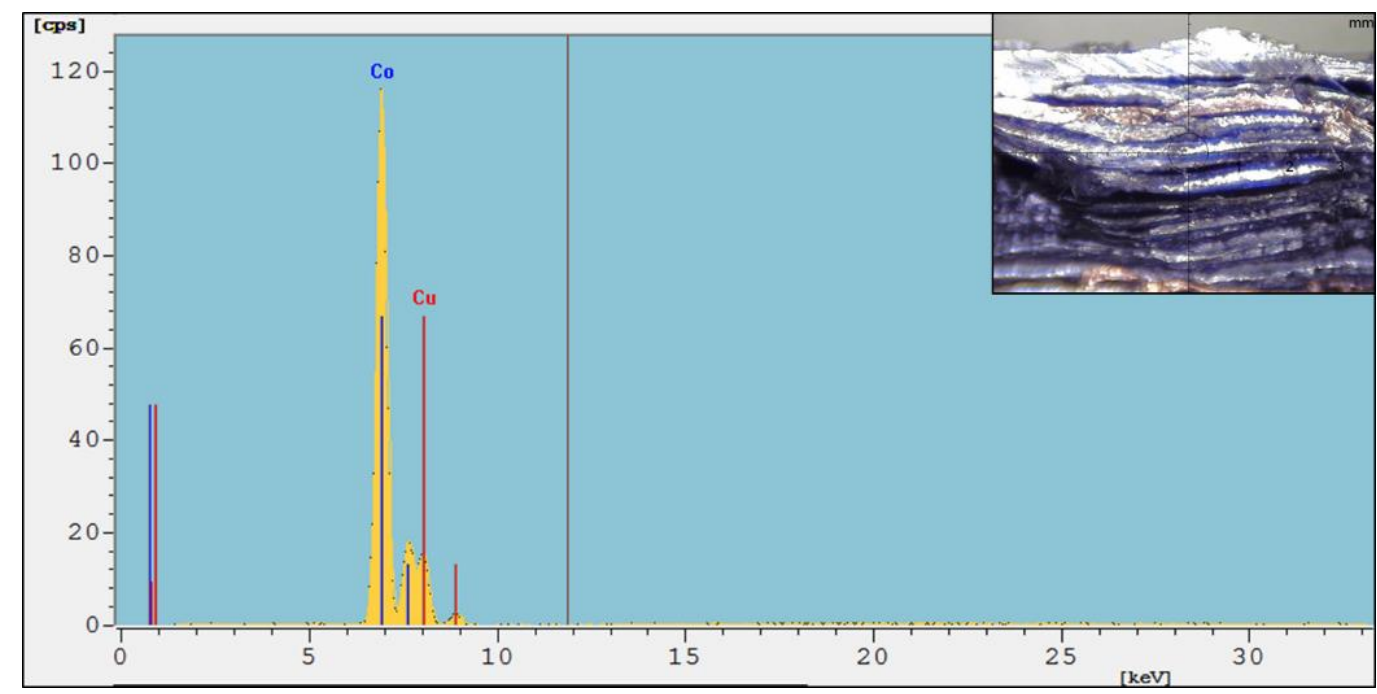

Figure 4.29 X-ray emission spectrum of electrodes in Nokia 6230i mobile phone Li-ion battery 


\section{Printed Circuit Boards (PCBs)}

This section outlines the common components in which CRMs have been identified in PCBs. Samples discussed include PCBs from TVs, fridges, STBs, phones, digital media players, cameras, LCD screens and HDDs.

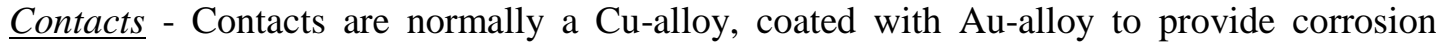
protection. ${ }^{198}$ Two main kinds of contacts were found: those coated directly onto PCBs such as the edge contacts on PC PCI cards (e.g. graphics cards - Figure 4.30); and those in connectors soldered to PCBs such as HDMI, VGA, and USB ports (Figure 4.31). The presence of gold was confirmed in by XRF in all contacts which appeared golden to the naked eye (Figure 4.32). On occasion, Pd was also found in combination with gold, suggesting Pd is used as an alloying element in the Au-alloy coating.

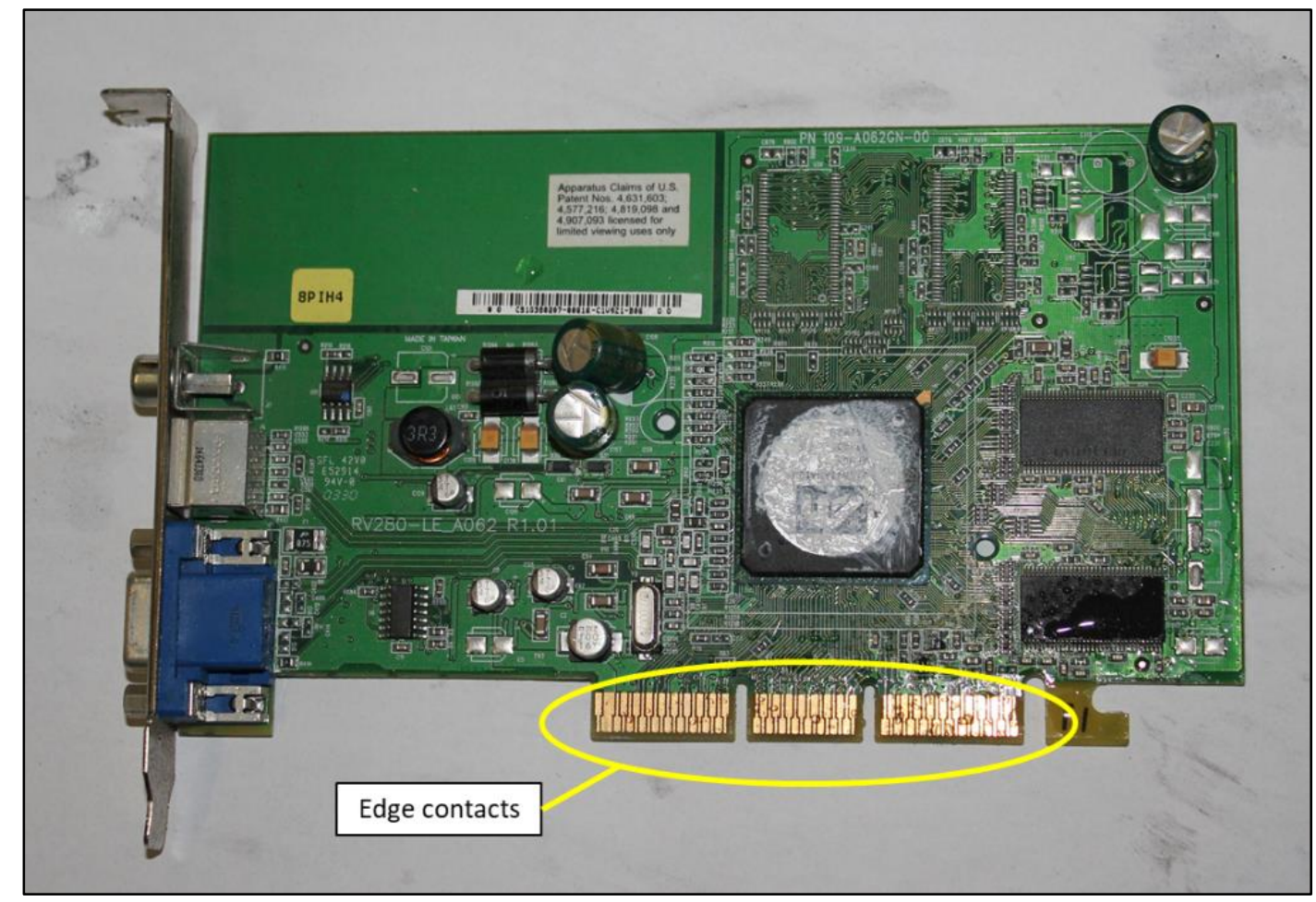

Figure 4.30 Edge contacts on a PC graphics card 


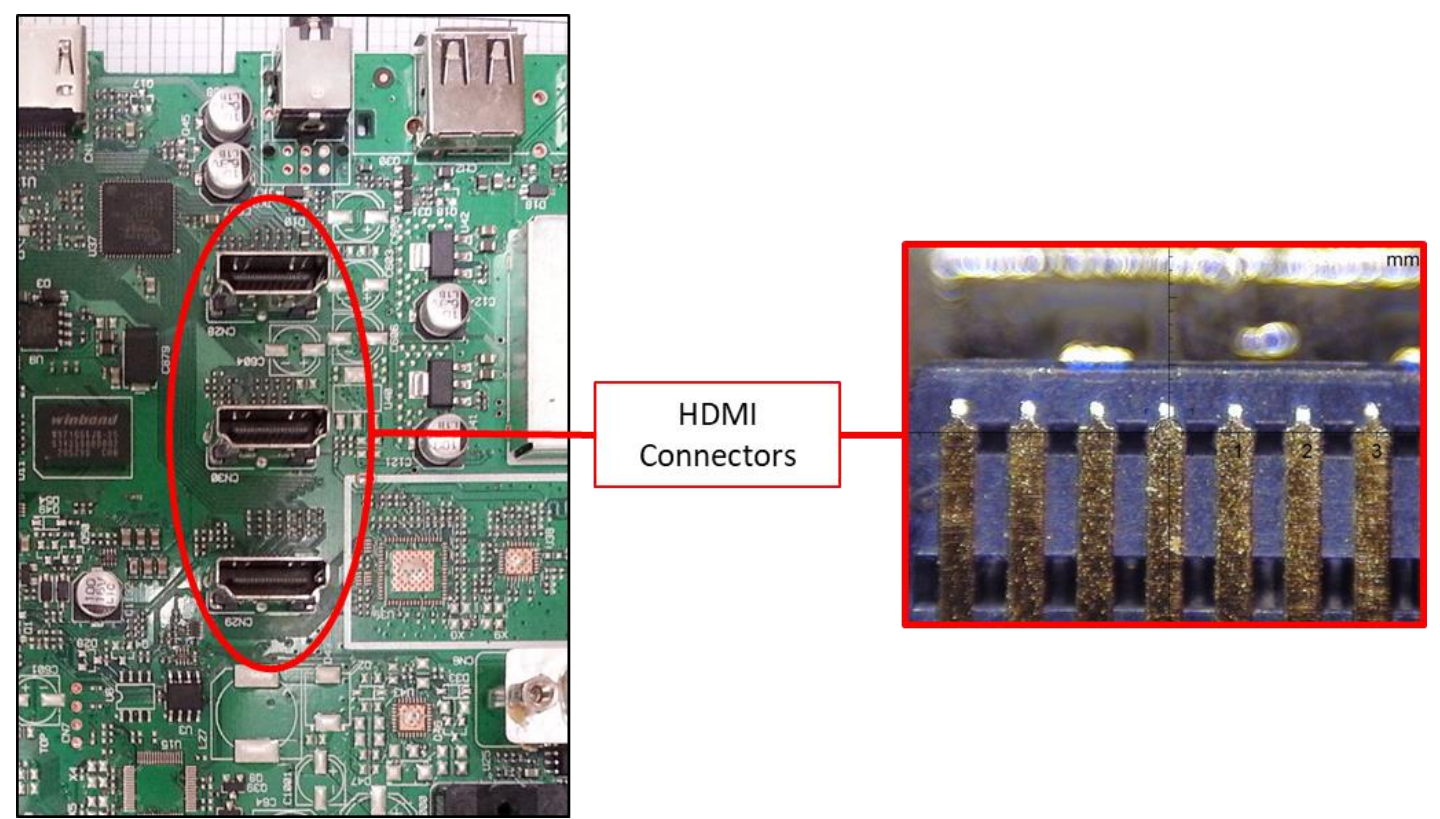

Figure 4.31 HDMI connectors on a TV PCB (left) and the gold coated contacts within (right)

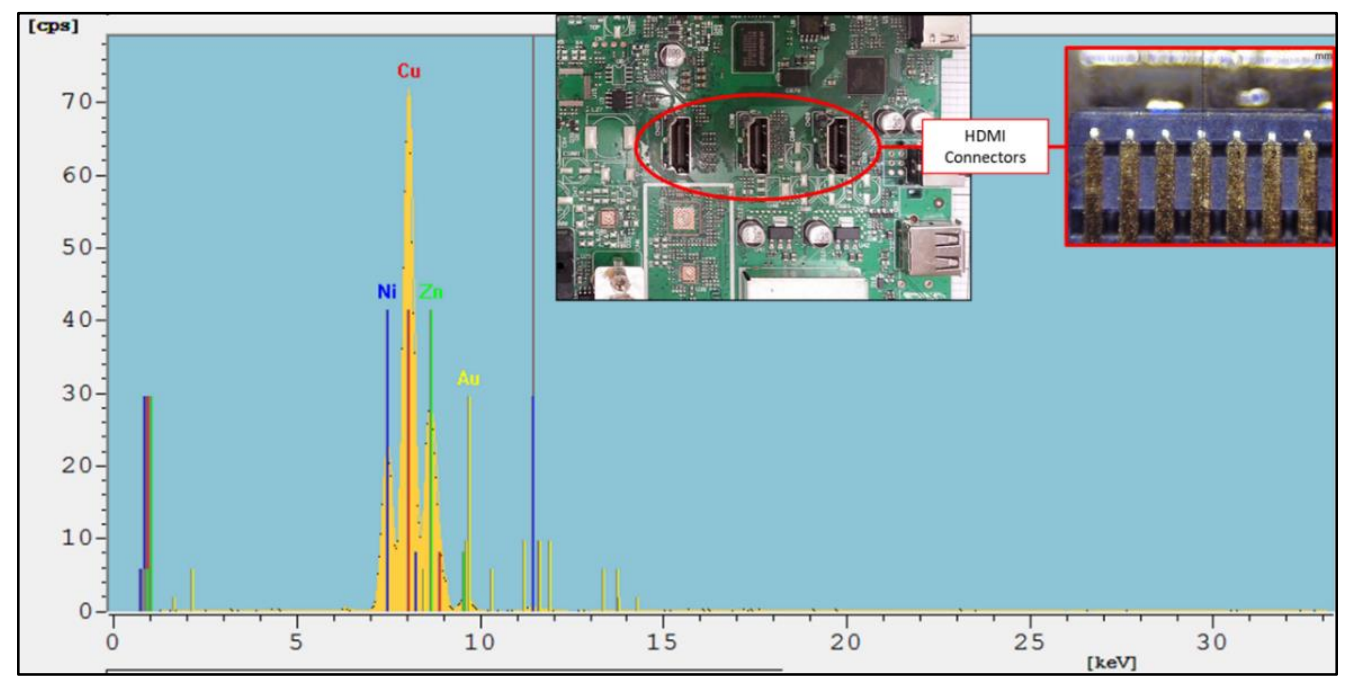

Figure 4.32 HDMI ports on a TV PCB and their X-ray emission spectrum indicating the presence of gold $(9.71 \mathrm{keV})$

Surface Gold - Many of the PCBs found in the samples had large areas on their surfaces coated with Au. This was prevalent in mobile phones and the PCBs from LCD screens (Figure 4.33). XRF analyses confirmed that the alloy was similar in composition to that used in PCB contacts PCBs, always containing Au, with a small quantity of Pd on occasion. 


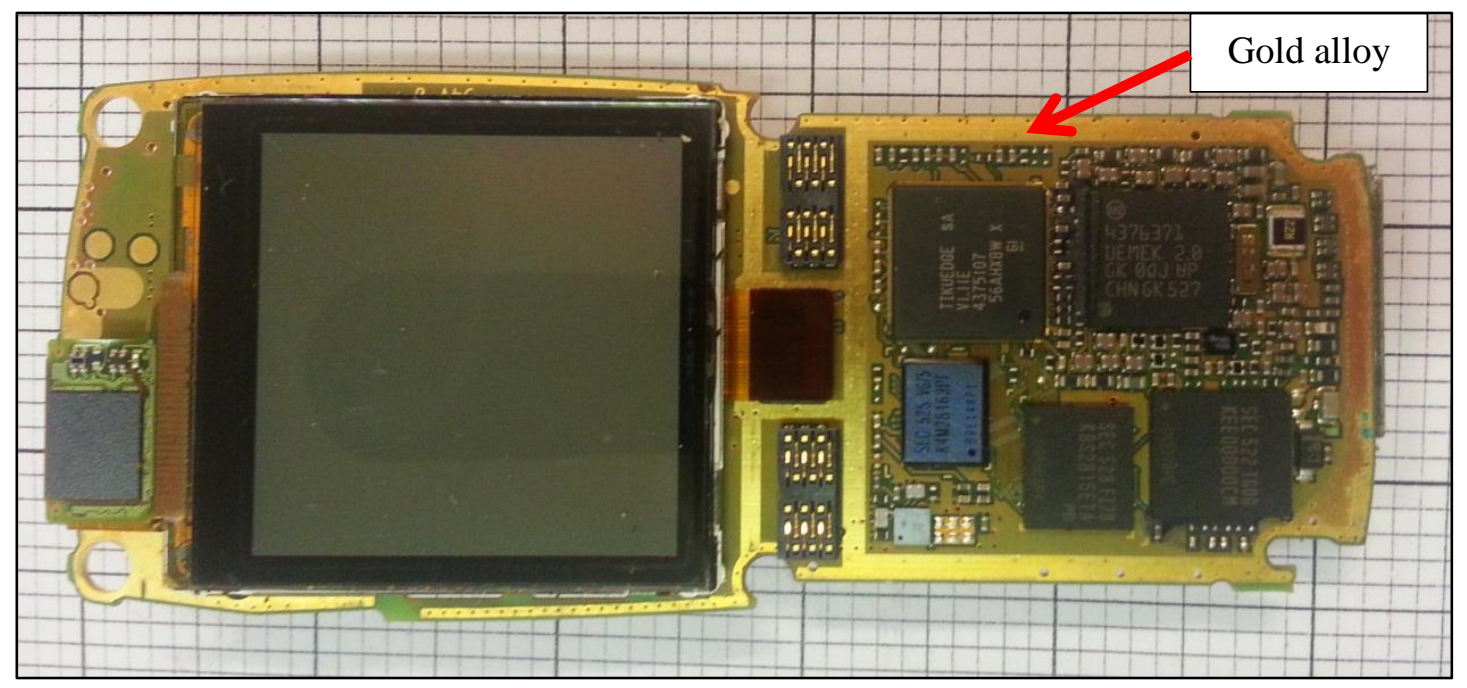

Figure 4.33 Plated Au on the surface of mobile phone PCB

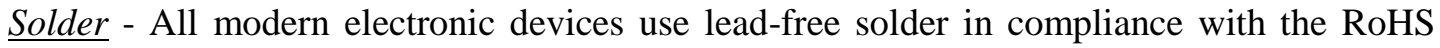
directive. These solders contain a Ag-Sn alloy, as shown in XRF spectra of PCBs from cameras, phones, TVs, LCDs and MP3 players (Figure 4.34).

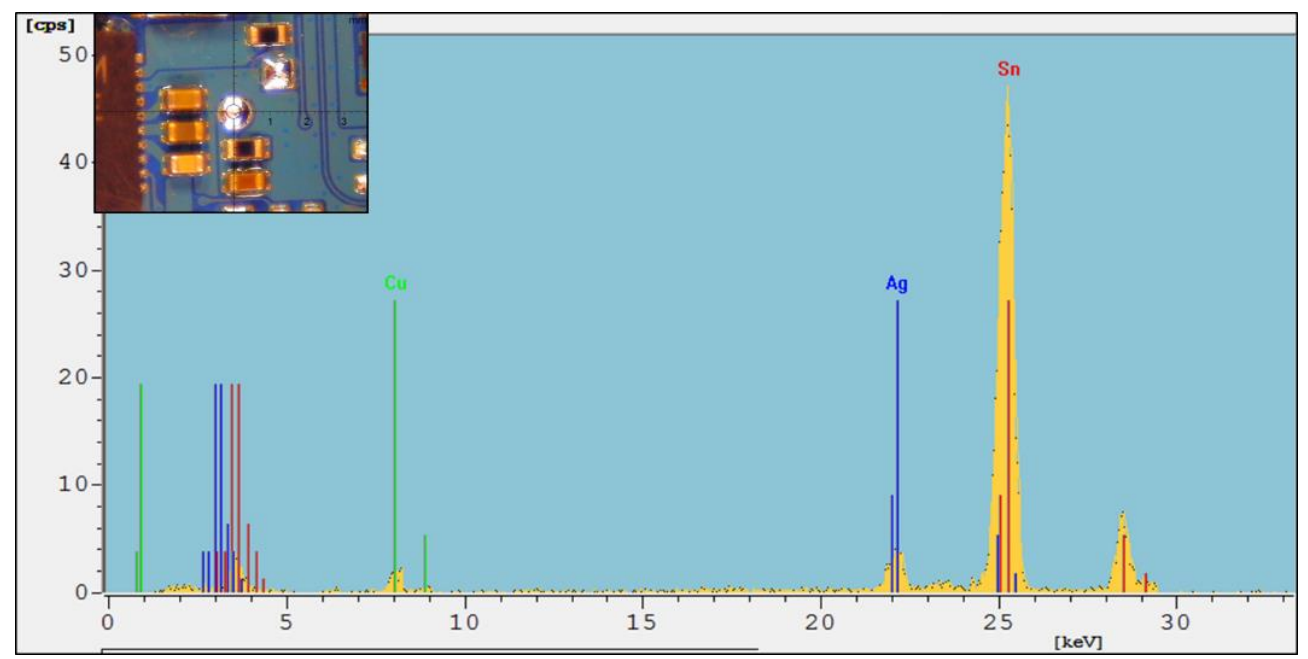

Figure 4.34 X-ray emission spectrum of Pb free solder containing Ag on the PCB of a digital media player (MP3 player)

\section{Surface Mount Device (SMD)Components}

Chip Resistors - The most commonly encountered SMDs were chip resistors (Figure 4.35) found in all PCBs analysed. These vary in size but are always black and frequently numbered on their surface. XRF analysis of chip resistors shows $\mathrm{Ag}, \mathrm{Ru}$ and $\mathrm{Sb}$ with almost no exceptions, and Pd in approximately half of those analysed. Some of the larger resistors which less common also Co (Figure 4.36). 


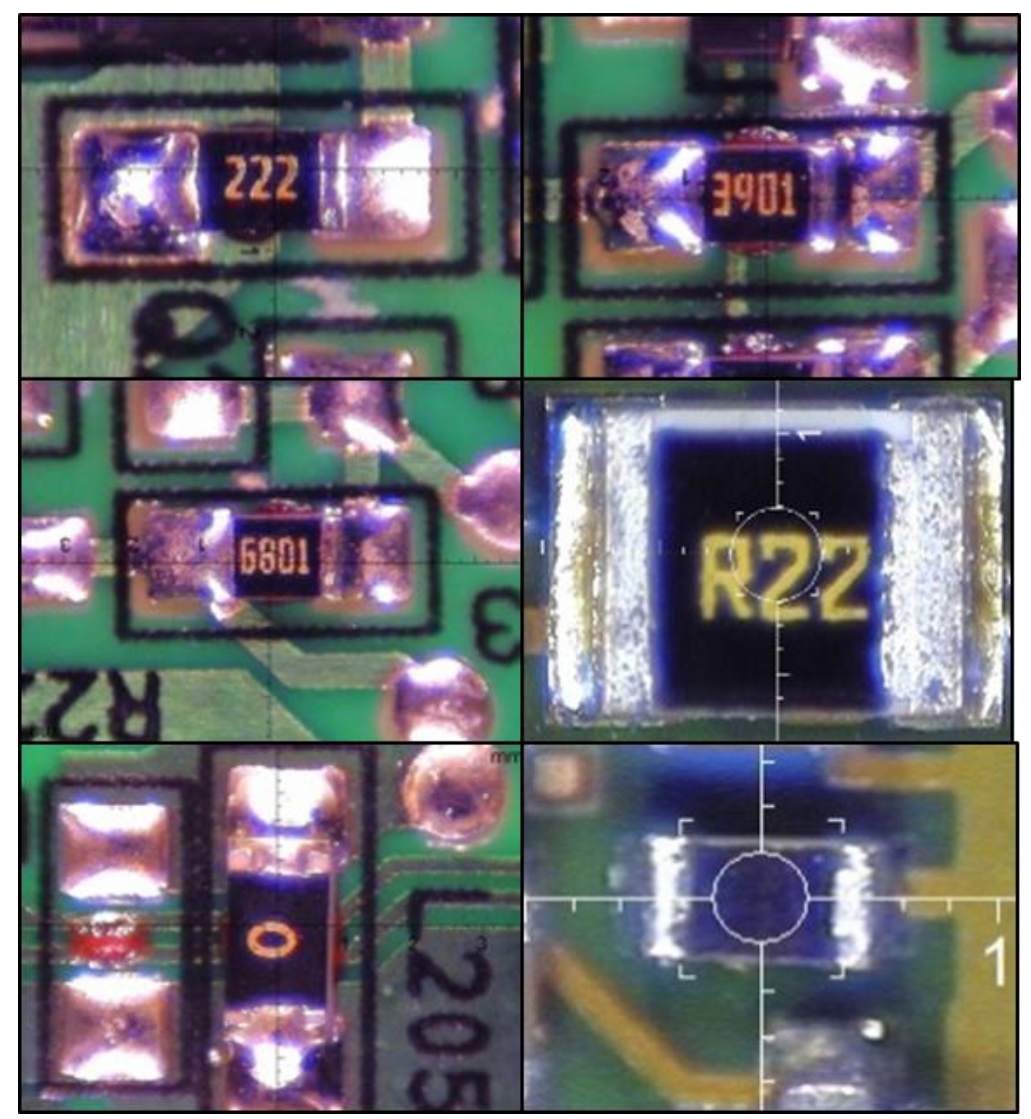

Figure 4.35 Examples of surface mount chip resistors

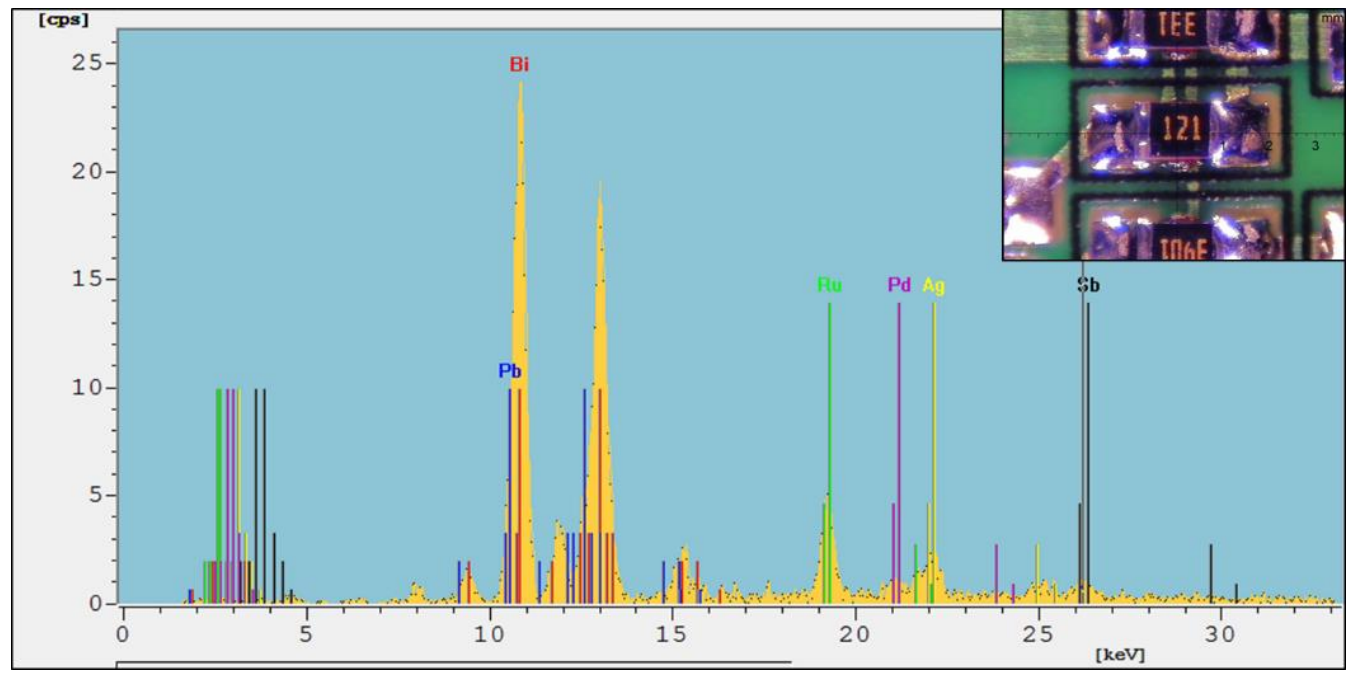

Figure $4.36 \mathrm{X}$-ray emission spectrum of a chip resistor $S M D$ containing $R u, P d, A g \& S b$

Integrated Circuits (ICs) - ICs were encountered in every PCB sample (Figure 4.37). XRF analysis revealed ICs to always contain Au \& Ag (Figure 4.38), often Sb, and occasionally Pd. Surprisingly, no tungsten was identified even in ground samples, which is a known interconnect material used in ICs. 


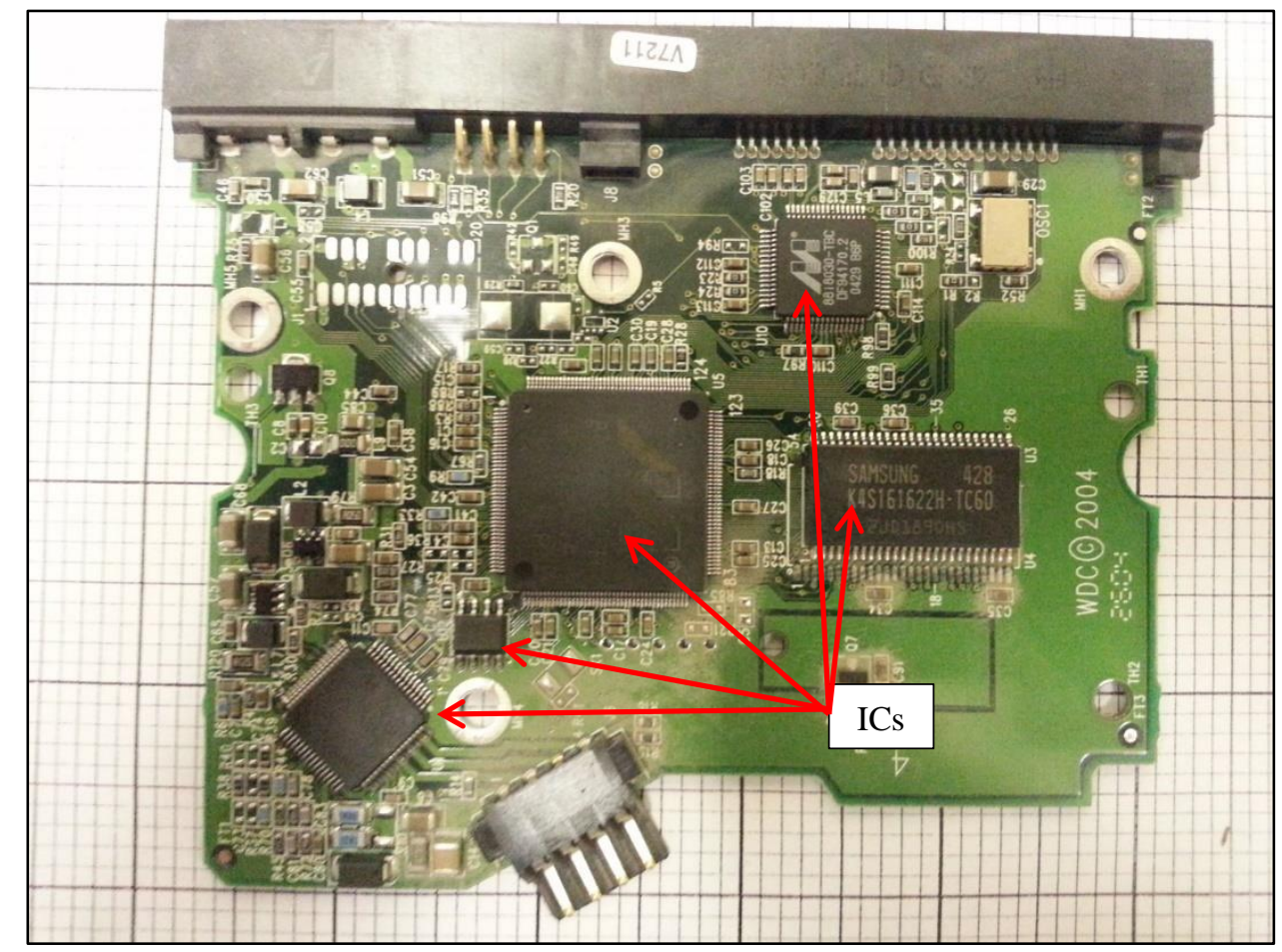

Figure 4.37 ICs on HDD PCB

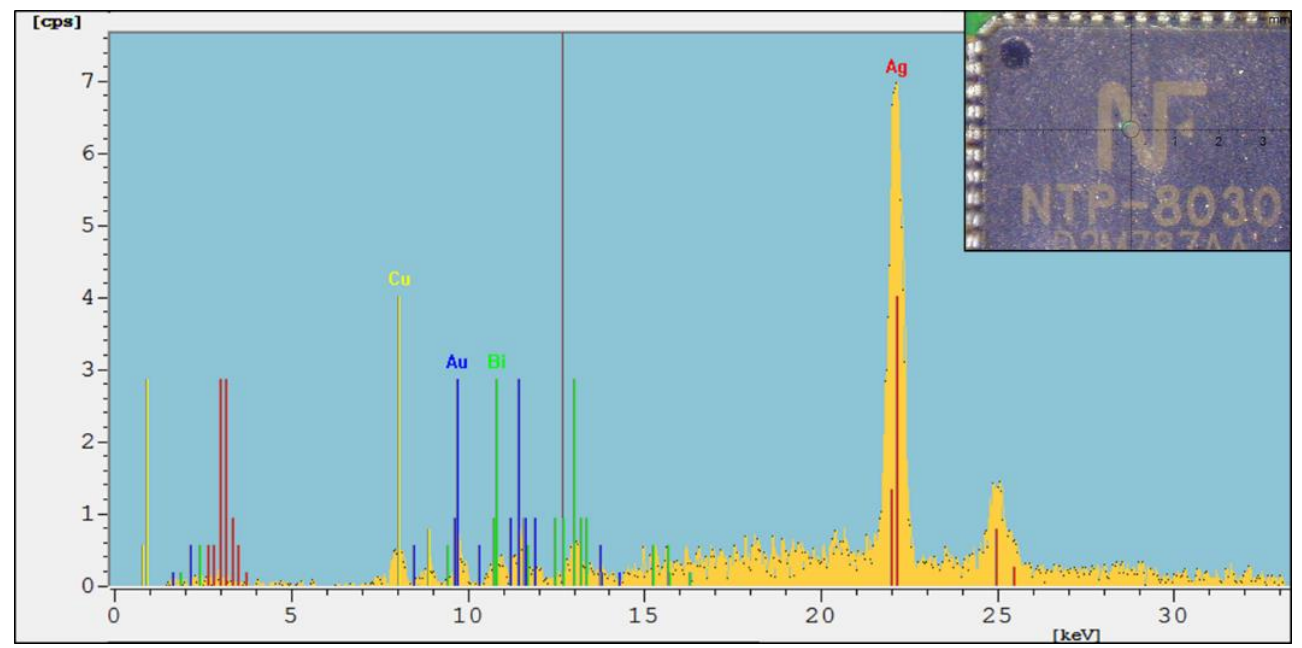

Figure $4.38 X$-ray emission spectrum of an IC showing the presence of $A u \& A g$

Multi-layered Ceramic Capacitors (MLCCs) - These SMDs are very common on PCBs, occurring in a variety of sizes and colours, with varying CRM content. XRF surface scanning of MLCCs revealed that $\mathrm{Y}$ is present in brown coloured MLCCs (Figure 4.39). This is unsurprising as $\mathrm{Y}$ is used as an additive in $\mathrm{BaTiO}_{3}$ to control formation of the appropriate "core shell' microstructure for MLCCs. ${ }^{199}$ Table 4.5 shows the Y content of two sizes of typical capacitors determined by ICP-MS . 


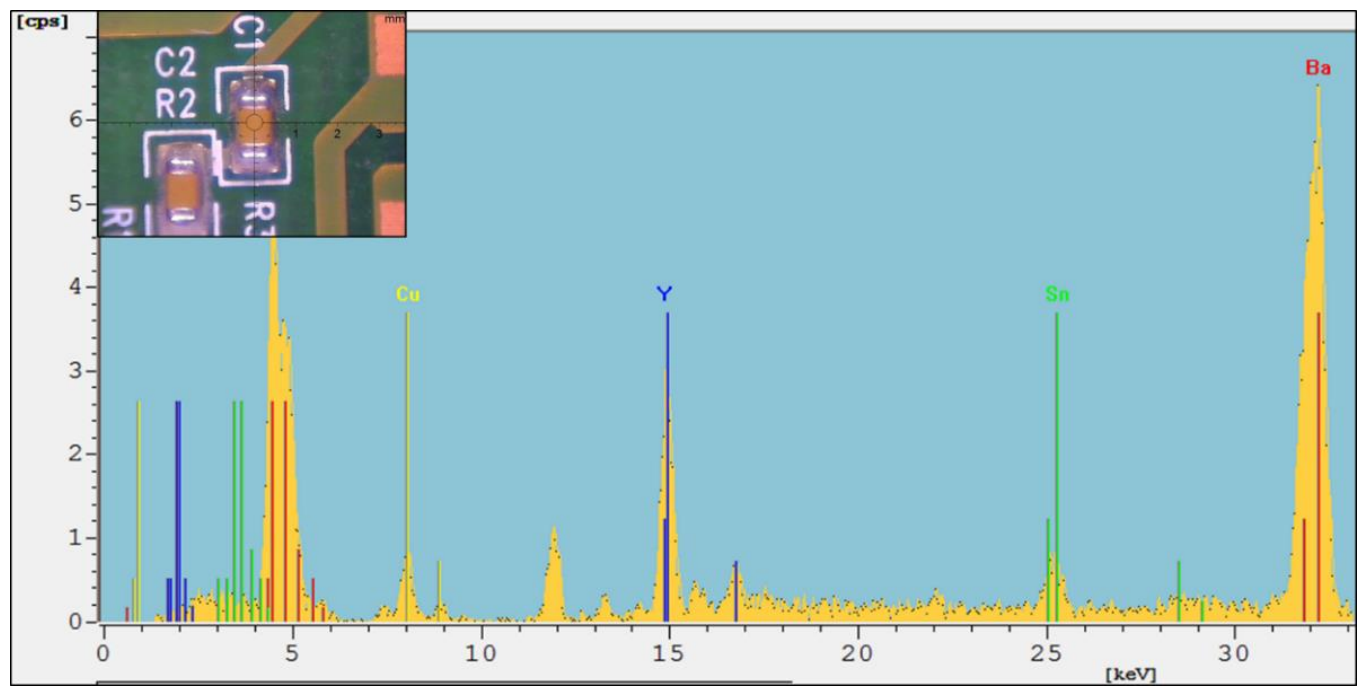

Figure 4.39 Typical capacitors and XRF analysis showing presence of yttrium (Y)

Table 4.5 Yttrium content in capacitors

\begin{tabular}{lc}
\hline Size & Y content $(w t \%)$ \\
\hline $0.55 \times 0.25 \mathrm{~mm}$ & $0.3 \%$ \\
$0.90 \times 0.60 \mathrm{~mm}$ & $0.8 \%$ \\
\hline
\end{tabular}

Less frequently encountered, but prevalent in highly miniaturised electronics such as mobile phones and MP3 players were Nb containing MLCCs. Nb too has been used as an additive to $\mathrm{BaTiO}_{3}$ to control microstructure formation. ${ }^{199}$ Examples of CRM found with $\mathrm{Nb}$ in MLCCs include: purple and blue MLCCs found to contain Pd (Figure 4.40); gold-coloured MLCCs containing Ag (Figure 4.41); and red MLCCs containing Pd (Figure 4.42). Off-white coloured SMDs of two sizes $(1.8 \mathrm{~mm} \times 1.1 \mathrm{~mm} \& 0.9 \mathrm{~mm} \times 0.6 \mathrm{~mm})$ containing $\mathrm{Ir}$ were also found (Figure 4.43)

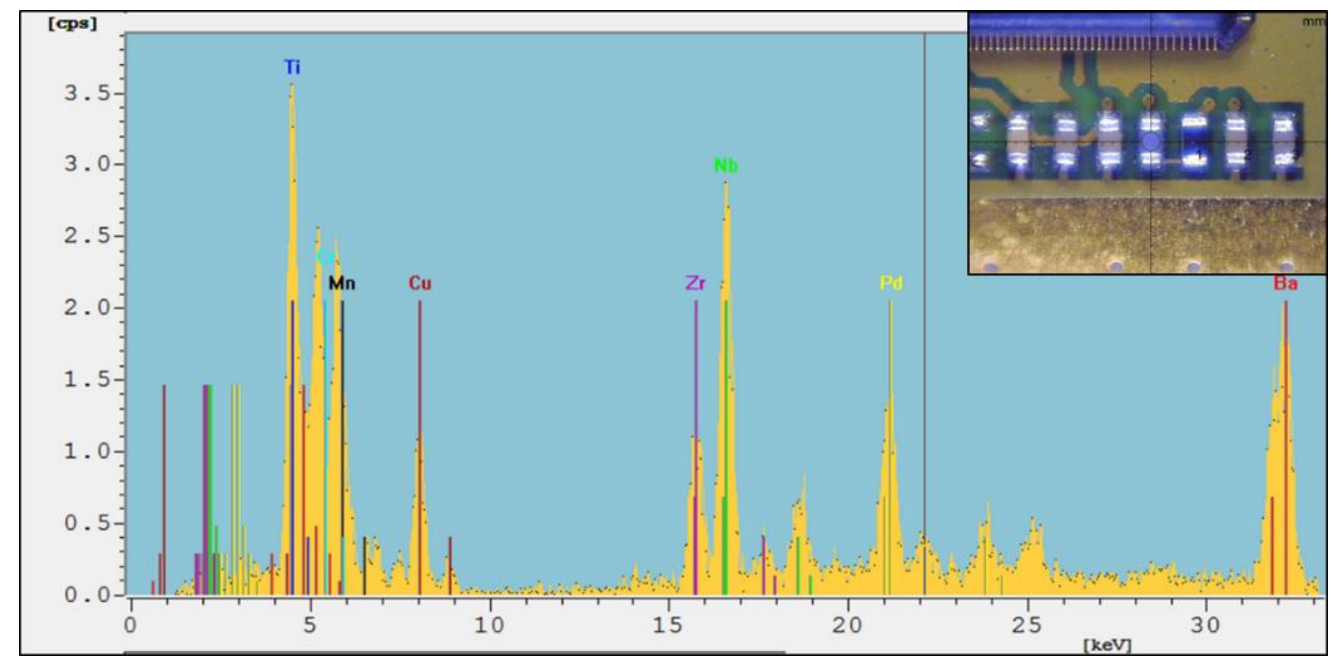

Figure 4.40 X-ray emission spectra of typical blue MLCC indicating the presence of Pd. 


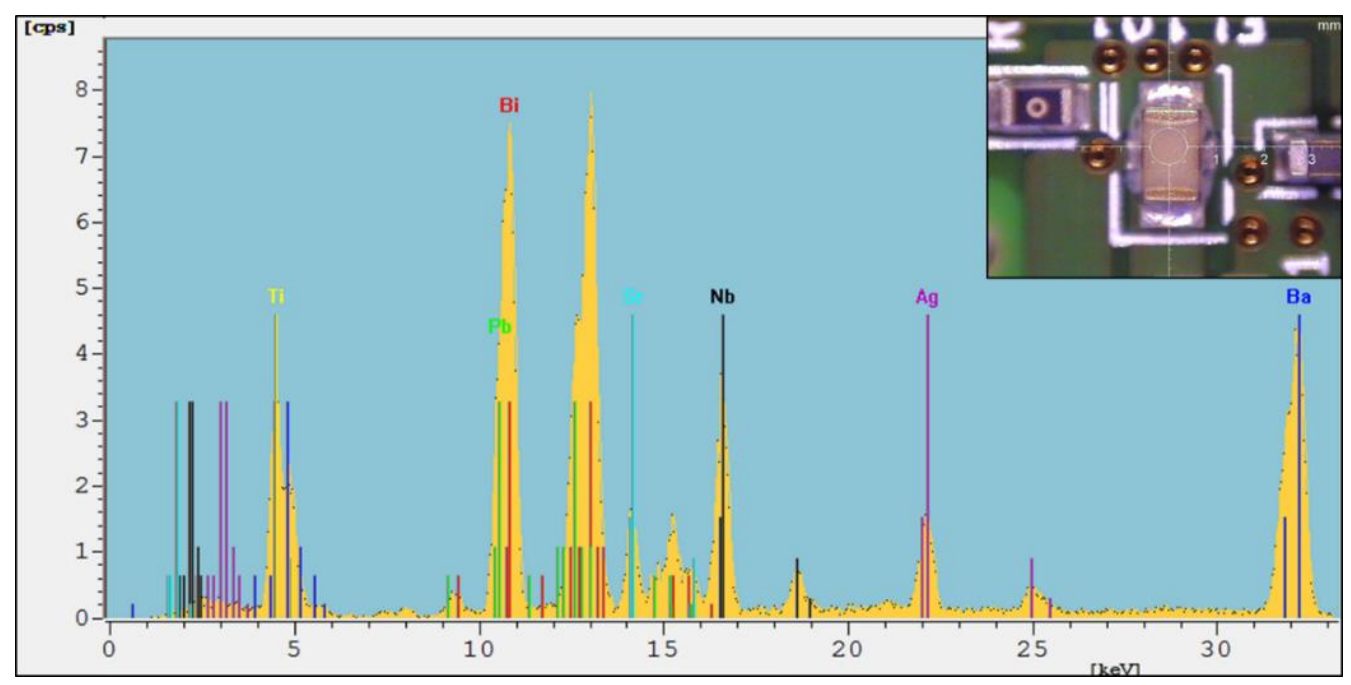

Figure 4.41 MLCC containing $\mathrm{Nb} \& A g$

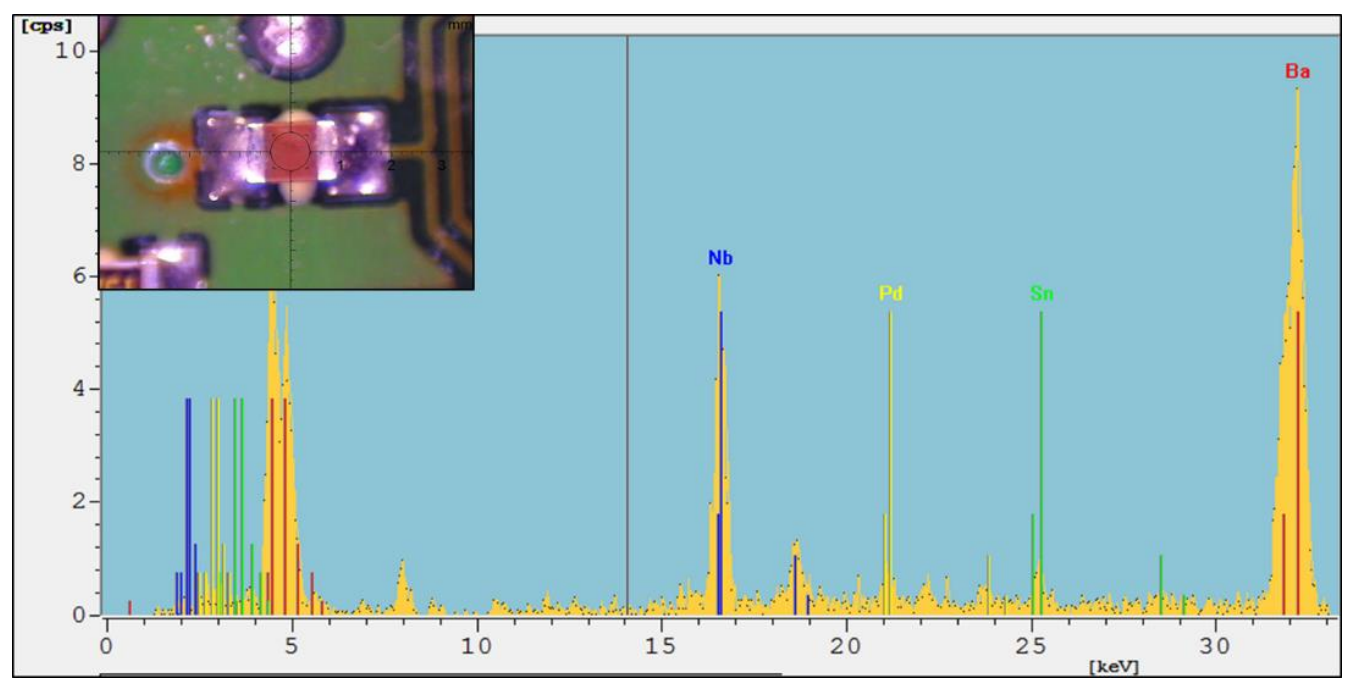

Figure 4.42 Red MLCC containing $\mathrm{Nb} \& \mathrm{Pd}$

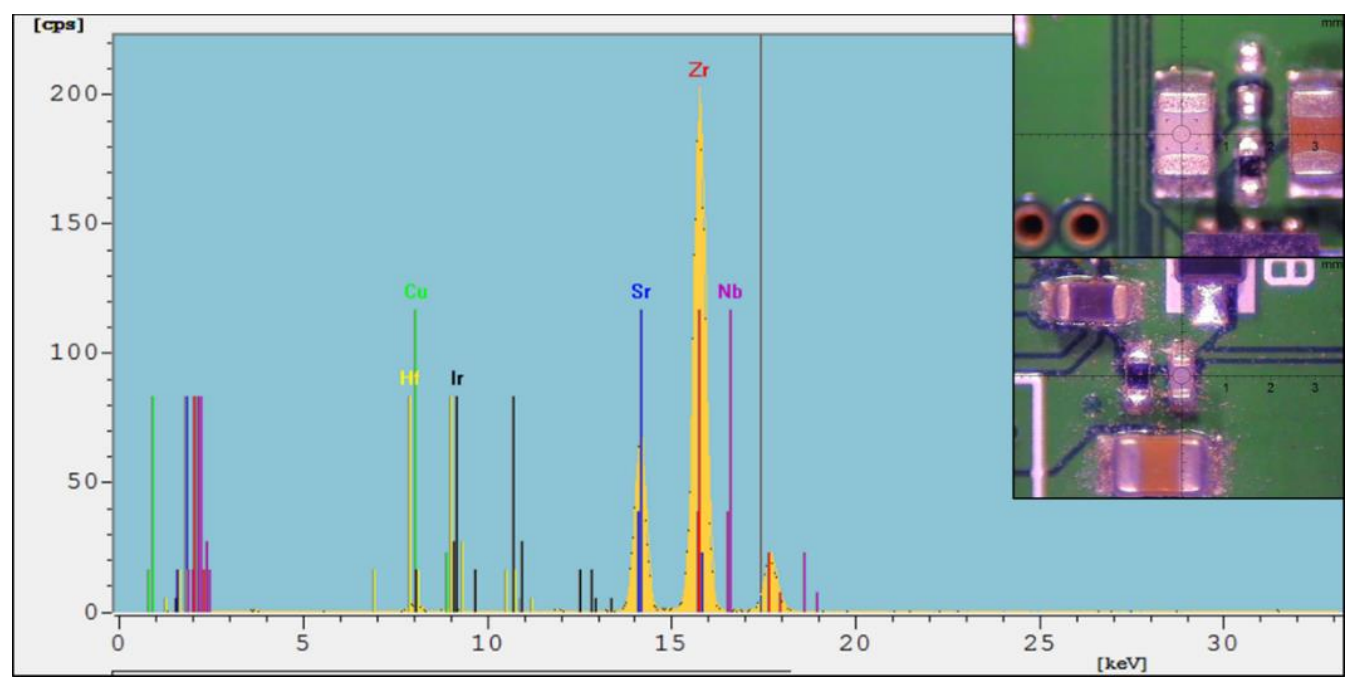

Figure 4.43 Typical x-ray emission spectrum of large (top) and small (bottom) MLCCs containing iridium $\mathrm{Nb} \& \mathrm{Ir}$ 
Transistors - Transistors are common in all PCBs. They generally appear in two forms: 3-pin small outline transistors, and standard small outline transistors. Standard small outline transistors were identified in PCBs of items such as HDDs and TVs, but were not present in highly miniaturised PCBs e.g. mobile phones, MP3 players etc. In these devices, smaller 3pin transistors are common. Standard small outline transistors consistently contain $\mathrm{Au}, \mathrm{Ag}$ and $\mathrm{Sb}$ (Figure 4.44). 3-pin small outline transistors of two varieties were identified in samples. Those most commonly found were black in colour and numbered on their surface, consistently found to contain $\mathrm{Au}, \mathrm{Ag}$ and $\mathrm{Sb}$ (Figure 4.45), just as their larger counterparts. A second variety of 3-pin transistor were found various colours (pink, green $\&$ yellow) on server boards and found to contain $\mathrm{Au}, \mathrm{Ag}$, and Ga (Figure 6.46).

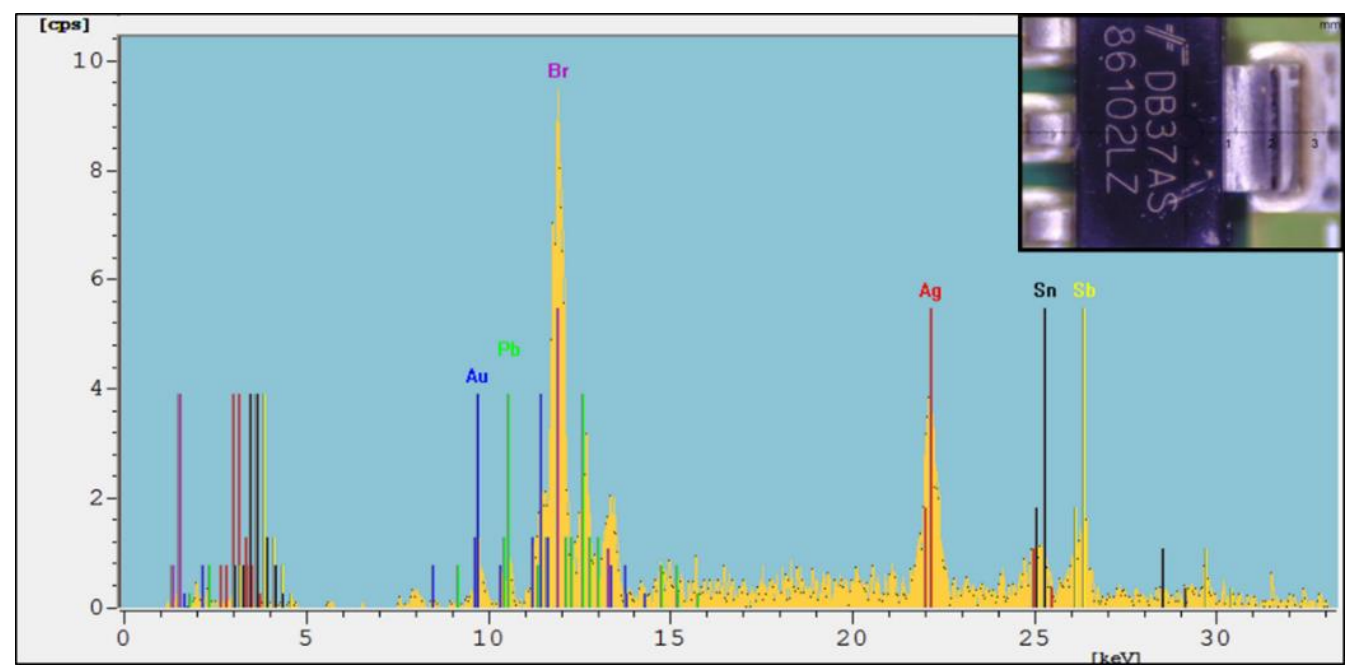

Figure 4.44 X-ray emission spectrum of small outline transistor containing Au, Ag and Sb

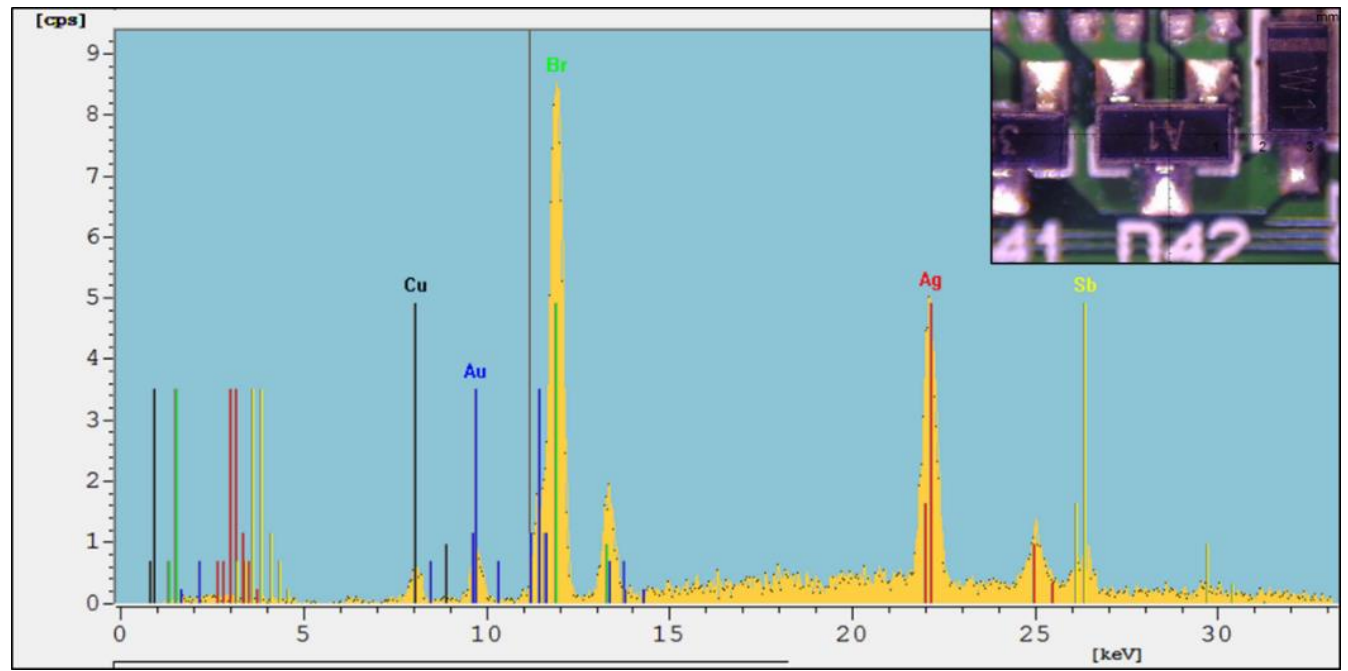

Figure 4.45 Typical SMD transistor containing Au, Ag and Sb 


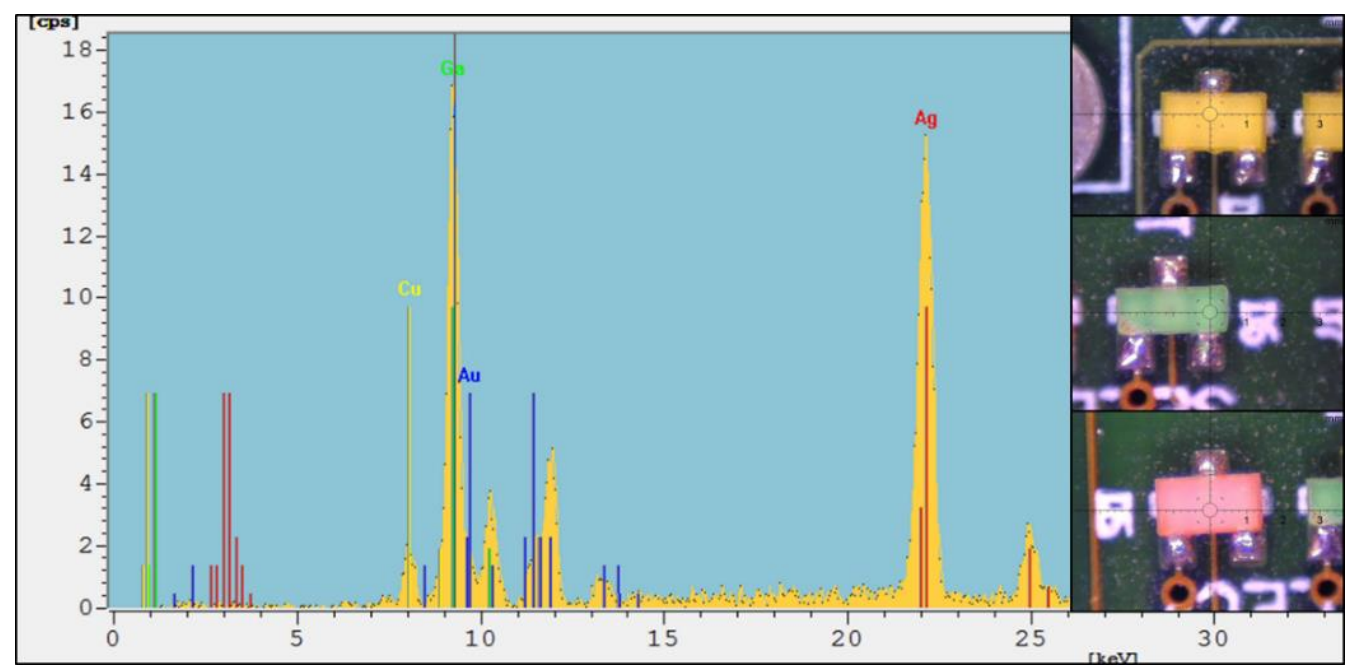

Figure 4.46 X-ray emission spectrum of coloured 3-pin small outline transistors containing Au, Ag, and Ga, present on server board sample.

Tantalum capacitors - Ta-capacitors occur frequently in miniaturised PCBs where high charge storage density is required, but not in TV \& Laptop LCD PCBs, TV \& Fridge power boards, and STB base boards. Where Ta-capacitors were present, they numbered 1-3 per PCB, with the exception of the server board sample which contained 15 of these SMDs. Ta-capacitors of difference sizes and capacitances are used. (Figure 4.47). To detect Ta in capacitors with XRF, the plastic of the encapsulation had first to be cut open (Figure 4.48), or the whole device had to be ground into powder. The Ta containing capacitor is encapsulated within moulded plastic containing $\mathrm{Sb}$ (probably as $\mathrm{SbO}_{3}$ ). Ag was also detected at the component terminals.

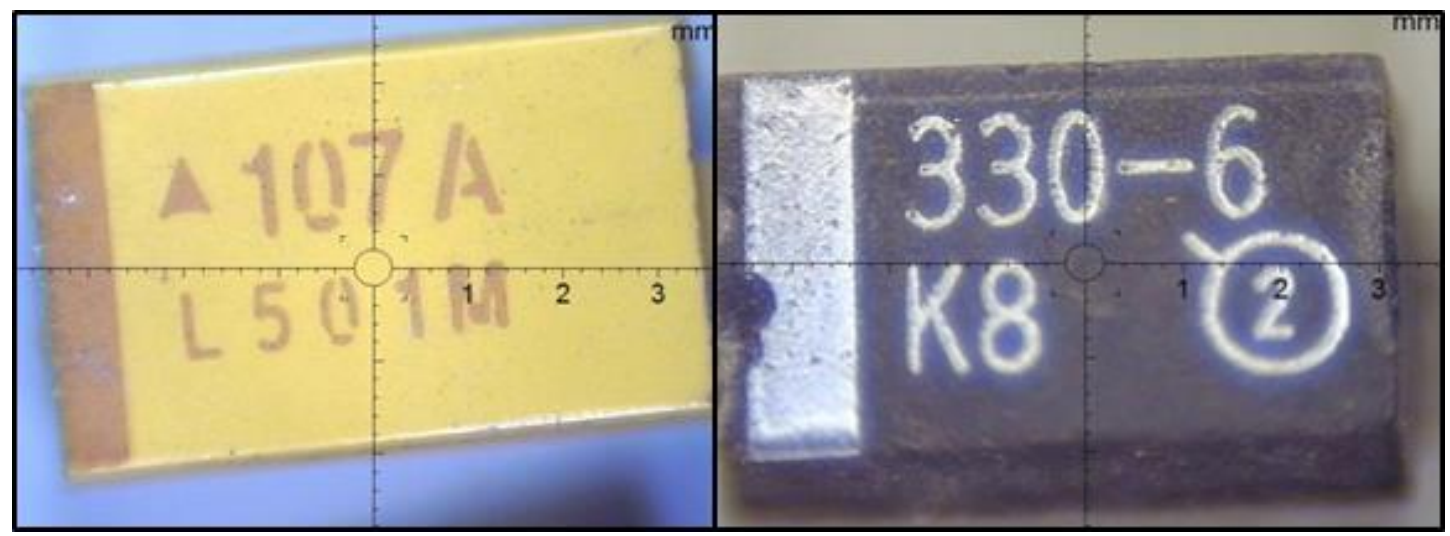

Figure 4.47 Typical tantalum capacitor SMDs from samples 


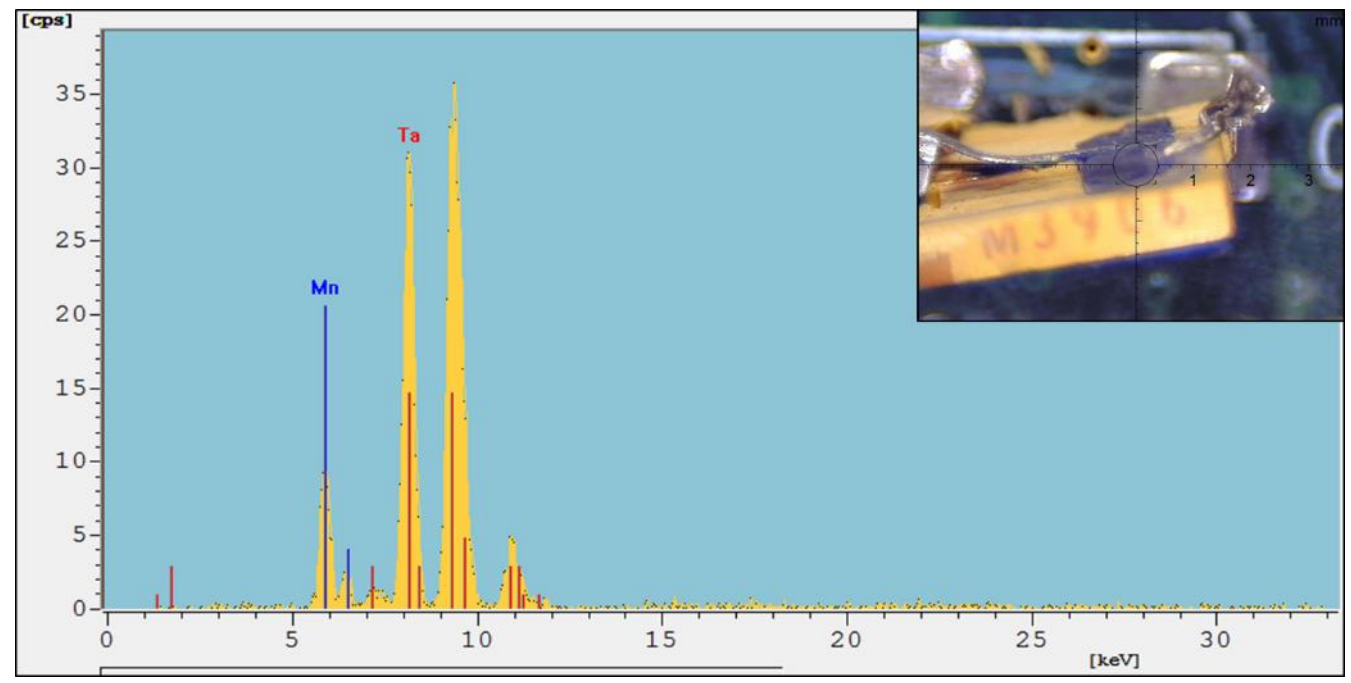

Figure $4.48 X$-ray mission spectrum of exposed Ta metal of typical Ta-capacitor SMD revealing the presence of and measured $x$-ray emission spectrum

As described in Appendix A, Ta capacitors were used to evaluate the performance of the XRF instrument. A typical capacitor (107 A, Figure 4.47) was ground to powder with inclusion of a known quantity of $\mathrm{Zn}$ as an internal standard for quantitative analysis. The results shown that Ta makes up $\sim 60 \mathrm{wt} \%$ of this capacitor (Table 4.6).

Table 4.6 Average CRM content of $100 \mu \mathrm{F} 10 \mathrm{~V} 107 \mathrm{~A}$ Ta-capacitors

\begin{tabular}{cccc}
\hline Mass of capacitor $(\mathrm{g})$ & Wt\% Ta & Wt\% Ag & Wt\% Sb \\
\hline 0.2887 & $62( \pm 9)$ & $3( \pm 1)$ & $1.6( \pm 0.5)$ \\
\hline
\end{tabular}

Chip Arrays - These are common to all PCBs examined with the exception of power boards.. XRF consistently confirmed the presence of $\mathrm{Ag}$ and either $\mathrm{Ru}$, or Pd with $\mathrm{Au}$ (Figure 4.49).

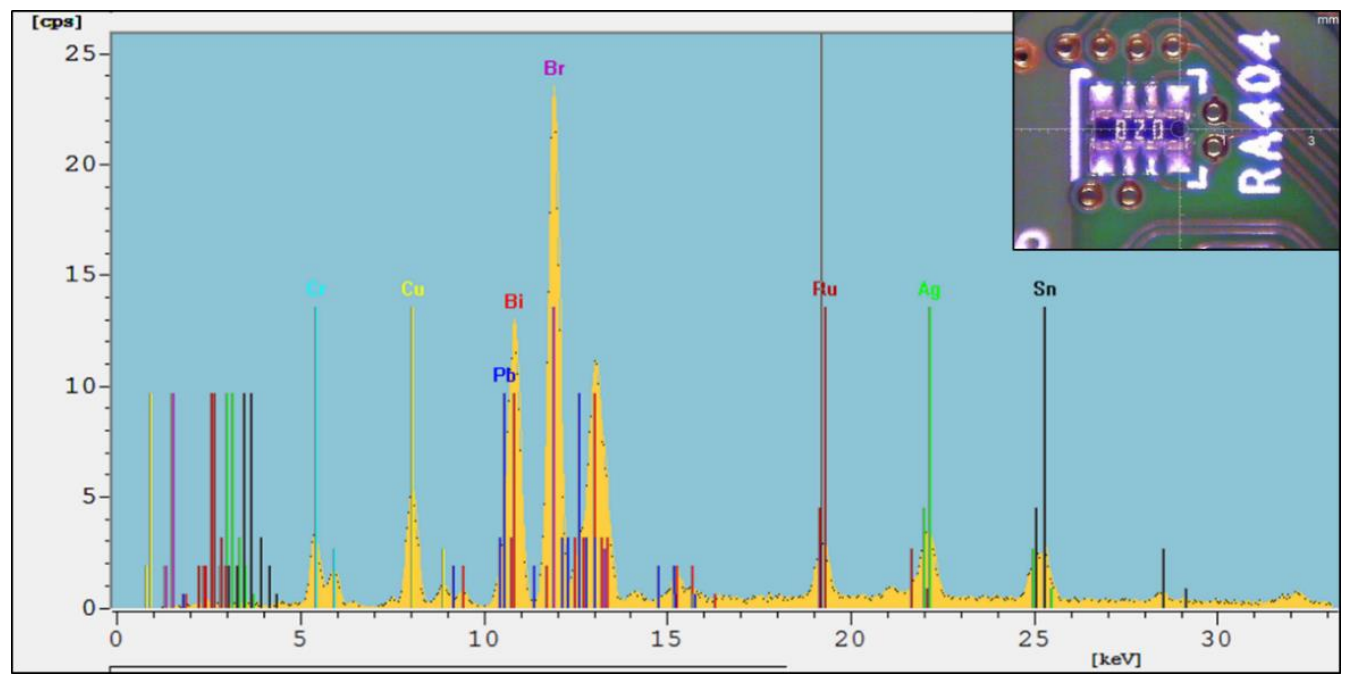

Figure 4.49X-ray emission spectrum of a typical chip array SMD containing Ag and Ru 
Inductors - These were mainly found on the PCBs of mobile phones, with infrequent occurrence on other miniaturised PCBs. Three different types were identified based on composition, distinguishable by colour (brown, grey and purple). Brown inductors contain Pd, $\mathrm{Ag}$ and $\mathrm{Nb}$ (Figure 4.50), there is $\mathrm{Ag}$ in grey inductors (Figure 4.51), and $\mathrm{Ag}$ and $\mathrm{Co}$ in purple inductors (Figure 4.52).

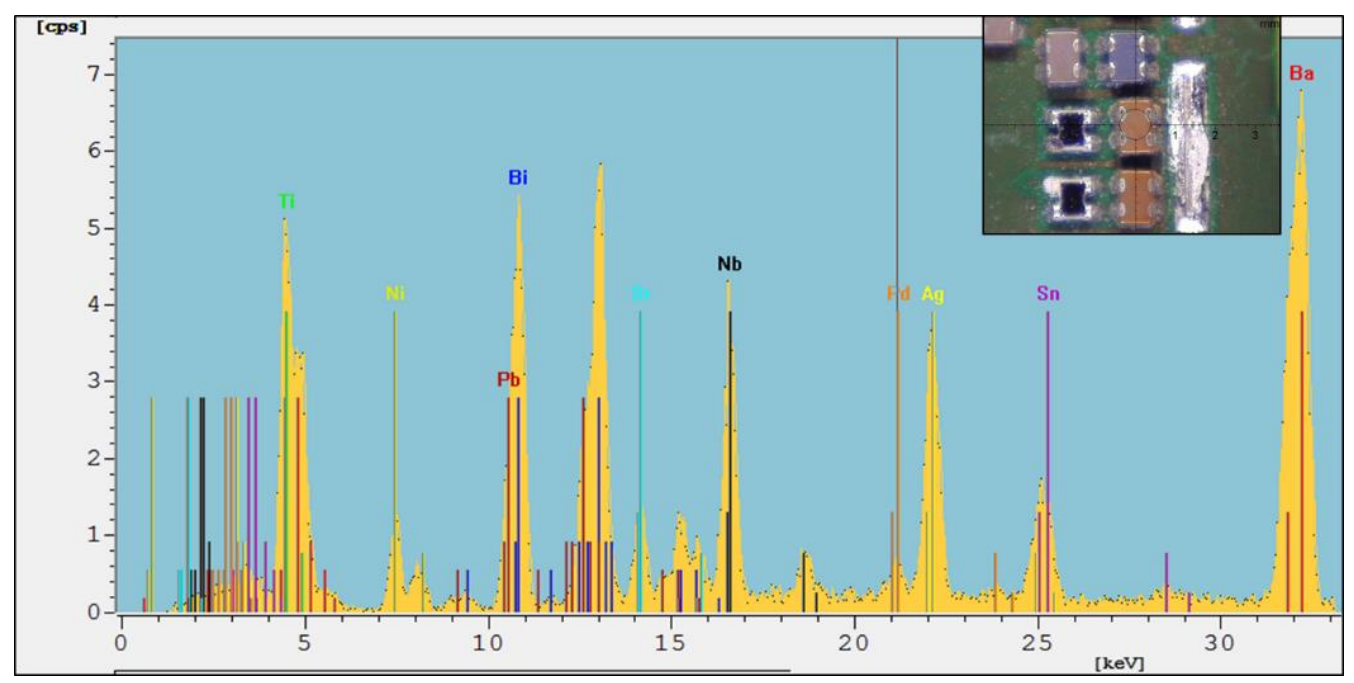

Figure 4.50 X-ray emission spectrum of brown inductors on mobile phone PCBs containing $N b, P d \&$ Ag

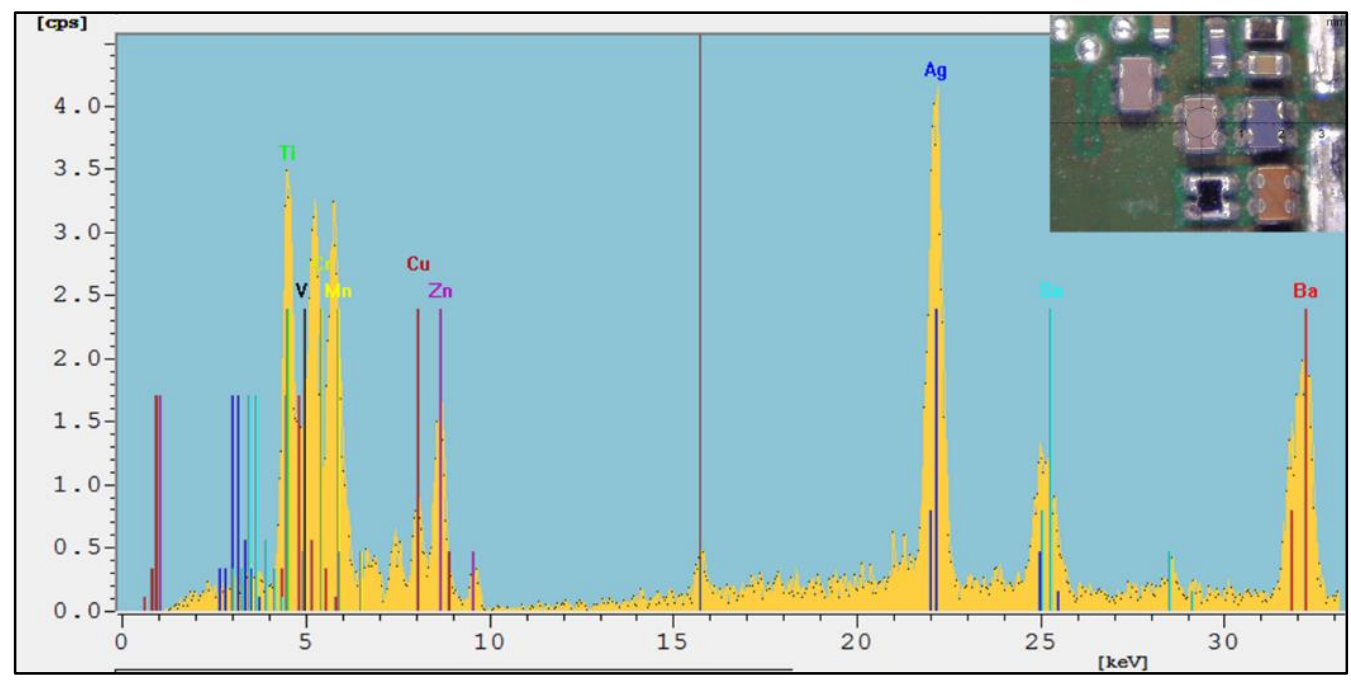

Figure 4.51 X-ray emission spectrum of grey inductors on mobile phone PCBs containing Ag 


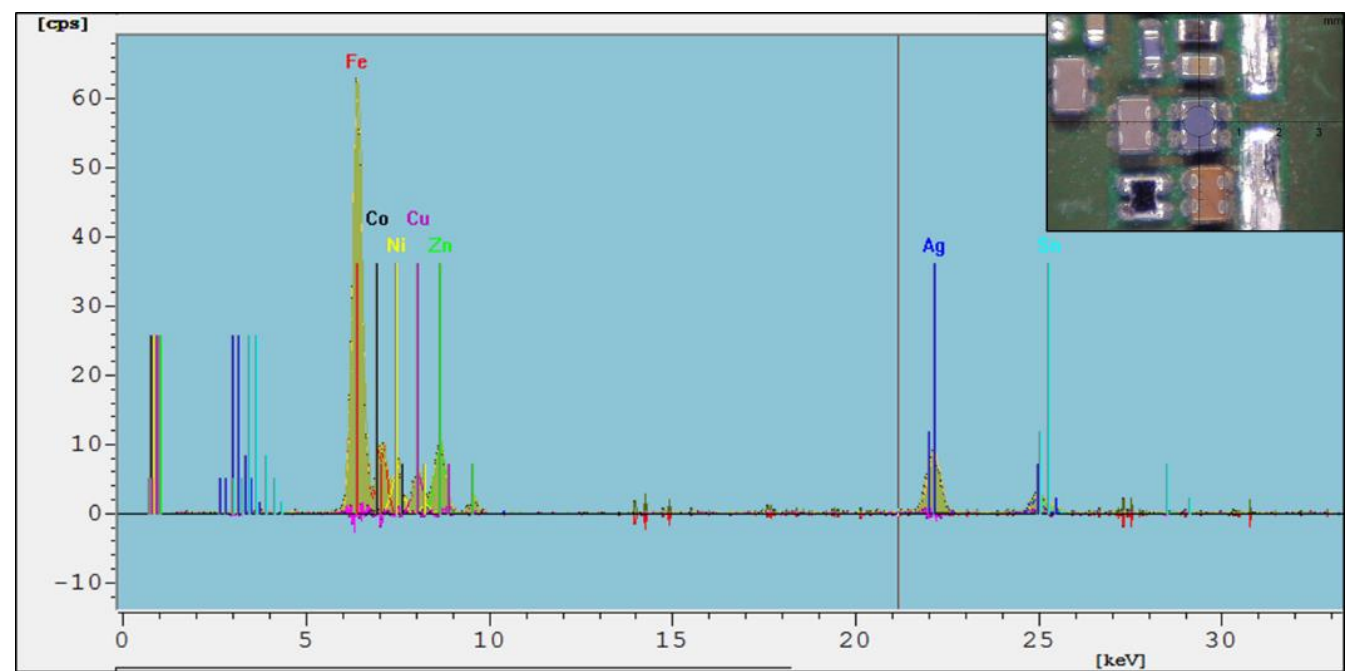

Figure 4.52 X-ray emission spectrum of purple inductors on mobile phone PCBs containing Ag \& Co

Polarized moulded body diodes - These SMDs are infrequently found. To the naked eye it is easy to confuse these devices with Ta-capacitors due to the shape of their moulded plastic encapsulation. Diodes were found to contain $\mathrm{Sb}$ within the encapsulation plastic, probably in $\mathrm{SbO}_{3}$ flame retardant (Figure 4.53).

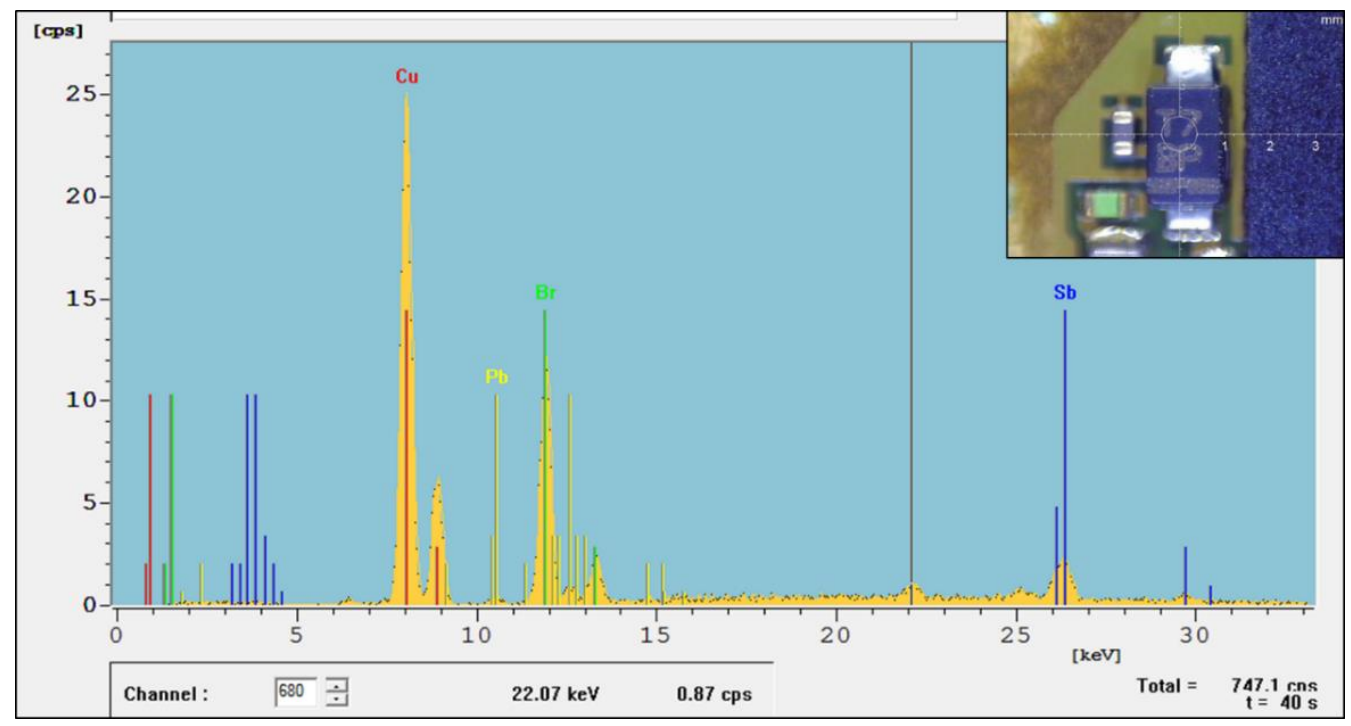

Figure 4.53 X-ray emission spectrum of typical polarized moulded body diode containing Sb

\section{PCB Component Summary}

A summary of CRMs found in PCB components is given in Table 4.7. 
Table 4.7 CRMs in PCB components

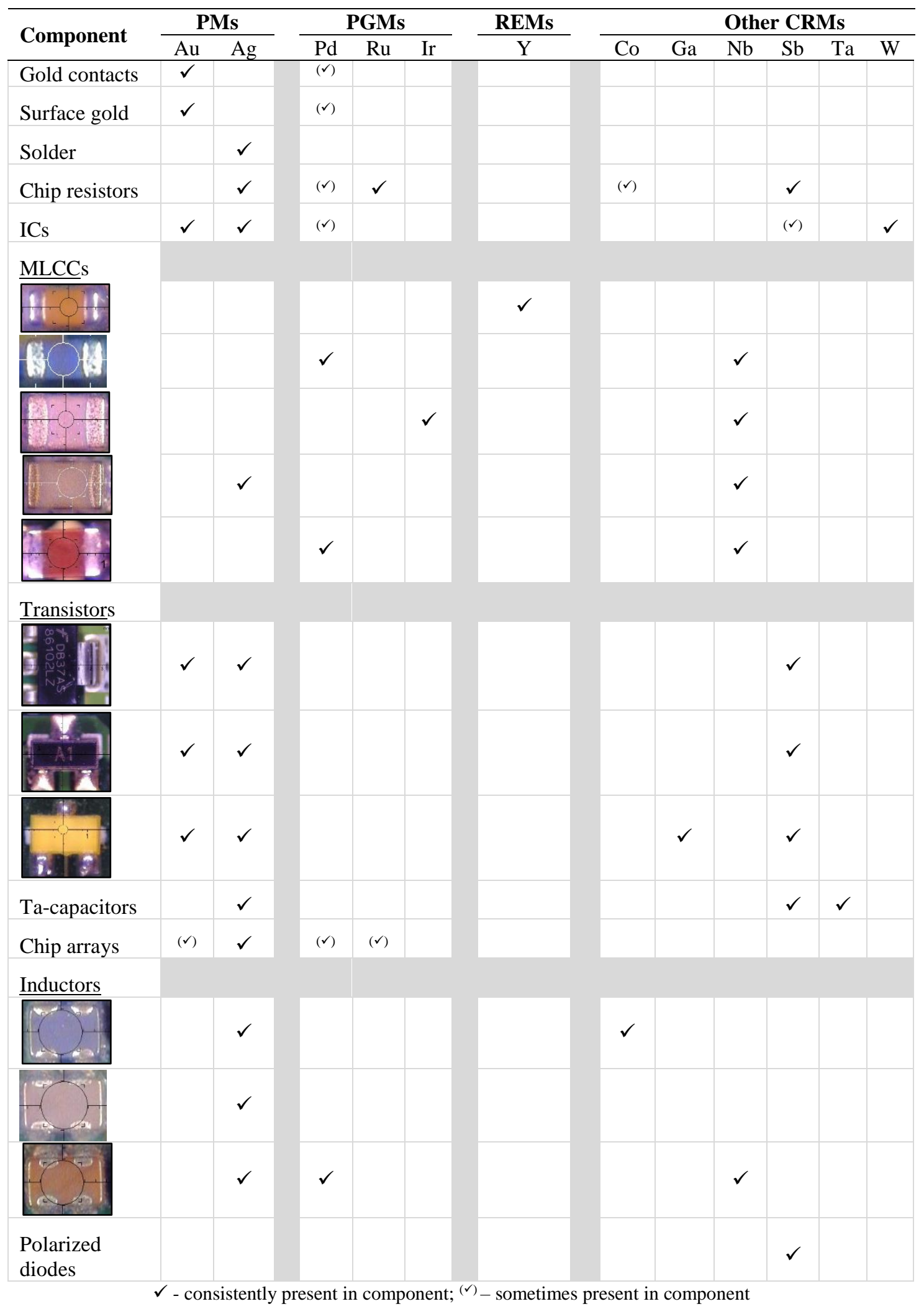


Using this data, it is possible to give a visual representation of the distribution of CRMs in PCBs based on the library of components established. Figure 4.54 shows the spatial distribution of CRMs in the main PCB of a TV.

\section{Colour Code: \begin{tabular}{|l|l|l|l|l|l|l|l|}
\hline Au & Ag & Pd & Ru & Y & Co & Sb & Ir \\
\hline
\end{tabular}}

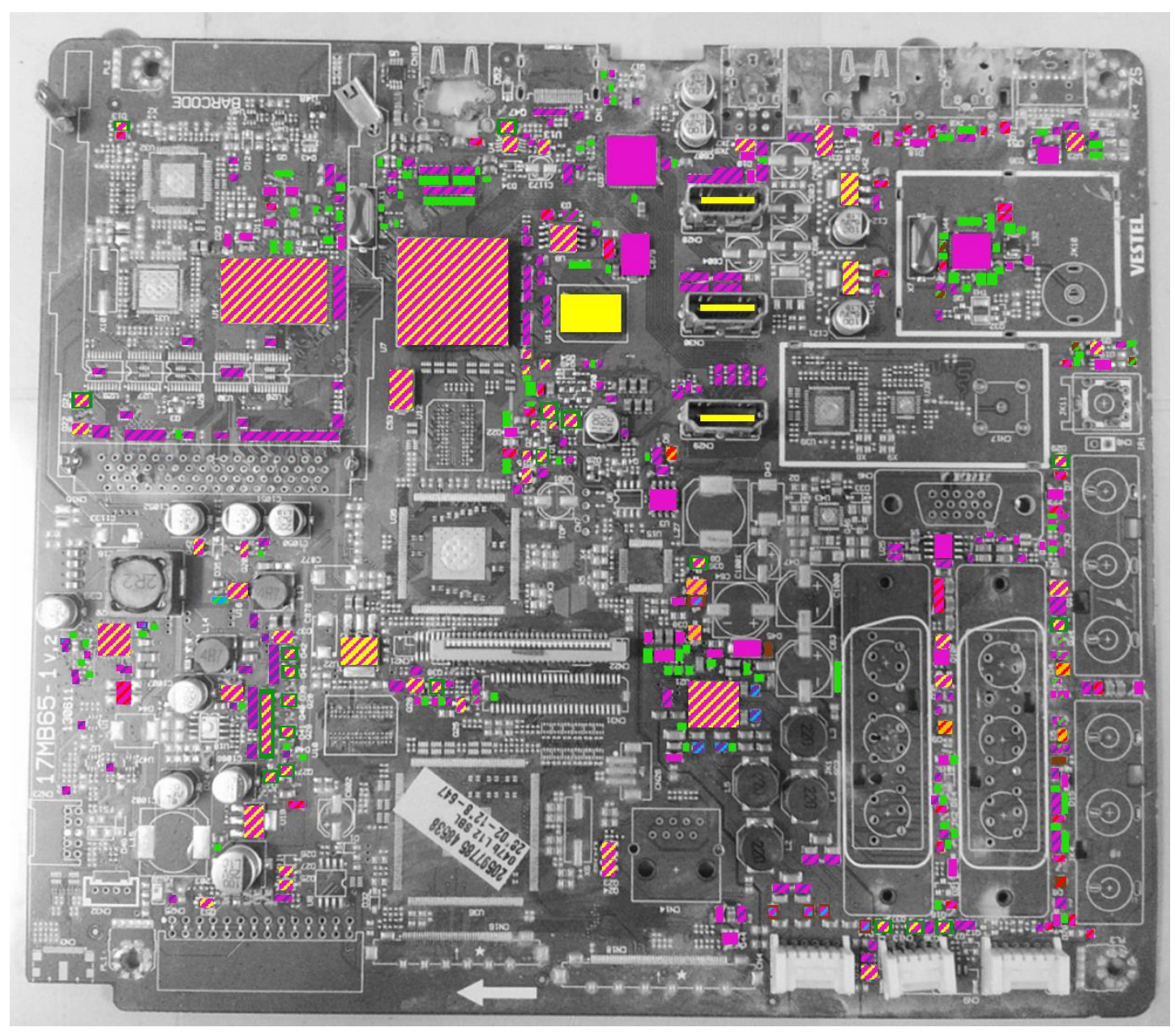

Figure 4.54 Colour coded CRM distribution in TV main PCB

It should be possible to develop software capable of analysing digital images of PCBs to identify and quantify components and calculate their value based upon knowledge of component compositions. Such software would be a powerful tool for rapidly identifying components on PCBs containing CRMs for selective disassemble and concentration, although this is outside the scope of this study. 


\section{Summary of CRMs identifies in WEEE samples.}

Table 4.8 summarises the occurrences of CRMs in WEEE samples identified. Overall, the greatest variety of CRMs in items are found within their PCBs, particularly in those items where miniaturisation to a significant extent is observed. In addition to the CRMs found in PCBs: Sb has been identified in plastics as a flame retardant, Co is present in magnets and ferrite cores of magnetrons, magnesium-alloy is used for laptop housings, $\mathrm{Y}$ is present in the phosphors of LCD CCFL backlights, and Nd is present in NIB magnets of HDDs.

Table 4.8 Summary of identified CRMs in trial samples

\begin{tabular}{ll}
\hline Critical Material & \\
\hline Antimony (Sb) & Was identified within plastics \& numerous SMD s, probably as $\mathrm{SbO}_{3}$ \\
Cobalt (Co) & $\begin{array}{l}\text { Co was identified in large concentrations ( 19-21 wt\%) within LIB cells, ferrous } \\
\text { alloys in magnetrons (56 mg total), and within SMDs. } \\
\text { Ga was identified within LEDs }\end{array}$ \\
Gallium (Ga) & Coated Mg-alloy laptop housings \\
Magnesium (Mg) & CCFLs \\
Nb & SMD Ta-capacitors \\
Tantalum (Ta) & Identified in ICs \\
Tungsten (W) & Found in phosphors of CCFLs (1.7 wt\% in CCFL) and in MLCC SMDs \\
Yttrium (Y) & NdFeB (NIB) magnets for HDDs, 35wt\% (11.4 g/HDD) \\
Neodymium (Nd) & Found in some MLCCs \\
Iridium (Ir) & Found in chip resistor and chip array SMDs \\
Ruthenium (Ru) & $\begin{array}{l}\text { Found in Au-alloy of contacts, solder, numerous SMDs (chip resistors, ICs, } \\
\text { Palladium (Pd) }\end{array}$ \\
MLCCs \& chip arrays) \\
Found in LEDs, contacts, coating alloys on PCBs, and numerous SMDs (ICs, \\
transistors, chip arrays) \\
Used in numerous SMDs (ICs, chip resistors, MLCCs, transistors, Ta-capacitors, \\
chip arrays \& inductors) and Pb-free solder
\end{tabular}

\subsubsection{Evaluation of processes for isolation of CRMs from WEEE}

As no CRMs were identified in power boards, and RSA security dongles have been found to be impossible to recycle in a cost-effective manner, these are not considered in this section. Due to the similar nature of the digital media device samples (i.e. mobile phones, MP3 players and cameras) disassembly processes for these are considered together.

\section{Product Disassembly, analysis of components and CRM recycling potential}

The following section outlines the disassembly methods used on each of the samples, an assessment of the cost of the process, and where routes for isolated materials could be found, an assessment of the value of material fractions generated based on quantities of CRMs in isolated fractions, as determined by XRF, AAS and ICP-MS analysis. PCBs are considered collectively. 


\section{Sanyo domestic microwave magnetron}

Disassembly - Following confirmation of the presence of Co in magnets, three attempts were made to isolate them from whole magnetrons. The first, which was to unscrew the magnetron housing and remove the magnetron for disassembly, proved time consuming, as the housing did not easily separate because of the way its parts are fasten together (Figure 4.55). The second attempt was to smash the magnets with a hammer to allow their isolation in pieces. This process also proved unsuitable due to the attraction of the magnet fragments to the steel housing, preventing them from falling out of the housing once broken. The third attempt was to strike the magnetron housing with a hammer, deforming it sufficiently to removed it and access the magnets. This was the fastest and most suitable method, a single hammer strike enabling quick isolation of whole magnets.

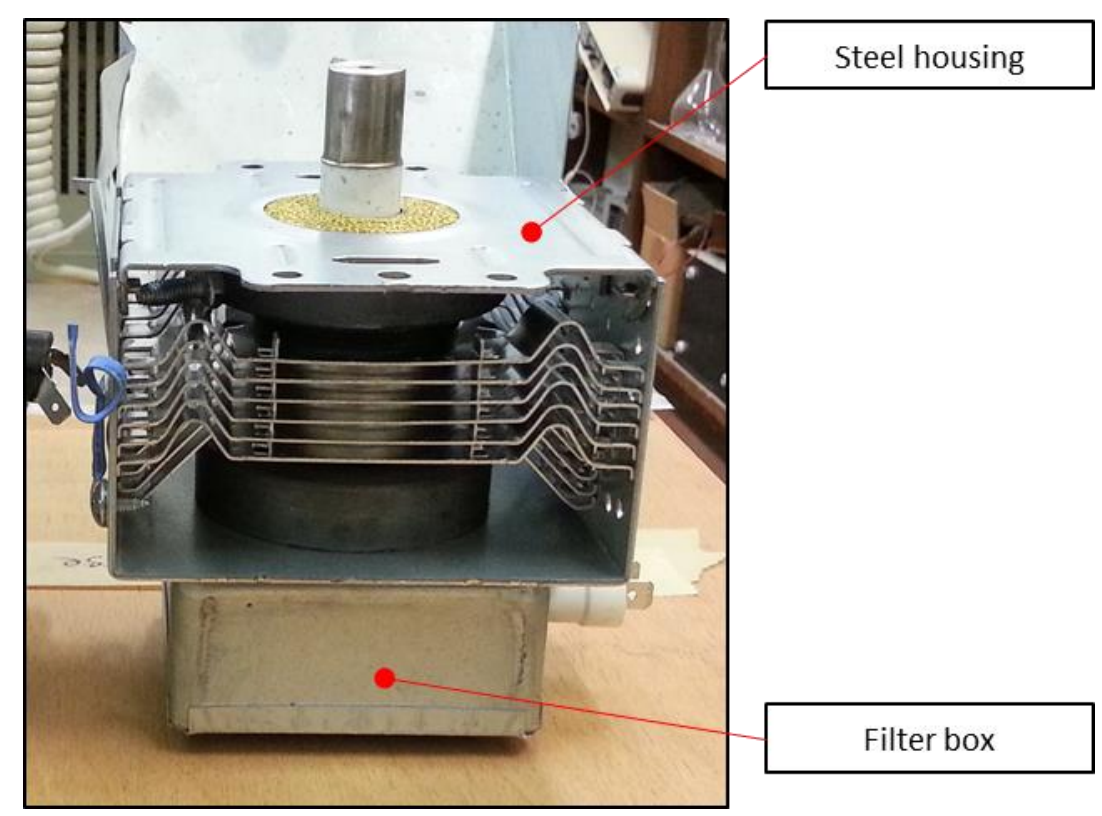

Figure 4.55 Sanyo domestic microwave magnetron

Three methods were attempted to isolate ferrite cores from the filter box, which is not easy to open because of the way the cover is fastened. Opening the filter box is therefore time consuming and costly. An attempt was made to break open the filter box with a hammer prior to removal of the magnets. Isolation of the cores following removal of the magnets was however found to be a better method. Once the magnetron is removed from its housing, copper wires forming the coils of the filter were left protruding from the filter box. Simply pulling these wires out of the filter box resulted in the fastest isolation of both magnets and ferrite cores, in a time of $120 \mathrm{~s}$. 
Recovery Potential - AAS analysis revealed that magnets and ferrite cores contain 22.1( \pm 0.3$)$ $\mathrm{mg}(0.01 \mathrm{wt} \%)$ and $6.24( \pm 0.03) \mathrm{mg}(0.5 \mathrm{wt} \%)$ of Co respectively, totalling $56 \mathrm{mg}$ of Co per device. An attempt was made to contact Swift Levick Magnets Europe, the Euro branch of the US company Arnold Magnetic Technologies, who were reported as the only company in the UK recycling cobalt-based magnetic alloys. ${ }^{191}$ The company had gone into liquidation and no alternative process was identified. At the time of this study (2012-2013) magnetrons were shipped from Metech as whole items with other small domestic appliances for automated recycling to recovery their scrap metal value. ${ }^{22}$ Given the recovery route employed, the magnetic alloy will adhere to the surfaces of ferrous fractions which are sent to scrap steel processes. The most common route for such material is for use in steel making as a coolant. The Co therefore will partition to the metal phase in BOF and EAF processes becoming alloyed with steel. ${ }^{118}$ The fate of the ferrite cores is likely to be the same. Co cannot be separated from ferrous materials at EoL through traditional recycling routes, and so no additional value can be derived from isolating Co bearing components of magnetrons in material streams of their own.

\section{Hard Disk Drives (HDDs)}

Disassembly - Manual disassembly was carried out with a standard precision screw driver. The PCB was isolated from the HDD casing in a time of 55 seconds. The sheet aluminium cover was removed exposing the internal components of drive. NIB magnet steel supports were removed from the housing. This was not straightforward requiring the magnet supports to be levered out one at a time (two magnets were present in each HDD). Total time required to open the drive and isolate magnets attached to their steel supports was $185 \mathrm{~s}$. No easy way was discovered to isolate the NIB magnets from their steel supports due to the force of attraction between the two. Isolation of HDD platters required an additional 73 seconds (Table 4.9).

Table 4.9 Hard disk drive disassembly times

\begin{tabular}{lc}
\hline Component & Time to isolate (sec) \\
\hline PCB & 55 \\
NIB magnets & 185 \\
NIB magnets and platters & 258 \\
PCB and NIB magnets & 240 \\
PCB, NIB magnets and platters & 313 \\
\hline
\end{tabular}

A faster way to gain access to the magnets inside HDDs may be to remove PCBs, and chill the cast aluminium housing, embrittling it so that it may be smashed, granting faster access to the 
magnets. Such a process would be analogous to the cryo-cracking method previously discussed. This would however require larger volumes of liquid nitrogen per HDD than will be required to treat mobile phones, camera and MP3 players due to the large size of HDD in comparison to these devices.

Recovery Potential ICP analysis confirm each magnet contains $5.7( \pm 0.2) \mathrm{g}$ of $\mathrm{Nd}$, $34.9 \pm 0.9 \mathrm{wt} \%, \sim 4-5 \%$ higher than indicated in $2 \frac{1 / 2}{2}$ inch HDDs by the Öko-Institut e.V. ${ }^{19}$ This equates to $11.4( \pm 0.3) \mathrm{g}$ of $\mathrm{Nd}$ per $3 \frac{1}{2} \mathrm{inch} \mathrm{HDD}$.

At the time of this study (2012) it had been frequently stated that no commercial processes existed for recovery of REMs from HDD magnets. ${ }^{191}$ However, discussions with one company revealed that there were markets for refining NIB, developed for processing production scrap from NIB manufacturing. Refinement is possible provided a sufficient quantity can be accumulated. It is speculated that a viable quantity for refining was $\sim 300$ tonnes. This quantity equates to approximately 184,000 NIB magnets, which would require approximately 92,000 HDD to be manually disassembled from hard drives (presuming two NIB magnets are present in every HDD).

At the time of this study much research was underway to develop a process for HDD NIB recycling, and to date there has been much progress. The hydrogen decrepitation method has received a great deal of publicity as a 'closed loop' process which enables production of new magnets by sintering recovered material. Much research is underway to improve this process. ${ }^{200-204}$ In an attempt to enable cost-effective isolation of the magnets from HDDs, the US Critical Materials Institute has developed a robot capable of 'punching' the magnets from drives with high throughput. ${ }^{58}$ Both processes allow magnetic materials to be isolated from HDDs whilst avoiding the major barrier represented by high cost of manual isolation of magnets from drives. Numerous other processes have been investigated for recovery of REMs from isolated NIB magnets as reviewed by Yang et al., 2017. ${ }^{205}$

\section{Set top box front panel PCBs}

Manual Disassembly - The LEDs of the boards (two blue, three green) were simply removed from the PCB with pliers within 20 seconds.

Recovery potential - It was not possible to find any commercial operations recovering Ga from LEDs, and it has been reported that no such process exists in the UK. Indeed, today the only recycling of $\mathrm{Ga}$ which occurs at commercial scale is from primary production. LED recycling is problematic. Small quantities of metals are used in each tiny device, which is 
encapsulated. This results in problems with accessing the trace amounts of low value metals for recovery. In addition, an enormous number of LEDs would have to be isolated to amass a sufficient quantity for refining (were such a process available). Recent studies have shown that due to technical complexity of recycling Ga from LEDs, and recycling barriers throughout the whole recycling chain, no implementation of commercial recycling is likely in the near future. ${ }^{206}$ It is therefore not viable to implement removal of LEDs from STB boards (or any other device). These relatively low grade FR-2 PCBs will be sent for smelting to recover $\mathrm{Cu}$ and any PMs, with LEDs in place resulting in loss of Ga to slag in the process. ${ }^{118}$ The FP7 funded cycLED project, which aimed at improving the resource-efficiency of LED products, concludes that it is uneconomical at this time to recover any of the CRMs in LEDs (including In, PMs, Ga and REMs). This is due to cots barriers resulting from the dissipative nature of LED applications in WEEE, prohibitive costs of manual isolation, and losses through unintended co-separation in automated recycling. ${ }^{207}$ LEDs therefore are an example of the dissipative application of CRMs resulting in non-viable recovery at end-of-life.

\section{Sony Li-ion laptop batteries}

Manual disassembly - Eleven samples of the Sony Li-ion battery packs from laptops were collected. These are disassembled from laptops as part of the standard recycling procedure employed by Metech. This is necessary to comply with depollution aspects of WEEE legislation. The question arose - could greater value be generated from disassembly of the battery packs into cells and component parts, rather than sending whole battery packs for recycling via a compliance scheme? Each unit contains six Li-ion cells. The cells were accessed by unscrewing the plastic housing and removing them from their plastic wrap. This process took 157 seconds. Included in the battery pack along with cylindrical Li-ion cells was a small PCB which was isolated in 5 minutes from the housing. This process was difficult as PCBs are permanently bonded in place with adhesive (Table 4.10).

Table 4.10 Times taken for manual disassembly of CRM bearing components of Li-ion battery packs

\begin{tabular}{lc}
\hline Unit & Time taken for isolation (s) \\
\hline Cells from whole unit & 157 \\
PCB & 291 \\
\hline
\end{tabular}

\section{Recycling potential}

$\underline{\text { Li-ion cells }}$ - There is no capacity to recycle Li-ion batteries in the UK. Three national battery compliance schemes were contacted (G\&P, Clarity Environmental, and Budget Pack), to investigate recovery routes in Europe (Holland, Belgium, Germany). Compliance schemes 
were found to be the only possible route to recycling for Li-ion batteries. European battery smelters (e.g. Umicore) have minimum quantities they will accept for refining, and compliance schemes perform a crucial function in accumulating Li-ion batteries from across the UK, achieving sufficient quantities to ship. The transportation of $\mathrm{Li}$-ion batteries must be conducted in accordance with UK and international regulation. ${ }^{208}$ Compliance with these regulation results in high logistics costs.

The value of the batteries to recyclers was found to fluctuate greatly depending on the type of battery and its cobalt content. ${ }^{209,210}$ For this reason the Co content of batteries was measured by AAS (Table 4.11). Each cell was found to contain 8.30g of Co, $18.9 \mathrm{wt} \%$, i.e. $49.8 \mathrm{~g}$ (15.8 wt $\%)$ Co in the whole battery pack.

Table 4.11 Cobalt content of Laptop Li-ion batteries

\begin{tabular}{lcc}
\hline \multirow{2}{*}{ Sample } & \multicolumn{2}{c}{ Cobalt content } \\
\cline { 2 - 3 } & $(\mathrm{g})$ & $(\mathrm{Wt} \%)$ \\
\hline Single cylinder Li-ion battery Cell & $8.30( \pm 0.01)$ & 18.9 \\
Whole battery pack & $49.82( \pm 0.06)$ & 15.8 \\
\hline
\end{tabular}

Great fluctuations in value have also resulted from volatile market values of Co, which had shown a net decrease over the two years previous to this study (2010-2012). Metech indicated that they used to regularly receive $£ 30 /$ tonne rebate for L-ion batteries, but at the time of the study received variable prices upon request. ${ }^{211} \mathrm{Li}$-ion batteries represent a 'pure cost' to UK recyclers with a consignment fee of $£ 80$ being charged. Depending on battery chemistry and how costly they are to process, an additional charge may be incurred. Primary Li button cells such as the type found in the RSA security dongle and on PC motherboards are most costly to recyclers, costing up to $£ 2000 /$ tonne to have processed.

When Co prices were high, packet batteries and laptop batteries fetched a price at refineries of $£ 100 /$ tonne but currently (2017) no revenue from recovery is passed down the recycling process chain to recyclers. To see a positive value at refineries, 13-18 tonnes of batteries must be accumulated, which is unlikely to be viable for any one company performing manual preprocessing. If a single recycler could amass such a quantity of batteries then a profit may be generated if materials were sent directly to refineries, by-passing compliance schemes. Some older Li-ion batteries may fetch a profit due to their size and high Co content, but none of this type were included in the trial samples.

Due to regulations regarding handling and treatment of waste batteries, the Co content of the Li-ion batteries is recovered. However, graphite (another critical material) present in cells is 
consumed in pyrometallurgical recovery. An alternative route for laptop batteries posing significant benefits in terms of critical materials conservation and revenue generation is sale for re-use in refurbished laptops or as replacements for products with users, once their functionality and storage capacity had been tested and verified as suitable.

As no value can be derived from the LIB cells, and isolation of their PCB is costly due to permanent bonding with the housing, it was deemed economically unfeasible to disassemble laptop Li-ion battery packs.

\section{HP laptop screens}

WRAP has published a study on end-of-life treatment of LCD displays, which focused on manual disassembly and shredding. ${ }^{212}$ As manual isolation is costly in the UK, and shredding is disadvantageous in terms of recovery efficiencies for CRMs, methods were investigated which augment manual processes to achieve more rapid disassembly.

Disassembly Manual disassembly of the LCD sandwich from its housing was easily achieved by removal of a series of screws and releasing a series of clip fasteners with the flat end of a screwdriver. This allowed the front bezel of the housing to be removed and the LCD sandwich to be unscrewed and removed from the housing. The main PCB was simply pulled from the back of the LCD sandwich, and the minor PCB detached from the bottom. The aluminium frame of the LCD sandwich in which the cold cathode fluorescent lamp (CCFL) is located was also unscrewed. This was detached by snipping through wires connecting it to the LCD. Times taken to isolate the CRM bearing parts of the screen using manual disassembly are given in Table 4.12.

Table 4.12 Time to manually isolate CRM bearing components of LCD displays

\begin{tabular}{lcc}
\hline Component & Time to isolate (sec) & Cost $(\mathfrak{f})$ \\
\hline AZ91D Mg-alloy housing & 180 & 0.79 \\
Minor PCB & 224 & 0.99 \\
Major PCB & 248 & 1.09 \\
CCFL & 390 & 1.72 \\
\hline
\end{tabular}

Disk Cutting - An attempt was made to isolate the CCFL and PCBs from the rest of the LCD unit by cutting the bottom section from the screen. This was followed by removal of the two side edges of the housing to open it for easy removal of PCBs. The first attempt to remove the bottom of the screen used a Dremel handheld multi-tool with rotating cutting disk attachment. This was extremely time consuming and after 10 minutes very little progress was achieved. The second attempt used the rotating cutting disk of a wet tile cutter to saw directly through 
the screen parallel to its bottom edge, isolating the piece of housing containing the CCFL (Figure 4.56), which was prized open with a screw driver to access to the CCFL in a total time of $241 \mathrm{~s}$. Repeated attempts always resulted in the CCFL being broken. This shows the method to be unsuitable, resulting in release of $\mathrm{Hg}$ from the CCFL posing an environmental $\mathrm{H} \& \mathrm{~S}$ hazard. ${ }^{212,213}$ Removal and opening of the remaining two sides of the housing to isolate PCBs required an additional 141 seconds. A faster method of gaining access to the PCBs was to cut through the top and side edges of the housing avoiding the LCD sandwich inside. Using this method both PCBs were isolated from whole displays in a time of 154 seconds.

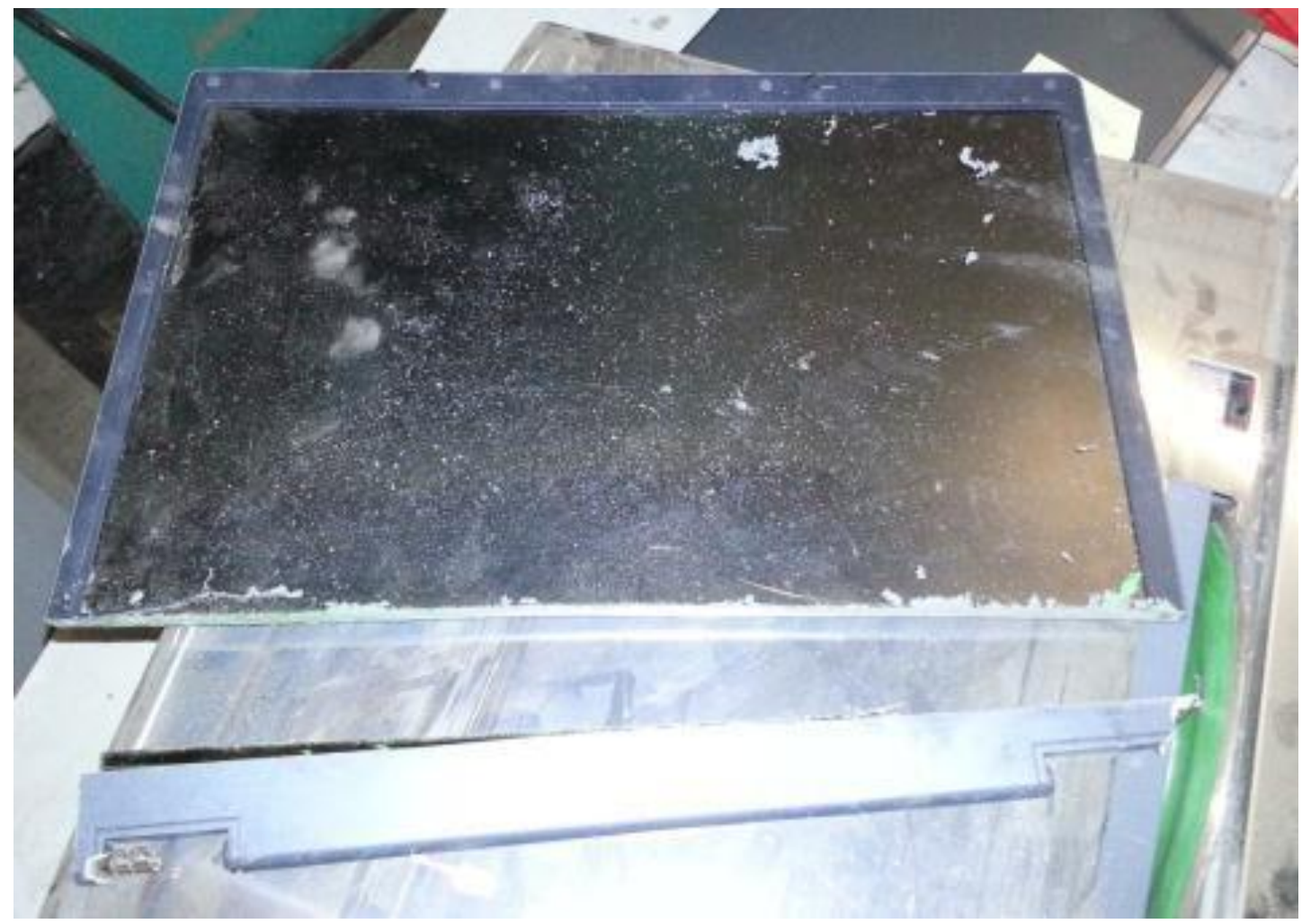

Figure 4.56 LCD screen with the CCFL containing portion cut away

Guillotine Use of the guillotine to remove the top and side edges of the housing allowed the PCBs to be isolated in 65 seconds. This was by far the fastest method of opening the housings (Table 4.13) and requires no electricity, unlike the tile cutter.

Table 4.13 Comparison of best times for LCD screen disassembly methods.

\begin{tabular}{llll}
\hline Method & $\begin{array}{l}\text { Isolation } \\
\text { CCFL (s) }\end{array}$ & $\begin{array}{l}\text { of } \\
\text { PCBs (s) }\end{array}$ & $\begin{array}{l}\text { Isolation of CCFL \& } \\
\text { PCBs (s) }\end{array}$ \\
\hline Manual disassembly & - & 248 & 390 \\
Tile cutter & 241 & 154 & 382 \\
Guillotine & - & 65 & - \\
\hline
\end{tabular}




\section{Recovery Potential}

Housing - Although the Mg-alloy housing is easily isolated using the guillotine, no companies in Europe processed painted magnesium alloy laptop housings. Most die casters in Europe would only process internal production scrap, which gives foundries a guaranteed source of supply of known composition at a stable price.

Magnesium Elektron in Manchester provide a recycling service for Mg processing scrap, but do not deal with post-consumer waste due to contamination issues. The paint from magnesium alloy laptop housings is likely to release dioxins which are not fully removed by the abatement plant. The presence of any copper wiring or stainless steel bolts/fixings would also downgrade the value of the scrap. This contamination would therefore mean that only small volumes of this type of material at a time may be accommodated in the process. Removal of such contamination from the housing would be costly. It is also questionable whether sufficient capacity exists to accommodate the volumes of Mg-alloy laptop housings that will enter the waste stream.

The recycling of coated $\mathrm{Mg}$-alloy is problematic due to the difficulties encountered with complete removal of coatings using thermal processes of de-coating. ${ }^{214-216}$ The only company identified to successfully process painted magnesium alloy laptop housings is Fujitsu Group in Japan. ${ }^{217}$ They became the first in the world to achieve practical application of a technology for recycling $\mathrm{Mg}$-alloy from housings of notebook computers collected in-house, and they began employing the technology in product manufacturing in Autumn of 2002. The paint is stripped off using solution treatment, and $\mathrm{Al}$ and $\mathrm{Mn}$ are added during re-melting to increase strength and corrosion resistance of the recycled alloy. The performance of the recycled alloy is almost equal to that made using virgin materials. ${ }^{218}$ Shipping to Japan would require large quantities of laptop housings to be accumulated, and for this reason it is not a viable route to recovery for Metech. The problem of scale is less of a concern for specialist LCD recyclers who receive large quantities of screens and housings from AATF's.

CCFL: The presence of $\mathrm{Hg}$, which is controlled by the RoHS directive, will complicate CCFL recycling and cause the process to be costly, requiring atmospheric monitoring and extraction systems, as well as special licensing. It is unlikely Metech would implement such a process given the volumes of material available to them and their inability to derive value from the CCFL. Metech will therefore continue to send whole laptop screens to specialist processors.

\section{Digital Media Devices}

The vast majority of recoverable value and CRMs found in MP3 players, mobile phones and digital cameras is concentrated in PCBs which are constructed from common SMD 
components. For this reason, these products have been grouped into this single section. In addition to high-grade PCBs, all these items contain similar screens and packet Li-ion batteries. The PCBs are high-grade but despite this, they are not disassembled by Metech due to the costly nature of disassembly.

\section{Disassembly}

MP3 players - Complete manual disassembly of a Sony NWZ-s639F digital media player (MP3 player) into its parts was achieved in $105 \mathrm{~s}$ using a power screw driver. The time taken to isolate each of the CRM bearing parts is indicated in Table 4.14:

Table 4.14 MP3 player disassembly times

\begin{tabular}{lcc}
\hline Component & Time to isolate (sec) & Cost (£) \\
\hline Li-ion battery & 52 & 0.23 \\
Major PCB & 62 & 0.27 \\
Screen & 94 & 0.41 \\
Minor PCB & 105 & 0.46 \\
\hline
\end{tabular}

Cryo-cracking with liquid nitrogen was attempted on a similar Sony Walkman digital media player (Figure 4.57). Costs associated with use of liquid nitrogen are given in Table 4.17. The sheet aluminium casing on this player was not easily removed by this method. Repeated submersions were necessary before the casing was eventually opened by hammering. Time for isolation of the PCB was $\sim 4$ minutes. Repeated impact resulted in detachment of the larger ICs from the rest of the board. This made their recovery from debris generated in the process difficult. Failing to recover detached ICs results in loss of potentially recoverable value from PCBs along with CRMs. While the method is suitable for plastic digital media players, it is unsuitable for those with metallic casings.

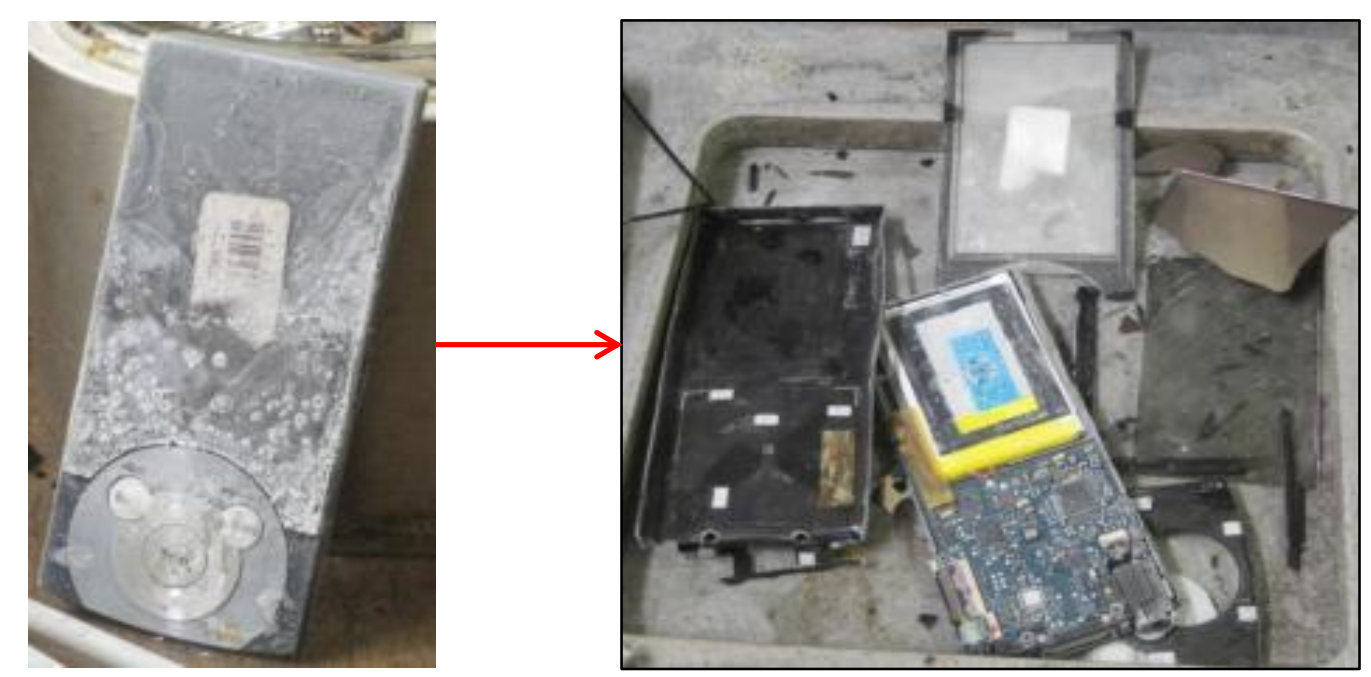

Figure 4.57 Liquid nitrogen cooling and smashing of MP3 player 
Digital Cameras - Complete manual disassembly of the Sanyo X1220 digital camera with a power screw driver was achieved in $166 \mathrm{~s}$. The times taken to isolate each of these components is indicated in Table 4.15.

Table 4.15 Digital camera disassembly times

\begin{tabular}{lcc}
\hline Component & Time to isolate (sec) & cost $(\mathfrak{f})$ \\
\hline Li-ion battery & 8 & 0.035 \\
Screen & 120 & 0.53 \\
PCB & 166 & 0.73 \\
\hline
\end{tabular}

Digital cameras exhibited mixed performances in cryo-cracking. Plastic casings are easily shattered after $20 \mathrm{~s}$ submersions in liquid nitrogen (Figure 4.58). If the PCBs are located near the rear (screen side) surface of the device, shattering the case in this way enables easy isolation. However, several of the cameras examined had PCBs located deeper within the structure of the camera behind other components, making isolation following cryo-cracking more difficult, requiring removal of additional screws, components and internal structural parts. One camera had an aluminium front casing which absorbed much of the impact when smashing, making removal of the plastic case more difficult. 


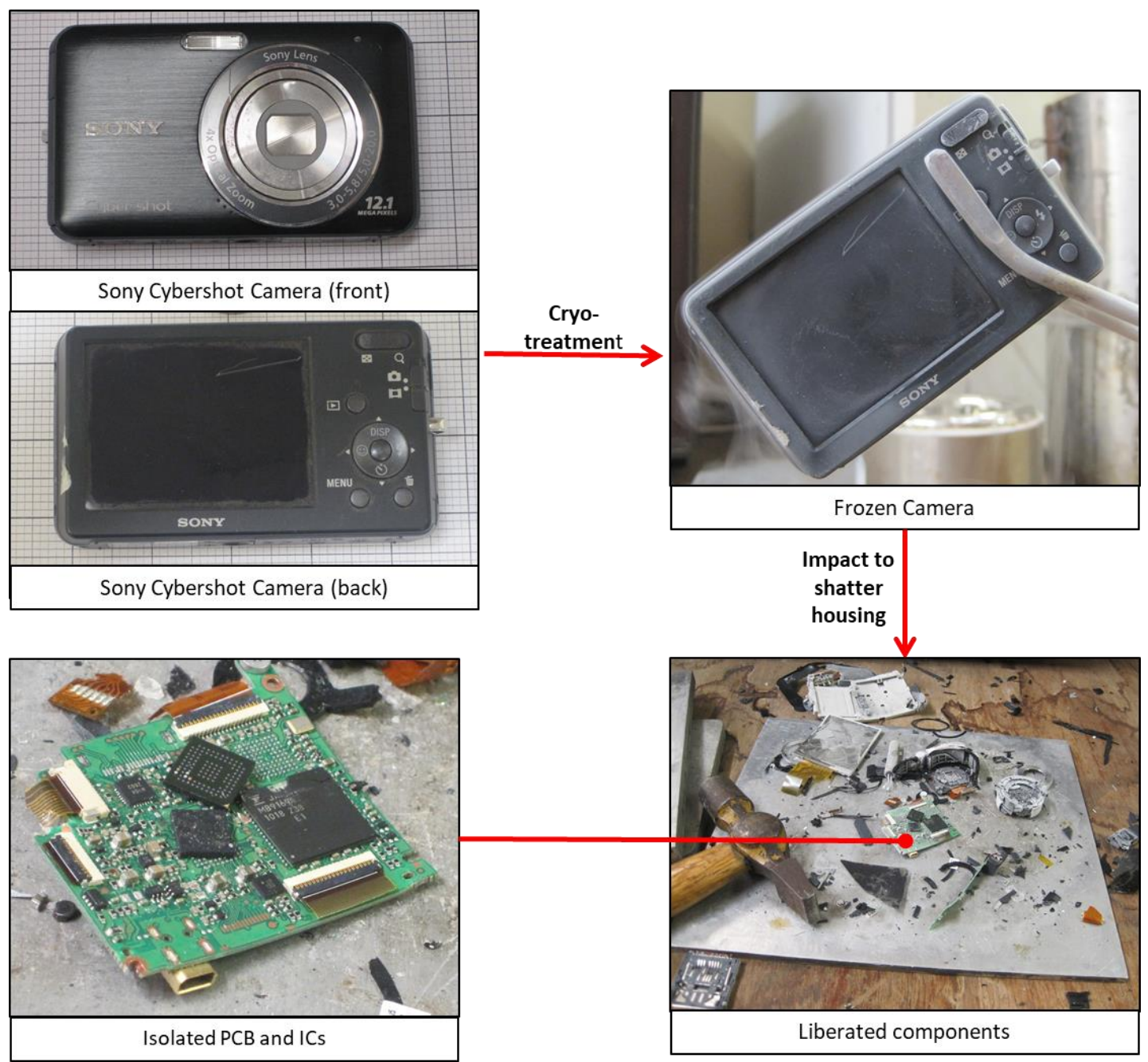

Figure 4.58 Cryo-cracking of a Sony Cybershot digital camera to isolate its PCB

Times for disassembly achieved with cryo-cracking for cameras with plastic cases are comparable to those for MP3 players, $\sim 30$ s total. Cryo-cracking was also attempted on a digital video camera (Figure 4.59), which had proved problematic to disassemble. There are multiple PCBs in the device housed in different sections. These are relatively small as the functionality of the device is split between PCBs in the different parts of the camera. Isolation of all PCBs is time consuming. To achieve liberation of all components with cryo-cracking, repeated submersion in liquid nitrogen were required to shatter each housing section and isolate PCBs separately. PCBs were fastened to the device so isolation required further manual disassembly. The design of the device therefore prohibits easy isolation of PCBs through manual disassembly and cryo-cracking. A total of 60 s submersion time was required, after which PCBs were still not easily isolated. 


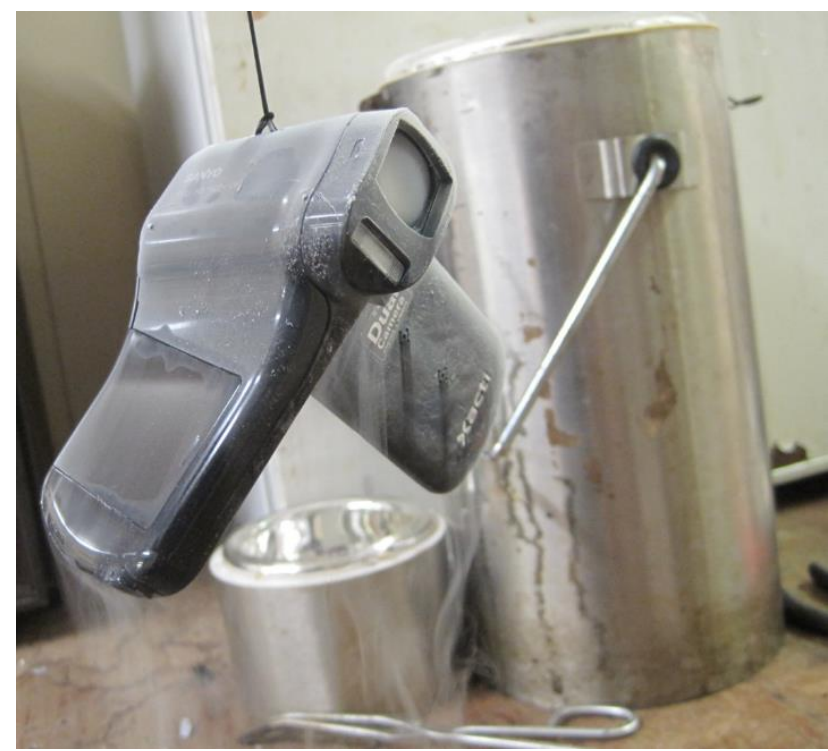

Figure 4.59 Sony Xacti Full HD 10 MP digital video camera sample post cryo-treatment

Mobile phones - A Nokia 6230i was selected for disassembly as a representative sample of the mobile phones currently received by Metech for treatment. Complete manual disassembly of the Nokia 6230i mobile phone sample to its CRM bearing components was achieved in $87 \mathrm{~s}$ with a powered screw driver. The time taken to isolate each of the constituent CRM bearing parts is indicated in Table 4.16 .

Table 4.16 Time to isolate CRM bearing components of mobile phones using manual disassembly

\begin{tabular}{|c|c|c|}
\hline Component & Time to isolate (sec) & Cost (£) \\
\hline SD Card & 8 & 0.04 \\
\hline PCB & 87 & 0.38 \\
\hline Screen & 87 & 0.38 \\
\hline Speaker Magnet & 98 & 0.43 \\
\hline
\end{tabular}

Casings of mobile phones were easily shattered following submersion in liquid nitrogen, and since very little of the structure of the devices was metallic the PCBs could be rapidly isolated. (Figure 4.60). Some loss of ICs occurred but the problem is not as serious as in items where repeated hammering is necessary to gain access to the PCBs. Cryo-cracking was well suited to isolation of PCBs from mobile phones, requiring only 20 s submersions in liq. nitrogen and a further 10 seconds of hammering to isolate the board. 


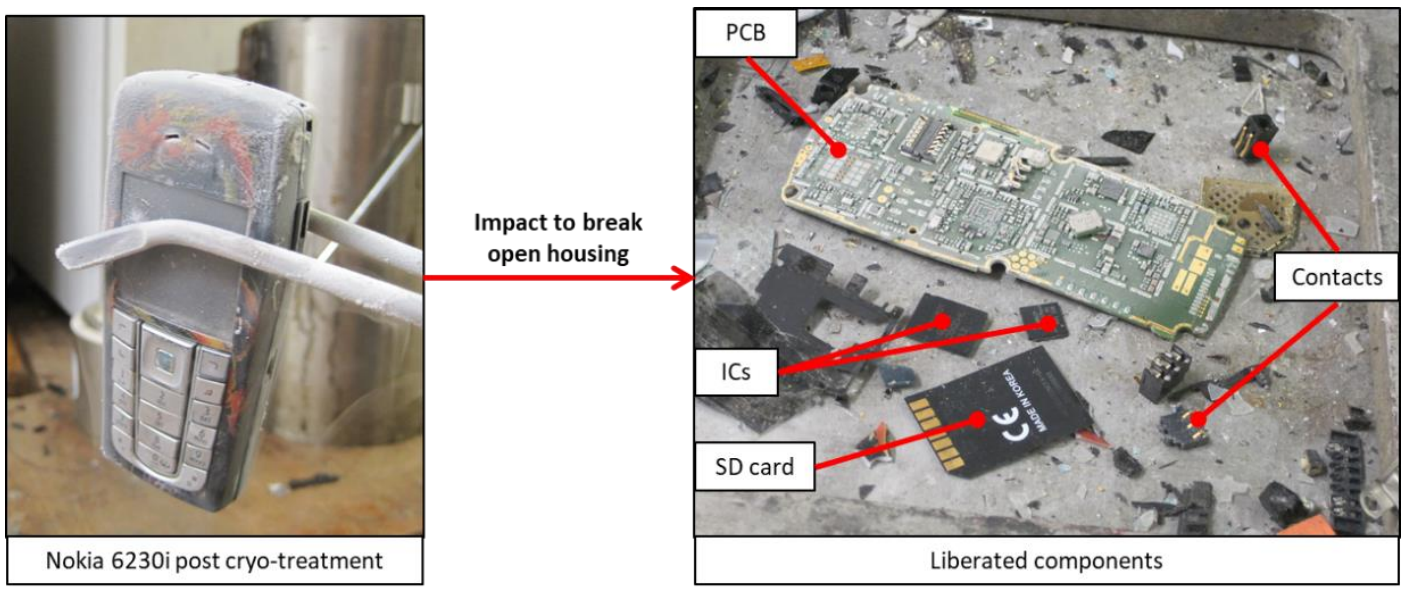

Figure 4.60. Cryo-cracking of Nokia 6310i mobile phones sample to isolate SM bearing components

\section{General comments on disassembly methods}

The equipment costs for manual disassembly are low. However, this process is time consuming resulting in high labour costs. Cryo-cracking enabled rapid isolation of high grade PCBs and lithium-ion batteries from digital media devices. Time trial results for isolation of PCBs with cryo-cracking are given in Table 4.17. Based on the time taken to perform the procedure, and costs of equipment and liquid nitrogen, the total cost of the process was $\sim 10 \mathrm{p} / \mathrm{sample}$. This method was well suited to items with plastic housings and no major metallic structural features, e.g. mobile phones. Difficulty was encountered for items which have metallic structural features which remain ductile following cooling and obscure access to PCBs once housings are removed. However, consideration of H\&S will be necessary before any industrial process based on this method can be implemented due to use of liquid nitrogen and the issue of flying fragments from items.

Table 4.17 Results of product disassembly following liquid nitrogen treatment and cracking

\begin{tabular}{cccccc}
\hline Item & Model & $\begin{array}{c}\text { Item } \\
\text { Mass } \\
(\mathbf{g})\end{array}$ & $\begin{array}{c}\text { PCB } \\
\text { mass } \\
(\mathbf{g})\end{array}$ & $\begin{array}{c}\text { Immersion } \\
\text { time } \\
(\mathbf{s})\end{array}$ & $\begin{array}{c}\text { Cost of } \\
\text { process } \\
(\mathfrak{f})\end{array}$ \\
\hline Digital Camera & $\begin{array}{c}\text { Sony Cyber Shot: Steady } \\
\text { shot DSSC-W310 12.1 } \\
\text { MP (no battery) }\end{array}$ & 115.99 & 6.10 & 20 & 0.102 \\
& & & & \\
Mobile Phone & Nokia 6350i (no battery) & 75.8 & 12.99 & 20 & 0.102 \\
$\begin{array}{c}\text { Digital media } \\
\text { player }\end{array}$ & $\begin{array}{c}\text { Sony Walkman NWZ- } \\
\text { A845 (including battery) }\end{array}$ & 59.37 & 6.91 & 20 & 0.102 \\
$\begin{array}{c}\text { Video camera } \\
\text { Sony Xacti Full HD 10 } \\
\text { MP (no battery) }\end{array}$ & 115.24 & 11.06 & $60 \mathrm{~s}^{*}$ & \\
\hline
\end{tabular}

* Required 3 submersions before all PCBs could be liberated 
An enquiry to BOC revealed this process would cost around $£ 3,000$ investment for a liquid nitrogen dewar, with consumable costs of liquid nitrogen $£ 2.29 / \mathrm{L}$ (sufficient to process $\sim 200$ products per litre of liq. nitrogen). ${ }^{219} \mathrm{~A}$ breakdown of sample disassembly costs is given in Table 4.18.s

Table 4.18 Breakdown of costs for cryo-cracking

\begin{tabular}{lc}
\hline l00 L of liq. nitrogen & $£ 229.00$ \\
Delivery charge & $£ 48.85$ \\
\hline Total cost & $£ 277.85$ \\
Units processed & 20,000 \\
\hline \hline Liq. Nitrogen cost per item & $£ 0.0139$ \\
\hline
\end{tabular}

Liquid nitrogen is costly and it may not be necessary to cool items to $-178^{\circ} \mathrm{C}$. Investigation of embrittlement temperatures of commonly used WEEE plastics (e.g. PC-ABS) will provide data upon which it may be possible to develop less costly alternative cryo-cracking methods, e.g. using solid $\mathrm{CO}_{2}$. Design and material composition of items should be assessed to determine suitability of individual items for cryo-cracking. This method could be scaled up for industrial use by freezing multiple items simultaneously and feeding them into an automated process to shatter casings while limiting losses of CRM bearing components. A jaw crusher may be a good solution. ${ }^{220}$

\section{CRM recovery potential}

Li-Ion batteries - The inability to derive additional value from LIBs has been discussed previously with regard to laptop battery packs. Co content for LIB types encountered in samples as determined by AAS is given in Table 4.19.

Table 4.19 Cobalt content of Li-ion batteries

\begin{tabular}{lcc}
\hline \multirow{2}{*}{ Battery } & \multicolumn{2}{c}{ Co content } \\
\cline { 2 - 3 } & $(\mathrm{g})$ & $\mathrm{Wt} \%$ \\
\hline Cylinder Li-ion & $8.30( \pm 0.01)$ & $18.94( \pm 0.02)$ \\
MP3 packet Li-ion & $2.46( \pm 0.02)$ & $20.8( \pm 0.2)$ \\
Mobile phone packet Li-ion & $4.17( \pm 0.01)$ & $19.44( \pm 0.07)$ \\
\hline
\end{tabular}

Speaker magnets - ICP-MS analysis of the magnets showed each contains $133( \pm 3) \mathrm{mg}$ of $\mathrm{Nd}$, equivalent to $27.9( \pm 0.7) \mathrm{wt} \%$. This concentration is comparable with NIB HDD magnets from notebook computers reported by the Öko-Institut e.V. ${ }^{19}$ No commercial recovery route for these items could be found, and a great deal of time and effort is required to isolate them from 
casings of the device. As discussed previously, at the time of the trial (2012-13) there was little chance of recovery of $\mathrm{Nd}$ because of a lack of available recovery infrastructure.

Screens - As previously discussed, at the time of thia study no organizations were operating recovery processes targeting indium in LCDs. Since the time of this trial, a cross-current leaching method has been developed by Rocchetti et al., which achieves economic recovery of In from LCDs. ${ }^{221,222}$ Dissolution of ITO from multiple screens in a single volume of nitric acid enables an economic quantity for recovery to be collected. How close this process is to commercialisation is unclear, and what quantity of screens would need to be amassed to ship for refining in Italy is also unknown. It is likely that limitations of scale will mean that Metech continue to send LCDs to specialist processors in the UK, who may then send larger quantities to this process once commercialised. SMDs and gold connectors on the 'flexible PCB' type connectors of screens can be included with PCBs for downstream recovery.

PCBs - Most waste PCBs are smelted to recover $\mathrm{Cu}$, PMs and PGMs. There are economic and thermodynamic limitations to viable recovery of other CRMs in this process. The way in which metals partition between the liquid copper and slag phases during smelting is important in determining recycling potential. ${ }^{118}$ Metals which do not dissolved in liquid copper have no chance of recovery in subsequent refining processes. Umicore's integrated smelting facility in Hoboken, Belgium is the most advanced plant of its kind, and in addition to the normal metals recovered $\mathrm{Sb}$ and In are target metals for recovery. Many of the PCBs from the UK find their way to this process, although there is competition for material between European and Asian refiners, and In and Sb may not be recovered from material sent to alternative recovery processes. Co, Ga, Ge, Y, Ta and $\mathrm{W}$ in PCBs are not recovered. For these reasons, there is little prospect of recovering CRMs from PCBs other than PMs, PGMs, Sb and In.

For CRMs in PCB components which are not targeted in recovery, isolation of these components from $\mathrm{PCBs}$ prior to smelting is the only viable option to prevent loss of these materials, and improve prospects for their eventual recovery. This strategy has been advocated by others for recovering CRMs from LEDs, Ta-capacitors, and Y from MLCCs. ${ }^{207,} 223$ Correspondence with Delta Products, professionals dealing in minor metals recycling, revealed that a market exists for recovery of Ta from capacitors; however this is only viable for minimum quantities of $500 \mathrm{~kg} .{ }^{224}$ It was advised that the value of such a material would be equivalent to that of tantalite ore - $£ 40-50 \mathrm{k}$ per tonne at the time of the trials. Ta capacitors weigh $\sim 0.25 \mathrm{~g}$ each, meaning $\sim 2$ million capacitors would have to be isolated from PCBs to achieve a viable quantity for refining, requiring $\sim 2,700$ man hours at a cost of $\sim £ 44 \mathrm{k}(\sim £ 88 \mathrm{k} / \mathrm{t})$ to Metech. This strategy is not economically viable for Metech using manual isolation.. 
Capacitor Grade Ta powder is more valuable, $\sim £ 100 \mathrm{k} / \mathrm{t}$. If a process of isolating Ta from capacitors could be established, this may provide sufficient value to justify their isolation. Automation could also reduce costs of isolating capacitors, improving the economics of recovery. The process of isolating $500 \mathrm{~kg}$ of capacitors would take years at Metech with a long delay to any financial return seen given levels of material throughput at the time of the trial. This would be detrimental to cash-flow, and so such a process is unlikely to be adopted my recyclers operating at similar scales. Prospects for Ta recovery are better in organisations dealing with larger volumes of PCBs at high throughput, such as refiners, where isolation of Ta-capacitors from PCBs prior to smelting is a potential pathway to enhanced CRM recovery rates and avoid dissipative losses in recycling.

There is no economic justification for adopting equivalent processes to isolate LEDs (which contain Ga, REMs \& PMs), or MLCCs (which contain Y), because no viable recovery options exist. It is important to point out that where SMDs contain both target and non-target materials of smelting processes, a dichotomy exists between the requirement to retain CRMs within the economy, and the desire to derive the maximum possible economic value from WEEE. Stockpiling of SMDs containing non-target CRMs of existing smelting processes in anticipation of a possible future recovery solution, when components have a high derivable value through smelting is not sensible for recyclers when the vast majority of material value can be exploited in current processes.

\section{PCB component isolation methods}

CRMs are often found in surface components and edge contacts of PCBs. Enhancing the value derived from recovery of these materials may be possible by isolating them from PCBs, thus concentrating them into a lower mass fraction. This will reduce refining costs (see Section 1.5 re refining costs). It is also generally true that recovery efficiency increases with target material concentration. ${ }^{106}$

\section{Contacts}

Contacts soldered to boards - Manual isolation of gold contacts from the PCB and housing of the Nokia 6230i with pliers was achieved in 60 seconds at a cost of $£ 0.26$ (Figure 4.61). AAS analysis revealed the contacts contained $3.82( \pm 0.04) \mathrm{mg}$ of gold in total and the isolated contacts fraction contains $\sim 1300 \mathrm{ppm}$ of Au. 

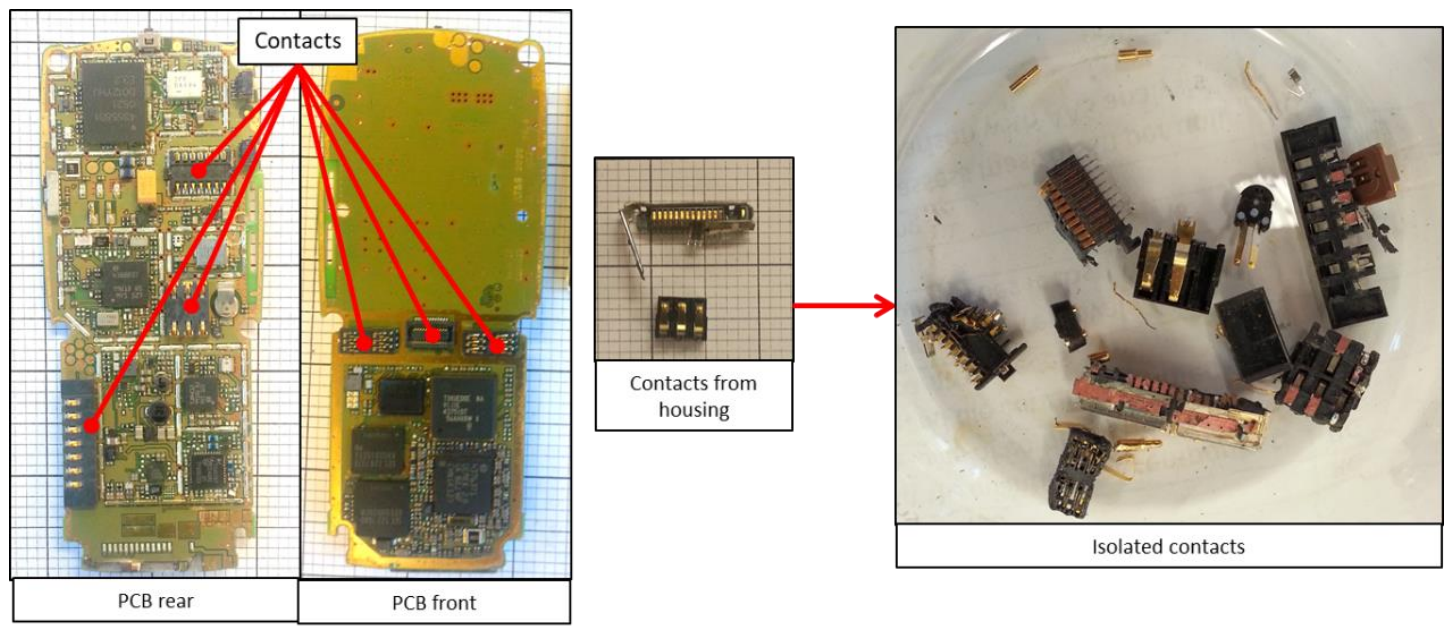

Figure 4.61 Isolation of contacts from Nokia 6230i mobile phone PCB and housing

$\underline{P C B}$ edge contacts - Both the guillotine and the tile cutter were used to separate the contacts deposited directly onto the PCB of a series of samples -graphics cards, TV LCD PCBs, server boards and Ethernet cards (Figure 4.62). The quantities of gold \& copper found in each were analysed with AAS to establish their value (Table 4.20).

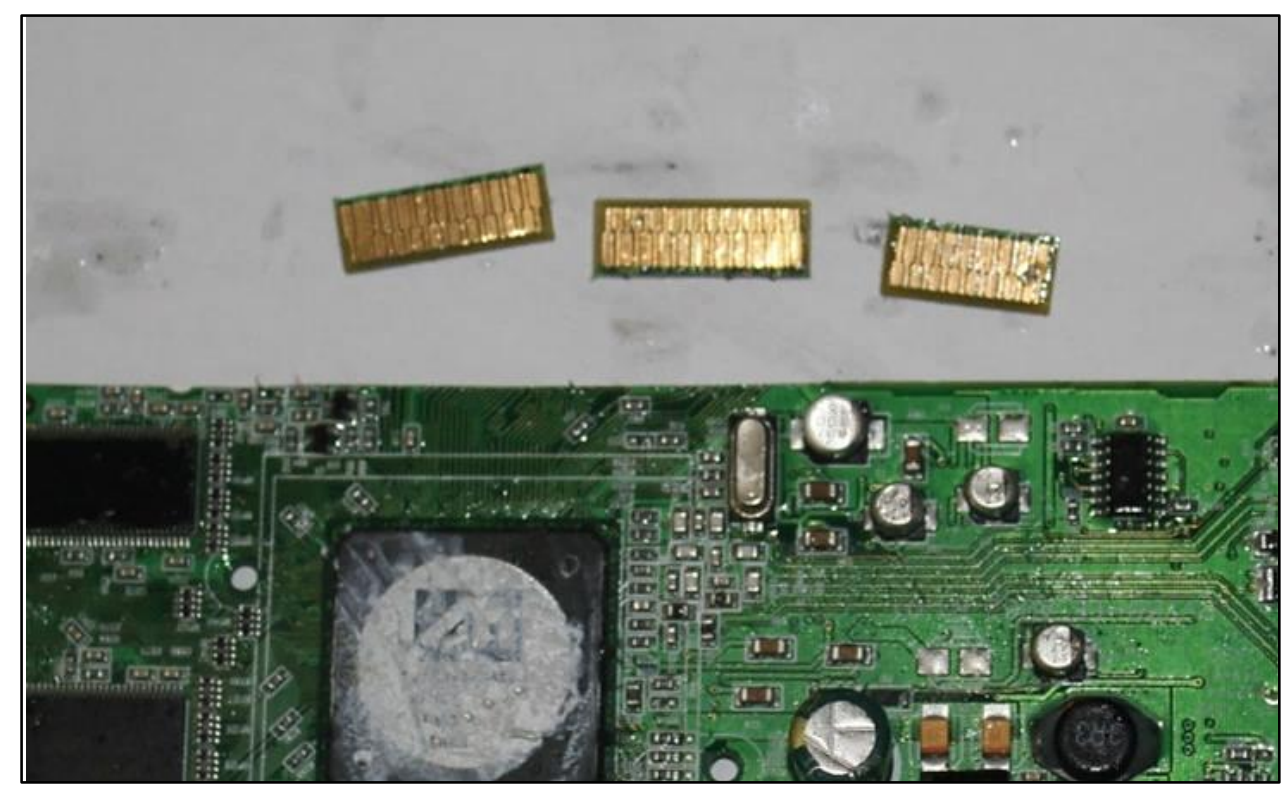

Figure 4.62 Gold edge connectors removed from graphics card

Table 4.20 Gold and copper quantity in samples and the value of these metals (based on market values, not revenue received from refiners)

\begin{tabular}{lccc}
\hline Contacts from item & Au content $(\mathbf{m g})$ & Cu content $(\mathbf{m g})$ & Total value $(\mathfrak{£})$ \\
\hline TV LCD PCB & 1.16 & 1363 & 0.045 \\
Ethernet card & 4.11 & 231 & 0.137 \\
Server board & 22.2 & 408 & 0.736 \\
Graphic card & 2.95 & 63.2 & 0.098 \\
\hline
\end{tabular}


Guillotine - Table 4.21 shows times takes to cut the edge connector from a board and the costs of carrying out this operation. The method allowed rapid isolation of gold coated sections of PCBs and was a good tool for large mass high volume sections where the high value parts on boards such as edge connectors can be easily removed and bulked together. PCB guillotines are readily available. This process could possibly be automated by using image recognition of the gold edge connectors to align them with a guillotine blade.

Table 4.21 Time to isolate gold edge contacts from PCB devices using a guillotine and cost of operation

\begin{tabular}{ccc}
\hline PCB & $\begin{array}{c}\text { Contacts separation time } \\
\text { per board (s) }\end{array}$ & Cost per board (£) \\
\hline TV LCD PCB & 8 & 0.0352 \\
Graphics card & 4 & 0.0176 \\
Server board & 6 & 0.0264 \\
Ethernet card & 4 & 0.0176 \\
\hline
\end{tabular}

Wet Tile cutter - Despite this being a powered cutter it still took as long to remove edge contacts as the guillotine (Table 4.22). The tile cutter allows more intricate cutting of boards than the guillotine, and will cut through boards over $2 \mathrm{~mm}$ thick and $200 \mathrm{~mm}$ wide. However, this method presents more health and safety issues than the guillotine as it is dangerous for the user if care is not taken; it is noisy and the waste water will contain a slurry of toxic PCB dust which will have to be properly disposed of. It also has higher investment and running costs: The cutter costs $£ 400$, and replacement blades are £25-35 each. The power consumption during cutting was $880 \mathrm{~W}$.

Table 4.22 Time to isolate gold edge contacts from PCB devices using a wet tile cutter and cost of the operation (based on energy demand and time of process)

\begin{tabular}{ccc}
\hline PCB & Contacts separation time $(\mathbf{s})$ & Cost per board $(\mathfrak{f})$ \\
\hline TV LCD PCB & 8 & 0.037 \\
Graphics card & 4 & 0.019 \\
Server board & 6 & 0.028 \\
Ethernet card & 4 & 0.019 \\
\hline
\end{tabular}

The Rubi ND-200 wet saw selected is suitable for industrial use and has the following advantages: It has a cutting table large enough to accommodate larger boards than the guillotine; clearance between the cutting table and the cutting wheel guard is adjustable and sufficient to allow it to cut around and/or through the majority of components found on PCBs; 
the water supresses the generation of PCB dust and it can be topped up with a jug or hose rather than requiring permanent plumbing; and spare parts are readily available.

On a commercial scale, areas identified as containing concentrations of target materials could be cut away from the rest of the PCB using a guillotine, wet circular saw, laser, snips, a punch or a water jet. It is likely that this method would be used in conjunction with manual selective disassembly to speed up the process.

\section{SMDs}

Manual isolation - Manual selective disassembly of SMDs from PCBs involved no specialist equipment or initial capital outlay. Standard tools were used to successfully scrape or pull SMDs from PCBs. The results of times taken for removal of all surface components from MP3 player and LCD PCBs are given in Table 4.23. This method, used in conjunction with the image library would allow quick identification and 'cherry picking' of CRM bearing SMDs from PCBs prior to sending PCBs for recovery. This would be particularly advantageous for recovery of Ta capacitors, and components containing other CRMs such as Ge and Ga which are lost in smelting processes. However, as a means of depopulating entire PCBs, the method is time consuming and costly. For this reason, the method was only tested on two samples before being abandoned.

Table 4.23 Time taken for manual removal of components from PCBs and process cost

\begin{tabular}{lcc}
\hline PCB & Time taken $(\mathbf{s})$ & Cost of process $(\mathfrak{£})$ \\
\hline MP3 player main PCB & 240 & 1.06 \\
LCD main PCB & 600 & 2.64 \\
\hline
\end{tabular}

\section{De-soldering of PCBs (thermal methods)}

Blow Torch - This method has low running costs: The Dremel Versaflam was available from $£ 30$ and the butane gas fuel is available in $200 \mathrm{ml}$ canisters at a cost of approximately $£ 2$. The cost of gas is likely to be a low operational cost in any scaled-up process as a canister refills the Versaflame many times, which was not necessary over the duration of the trial. However, it was difficult to avoid burning boards with this method which presents a H\&S issue. In addition, removing components from a whole board with this method was time consuming, requiring operators to slowly work their way across PCB surfaces to remove all SMDs. Results of time trials on PCBs using this method and the associated costs of the process are shown in Table 4.24. 
Table 4.24 Time taken for isolation of components from PCBs and process cost

\begin{tabular}{lcc}
\hline PCB & Time taken $(\mathbf{s})$ & Cost of process $(\mathfrak{E})$ \\
\hline MP3 player main PCB & 36 & 0.1584 \\
Mobile Phone & 120 & 0.5279 \\
Camera & 266 & 1.170 \\
LCD main PCB & 60 & 0.2640 \\
\hline
\end{tabular}

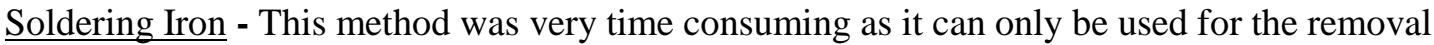
of one component at a time. Therefore, it is not a viable option to recover components for recycling on an industrial scale.

Paint Stripping Gun - Typically, the $2 \mathrm{~kW}$ heat gun took $15-30 \mathrm{~s}$ to reach a sufficient temperature to soften solder. Solder around individual components took a further 3-5 seconds to soften sufficiently to remove them. A disadvantage of this method was that the temperature of the gun could not be set and therefore over-heating and burning of SMDs and PCBs may occur, liberating hazardous emissions and posing a H\&S issue. Running costs were high compared to other thermal methods, and the process was time consuming (Table 4.25).

Table 4.25 Time taken for isolation of components from PCBs, energy demand and process cost using a paint stripping gun.

\begin{tabular}{|c|c|c|c|}
\hline PCB & Time taken (s) & Energy cost (£) & Cost of process (f) \\
\hline MP3 player main $\mathrm{PCB}$ & 60 & 0.003182 & 0.2671 \\
\hline Mobile Phone & 180 & 0.009546 & 0.8014 \\
\hline Camera & 300 & 0.01591 & 1.336 \\
\hline LCD main PCB & 120 & 0.006364 & 0.5343 \\
\hline
\end{tabular}

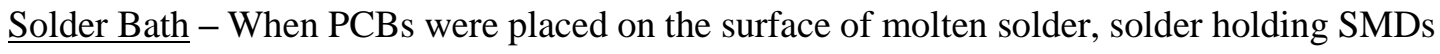
to PCBs softened sufficiently for component removal in 10-20 s. This method could also be used with double sided boards as the majority of SMDs on the underside of PCBs in contact with the liquid solder surface remained in place until removed from the solder bath. Those that did detach remained floating on the surface of the molten solder and were easily sieved out again. Table 4.26 shows times taken to remove SMDs from PCBs, cost of energy consumed and the total cost of the process.

During use, the solder bath used $500 \mathrm{~W}$. It allowed rapid removal of components from a PCB without destroying them. The solder bath system could be scaled up for industrial use as there are many larger sizes available. Today, component removal from PCBs is a common practice 
on manual recycling lines in China. With a larger bath a greater number of boards could be processed simultaneously, potentially reducing operational costs. Automation of the process could potentially increase the cost benefit and speed. However, the equipment cost ( $£ 3 \mathrm{k})$ and increased energy required to heat a larger volume of solder $(\sim 3-5 \mathrm{~kW})$ would need to be taken into account. The solder cost will be dependent upon the solder type. Lead solder can cost up to $£ 40 / \mathrm{kg}$, which should last for hundreds of boards. It would require occasional topping up of the solder during operation.

Table 4.26 Time for isolation of SMDs from the surfaces of PCBs using a solder bath

\begin{tabular}{lccc}
\hline PCB & Time taken $(\mathbf{s})$ & Energy cost $(\mathfrak{£})$ & Cost of process $(\mathfrak{f})$ \\
\hline MP3 player main PCB & 15 & 0.000199 & 0.0662 \\
Mobile Phone & 20 & 0.000265 & 0.0883 \\
Camera & 20 & 0.000265 & 0.088 \\
LCD main PCB & 15 & 0.000199 & 0.0662 \\
\hline
\end{tabular}

NIR oven - The NIR oven used was successful at softening solder for removal of SMDs from PCBs. However, difficulty was encountered when attempting to find the correct balance of power and travel rate through the overn to soften solder without burning PCBs, which results in toxic emissions and environmental H\&S issues. Further research would be necessary to optimize the operational parameters to soften solder without burning boards, and it is likely that these setting will vary depending on the alloy of solder employed on boards and the IR absorbing nature of the PCB substrate. For this reason, the method was found to be unsuitable for adoption by Metech. The high throughput possible lends itself to adoption at industrial scale however, and although outside the scope of this project, with further research, NIR ovens could be adopted for large scale depopulation of waste PCBs. The nominal power of the NIR investigated was $25 \mathrm{~kW}$. The machine cost $£ 25 \mathrm{k}$ plus installation costs, e.g. extractor fans, fume hoods, and safety curtains.

Value of isolated components - To determine cost benefit of component isolation with each technique it was necessary to analyse the samples of isolated components from each PCB and establish their value. Isolated components from PCBs were therefore sent to BASF metals recycling (Cinderford) where Metech were sending PMR materials for refining. BASF conducted precious metal assay on the materials, and provided the results and refining terms from which a value for each batch of isolated components was calculated (Table 4.27). 
Table 4.27 Value of isolated components from PCB samples when sent for refining

\begin{tabular}{|c|c|c|c|c|c|c|c|c|}
\hline \multirow{2}{*}{$\begin{array}{c}\text { Source of } \\
\text { components }\end{array}$} & \multirow{2}{*}{$\begin{array}{l}\text { Mass } \\
(\mathrm{g})\end{array}$} & & \multicolumn{4}{|c|}{ Metal } & \multirow{2}{*}{ Total } & \multirow{2}{*}{$\begin{array}{c}\text { Treatment } \\
\text { Charge }(£ / t)\end{array}$} \\
\hline & & & Au & Ag & Pd & $\mathbf{C u}$ & & \\
\hline \multirow{7}{*}{ MP3 player } & \multirow{7}{*}{2.8169} & Recovery efficiency & $98 \%$ & $95 \%$ & $85 \%$ & $98 \%$ & & \multirow{7}{*}{1,800} \\
\hline & & Refining Charge $(£ / k g)$ & 200 & 25 & 475 & - & & \\
\hline & & Metal content $(\mathrm{Wt} \%)$ & 0.16 & 0.29 & 0.011 & 10.8 & & \\
\hline & & Metal content $(\mathrm{g} / \mathrm{t})$ & 1,600 & 2,900 & 110 & 108,000 & & \\
\hline & & Value of contained metal $(£ / t)$ & 57,544 & 2,015 & 1,579 & 590 & 61,728 & \\
\hline & & Refining charge $(£ / t))$ & 320 & 72.5 & 52.25 & - & 444.75 & \\
\hline & & Value of recovered metal $(£ / t)$ & 56,393 & 1,914 & 1,342 & 578 & 60,228 & \\
\hline \multirow{5}{*}{ Mobile phone } & \multirow{5}{*}{3.4114} & Metal content $(\mathrm{Wt} \%)$ & 0.146 & 0.131 & 0.066 & 16 & & \multirow{5}{*}{1,800} \\
\hline & & Metal content $(\mathrm{g} / \mathrm{t})$ & 1,460 & 1,310 & 660 & 160,000 & & \\
\hline & & Value of contained metal $(£ / t)$ & 52,509 & 910 & 9,475 & 873 & 63,768 & \\
\hline & & Refining charge $(£ / t))$ & 292 & 32.75 & 314 & - & 638.25 & \\
\hline & & Value of recovered metal $(£ / t)$ & 51,459 & 865 & 8,054 & 856 & 61,234 & \\
\hline \multirow{5}{*}{ Camera } & \multirow{5}{*}{2.1410} & Metal content $(\mathrm{Wt} \%)$ & 0.047 & 0.08 & 0.004 & 24.7 & & \multirow{5}{*}{1,800} \\
\hline & & Metal content (g/t) & 470 & 800 & 40 & 247,000 & & \\
\hline & & Value of contained metal $(£ / t)$ & 16,903 & 556 & 574 & 1,349 & 19,382 & \\
\hline & & Refining charge $(£ / t))$ & 94 & 20 & 19 & - & 133 & \\
\hline & & Value of recovered metal $(£ / t)$ & 16,566 & 528 & 488 & 1,322 & 18,903 & \\
\hline \multirow{5}{*}{ LCD PCB } & \multirow{5}{*}{1.288} & Metal content $(\mathrm{Wt} \%)$ & 0.115 & 0.29 & 0.014 & 19.6 & & \multirow{5}{*}{1,800} \\
\hline & & Metal content $(\mathrm{g} / \mathrm{t})$ & 1,150 & 2,900 & 140 & 196,000 & & \\
\hline & & Value of contained metal $(£ / t)$ & 41,360 & 2,015 & 2,010 & 1,070 & 46,455 & \\
\hline & & Refining charge $(£ / t))$ & 230 & 72.5 & 66.5 & - & 369 & \\
\hline & & Value of recovered metal $(£ / t)$ & 40,533 & 1,914 & 1,708 & 1,049 & 45,204 & \\
\hline
\end{tabular}

Market values of metals used: $\mathrm{Au}=£ 35.9652 / \mathrm{g} ; \mathrm{Ag}=£ 0.6949 / \mathrm{g} ; \mathrm{Pd}=£ 14.3566 / \mathrm{g} ; \mathrm{Cu}=£ 5.46 / \mathrm{kg}$. Terms of refining: $£ 1,800 / \mathrm{t}$ treatment charg then made on recovered metals (metal content of material x recovery efficiency) less refining charges for Au, Ag and Pd content of material 


\section{Methods used to concentrate CRM content in the isolated components}

\section{Microwave ashing}

Dry ashing procedures using traditional muffle furnaces are time consuming, requiring hours to fully ash samples. When plastic/resin samples are to be treated, a pre-ashing process is also necessary which adds to process costs. However, microwave ashing uses microwave absorbing silicon carbide elements and samples can be ashed in less than an hour with no pre-treatment necessary. For this reason, ashing of isolated CRM bearing samples was trialled with isolated edge contacts from PCB samples. There are numerous suppliers of microwave muffle furnaces which cost in the region of $£ 10-15 \mathrm{k}$ depending on required capacity.

Gold edge contacts isolated from PCBs using the guillotine and tile cutter were ashed (Figure 4.63). The mass reduction achieved and corresponding savings in treatment charges at the refinery are given in Table 4.28. Au \& $\mathrm{Cu}$ content of isolated contact samples was determined by AAS, the results of which with calculated value increase for Metech associated with ashing 1 tonne of each based on BASF's refining terms are given in Table 4.29.

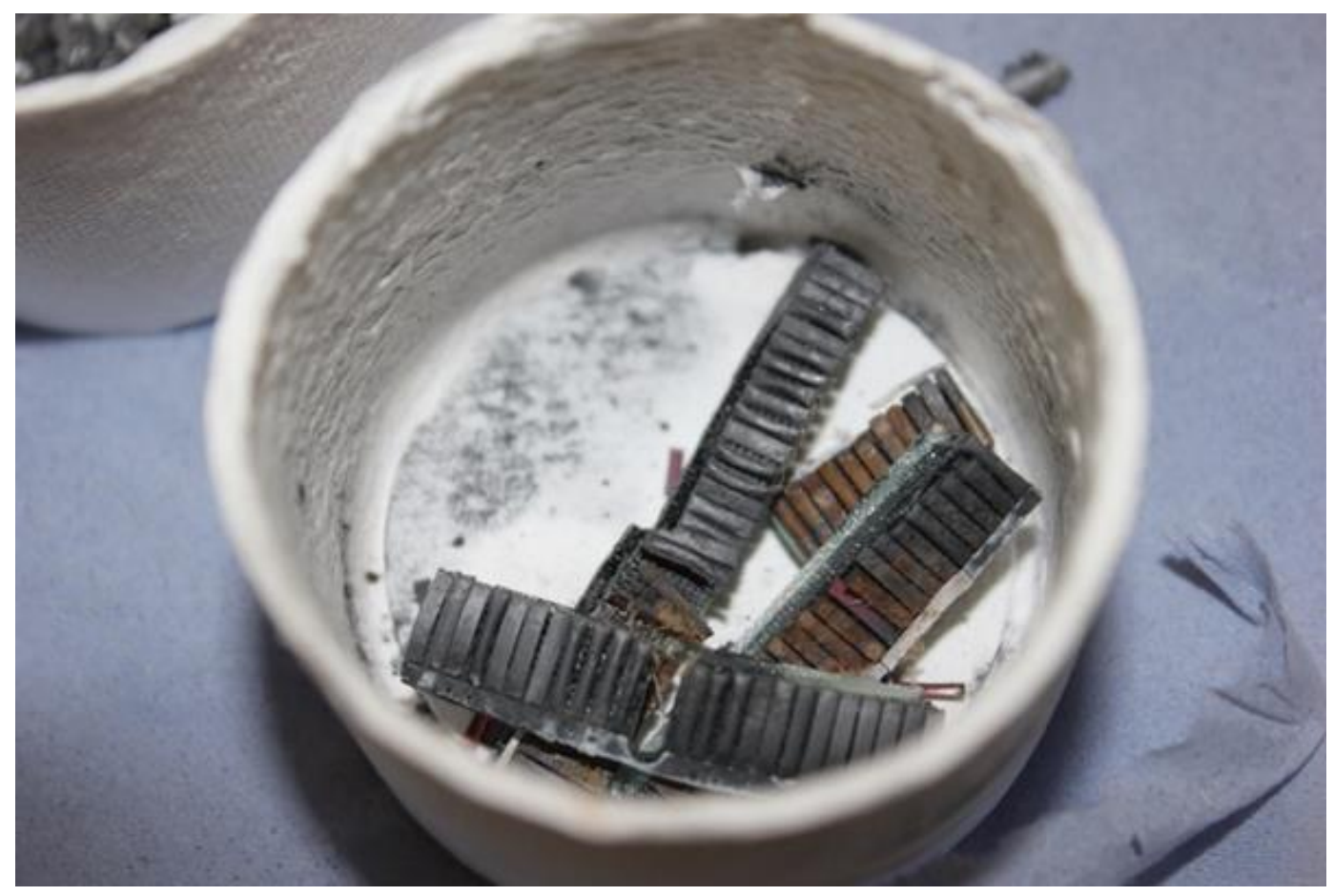

Figure 4.63 Ashed gold edge contacts isolated from graphics card PCB 
Table 4.28 Mass reductions and savings on treatment charges achieved for materials with microv

\begin{tabular}{lccccr}
\hline Samples & $\begin{array}{c}\text { Mass } \\
(\mathrm{g})\end{array}$ & $\begin{array}{c}\text { Ashed } \\
\text { sample } \\
\text { mass }(\mathrm{g})\end{array}$ & $\begin{array}{c}\text { Mass } \\
\text { reduction } \\
(\mathrm{g})\end{array}$ & $\begin{array}{c}\text { \% of } \\
\text { original } \\
\text { mass }\end{array}$ & $\begin{array}{c}\text { Saving } \\
\text { treatm } \\
\text { charge }\end{array}$ \\
\hline TV board contacts & 3.3804 & 2.7471 & 0.6333 & $81 \%$ & 337 \\
Graphics card contacts & 1.752 & 1.1705 & 0.5815 & $67 \%$ & 597 \\
Server board contacts & 3.108 & 1.7329 & 1.3751 & $56 \%$ & 796 \\
Ethernet Card contacts & 1.701 & 0.8293 & 0.8717 & $49 \%$ & 922 \\
\hline
\end{tabular}

Table 4.29 Value gained from microwave ashing of 1 tonne of isolated contacts from samp

\begin{tabular}{|c|c|c|c|c|c|c|c|}
\hline Sample & $\begin{array}{c}\text { Mass } \\
(\mathrm{t})\end{array}$ & $\begin{array}{l}{[\mathrm{Au}]} \\
(\mathrm{ppm})\end{array}$ & $\begin{array}{l}{[\mathrm{Cu}]} \\
(\mathrm{kg} / \mathrm{t})\end{array}$ & $\begin{array}{c}\text { Refining } \\
\text { charges }(£)\end{array}$ & $\begin{array}{l}\text { Recovered Au } \\
\text { value }(\mathfrak{f})\end{array}$ & $\begin{array}{c}\text { Recovered Cu } \\
\text { value }(£)\end{array}$ & $\begin{array}{c}\text { Total charge } \\
(£)\end{array}$ \\
\hline TV board contact & 1 & 345 & 403 & 69 & 12,151 & 2,157 & 1,869 \\
\hline Ashed TV board contacts & 0.812 & 424 & 496 & 69 & 12,151 & 2,157 & 1,532 \\
\hline Graphics card contacts & 1 & 2,350 & 132 & 470 & 82,812 & 706 & 2,270 \\
\hline Ashed graphics card contacts & 0.668 & 3,517 & 198 & 470 & 82,812 & 706 & 1,672 \\
\hline Server board contacts & 1 & 7,158 & 131 & 1,432 & 252,289 & 702 & 3,232 \\
\hline Ashed server board contacts & 0.557 & 12,838 & 235 & 1,432 & 252,289 & 702 & 2,435 \\
\hline Ethernet card contacts & 1 & 1,734 & 37.2 & 347 & 61,126 & 199 & 2,147 \\
\hline Ashed ethernet card contacts & 0.487 & 3,557 & 76.2 & 347 & 61,126 & 199 & 1,224 \\
\hline
\end{tabular}


The CEM microwave ashing oven costs $£ 10-12 \mathrm{k}$. Each run could hold $50 \mathrm{~g}$ of ashable material and consumed $1.06 \mathrm{kWh}$ of electricity. The process significantly reduced volumes and masses of boards which could result in significant logistics savings when transporting materials to smelters in Europe. However, smelters will only accept minimum loads of $500 \mathrm{~kg}$ for refining, which could require ashing of $>285,000$ graphics card edge connectors and result in considerable expenditure before a return on processed material is received from refiners. This would be detrimental to healthy cash flow, particularly for smaller recyclers. This process also requires ventilation for the release of smoke which presents potential environmental and H\&S issues. Any industrial process based on microwave ashing will require investment in a scrubbing system for removal of hazardous compounds evolved in the process, adding considerably to the capital investment required.

\section{$\underline{\text { Sieving }}$}

The results of sieving are indicated in Table 4.30. All fractions produced were predominantly composed of PM and PGM bearing components in which these metals represent the most significant value by far. Each of the fractions can be recovered in the same downstream PM recovery process (either hydrometallurgical or pyrometallurgical). It is interesting to note that Ta capacitors are isolated in the 9.52-2.80 $\mathrm{mm}$ fraction. Were a viable downstream recovery option available for Ta in these components, then this seems a promising way of isolating them from large volumes of mixed SMDs. Although included in a fraction with PM rich components, the process of isolating the Ta-capacitors from these fractions may be easier than attempting to sort them from all components. This sorting may be possible with automated optical recognition systems, or XRF based intelligent sorting systems. The size range of components in this fraction is also relatively large, and use of more sieves with mesh sizes within this range may result in greater concentration of Ta within a narrower size range fraction. Loading the stack of sieves with SMDs and shaking for 60s incurrs a cost of $£ 0.13$. Further investigation into the value per unit mass of each fraction may reveal a cost benefit to the process if fractions of significantly different grade can be generated, through avoidance of treatment charges at refineries on higher grade fractions. 
Table 4.30 SMDs and CRMs contained in sieved fractions

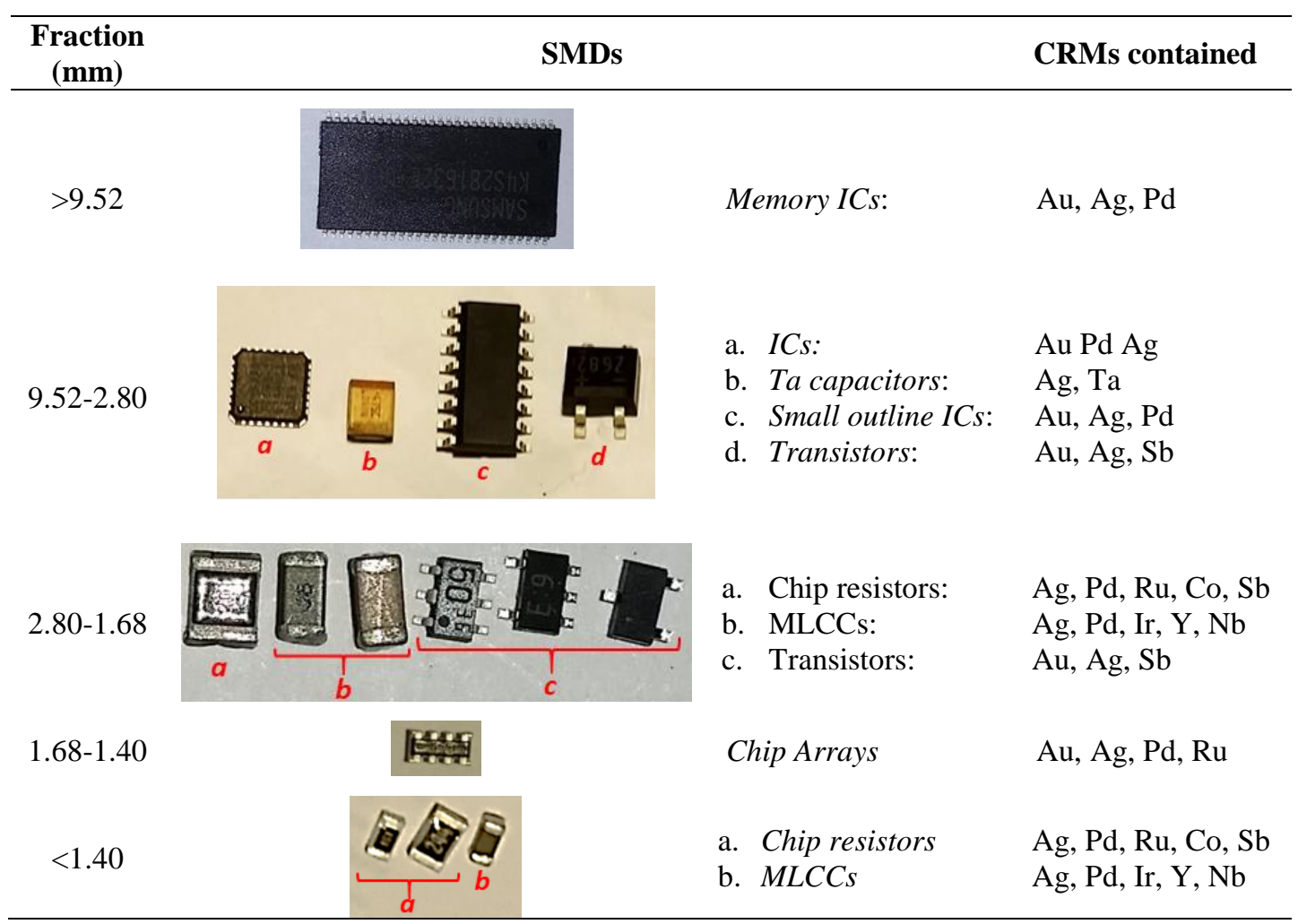

\subsubsection{Cost Benefit Analysis}

\section{Isolating the Gold Contacts from Boards}

CBA was conducted for methods used to isolate the gold edge contacts of PCBs generated as part of Metech's standard manual disassembly operations. The components considered were: TV main PCBs, graphics cards, server boards and ethernet cards. The guillotine and tile cutting operations were analysed alone, and in combination with the microwave ashing processes used to concentrate value in the contacts. The results are presented in Table $4.31 \& 4.32$.

The increase in revenue seen for materials as a result of reduced mass based treatment charges at the refinery does not compensate for the costs of electricity and labour incurred by ashings. 
Table 4.31 No of each PCB sample investigated required to generate $500 \mathrm{~kg}$ of ash from contacts, calculated costs of isolating cont received for this material based on BASF refining charges (issued to Metech in 2012).

\begin{tabular}{|c|c|c|c|c|c|c|}
\hline \multirow{2}{*}{ PCB } & \multirow{2}{*}{$\begin{array}{c}\text { No } \\
\text { processed }\end{array}$} & \multirow{2}{*}{ Operation } & \multicolumn{3}{|c|}{ Costs (f) } & \multirow{2}{*}{$\begin{array}{c}\text { Revenue for mat } \\
(\mathfrak{f})\end{array}$} \\
\hline & & & Labour & Power & Total & \\
\hline TV board contact & 182,010 & Guillotine separation & 6,406 & - & 6,406 & 7,653 \\
\hline Graphics card contacts & 427,168 & Guillotine separation & 7,517 & - & 7,517 & 60,806 \\
\hline Server board contacts & 288,534 & Guillotine separation & 7,616 & - & 7,616 & 223,975 \\
\hline Ethernet Card contact & 602,918 & Guillotine separation & 10,610 & - & 10,610 & 60,691 \\
\hline TV board ce & 182,010 & Tile cutte & 6,406 & 33.98 & 6,440 & 7,653 \\
\hline Graphics card contacts & 427,168 & Tile cutter separation & 7,517 & 39.87 & 7,557 & 60,806 \\
\hline Server board contacts & 288,534 & Tile cutter separation & 7,616 & 40.40 & 7,657 & 223,975 \\
\hline Ethernet Card contact & 602,918 & Tile cutter separation & 10,610 & 56.28 & 10,666 & 60,691 \\
\hline
\end{tabular}

* does not consider the difference in revenues obtained from material flows of whole samples with contacts attached, and separate

Table 4.32 Cost-benefit analysis of isolating and ashing contacts to produce $500 \mathrm{~kg}$ of ash from the quantities of analyse

\begin{tabular}{|c|c|c|c|c|c|c|c|}
\hline \multirow{2}{*}{ PCB } & \multirow{2}{*}{ Operation } & \multirow{2}{*}{$\begin{array}{c}\text { Units } \\
\text { processed }\end{array}$} & \multicolumn{3}{|c|}{ Ashing costs (f) } & \multirow{2}{*}{$\begin{array}{c}\text { Total } \\
\text { Cost }(\mathfrak{f})\end{array}$} & \multirow{2}{*}{$\begin{array}{l}\text { Reve } \\
\text { mate }\end{array}$} \\
\hline & & & Labour & Power & Total & & \\
\hline TV board contact & Guillotine separation + ashing & 182,010 & 6,496 & 1,245 & 7,742 & 14,147 & \\
\hline Graphics card contacts & Guillotine separation + ashing & 427,168 & 7,902 & 1,515 & 9,417 & 16,934 & \\
\hline Server board contacts & Guillotine separation + ashing & 288,534 & 9,469 & 1,815 & 11,283 & 18,900 & \\
\hline Ethernet Card contact & Guillotine separation + ashing & 602,918 & 10,829 & 2,075 & 12,904 & 23,514 & \\
\hline TV board contact & Tile cutter separation & 182,010 & 6,496 & 1,245 & 7,742 & 14,181 & \\
\hline Graphics card contacts & Tile cutter separation & 427,168 & 7,902 & 1,515 & 9,417 & 16,974 & \\
\hline Server board contacts & Tile cutter separation & 288,534 & 9,469 & 1,815 & 11,283 & 18,940 & \\
\hline Ethernet Card contact & Tile cutter separation & 602,918 & 10,829 & 2,075 & 12,904 & 23,570 & \\
\hline
\end{tabular}




\section{Disassembly of items for isolation of PCBs and SMDs}

Costs associated with disassembly of samples to isolate PCBs are given in Table 4.33 Manual disassembly is prohibitively expensive. The guillotine is much cheaper than the tile cutter for opening LCD housing.

Table 4.33 Costs for isolation of PCBs from items using various disassembly techniques

\begin{tabular}{lcccc}
\hline Item & Manual Disassembly & Cryo-cracking & Guillotine & Tile cutter \\
\hline MP3 player & $£ 0.46$ & $£ 0.10$ & - & - \\
Mobile phone & $£ 0.38$ & $£ 0.10$ & - & - \\
Camera & $£ 0.73$ & $£ 0.10$ & - & - \\
LCD PCB & $£ 1.09$ & - & $£ 0.29$ & $£ 0.68$ \\
\hline
\end{tabular}

The costs for isolation of SMDs from PCB samples are given in Table 4.34. The least expensive method by far is use of the solder bath. This method is also fastest with best outcome in terms of damage caused to components resulting in CRM losses.

The most cost-effective method for isolating components from whole samples is to use cryocracking for digital media devices with plastic housings, the guillotine for the HP laptop LCDs, and the solder bath for removal of SMDs. Combining the costs calculated so far with the calculated revenues for isolated SMDs from each device provides the total cost benefit of this process for the samples examined (Table 4.35). The proposed processes would only be cost effective for use on mobile phones, giving a cost benefit of $\sim £ 3 \mathrm{k} /$ tonne of isolated SMDs, or $\sim 10$ p/phone. The higher value of phone components being sufficient to recoup costs incurred. Careful consideration of the recoverable value in mobile phones of different models should also be taken into account before the decision is made to utilise this process on any one particular model. 
Table 4.34 Per item costs of isolating SMDs from PCBs with various techniques

\begin{tabular}{|c|c|c|c|c|c|c|c|c|c|c|}
\hline \multirow[b]{2}{*}{ PCB } & \multicolumn{2}{|c|}{ Manual disassembly } & \multicolumn{2}{|c|}{ Blow torch } & \multicolumn{3}{|c|}{ Heat Gun } & \multicolumn{3}{|c|}{ Solder bath } \\
\hline & $\begin{array}{c}\text { Time } \\
(\mathrm{s})\end{array}$ & $\begin{array}{c}\text { Cost } \\
(\mathfrak{f})\end{array}$ & $\begin{array}{c}\text { Time } \\
\text { (s) }\end{array}$ & $\begin{array}{c}\text { Cost } \\
(\mathfrak{f})\end{array}$ & $\begin{array}{c}\text { Time } \\
\text { (s) }\end{array}$ & $\begin{array}{c}\text { Energy } \\
(\mathbf{k W h})\end{array}$ & $\begin{array}{c}\text { Cost } \\
(\mathfrak{f})\end{array}$ & $\begin{array}{c}\text { Time } \\
\text { (s) }\end{array}$ & $\begin{array}{c}\text { Energy } \\
\text { (Wh) }\end{array}$ & \\
\hline MP3 player & 240 & 1.06 & 36 & 0.16 & 60 & 0.033 & 0.27 & 15 & 2.08 & \\
\hline Mobile phone & 600 & 2.64 & 120 & 0.53 & 180 & 0.100 & 0.80 & 20 & 2.78 & \\
\hline Camera & 620 & 2.73 & 266 & 1.17 & 300 & 0.167 & 1.34 & 20 & 2.78 & \\
\hline LCD main PCB & 600 & 2.64 & 60 & 0.26 & 120 & 0.067 & 0.53 & 15 & 2.08 & \\
\hline
\end{tabular}

Table 4.35 Cost benefit of best cost options for disassembly and isolation of SMDs from PCBs in w

\begin{tabular}{|c|c|c|c|c|}
\hline Item & Process & Cost of isolating SMDs (£) & Revenue from SMDs (£) & per iten \\
\hline MP3 player & cryo-cracking \& solder bath & $£ 0.168$ & 0.163 & -0.005 \\
\hline Mobile phone & cryo-cracking \& solder bath & $£ 0.190$ & 0.201 & 0.010 \\
\hline Camera & cryo-cracking \& solder bath & $£ 0.190$ & 0.036 & -0.154 \\
\hline LCD PCB & guillotine \& solder bath & $£ 0.352$ & 0.055 & -0.297 \\
\hline
\end{tabular}




\subsection{Conclusions}

PCBs contain the largest variety of CRMs, particularly those of items which have been significantly miniaturised in recent years to increase functionality in smaller devices such as mobile phones.

XRF combined with digital imaging has proven useful for building a library of component images and x-ray emission spectra to inform future recycling processes which target CRMs for recovery. PCBs contain the largest variety of CRMs, particularly those of items which have been significantly miniaturised in recent years to increase functionality in smaller devices such as mobile phones.

In a study of pre-processing techniques that might enable greater value generation from WEEE, cryo-cracking of high value products whose manual disassembly is problematic has shown potential as a means of diverting $\mathrm{CRM}$ rich $\mathrm{PCBs}$ from automated recycling processes in which CRMs are dissipated and lost through unintended co-separation. The use of a guillotine to break through plastic housings and reduce costs associated with isolating CRM bearing components also shows potential. Isolation of SMDs from PCBs is best achieved by use of a solder bath; other thermal methods investigated are more costly, and/or present environmental H\&S issues from burning PCBs if care is not taken.

The use of microwave ashing as a means of pre-concentrating CRMs in samples shows negative cost benefit showing its application for concentration of copper and $\mathrm{Au}$ in isolated edge contacts prior to refining. However, the method could potentially be suitable for components subject to such mass based treatment charges which high organic content. Ashing to remove the epoxy component from whole PCBs may result in sufficient logistics and refining charge savings to grant a cost benefit. This technique may therefore prove a suitable means for 'upgrading' of materials which contain PMs, PGMs and copper but fall below cutoff grades for PM recovery processes, facilitating greater recovery rates of these CRMs from WEEE.

Although CRMs have been identified throughout these electrical/electronic devices, many barriers exist to enhancing rates of recovery. While PMs and PGMs are recovered with high efficiency in smelting processes for PCBs, in general the dissipative nature of the application of CRMs in electronics seriously limits the potential for their recovery. Of the other CRMs identified in PCBs many partition into the slag during smelting for PMs/PGMs, rather than into the copper bullion collected for further refinement. CRMs identified in samples that are irrecoverably lost are Ga, Ge, Ta, Y, and Co. It is possible to isolate SMDs containing these 
CRMs prior to smelting. However, at this time, there is little economic justification for the additional cost of such processes because of a lack of available recovery infrastructure for CRMs, and the fact that they are often found in combination with more valuable copper and PMs which are recoverable by smelting, but in a process in which other CRMs are lost. On solution may be to pursue development of hydrometallurgical recovery methods, through which losses of CRMs in smelting can be avoided, and through which a wider range of CRMs may be recovered. Lack of recovery infrastructure so far has resulted from the low recoverable value of CRMs in WEEE, due to low concentrations present and low market values, and although recycling of CRMs is fairly common from production scrap and other wastes, recovery from post-consumer wastes is rare. A 'chicken \& egg' scenario exists in which isolating CRMs from WEEE in appropriate fractions for downstream recovery is not viable due to lack of recovery infrastructure, and recovery infrastructure is not in place as insufficient quantities of suitable WEEE derived materials are currently available to feed such a process. Price volatility for CRMs also results in greater risk to investment in CRM recovery processes. Poor design for disassembly also results in high costs associated with isolating CRM bearing component. The RSA security dongles investigated here are an example of poor design significantly limiting recycling potential. 


\section{Platinized counter-electrodes for dye- sensitised solar cells from waste thermocouples: a case study for resource-efficiency, industrial symbiosis and circular economy}

\section{Chapter summary}

This thesis so far has dealt with the issue of how value can be exploited from WEEE through traditional recycling pathways. It will be shown in this chapter that through industrial symbiosis in which waste materials become raw materials for manufacturing, greater value can be generated from materials in WEEE than through traditional recycling process chains, resulting in sufficient economic advantage to drive recovery which is not economically viable through traditional recycling pathways. This demonstrates the greater value generation potential afforded by circular economy, in which 'wastes' are viewed as resources.

Future widespread deployment of photovoltaic $(P V)$ technologies will make a significant contribution to sustainable energy generation, but the scale of future PV deployment will most likely be limited by access to 'critical' raw materials (CRMs). Circular economy represents a path for sustainable economic development with associated economic, environmental and social benefits. Adoption of circular practices by the PV industry such as industrial symbiosis will enhance access to CRMs through recycling, and reduce energy payback times, materials costs, levelised costs of energy generation (LCOE) and energy payback times (EPBT) for PV. Industrial symbiosis also enables partner organizations to valorise wastes and materials contained therein which may be uneconomical to recover through traditional recycling routes such as CRMs used in dissipative applications e.g. platinum in waste thermocouples (WTCs) filaments. Here, on a very small scale, the means to overcome these barriers is demonstrated through a proposed industrial symbiosis between Weartech International Ltd and the SPECIFIC IKC in South Wales (Figure 5.1). Small quantities of platinum in WTC filaments generated by Weartech ( 43,000/year) can be economically recovered by conversion to chloroplatinic acid - an 'added value' product, for use by SPECIFIC in fabrication of dyesensitised solar cell (DSSC) counter-electrode catalysts. $91 \pm 3 \%$ recovery of platinum from filaments as chloroplatinic acid hydrate $(48.6 \pm 1.6 \mathrm{wt} \% \mathrm{Pt})$ has been achieved by aqua regia digestion of manually isolated filaments. Cost-benefit analysis shows the proposed process derives sufficient value from WTCs to cover Weartech's landfill costs for WTCs; provide $\sim 5$ 
days employment; and provide 63\% materials cost savings for SPECIFIC in comparison to purchasing chloroplatinic acid. Suitability of WTC derived chloroplatinic acid for DSSC catalysts has been verified. EIS has shown counter electrodes created from 3 sources of chloroplatinic acid (WTCs, 99.998\% Pt wire and purchased chloroplatinic acid) to have comparable charge transfer resistances $\left(R_{C T}\right)$ and exchange current densities $\left(j_{0}\right)$. The proposed process would divert $>50$ g/year of platinum from landfill, enough to produce $\sim 500$ $\mathrm{m}^{2}$ of DSSCs and supply clean energy for 12 homes in South Wales, and avoid up to $1400 \mathrm{~kg}$ $\mathrm{CO}_{2}$ in emissions associated with primary production of an equivalent quantity of platinum.

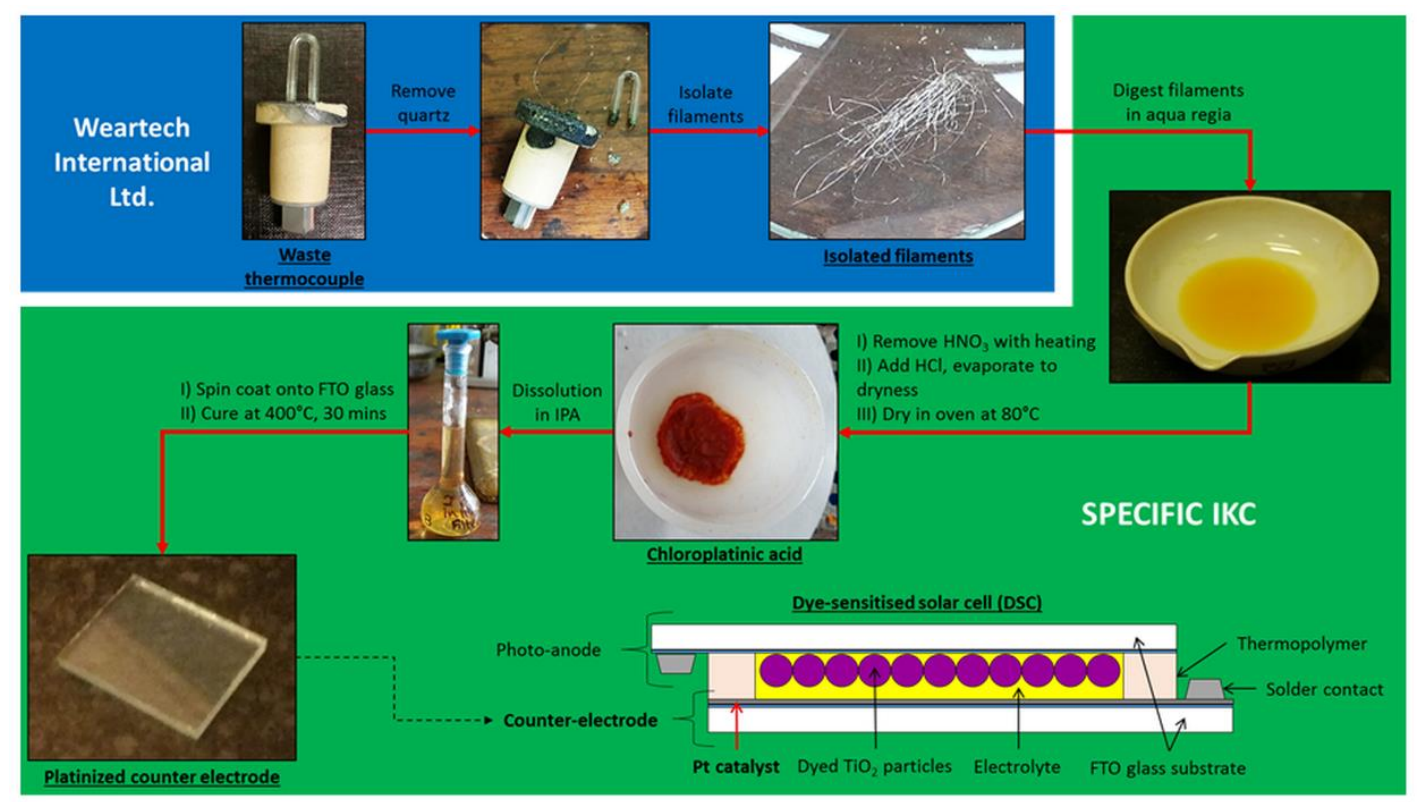

Figure 5.1 Industrial symbiosis process for fabrication of dye-sensitised solar cell (DSSC) counterelectrode catalysts from waste thermocouples

\subsection{Introduction}

The world faces the limits of current 'linear' economic models due to resource security issues, growing population and increasing per-capita consumption. Transition is necessary to a new 'circular economy': a resource-efficient industrial economy which decouples economic growth from resource consumption. ${ }^{47,225,226}$ In a circular economy the concept of 'waste' is replaced with one of 'resource' and process waste is reduced through industrial symbiosis, whereby process waste becomes the feedstock of another process. In this way materials are retained in an economically productive capacity, cascading across multiple product lifecycles. Enhanced resource-efficiency will deliver synergistic economic, environmental and social benefits ${ }^{47}$. Of significant importance to sustainable economic growth and transition to a low- 
carbon society is a secure supply of critical raw materials (CRMs), ${ }^{53,100,188,227}$ and widespread deployment of low-carbon renewable energy technologies. CRMs face future supply bottlenecks and their global recovery rates are currently low (Figure 5.2).${ }^{60,118}$ CRMs are vital to the functionality of many low-carbon technologies upon which reductions in global emissions and achieving sustainability with our planet rely. ${ }^{20,53,100,118}$ The scale of deployment of renewable technologies, particularly photovoltaics (PV) may well be constrained by access to CRMs, and increasing CRM recovery rates will be crucial if demand for wide-scale deployment of sustainable technologies is to be met. ${ }^{187,228,229}$

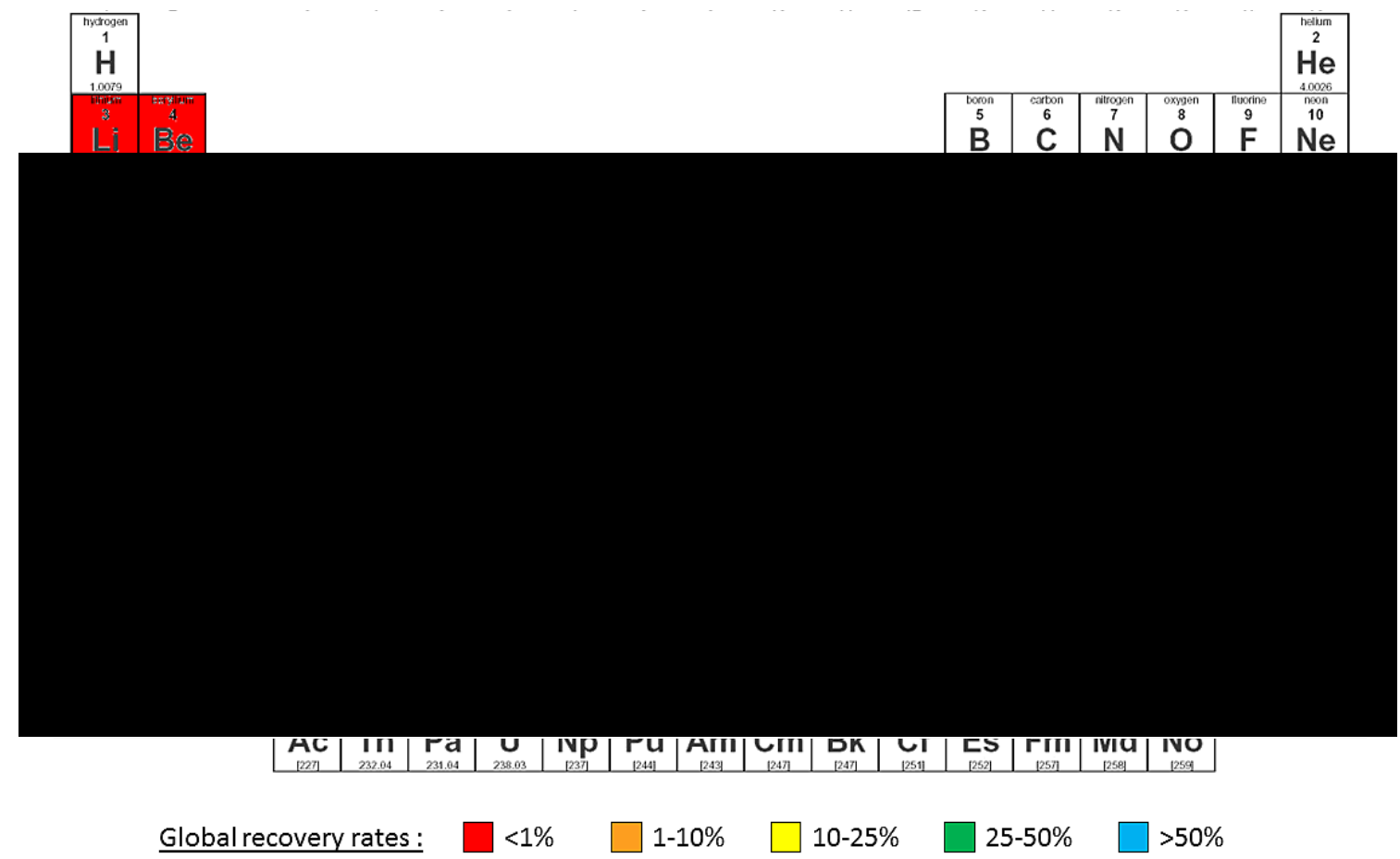

Figure 5.2 Recycling rates of metals highlighted as critical in recent assessments (adapted from Graedel et al., 2011; Reck \& Graedel, 2012) 21, 53, 60,100, 118

Part of the reason for low recovery rates, even for valuable metals like $\mathrm{Pt}$, is the use of these metals in dissipative applications, i.e. where they are present in low concentrations and the consequent high costs of recovery and low total recoverable value make traditional recycling routes unfeasible, resulting in loss of these elements from the economy. ${ }^{62,230-233}$

Platinum is a CRM with high economic value (> \$37/g LME 10/7/15, converted from GBP at $1.32 \$ / £$.) reflecting its low abundance in the Earth's crust as well as the economic and environmental costs of its production $\left(13,954 \mathrm{t} \mathrm{CO}_{2} / \mathrm{t}\right.$ of metal i.e. 4,100 times greater than copper). ${ }^{16}$ Table 5.1 gives global demand and recycling rates for platinum by end use sector. The high value of platinum has driven efficient recycling from applications such as industrial 
chemical catalysts and bullion where use and management of these materials is carefully controlled and where recycling rates are very high, $\sim 95 \%$. On the other hand, recycling rates from electronics are very low, $0-5 \%$, mainly due to the dissipative use of platinum in electronics, low global collection rates for WEEE, and poor product design for recycling \& recovery. ${ }^{234}$

Table 5.1 Global demand for platinum by end use sector in 2015 and estimated end-of-life (EoL) recycling rates

\begin{tabular}{|c|c|c|c|}
\hline \multirow{2}{*}{ Application } & \multicolumn{2}{|l|}{ Global Demand } & \multirow{2}{*}{ Recycling Rate $(\%)^{59,}, 189$} \\
\hline & (mass/Troy Oz) $10^{-3} 153$ & $\%$ of demand & \\
\hline Autocatalyst & 3,011 & $40 \%$ & $50-55$ \\
\hline Jewellery & 2,456 & $32 \%$ & $90-100$ \\
\hline Industrial total & 1,629 & $22 \%$ & $80-90$ \\
\hline Chemical & 494 & $7 \%$ & $\leq 90^{\mathrm{a}}$ \\
\hline Electronics & 151 & $2 \%$ & $0-5$ \\
\hline Glass & 163 & $2 \%$ & \\
\hline Petroleum & 100 & $1 \%$ & $\leq 90^{\mathrm{a}}$ \\
\hline Other Industrial & 721 & $10 \%$ & $10-20^{\mathrm{b}}$ \\
\hline Retail Investment & 474 & $6 \%$ & $90-100^{c}$ \\
\hline Total & 7,570 & & $60-70^{\mathrm{b}}$ \\
\hline
\end{tabular}

a recovery from catalysts used by this sector; $b$ Includes sensors, crucibles, medical/dental and decorative applications; c excludes coins and bullion- no typical end-of-life management, reuse is common

Low recycling rates are typical for many of the CRMs in electronics, where they are often used in trace amounts within complex systems. If cost-effective recovery of CRMs in WEEE could be achieved, then utilisation of this rich secondary resource could mitigate the issue of CRM

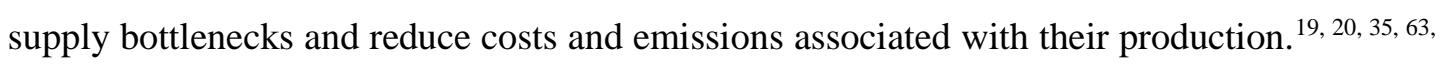
206, 222, 235, 236 Recycling of materials from WEEE for fabrication of solar cells is not only environmentally beneficial in comparison to primary resource based production, but offers a pathway to reduced energy payback times (EPBT), reduce levelised cost of energy generation (LCOE), and enhanced competitiveness and intangible value for PV manufacturers in a global market in which consumers are becoming increasingly environmentally aware. ${ }^{61,237,238}$

A significant application of platinum in industrial electronics is in thermocouple wire. The thermocouple temperature sensors market is estimated at $\$ 1.6$ billion by 2018 with a current growth rate of $6.6 \%$ year over the period 2014-2020. ${ }^{239}$ Measurement of molten metal temperatures in foundries and steel plants is almost exclusively conducted using single use thermocouples (Figure 5.3) containing a pair of filaments, one of which is either pure platinum or PtRh6\% alloy, and the other is platinum alloyed with 10-30\% Rh depending on the required working temperature range. Recovery of platinum group metals (PGMs) from waste thermocouples (WTCs) is hindered by the small quantities of PGMs present in each thermocouple. ${ }^{240}$ However, opportunities exist within circular economy for innovative 
recovery models which valorise materials that may otherwise be lost when recovery through traditional recycling chains is not economic. ${ }^{72}$ As we show here, a circular economy approach involving industrial symbiosis i.e. an association between two or more industrial facilities or companies in which the wastes or by-products of one become the raw materials for another, ${ }^{71}$ may be used to drive cost effective recovery of platinum from waste thermocouples (Figure 5.4).

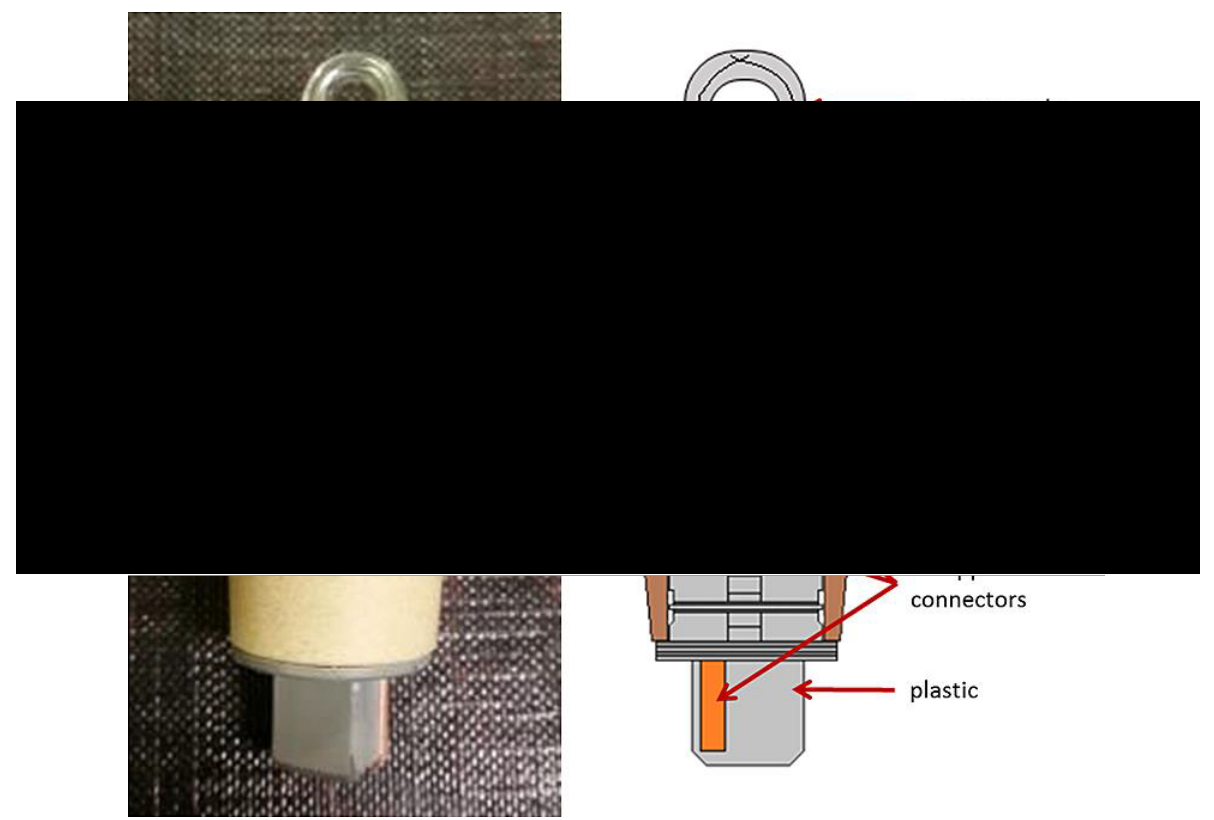

Figure 5.3 Waste Heraeus Electro-Nite Positherm expendable immersion thermocouple (schematic diagram adapted from Van der Perre $)^{241}$

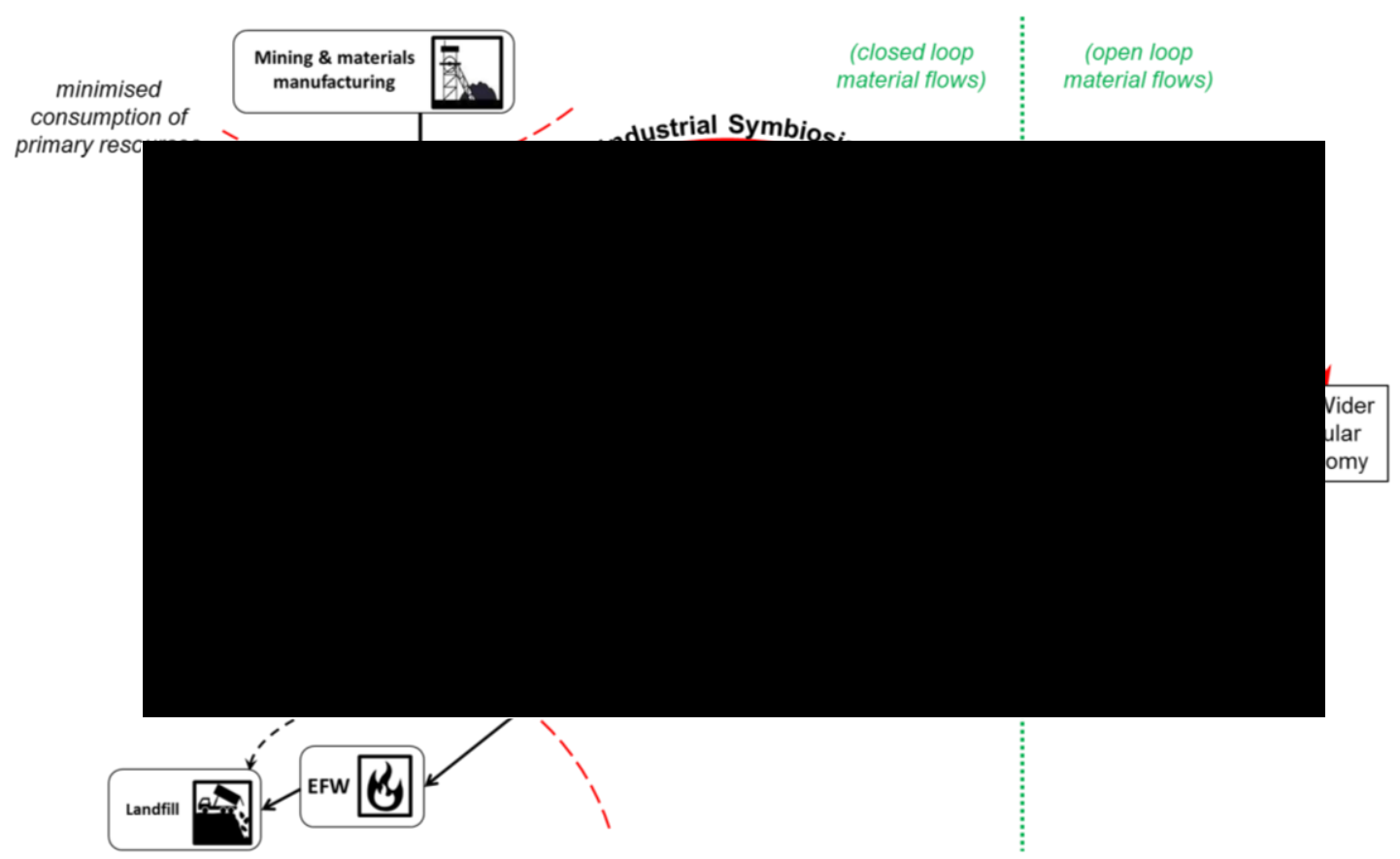

Figure 5.4 Material flows in a circular economy drawing on secondary raw materials from the wider circular economy through industrial symbiosis (adapted from Ellen McArthur Foundation, 2013) ${ }^{47}$ 


\subsubsection{Industrial symbiosis strategy}

Chloroplatinic acid is an important precursor in many applications of platinum. ${ }^{242}$ It is more expensive than platinum $(\$ 102 / \mathrm{g}$ at $\sim 38 \mathrm{wt} \% \mathrm{Pt})$, and conversion of the $\mathrm{Pt}$ in waste thermocouples to chloroplatinic acid, an 'added value' Pt compound, could significantly increase the derivable value from $\mathrm{Pt}$ in the filaments. Following manual isolation of $\mathrm{Pt}$ filaments from WTCs it is possible to convert these to chloroplatinic acid by digestion in aqua regia $^{243}$ through reaction 5.1, followed by removal of nitric acid from the resulting solution by boiling through reaction 5.2, and evaporation to dryness following addition of $\mathrm{HCl}$ to yield solid $\mathrm{H}_{2} \mathrm{PtCl}_{6} \cdot \mathrm{XH}_{2} \mathrm{O}$. ${ }^{243}$

$$
\begin{gathered}
\mathrm{Pt}+8 \mathrm{HCl}+2 \mathrm{HNO}_{3} \rightarrow \mathrm{H}_{2} \mathrm{PtCl}_{6}+4 \mathrm{H}_{2} \mathrm{O}+2 \mathrm{NOCl} \\
3 \mathrm{HCl}+\mathrm{HNO}_{3} \rightarrow \mathrm{Cl}_{2}+2 \mathrm{H}_{2} \mathrm{O}+\mathrm{NOCl}
\end{gathered}
$$

This simple process requires no specialist equipment other than a fume hood, producing no solid or liquid waste other than WTCs minus filaments. The potential for small scale recovery close to the source of generation of WTCs offers interesting possibilities at low economic and environmental cost in comparison to traditional recycling routes. If a suitable partner organization could be found that uses chloroplatinic acid, then an industrial symbiosis strategy could potentially lead to: increased levels of PGM recovery from end-of-life devices; reduced waste management costs for producers of WTCs; mitigation of materials criticality issues; and reduced environmental and economic costs for organisations using chloroplatinic acid.

Weartech International Ltd. operate a foundry in Baglan, South Wales and produce $>43,000$ WTCs annually. Conscious of resource security concerns over PGMs, Weartech sought a recycling route which recovers PGMs from WTCs, which are currently (2015) landfilled at a cost of $\$ 158.40 /$ tonne ( $\$ 132 /$ tonne landfill tax $+\$ 26.40$ gate fee) for lack of viable recycling options. A potential partner for this industrial symbiosis is the SPECIFIC Innovation and Knowledge Centre in Swansea University, which conducts research into optimisation of dyesensitised solar cell (DSSC) performance and scale up of roll-to-roll production (R2R). Currently, SPECIFIC purchases chloroplatinic acid, in $1 \mathrm{~g}$ quantities, for fabrication of DSSC catalysts $^{244}$ (Figure 5.5) at their site in the Baglan Energy Park, $<2$ miles from Weartech. 


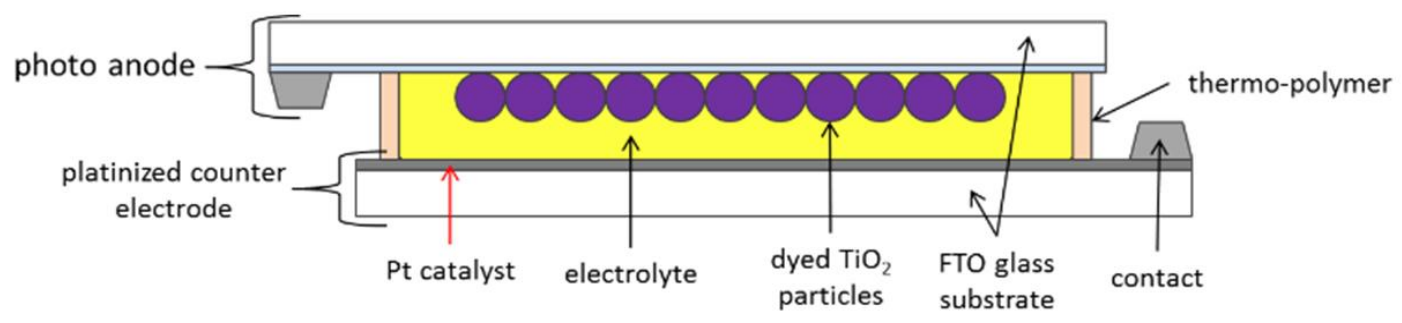

Figure 5.5 Structure of a Dye-Sensitised Solar Cell (DSSC)

To verify the suitability of WTCs as a source of platinum for SPECIFIC and the appropriateness of the industrial symbiosis strategy with Weartech, the following question must be answered: can chloroplatinic acid of sufficient quality for use in DSSCs be synthesised from WTCs in sufficient quantity and at sufficient cost-benefit to justify the process? Here we show that the answer to this question is 'yes' and that the barriers to recovery of Pt from this dissipative application in thermocouples may be overcome by local industrial symbiosis. We also use this example to illustrate the benefits of the circular economy approach.

Although here we focus on the example of Pt from WTCs for DSSC counter-electrode catalysts in a relatively small scale local operation between Weartech and SPECIFIC, chloroplatinic acid is important in numerous applications where platinum deposition is required. A major example for low-carbon technologies is in the preparation of catalysts for fuel cells. ${ }^{245-247} \mathrm{Pt}$ is also only one of the many elements facing future supply bottlenecks that may be sourced from available 'wastes'. In addition, small-scale local operations such as this are of significant importance to a healthy circular economy. Economies require a balance of various size businesses to thrive in the long term with larger enterprises bringing volume and efficiency and smaller ones offering alternative models when crises occur. ${ }^{248}$ A multitude of similarly sized operations throughout the circular economy could deliver materials cost savings and domestic sources of CRMs for manufacturing as shown by this example, while providing economic resilience through diversity. ${ }^{47}$

\subsection{Experimental}

A $5.5 \mathrm{~kg}$ sample of WTCs was obtained from Weartech International Ltd., and was cone and quartered to give a representative sub samples of 82 individual WTCs for further study. Details of analytical techniques employed are outlined in section 2.2. Diameters of filaments were measured from SEM images, and alloy composition of filaments was measured using $\mathrm{SEM} / \mathrm{EDS}$. Pt concentration in chloroplatinic acid samples is given as the average of results from ICP-OES and AAS analysis. EIS is used to evaluate electrochemical performance of DSSC catalysts created from chloroplatinic acid from different sources. 


\subsubsection{Chloroplatinic acid synthesis}

$\sim 100 \mathrm{mg}$ of platinum filaments, or wire, was digested by boiling in $10 \mathrm{ml}$ of aqua regia (3:1 $\mathrm{HCl} / \mathrm{HNO}_{3}$ ) for 3 hours. Following digestion, solutions were evaporated to near dryness to remove nitric acid, before dissolution of the residue in $10 \mathrm{ml} \mathrm{1:1} \mathrm{conc.} \mathrm{HCl}$ : water. Chloroplatinic acid was obtained from $5 \mathrm{ml}$ of this solution by evaporation to dryness with further drying at $80{ }^{\circ} \mathrm{C}$ for 12 hours (Figure 5.6, stage 2). The remaining $5 \mathrm{ml}$ of solution was retained for analysis and fabrication of DSSC counter electrodes.
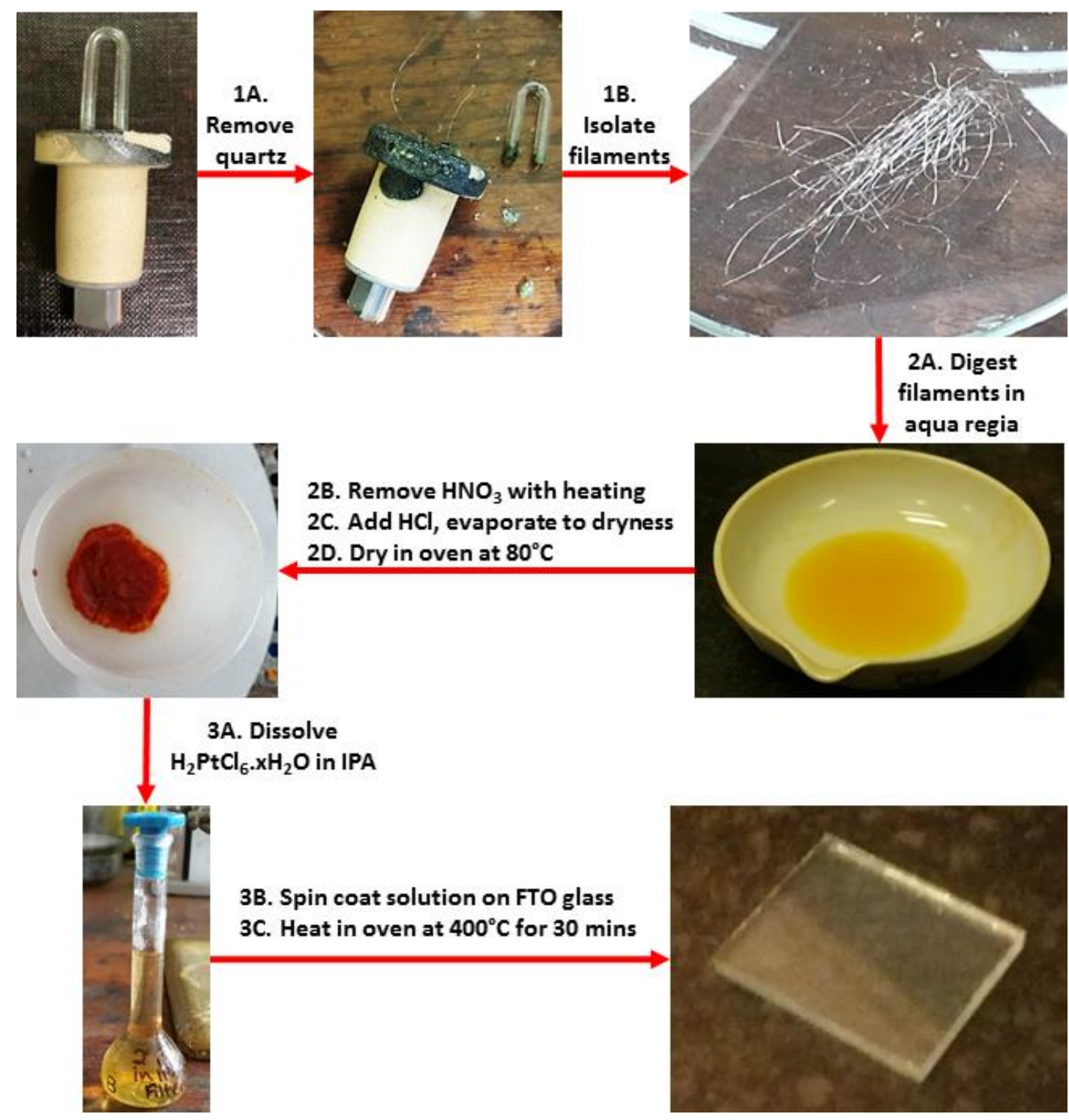

Figure 5.6 Procedure for synthesising DSSC counter electrodes from waste thermocouples (WTCs). The process includes 3 stages: 1) isolation of Pt filaments from WTCs; 2) synthesis of chloroplatinic acid from filaments; 3 ) creation of DSSC counter electrodes 


\subsubsection{Fabrication and characterization of platinized counter-electrodes}

NSG Tec7 FTO glass (7 $\Omega / \mathrm{sq})$ was cut into $25 \mathrm{~mm}$ x $15 \mathrm{~mm}$ pieces. A small hole was drilled into the non-conductive surface adjacent to the upper right hand corner of a $1 \mathrm{~cm}^{2}$ area at their centre to allow filling of cells with electrolyte. The glass was cleaned with distilled water \& detergent, rinsing with distilled water and then IPA, before drying with compressed air. 100 $\mu \mathrm{L}$ of $\sim 5 \mathrm{mM}$ chloroplatinic acid in IPA was spin coated onto the FTO at 2,000 rpm for $40 \mathrm{~s}$, accelerating at $2,000 \mathrm{rpm} / \mathrm{s}$ and heated to $400{ }^{\circ} \mathrm{C}$ for $30 \mathrm{mins}$ to create the platinized electrode (Figure 5.6, stage 3). Pairs of CEs were used to create 'dummy cells' (Figure 5.7), ${ }^{138-142}$ sealed together with a $1 \mathrm{~cm} \times 1 \mathrm{~cm}, 25 \mu \mathrm{m}$ Surlyn ${ }^{\mathrm{TM}}$ gasket. Cells were filled with electrolyte $(0.8 \mathrm{M}$ 1-methyl-3-propylimidazolium iodide, $0.3 \mathrm{M}$ benzimidazole, $0.1 \mathrm{M}$ iodine, and $0.05 \mathrm{M}$ guanidinium thiocyanate in 3-methoxypropionitrile) via channels drilled through the glass. Cells were sealed with Surlyn ${ }^{\text {TM }}$ and a glass slip. An MBR electronics USS-9210 ultrasonic soldering system was used to apply CERASOLZER GS155 solder to the conductive side of each electrode at its terminus. Charge transfer resistances $\left(R_{\mathrm{CT}}\right)$ and double layer capacitances $\left(\mathrm{C}_{\mathrm{dl}}\right)$ of the electrodes were measured using electrical impedance spectroscopy (EIS) on the dummy cells. The area tested by EIS analysis was $1 \mathrm{~cm}^{2}$ per electrode as defined by the Surlyn gasket. Exchange current densities at each electrode $\left(j_{0}\right)$ were calculated from measured $R_{C T}$ values using equation 5.1, where $\mathrm{F}$ is Faraday's constant, $T$ is temperature, $R$ the gas constant, and $n$ the number of electrons involved in the reaction. ${ }^{249}$

$$
R_{C T}=\frac{R T}{j_{0} n F}
$$

Equation 5.1

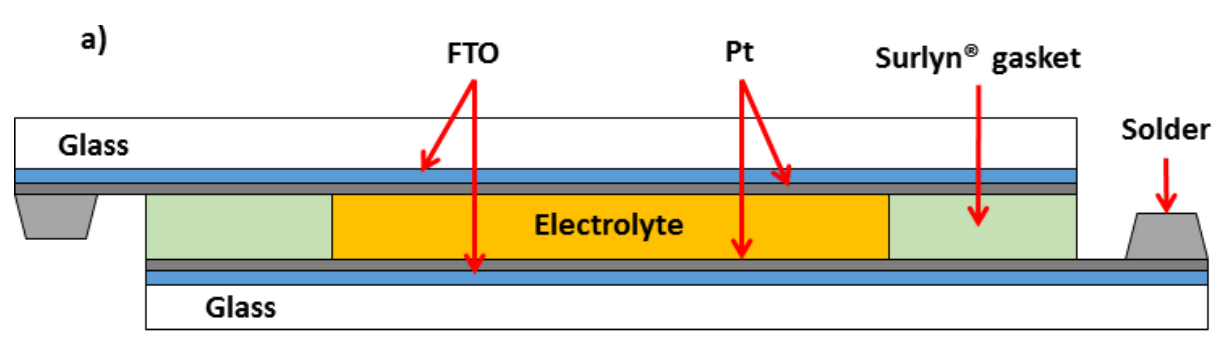

Figure 5.7 Electrochemical symmetrical 'dummy cells' used for EIS measurements

\subsection{Result and Discussion}

\subsubsection{Isolation of filaments from waste thermocouples}

Inspection of the sample from Weartech revealed the physical condition of WTCs to be variable with some in close to 'as new' condition (e.g. Figure 5.3) and others damaged extensively with some cracked, and/or quartz missing, and/or caked in the alloy they were 
used to measure (Figure 5.8) Despite damage, most WTCs were found to contain both filaments in their entirety and WTCs without filaments were rare.

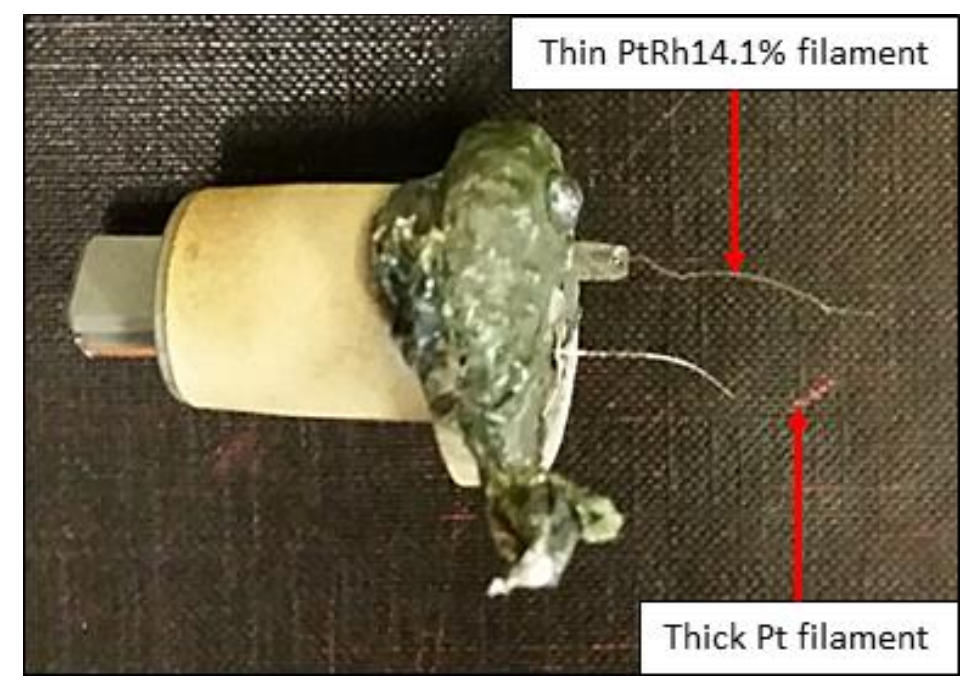

Figure 5.8 Waste thermocouple in poor condition with exposed filaments and alloy compositions as determined by SEM/EDS.

The quartz tube was removed with pliers to access the base of filaments protruding from the refractory cement. Filaments were snipped as close to their base as possible to minimise the quantity remaining within the refractory cement of WTCs (Figure 5.6, stage 1). This process was timed, and masses of isolated filaments were recorded for subsequent costing exercises. Microscopic analysis of isolated filaments revealed each WTC contained 2 filaments of different diameters, larger filaments with a mass of $1.1( \pm 0.3) \mathrm{mg}$ and diameter of $\sim 74 \mu \mathrm{m}$, and lighter thinner filaments with a mass of $0.4( \pm 0.1) \mathrm{mg}$ and diameter of $40.5 \mu \mathrm{m}$. Diameters of thin and thick filaments were measured from images at 1,200 to 1,800 times magnification respectively (Figure 5.9). SEM/EDS analysis was used to determine alloy composition of the filaments (Figure 5.11). 

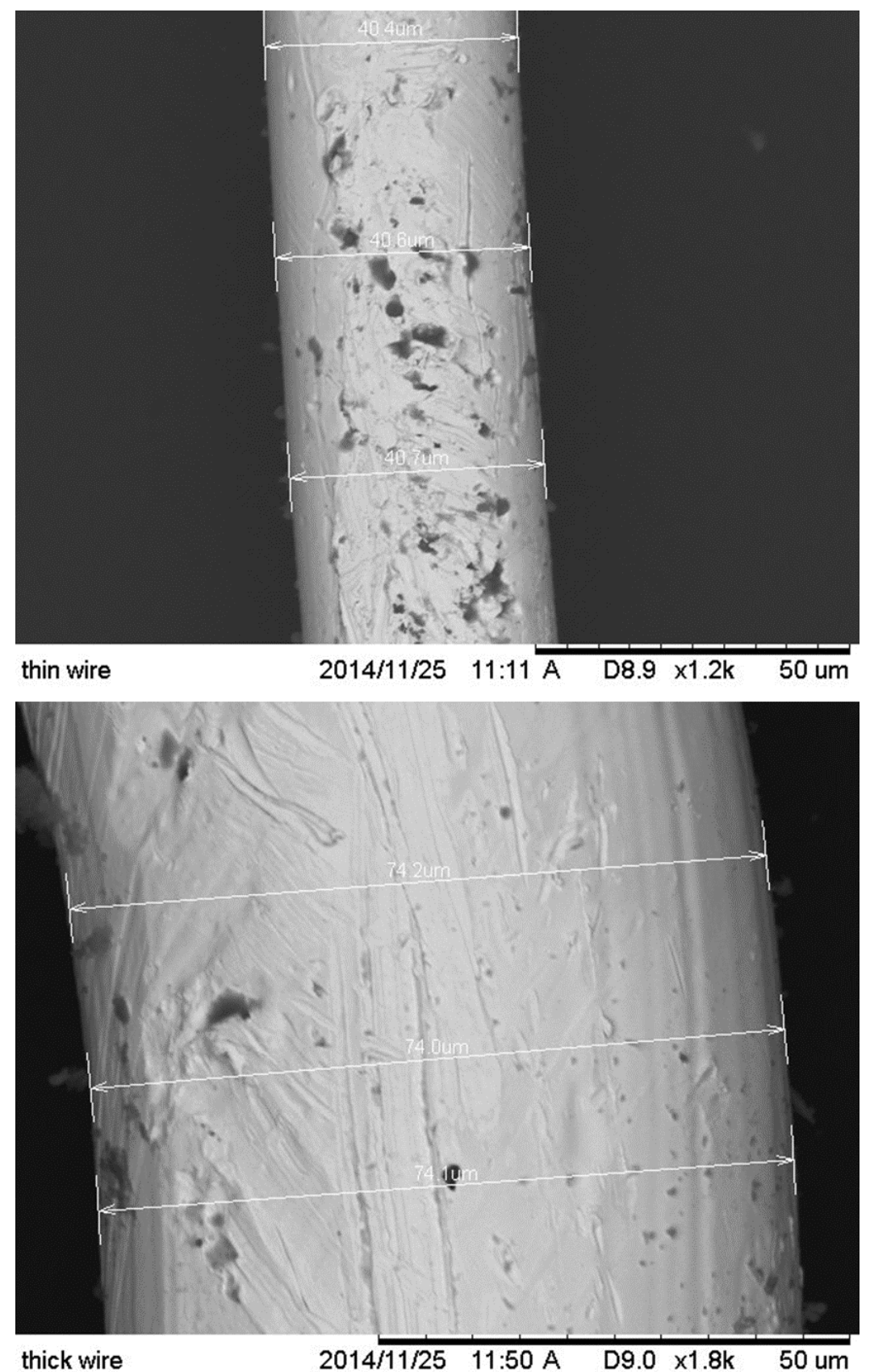

Figure 5.9 Diameter measurements of thin (left) and thick (right) waste thermocouple filaments from SEM images at 1,200 and 1,800 time magnification respectively

Alloy compositions of filaments were generated using Bruker Quantax software from X-ray fluorescence signals corresponding to L \& $\mathrm{M}$ transitions for $\mathrm{Pt}$, and $\mathrm{L}$ transitions for $\mathrm{Rh}$ in the resulting emission spectra of samples. SEM/EDS analysis revealed that heavier filaments are $\sim 100 \% \mathrm{Pt}$ (Figure 5.10), and lighter filaments are $\sim \mathrm{PtRh} 14 \%$ alloy (Figure 5.11), (close to the alloy composition of PtRh13\% given for ANSI-type R thermocouple filaments in the manufacturer specification and within the tolerance of the EDS). ${ }^{241}$ XRF analysis confirmed the thicker filament to be $100 \% \mathrm{Pt}$ and the lighter filament to be PtRh12.75\% alloy. Assuming 
filaments are present in a 1:1 length ratio, then the average composition of filaments is $\mathrm{Pt} 96 \% \mathrm{Rh} 4 \%$.
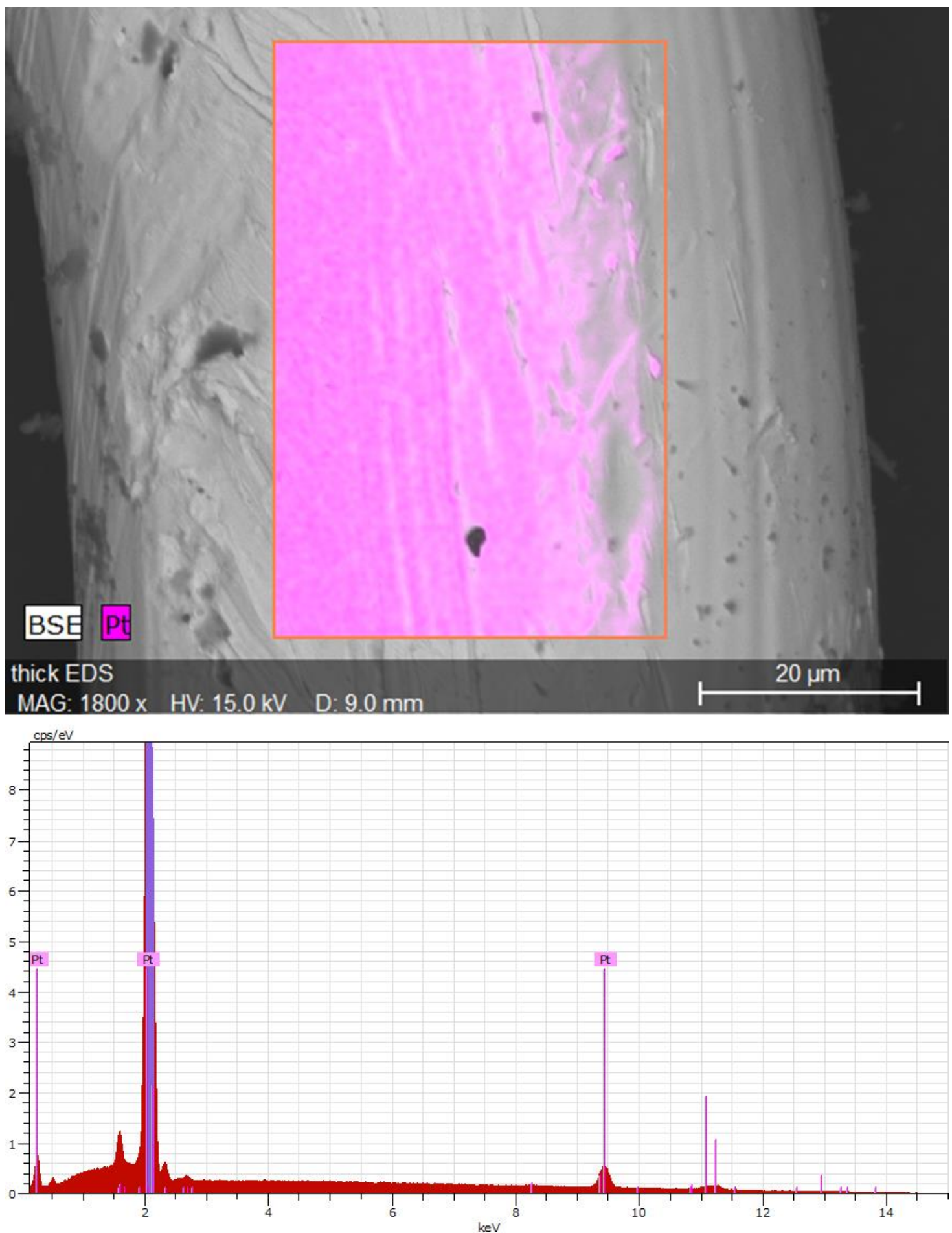

Figure 5.10 Elemental map of Pt in thin waste thermocouple filament (top) with corresponding X-ray emission spectra and alloy composition analysis (bottom) produced using SEM/EDS 

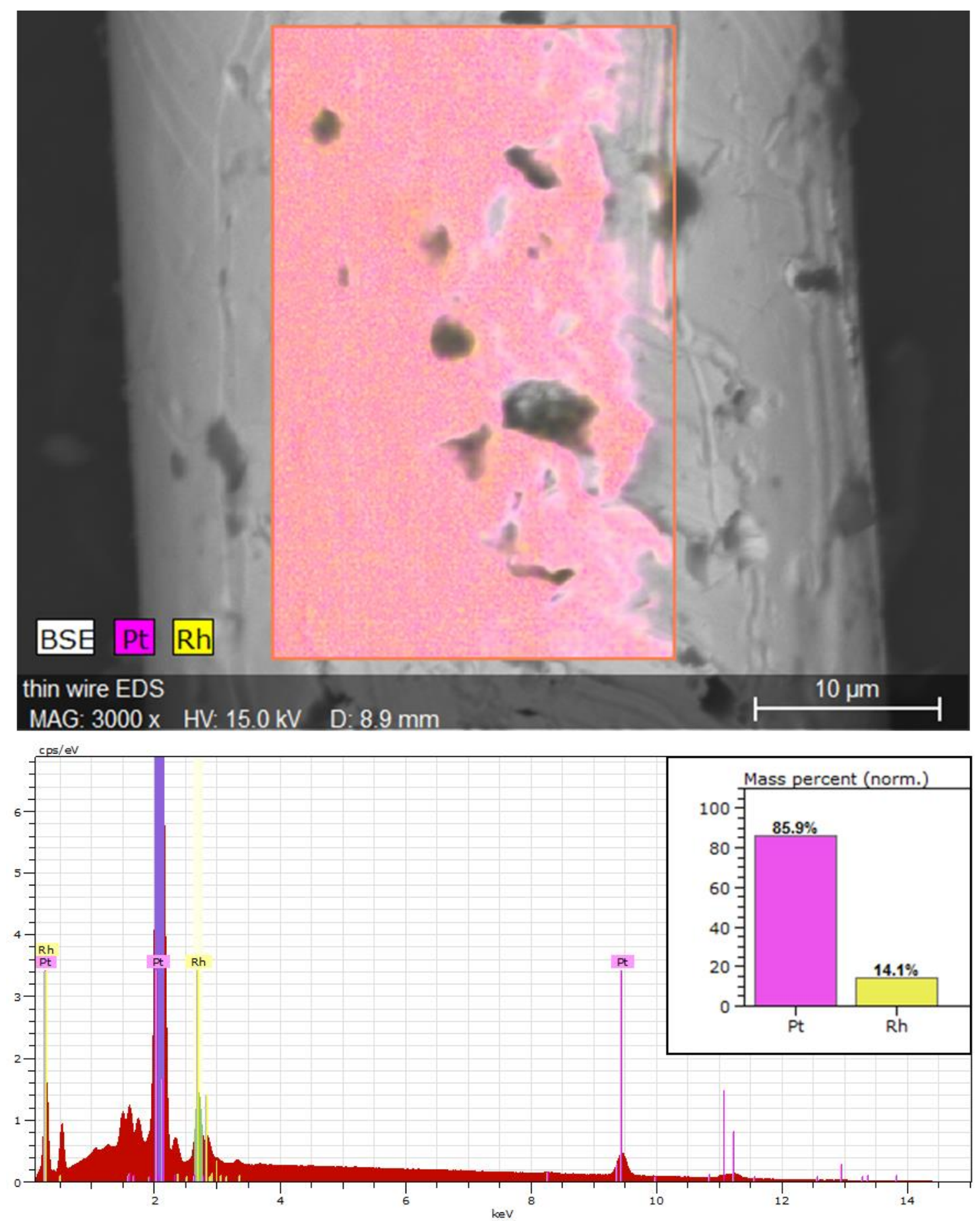

Figure 5.11 Elemental map of Pt and Rh in thin waste thermocouple filament (top) with corresponding $X$-ray emission spectra and alloy composition analysis (bottom) produced using SEM/EDS/

WTCs have an average PGM concentration of $\sim 120 \mathrm{ppm}$, considerably higher than the majority of ores refined for Pt production (Nishnij-Tagil, Russia: 10-20 ppm; South African Bushveld complex: 3-20 ppm). ${ }^{243} 97.3( \pm 0.3) \mathrm{mg}$ of Pt bearing filaments were isolated from the 82 WTC sub-samples, an average of $1.2 \mathrm{mg}$ per thermocouple. 


\subsubsection{Chloroplatinic acid}

AAS analysis of the solution after filament digestion shows the average Pt content of WTC filaments to be $95( \pm 1) \%$, in line with expectation. Table 5.2 gives details of yields and metal content for chloroplatinic acid synthesised from thermocouple filaments $99.998 \% \mathrm{Pt}$ wire for comparison. Both chloroplatinic acid samples synthesised have significantly higher Pt content than purchased chloroplatinic acid currently purchased used by SPECIFIC $(38.6( \pm 0.2) \mathrm{wt} \%$ $\mathrm{Pt}$ ), probably as a result of the oven drying at $80^{\circ} \mathrm{C}$.

Table 5.2 PGM content of chloroplatinic acid and Pt recovery yield from WTCs and 99.998\% Pt wire

\begin{tabular}{lll}
\hline & \multicolumn{2}{c}{ Pt source } \\
\cline { 2 - 3 } & WTCs & Pt wire \\
\hline Metal Digested & $97.3( \pm 0.3)$ & $97.3( \pm 0.3)$ \\
Mass (mg) & $95( \pm 1)$ & 100 \\
Wt\%Pt & & \\
Chloroplatinic acid product & & \\
Mass (mg) & $173.2( \pm 0.3)$ & $204.0( \pm 0.3)$ \\
Wt\%Pt & $49( \pm 2)$ & $47( \pm 1)$ \\
& & \\
Pt recovered in product & & $96( \pm 2)$ \\
Yield (mg) & & $99( \pm 3)$ \\
\hline
\end{tabular}

\subsubsection{Assessment of suitability for DSSC electrode manufacture}

The role of the catalyst in a DSSC is to catalyse the reduction of tri-iodide to iodide at the counter electrode, reaction 5.3. This allows the iodide to regenerate the dye in the reverse reaction at the working electrode. The charge transfer resistance $\left(R_{\mathrm{CT}}\right)$ for the tri-iodide to iodide reaction can be measured using EIS (as discussed in section 2.2.7) and is indicative of the efficiency of the catalytic reaction.

$$
\mathrm{I}_{3}^{-}+2 \mathrm{e}^{-} \rightarrow 3 \mathrm{I}^{-}
$$

The mean values of $R_{\mathrm{CT}}$ for dummy cells created from chloroplatinic acid derived from different sources are given in Table 5.3. The slightly higher $R_{\mathrm{CT}}$ value observed for cells made from purchased chloroplatinic acid is likely due to a lower quantity of platinum deposited on the electrode surface as a result of the slightly lower concentration Pt solution used, possibly as a consequence of deliquescence of commercial chloroplatinic acid sample during weighing due to its highly hygroscopic nature. Column $j_{0} /[\mathrm{Pt}]$ gives exchange current densities for the deposited Pt layer per unit concentration of Pt in IPA used to deposit the layer and within experimental error the results are the same. 
Table 5.3 Average charge transfer resistances $\left(R_{C T}\right)$, double layer capacitances $\left(C_{d l}\right)$ and exchange current densities $\left(j_{0}\right)$ at the electrolyte | electrode interface for electrodes in 'dummy cells' created with platinum from different source; and corresponding jo expressed per unit concentration of Pt in IPA used for creation of electrodes ( $\mathrm{j} /[\mathrm{Pt}])$.

\begin{tabular}{|c|c|c|c|c|c|}
\hline $\begin{array}{c}\text { Chlorplatinic acid } \\
\text { source }\end{array}$ & $\begin{array}{l}{[\mathrm{Pt}] \text { in IPA }} \\
(\mathbf{m M})\end{array}$ & $\begin{array}{c}\text { RCT } \\
\left(\Omega \mathbf{c m}^{-2}\right)\end{array}$ & $\begin{array}{c}\mathrm{C}_{\mathrm{dl}} \\
(\boldsymbol{\mu} \mathbf{F})\end{array}$ & $\begin{array}{c}j_{0} \\
\left(\mathbf{m A ~} \mathbf{~ c m}^{-2}\right) \\
\end{array}$ & $\begin{array}{c}j_{o} /[\mathrm{Pt}] \\
\left(\mathbf{m A} \mathbf{~ c m}^{-2} \mathbf{M}^{-1}\right) \\
\end{array}$ \\
\hline \multirow{3}{*}{$\begin{array}{ll}- & \text { Pt wire } \\
\text { - } & \text { WTC } \\
- & \text { Purchased from } \\
& \text { Sigma-Aldrich }\end{array}$} & $4.41( \pm 0.06)$ & $5.15( \pm 0.31)$ & $10.05( \pm 0.46)$ & $2.47( \pm 0.15)$ & $0.56( \pm 0.03)$ \\
\hline & $4.70( \pm 0.06)$ & $4.80( \pm 0.58)$ & $10.73( \pm 0.55)$ & $2.65( \pm 0.32)$ & $0.56( \pm 0.07)$ \\
\hline & $3.84( \pm 0.05)$ & $7.00( \pm 0.56)$ & $9.20( \pm 0.23)$ & $1.82( \pm 0.15)$ & $0.47( \pm 0.04)$ \\
\hline
\end{tabular}

Errors are standard error on the measn, $(\mathrm{N}=6)$

The data in Table 5.3 show that all three sources of Pt are equally suitable for the preparation of DSSC electrodes. The presence of Rh and any other contaminants in WTC derived chloroplatinic acid does not have any detrimental effect on the catalytic activity of platinized electrodes prepared using chloroplatinic acid from waste thermocouples. It was not possible to identify localised metal deposits on the electrodes using SEM across the 5,000-100,000x magnification range (the size range of $\sim 1 \mu \mathrm{m}$ to $\sim 10 \mathrm{~nm}$ ), and so we presume the metal particles to be smaller than $\sim 10 \mathrm{~nm}$, but what is most important for this work is that across this magnification range SEM showed no gross differences in electrodes made from recycled thermocouples compared to those made from pure Pt wire or purchased chloroplatinic acid.

\subsubsection{Supply and environmental impact}

Weartech International Ltd currently generate $~ 43,340$ WTCs per year, which would yield $51.4( \pm 0.2) \mathrm{g}$ of filaments, more than enough to support the current activities of SPECIFIC. Recovery of this quantity of Pt offsets demand for an equivalent quantity from primary sources, negating the requirement to mine and process between 2.5-17 tonne of ore and saving 200-1400 $\mathrm{kg} \mathrm{CO}_{2}$. Counter-electrodes for highly efficient DSSCs require 5-50 $\mu \mathrm{g} / \mathrm{cm}^{2}$ of $\mathrm{Pt},{ }^{142,250,251}$ therefore Weartech's WTCs could be used to create $100-1,000 \mathrm{~m}^{2}$ of electrodes for DSSC annually. $500 \mathrm{~m}^{2}$ of DSSCs is sufficient to provide enough solar electricity for $\sim 12$ households in South Wales, based on the local solar irradiance and a cell efficiency of $\sim 12 \%$.

\subsubsection{Cost benefit analysis}

The potential industrial symbiosis operation is indicated in Figure 5.12, and associated costs are given in Table 5.4 with a cost benefit analysis given in Table 5.5. It is presumed that associated labour and overhead costs will be in line with those of Metech Recycling (UK) Ltd who operate a WEEE recycling facility near SPECIFIC and Weartech, and employ staff to conduct manual recycling processes at an overhead rate of $\$ 20.91 /$ hour. Times for processing WTCs were scaled up from the time taken to process the 82 WTC samples. WTC filaments are then sold to SPECIFIC for conversion to chloroplatinic acid at a price which covers the 
costs of this operation and disposal of processed WTCs in landfill (\$158.40/tonne). Cost analysis for chloroplatinic acid synthesis by SPECIFIC takes account of: chemical costs (HCl ACS reagent grade, $2.5 \mathrm{~L}$ : \$75.11; $\mathrm{HNO}_{3}$ ACS reagent grade, $\left.2.5 \mathrm{~L}: \$ 115.63\right)$; transport costs $(\$ 0.59 / \mathrm{mile})$; and electricity costs $(\$ 0.20 / \mathrm{kWh}$, UK average 2015). Costs incurred for chloroplatinic acid synthesis are scaled up from production of $1 \mathrm{~g}$ of chloroplatinic acid. Cost of electricity for the oven used to dry the sample was not included as the oven used is in operation 24 hours a day regardless. No waste disposal costs are incurred in the chloroplatinic acid production as the process produces no solid or liquid waste. Labour costs for this process are not included in the cost benefit as minimal staff time is required for addition of acids to the process and during the digestion process and drying of the product. Administrative overheads for SPECIFIC associated with the process of procuring chloroplatinic acid are not included. A cost-benefit analysis was conducted by comparison of the total price of filaments and cost of chloroplatinic acid synthesis (per g), with the cost of purchasing chloroplatinic acid in $1 \mathrm{~g}$ and $25 \mathrm{~g}$ lots from Sigma-Aldrich.

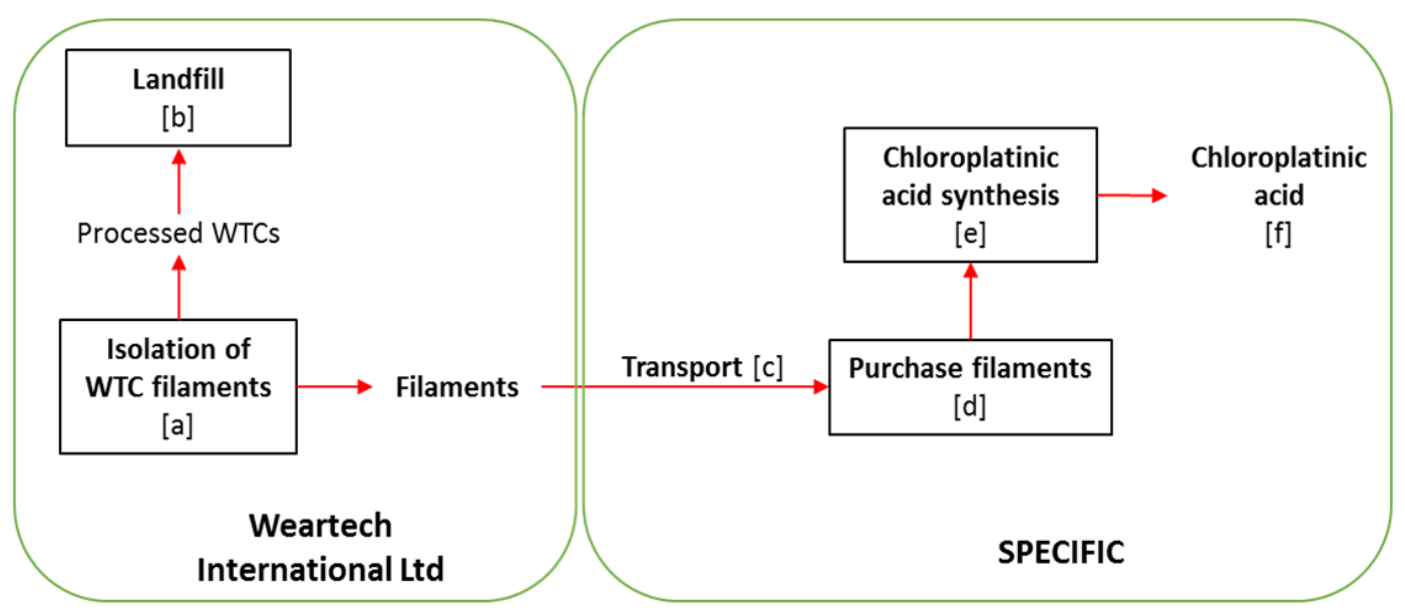

Figure 5.12 Industrial symbiosis operations and associated costs of each operation indicated by letters in square brackets: [a] Labour and overheads; [b] Landfill tax + gate fee; [c] transport; [d] price of filaments ([a+b]); [e] chemicals + energy; [f] total cost of chloroplatinic acid $([c+d+e])$

Table 5.4 Cost analysis for production of $1 \mathrm{~g}$ chloroplatinic acid from WTCs

\begin{tabular}{|c|c|c|c|c|}
\hline Operation & Costs & Weartech Costs & $\begin{array}{c}\text { SPECIFIC } \\
\text { Costs }\end{array}$ & $\begin{array}{c}\% \text { of Total } \\
\text { Cost }\end{array}$ \\
\hline [a] & Labour \& overheads & $\$ 41.25$ & & 87.0 \\
\hline [b] & Landfill & $\$ 0.73$ & & 1.5 \\
\hline [c] & Transport & & $\$ 0.32$ & 0.7 \\
\hline \multirow[t]{2}{*}{ [d] } & Price of filaments $([\mathrm{a}+\mathrm{b}])$ & & $\$ 41.98$ & 88.6 \\
\hline & Chemicals & & $\$ 3.70$ & 7.8 \\
\hline [e] & Energy & & $\$ 1.39$ & 2.9 \\
\hline [f] & Total cost $([c+d+e])$ & & $\$ 47.39$ & \\
\hline
\end{tabular}


Table 5.5 Comparative cost of platinum as chloroplatinic acid synthesised from WTCs and purchased in minimum and maximum available quantities.

\begin{tabular}{|c|c|c|c|c|}
\hline Pt Source & Cost $(\$ / g)$ & Wt\%Pt & $P t \operatorname{cost}(\$ / g)$ & Cost Benefit (\$) \\
\hline WTC & 47.39 & 48.6 & 97.51 & \\
\hline Purchased $\mathrm{H}_{2} \mathrm{PtCl}_{6} . \mathrm{xH}_{2} \mathrm{O}$ & & 38.6 & & \\
\hline $1 \mathrm{~g}$ quantity & $102.56^{252}$ & & 265.71 & 168.20 \\
\hline 25 g quantity & $41.03^{252}$ & & 106.28 & 8.77 \\
\hline
\end{tabular}

The cost of producing WTC derived chloroplatinic acid for SPECIFIC was calculated to be $\$ 47.39 / \mathrm{g}$ (Table 5.4), less than half the current price of chloroplatinic acid used by SPECIFIC when purchased in $1 \mathrm{~g}$ quantities (Table 5.5). The majority of cost is incurred in the manual isolation of filaments at Weartech. Despite this, there is sufficient cost benefit to justify $\sim 5$ days of employment per year at $37 \mathrm{hrs} /$ week on UK national living wage, whilst also covering landfill costs.

Process costs could be reduced further by an increase of scale by extension of the process to take waste thermocouples from other foundries, steel works and glass works, along with the development of semi-automated isolation of WTC filaments. Although traditional automated recycling processes (grinding, with subsequent eddy current and density separation processes) without prior removal of filaments would render efficient recovery of Pt impossible, ${ }^{18,106,116 \text {, }}$ ${ }^{253}$ a semi-automated process for isolation of filaments from whole WTCs could accelerate the process whilst reducing costs. In addition, chemical costs - the second most significant in the process, could be reduced by processing greater numbers of filaments simultaneously. Although $10 \mathrm{ml}$ of aqua regia was used in this case, a lower solid/liquid ratio could still achieve efficient digestion.

Additional cost benefit may be achievable through automated recycling of the remaining portions of WTCs. Comminution followed by eddy current and density separation techniques could produce $\mathrm{Cu}$, plastic and refractory oxide fractions for recycling could valorise this material for Weartech which would generate additional revenue whilst eliminating landfill cost for Weartech.

\subsection{Conclusions}

Manual isolation of filaments from waste thermocouples (WTCs) with subsequent digestion in aqua regia and precipitation from $\mathrm{HCl}$ yields chloroplatinic acid of sufficient quality for use in production of platinized electrodes for dye-sensitised solar cells (DSSCs). This is achievable at sufficient cost benefit to justify implementation of the proposed industrial symbiosis 
strategy between Weartech (WTC producers) and SPECIFIC (consumers of chloroplatinic acid). Through conversion of WTC filaments to the 'added value' product, chloroplatinic acid, sufficient value is derivable to cover Weartech's landfill costs; provide $\sim 5$ days employment; and enable SPECIFIC to achieve 63\% materials cost saving on chloroplatinic acid in comparison to the current scenario in which chloroplatinic acid is purchased from SigmaAldrich.

In addition, $>50 \mathrm{~g}$ of $\mathrm{Pt}$ can be diverted from landfill annually. This enhances resourceefficiency and mitigates materials criticality issues by decoupling supply from primary production. Recovery of this quantity of Pt offsets demand for an equivalent quantity from primary sources, negating the requirement to mine and process between 2.5-17 tonne of ore and saving 200-1400 $\mathrm{kg} \mathrm{CO}_{2}$. Additional environmental benefits result from avoidance of long global logistics chains necessary for supply of chloroplatinic acid through traditional routes. Enough chloroplatinic acid can be synthesised annually from this single foundry alone to produce $\sim 500 \mathrm{~m}^{2}$ of DSSCs, sufficient to make the electrodes necessary for solar cells to supply clean energy for $\sim 12$ households in South Wales. Utilisation of secondary materials from within the circular economy in this way will result in reduced energy payback times (EPBTs) and cumulative energy demand (CED) for photovoltaics whilst reducing the cost and emissions associated with electricity generated..$^{238}$

The proposed strategy exemplifies the environmental, economic and social benefits available through adoption of circular practices and industrial symbiosis, which make use of secondary materials available within the local economy by valorising wastes. The general approach taken provides a mechanism to improve CRM recovery rates from WEEE and other wastes which are uneconomic to process through traditional recycling pathways. 


\title{
6 Third Generation Photovoltaics - Early Intervention for Circular Economy and a Sustainable Future
}

\begin{abstract}
"We need to accept the fact that natural resources are limited and that we are not using them in a sustainable way. Growing population and per capita consumption are challenging the very essence of our consumer society. Our responsibility, individual and collective, is increasing and is incomparable to the responsibility mankind was facing a century ago. Change is unavoidable. Change in the way we produce, consume, in the way we live... It is essential for our future industrial competitiveness not only that we produce products using less raw materials, less energy and less water, but also that we are able to replace virgin materials and imports with supplies of secondary raw materials where they are available, and that we produce products that can be re-used, repaired, refurbished and recycled. This is what we mean by circular economy"
\end{abstract}

Janez Potočnik 225

European Commissioner for Environment, 2010-2014)

\section{Chapter summary}

Work presented so far focuses on recovering the inherent value of WEEE through means which are very much 'reactionary' i.e. using end-of-life (EoL) practice alone to recover value and manage potential environmental impacts. There has been no consideration of how greater value generation and superior EoL outcomes for future EEE may be achieved through feedback of knowledge from EoL operations to other stages of product lifecycles including design \& manufacturing. This chapter introduces the concept of lifecycle optimisation for circular economy, in which EoL treatment will be very much 'pre-meditated' rather than reactionary, with products and components designed to accommodate upgrade, repair, refurbishment, remanufacturing and recycling. This results in superior 'circularity', with enhanced retention of items, components and materials within the economy through multiple successive product generations resulting in greater resource-efficiency. This enhancement of the industrial ecology of EEE is necessary in the interests of global sustainability and mitigation of climate change and global resource security issues. Here, lifecycle optimisation for emerging printable photovoltaic (PPV) technologies is considered as an example of how this may be achieved for EEE. ${ }^{238}$

$P P V$, which include dye-sensitised solar cells (DSSCs), organic photovoltaics (OPV), and perovskite solar cells, are promising green energy technologies in their infancy which hold the promise of low cost energy generation for the future. At this early stage in development, full lifecycle optimisation, taking account of all parts of the product lifecycle, must be 
conducted to maximise the resource-efficiency benefits associated with the use of PPV and create truly sustainable renewable energy technologies. Here we examine the advantages of lifecycle optimisation for 3GPV technologies along with key aspects of design; materials selection; manufacturing processes; likely applications of the technologies; and potential recycling and refurbishment strategies. In so doing, features which are conducive to circular economy and challenges for resource-efficiency for these technologies are identified along with potential solutions and priority areas for future research in the field.

The findings detailed in this chapter resulted from a multi-disciplinary sandpit organised and co-chaired by the author entitled: '3rd Generation Photovoltaics: Early Intervention for Circular Economy and a Sustainable Future', hosted at the SPECIFIC IKC, Baglan, South Wales on 7th-8th of April 2016. The aim of the 2-day sandpit with stakeholders involved in all stages of PV life cycles including manufacturers, academics, resource managers, circular business model and eco-design specialists. The purpose of the sandpit was to develop a framework for future $R \& D$ which promotes whole lifecycle considerations informed by experts from all stages of the product lifecycle, whilst creating new and strengthening existing collaborations for future funding applications to pursue the progress in this area.

\subsection{Introduction}

We are on the brink of significant climate change and face the limits of current linear economic models. Transition is necessary to a resource efficient 'circular economy' (CE) with widespread deployment of sustainable green energy technologies. Embracing the waste hierarchy for EoL EEE and prioritising reuse and repair > refurbishment/upgrade > remanufacturing > recycling, results in greatest resource-efficiency benefits and larger savings in embedded costs (economic and environmental) of products and components. This is the basis of the superior economic value potential of CE. Whenever costs of reverse logistics and returning products to market are lower than production costs in linear models, circular systems afford greater value than linear systems.

The economic benefits of CE will also increase over time as prices of primary raw materials rise ${ }^{49}$ as seen for CRMs in recent years. CE presents opportunities for substitution of virgin materials by cascading products, components and materials across multiple product lifecycles. Resource-efficiency benefits result from using cascading materials in new applications; more of the embodied costs (labour, energy, materials) are retained than in traditional recycling pathways. This creates opportunities for organisations to valorise 'waste' through industrial 
symbiosis, as described in Chapter 5. Resource-efficiency gains from use of post-consumer materials/components are enabled by design for disassembly and materials separation, which reduces costs of reverse cycles and maintains materials quality and longevity within the circular economy. ${ }^{47}$ Environmental impacts associated with the use of toxic materials in devices can also be mitigated with appropriate circular practices. Additionally, intangible company assets such as brand value may be enhanced as consumers become increasingly environmentally aware. ${ }^{49}$

Growing recognition of these benefits, rising/volatile resource prices $^{49}$ global resource criticality concerns, ${ }^{53}$ and rising production costs has made CE an attractive prospect, particularly for manufacturers who rely on supplies of critical raw materials (CRMs). Organizations such as First Solar who produce CdTe PV have therefore adopted business models that unlock the power of $\mathrm{CE}$ and generate value through development of appropriate recycling technologies, long product lifecycles, and linking value chains with other industries and supply chain partners. ${ }^{254}$

Commercial viability of photovoltaics (PV) is based on the levelised cost of electricity (LCOE) generated, determined by the power conversion efficiency (PCE), cost and lifetime of PV products (Figure 6.1). Resource-efficiency benefits afforded by CE can potentially reduce the economic and environmental costs of module production, enhancing commercial viability and increasing competitiveness with other energy technologies. The use of recovered components and materials can reduce module costs; and design for refurbishment, upgrade, repair and remanufacturing extends module lifetimes. Energy payback time (EPBT), emissions associated with electricity generation $\left(\mathrm{CO}_{2} \mathrm{eq} / \mathrm{kWh}\right)$ and the cost of energy generation $(\$ / \mathrm{Wp})$ can all be reduced through adoption of circular practices. Studies have shown that EPBT for Si wafer based PV technologies are reduced by half through use of recycled materials. ${ }^{255}$ For CdTe PV, it has been predicted that as power conversion efficiency increases and volumes of EoL modules increase, demand for CdTe for PV could be satisfied by secondary supplies from EoL modules alone. ${ }^{256}$ The value of take-back and recovery in this case is enormous. The magnitude of these benefits resulting from lifecycle optimisation is determined by the effectiveness of eco-design, which is greatest when implemented at the earliest possible stage in development of new technologies and performed in collaboration with experts from all parties involved in product lifecycles. 


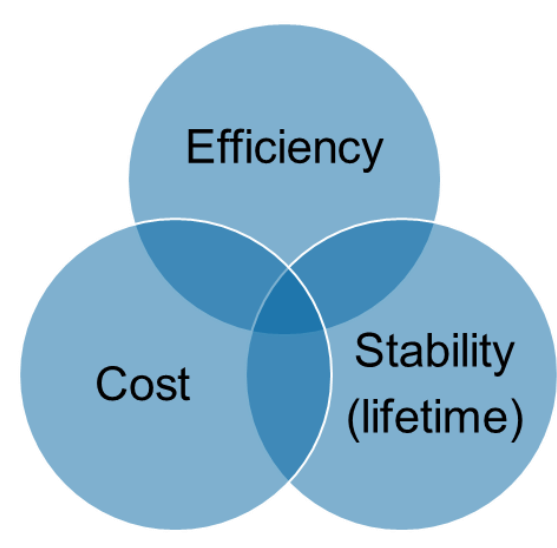

Figure 6.1 The commercialisation challenge for photovoltaics

$3^{\text {rd }}$ generation photovoltaics (3GPV) include dye-sensitised solar cells (DSSC), ${ }^{257}$ organic photovoltaics (OPV), ${ }^{258}$ and lead halide perovskite solar cells ${ }^{259}$ which are promising low cost green energy technologies in their infancy. The advantages 3GPV offer over previous PV technologies include: the use of low-cost abundant materials; the possibility of roll-to-roll (R2R) printing technologies for manufacturing; flexible light-weight devices; suitability for building and product integration; superior performance in diffuse light conditions; and a range of aesthetic possibilities such as tuneable colour and transparency. As a technology in its infancy, 3GPV has high potential for lifecycle optimised for CE. In addition to appropriate business models and reverse logistics systems, enabling technologies and product design will also require careful consideration and optimisation.

Although viewed as a 'green' technology, 3GPV has environmental impacts associated with its production and may contain hazardous components. Widespread deployment will require a continued supply of CRMs and full lifecycle optimisation for CE is necessary at this early stage in development to create truly sustainable technologies. This includes: minimization of environmental impacts associated with production; development of EoL strategies and processes; design for longevity; cradle-to-cradle design optimisation; selection of low impact materials; and substitution of primary resources and CRMs. This is achievable through the process of eco-design ${ }^{168}$ which considers: selection of low impact materials; optimisation of production techniques and reductions in material usage; optimisation of business models and logistics systems; reduction of impacts during use; optimisation of initial life stages of products through design for longevity, upgrade and repair; and optimisation of end-of-life systems. ${ }^{122,260,261}$ Full lifecycle optimisation also requires cross-sector collaboration between all parties involved in product lifecycles including academics, manufacturers, waste managers, designers and logisticians, to enable cascades of reuse, remanufacturing and recycling, and to 
ensure appropriate circular flows of products and materials for optimal economic, environmental and social outcomes.

Here we present an assessment of key features of design, production, reuse and recycling of $3 \mathrm{GPV}$, and the future priority research areas necessary to ensure truly sustainable photovoltaic energy generation. These include: likely applications, architectures and manufacturing processes; attributes of these technologies which are conducive to circularity; appropriate materials selection; potential recycling/remanufacturing processes \& strategies; and potential barriers to circularity/sustainability.

\subsection{Current PV generation technology}

First generation crystalline silicon (c-Si) PV devices are the dominant product on the market today (>90\% market share), due to high PCE and stability. Devices are however fragile, expensive and have relatively high embodied energy compared to successive generations of PV. ${ }^{262}$ Second generation 'thin-film' technologies, which include amorphous silicon (a-Si), cadmium tellurinde (CdTe), copper-indium-sellenide (CIS) and copper-indium-galliumdiselinide (CIGS), have begun gaining market share. They accounted for $\sim 7 \%$ of global PV production in 2015, and some projections show an increase to $50 \%$ by $2030 .{ }^{228}$ Although offering lower PCEs than c-Si PV, $2^{\text {nd }}$ generation PV requires less materials and energy for manufacturing and offers lower cost electricity generation, short energy payback times (EPBT) and reduced emissions associated with electricity generation. ${ }^{263}$ In addition, flexible devices can be created. Despite these advantages, manufacturing involves costly vacuum processes and devices contain toxic materials (e.g. $\mathrm{Cd}, \mathrm{Pb}$ ) and CRMs (e.g. In, Ga, Te), which may limit widespread deployment of these technologies. 256

In light of these issues, we are now witnessing the emergence of 3GPV, or printable PV (PPV), which are thin-film devices based on molecular photoactive layers, potentially manufactured from earth abundant materials using cheap roll-to-roll (R2R) production. These technologies have attracted an enormous amount of research over the past decade. ${ }^{264}$ Early versions of DSSC and OPV devices for niche applications are now commercially available, although much research into new materials, improved device performance and superior manufacturing processes is on-going. Perovskite solar cells, ${ }^{265}$ the newest of the PPV technologies, are yet to emerge on the market because of issues of stability. However, lab based devices have reached efficiencies of $22.1 \%,{ }^{264}$ which is comparable with record cell efficiencies for competing thin- 
film technologies. Perovskite cells hold the promise of low cost and high efficiencies if stability issues can be addressed.

\subsection{Architectures and operation}

3GPV modules are composed of individual solar cells, which are electrically connected and encapsulated in ethylene vinyl acetate (EVA) or glass to form flexible or rigid modules respectively. The various $3 \mathrm{GPV}$ technologies share common features in terms of cell architectures and materials sets. Substrate and electrode materials are common across all technologies as are encapsulating materials. Some of the active materials are common across technologies, although a wide range of active materials and architectures have been explored in research, complicating the issue of materials selection in future devices. Here we limit discussion to 'sandwich' cells (Figure 6.2), with working and counter electrodes on different substrates.

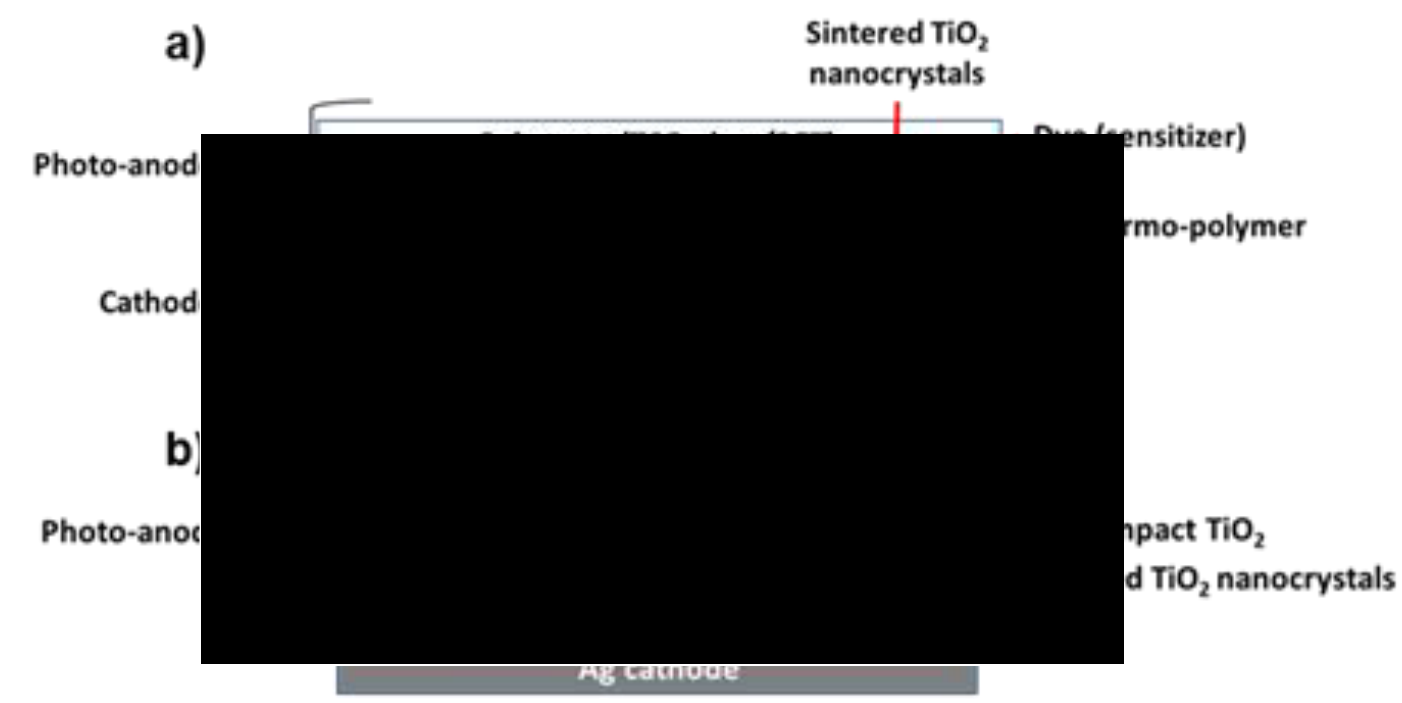

Figure 6.2 a) Dye-sensitised solar cell (DSSC - Grätzel Cell); b) solid-state dye-sensitised solar cell (ssDSSC) (adapted from Snaith, 2013) ${ }^{265}$

DSSCs have been created with various architectures and materials sets. ${ }^{244}$ Generally, these are composed of two electrodes, the photo-anode, and a cathodic counter electrode (Figure 6.2a). One electrode must be transparent e.g. coated glass, or a plastic such as polyethylene terephthalate (PET) for flexible devices. The second electrode can be made of either a similar substrate or a metal foil. 
The photo-anode is composed of a substrate coated with a mesoscopic layer of a semiconductor, commonly $\mathrm{TiO}_{2}$, which is dyed with a sensitiser species capable of absorbing visible light. The counter electrode is either a transparent conductive oxide (TCO) coated substrate, with a catalytic layer of Pt, or a metallic substrate such as Ti. Electrodes are sealed together with a thermopolymer, and the cavity between is filled with a liquid electrolyte of an iodine/triiodide or cobalt complex redox couple. ${ }^{266}$ When illuminated, absorption of a photon by the dye creates an exciton (electron-hole pair). Rapid injection of the electron from the dye into the conduction band of titania follows, enabling the electron to diffuse through the TCO of the anode, around a circuit, and back to the counter electrode. The oxidized dye is reduced by the electrolyte, which is in turn, reduced at the Pt of the counter electrode in the reverse reaction 6.1. DSSCs are commercially available.

$$
\mathrm{I}^{-}+2 \mathrm{e}^{-} \rightarrow 3 \mathrm{I}^{-}
$$

Solid-state DSSCs (ssDSSCs) (Figure 6.1b) are not yet commercialised. Their structure and function is analogous to liquid DSSCs with the electrolyte replaced by a solid hole transport material. These can be organic or inorganic species capable of reducing oxidised dye species and transporting the exciton hole to the counter electrode. A compact titania layer is also used between mesoporous titania and the TCO on the anode which functions as an electron transport layer (ETL) and blocking layer preventing short circuiting through contact of the hole transport materials with the transparent conductor of the anode. Devices use fluorine doped tin oxide (FTO) glass or indium doped tin oxide (ITO) PET as transparent electrode substrates, and metal laminates as counter-electrodes such as Ti. The catalytic Pt layer is not necessary in a ssDSSC.

Perovskite solar cells use methylammonium lead halides $\left(\mathrm{CH}_{3} \mathrm{NH}_{3} \mathrm{PbX} ; \mathrm{X}=\mathrm{Cl}, \mathrm{I}\right.$ or $\left.\mathrm{Br}\right)$ as absorbers. These crystallise in a perovskite structure which gives the cells their names. The first perovskite cells used DSSC type architectures with perovskite infiltrated in a titania scaffold as a sensitiser, referred to as mesosuperstructured solar cells (MSSCs) (Figure 6.3a). ${ }^{267}$ Later it was found that perovskites themselves function as excellent electron transport materials ${ }^{268,269}$ and porous perovskite heterojunction (Figure 6.3b) and perovskite p-i-n heterojunction cells, (Figure 6.3c) without titania/alumina scaffolds, have been created. ${ }^{265}$ The most efficient devices use Au as a contact. Both organic and inorganic hole transport materials have been used.. ${ }^{270,271}$ 


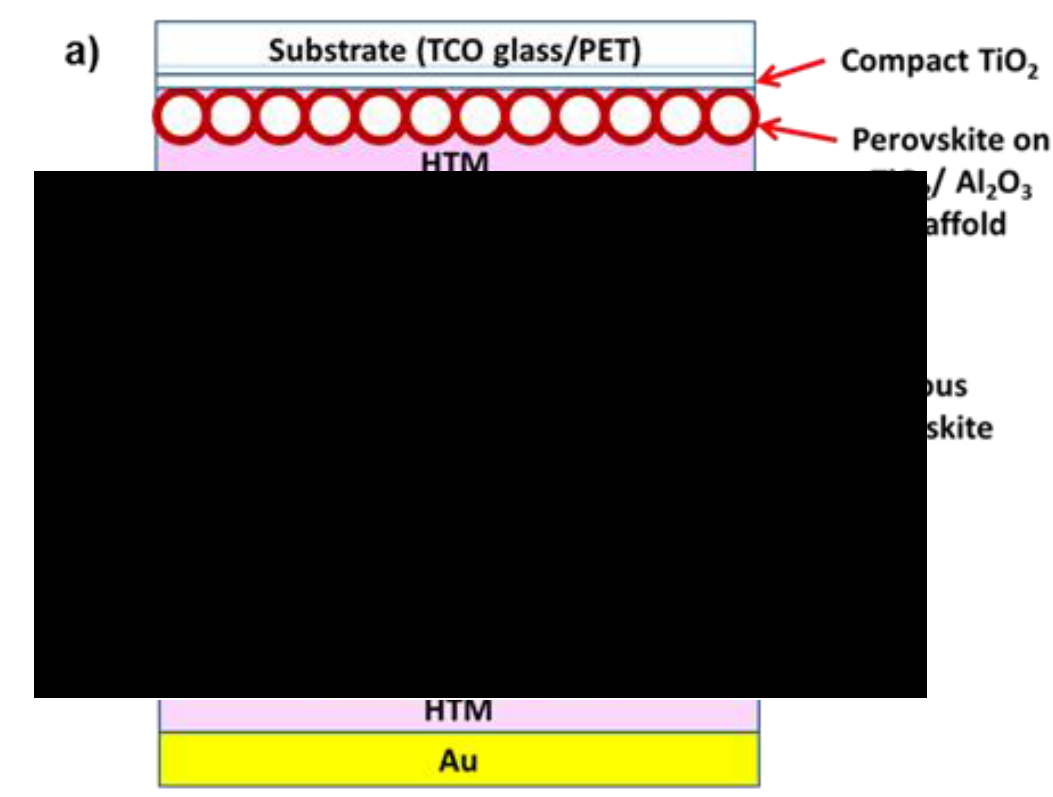

Figure 6.3 Example architectures of Peroskite solar cells: a) perovskite based mesosuperstructured solar cell (MSSC); b) porous perovskite heterojunction; c) perovskite $p$-i-n heterojunction (adapted from Snaith, 2013; ${ }^{265}$ HTM: hole transport material

Between the electrodes of OPV devices are two organic materials with extended conjugated $\pi$-orbital systems. The first is generally a polymer, such as 3-hexylpolythiophene (P3HT), which acts as a light absorber. The second functions as an ETL, typically a fullerene compound such as phenyl-C61-butyric methyl ester (PCBM). These organic materials can be deposited as individual thin films (Figure 6.4), or can be combined and deposited as a single film polymer blend. ${ }^{272}$

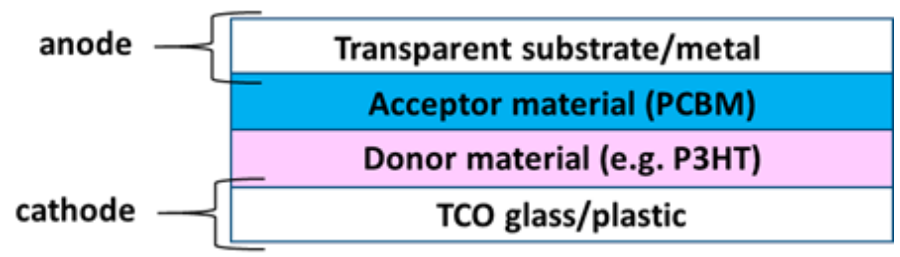

Figure 6.4 Organic photovoltaic (OPV) solar cell device architecture

\subsection{Applications of 3GPV}

Currently, poor stability and low conversion efficiencies prevent the production of commercially viable large area $3 \mathrm{GPV}$ cells, and commercial 3GPV devices emerging on the market are for niche applications. Building integrated photovoltaics (BIPV) to date have been mounted on south facing roofs to achieve good efficiency. $3 \mathrm{GPV}$ can be applied vertically to 
walls and windows. Retrofitting existing buildings which were not designed to bear the additional weight of other forms of PV is also possible. Interesting new product integration possibilities for $3 \mathrm{GPV}$ also exist, particularly at the dawn of the internet of things where electronics will communicate wirelessly and require off-grid energy supply. Additional possibilities exist for combinations with existing PV technologies in tandem devices with enhanced PCEs. ${ }^{273}$

In the immediate future DSSCs will likely find most use in integrated applications. One example is the GCell produced in Wales. ${ }^{274}$ This flexible product has found applications in solar backpacks for charging consumer electronics, keyboard folios for iPads and more recently in the first energy harvesting ibeacon, designed for indoor use (Figure 6.5). Sony has produced prototypes of their indoor Hana-Akari (flower lamps), which use glass based DSSCs to charge batteries to power LEDs (Figure 6.6). An example of BIPV DSSCs include the façade of the SwissTech Conference Centre (Figure 6.7).

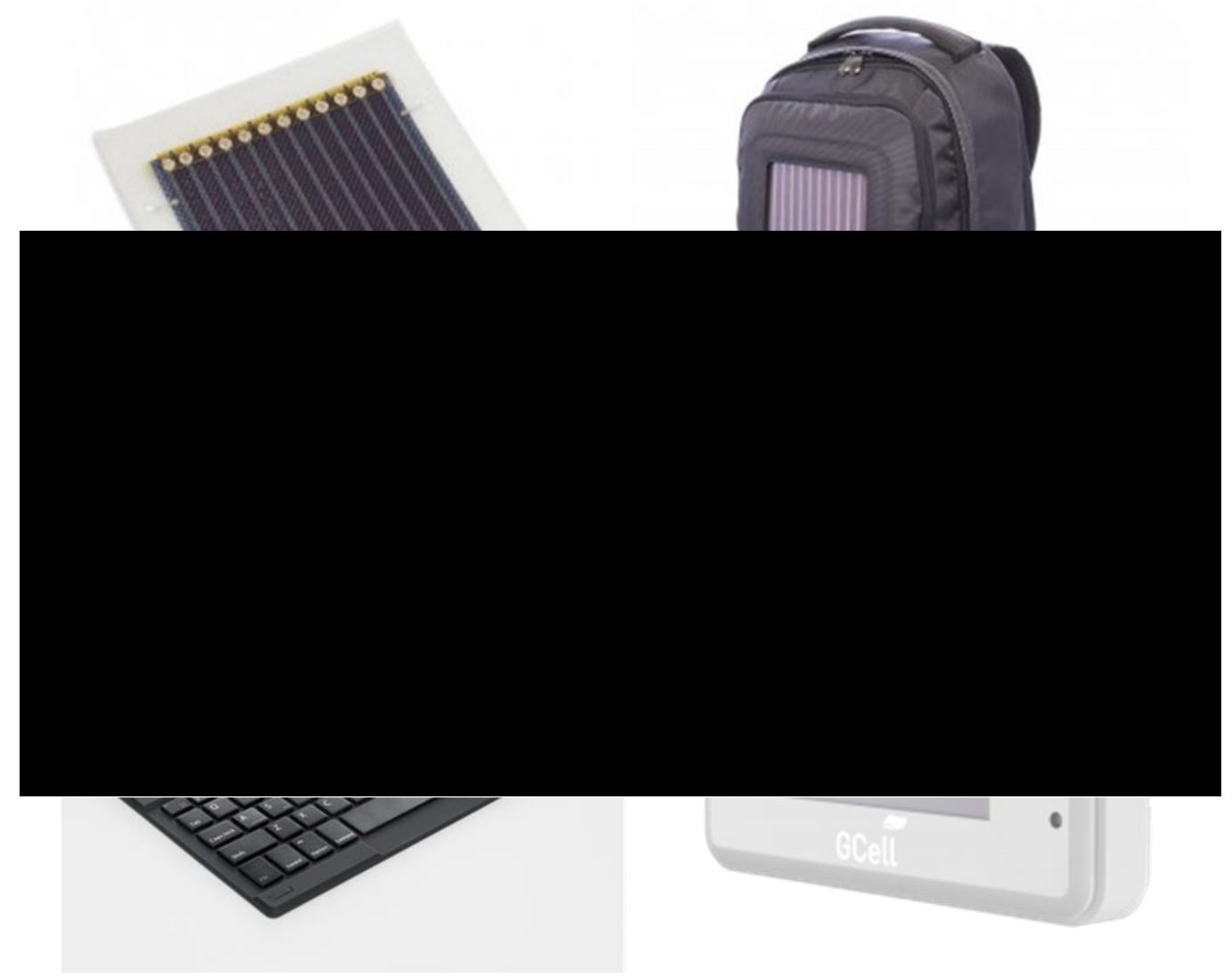

Figure 6.5 The DSSC 'GCell' produced by G24Power (top left) and its product intergrated applications in: solar backpacks (top right), ipad keyboard folios (bottom left), and ibeacons (bottom right); images coutesy of www.gcell.com ${ }^{274}$ 


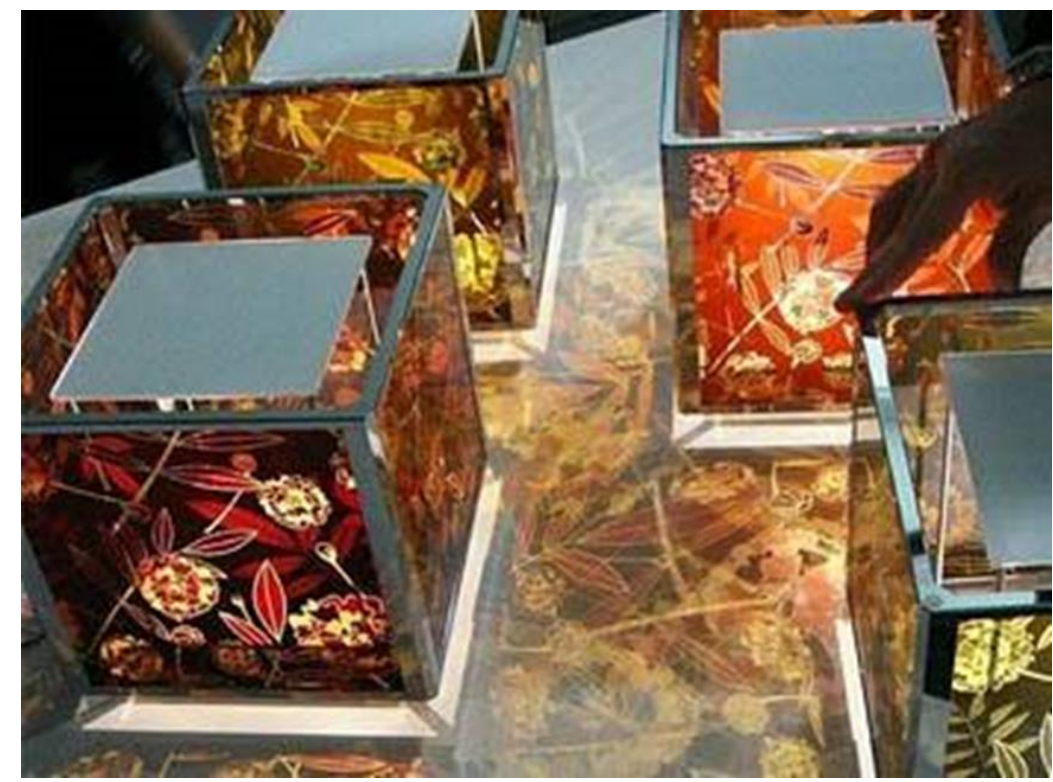

Figure 6.6 Sony Hana-Akari (flower lamps) for indoor use powered by glass based DSSCs in the external flower decorations.

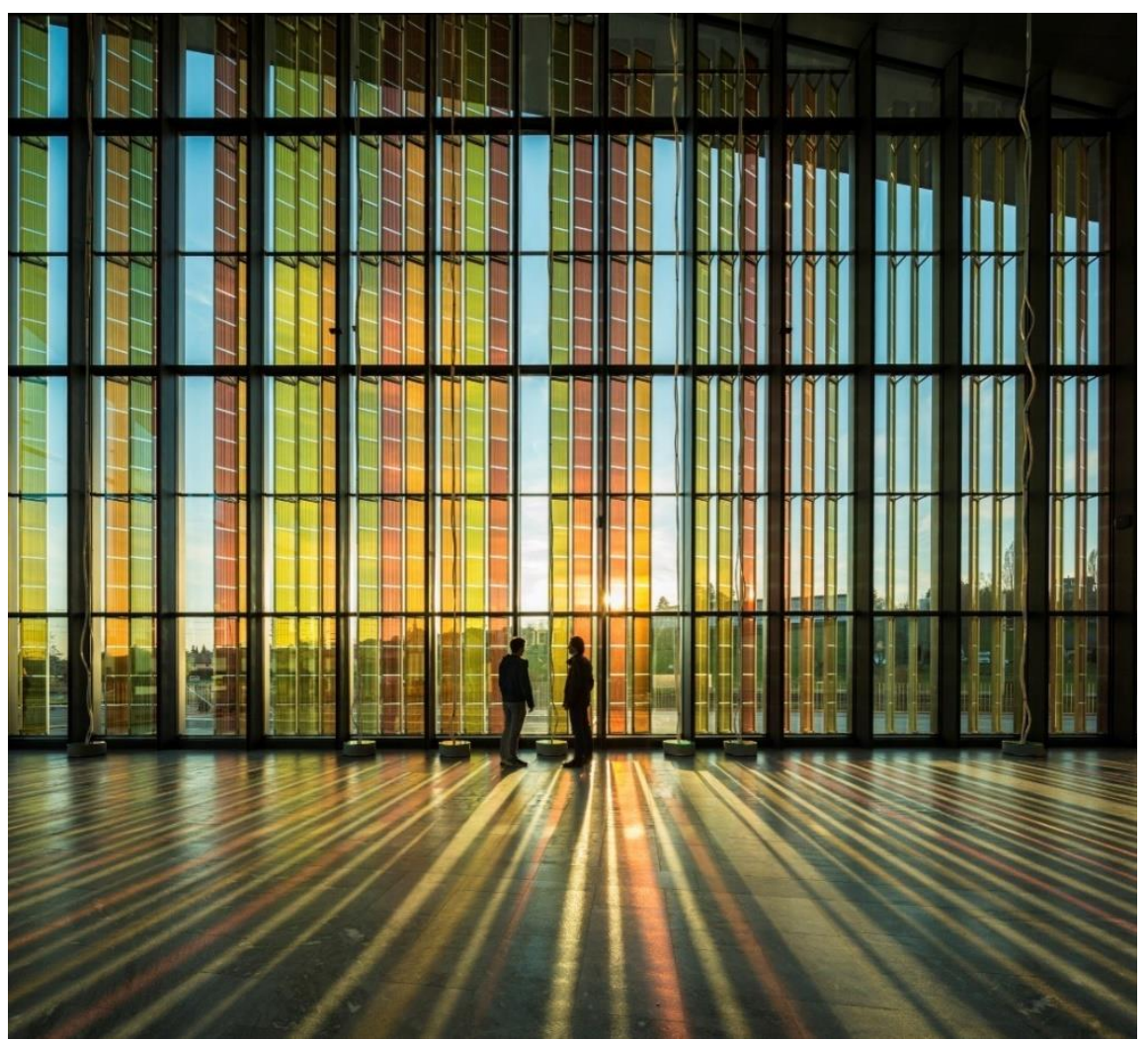

Figure 6.7 Coloured DSSC façade of the Swisstech Convention Centre

The first perovskite products will probably be 'tandem cells', in which a perovskite device is combined with existing PV technologies. This is the goal of Oxford PV who are developing 
and commercialising thin film perovskite solar cells for printing directly onto Si or CIGS modules (Figure 6.8).

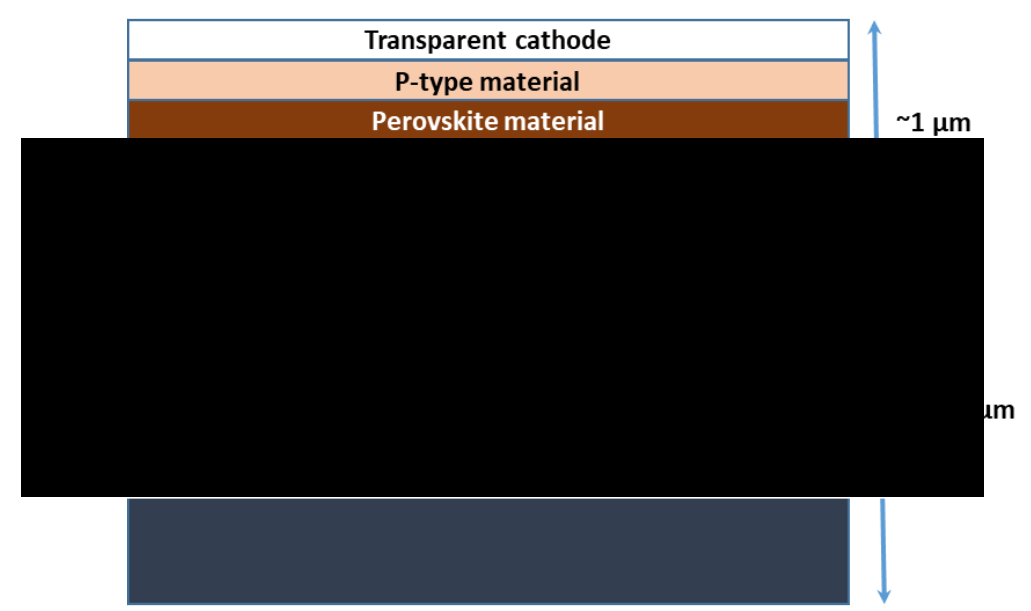

Figure 6.8 Structure of a tandem perovskite-Si solar cell (adapted from Oxford PV) 275

Flexible OPV products have emerged on the market including Heliatek's Heliafilm ${ }^{\circledR}$ for use in BIPV applications, and in the automotive sector for integration with car roofs. ${ }^{276}$ Solar phone chargers, solar adhesive tapes and flexible solar foils are also commercially available (Figure 6.9). ${ }^{277}$ Such products are suited to retrofitting of buildings, windows and consumer electronics.

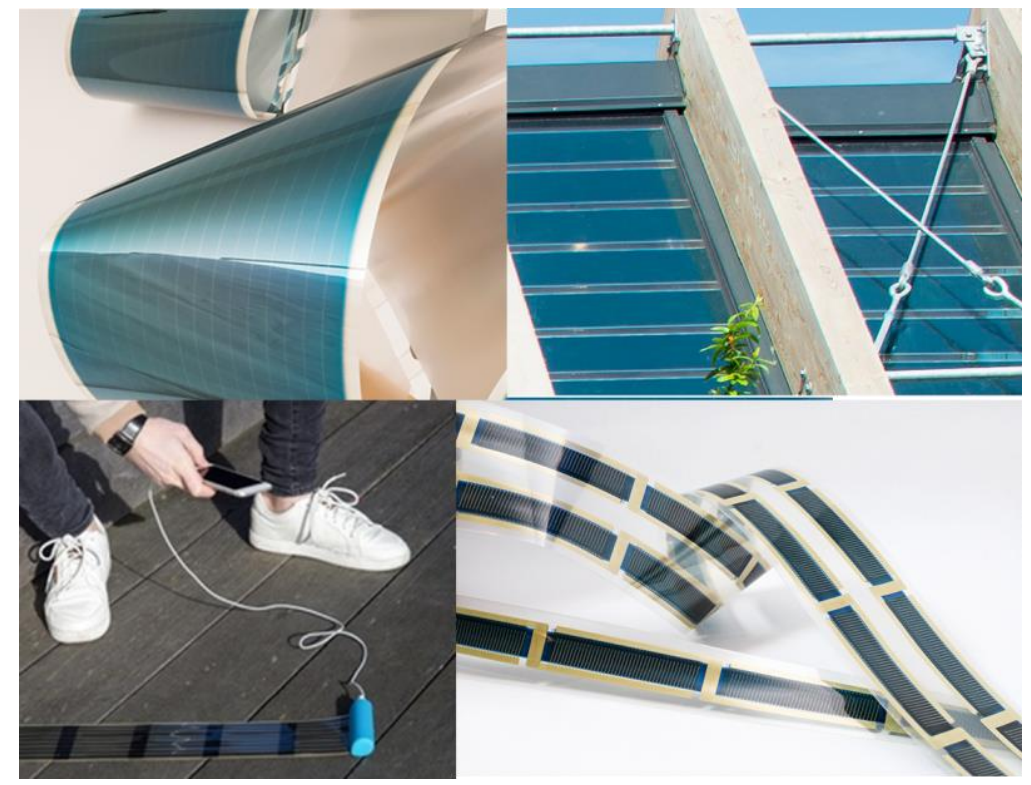

Figure 6.9 Heliatek's OPV Heliafilms ${ }^{\circledR}$ (top left) suitable for: retrofitting buildings (top right; , product integration in mobile phone chargers (bottom left), and solar tape for retrofitting products (bottom right). ${ }^{276}$ 


\subsection{Manufacturing processes}

3GPV is cheap because it can be made using R2R production with solution deposition of materials on flexible substrates. ${ }^{278}$ Rolls of substrate are run through a series of sequential deposition techniques in which the layers of the cell are deposited as thin films (10 nm - 10 $\mu \mathrm{m}$ ), with the final coated product recoiled at the end of the line (Figure 6.10). The result is rapid production at relatively low cost. Substrates include metals, such as steel for functionalised building envelopes, and ITO PET for transparent devices. A variety of solution based coating techniques are possible including: bar coating, screen printing, spray deposition, dip coating, slot-die coating and inkjet printing. Coating is followed by thermal treatment to drive-off solvents and cure films. Convection ovens and hot plates have been employed, but higher throughputs, shorter processing times and greater energy efficiency is achievable with NIR curing. ${ }^{279}$ Photonic flash annealing is another promising option currently under research. ${ }^{280}$ For glass-based devices, R2R is not a possibility and glass must be processed in sheets.

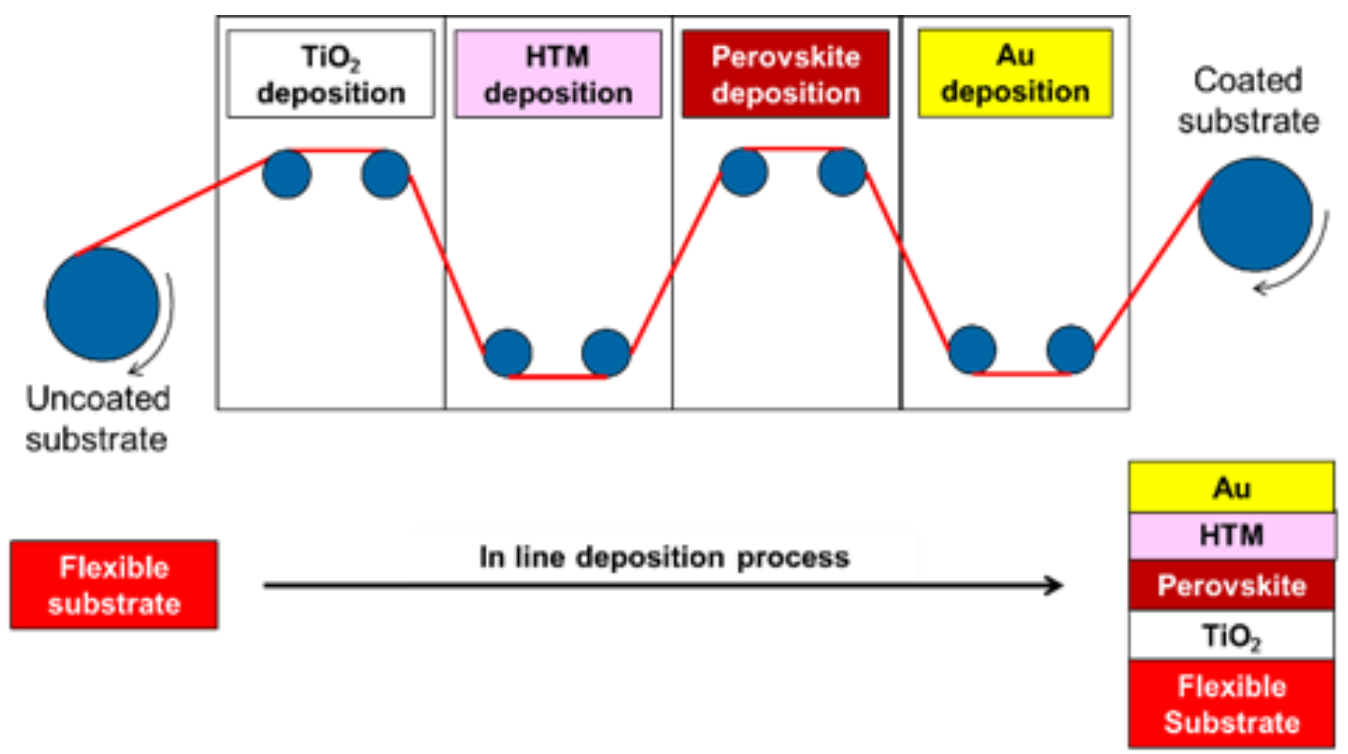

Figure 6.10 Principle of roll-to-roll production of planar p-i-n perovskite cell as shown in Figure 6.3c.

Solution deposition of active materials allows precise quantities of materials to be deposited, with little waste resulting from each print. This is in contrast to the large volumes of waste generated in producing Si wafers which is notoriously problematic to recycle. ${ }^{229}$ Despite the suitability of R2R production for resource-efficient manufacturing, there are still issues which 
need to be addressed: some components, such as gold cathodes, require vacuum deposition techniques which are relatively high in cost, energy demand, and material wastage; masks used in deposition processes retain material; there is the waste issue of production scrap; and solution based processing involves solvent loss. Many of the solvents used, such as DMF and chlorobenzene are hazardous and much research is underway to replace these. New environmentally-friendly solvents are becoming commercially available, such as Cyrene ${ }^{\circledR}$ derived from wood cellulose, which exhibits similar solvent properties to DMF and may be suitable for use in the manufacture of perovskite cells. ${ }^{281,282}$

A particular advantage of solution deposition is that devices manufactured in this way are often capable of being recycled by 'reverse manufacturing', i.e. selective sequential dissolution of the layers using the same solvents as used for deposition. Solutions of recovered materials may then be used to manufacture new cells. This has been demonstrated at lab scale for perovskite cells by Binek et al. (Figure 6.11). ${ }^{283}$

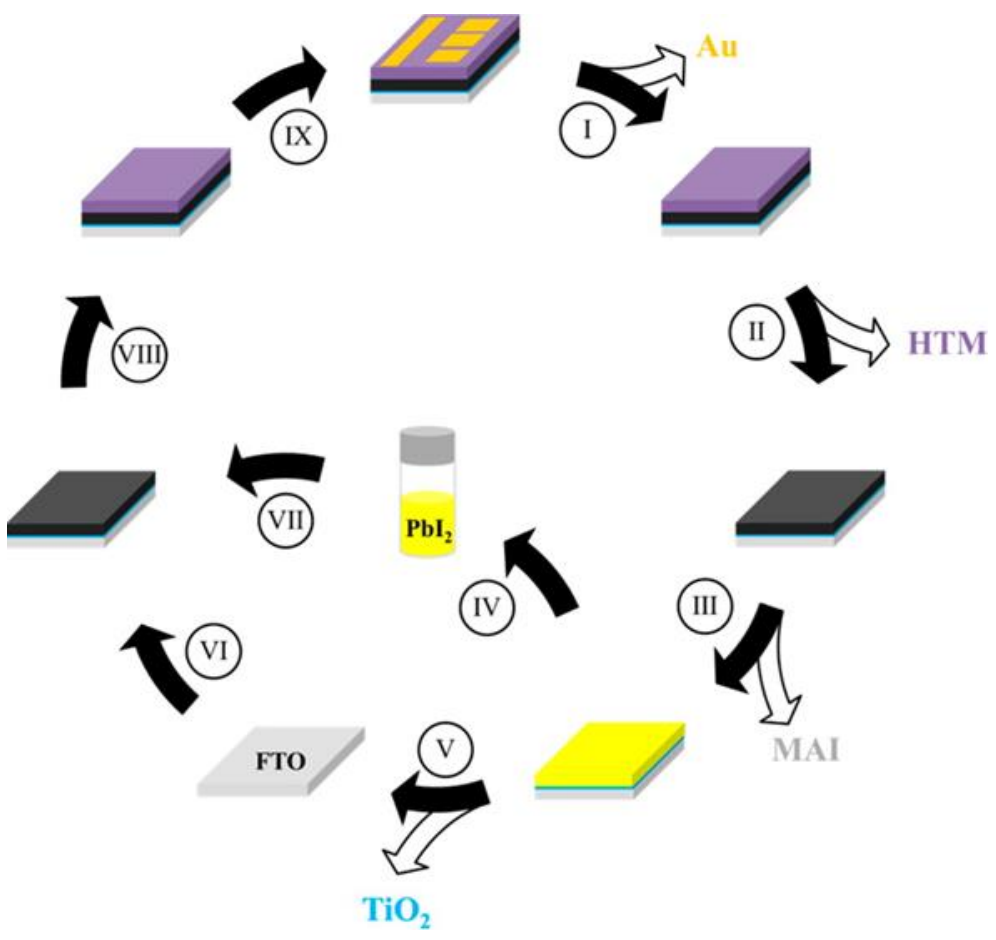

Figure 6.11 Recycling procedure for perovskite solar cells. (I) Removal of Au electrode with adhesive tape. (II) Removal of the HTM by immersing in chlorobenzene. (III) Transformation of the perovskite into methylammonium iodide (MAI) and $\mathrm{PbI}_{2}$ and extraction of MAI in water. (IV, V) Removal of $\mathrm{PbI}_{2}$ and $\mathrm{TiO}_{2}$ using dimethylformamide (DMF). (VI) Preparation of a new $\mathrm{TiO}_{2}$ film. (VII) Formation of the perovskite film on recycled FTO from recycled $\mathrm{PbI}_{2}$. (VIII) Preparation of the HTM layer. (IX) Evaporation of the Au top electrode. (Reprinted with permission from Binek et al., ${ }^{283}$ Copyright (2016) American Chemical Society). 


\subsection{Materials selection}

In the interest of circular economy and sustainable economic development, materials selection must take account of compatibility with EoL processes. Numerous studies have shown that substrates represent a large proportion of the embodied environmental and economic costs of PV cells, ${ }^{258,283-286}$ and that these costs are lower for PET substrates than glass. ${ }^{257}$ Recovering substrates for reuse is therefore important and delamination of modules is necessary. Mechanical delamination usually destroys substrates and thermal delamination degrades PET. ${ }^{229}$ LCA has also shown that laminate materials represent a significant proportion of the embodied costs of thin-film modules ${ }^{286}$ and so their recovery is also desirable. A requirement therefore exists to develop new delamination methods, new laminate materials, or alternative flexible transparent substrates which are compatible with thermal delamination. Chemical dissolution of EVA with sulfuric acid, and lactic acid has been demonstrated for second generation PV modules. There is also a case for the use of glass (flexible glass is available although expensive and fragile) in favour of PET despite its higher embodied cost, because of net resource-efficiency benefits over time resulting from viable recovery and reuse of glass substrates using existing delamination processes. Active materials may also be degraded, particularly in thermal delamination processes, and consideration of the benefit of their recovery by mechanical or chemical delamination in preference to thermal delamination is also necessary. The use of metals as electrodes also requires careful consideration. These are readily recyclable, however their initial deposition in devices is usually by relatively high cost, high energy, high waste, vapour deposition techniques, unless cells are printed directly onto metallic substrates. ${ }^{287}$

3GPV technologies use numerous critical raw materials (CRMs). The traditional TCO used in devices has been ITO. However, due to rising prices and the global criticality of indium and demand for ITO for flat screens, this has been replaced by FTO on glass in solar cell applications. Replacement with FTO on PET has been problematic because the high temperature physical vapour deposition (PVD) process used for FTO deposition degrades PET. Mitigation strategies enabling ITO substitution may result from research into new low temperature methods for deposition of TCOs, such as RF magnetron sputtering. ${ }^{288-290}$ Substitution with graphene coated PET may be a solution, however its cost is currently prohibitive for commercial application. Carbon-nanotubes which can be printed on substrates have also been explored as TCO alternatives. ${ }^{291}$ Another CRM issue arises from the use of Ru in dyes for DSSCs, ${ }^{244}$ and there is much work on fully organic dyes as replacements. ${ }^{292}$ However, further investigation is necessary into their degradation mechanisms and whether they can, after degradation, be converted easily back into their functional forms for reuse. 
An alternative strategy to substitution for mitigating resource criticality issues is to decouple supply from primary production by developing secondary supplies from within the circular economy, including supplies from EoL devices and cascaded materials derived from wastes available within the circular economy (industrial symbiosis). Examples of lab scale processes for production of $3 \mathrm{GPV}$ materials from waste include the production of perovskites from $\mathrm{Pb}$ acid car batteries, ${ }^{237}$ production of carbon based counter electrodes from batteries, ${ }^{293}$ use of conductive glass from LCD screens as DSSC counter electrodes, ${ }^{294,} 295$ and the generation of platinized counter electrodes for DSSCs from waste thermocouples described in the previous chapter. ${ }^{230}$ The use of WEEE as a raw material may lead to significant materials costs savings and even additional revenue for PV manufacturers. Producers of such WEEE, particularly the items described here which result in disposal costs for those producing them, may be happy to pay PV manufacturers to take such material if the cost is below current disposal costs.

Biologically derived alternatives to oil based plastics are an attractive prospect. Transparent flexible substrates composed of cellulose nanocrystals (CNCs) have been used in OPV cells and shown to have high transparency and suitable surface roughness for this application (relatively high surface roughness has hampered efforts to fabricate viable devices based on paper substrates). ${ }^{296}$ In addition, OPV devices on CNC substrates have been shown to be readily recyclable because due to their solubility in water. ${ }^{297}$ Where plant derived materials are used, the carbon sequestration benefit will also contribute to reducing GHG emissions associated with electricity generation.

\subsection{Product integration}

It is likely that the lifetimes of buildings will be considerably longer than the target lifetime of 25-30 years for 3GPV. Full integration into buildings therefore presents issues once modules degrade and reach EoL, and a 'roll-on/roll-off' approach may be useful. OPV lifetimes (currently $\sim 5$ years), are more aligned with those of consumer electronics products and so these devices may be most suitable for product integrated applications. DSSCs on the other hand present an interesting opportunity in terms of in-situ refurbishment. DSSCs fail due to degradation of electrolytes and dyes. But modified cells (Figure 6.12) can be flushed of electrolyte with dilute $\mathrm{NaOH}$ and re-dyed in a period of 5 mins with no loss of functionality, in-fact evidence suggests that this treatment enhances PCEs in comparison to virgin cells. ${ }^{298-}$ ${ }^{300}$ Modification of DSSC design to accommodate a re-dying process presents possibilities for in-situ refurbishment and upgrade in BIPV applications. 


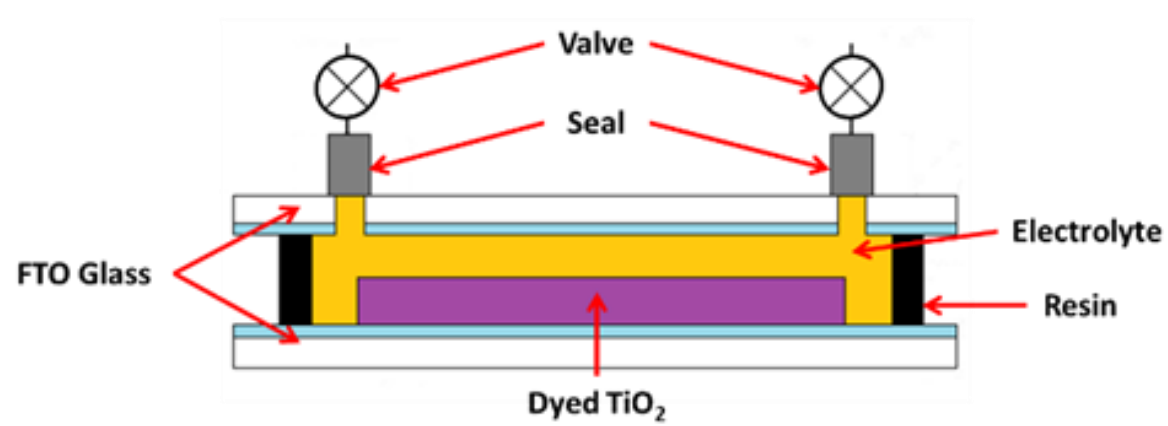

Figure 6.12 Modified DSSC for replacement of dye and electrolyte.

Product integration of PV will also results in complex EoL issues. It is likely that much $3 \mathrm{GPV}$ will end up incorporated into low value small domestic appliances that are commonly recycled with automated pre-processing in which shredding will result in dissipation and loss of PV materials. To address this issue, design optimisation of EEE so that PV can be easily isolated from appliances at EoL is necessary. This will require cooperation between PV manufacturers and their clients, EEE manufacturers who purchase PV for integration into products. The cost benefit of returning PV from where it is globally distributed to EEE manufacturers for reuse in new/refurbished products or PV producers for recycling is likely to be poor due to the low inherent material value of $3 \mathrm{GPV}$ devices small enough for product integration. The 'refurbishability' of DSSCs again presents interesting opportunities for product integration. As DSSCs could potentially be 'recharged' they could retain value after degradation which may justify isolation from products and return to manufacturers for refurbishment using reverse logistics system resembling those currently used for printer cartridges for example. This 're-charging' strategy for DSSCs also presents opportunities for upgrade as new dyes become available which enhance the PCE of cells.

\subsection{Conclusion}

3GPV technologies hold great potential as a sustainable renewable energy source for the future. With full lifecycle optimisation for circular economy enabled by eco-design which takes account of EoL, reuse of substrates, laminates and active materials in successive product generations may be possible. Materials costs savings achieved by this, combined with the possibilities of upgrade and refurbishment to extend product lifetimes could result in the lowest levelised cost of electricity from PV technology to date.

DSSCs show great potential as the first 'refurbishable'/upgradable PV device due to the ability to replace dyes and electrolytes repeatedly with no observable loss in functionality over many 
product generations. The numerous aesthetic possibilities for $3 \mathrm{GPV}$ technologies suggest interesting possibilities in building and product integration.

Priority research areas to enable full lifecycle optimisation include: methods of module lamination/delamination which do not degrade material components; substitution of CRMs; processes for generation of secondary resources from 'wastes' available within the $\mathrm{CE}$ and EoL of cells; development of biologically derived cell components such as CNC based substrates; methods which enhance resource and energy efficiency of R2R manufacturing such as solvent capture and recovery of production scrap; and product design for modularity, recyclability, reusability, refurbishability, upgradability and remanufacture.

The examples discussed for PPV highlight the value of intervention at the earliest opportunity in development of EEE to optimise entire product lifecycles for circular economy. This will avoid the complex problems associated with managing current WEEE arising when technologies reach EoL and enhance the industrial ecology of the EEE industry. This enables greater value from technologies past the point of initial sale, a crucial aspect to enhancing resource-efficiency through circular economy. Consideration of EoL strategy, product longevity, and integration with the wider circular economy when designing products in tandem with manufacturing and EoL processes will lead to cost-effective reuse of products and components, and more efficient recycling of materials. The resulting greater value generation from EEE at EoL could drive enhanced resource-efficiency, thereby facilitating sustainable economic development and a solution to the global WEEE problem for the future. 


\section{Sustainable Solar Energy Storage for Rural Africa}

\section{Chapter summary}

Previous chapters have demonstrated that greater resource-efficiency and value generation from WEEE may be achieved through lifecycle optimisation of EEE for circular economy. However, a transition towards circular economy will be a gradual and continuous process, which will have little immediate impact on the global WEEE problem. In the short term, consumers themselves have a role to play through selection of the most appropriate EEE for given applications. This requires informed consideration of product design and materials contained, as well as available infrastructure for extending the working life of items and managing items appropriately at EoL for best resource-efficiency outcomes. This is not a simple task, as often consumers and those responsible for procurement within large organisations do not possess sufficient knowledge or expertise to make informed decisions on the matter, or easily differentiate between competing technologies. Lack of knowledge by user of EEE may also result in poor operation resulting in shortened product lifetimes, and management and disposal at end-of-life in an inappropriate manor.

Photovoltaics, notably silicon $P V$, are becoming increasingly important in providing localised energy for rural communities in Africa. Although viewed as 'green', these technologies have environmental impacts associated with their production, use, and disposal, and full lifecycle optimisation for the circular economy is necessary to make every aspect of these technologies truly sustainable. One of the key questions when considering installation of solar energy systems is what is the best energy storage technology to use. Here various battery technologies are discussed in terms of their suitability for circular economy and functionality for small scale domestic installation in South Africa. This discussion is within a framework for selection of appropriate battery technologies for use in PV systems for off-grid electrification of rural sub-Saharan Africa. Using South Africa as an example location, selection is informed by careful examination of materials content, cost, local available skills \& knowledge, and infrastructure for management and processing of batteries in a manner conducive to circular economy and greatest benefit for local economies and communities. ${ }^{301}$ 


\subsection{Introduction}

In 2016, 10\% of households in South Africa had no access to electricity. ${ }^{302}$ This equates to around 5.5 million people from a population of 55 million. Most of these households are in rural areas. ${ }^{303}$ In January 2017 the author co-organised and chaired a workshop: 'Eco-Design of Printable Photovoltaics - the Building of an African Infrastructure' in Durban, South Africa, to discuss the multidisciplinary research necessary to develop a functioning circular economy for photovoltaic solar energy systems for rural Africa. The key question which arose from the workshop, more significant than choice of solar cell technology was: "What is the most suitable battery technology for sustainable solar energy storage for small scale domestic use in rural Africa?"

In Swansea University the SPECIFIC Innovation and Knowledge Centre (www.specific.eu.com) team has constructed a building on which new technologies for solar energy collection and storage are monitored and validated. This $\sim 200 \mathrm{~m}^{2}$ building demonstrates the 'buildings as power stations' principles under development by SPECIFIC using technologies embedded into building envelopes to generate, store, and release energy. ${ }^{304}$ The building has a $17 \mathrm{kWp}$ roof integrated photovoltaic $(\mathrm{PV})$ installation, transpired solar air collectors integrated into the external south wall for heating, and $60 \mathrm{kWh}$ capacity of aqueous hybrid ion batteries (C2C certified) for clean and safe energy storage (Figure 7.1) ${ }^{305}$ In Africa, a team from Swansea University installed a small scale off-grid solar energy structure with 1.4 $\mathrm{kWp}$ of integrated PV in an orphanage in Mutende, Lulamba, Zambia (Figure 7.2). ${ }^{306}$ Two 12 V, 102 Ah lead-acid batteries were used for electricity storage. They seemed an obvious choice given they were readily available, easily replaced, durable, and provided the required capacity. But was it the best choice technology? 


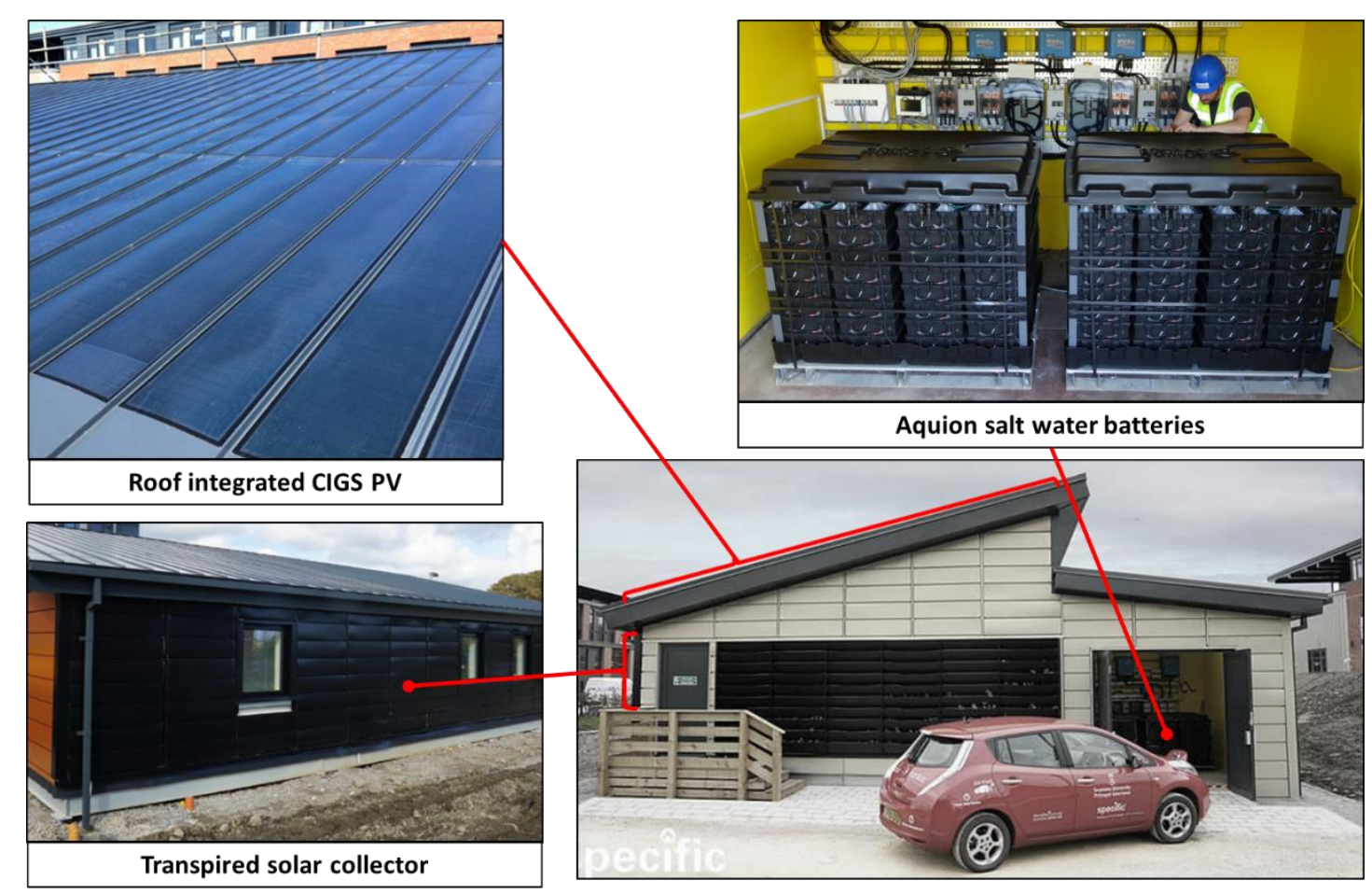

Figure 7.1 Integrated technologies on the SPECIFIC 'Active Classroom'

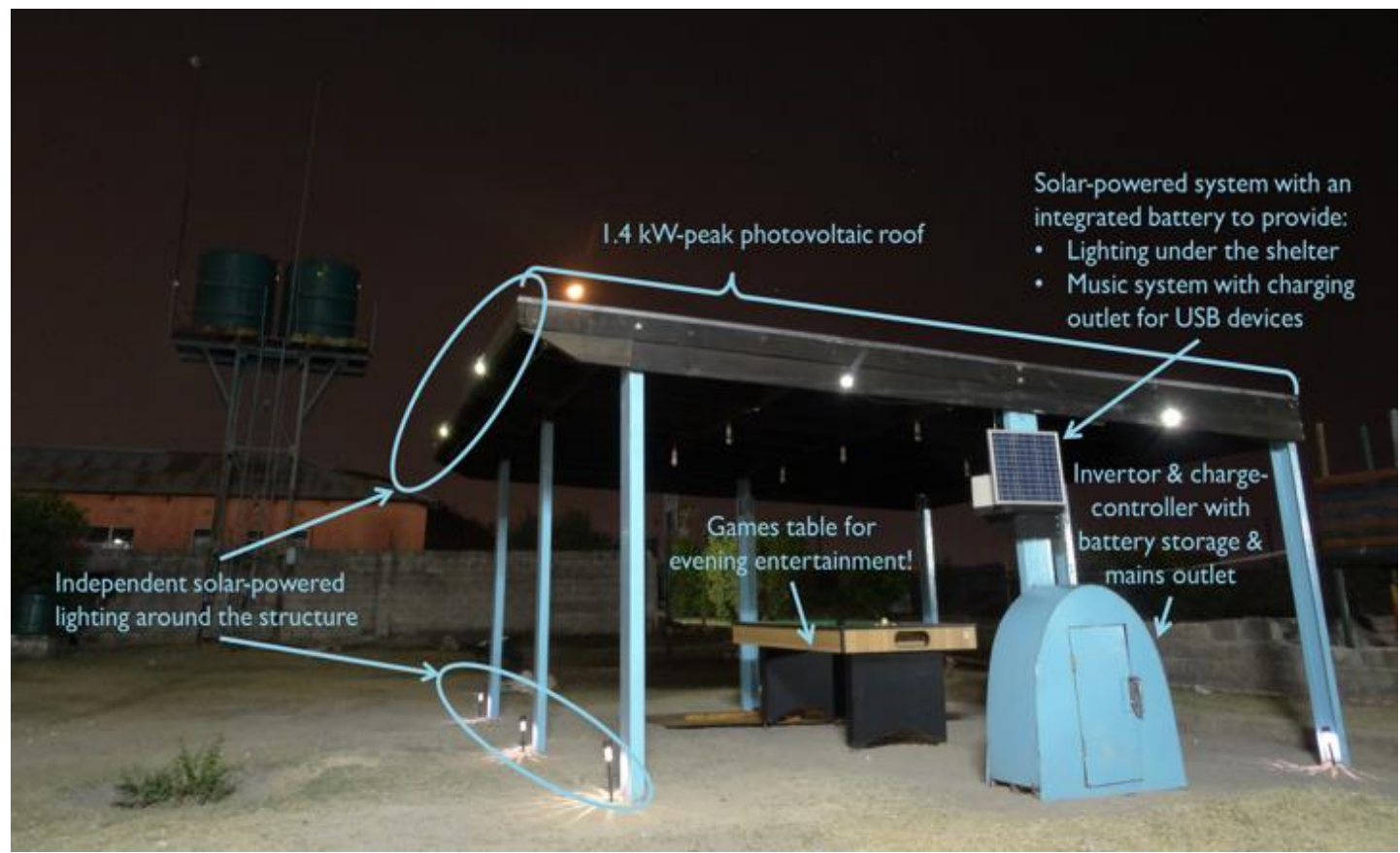

Figure 7.2 Off-grid solar energy structure with $1.4 \mathrm{kWp}$ of integrated PV in an orphanage in Mutende, Lulamba, Zambia

\subsection{Defining the System}

To establish the specification of off-grid systems, the energy requirements of households to be met must first be defined. In recent years, South African energy suppliers introduced a scheme 
to provide $50 \mathrm{kWh} /$ month 'free basic electricity' to grid-connected households, with a plan to develop off-grid solar powered systems providing $50 \mathrm{kWh} /$ month to rural households. ${ }^{303} 50$ $\mathrm{kWh} /$ month is $\sim 1.67 \mathrm{kWh} /$ day, significantly lower than the average daily consumption of $\sim 8$ $\mathrm{kWh}$ /day by grid connected homes in South Africa, but sufficient for basic commodities such as lighting TV, radio, cell phone charging, washing, and possibly refrigeration (Table 7.1).

Table 7.1 Example daily energy consumption for small domestic appliances

\begin{tabular}{lcc}
\hline Item & Usage & Energy consumption (kWh/day) \\
\hline TV (32 inch LCD) & $5 \mathrm{~h}$ & 0.35 \\
Small DC fridge $(50 \mathrm{~W})$ & $24 \mathrm{~h}$ & 1.2 \\
Compact fluorescent light (one $14 \mathrm{~W}$ bulb) & $5 \mathrm{~h}$ & 0.07 \\
Cell phone charger $(5 \mathrm{~W})$ & $3 \mathrm{~h}$ & 0.01 \\
Washing machine $(500 \mathrm{~W})$ & $1 \mathrm{~h}$ & 0.5 \\
\hline Total Energy Consumption & & $\mathbf{2 . 1 3}$ \\
\hline
\end{tabular}

If $90 \%$ of this energy was required overnight, then $\sim 1.5 \mathrm{kWh}$ energy storage is needed. Allowing for an annual load growth of $2 \%$ /year over the 20 year lifetime of the PV system requires $\sim 2.1 \mathrm{kWh}$ of battery storage. (It is interesting to note that increase in electricity demand is such that this year, 2017, the proposed on-grid 'free electricity' allocation in Durban is $65 \mathrm{kWh} /$ month, $\sim 2.1 \mathrm{kWh} /$ day). Battery technology is developing rapidly; here the focus is on systems which could be put in place immediately.

Operational conditions have a strong influence on battery performance and lifetime, and South Africa is a tough environment in terms of ambient temperatures both day-to-day and over the year. To mitigate this, it is proposed that batteries be buried at a depth of $\sim 1.5 \mathrm{~m}$ where the insulation and thermal lag due to soil cover, is expected to limit temperature variations to $\sim 12$ $25{ }^{\circ} \mathrm{C} \cdot{ }^{307}$

To give some idea of what is involved, the battery and photovoltaic requirements have been modelled using the Photovoltaic Geographical Information System ${ }^{308}$ with Durban as an example location. The cost of a system capable of generating a minimum of $50 \mathrm{kWh} / \mathrm{month}$ has been estimated. A $600 \mathrm{Wp}$ photovoltaic system (optimised in terms of orientation and angle) coupled with a $12 \mathrm{~V}, 250$ Ah lead-acid battery with a minimum state of charge cut-off limit of $40 \%$ and a daily power consumption of $2 \mathrm{kWh}$ would yield an average of 56.5 $\mathrm{kWh} / \mathrm{month}$ and $678 \mathrm{kWh} /$ year. Using a value of $1.3928 \mathrm{R} / \mathrm{kWh}(0.096 € / \mathrm{kWh})$ as the cost of electricity (provided by Prof. Bice S. Martincigh, School of Chemistry \& Physics, University of KwaZulu-Natal, Durban, RSA, as a typical cost for electricity in Durban) gives a yearly value of $€ 65.09$ for the $678 \mathrm{kWh}$. The estimated cost of this system is: $0.34 € / \mathrm{W}$ for a multi- 
Si module, ${ }^{309}$ totalling $€ 205.32$ for $600 \mathrm{Wp}$; $€ 345.15$ for a $250 \mathrm{Ah}$ lead-acid battery based on commercially available batteries in South Africa; and $€ 65$ for a charge controller and cables. This gives a total initial system cost of $€ 615$. Over a period of 20 years, given the typical number of cycles from a lead-acid battery, the battery would need to be replaced 4 times ( 5 batteries in total), roughly once every 4 years, bringing the 20 -year cost of the system to $€ 1,996$. Figure 7.3 shows the cost of the system over time with these battery changes and the value of the electricity produced assuming all costs remain constant. It is evident from that most of the system cost is attributable to the batteries $(\sim 87 \%)$. To make the system cost effective, reduction of lifecycle cost is necessary. The role of circular economy as a means of reducing these estimated costs is discussed below.
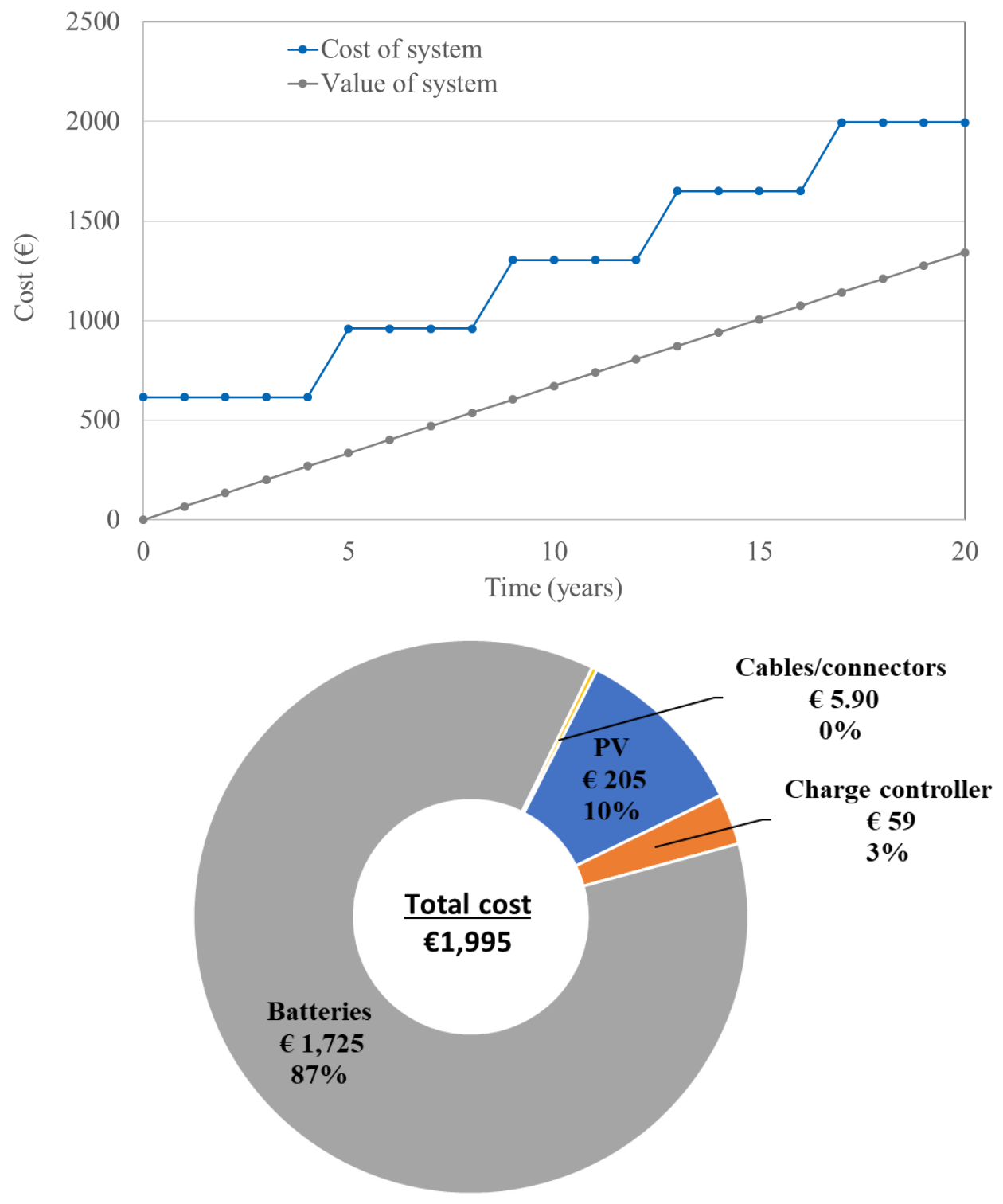

Figure 7.3 An estimated cost of an off-grid DC, PV system in Durban, South Africa, consisting of 600 Wp silicon module, a $12 \mathrm{~V}, 250$ Ah lead-acid battery and a charge controller and cables (top); and breakdown of cost of system ( $€$ ) over 20 years (bottom) 


\subsection{Battery Choices \& Cost}

Four main battery technologies dominate stationary energy storage applications (Table 7.2). ${ }^{310}$ Lithium ion (Li-ion) batteries represent the majority of installed storage capacity and are commonly used in domestic photovoltaic systems. The merits of lead-acid batteries for application in rural Africa have already been highlighted in the discussion of their use for the Swansea University Zambia project. Vanadium redox flow batteries (VRFB), require pumps for electrolyte flow and additional energy and storage capacity to support this. This along with the additional mechanical complexity of VRFB systems makes them unsuitable for small-scale applications. High temperature $\mathrm{NaNiCl}$ batteries are unsuitable because of the hazards associated with molten metal electrodes.

Table 7.2 Globally installed stationary energy storage capacity by battery type (data from Baumann et al. $)^{310}$

\begin{tabular}{lcc}
\hline \multirow{2}{*}{ Battery Technology } & \multicolumn{2}{c}{ Installed Capacity } \\
\cline { 2 - 3 } & $(\mathrm{MW})$ & $(\mathrm{GWh})$ \\
\hline Li-ion & $\sim 1300$ & 1.27 \\
Hight temperature NaNiCl & 171 & 1.01 \\
Valve regulated lead-acid (VRLA) & 196 & 0.173 \\
Vanadium Redox-flow batteries (VRFB) & 114 & 412 \\
\hline
\end{tabular}

Cost is of paramount importance for application in rural South Africa, and Table 7.3 compares costs for three candidate technologies for the proposed system i.e. lead-acid, Li-ion batteries and the Aquion saltwater battery (Table 6.3). Commercially available lead-acid batteries are significantly cheaper than LFP or Aquion batteries ( 10 and 5 times respectively). The easy availability and low capital investment costs of lead-acid batteries are very attractive, but leadacid has comparatively low cycle lives, in comparison to Li-ion and Aquion batteries and a high sensitivity to deep discharge. Significant oversizing of capacity is therefore required. And the relatively short lifetimes for lead-acid batteries mean that these must be replaced 4 times over the lifetime of the proposed system, resulting in a total cost of $€ 1,726$, which is still only one quarter and one third the price of the longer lifetime LFPs $(€ 7,104)$ Aquion batteries $(€ 5,040)$ respectively.

Table 7.3 Comparative costs of commercially available batteries over 20-year lifetime of system

\begin{tabular}{cccc}
\hline Battery & $\begin{array}{c}\text { Cost } \\
(\boldsymbol{\epsilon} / \mathbf{A h})\end{array}$ & No. replacements & $\begin{array}{c}\text { Total cost } \\
(\boldsymbol{\Theta})\end{array}$ \\
\hline VRLA & 1.38 & 4 & 1,726 \\
LFP* & 14.21 & 1 & 7,104 \\
Aquion & 6.72 & 2 & 5,040 \\
\hline *LFYP - lithium-iron-yttrium-phosphate battery; VRLA - valve \\
\multicolumn{4}{c}{ regulated lead acid battery }
\end{tabular}


Of course, economies of scale and direct bulk purchase from manufacturers may result in lower these battery prices and the use of a 'circular economy' approach can give cost savings by: utilising remanufactured, refurbished or repurposed batteries; purchasing batteries manufactured from recovered materials (obtained at lower cost than virgin materials); and valorising end-of-life batteries to recoup costs by diverting them to refurbishment, remanufacturing and recycling processes.

\subsection{Carbon Footprint and Lifecycle Impact Consideration}

A comparison of carbon footprints of lead-acid and different types of Li-ion battery production for the system is given in Figure 7.4 (equivalent data for Aquion batteries could not be found). When the need for replacement batteries is considered, lead-acid batteries account for a greater contribution to the carbon footprint of the system than Li-ion alternatives, except for lithium titanate batteries (LTO). Lithium-nickel-cobalt-aluminium batteries (NCA) would contribute least to the carbon footprint of the system over its lifetime. It should be noted that, utilisation of recovered materials for battery manufacturing would reduce emissions associated with production.

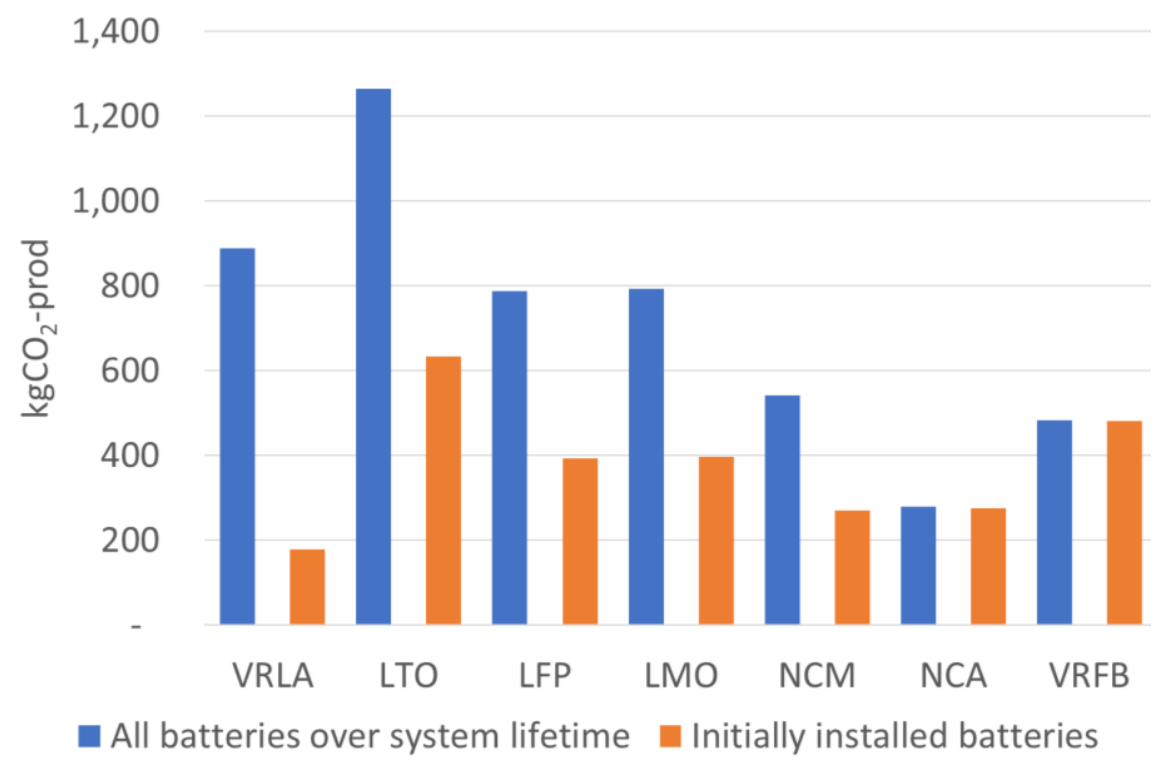

Figure 7.4 Carbon footprint of batteries for the system including replacements (based on data from Baumann et al. ${ }^{310}$. VRLA - valve regulated lead-acid; LTO - lithium-iron-phosphate with lithium titanate anode; LFP - lithium-iron-phosphate with carbon anode; LMO - lithium-manganese-oxide; NCM - lithium-nickel-cobalt-manganese; NCA - lithium nickel-cobalt-aluminium-oxide).

Carbon footprint of the production of batteries is useful for a comparison of global warming potential. However, this is a limited picture of the environmental impacts of batteries. Further consideration of emissions during production from primary resources should be made e.g. the 
production of Li-ion batteries from primary raw materials results in considerable $\mathrm{SO}_{2}$ emissions and water contamination. ${ }^{311}$

Additional consideration of the hazardous nature of materials within batteries and their potential impacts if improperly managed during use and end-of-life is necessary. Issues relating to end-of-life of $\mathrm{Li}$-ion batteries arise from their metal content e.g. Co present in cathodic materials; fluorine, arsenic or sulfonated compounds present in electrolytes; and the extremely reactive alkali metal - Li. Improper treatment of lead-acid batteries at end of life results in the release of $\mathrm{Pb}$ and sulfuric acid to the environment. These materials can directly impact human health through contamination of water \& soil and accumulate in food chains when batteries are landfilled or recycled improperly. ${ }^{117}$ This is of particular concern for rural of sub-Saharan Africa where batteries are installed in isolated underdeveloped area making collection problematic and recycling practices lack basic precautions to prevent emissions of these substance to the workplace and environment, and those recycling facilities that are licensed face serious competition from the informal sector which formerly consisted of small scale backyard recycling operations which are now increasingly replaced by industrial scale informal smelters. ${ }^{312,313} \mathrm{~Pb}$-poisoning of workers is common and fatal in some cases.

The issue of hazardous waste arising from increased deployment of batteries for solar home systems in Africa is significant. In 2016, 1.232 million tonnes of Pb-acid batteries were shipped to Africa containing $>800,000$ tonnes of $\mathrm{Pb}$ (equivalent to $10 \%$ of global production). ${ }^{312}$ The African Renewable Energy Initiative that was launched in 2015 has a 300 gigawatt target for 2030, and solar will form a major part of installed capacity. Nigeria alone has a target of $30 \mathrm{GW}$ of installed solar capacity, which will require an initial installation of over 40 million batteries, and if $\mathrm{Pb}$-acid batteries are used to support the systems, 280 million batteries will have to be installed, recovered and recycled over the lifetime of these systems. ${ }^{314}$

The discussion so far suggests lead-acid batteries are the cheapest option by a significant margin, however their use results in greater global warming potential over the 20 -year lifetime of the system than most Li-ion alternatives.

Optimum battery use requires some knowledge of the technology, as does proper handling of waste batteries. ${ }^{312}$ Thus, any system installation also requires: i) an additional basic education and training package on the benefits of solar energy, and proper operation, maintenance and replacement of components; and ii) full system performance monitoring and analysis for problem/fault prediction/finding. 


\subsection{Resource-efficiency and Circular Economy}

\subsubsection{Critical Materials}

Critical raw materials (CRMs) used in batteries are shown in Table 7.4 with their current supply risk index from the British Geological Survey. The high supply risk associated with vanadium may present future resource security issues for VRFBs, further justifying its elimination as a potentially suitable technology for the proposed application. Li-ion batteries face resource security issues due to $\mathrm{Li}$, Co and graphite, as do Aquion cells which contain graphite. In the interests of global resource security, it is questionable whether technologies containing CRMs should be utilised without further consideration of available infrastructure to support collection and closed-loop recycling, refurbishment and remanufacturing. Leadacid batteries contain no CRMs.

Table 7.4 Supply risks of materials in batteries, those highlighted in orange have been identified as CRMs in recent assesments

\begin{tabular}{ccl}
\hline Element & Relative supply risk index $^{\mathbf{3 1 5}}$ & Relevant battery technology \\
\hline $\mathrm{V}$ & 8.6 & VRFB \\
$\mathrm{Co}$ & 8.1 & LIBs (NMC and NCA) \\
$\mathrm{Li}$ & 7.6 & All LIBs \\
$\mathrm{Graphite}$ & 7.4 & LIBs (LFP, LMO, NMC, and NCA), Aquion \\
\hline $\mathrm{Mn}$ & 5.7 & LIBs (LMO NMC), Aquion \\
$\mathrm{Ni}$ & 5.7 & LIBs (NMC and NCA) \\
$\mathrm{Pb}$ & 5.5 & VRLA \\
$\mathrm{Fe}$ & 5.2 & LIBs \\
$\mathrm{Ti}$ & 4.8 & LIBs (LTO) \\
$\mathrm{Al}$ & 4.8 & LIB \\
\hline
\end{tabular}

Supply risk index runs from 1 (very low risk) to 10 (very high risk); LIBs - Li-ion batteries; highlighted elements included in EU20 critical list.

\subsubsection{End-of-life prospects \& compatibility with circular economy}

Closed-loop recycling of lead-acid batteries is well established in South Africa. First National Batteries operate a network of collection points across South Africa, which divert lead-acid batteries to their smelting facility in Benoni for recycling. Recovered $\mathrm{Pb}$ and plastics are used to manufacture new batteries with optimised design for disassembly. ${ }^{316}$ This suggests end-oflife costs will be low in comparison to other batteries which cannot be recycled domestically, and that lead-acid batteries are an appropriate choice for circular economy in South Africa, with environmental, economic and social benefits afforded through closed-loop retention of the materials within the South African economy. Materials cost savings resulting from use of recovered components/materials should rise with volumes of lead-acid-batteries recycled in the future, resulting in cheaper batteries for the proposed photovoltaic system. Several 
businesses within South Africa operate a lead-acid battery reconditioning service which reverses the sulfation process that limits their working life. This presents opportunities to extend the longevity of lead-acid batteries and reduce battery replacement costs over the system lifetime. With this infrastructure in place, opportunities should be explored for utilisation of automotive lead-acid batteries in a second life for the proposed systems which may be obtained and refurbished at lower cost than they can be purchased, with associated environmental, social and economic benefits resulting from the initiation of new industry.

To the best of the author's knowledge no Li-ion battery recycling exists in Africa. Li-ion batteries are collected and shipped to Europe for recycling, at considerable economic \& environmental cost. South Africa also has no Li-ion battery manufacturing through which to valorise recovered materials in closed-loop material flows. ${ }^{317}$ This indicates Li-ion end-of-life costs in South Africa will be comparatively high with significant logistics costs incurred and little of the social and economic value inherent in $\mathrm{Li}$-ion batteries exploited within South African local economies. High costs increase the likelihood of improper end-of-life management, and the resulting potential for impacts on health and the environment is high. However, the South African government has funded research seeking to develop domestic Liion battery recycling. ${ }^{317}$ Were such an industry to emerge, the derivable economic, environmental and social benefits from Li-ion battery recycling within South Africa could be improved significantly. No future prospects for Li-ion refurbishment exist at this time. It may be possible to source used automotive batteries for reuse in the proposed system at reduced costs with associated environmental benefits to the proposed system. ${ }^{318}$ As an emerging technology yet to be deployed in Africa, Aquion batteries have few prospects for end-of-life treatment within the continent in the near future.

\subsection{Conclusion}

From this preliminary examination of battery technologies for sustainable small scale (50 $\mathrm{kWh} /$ month) domestic installation in South Africa it seems that the current best choice is leadacid batteries despite lower efficiencies and lifetimes than Li-ion and Aquion batteries. This is justified by the ready availability of lead-acid batteries in South Africa through domestic manufacturing; low cost; and existing infrastructure for refurbishment and closed-loop recycling. To maximise the circular economy benefits and achieve economic viability for the proposed PV system, South African energy companies should engage key organisations involved at all stages of lead-acid battery lifecycles, and consider appropriate business models 
to maximise return of batteries at end-of-life such as 'lease and takeback' schemes or deposit schemes for batteries.

Future developments for Li-ion batteries initiated by the South African government may in time enhance the benefits of Li-ions for this application, however high costs, critical materials issues and poor prospects for refurbishment and remanufacturing cast doubt over the suitability of this technology for the proposed system.

Whichever battery technology is used, optimum battery use and management over their lifecycles requires knowledge of the technology. Thus, any system installation also requires an additional basic education and training package on the benefits of solar energy and the proper operation, maintenance and replacement of components; and full system performance monitoring and analysis for problem/fault prediction/finding.

This evaluation is South Africa specific, and based upon local availability of systems and infrastructure to support circular economy around batteries for PV systems. The primary purpose of the work presented is not to identify the most appropriate battery technology for the circular economy for the whole of sub-sahran Africa through examination of the South African context, but to stimulate consideration of the issue by others within the framework applied here, taking account of geographically specific considerations so that optimum solutions to battery selection and end-of-life management can be developed and key areas for further development to support circular economy around off-grid PV systems. 


\section{Conclusion}

The 'global WEEE problem' represents a significant sustainability challenge for humanity. Most of the 41.8 million tonnes of WEEE generated annually around the globe is still unaccounted for and its improper treatment by informal sectors has resulted in significant impacts on the environment and human health.

Initially, this EngD project was focussed on the measurement and assessment of value in WEEE for a local firm, Metech Recycling (UK) Ltd to inform operations and increase the value derived from recycling. However, over the period of study, the author became increasingly convinced that this, while important work, is only a small part of the solution to the global WEEE problem. The emphasis of the project shifted over time in recognition of the fact that linear throughput of materials used by the EEE industry to manufacture new products which replace WEEE represents a resource-efficiency crisis that must be urgently addressed, and that the answer to the 'Global WEEE problem' lies in the replacement of current linear economic models with circular economy.

The study of temporal trends in precious metal content of WEEE described in Chapter 3 shows that quantities of gold and silver in DRAM modules have been stable over the time period 2000-2010, but that copper content has risen, and a very significant decrease in palladium content has occurred as a result of miniaturisation of MLCCs, although Pd content of modules (and other PCBs utilising MLCCs) may eventually begin to slowly rise again when additional MLCCs are incorporated to support advanced functionality. Global sales of desktop PCs and therefore DRAM modules are diminishing as laptops and tablets gain greater market share. Thus, the total size of the 'reserve' of metals in PC DRAM modules is decreasing and therefore so is their recycling potential. Based on projections of global PC sales, it is likely that the annual number of waste DRAM modules produced will decreased in proportion with PC sales.

Gold and silver make up the major value fraction of recoverable value in computers, laptops and tablets, and were there to be a decrease in content of these metals in the future, then major discrepancies between recycling potential projections and the resulting future reality could arise, to the detriment of waste management operations and legislation based upon such projections. The immediate technological changes which may lead to a reduction in gold and silver content are continuing miniaturisation and thrifting in manufacturing, and cloud based computing and data storage. Miniaturisation may well decrease the absolute gold content of a device, but miniaturisation of the whole device may result in sustained or even increases in 
recycling 'grade' of devices overall. Similarly, while cloud based computing will decrease the required functionality of PCs, laptops and tablets and therefore the material value, this will be accompanied by increased volumes of servers in data centres which generally represent higher grade material than PCs. This may mean the shift to cloud computing results in a concentration of precious metals in data centres which may make recycling more efficient.

When considering recovery of critical raw materials from WEEE as discussed in Chapter 4, XRF combined with digital imaging has proven useful for building a library of component images and $\mathrm{x}$-ray emission spectra to inform future recycling processes which target CRMs for recovery.

Cryo-cracking of high value products whose manual disassembly is problematic may be useful as a means of diverting CRM rich PCBs from automated recycling processes in which CRMs are dissipated and lost through unintended co-separation. The use of a guillotine to break through plastic housings and reduce costs associated with isolating CRM bearing components also shows potential, while isolation of SMDs from PCBs is best achieved by use of a solder bath. The use of microwave ashing to pre-concentrating CRMs can provide sufficient cost benefit through mass reduction prior to refining which incurs mass based treatment charges.

While CRMs have been identified throughout the WEEE samples examined, the dissipative nature of applications of CRMs in EEE is a major barrier to recovery. Many CRMs in PCBs partition into slag during smelting for precious metals recovery, rather than into the liquid copper carrier metal for refinement from collected copper bullion. CRMs which are irrecoverably lost this way include $\mathrm{Ga}, \mathrm{Ge}, \mathrm{Ta}, \mathrm{Y}$, and Co. It is possible to isolate SMDs containing these CRMs prior to smelting, but there is little economic justification for the additional cost of this because of a lack of available recovery infrastructure for CRMs, and the fact that the more valuable copper and PMs are recoverable simply by smelting, even though this results in permanent losses of the other CRMs. One solution may be to pursue development of hydrometallurgical recovery methods through which a wider range of CRMs may be recovered. A 'chicken \& egg' scenario exists currently in which isolating CRMs from WEEE in appropriate fractions for downstream recovery is not viable due to lack of recovery infrastructure to valorise CRMs in those fractions, and recovery infrastructure is not in place as insufficient quantities of suitable WEEE derived materials are currently available to feed such processes. Poor design of EEE for disassembly also results in high costs associated with isolating CRM bearing components from WEEE, another barrier to their recovery.

One important feature of circular economy is industrial symbiosis in which waste from one process becomes feedstock for another, and the work described in Chapter 5 on platinized 
counter-electrodes for dye-sensitised solar cells from waste thermocouples develops a local example of this. The process developed essentially 'activates' waste thermocouples (WTCs) produced by Weartech as a cost-effective source of chloroplatinic acid for production of platinized electrodes for dye-sensitised solar cells (DSSCs) in Swansea University SPECIFICIKC labs. Sufficient value is derivable to cover Weartech's landfill costs; provide $\sim 5$ days employment; and enable SPECIFIC to achieve 63\% materials cost saving on chloroplatinic acid in comparison to buying from Sigma-Aldrich. $~ 50 \mathrm{~g}$ of Pt can be diverted from landfill annually negating the requirement to mine and process between 2.5-17 tonne of ore and saving 200-1400 $\mathrm{kg} \mathrm{CO}_{2}$. The proposed strategy exemplifies the environmental, economic and social benefits available through adoption of circular practices and industrial symbiosis, which make use of secondary materials available within the local economy as secondary CRM sources by valorising them to a greater extent than can be achieved through traditional recycling chains, thereby improve CRM recovery rates from WEEE. The principle of direct generation of addedvalue precursor compounds for manufacturing to enhance derivable value from materials in wastes is applicable to other materials and waste streams which are otherwise uneconomic to process through traditional recycling pathways. This highlights the importance of industrial symbiosis as a means to enhance recovery of CRMs and other metals used in dissipative applications in general.

The work on early intervention for circular economy for third generation photovoltaics decribed in Chapter 6 identifies the need for eco-design at the earliest stages of product development. Although photovoltaics represent a 'green' energy solution, whole lifecycle optimisation for circular economy of photovoltaic systems is necessary before this technology can be considered truly 'sustainable'. Priority research areas to enable full lifecycle optimisation include: methods of module lamination/delamination which do not degrade material components; substitution of CRMs; processes for generation of secondary resources from 'wastes' available within the CE and EoL modules and solar cells; development of biologically derived cell components such as CNC based substrates; and methods which enhance resource and energy efficiency of R2R manufacturing such as solvent capture and recovery of production scrap. Consideration of EoL strategy, product longevity, and integration with the wider circular economy when designing products in tandem with manufacturing and EoL processes will lead to cost-effective reuse and remanufacturing of products and components, and more efficient recycling of materials. The resulting greater value generation from EEE at EoL could drive enhanced resource-efficiency, thereby facilitating sustainable economic development and a solution to the global WEEE problem for the future. 
Along with the generation of solar energy there is a need for energy storage, and sustainable energy storage is at least as important as sustainable generation, particularly for small scale generation. From the preliminary examination of battery technologies for sustainable small scale off-grid domestic PV installation in South Africa described in Chapter 7, lead-acid batteries are determined to be the best choice because of low cost, domestic manufacturing, and an existing infrastructure for collection, refurbishment and closed-loop recycling into new products with design for disassembly. $\mathrm{Pb}$-acid batteries are more compatible with existing local knowledge, skills and infrastructure to support circular economy than alternative battery technologies, so greater potential exists to generate economic, social and environmental value from $\mathrm{Pb}$-acid batteries than alternative technologies. Optimum battery use and management throughout their lifecycles to maximise their longevity and retention of batteries and constituent materials requires knowledge of the technology. Basic education and training on the benefits of solar energy and the proper operation, maintenance and replacement of components must therefore accompany any system installations to ensure component longevity is maintained, and that EoL components are managed appropriately. Full system performance monitoring and analysis for problem/fault prediction/finding is also required. While this evaluation was specific to South Africa the framework for evaluation which takes account of local conditions is generally applicable to all EEE. It is hoped this work will stimulate consideration of these issue by others so that optimum solutions to battery selection and end-of-life management can be developed. Key areas for further development to support circular economy around off-grid PV systems include investigation of appropriate reverselogistics and business models which suit low population density rural areas and will facilitate maximum collection rates of EoL PV system components, suitable legislation to govern EoL management of system components, and a thorough evaluation of how the informal sector may be incorporated into a functioning circular economy.

In the broader context, circular economy represents a solution to the global WEEE problem and a means to enhance the industrial ecology of the EEE industry. By effective exploitation of the inherent value of WEEE, significant economic, environmental and social benefits can be derived, whilst limiting detrimental impacts resulting from its hazardous nature.

Treatment processes which are conducive to circular economy provide opportunities for greater valorisation of WEEE than is possible through traditional recycling process chains. Upgrade, refurbishment and repair of WEEE extends its life so that it can be reused. This, as well as remanufacturing with retention of as many original components as possible; cascading components and materials across product lifecycles; and recycling with the greatest efficiency possible including industrial symbiosis approaches, conserves primary raw materials and 
natural capital and retains materials within WEEE in an economically productive capacity. Greater value generation afforded through such processes provides a financial incentive which may deter illegal exports, and reduce quantities of WEEE processed in 'back yard' recycling operations reducing impacts on human health and the environmental resulting from WEEE. The extent to which economic value is exploited form WEEE determines the magnitude of associated environmental and social benefits, and so the waste hierarchy should be embraced and prioritised.

Circular material flows require sufficient economic value to be derived from WEEE at EoL to finance the costs of necessary logistics and processing. Reuse, remanufacturing and recycling must all exploit as much of the inherent value of WEEE as possible. To date, little remanufacturing of WEEE occurs and effective reuse of WEEE and its components is often prohibited by inadequate product design. To overcome these barriers in the future, full lifecycle optimisation of EEE will be necessary to ensure that problems associated with management of WEEE today, are avoided in the future. To enable this, eco-design of products to accommodate efficient disassembly, upgrade, refurbishment, remanufacturing and component/materials recovery must be undertaken. Manufacturing and remanufacturing must be optimised in tandem with recycling so that recovered materials from EoL products can be efficiently cycled back into manufacturing. Product design must also accommodate this. Complete lifecycle optimisation such as this is most effective when intervention at the earliest possible stage in design of new products occurs. This should take account of suitable secondary materials available for manufacturing, compatibility of materials with recycling and remanufacturing processes, substitution of CRMs where possible, failure mechanisms of technologies, and the applications of technologies. Appropriate reverse-logistics and business models are essential to ensure efficient collection so circular material and product cycles are realised from which maximum value can be exploited. This is only possible with a collaborative multi-disciplinary approach involving experts and stakeholders in all aspects and stages of product lifecycles.

For existing WEEE which has not benefited from lifecycle optimisation and eco-design for reuse, upgrade, repair and remanufacturing, efficient recycling of all materials, particularly precious metals in PCBs which represent the greatest part of recoverable economic and environmental value, is of paramount importance. The economic value afforded through recovery of these materials grants viable recovery of other strategically and environmentally important components such as CRMs which on their own are of insufficient value for economic recovery. Effective recovery of these materials is possible only with costly manual pre-processing, and so exploiting the maximum value from items is necessary to ensure as 
much WEEE as possible falls above the cut-off grades for manual processes. Without viable manual processing, the derivable economic value and efficiency with which CRMs and other 'high-tech' metals can be recovered is limited.

Exploitation of the maximum value of WEEE through recycling relies on knowledge of the total available quantities of target materials in the waste stream, and their location within individual WEEE items. This information about the 'urban mine' is analogous to 'prospecting' of primary resources when planning mining operations. Detailed knowledge of the material composition of future WEEE and material value contained therein, and volumes of specific categories and types of items that will be available in the future waste stream is required. This will inform planning of future recycling strategies capable of exploiting the maximum possible material value from WEEE. As the nature of the future waste stream cannot be known with certainty, predictions must be made based upon available knowledge of current trends in manufacturing practices, lifetime distribution data for EEE products, likely changes in consumption patterns following the emergence of new technologies onto the market, and data on quantities of EEE placed on the market. This must take account of temporal trends in manufacturing which may result in reductions in precious metal content in WEEE, which in combination with rising costs of recycling could result in major barriers to manual recycling of WEEE in the future. Data on historic temporal trends in material composition of items can be used to improve the accuracy of such predictions which currently use static compositional data for existing items.

In the interests of sustainable economic development, the strategic value of WEEE must also be exploited to a far greater extent through efficient recovery of CRMs. In general, the low economic value of CRMs and dissipative nature of their applications in EEE renders recovery economically unviable through traditional recycling processes. Shredding of WEEE results in dissipative losses of CRMs through unintended co-separation with bulk material fractions, so processes are necessary which enable isolation of CRM bearing components from WEEE in their own distinct material streams for recovery. At this time, a lack of such processes as well as those for recovery of CRMs identified in WEEE prevents viable recovery.

Enhancing CRM recovery rates from WEEE requires efforts to reduce pre-processing costs for isolating and concentrating CRM bearing components from WEEE, and to enhance the derivable value from CRMs through recovery. Innovative separation processes for isolated components, such as sieving with further mechanical separation which avoids shredding, or intelligent liberation/sorting systems which make use of optical recognition and established knowledge of CRM content of components, or online XRF analysis to identify and isolate 
CRM bearing components in WEEE, are required. Processes which enable cost-effective recovery of CRMs from output fractions of pre-processing must also be commercialised so that isolated CRM bearing fractions from WEEE may be directed to them. Hydrometallurgical processes offer superior control and flexibility in this regard than established pyrometallurgical recovery processes. In addition, recovery processes which derive greater value through circular economy are required for viable recovery for materials that are currently lost due to low economic value.

Demand for CRMs from the EEE sector is a major contributing factor to the critical status of high-tech metals. One method of addressing materials criticality is to harvest and reuse components, offsetting demand for virgin components manufactured from primary raw materials. This more 'closed-loop strategy' may result in greater value generation from the isolated components. In the future, cost-effective isolation of such components may be achieved through robotic automation should sufficient quantities of similar items be gathered to justify the capital investment. One example of such a technology is Apples's 'Liam' the robot, designed to disassemble Apple's own products. It will be difficult to stimulate a demand for such second-hand components who's functionality may be questionable, and who's cost is likely to be comparable to, if not higher than new components manufactured at massive scale. The solder bath method could be used to recover components from PCBs for this purpose, however, further study of the impact of this high temperature process on functionality of recovered components is necessary.

The value available through circular economy and cascading materials across product cycles may be sufficient to drive CRM recovery in the future. Added-value opportunities through industrial symbiosis (and other cascades of reuse) in which CRM bearing components of WEEE are used as feedstocks for manufacturing holds the potential to generate sufficient value for economic CRM recovery. Such opportunities will enhance derivable value from WEEE and therefore lower cut-off-grades for manual recycling, whilst improving global resourceefficiency and mitigating resource criticality for manufacturing. Local industrial symbiosis solutions may also reduce the environmental impacts of materials recycling when economic and environmental costs associated with logistics throughout existing global recycling process chains are avoided. Processes which cycle materials into emerging 'green' technologies are particularly valuable in terms of sustainable economic development. Clean energy technologies, including wind turbines, electric vehicles, photovoltaic cells and fluorescent lighting, currently consume about $20 \%$ of global CRM production, growth of emerging technologies will increase this quantity significantly in the future. Securing secondary sources of CRMs for such applications will be crucial for reducing global emissions and combating 
climate change. As such, new strategies for generating additional value from CRMs in WEEE through recovery for use as raw materials for clean-energy technologies will be essential to meet demand and increase the resilience of these emerging industries to global resource security issues, thereby safeguarding jobs and futureproofing technologies.

The issue of criticality is one that can be addressed through a number of strategies apart from enhanced recovery. Research into suitable substitutes for CRMs should be explored, as well as new manufacturing processes which enable utilisation of alternative materials in mass production The use of FTO in solar cells in place of ITO containing critical indium is a good example of an industry reacting to the issue of 'resource criticality'. Optimisation of logistics (and reverse-logistics) to minimise damage of products on their way to the market place, and during take-back will also result in less generation of WEEE and reduce demand for virgin CRMs.

The EC's ongoing Eco-design Working Plan initiated as part of the Circular Economy Package aims to investigate measures for implementation which would mandate appropriate product design measures to support circular economy. ${ }^{319}$ This opportunity to establish overarching legislation to drive progress in this area should be capitalised upon. This would result in legislative pressure on OEMs to adopt such practices in addition to existing extended producer responsibility (EPR) imposed by the WEEE directive. This is particularly important when the implementation of EPR as collective producer responsibility (CPR) is common. Where possible, CPR systems of compliance should be altered to individual producer responsibility (IPR) systems, providing a direct economic incentive to OEMs to design products in a way which reduces EoL costs by enabling reuse, upgrade, repair, remanufacturing and efficient recycling.

Major impacts on the global WEEE problem through exploitation of the inherent value of the waste stream must be facilitated by significant improvements in collection efficiencies and diversion of collected WEEE from inappropriate recycling practices in the developing world. Education about the WEEE problem at hand is also vital to ensure that we, the users of EEE, make appropriate decisions when it comes to disposal of items. Equally valuable is the education of those involved in informal WEEE sectors in the developing world so that they may take measures to protect themselves and the environment from the hazards associated with materials from WEEE. Such education would go a long way to motivating those involved in the informal WEEE sector to make more responsible choices concerning the practices they use to treat WEEE. Many who conduct backyard-recycling do so out of necessity, with few alternative employment opportunities available to them. It is therefore essential that solutions 
to the global WEEE problem embrace the informal sector, and that value exploited from WEEE is shared amongst all parties involved in the lifecycle of EEE to the benefit of global society and the environment. 


\section{Appendix A - Evaluation of Fischerscope XDAL}

This section outlines assessments made of the Fischerscope XDAL instrument's ability to detect and quantify CRMs in WEEE samples.

\section{Qualitative analysis: direct measurement of CRMs in devices}

Standard spectra of CRMs were obtained from metal foils of Pd, Pt, Au, Ge, Co, Ta, Ag, W, $\mathrm{In}, \mathrm{Mg}, \mathrm{Nb}$ and $\mathrm{Y}$. Attempts were made to detect these foils through i) $0.1 \mathrm{~mm} \mathrm{Al}$ foil and ii)

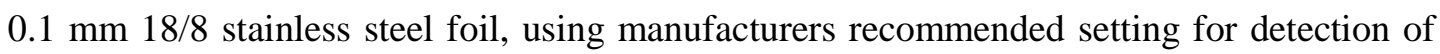
trace metals. (50 keV with a beam diameter of $0.3 \mathrm{~mm}$ and $\mathrm{Al}$ primary filter applied). The results are given in Table A.1. All elements tested were visible through aluminium, with reduced peak intensity in the cases of $\mathrm{W}, \mathrm{Nb}$ and $\mathrm{Y}$. Through the stainless-steel foil only Ag, In and Ge were visible. Results suggest that it may be necessary to cut open encapsulated components or grind them to powder before scanning. The same may be true for SMDs encapsulated in glass, plastic, or ceramic.

Table A.1 Detection of metals through steel and aluminium foils.

\begin{tabular}{|c|c|c|c|c|}
\hline CRM & $\begin{array}{l}\text { Nature of } \\
\text { electronic } \\
\text { transitions }\end{array}$ & $\begin{array}{c}\text { Emission } \\
\text { energy } \\
(\mathrm{keV})\end{array}$ & $\begin{array}{l}\text { Visibility through } \\
0.1 \mathrm{~mm} \text { Al foil }\end{array}$ & $\begin{array}{l}\text { Visibility through } 0.1 \mathrm{~mm} \quad 18 / 8 \\
\text { stainless steel }\end{array}$ \\
\hline $\mathrm{Pd}$ & $\begin{array}{l}\mathrm{K} \alpha_{1} \\
\mathrm{~K} \beta_{1}\end{array}$ & $\begin{array}{l}21.18 \\
23.82\end{array}$ & Clearly visible & $\begin{array}{l}\text { Clearly visible with diminished peak } \\
\text { height }\end{array}$ \\
\hline $\mathrm{Pt}$ & $\begin{array}{l}\mathrm{L} \alpha_{1} \\
\mathrm{~L} \beta_{1}\end{array}$ & $\begin{array}{c}9.42 \\
11.07\end{array}$ & Clearly visible & Not visible \\
\hline $\mathrm{Au}$ & $\begin{array}{l}\mathrm{L} \alpha_{1} \\
\mathrm{~L} \beta_{1}\end{array}$ & $\begin{array}{c}9.71 \\
11.44\end{array}$ & Clearly visible & Not visible \\
\hline Ge & $\begin{array}{l}\mathrm{K} \alpha_{1} \\
\mathrm{~K} \beta_{1}\end{array}$ & $\begin{array}{c}9.89 \\
10.98\end{array}$ & Clearly visible & $\begin{array}{l}\text { Barely visible (only with maximum } \\
\text { expansion and zoom on spectra) }\end{array}$ \\
\hline Co & $\begin{array}{l}\mathrm{K} \alpha_{1} \\
\mathrm{~K} \beta_{1}\end{array}$ & $\begin{array}{l}6.93 \\
7.65\end{array}$ & Clearly visible & Not visible \\
\hline $\mathrm{Ta}$ & $\begin{array}{l}\mathrm{L} \alpha_{1} \\
\mathrm{~L} \beta_{1}\end{array}$ & $\begin{array}{l}8.15 \\
9.34\end{array}$ & Clearly visible & Not visible \\
\hline $\mathrm{Ag}$ & $\begin{array}{l}\mathrm{K} \alpha_{1} \\
\mathrm{~K} \beta_{1}\end{array}$ & $\begin{array}{l}22.16 \\
24.94\end{array}$ & Clearly visible & $\begin{array}{l}\text { Clearly visible (diminished peak } \\
\text { height) }\end{array}$ \\
\hline $\mathrm{W}$ & $\begin{array}{l}\mathrm{L} \alpha_{1} \\
\mathrm{~L} \beta_{1}\end{array}$ & $\begin{array}{l}8.40 \\
9.67\end{array}$ & $\begin{array}{l}\text { Clearly visible with } \\
\text { reduced } L_{\alpha} L_{\beta} \text { peak } \\
\text { ratio }\end{array}$ & Not visible \\
\hline In & $\begin{array}{l}\mathrm{K} \alpha_{1} \\
\mathrm{~K} \beta_{1}\end{array}$ & $\begin{array}{l}24.21 \\
22.28\end{array}$ & Clearly Visible & Visible \\
\hline $\mathrm{Nb}$ & $\begin{array}{l}\mathrm{K} \alpha_{1} \\
\mathrm{~K} \beta_{1}\end{array}$ & $\begin{array}{l}16.62 \\
18.63\end{array}$ & Visible & Small peaks visible \\
\hline $\mathrm{Y}$ & $\begin{array}{l}K \alpha_{1} \\
K \beta_{1}\end{array}$ & $\begin{array}{l}14.60 \\
16.74\end{array}$ & Visible & Not clearly visible \\
\hline
\end{tabular}




\section{Quantitative analysis of powder samples}

\section{A) Relative composition}

To investigate the precision, accuracy and limitations of the XDAL in determination of relative masses of elements, and to evaluate different methods of quantification, ground samples of Ta capacitors were analysed.

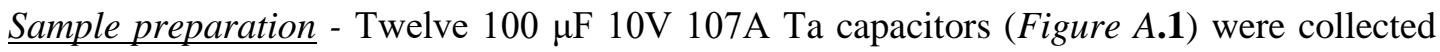
from PCBs (total mass $=1.7242 \mathrm{~g}$ ) and ground to a powder (for details of grinding procedure see section 2.1.1). To assess precision of the XDAL in measuring rel.\% composition of target materials over a range of concentrations, the TaCap powder was mixed and ground with graphite to give samples of: 50/50; 25/50 and 10/50, TaCap/graphite, w/w.

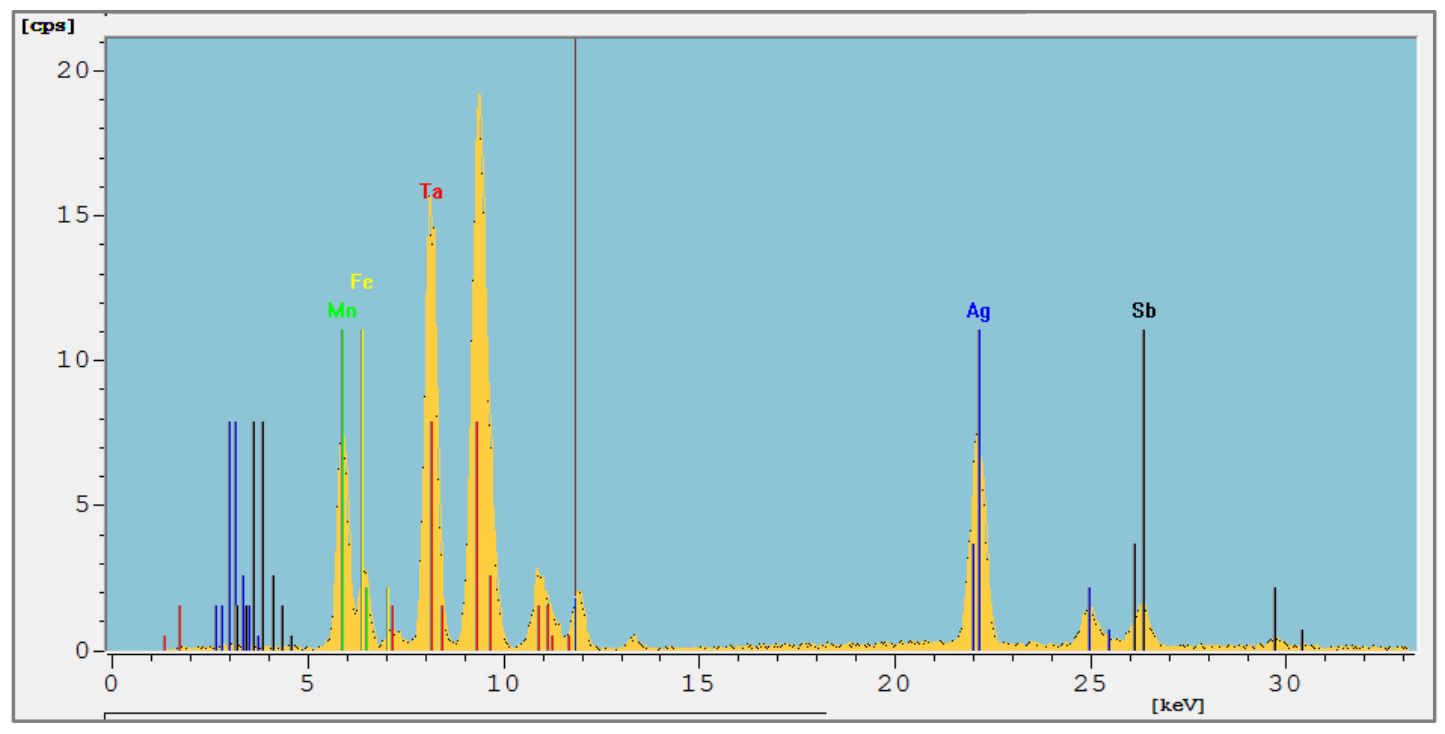

Figure A.1 X-ray emission spectrum of 100\%-TaCap powder

Effect of scan time - Figure A.2 shows spectra at 2s and 200s scan times. Results from repeat XRF analyses of the same area of the 100\%-TaCap powder, are given in Table A.2. Measured composition stabilises by about 16 scans (Figure A.2 and Figure A.3). 


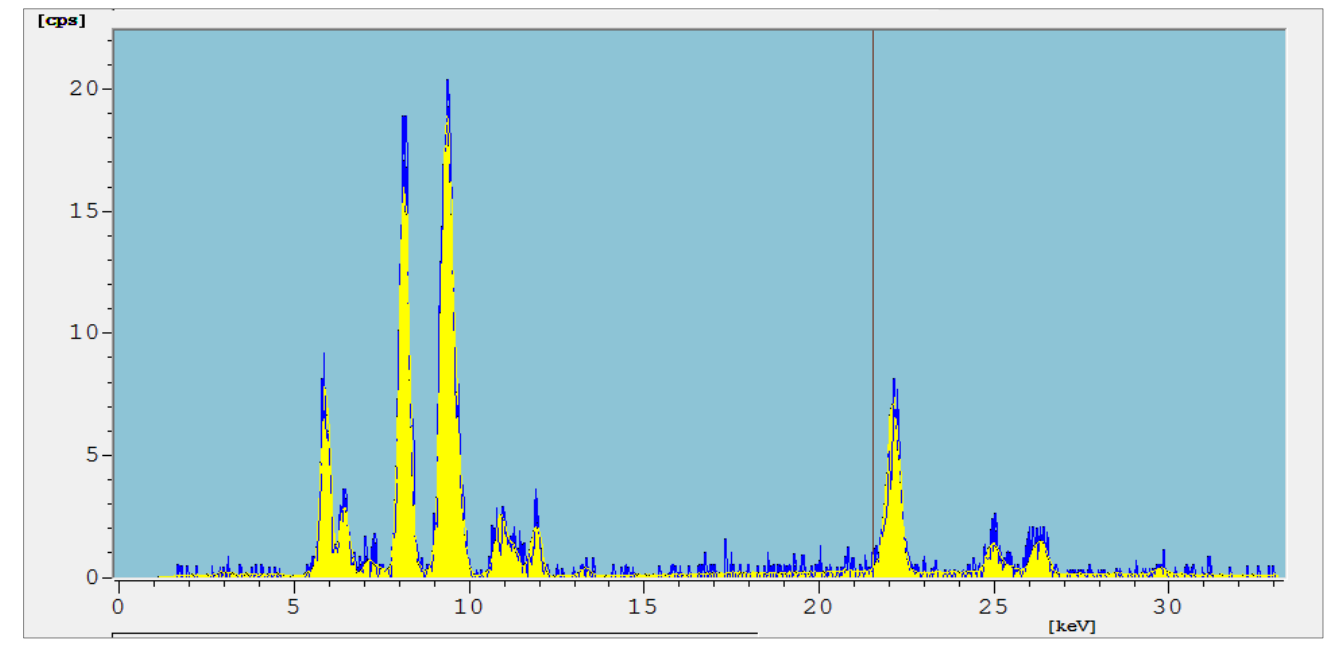

Figure A.2 Comparison of spectra obtained from the same area of $100 \%$ TaCap with $2 s$ (blue) and 300 s (yellow) scan times, showing improved $S / N$ with increased scan time; blue 2 scan time spectra is jagged and noisy compared to the yellow 300s scan

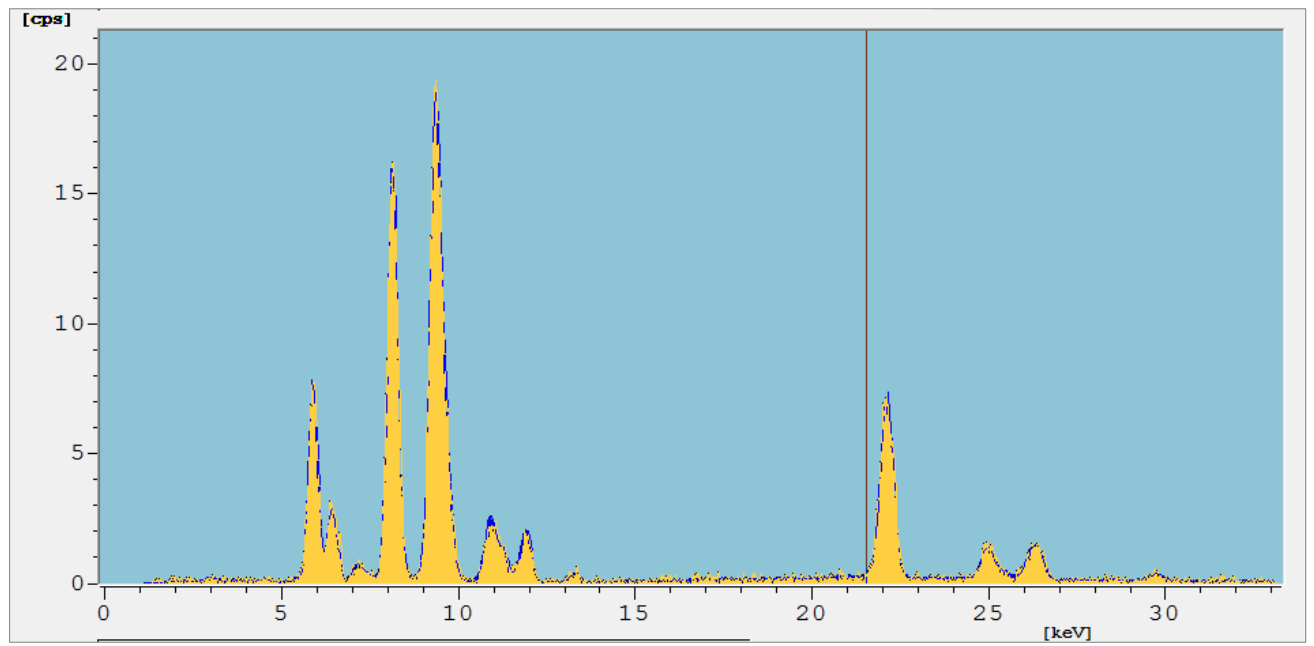

Figure A.3 Comparison of X-ray emission spectra of 100\%-TaCap powder measured with 16s (yellow) and 300s (blue) scan times

Table A.2 Variations in measured relative mass percentages (rel. \%) of detected elements in 100\%TaCap powder with scan time.

\begin{tabular}{cccccc}
\hline Scan time (s) & Ta & $\mathbf{A g}$ & $\mathbf{M n}$ & $\mathbf{F e}$ & $\mathbf{S b}$ \\
\hline 2 & 60.50 & 5.97 & 25.09 & 5.00 & 3.44 \\
4 & 58.43 & 6.59 & 29.47 & 3.57 & 1.94 \\
8 & 57.84 & 6.31 & 29.98 & 3.22 & 2.64 \\
16 & 57.54 & 6.75 & 28.91 & 3.98 & 2.82 \\
32 & 57.47 & 6.42 & 29.58 & 3.78 & 2.75 \\
64 & 57.51 & 6.57 & 29.43 & 3.67 & 2.82 \\
132 & 57.95 & 6.72 & 28.74 & 3.86 & 2.73 \\
300 & 57.59 & 6.57 & 29.08 & 3.98 & 2.78 \\
\hline
\end{tabular}


Instrument precision - Table A.3 gives compositions from 6 repeat measurements using 16s scan times on a single region of $100 \%$-TaCap powder. The instrument precision for determination of each element decreases with elemental \% composition; it is typically a few $10^{-1} \%$ for elements of high $\%$ composition and up to $\sim 10 \%$ for elements which make up only a few $\%$ of the sample composition.

Table A.3 Repeat measurements of relative masses of detected elements in a single area of 100\%TaCap powder using 16s scans.

\begin{tabular}{cccccc}
\hline \multirow{2}{*}{ Scan } & \multicolumn{5}{c}{ Relative mass percentage \% } \\
\cline { 2 - 6 } & Ta & Ag & Mn & Fe & Sb \\
\hline 1 & 57.54 & 6.75 & 28.91 & 3.98 & 2.82 \\
2 & 57.60 & 6.64 & 29.35 & 3.83 & 2.58 \\
3 & 57.56 & 6.51 & 29.03 & 3.89 & 3.01 \\
4 & 57.97 & 6.52 & 28.68 & 4.05 & 2.78 \\
5 & 57.81 & 6.45 & 29.52 & 3.58 & 2.65 \\
6 & 57.65 & 6.40 & 28.82 & 4.51 & 2.62 \\
\hline Mean & 57.69 & 6.55 & 29.05 & 3.97 & 2.74 \\
SD & 0.17 & 0.13 & 0.32 & 0.31 & 0.16 \\
RSD & $0.3 \%$ & $2.0 \%$ & $1.1 \%$ & $7.8 \%$ & $5.9 \%$ \\
\hline
\end{tabular}

Evaluating Method Precision - Table A.4 shows the results obtained from $16 \mathrm{~s}$ scans at 12 different sites in a single sample of $100 \%$-TaCap powder. Copper was only automatically detected at site 12, and results for each element show high 15-60\% relative standard deviations across the 12 sites. To investigate how relative abundance of elements in samples may affected the precision of results, the process was repeated for 50\%- and 25\%-TaCap powders in order to compare results with those obtained for 100\%-TaCap powder (Table A.5). $\mathrm{Cu}$ was not detected in 50\%-TaCap powder, and was detected at only one site in $25 \%$-TaCap powder. $\mathrm{Sb}$ was not detected in 25\%-TaCap powder. It is interesting to note that for the $25 \%$-TaCap powder the RSD of results obtained for all detected elements has decreased greatly. This may be due to the method of producing the 25\%-TaCap powder which may produce a more homogeneous sample because of the addition of graphite as a diluent with additional grinding.

Sampling and sample heterogeneity dominates analytical precision for these powder analyses. Grinding of samples for quantitative analysis of CRMs using the XDAL with this method will not result in quantitative data of suitable precision to base recycling process changes upon. The instrument is therefore suitable for qualitative identification of CRMs only. 
Table A.4 Relative percentage masses of detected elements at 12 sites of 100\%-TaCap powder measured with 16 s scan times.

\begin{tabular}{|c|c|c|c|c|c|c|}
\hline \multirow{2}{*}{ Area } & \multicolumn{6}{|c|}{ Rel. \% } \\
\hline & $\mathbf{T a}$ & Ag & Mn & $\mathbf{F e}$ & Sb & $\mathrm{Cu}$ \\
\hline 1 & 57.69 & 6.55 & 29.05 & 3.97 & 2.74 & ND \\
\hline 2 & 68.74 & 3.28 & 22.13 & 3.34 & 2.51 & ND \\
\hline 3 & 68.91 & 3.18 & 21.9 & 2.95 & 3.06 & ND \\
\hline 4 & 67.73 & 1.64 & 25.13 & 4.06 & 1.44 & ND \\
\hline 5 & 65.96 & 3.28 & 26.34 & 2.7 & 1.71 & ND \\
\hline 6 & 62.97 & 1.39 & 28.17 & 5.81 & 1.68 & ND \\
\hline 7 & 57.37 & 0.96 & 32.83 & 7.15 & 1.7 & ND \\
\hline 8 & 58.64 & 3.42 & 28.19 & 6.26 & 3.49 & ND \\
\hline 9 & 52.1 & 2.43 & 30.42 & 10.67 & 4.38 & ND \\
\hline 10 & 65.16 & 1.6 & 23.19 & 6.74 & 2.59 & ND \\
\hline 11 & 41.54 & 1.81 & 39.06 & 15.81 & 1.78 & ND \\
\hline 12 & 46.82 & 2.08 & 37.44 & 8.6 & ND & 3.19 \\
\hline Mean & 59 & 2.6 & 29 & 7 & 2.3 & \\
\hline SD & 9 & 1.5 & 6 & 4 & 1 & \\
\hline RSD & $15 \%$ & $57 \%$ & $19 \%$ & $58 \%$ & $50 \%$ & \\
\hline Sem & 3 & 0.5 & 2 & 1 & 0.3 & \\
\hline
\end{tabular}

ND: not detected; SD: standard deviation ( $\mathrm{n}=12)$; RSD: relative standard deviation; sem: standard error of the mean.

Table A.5 Average relative mass percentage composition of elements calculated from 12 repeat $16 \mathrm{~s}$ scans on different areas of samples

\begin{tabular}{cccccccc}
\hline \multirow{2}{*}{ Sample } & Result & \multicolumn{7}{c}{ Relative percentage mass (\%) } \\
\cline { 3 - 7 } & & Ta & Ag & Mn & Fe & Sb & Cu \\
\hline \multirow{2}{*}{$\begin{array}{c}\text { 100\%-TaCap } \\
\text { powder }\end{array}$} & Mean & 59.5 & 2.6 & 28.7 & 6.5 & 2.3 & 3.2 \\
& RSD & 8.9 & 1.5 & 5.6 & 3.8 & 1.1 & 3.2 \\
& & $15 \%$ & $57 \%$ & $19 \%$ & $58 \%$ & $50 \%$ & $100 \%$ \\
50\%-TaCap & Mean & 59.7 & 3.0 & 27.2 & 5.8 & 3.4 & ND \\
powder & SD & 7.8 & 0.8 & 5.3 & 3.9 & 1.6 & \\
& RSD & $13.1 \%$ & $26.1 \%$ & $19.4 \%$ & $67.0 \%$ & $47.1 \%$ & \\
25\%-TaCap & Mean & 60.74 & 2.79 & 29.66 & 6.57 & ND & 1.09 \\
powder & SD & 1.34 & 0.33 & 1.44 & 0.75 & & - \\
& RSD & $2.2 \%$ & $11.9 \%$ & $4.9 \%$ & $11.4 \%$ & & - \\
\hline
\end{tabular}

\section{B) Absolute composition - use of an internal standard}

Quantitative Analysis by Inclusion of Metallic Zn internal standard - 1.1272 g of metallic zinc was added to $1.7242 \mathrm{~g}$ of $100 \%$-TaCap powder and ground for $15 \mathrm{mins}$, giving a sample with $\mathrm{Zn}$ present at $39.5 \mathrm{wt} \%$ (Figure A.4) whose spectra obtained with 32s scan time is given in Figure A.5. 


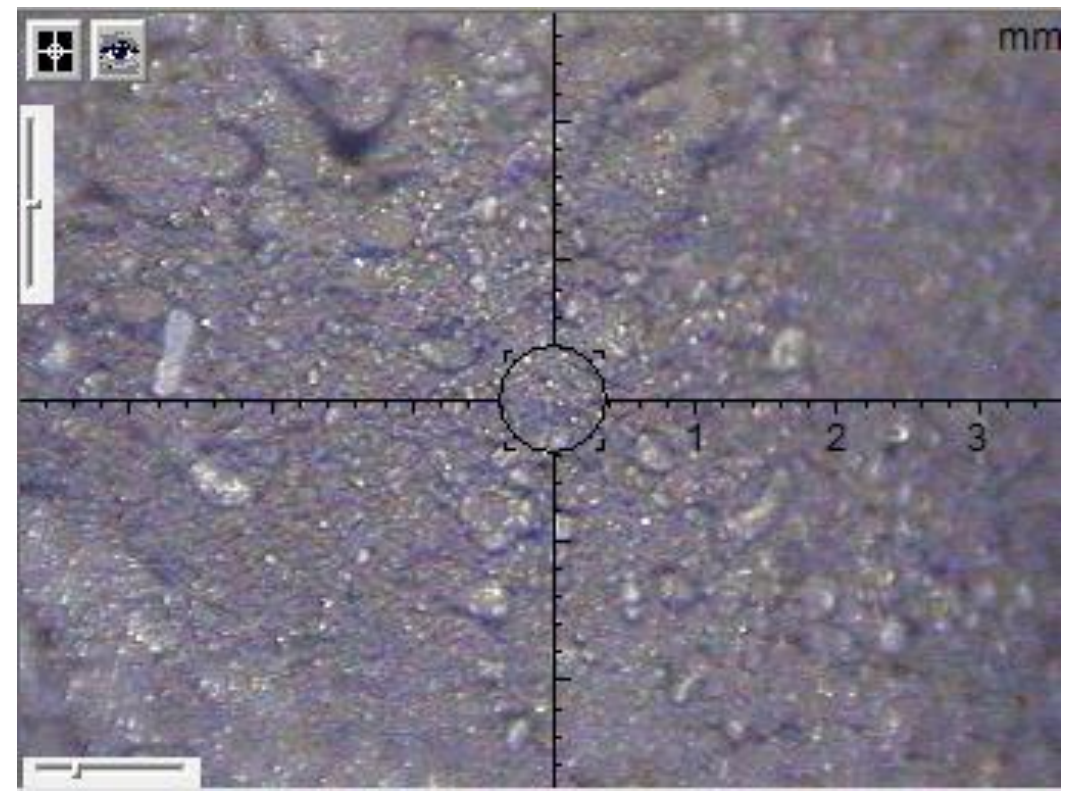

Figure A.4 Ground Ta capacitor powder containing 39.5 wt\% metallic $\mathrm{Zn}$

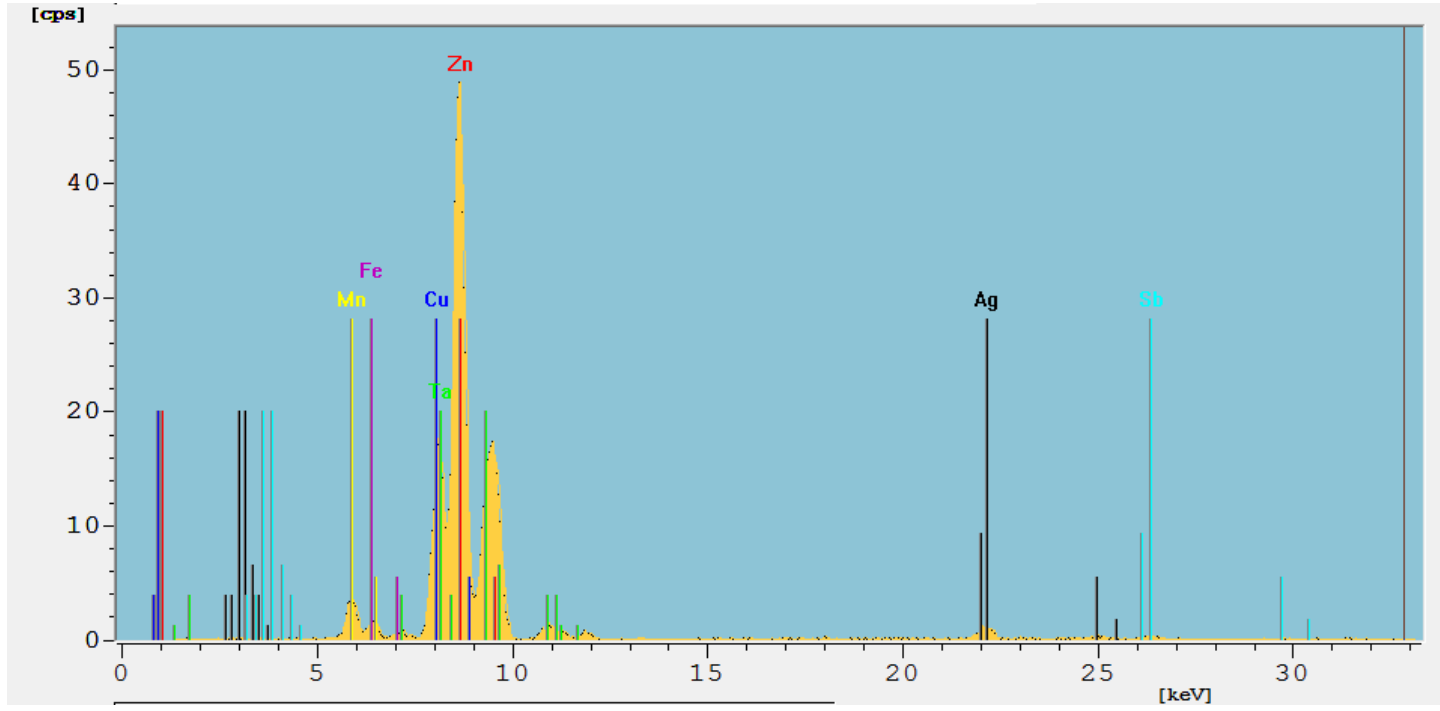

Figure A.5 X-ray emission spectrum of 35.9 wt\%-Zn sample obtained with 32 s scan time

12 areas of the powder were analysed using 32s scans (Table A.6). The standard deviation (SD) of results obtained for rel.\% composition across all areas scanned are large (22-96\%). The behaviour of metallic $\mathrm{Zn}$, which is soft and ductile, during grinding may go some way to account for this. Close inspection of the $39.5 \mathrm{wt} \%-\mathrm{Zn}$ sample (Figure A.4) revealed poor homogeneity, with large particles of $\mathrm{Zn}$ dispersed throughout the finer TaCap powder. The problem of beam size relative to particle size is worsened by this. More precise results may be obtained if a brittle salt of $\mathrm{Zn}$ with better grinding performance were used. 
Table A.6 rel.\% of elements detected in 12 sample locations in $39.5 \%$ Zn sample using 32 s scan times and calculated wt\% of elements in the sample.

\begin{tabular}{|c|c|c|c|c|c|c|c|}
\hline \multirow{2}{*}{ Scan } & \multicolumn{7}{|c|}{ Relative percentage masses $(\%)$} \\
\hline & $\mathbf{Z n}$ & $\mathbf{C u}$ & Ta & Mn & Ag & $\mathbf{F e}$ & Sb \\
\hline 1 & 44.44 & 5.97 & 36.77 & 9.57 & 1.06 & 1.69 & 0.51 \\
\hline 2 & 45.33 & 2.73 & 38.1 & 9.82 & 1.68 & 1.64 & 0.7 \\
\hline 3 & 68.96 & 2.21 & 20.39 & 6.38 & 0.81 & 1.01 & 0.25 \\
\hline 4 & 11.56 & 1.75 & 49.95 & 22.17 & 6.5 & 4.76 & 3.31 \\
\hline 5 & 38.97 & 6.29 & 40.49 & 10.54 & 1.26 & 1.68 & 0.77 \\
\hline 6 & 25.02 & 13.98 & 45.71 & 11.02 & 1.3 & 2.23 & 0.74 \\
\hline 7 & 48.33 & 6.79 & 32.35 & 9.4 & 1.02 & 1.7 & 0.41 \\
\hline 8 & 38.54 & 5.7 & 40.54 & 11.49 & 1.46 & 1.62 & 0.65 \\
\hline 9 & 43.88 & 4.2 & 37.79 & 10.46 & 1.41 & 1.58 & 0.69 \\
\hline 10 & 52.74 & 1.73 & 33.02 & 9.1 & 1.33 & 1.47 & 0.62 \\
\hline 11 & 52.71 & 1.27 & 32.6 & 9.88 & 1.37 & 1.51 & 0.67 \\
\hline 12 & 10.07 & 1.58 & 51.05 & 22.62 & 6.52 & 5.56 & 2.6 \\
\hline mean rel. $\%$ & 40 & 5 & 38 & 12 & 2.1 & 2.2 & 1.0 \\
\hline SD & 17 & 3.6 & 8.4 & 5 & 2.1 & 1.4 & 0.9 \\
\hline RSD & $43 \%$ & $80 \%$ & $22 \%$ & $43 \%$ & $96 \%$ & $64 \%$ & $95 \%$ \\
\hline sem & 5 & 1 & 3 & 2 & 0.6 & 0.4 & 0.3 \\
\hline $\mathrm{Wt} \%$ in sample & 39.5 & 4 & 38 & 12 & 2 & 2 & 1.0 \\
\hline$\%$ error & $0.4 \%$ & $27 \%$ & $15 \%$ & $18 \%$ & $32 \%$ & $23 \%$ & $31 \%$ \\
\hline Error & 0.2 & 1 & 5 & 2 & 1 & 1 & 0.3 \\
\hline mass in sample & $1.1272^{\#}$ & 0.13 & 1.1 & 0.33 & 0.06 & 0.06 & 0.03 \\
\hline$\%$ error & $0.03 \%$ & $27 \%$ & $15 \%$ & $18 \%$ & $32 \%$ & $23 \%$ & $31 \%$ \\
\hline Error & 0.0003 & 0.03 & 0.2 & 0.06 & 0.02 & 0.01 & 0.01 \\
\hline $\mathrm{Wt} \%$ in $100 \%$-TaCap powder & & 7 & 62 & 19 & 3 & 3.6 & 1.6 \\
\hline$\%$ error & & $27 \%$ & $15 \%$ & $18 \%$ & $32 \%$ & $23 \%$ & $31 \%$ \\
\hline Error & & 2 & 9 & 4 & 1 & 0.8 & 0.5 \\
\hline
\end{tabular}

SD: standard deviation $(n=12)$; RSD: relative standard deviation; sem: standard error of the mean; \% error - error expressed as $\%$ of result.

It quickly became clear that while XRF is an excellent rapid qualitative and/or semiquantitative method, much calibration and preliminary standardisation work would be required before XRF could be used as a reliable quantitative technique with a precision comparable to other methods, such as AAS. Therefore, the decision was taken to use XRF for rapid qualitative/semi-quantitative analysis only, and to use methods such as AAS and ICP-OES and/or ICP-MS for quantitative analysis. AAS analysis of $0.1 \mathrm{~g}$ samples of the powder ( $5 \%$ of total sample mass) gives results with a RSD of $\sim 5 \%$. This reflects, in part at least, the fact that the larger sample sizes used in AAS are more representative of the overall sample composition than that imaged in a $1 \mathrm{~mm}$ diameter beam. XRF instruments with beam widths of $40 \mathrm{~mm}$ such as the PANalytical CubiX XRF are available and would be more suited to quantitative analysis of powder samples. 


\section{Glossary}

\begin{tabular}{ll}
\hline Term & Definition \\
\hline $3^{\text {rd }}$ generation, or & An emerging set of thin-film photovoltaic technologies which can be \\
printable photovoltaics & manufactured using printing and which includes dye-sensitized solar cells \\
(3GPV or PPV) & (DSSCs), organic PV (OPV) and perovskite solar cells. ${ }^{238}$
\end{tabular}

Cascading components materials and

Eco-design

EEE

Industrial symbiosis

Industrial Ecology ${ }^{\mathrm{a}}$

LCA analysis) (lifecycle

Recycling

Refurbishment

Remanufacturing

Reuse

Urban mining

WEEE (e-waste)
"This involves a series of new applications for constituent materials/components of a product, each providing a fresh use and additional commercial benefit..

"Any household or business item with circuitry or electrical components with power or battery supply". 6

"An association between two or more industrial facilities or companies in which the wastes or by-products of one become the raw materials for another". 71

"Industrial ecology can be best defined as the totality or the pattern of relationships between various industrial activities, their products, and the environment. Traditional ecological activities have focused on two time aspects of interactions between the industrial activities and the environment - the past and the present. Industrial ecology, a systems view of the environment, pertains to the future". 320

A systematic set of procedures for compiling and examining the inputs and outputs of materials and energy and the associated environmental impacts directly attributable to the functioning of a product or service system throughout its life cycle.

"process of recovering materials for the original purpose or for other purposes, excluding energy recovery" 47

"A process of returning a product to good working condition by replacing or repairing major components that are faulty or close to failure, and making 'cosmetic' changes to update the appearance of a product, such as cleaning, changing fabric, painting or refinishing." 47

"A process of disassembly and recovery at the subassembly or component level. Functioning, reusable parts are taken out of a used product and rebuilt into a new one. This process includes quality assurance and potential enhancements or changes to the components." 47

"The use of a product again for the same purpose in its original form or with little enhancement or change". ${ }^{47}$

"the process of reclaiming compounds and elements from any kind of anthropogenic stocks, including buildings, infrastructure, industries, products (in and out of use), environmental media receiving anthropogenic emissions, etc. The stocked materials may represent a significant source of resources, with concentrations of elements often comparable to or exceeding natural stocks. As for natural ores, extraction and processing of anthropogenic stocks is necessary and the generation of an economic benefit is essential. For these reasons, urban mining originally focussed on electrical and electronic wastes (WEEE) which contain relatively high concentrations of expensive metals and rare earth elements." 119

"E-waste is a terms used to cover items of all types of electrical and electronic equipment (EEE) and its parts that have been discarded by the owner as waste without the intention for re-use". 6

\footnotetext{
${ }^{a}$ there is no one definition of industrial ecology as a complex multi-disciplinary subject. For an excellent overview of the subject and further definitions see Garner and Keoleian ${ }^{321}$
} 


\section{References}

1. I. Dalrymple, N. Wright, R. Kellner, N. Bains, K. Geraghty, M. Goosey and L. Lightfoot, An integrated approach to electronic waste (WEEE) recycling, Circuit World, 2007, 33, 52-58.

2. F. O. Ongondo, I. D. Williams and T. J. Cherrett, How are WEEE doing? A global review of the management of electrical and electronic wastes, Waste Management, 2011, 31, 714-730.

3. R. Widmer, H. Oswald-Krapf, D. Sinha-Khetriwal, M. Schnellmann and H. Böni, Global perspectives on e-waste, Environmental Impact Assessment Review, 2005, 25, 436-458.

4. EU, Directive 2012/19/EU of the European Parliament and of the Council of 4 July 2012 on waste electrical and electronic equipment (WEEE) (recast), Official Journal of the European Union, 2012, 55, 38.

5. EU, Directive 2002/96/EC of the European Parliament and of the council of 27 January 2003 on waste electrical and electronic equipment (WEEE), Official Journal of the European Union; 2003, L 37/24, 24-38.

6. StEP Initiative, Solving the E-waste Problem (StEP): What is e-waste?, United Nations University, 2014.

7. OECD, Extended Producer Responsibility, OECD Publishing, OECD iLibrary, 2001.

8. J. Pucket, L. Byster, S. Westervelt, R. Gutierrez, S. Davis, A. Hussain and M. Dutta, Exporting Harm: The high-tech trashing of Asia, The Basel Action Network (BAN), Silicon Valley Toxics Coalition (SVTC), Seattle, WA, 2002.

9. EU, Directive 2008/98/EC of the European Paliament and of the Council of 19 November 2008 on Waste and Repealing certain Directives (Waste Framework Directive), Official Journal of the European Union, 2008, L312, 3-22.

10. StEP Initiative, Solving the E-Waste Problem (Step) White Paper: One Global Definition of E-waste, United Nations University; 2015.

11. Statistical Office of the European Communities, EUROSTAT: Waste electrical and electronic equipment (online data code: env_waselee), http://appsso.eurostat.ec.europa.eu/nui/show.do?; 2016, query=BOOKMARK_DS185466_QID_18806AE9_UID_-

3F171EB0\&layout=TIME,C,X,0;GEO,L,Y,0;WASTE,L,Z,0;WST_OPER,L,Z,1;UN IT,L,Z,2;INDICATORS,C,Z,3;\&zSelection=DS-

185466INDICATORS,OBS_FLAG;DS-185466WST_OPER,RCY_REU;DS185466WASTE,EE_LHA;DS-185466UNIT,T;\&rankName1=WST-OPER_1_2_1_2\&rankName2=WASTE_1_2_-1_2\&rankName3=INDICATORS_1_2_$1 \_2 \&$ rankName4=UNIT_1_2_1_2\&rankName5=TIME_1_0_0_0\&rankName6=GEO_1_2_0_1\&pprRK=FIRST\&p $\mathrm{prSO}=\mathrm{CUSTOM} \& \mathrm{ppcRK}=\mathrm{FIRST} \& \mathrm{ppcSO}=\mathrm{ASC} \& \operatorname{sortC}=\mathrm{ASC}-1$-FIRST\&rStp$=$ (accessed 26/9/17) 
12. A. Tuncuk, V. Stazi, A. Akcil, E. Y. Yazici and H. Deveci, Aqueous metal recovery techniques from e-scrap: Hydrometallurgy in recycling, Minerals Engineering, 2012, 25, 28-37.

13. J. C. Arnold, T. Watson, S. Alston, M. Carnie and C. Glover, The use of FTIR mapping to assess phase distribution in mixed and recycled WEEE plastics, Polymer Testing, 2010, 29, 459-470.

14. A. Buekens and J. Yang, Recycling of WEEE plastics: A review, Journal of Material Cycles and Waste Management, 2014, 16, 415-434.

15. X. Yang, L. Sun, J. Xiang, S. Hu and S. Su, Pyrolysis and dehalogenation of plastics from waste electrical and electronic equipment (WEEE): A review, Waste Management, 2013, 33, 462-473.

16. M. Schluep, C. Hagelueken, R. Kuehr, F. Magalini, C. Maurer, C. Meskers, E. Mueller and F. Wang, Sustainable innovation and technology transfer industrial sector studies: Recycling-from e-waste to resources, United Nations Environment Programme \& United Nations University, Bonn, Germany, 2009.

17. EMPA, ewasteguide.info, 2016, http://ewasteguide.info/node/4074 (accessed 19/9/2016).

18. C. Hagelüken, Improving metal returns and eco-efficiency in electronics recycling A holistic approach for interface optimisation between pre-processing and integrated metals smelting and refining, Proceedings of the 2006 IEEE International Symposium on Electronics \& the Environment, San Francisco, 2006.

19. M. Buchert, A. Manhart, D. Bleher and D. Pingel, Recycling Raw Materials from Waste Electronic Equipment, Oko Institute e.V., 2012.

20. M. Buchert, D. Schuler and D. Bleher, Critical metals for future sustainable technologies and their recycling potential, Oko-Institut \& UNEP \& UNU, Darmstadt, Germany, 2008.

21. P. Harfield, C. Harris and C. Sanders, Mapping Critical Resources for Wales: Literature Review, Ecodesign Centre Wales, 2014.

22. R. G. Charles, The Assesment of Ferrous and Non-Ferrous Metals in WEEE, MRes Thesis, Swansea University, 2012.

23. L. S. Morf, J. Tremp, R. Gloor, Y. Huber, M. Stengele and M. Zennegg; Brominated flame retardants in waste electrical and electronic equipment: substance flows in a recycling plant, Environmental Science \& Technology, 2005, 39, 8691-8699.

24. O. Tsydenova and M. Bengtsson, Chemical hazards associated with treatment of waste electrical and electronic equipment, Waste Management, 2011, 31, 45-58.

25. B. H. Robinson; E-waste, An assessment of global production and environmental impacts, Science of the Total Environment, 2009, 408, 183-191. 
26. EMPA - Swiss Federal Laboratories for Materials Science and Technology, Hazardous Substances in e-waste, 2012, http://ewasteguide.info/hazardoussubstances (accessed 18/2/2012).

27. N. Singh, J. Li and X. Zeng, Solutions and challenges in recycling waste cathode-ray tubes, Journal of Cleaner Production, 2016, 133, 188-200.

28. T. P. Taylor, M. Ding, D. S. Ehler, T. M. Foreman, J. P. Kaszuba and N. N. Sauer; Beryllium in the environment: A review, Journal of Environmental Science and Health - Part A Toxic/Hazardous Substances and Environmental Engineering, 2003, 38, 439-469.

29. Greenpeace, Greenpeace Briefing: TOXIC TECH - The Dangerous Chemicals in Electronic Products, Greenpeace International, 2005.

30. M. S. Sodhi and B. Reimer, Models for recycling electronics end-of-life products, $O R$ Spektrum, 2001, 23, 97-115.

31. UNU-ISP, StEP, E-Waste World Map, 2014, http://www.step-initiative.org/step-ewaste-world-map.html (accessed 02/10/2015).

32. I. O. Ogunniyi, M. K. G. Vermaak and D. R. Groot, Chemical composition and liberation characterization of printed circuit board comminution fines for beneficiation investigations, Waste Management, 2009, 29, 2140-2146.

33. W. J. Hall and P. T. Williams, Separation and recovery of materials from scrap printed circuit boards, Resources, Conservation and Recycling, 2007, 51, 691-709.

34. M. Oguchi, S. Murakami, H. Sakanakura, A. Kida and T. Kameya, A preliminary categorization of end-of-life electrical and electronic equipment as secondary metal resources, Waste Management, 2011, 31, 2150-2160.

35. S. N. M. Menikpura, A. Santo and Y. Hotta, Assessing the climate co-benefits from Waste Electrical and Electronic Equipment (WEEE) recycling in Japan, Journal of Cleaner Production, 2014, 74, 183-190.

36. M. Goosey, in Electronic Waste Management - Design, Analysis and Application, eds. R. E. Hester and R. M. Harrison, The Royal Society of Chemistry, Cambridge, 2009, pp. 4-39.

37. WRAP, Switched on to value: Powering business change, WRAP, 2017.

38. A. Kumar, M. Holuszko and D. C. R. Espinosa; E-waste: An overview on generation, collection, legislation and recycling practices, Resources, Conservation and Recycling, 2017, 122, 32-42.

39. UNEP, Press Release - Basel Conference Addresses Electronic Wastes Challenge; 2006

http://www.unep.org/Documents.Multilingual/Default.asp?DocumentID=485\&Artic leID $=5431 \& l=e n)($ accessed 21/9/12). 
40. Y. Kalmykova, J. Patrício, L. Rosado and P. E. Berg, Out with the old, out with the new - The effect of transitions in TVs and monitors technology on consumption and WEEE generation in Sweden 1996-2014 Waste Management, 2015, 46, 511-522.

41. Statista, The "Slow iPhone" Phenomenon, 2015 https://www.statista.com/chart/2514/iphone-releases/ (accessed 26/9/2015).

42. S. Mullainathan, Hold the Phone: A Big-Data Conundrum, TheUpShot, The New York Times (online), 27/7/2014, http://www.nytimes.com/2014/07/27/upshot/holdthe-phone-a-big-data-conundrum.html?_r=0 (accessed 26/9/17).

43. M. Cobbing, Toxic Tech: Not in our Backyard - Uncovering the Hidden Flows of ewaste; Greenpeace, Amsterdam, 2008.

44. S. Wilkinson, N. Duffy; M. Crowe and K. Nolan, Waste from Electrical and Electronic Equipment, Environmental Protection Agency, Ireland, 2001.

45. K. Grant, F. C. Goldizen, P. D. Sly, M.-N. Brune, M. Neira, M. van den Berg and R. E. Norman, Health consequences of exposure to e-waste: a systematic review, The Lancet Global Health, 2013, 1, e350-e361.

46. S. Schwartzer, A. De Bono, G. Giuliani, S. Kluser and P. Peduzzi, E-waste, the hidden side of IT equipment's manufacturing and use, UNEP \& GRID Europe, Nairobi, Kenya, 2005.

47. Ellen MacArthur Foundation, Towards a Circular Economy-Economics and business rationale for an accelerated transition, Ellen MacArthur Foundation, 2013 https://www.ellenmacarthurfoundation.org/assets/downloads/publications/Ellen-

MacArthur-Foundation-Towards-the-Circular-Economy-vol.1.pdf (accessed 26/9/17).

48. Brundtland Commision, Our Common future, Oxford University Press, Oxford, 1987.

49. P. Lacy, J. Keeble, R. McNamara and J. Rutqvist, Circular Advantage: Innovative Business Models and Technologies to Create Value in a World Without Limits to Growth, Accenture Strategy, Accenture, 2014.

50. UNEP, Global Environemental Outlook (GEO5) - Environement for the Future we Want, Progress Press Ltd, Malta, 2012.

51. Oakdene Hollins, Study into the feasibility of protecting and recovering critical raw materials through infrastructure development in the south east of England - Final Report, EPOW, Environment Agency, 2011.

52. D. Peck, P. Kandachar and E. Tempelman, Critical materials from a product design perspective, Materials \& Design, 2015, 65, 147-159.

53. The ad-hoc Working Group, Report on Critical Raw Materials for the EU-Report of the Ad hoc Working Group on defining critical raw materials, European Commission, 2014.

54. M. Frenzel, J. Kullik, M. A. Reuter and J. Gutzmer, Raw material 'criticality' - sense or nonsense?, Journal of Physics D: Applied Physics, 2017, 50, 123002. 
55. J J. H. Clark, T. J. Farmer, L. Herrero-Davila and J. Sherwood, Circular economy design considerations for research and process development in the chemical sciences, Green Chemistry, 2016, 18, 3914-3934.

56. L. T. Peiró, G. V. Méndez and R. U. Ayres, Material Flow Analysis of Scarce Metals: Sources, Functions, End-Uses and Aspects for Future Supply, Environmental Science \& Technology, 2013, 47, 2939-2947.

57. M. Reuter, C. Hudson, A. van Schaik, K. Heiskanen, C. Meskers and C. Hagelueken, Metal Recycling: Opportunities, Limits, Infrstructure, A Report of the Working Group on the Global Metal Flows to the International Resource Panel., UNEP, 2013.

58. A. H. King, When agendas align: Critical materials and green electronics, Electronics

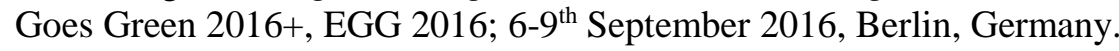

59. T. E. Graedel, J. Allwood, J.-P. Birat, M. Buchert, C. Hagelueken, B. K. Reck, S. F. Sibley and G. Sonnemann, Recycling Rates of Metals - A status Report, UNEP, 2011.

60. T. E. Graedel, J. Allwood, J.-P. Birat, M. Buchert, C. Hagelüken, B. K. Reck, S. F. Sibley and G. Sonnemann, What Do We Know About Metal Recycling Rates?, Journal of Industrial Ecology, 2011, 15, 355-366.

61. P. Viebahn, O. Soukup, S. Samadi, J. Teubler, K. Wiesen and M. Ritthoff, Assessing the need for critical minerals to shift the German energy system towards a high proportion of renewables, Renewable and Sustainable Energy Reviews, 2015, 49, 655671.

62. T. Zimmermann and S. Gößling-Reisemann, Assessing the need for critical minerals to shift the German energy system towards a high proportion of renewables, Science of The Total Environment, 2013, 461-462, 774-780.

63. J. Huisman, H. Habib, M. G. Brechu, S. Downes, L. Herreras, A. N. Løvik, P. Wäger, D. Cassard, F. Tertre, P. Mählitz, S. Rotter, P. Chancerel and M. L. Söderman, ProSUM: Prospecting secondary Raw Materials in the Urban Mine and Mining Wastes, Electronics Goes Green 2016+, EGG 2016, 6-9 ${ }^{\text {th }}$ September 2016, Berlin, Germany.

64. F. O. Ongondo, I. D. Williams and G. Whitlock, Distinct Urban Mines: Exploiting secondary resources in unique anthropogenic spaces, Waste Management, 2015, 45, 4-9.

65. Z. Sun, Y. Xiao, H. Agterhuis, J. Sietsma and Y. Yang, Recycling of metals from urban mines - a strategic evaluation, Journal of Cleaner Production, 2016, 112, Part 4, 2977-2987.

66. A. Golev, D. R. Schmeda-Lopez, S. K. Smart, G. D. Corder and E. W. McFarland, Where next on e-waste in Australia?, Waste Management, 2016, 58, 348-358.

67. M. Bigum, L. Brogaard and T. H. Christensen, Metal recovery from high-grade WEEE: A life cycle assessment, Journal of Hazardous Materials, 2012, 207-208, 814. 
68. M. E. Slade, The effects of higher energy prices and declining ore quality: Copperaluminium substitution and recycling in the USA, Resources Policy, 1980, 6, 223-239.

69. C. Hagelüken and C. Meskers, Mining our computers - opportunities and challenges to recover scarce and valuable metals from end-of-life electronic devices, Electronics Goes Green 2008+, EGG 2008, 7-10 ${ }^{\text {th }}$ September 2008, Berlin, Germany, 2008.

70. G. Bunting, L. Huxtable and M. Clement, Strategic Solutions for Resource Efficiency, 13th International Conference of the Greening of Industry Network - Integration and Communication: A Clear Route to sustainability?, 2-6 ${ }^{\text {th }}$ July 2006, Cardiff, Wales, UK.

71. WRAP, What is Industrial Symbiosis?, 2015 http://www.wrap.org.uk/content/whatindustrial-symbiosis, (accessed 22/9/2015).

72. L. Chamberlin, E. Jasmin and A. Raksit, Wales and the Circular Economy: Favourable system conditions and economic opportunitie, Ellen McArthur Foundation on behalf of the Waste and Resources Action Programme (WRAP) and the Welsh Government, 2013.

73. J J. Morgan and P. Mitchell, Employment and the circular economy, Green Alliance and WRAP, London, UK, 2015.

74. EC, Report from the commission to the Europeean Parliament, the Council, the European Economic and Social Committee and the Committee of the regions on the implementation of the Circular Economy Action Plan, European Commission, Brussels, 2017.

75. F. O. Ongondo, I. D. Williams, J. Dietrich and C. Carroll, ICT reuse in socio-economic enterprises, Waste Management, 2013, 33, 2600-2606.

76. I. D. Williams and P. J. Shaw, Reuse: Fashion or future?, Waste Management, 2017, 60, $1-2$.

77. H.-Y. Kang and J. M. Schoenung, Electronic waste recycling: A review of U.S. infrastructure and technology options, Resources, Conservation and Recycling, 2005, 45, 368-400.

78. J J. Cui and L. Zhang, Metallurgical recovery of metals from electronic waste: A review, Journal of Hazardous Materials, 2008, 158, 228-256.

79. K. Tanong, J. F. Blais and G. Mercier, Metal recycling technologies for battery waste, Recent Patents on Engineering, 2014, 8, 13-23.

80. J. Xu, H. R. Thomas, R. W. Francis, K. R. Lum, J. Wang and B. Liang, A review of processes and technologies for the recycling of lithium-ion secondary batteries, Journal of Power Sources, 2008, 177, 512-527.

81. L. Zhang and $\mathrm{Z}$. Xu, A review of current progress of recycling technologies for metals from waste electrical and electronic equipment, Journal of Cleaner Production, 2016, 127, 19-36. 
82. A. Khaliq, M. Rhamdhani, G. Brooks and S. Masood, Metal Extraction Processes for Electronic Waste and Existing Industrial Routes: A Review and Australian Perspective, Resources, 2014, 3, 152.

83. EU, Directive 2011/65/EU of the European Parliament and of The Council of 8 June 2011 on the restriction of the use of certain hazardous substances in electrical and electronic equipment (recast), Official Journal of the European Union, 2011, 1.7.2011, 88-110.84. EU, Journal, 2003, 13.2.2003, 19-23.

85. DEFRA, Guidance on Best Available Treatment Recovery and Recycling Techniques (BATRRT) and treatment of Waste Electrical and Electronic Equipment (WEEE), Department for Environment, Food and Rural Affairs, London, UK, 2006.

86. EC, Proposal for a Directive of the European parliament and of the council amending Directives 2000/53/EC on end-of-life vehicles, 2006/66/EC on batteries and accumulators and waste batteries and accumulators, and 2012/19/EC on waste electrical and electronic equipment, $\operatorname{COM(2015)} 593$ final, Brussels, 2/12/2015.

87. D. Bleiwas and T. Kelly, USGS Fact Sheet 060-01 - Obsolete Computers, "Gold Mine," or High-Tech Trash? Resource Recovery from Recycling, USGS, Eastern Publications Group Web Team, 2012 http://pubs.usgs.gov/factsheet/fs060-01/ (accessed 27/917).

88. California Department of Toxic Substances Control, Restrictions on the use of Certain Hazardous Substances (RoHS) in Electronic Devices, State of California, 2010 http://www.dtsc.ca.gov/HazardousWaste/rohs.cfm (accessed 27/9/17).

89. Ministry of Environment and Urban Development, Regulation on the control of Waste Electrical and Electronic Equipments, Official Gazette, 22 May 2012.

90. Washington State Department of Ecology, First year of Washington's electronics recycling program collects over 38 million pounds, Department of Ecology Nres release; Access Washington - Official State Government Website, 2010 http://www.ecy.wa.gov/news/2010news/2010-012.html (accessed 31/8/12).

91. B. Kuschnik, The European Union's Energy Using Produt - EuP - Directive 2005/32/EC: Taking Transnational Eco-Product Design Regulation One Step Further, Templ. Journal of Science Technology and Environmental Law, 2008, 27, 1-33.

92. METI, Home Appliance Recycling Law (HARL), Japanese Ministry of Economy, Trade and Industry, 2015 http://www.meti.go.jp/policy/recycle/main/english/law/home.html (accessed 27/9/17).

93. RoHSGuide.com, RoHS Guide Compliance, 2015 http://www.rohsguide.com/rohsfuture.htm, (accessed 27/9/17).

94. R. Spiegel, India Passes WEEE and RoHS Regulations, Green Scene, 2011 http://www.designnews.com/author.asp?section_id=1387\&doc_id=230705 (accessed 27/9/15). 
95. M. Liddane, Ghana is the Shining Star for e-Waste Law in Africa, compliance \& risks, $2016 \mathrm{http} / / / \mathrm{blog}$.complianceandrisks.com/ghana-is-the-shining-star-for-e-waste-lawin-africa/ (accessed 27/9/17).

96. A. Morris and G. Metternicht, Assessing effectiveness of WEEE management policy in Australia, Journal of Environmental Management, 2016, 181, 218-230.

97. EC, A resource-efficient Europe - Flagship initiative under the Europe 2020 Strategy, COM(2011) 21, Brussels; 2011..

98. EC, Communication from the commission to the European Parliament, the Council, the European Economic and Social Committee of the Regions - Tackling the Challenges in Commodity Markets and on Raw Materials, COM(2011) 25 final, Brussels, 2012.

99. K. Binnemans, P. T. Jones, B. Blanpain, T. Van Gerven, Y. Yang, A. Walton and M. Buchert, Recycling of rare earths: a critical review, Journal of Cleaner Production, 2013, 51, 1-22.

100. EC; Critical Raw Materials for the EU - Report of the Working Group on defining Critical Raw Materials, European Commision, Department of Enterprise and Industry, 2010.

101. F. Cucchiella, I. D'Adamo, S. C. Lenny Koh and P. Rosa, Recycling of WEEEs: An economic assessment of present and future e-waste streams, Renewable and Sustainable Energy Reviews, 2015, 51, 263-272.

102. M. S. Gasser and M. I. Aly, Separation and recovery of rare earth elements from spent nickel-metal-hydride batteries using synthetic adsorbent, International Journal of Mineral Processing, 2013, 121, 31-38.

103. H. Hasegawa, I. M. M. Rahman, Y. Egawa, H. Sawai, Z. A. Begum, T. Maki and S. Mizutani, Recovery of indium from end-of-life liquid-crystal display panels using aminopolycarboxylate chelants with the aid of mechanochemical treatment, Microchemical Journal, 2013, 106, 289-294.

104. A. K. Jha, M. K. Jha, A. Kumari, S. K. Sahu, V. Kumar and B. D. Pandey, Selective separation and recovery of cobalt from leach liquor of discarded Li-ion batteries using thiophosphinic extractant, Separation and Purification Technology, 2013, 104, 160166.

105. Hugh Smith, Mike Jefferson, Jeff Weeks, Gordon Day and N. Swierczynska, Strategic Raw Materials, Recovery Capacity and Technologies, WRAP, 2012.

106. P. Chancerel, C. E. M. Meskers, C. Hagelüken and V. S. Rotter, Assessment of Precious Metal Flows During Preprocessing of Waste Electrical and Electronic Equipment, Journal of Industrial Ecology, 2009, 13, 791-810.

107. J. Cui and E. Forssberg, Mechanical recycling of waste electric and electronic equipment: a review, Journal of Hazardous Materials, 2003, 99, 243-263. 
108. A. Terazono, M. Oguchi, S. Kuzuhara, R. P. Medina and F. C. J. Ballesteros, Survey of Material Recovered by Informal E-waste Processing in the Philippines, Electronics Goes Green 2016+, EGG 2016; 6-9 $9^{\text {th }}$ September 2016, Berlin, Germany.

109. D. Parker and J. Arendorf, Mapping consumption and waste of raw materials in electrical products in the UK, WRAP, 2012.

110. T. Anand and N. Ha, Implementing challenges of extended producer responsibility, International Journal of Civil Engineering and Technology, 2017, 8, 1188-1194.

111. D. Kell, Recycling \& Recovery, in Electronic Waste Management, eds. Hester R. E., Harrison, R.M., RSC Publishing, Cambridge, 2009, ch. 4, pp. 91-110.

112. V. K. Garlapati, E-waste in India and developed countries: Management, recycling, business and biotechnological initiatives, Renewable and Sustainable Energy Reviews, 2016, 54, 874-881.

113. M. Bigum, C. Petersen, T. H. Christensen and C. Scheutz, WEEE and portable batteries in residual household waste: Quantification and characterisation of misplaced waste, Waste Management, 2013, 33, 2372-2380.

114. E. Bridgewater, Defra EV0801 National compositional estimates for local authority collected waste and recycling in England, 2010/11, DEFRA, 2012.

115. Environment Agency, Statistics - Waste electrical and electronic equipment in the UK, 2015 https://www.gov.uk/government/statistics/waste-electrical-and-electronicequipment-in-the-uk-2013 (accessed 27/9/17).

116. C. Hagelüken and C. Meskers, Recycling of technology metals; in E-Waste Management, From Waste to Resource, eds. K. Hieronymi, R. Kahhat and E. Williams, Routledge, Oxon, 2012, ch. 3, pp. 63-64.

117. A. Cesaro, A. Marra, V. Belgiorno and M. Guida, Effectiveness of WEEE mechanical treatment: Separation yields and recovered material toxicity, Journal of Cleaner Production, 2017, 142, 2656-2662.

118. B. K. Reck and T. E. Graedel, Challenges in Metal Recycling, Science, 2012, 337, 690-695.

119. R. Cossu and I. D. Williams, Urban mining: Concepts, terminology, challenges, Waste Management, 2015, 45, 1-3.

120. O. O. Akinade, L. O. Oyedele, S. O. Ajayi, M. Bilal, H. A. Alaka, H. A. Owolabi, S. A. Bello, B. E. Jaiyeoba and K. O. Kadiri, Design for Deconstruction (DfD): Critical success factors for diverting end-of-life waste from landfills, Waste Management, 2017, 60, 3-13.

121. I. Oehme, K. Sperlich, R. Kohlmeyer, S. Prakash, K. Sander and C. Clemm, Strengthening material efficiency of electrical and electronic equipment, Electronics Goes Green 2016+, EGG 2016, 6-9 ${ }^{\text {th }}$ September 2016, Berlin, Germany. 
122. J. L. Ospina, M. Paul, S. Hickey, I. vidorreta and M. Yang, D4R laptop - Industrial networks and eco-design to maximise reuse and eliminate waste, Electronics Goes Green 2012+, EGG 2012, 9-12 ${ }^{\text {th }}$ September 2012, Berlin, Germany.

123. M. A. Reuter, Limits of design for recycling and "sustainability": A review, Waste and Biomass Valorization, 2011, 2, 183-208.

124. A. Manhart, International Cooperation for Metal Recycling From Waste Electrical and Electronic Equipment, Journal of Industrial Ecology, 2011, 15, 13-30.

125. M. Colledani, G. Copani and T. Tolio, De-manufacturing Systems, Procedia CIRP, 2014, 17, 14-19.

126. R. Crozier, Robots put to work on e-waste, Science X Network, 2014 https://phys.org/news/2014-11-robots-e-waste.html, (accessed 29/8/17).

127. C. Rujanavech, J. Lessard, S. Chandler, S. Shannon, J. Dahmus and R. Guzzo, Liam An Innovation Story, Apple Inc., September 2016.

128. S. Zhang and E. Forssberg, Intelligent Liberation and classification of electronic scrap, Powder Technology, 1999, 105, 295-301.

129. T. Ernst, R. Popp, M. Wolf and R. van Eldik, Analysis of eco-relevant elements and noble metals in printed wiring boards using AAS, ICP-AES and EDXRF, Analytical and Bioanalytical Chemistry, 2003, 375, 805-814.

130. H. M. Veit, A. M. Bernardes, J. Z. Ferreira, J. A. S. Tenório and C. d. F. Malfatti, Recovery of copper from printed circuit boards scraps by mechanical processing and electrometallurgy, Journal of Hazardous Materials, 2006, 137, 1704-1709.

131. L. H. Yamane, V. T. de Moraes, D. C. R. Espinosa and J. A. S. Tenório, Recycling of WEEE: Characterization of spent printed circuit boards from mobile phones and computers, Waste Management., 2011, 31, 2553-2558.

132. D. A. Skoog, D. M. West, F. J. Holler and S. R. Crouch, Fundamentals of Analytical Chemistry $\left(9^{\text {th }}\right.$ Ed. international $)$, Brooks/Cole - Cengage Learning, Toronto, Canada, 2014.

133. Y. J. Park and D. J. Fray, Recovery of high purity precious metals from printed circuit boards, Journal of Hazardous Materials, 2009, 164, 1152-1158.

134. D. A. Skoog, D. M. West, F. J. Holler and S. R. Crouch, Standard Addition Methods, in Skoog and West's Fundamentals of Analytical Chemistry, $9^{\text {th }}$ Ed. international; Brooks/Cole - Cengage Learning, Toronto, Canada, 2014. Ch. 8, p. 185.

135. F. W. Fifield, Principles and practice of analytical chemistry, Chapman and Hall, London, 5th edn., 2000.

136. J. Bisquert and F. Fabregat-Santeago, Impedance Spectroscopy: A General Introduction and Application to Dye-Sensitized Solar Cells, in Dye Sensitised Solar cells, 2nd ed., ed. K. Kalyanasundaram, EPFL press, 2010, ch. 12, pp. 457-550. 
137 J. Baker, Development and characterisation of graphene ink catalysts for use in dye sensitised solar cells, Ph.D thesis - Swansea University, 2014.

138. X. Fang, T. Ma, G. Guan, M. Akiyama and E. Abe, Performances characteristics of dye-sensitized solar cells based on counter electrodes with Pt films of different thickness, Journal of Photochemistry and Photobiology A: Chemistry, 2004, 164, 179182.

139. X. Fang, T. Ma, G. Guan, M. Akiyama, T. Kida and E. Abe, Effect of the thickness of the Pt film coated on a counter electrode on the performance of a dye-sensitized solar cell, Journal of Electroanalytical Chemistry, 2004, 570, 257-263.

140. A. Hauch and A. Georg, Diffusion in the electrolyte and charge-transfer reaction at the platinum electrode in dye-sensitized solar cells, Electrochimica Acta, 2001, 46, 3457-3466.

141. J. Baker, J. D. McGettrick, D. T. Gethin and T. M. Watson, Impedance characteristics of transparent GNP-Pt ink catalysts for flexible dye sensitized solar cells, Journal of the Electrochemical Society, 2015, 162, H564-H569.

142. M. Liberatore, F. Decker, L. Burtone, V. Zardetto, T. M. Brown, A. Reale and A. Di Carlo, Using EIS for diagnosis of dye-sensitized solar cells performance, Journal of Applied Electrochemistry, 2009, 39, 2291-2295.

143. C. Chatfield, Statistics for Technolgy, 3rd Ed., CRC Press LLC, London, 1983.

144. R. G. Charles, P. Douglas, I. L. Hallin, I. Matthews and G. Liversage, An investigation of trends in precious metal and copper content of RAM modules in WEEE: Implications for long term recycling potential, Waste Management, 2017, 60, 505520.

145. X. Zeng, R. Gong, W. Q. Chen and J. Li, Uncovering the Recycling Potential of "new" WEEE in China, Environmental Science and Technology, 2016, 50, 1347-1358.

146. Morley N. and Eatherley D., Materials Security: Ensuring resource availability for the UK Economy, Network, Resource Efficiency Knowledge Transfer Network, Chester, 2008.

147. L. Schneider, M. Berger, E. Schüler-Hainsch, S. Knöfel, K. Ruhland, J. Mosig, V. Bach and M. Finkbeiner, The economic resource scarcity potential (ESP) for evaluating resource use based on life cycle assessment, The International Journal of Life Cycle Assessment, 2014, 19, 601-610.

148. N. T. Nassar, R. Barr, M. Browning, Z. Diao, E. Friedlander, E. M. Harper, C. Henly, G. Kavlak, S. Kwatra, C. Jun, S. Warren, M. Y. Yang and T. E. Graedel, Methodology of metal criticality determination, Environmental Science and Technology, 2012, 46, 1071-1078.

149. DEFRA and BIS, Resource Security Action Plan: Making the most of valuable materials, DEFRA, London, 2012.

150. R. O'Connell, W. Tankard, C. Alexander, R. Strachan, S. Nambiath, S. Litosh, J. Tourney, J. Wiebe, L. Wong, E. Rannestad, S. Li, N. Scott-Gray, D. Aranda, G. 
Rodwell, A. Ji, H. Cheng, T. Imangaliyeva and B. Salmon, GFMS Gold Survey 2016, Thompson Reuters, London, UK, 2016.

151. R. O'Connell, W. Tankard, C. Alexander, R. Strachan, S. Namblath, K. Norton, S. Litosh, J. Wiebe, W. Yao, E. Rannestad, S. Li, T. Imangaliyeva, N. Scott-Gray, G. Rodwell, A. Ji and H. Cheng, The Silver Institute - World Silver Survey 2016, Thompson Reuters, London, UK, 2016.

152. P. Dias, S. Javimczik, M. Benevit, H. Veit and A. M. Bernardes, Recycling WEEE: Extraction and concentration of silver from waste crystalline silicon photovoltaic modules, Waste Management, 2016, 57, 220-225.

153. R. O'Connell, W. Tankard, C. Alexander, R. Strachan, S. Nambiath, S. Litosh, J. Tourney, J. Wiebe, L. Wong, E. Rannestad, S. Li, D. Aranda, N. Scott-Gray, T. Imangaliyeva, G. Rodwell, A. Ji, H. Cheng and B. Salmon, GFMS Platinum and Palladium Survey, Thompson Reuters, London, UK, 2016.

154. R. O'Connell, B. Alway, B. Sischka, K. Norton, W. Yao, L. Wong, V. Bakourou and B. Salmon, GFMS Copper Survey 2016, Thompson Reuters, London, UK, 2016.

155. R. O'Connell, B. Alway, A. Leyland, R. Strachan, K. Norton, D. Chan, L. Wong, W. Yao, R. Sun, D. Aranda, J. Aragon, B. Salmon and M. Troman-Taylor, GFMS Copper Survey 2015, Thompson Reuters, London, UK, 2015.

156. C. Hagelüken and C. W. Corti, Recycling of gold from electronics: Cost-effective use through 'design for recycling', Gold Bulletin, 2010, 43, 209-220.

157. JORC, Australasian Code for Reporting of Exploration Results, Mineral resources and Ore Reserves - The JORC CODE - 2012 ed., Joint Ore Reserves Committee, AusIMM, 2012.

158. J. T. Cuddington, An analogy between secondary and primary metals production, Resources Policy, 2008, 33, 48-49.

159. K. I. Takahashi, M. Tsuda, J. Nakamura, K. Otabe, M. Tsuruoka, Y. Matsuno and Y. Adachi, Elementary analysis of mobile phones for optimizing end-of-life scenarios, 2009 Ieee International Symposium on Sustainable Systems and Technology, 18-20 ${ }^{\text {th }}$ May 2009, Tempe, AZ, 154-146.

160. J. Powell, A silver (and gold) lining in the Brexit vote, resource-recycling.com, 2017, http://resource-recycling.com/node/7641 (accessed 25/8/17).

161. R. O'Connell, W. Tankard, C. Alexander, R. Strachan, S. Nambiath, S. Litosh, J. Tourney, J. Wiebe, E. Rannestad, S. Li, D. Aranda, T. Imangaliyeva, N. Scott-Gray, A. Ji and B. Salmon, GFMS Gold Survey 2016 Q2 Update \& Outlook, Thompson Reuters, London, UK, 2016.

162. Recycling. Today, Analysis points to slowly rising disposal costs in the US; GIE Media, Inc., 2016, http://www.recyclingtoday.com/article/landfill-waste-recyclingcosts-ibisworld/ (accessed 14/10/2016). 
163. T. Risen, Americas Toxic Electronic Waste Trade; U.S. News \& World Report L.P, 2016, http://www.usnews.com/news/articles/2016-04-22/the-rising-cost-ofrecycling-not-exporting-electronic-waste (accessed 19/10/16).

164. A. Sepúlveda, M. Schluep, F. G. Renaud, M. Streicher, R. Kuehr, C. Hagelüken and A. C. Gerecke, A review of the environmental fate and effects of hazardous substances released from electrical and electronic equipments during recycling: Examples from China and India, Environmental Impact Assessment Review, 2010, 30, 28-41.

165. F. Magalini, F. Wang, J. Huisman, R. Kuehr, K. Balde, V. V. Straalen, M. Hestin, L. Lecerf, U. Sayman and O. Akpulat, Study on Collection Rates of Waste Electrical and Electronic Equipment (WEEE), possible measures to be initiated by the Commission as required by Article 7(4), 7(5), 7(6) and 7(7) of Directive 2012/19/EU on Waste Electrical and Electronic Equipment (WEEE), European Comission, Bonne, Germany, 2016.

166. X. Zeng and J. Li, Measuring the recyclability of e-waste: an innovative method and its implications, Journal of Cleaner Production, 2016, 131, 156-162.

167. J. Li, X. Zeng, M. Chen, O. A. Ogunseitan and A. Stevels, "Control-Alt-Delete": Rebooting Solutions for the E-Waste Problem, Environmental Science and Technology, 2015, 49, 7095-7108.

168. J. Li, X. Zeng and A. Stevels, Ecodesign in consumer electronics: Past, present, and future, Critical Reviews in Environmental Science and Technology, 2015, 45, 840860.

169. A. Stevels, J. Huisman, F. Wang, J. Li, B. Li and H. Duan, Take back and treatment of discarded electronics: a scientific update, Frontiers of Environmental Science and Engineering, 2013, 7, 1-8.

170. E. Williams, Future Perspectives on Electronic Scrap, in E-Waste Management: From Waste to Resource, eds. K. Hieronymi, R. Kahhat and E. Williams, Routledge, Oxon, 2012, p251.

171. J. Yang, B. Lu and C. Xu, WEEE flow and mitigating measures in China, Waste Management, 2008, 28, 1589-1597.

172. F. Cucchiella, I. D'Adamo, S. C. Lenny Koh and P. Rosa, A profitability assessment of European recycling processes treating printed circuit boards from waste electrical and electronic equipments, Renewable and Sustainable Energy Reviews, 2016, 64, 749-760.

173. J. Yu, E. Williams, M. Ju and Y. Yang, Forecasting global generation of obsolete personal computers, Environmental Science \& Technology, 2010, 44, 3232-3237.

174. B. Li, J. Yang, B. Lu and X. Song, Estimation of retired mobile phones generation in China: A comparative study on methodology, Waste Manage., 2015, 35, 247-254.

175. B. Li, J. X. Yang, B. Lü and X. L. Song, Generation estimation of waste electrical and electronic equipment: Methods review and selection strategy, Shengtai Xuebao/ Acta Ecologica Sinica, 2015, 35, 7965-7973. 
176. K. R. Polsson, Chronology of Personal Computers, 2014, http://pctimeline.info (accessed 22/7/17).

177. S. M. Mueller, Memory, in Upgrading and Repairing PCs 20th Ed., ed. G. Wiegand, Pearson Education, Inc., USA, 2011, Ch.6, pp315-366.

178. J. Ögren, T. Ögren, N. Edmundsson and M. Jonsson, Hardware Book (HwB), 2007 http://www.hardwarebook.info/Memory (accessed 27/9/17).

179. S. v. d. Walt, S. C. Colbert and G. Varoquaux, The Numpy Array: A structure for efficient numerical computation, Computing in Science \& Engineering, 2011, 13, 22 30.

170. J. D. Hunter, Matplotlib: A 2D graphics environment, Computing in Science and Engineering, 2007, 9, 99-104.

181. F. Pérez and B. E. Granger, IPython: A system for interactive scientific computing, Computing in Science and Engineering, 2007, 9, 21-29.

182. J. Cross, Precious metals in electronics: The winners and losers, The LBMA Precious Metals Conference 2004, Shanghai, China, 75-76

183. Statista, Global desktop PC shipments from 2010 to 2019 (in millions), Statista, 2015 http://www.statista.com/statistics/269044/worldwide-desktop-pc-shipments-forecast/ (accessed 27/9/17).

184. R. Hintemann and K. Fichter, Energy consumption and quantities of materials in German data centers, Electronics Goes Green 2012+(EGG), 9-12 Sept. 2012, Berlin, Germany., 2012.

185. Giraffe Innovations Ltd. and R. G. Charles, Recovery of Critical Raw Materials Wales, WRAP, Banbury, UK, 2013.

186. WRAP, CRM Recovery, 2016 http://www.criticalrawmaterialrecovery.eu/ (accessed 27/9/17).

187. R. L. Moss, E. Tzimas, H. Kara, P. Willis and J. Kooroshy, The potential risks from metals bottlenecks to the deployment of strategic energy technologies, Energy Policy, 2013, 55, 556-564.

188. The ad-hoc Working Group (a sub group of the Raw Materials Supply Group of the European Commision), Annex V to Report of the ad-hoc Working Group on defining critical raw materials, Eurpean Commission, Department of Enterprise and Industry, 2010.

189. EC, Report on Critical Raw Materials for the EU - Critical Raw Materials Profiles, Eurpean Commision, DG Enterprise and Industry, 2013.

190. House of commons Science and Technology committee, Strategically Important Metals - Fifth Report of Session 2010-12, The Stationery Office Limited, 2011.

191. B. Knott, A Study of the Recycling and Recovery Infrastructure for Materials Critical to the UK, Materials UK and KTN-Materials, 2011. 
192. V. Gente, F. La Marca, F. Lucci, P. Massacci and E. Pani, Cryo-comminution of plastic waste, Waste Management, 2004, 24, 663-672.

193. Z. Ullah, S. Atiq and S. Naseem, Influence of $\mathrm{Pb}$ doping on structural, electrical and magnetic properties of Sr-hexaferrites, Journal of Alloys and Compounds, 2013, 555, 263-267.

194. S. Zahi, M. Hashim and A. R. Daud, Preparation of Ni-Zn-Cu ferrite particles by solgel technique, Materials Letters, 2006, 60, 2803-2806.

195. D. Dupont and K. Binnemans, Rare-earth recycling using a functionalized ionic liquid for the selective dissolution and revalorization of $\mathrm{Y}_{2} \mathrm{O}_{3}: \mathrm{Eu}^{3+}$ from lamp phosphor waste, Green Chemistry, 2015, 17, 856-868.

196. M. J. Lain, Recycling of lithium ion cells and batteries, Journal of Power Sources, 2001, 97-98, 736-738.

197. P. Meshram, B. D. Pandey and T. R. Mankhand, Extraction of lithium from primary and secondary sources by pre-treatment, leaching and separation: A comprehensive review, Hydrometallurgy, 2014, 150, 192-208.

198. W. A. Fairweather, Alloy gold deposits for electronic applications, Gold Bulletin, 1977, 10, 15-20.

199. C.-H. Kim, K.-J. Park, Y.-J. Yoon, M.-H. Hong, J.-O. Hong and K.-H. Hur, Role of yttrium and magnesium in the formation of core-shell structure of $\mathrm{BaTiO}_{3}$ grains in MLCC, Journal of the European Ceramic Society, 2008, 28, 1213-1219.

200. A. Lixandru, I. Poenaru, K. Güth, R. Gauß and O. Gutfleisch, A systematic study of HDDR processing conditions for the recycling of end-of-life Nd-Fe-B magnets, Journal of Alloys and Compounds, 2017, 724, 51-61.

201. M. Szymański, B. Michalski, M. Leonowicz and Z. Miazga, Recycling of Nd-Fe-B magnets from scrap hard disc drives, Key Engineering Materials, 2016, 682, 308-313.

202. X. T. Li, M. Yue, W. Q. Liu, X. L. Li, X. F. Yi, X. L. Huang, D. T. Zhang and J. W. Chen, Large batch recycling of waste Nd-Fe-B magnets to manufacture sintered magnets with improved magnetic properties, Journal of Alloys and Compounds, 2015, 649, 656-660.

203. X. Li, M. Yue, M. Zakotnik, W. Liu, D. Zhang and T. Zuo, Regeneration of waste sintered Nd-Fe-B magnets to fabricate anisotropic bonded magnets, Journal of Rare Earths, 2015, 33, 736-739.

204. C. Li, M. Yue, W. Liu, T. Zuo, X. Yi, J. Chen, Z. Zhou and Y. Wu, Recycling of scrap sintered $\mathrm{Nd}-\mathrm{Fe}-\mathrm{B}$ magnets as anisotropic bonded magnets via hydrogen decrepitation process, Journal of Material Cycles and Waste Management, 2015, 17, 547-552.

205. Y. Yang, A. Walton, R. Sheridan, K. Güth, R. Gauß, O. Gutfleisch, M. Buchert, B.M. Steenari, T. Van Gerven, P. T. Jones and K. Binnemans, REE Recovery from Endof-Life NdFeB Permanent Magnet Scrap: A Critical Review, Journal of Sustainable Metallurgy, 2017, 3, 122-149. 
206. M. Ueberschaar, S. J. Otto and V. S. Rotter, Challenges for critical raw material recovery from WEEE - The case study of gallium, Waste Management, 2017, 60, 534545.

207. Fraunhofer IZM, cycLED - End of life, 2013 http://www.cycled.eu/End\%20of\%20life.html (accessed 27/9/17).

208. H. Huo, Y. Xing, M. Pecht, B. J. Züger, N. Khare and A. Vezzini, Safety Requirements for Transportation of Lithium Batteries, Energies, 2017, 10, 793.

209. L. Gaines, The future of automotive lithium-ion battery recycling: Charting a sustainable course, Sustainable Materials and Technologies, 2014, 1-2, 2-7.

210. L. Gaines and P. Nelson, Lithium-ion batteries: Examining material demand and recycling issues, EPD Congress 2010 - TMS 2010 Annual Meeting and Exhibition, 14-18 ${ }^{\text {th }}$ February 2010, Seattle, WA, USA, 809-816.

211. G. Liversage, Metech Recycling (UK) Ltd., personal communication, 2013.

212. J. Cryan, K. Freegard, L. Morrish and N. Myles, Demonstration of Flat Panel Display Recycling Technologies; WRAP, 2009.

213. T. J. McDonnell and K. S. Williams, The Location and Character of Mercury in LCD Backlights, WRAP, 2010.

214. C. Meskers, Coated magnesium - Designed for Sustainability? - PhD Thesis, Delft Technical University, 2008.

215. C. E. M. Meskers, Y. Xiao, R. Boom, U. Boin and M. A. Reuter, Evaluation of the recycling of coated magnesium using exergy analysis, Minerals Engineering, 2007, 20, 913-925.

216. C. Meskers, A. Kvithyld, M. Reuter and T. Engh, Thermal de-coating of magnesiuma first step towards recycling of coated magnesium, Magnesium Technology, 2006, 33-38.

217. K. Kimura, K. Nishii and M. Kawarada, Recycling magnesium alloy housings for notebook computers; Fujitsu Scientific and Technical Journal, 2002, 38, 102-111.

218. Magnesium Electron UK, Elektron Refining Technologies - Data Sheet 264, Magnesium Elektron, 2012.

219. E. Conway, BOC, personal communication, 2013.

220. Mount crusher Machine Co.. Ltd., Jaw crusher work principle and typical structure, 2013 http://www.crushermachine.org/crusher-machine/jaw-crusher.html (accesssed 23/7/17).

221. L. Rocchetti, A. Amato and F. Beolchini, Recovery of indium from liquid crystal displays, Journal of Cleaner Production, 2016, 116, 299-305. 
222. L. Rocchetti, A. Amato, V. Fonti, S. Ubaldini, I. De Michelis, B. Kopacek, F. Vegliò and F. Beolchini, Cross-current leaching of indium from end-of-life LCD panels, Waste Management, 2015, 42, 180-187.

223. H. Itoh, The Recent Trend Of E-waste Recycling And Rare Metal Recovery In Japan, WIT Transactions on Ecology and the Environment, 2014, 180, 12.

224. N. Trillwood, Delta Products, personal communication, 2013.

225. EC, Speaking points by Environment Commissioner Janez Potočnik on Circular Economy - Press conference on Circular Economy and Green Employment Initiative, Brussels, 2 July, Press Release, European Commission, 2014.

226. M. P. O'Connor, J. B. Zimmerman, P. T. Anastas and D. L. Plata, A strategy for material supply chain sustainability: Enabling a circular economy in the electronics industry through green engineering, ACS Sustainable Chemistry and Engineering, 2016, 4, 5879-5888.

227. Commision of the European Communities, Commission Staff Working Document accompanying the Communication from the Commission to the European Parliament and Council: The Raw Materials Initiative - meeting our critical needs for Growth and Jobs in Europe, COM(2008) 699, 2008.

228. A. Elshkaki and T. E. Graedel, Dynamic analysis of the global metals flows and stocks in electricity generation technologies, Journal of Cleaner Production, 2013, 59, 260273.

229. J. Tao and S. Yu, Review on feasible recycling pathways and technologies of solar photovoltaic modules, Solar Energy Materials and Solar Cells, 2015, 141, 108-124.

230. R. Charles, P.Douglas, T. Watson and D. Penney, From E-Waste to Green Energy: Waste as a Critical Material Source for Photovoltaic Technologies: A Case Study for Industrial Symbiosis, The Asian Conference on Sustainability, Energy and the Environment 2015, Kobe, Japan - Official Conference Proceeding, pp 427-439.

231. A. Reller, T. Bublies, T. Staudinger, I. Oswald, S. Meißner and M. Allen, The Mobile Phone: Powerful Communicator and Potential Metal Dissipator, GAIA - Ecological Perspectives for Science and Society, 2009, 18, 127-135.

232. V. S. Rotter and P. Chancerel, Recycling of critical resources - Upgrade introduction, Electronics Goes Green 2012+, EGG 2012; 9-12th September 2012, Berlin, Germany.

233. J. L. Richter and R. Koppejan, Extended producer responsibility for lamps in Nordic countries: best practices and challenges in closing material loops, Journal of Cleaner Production, 2016, 123, 167-179.

234. C. Hagelüken, Recycling the platinum group metals: A European perspective, Platinum Metals Review, 2012, 56, 29-35.

235. J. Hong, W. Shi, Y. Wang, W. Chen and X. Li, Life cycle assessment of electronic waste treatment, Waste Management, 2015, 38, 357-365. 
236. L. A. Diaz, T. E. Lister, J. A. Parkman and G. G. Clark, Comprehensive process for the recovery of value and critical materials from electronic waste, Journal of Cleaner Production, 2016, 125, 236-244.

237. P.-Y. Chen, J. Qi, M. T. Klug, X. Dang, P. T. Hammond and A. Belcher, Environmentally-responsible fabrication of efficient perovskite solar cells from recycled car batteries, Energy \& Environmental Science, 2014, 7, 3659-3665.

238. R. G. Charles, M. L. Davies and P. Douglas, Third generation photovoltaics-early intervention for circular economy and a sustainable future, Electronics Goes Green 2016+, EGG 2016, 6-9th September 2016, Berlin, Germany..

239. Mordor Intelligence, Global Thermocouple Temperature Sensors Market - By Type (Nickel alloy thermocouples, Platinum or rhodium alloy thermocouples, Tungsten or rhenium alloy thermocouples), Industry, Geography, Trends, Forecast - (2017 2022), Mordor Intelligence, 2017.

240. M. Godley, GC Metals, personal communication, 2014.

241. I. W. Van der Perre, Temperature Measurement in Liquid Metal, Heraeus, 2015 http://heraeus-electro-

nite.com/media/webmedia_local/media/downloads/steel_2/temperaturecontrol/tempe rature_wvdp_2000.pdf (accessed 22/9/17).

242. A. Chen and P. Holt-Hindle, Platinum-based nanostructured materials: synthesis, properties, and applications, Chemical Reviews, 2010, 110, 3767-3804.

243. H. Renner, G. Schlamp, I. Kleinwächter, E. Drost, H. M. Lüschow, P. Tews, P. Panster, M. Diehl, J. Lang, T. Kreuzer, A. Knödler, K. A. Starz, K. Dermann, J. Rothaut, R. Drieselmann, C. Peter and R. Schiele, Platinum Group Metals and Compounds, in Ullmann's Encyclopedia of Industrial Chemistry, Wiley-VCH Verlag $\mathrm{GmbH} \&$ Co. KGaA, 2001

244. A. Hagfeldt, G. Boschloo, L. Sun, L. Kloo and H. Pettersson, Dye-Sensitized Solar Cells, Chemical Reviews, 2010, 110, 6595-6663.

245. Z. He, J. Chen, D. Liu, H. Zhou and Y. Kuang, Electrodeposition of Pt-Ru nanoparticles on carbon nanotubes and their electrocatalytic properties for methanol electrooxidation, Diamond and Related Materials, 2004, 13, 1764-1770.

246. J. Prabhuram, X. Wang, C. L. Hui and I. M. Hsing, Synthesis and characterization of surfactant-stabilized Pt/C nanocatalysts for fuel cell applications, Journal of Physical Chemistry B, 2003, 107, 11057-11064.

247. A. Pozio, M. De Francesco, A. Cemmi, F. Cardellini and L. Giorgi, Comparison of high surface Pt/C catalysts by cyclic voltammetry, Journal of Power Sources, 2002, 105, 13-19.

248. R. E. Ulanowicz, S. J. Goerner, B. Lietaer and R. Gomez, Quantifying sustainability: resilience, efficiency and the return of information theory, Ecological Complexity, 2009, 6, 27-36. 
249. J. A. Baker, C. Worsley, H. K. H. Lee, R. N. Clark, W. C. Tsoi, G. Williams, D. A. Worsley, D. T. Gethin and T. M. Watson, Development of graphene nano-platelet ink for high voltage flexible dye sensitized solar cells with cobalt complex electrolytes; Advanced Engineering Materials, 2017, 19, 3.

250. C.-M. Chen, C.-H. Chen and T.-C. Wei, Chemical deposition of platinum on metallic sheets as counterelectrodes for dye-sensitized solar cells, Electrochimica Acta, 2010, 55, 1687-1695.

251. N. Papageorgiou, Counter-electrode function in nanocrystalline photoelectrochemical cell configurations, Coordination Chemistry Reviews, 2004, 248, 1421-1446.

252. Sigma-Aldrich, Chloroplatinic acid hydrate $\geq 99.9 \%$ trace metals basis, 2016 http://www.sigmaaldrich.com/catalog/product/aldrich/520896?lang=en\&region=GB (accessed 22/7/17).

253. A. Van schaik and M. A. Reuter, Shredding, sorting and recovery of metals from WEEE: linking design to resource efficiency, in Waste Electrical and Electronic Equipment (WEEE) Handbook, Woodhead Publishing, 2012, ch. 8, pp. 163-211.

254. First Solar, 2017 http://www.firstsolar.com/ (accessed 27/9/17).

255. A. Müller, K. Wambach and E. Alsema, Life cycle analysis of solar module recycling process, MRS Proceedings, 2005, 895, 0895-G03-07.

256. M. Marwede and A. Reller, Future recycling flows of tellurium from cadmium telluride photovoltaic waste, Resources, Conservation and Recycling, 2012, 69, 3549.

257. M. L. Parisi, S. Maranghi and R. Basosi, The evolution of the dye sensitized solar cells from Grätzel prototype to up-scaled solar applications: A life cycle assessment approach, Renewable and Sustainable Energy Reviews, 2014, 39, 124-138.

258. N. Espinosa, F. O. Lenzmann, S. Ryley, D. Angmo, M. Hösel, R. R. Søndergaard, D. Huss, S. Dafinger, S. Gritsch, J. M. Kroon, M. Jørgensen and F. C. Krebs, OPV for mobile applications: An evaluation of roll-to-roll processed indium and silver free polymer solar cells through analysis of life cycle, cost and layer quality using inline optical and functional inspection tools, Journal of Materials Chemistry A, 2013, 1, 7037-7049.

259. S. Brittman, G. W. P. Adhyaksa and E. C. Garnett, The expanding world of hybrid perovskites: materials properties and emerging applications, MRS communications, 2015, 5, 7-26.

260. NTUA, Manual on Eco-Design and End-of-Life management of Electronic Products, IPPTEL, 2007.

261. S. Ferrendier, F. Mathieux, G. Rebitzer, M. Simon and D. Froelich, Eco-design Guide - Environmentally Improved Product Design Case Studies of the European Electrical and Electronics Industry, ECOLIFE Thematic Network, Eco-design subgroup, 2002. 
262. J. Peng, L. Lu and H. Yang, Review on life cycle assessment of energy payback and greenhouse gas emission of solar photovoltaic systems, Renewable and Sustainable Energy Reviews, 2013, 19, 255-274.

263. V. Baharwani, N. Meena, A. Dubey, D. Sharma, U. Brighu and J. Mathur, Life cycle inventory and assessment of different solar photovoltaic systems, 2014 Power and Energy Systems Conference: Towards Sustainable Energy, PESTSE 2014, 13-15 March 2014, Bangalore, India.

264. NREL, Best Research Cell Efficiencies, 2016 http://www.nrel.gov/ncpv/images/efficiency_chart.jpg, (accessed 6/8/2016).

265. H. J. Snaith, Perovskites: the Emergence of a new era for low-cost, high-efficiency solar cells; The Journal of Physical Chemistry Letters, 2013, 4, 3623-3630.

266. A. Yella, H. W. Lee, H. N. Tsao, C. Yi, A. K. Chandiran, M. K. Nazeeruddin, E. W. G. Diau, C. Y. Yeh, S. M. Zakeeruddin and M. Grätzel, Porphyrin-sensitized solar cells with cobalt (II/III)-based redox electrolyte exceed 12 percent efficiency, Science, 2011, 334, 629-634.

267. D. Bryant, S. Wheeler, B. C. O'Regan, T. Watson, P. R. F. Barnes, D. Worsley and J. Durrant, Observable hysteresis at low temperature in "hysteresis free" organicinorganic lead halide perovskite solar cells; Journal of Physical Chemistry Letters, 2015, 6, 3190-3194.

268. S. Aharon, B. E. Cohen and L. Etgar, Hybrid lead halide iodide and lead halide bromide in efficient hole conductor free perovskite solar cell, The Journal of Physical Chemistry C, 2014, 118, 17160-17165.

269. L. Etgar, P. Gao, Z. Xue, Q. Peng, A. K. Chandiran, B. Liu, M. K. Nazeeruddin and M. Grätzel, Mesoscopic $\mathrm{CH}_{3} \mathrm{NH}_{3} \mathrm{PbI}_{3} / \mathrm{TiO}_{2}$ heterojunction solar cells, Journal of the American Chemical Society, 2012, 134, 17396-17399.

270. A. S. Subbiah, A. Halder, S. Ghosh, N. Mahuli, G. Hodes and S. K. Sarkar, Inorganic hole conducting layers for perovskite-based solar cells, The Journal of Physical Chemistry Letters, 2014, 5, 1748-1753.

271. P. Gao, M. Gratzel and M. K. Nazeeruddin, Organohalide lead perovskites for photovoltaic applications, Energy \& Environmental Science, 2014, 7, 2448-2463.

272. B. Kippelen and J.-L. Brédas, Organic photovoltaics, Energy \& Environmental Science, 2009, 2, 251-261.

273. R. Itten and M. Stucki, Highly efficient 3rd generation multi-junction solar cells using silicon heterojunction and perovskite tandem: Prospective life cycle environmental impacts, Energies, 2017, 10.

274. G24 Power Limited, GCell, 2016 http://gcell.com/ (accessed 22/9/17).

275. Oxford Photovoltaics, Tandem Cells - Boosting the performance of silicon or CIGS PV modules by 20\%, 2015 https://www.oxfordpv.com/Tandem-perovskite-solar-cells (accessed 27/9/17). 
276. Heliatek. Gmbh, Heliatek, http://www.heliatek.com/en/ (accessed 27/9/17).

277. infinityPV ApS, Infinity PV, 2017 https://www.infinitypv.com/, (accessed 22/9/17).

278. A. Shah, P. Torres, R. Tscharner, N. Wyrsch and H. Keppner, Photovoltaic technology: the case for thin-film solar cells, Science, 1999, 285, 692-698.

279. D. Bryant, I. Mabbett, P. Greenwood, T. Watson, M. Wijdekop and D. Worsley, Ultrafast near-infrared curing of PEDOT:PSS, Organic Electronics: physics, materials, applications, 2014, 15, 1126-1130.

280. J. Troughton, M. J. Carnie, M. L. Davies, C. Charbonneau, E. H. Jewell, D. A. Worsley and T. M. Watson, Photonic flash-annealing of lead halide perovskite solar cells in $1 \mathrm{~ms}$, Journal of Materials Chemistry A, 2016, 4, 3471-3476.

281. J. Sherwood, M. De bruyn, A. Constantinou, L. Moity, C. R. McElroy, T. J. Farmer, T. Duncan, W. Raverty, A. J. Hunt and J. H. Clark, Dihydrolevoglucosenone (Cyrene) as a bio-based alternative for dipolar aprotic solvents, Chemical Communications, 2014, 50, 9650-9652.

282. F. P. Byrne, S. Jin, G. Paggiola, T. H. M. Petchey, J. H. Clark, T. J. Farmer, A. J. Hunt, C. Robert McElroy and J. Sherwood, Tools and techniques for solvent selection: green solvent selection guides, Sustainable Chemical Processes, 2016, 4, 7.

283. A. Binek, M. L. Petrus, N. Huber, H. Bristow, Y. Hu, T. Bein and P. Docampo, Recycling perovskite solar cells to avoid lead waste; ACS Applied Materials \& Interfaces, 2016, 8, 12881-12886.

284. S. Lizin, S. Van Passel, E. De Schepper, W. Maes, L. Lutsen, J. Manca and D. Vanderzande, Life cycle analyses of organic photovoltaics: a review, Energy and Environmental Science, 2013, 6, 3136-3149.

285. M. L. Parisi, A. Sinicropi and R. Basosi, Life cycle assessment of gratzel-type cell production for non conventional photovoltaics from novel organic dyes, International Journal of Heat \& Technology, 2011, 29, 161-169.

286. M. J. De Wild-Scholten, Energy payback time and carbon footprint of commercial photovoltaic systems, Solar Energy Materials and Solar Cells, 2013, 119, 296-305.

287. M. Monteiro Lunardi, A. Wing Yi Ho-Baillie, J. P. Alvarez-Gaitan, S. Moore and R. Corkish, A life cycle assessment of perovskite/silicon tandem solar cells, Progress in Photovoltaics: Research and Applications, 2017, 25, 679-695.

288. C. Hudaya, J. H. Park and J. K. Lee, Effects of process parameters on sheet resistance uniformity of fluorine-doped tin oxide thin films, Nanoscale Research Letters, 2012, $7,17$.

289. Q. Xu, W. Shen, Q. Huang, Y. Yang, R. Tan, K. Zhu, N. Dai and W. Song, Flexible transparent conductive films on PET substrates with an AZO/AgNW/AZO sandwich structure, Journal of Materials Chemistry C, 2014, 2, 3750-3755. 
290. S. U. Park and J. H. Koh, Low temperature rf-sputtered in and al co-doped ZnO thin films deposited on flexible PET substrate, Ceramics International, 2014, 40, 1002110025 .

291. L. J. Brennan, M. T. Byrne, M. Bari and Y. K. Gun'ko, Carbon nanomaterials for dyesensitized solar cell applications: a bright future, Advanced Energy Materials, 2011, 1, 472-485.

292. A. Mishra, M. K. R. Fischer and P. Bäuerle, Metal-free organic dyes for dye-sensitized solar cells: from structure:property relationships to design rules, Angewandte Chemie International Edition, 2009, 48, 2474-2499.

293. G. Nair, M. Shafawi, M. Irwanto, M. I. Yusoff, M. Fitra and N. Mariun, Performance improvement of dye sensitized solar cell by using recycle material for counter electrode, Applied Mechanics and Materials, 2014, 446-447, 823-826.

294. M. Ayaz, J. K. Kasi, A. K. Kasi, Samiullah and M. Ali, Toward eco green energy: fabrication of DSSC from recycled phone screen, International Journal of Resistive Economics, 2016, 4, 17-22.

295. C. C. Chen, F. C. Chang, C. Y. Peng and H. P. Wang, Conducting glasses recovered from thin film transistor liquid crystal display wastes for dye-sensitized solar cell cathodes, Environmental Technology (United Kingdom), 2015, 36, 3008-3012.

296. H. A. Ribeiro, In situ decoration of gold nanoparticles on TiO2/cellulose nanocomposites: An application toward dye-sensitized solar cells on paper substrates, 2013 MRS Spring Meeting \& Exhibit, 1-5 ${ }^{\text {th }}$ April 2013, San Francisco, CA, USA.

297. Y. Zhou, T. M. Khan, J.-C. Liu, C. Fuentes-Hernandez, J. W. Shim, E. Najafabadi, J. P. Youngblood, R. J. Moon and B. Kippelen, Efficient recyclable organic solar cells on cellulose nanocrystal substrates with a conducting polymer top electrode deposited by film-transfer lamination, Organic Electronics, 2014, 15, 661-666.

298. P. J. Holliman, K. J. Al-Salihi, A. Connell, M. L. Davies, E. W. Jones and D. A. Worsley, Ultra-fast dye sensitisation and co-sensitisation for dye sensitized solar cells, RSC Advances, 2014, 4, 2515-2522.

299. P. J. Holliman, M. L. Davies, A. Connell, B. V. Velasco and T. M. Watson, Development of selective, ultra-fast multiple co-sensitization to control dye loading in dye-sensitized solar cells, Chemical Communications, 2010, 46, 7256-7258.

300. R.-T. Chen and C.-F. Liao, Evaluation and optimization to recycle used $\mathrm{TiO}_{2}$ photoelectrode for dye-sensitized solar cells, International Journal of Photoenergy, 2014, 2014, 7.

301. R. G. Charles, M. L. Davies, P. Douglas, S. M. Atiemo, M. Bates, A. Clews, I. Mabbett, B. S. Martincigh, E. T. Mombeshora, J. R. Morgan, V. O. Nyamori and D. A. Worsley, Sustainable Solar Energy Storage for Rural Africa, 10th International Conference on Sustainable Energy and Environmental Protection: Energy Storage Conference Proceedings, June 27-30 ${ }^{\text {th }} 2017$, Bled, Slovenia, p177. 
302. Africa News Agency, Nearly 90 percent of SA households have electricity, says Eskom; 2016 http://www.enca.com/south-africa/stats-show-that-nearly-90-percentof-sa-households-have-electricity-says-eskom (accessed 16/5/17).

303. N. Jamal, Options for the supply of electricity to rural homes in South Africa, Journal of Energy in Southern Africa, 2015, 26, 58-65.

304. SPECIFIC,

Active

Classroom,

http://www.specific.eu.com/assets/downloads/casestudy/Active_Classroom_Web_C

ase_Study.pdf (accessed 27/9/17).

305. blue.sky energy gmbh, Clean Energy Requires Clean Batteries, Aquion - the world's safest and cleanest energy storage for your home, 2014 http://www.blueskyenergy.eu/aquion-the-saltwater-battery/?gclid=CKmor_-O8tMCFe6_7QoduU4CRg (accessed 27/9/17).

306. Swansea University, Zambia Summer Expeditions, 2016 http://www.swansea.ac.uk/engineering/zambia/ (accessed 27/9/17).

307. D. Hillel, Introduction to Soil Physics, Academic Press Inc and Elsevier Inc., 2013.

308. European Commission Joint Research Centre, Photovoltaic Geographical Information System - $\quad$ Interactive Maps, 2017 http://re.jrc.ec.europa.eu/pvgis/apps4/pvest.php?map=africa\&lang=en (accessed 27/9/17).

309. EnergyTrend, EnergyTrend PV - PV Spot Price, TrendForce Corp., 2017 http://pv.energytrend.com/pricequotes.html (accessed 27/9/17).

310. M. Baumann, J. F. Peters, M. Weil and A. Grunwald, $\mathrm{CO}_{2}$ footprint and life-cycle costs of electrochemical energy storage for stationary grid applications; Energy Technology, 2017, 7, 1071-1083.

311. J. F. Peters and M. Weil, Providing a common base for life cycle assessments of LiIon batteries,Journal of Cleaner Production, 2018, 171, 704-713.

312. A. Manhart, T. Amera, G. Kuepouo, D. Mathai, S. Mng'anya and T. Schleicher, The deadly business - Findings from the Lead Recycling Africa Project., Öko-Institut e.V.; 2016.

313. Factor, PV/battery waste management in the context of rural electrification support on pv/battery waste management for a rural electrification program, Clean Energy Solutions Ceter, NREL, 2016.

314. P. Schroeder, Will solar PV create a wave of toxic battery waste in rural Africa?; Institute of Development Studies, 2016 http://www.ids.ac.uk/opinion/will-solar-pvcreate-a-wave-of-toxic-battery-waste-in-rural-africa (accessed 27/9/17).

$\begin{array}{llllll}\text { 315. British Geological } & \text { Survey, Risk }\end{array}$ https://www.bgs.ac.uk/mineralsuk/statistics/riskList.html (accessed 27/9/17).

316. ESI Africa, Recycling of Batteries in South Africa, 2013 https://www.esiafrica.com/news/recycling-of-batteries-in-south-africa/ (accessed 27/9/17). 
317. B. J. D. Knights and F. Saloojee, Lithium Battery Recycling - Keeping the future fully charged, Department of Environmental Affairs, Greenfund, Development Bank of South Africa, 2015.

318. L. Ahmadi, S. B. Young, M. Fowler, R. A. Fraser and M. A. Achachlouei, A cascaded life cycle: reuse of electric vehicle lithium-ion battery packs in energy storage systems, International Journal of Life Cycle Assessment, 2017, 22, 111-124.

319. EC, Communication from the commission - Ecodesign Working Plan 2016-2019, European Commission, Brussels, 2016.

320. L. W. Jelinski, T. E. Graedel, R. A. Laudise, D. W. McCall and C. K. N. Patel, Industrial ecology: concepts and approaches, Proceedings of the National Academy of Sciences, USA 1992, 89, 793-797.

321. A. Garner and G. Keoleian, Industrial Ecology: An Introduction, National Polution Prevention Center for Higher Education, 1995. 